

\section{Cornell aniversitp}

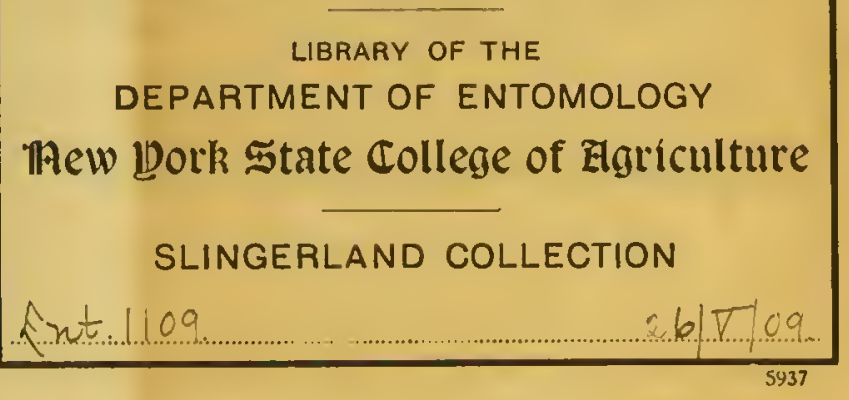

\section{SB $931 . \mathrm{G} 31$}

Cornell University Library

Notes pour servir a l'histoire des insec

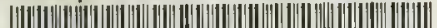


The date shows when this volume was taken. To renew this look copy llie call No. and give to the librarian.

HOME USE RULES.

All Books subject to Recall.

Books not used for instruction or rescarch are returnable within 4 wecks.

Volumes of periodicals and of paunplinlets are held in the library as much as possible. For special purposes they are givell out for a limited time.

Borrowers should not use their library privileges for the benefit of other persons.

Books not needed during recess periods sliould be returnerl to the library, or arrangements made for thicir return during borrower's abscnce, if wanted.

Books needed by more than one persois are held on the reserve list.

Books of special value and gift books, when the giver wishes it, are not allowed to eirculate.

Realers are asked to rcport all cases of books nuaked or muti. laterl.

Do not deface hooks by marks and writing. 

PUI li SERITR A LHISTUHEE

DE:

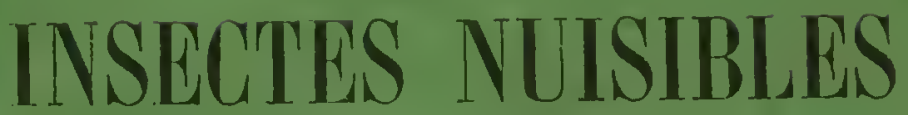

A ISAGRICULTURE

IIIS IL DIPARTEUEYT DE LA MOSELLE,

\section{Par d.-R. GEMIIN,}

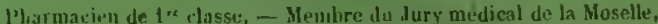
- Stecretaire du Conseil rentral d'hygiene dit departenent, - Nembre de la Societe

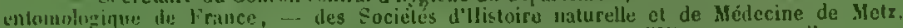
Correspondant des Sucielis linuriennes de Lyon, - d'Histoire naturell d. Luxembou'g, Entornologrique de Steftin, elc., etc.

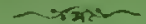

Numéro 1.

INTRODICTION.

necrorothen

Finrail du Journal de la Suciéti d'horticulture de la Hurelie.

acuenes.

\section{IIETZ}

Typographic de ROUSSEAU-PALLEZ, Éditeur',

Imprimetur de lie Socicté, Librairo du l'Acadétuie inupériale,

RUE VES CLERCS, 1$\}$. 


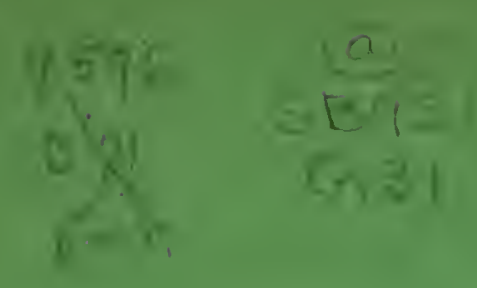




\title{
NOTES
}

\section{POUR SERVIR A L'HISTOIRE}

DES

\section{INSECTES \\ NUISIBLES}

\section{A L'AGRICULTURE}

\author{
DANS LE DÉPARTEMENT DE LA MOSELLE,
}

\section{Par J.-IB. Givand,}

Pharnacien de 1 "classe, - Mlembre du Jury médical do la Moselle,

- Secrélaire du Conseil central d'liygièno du département, - Membre de la Société entomologique do France, - des Sociêtes d'Histoiro naturelle et de Médecine de Metr, Correspondant des Sociétés linnéennes de Lyon, - d'Histoire nalurelle de Luxembourg, - Entonologique de Stellin, ete, etc.

\section{sainc \\ Numéro 1. \\ INTRODUCTION.}

\section{-coroxorom}

Extrail du Journal de la Sociéte d'horticultere de la loselle.

miseren

\section{IIIT T}

Typographie de ROUSSEAU-PALLEZ, Éditeur,

Imprimeur de la Socíté, Librairo de l'Académie imperiale,

RUE DES CIERCS, 14. 


$$
\begin{aligned}
& \frac{@}{\operatorname{seg31}} \stackrel{s / \xi_{i x}}{ } \\
& \text { G } 31
\end{aligned}
$$

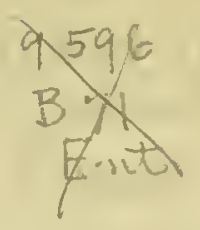

Ent. 1109 


\section{Notes pour servir à l'listoire des insectes nuisibles a) l'horticullure}

DANS LE DÉPARTEMENT DE IAA MOSEILE,

Par M. J.-B. Géhin, pharmacien de première classe à Melz, membre de la Sociélé d'horticulture de la Moselle, ctc.

\section{INTRODUCTION}

Aide-toi, le ciel l'aidern.

Poul beaucoup de gens, l'enlomologiste n'est encore autre chose qu'un original ayant la manie de chasser les insectes, de les pirfuer el de les classer 'dans une boîte avec des étiquettes portant des nous plus ou moins bizarres et baroques.

On ne saurait nicr ce fait qu'il y a encore des entomologues te celte nature, et quue beaucoup ont commencé par là. Mais il faut aussi admettre que leur nombre diminue à mesure que la science fait des progrces, el que, tôt ou tárd, les naturalistes qui persistent dians l'étude de l'entomologic finissent par' abandonner les spéculations théoriques pour éclairer ou approfondir quelque point pratique el appliqué de l'histoire des insectes.

Considéréc à ce dernier point de vue, l'histoire de ces petits animaıx présente un intérêt extrêmement puissant. En effet, si, après avoir étudie la forme et les fonctions de leurs organes, on cherche à pénétrer les mystères qui enveloppent l'accomplissement de leur's diverses métamorphoses, ou à connaître leurs mocurs, leurs instinets, leurs habitudes, on ne tarde pas à tronver l'existence de ces chétives créatures liće intimement à la culture du sol, à l'aulénagement des forêts, à la conservation des denrées alimentaires, elc., et par suite, à la fortune publique des nations. C'est par centaines de millions 
de francs qu'il fant comptcr les pertes annuelles causées à notre agriculture par les insectes nuisibles.

L'histoirc nous a conservé le sonvenir de véritables calamités jubliques causées par des sautcrelles, les vers, des chenilles, des inscctes. Selon toute probabilité, ce sont cncore, de nos jours, les inc̀mes espèces qui attaquent nos céréitles, nos vignes et nos forêts. Cependant il est bon de faire reunarquer que les noms en usage aujourd'hui par les entomologistes, ne correspondent pas toujours aux insectes sigualés par les auteurs ancicns; ainsi le Melolontha des Grecs n'est pas notre hanneton commun, mais lc Lethrus cephaloles de nos catalogues; celte espece cause souvent de grands ravages dans les vignes de la Hongrie. L'Ips, dont parle Homére dans l'Illiade, n'n pu, fautc de description, être rapporté à nos espèces connues; l'Ips de Strabon est la larve de notre É molpus vilis, que Columelle désigne sous le nom de Volucra'.

Lc Deutéronone, le Livre de Job, le Psalmiste, les prophètes Jonas, Amos, Joël, ctc., font déjả mention de vers et d'insectes nombreux altacuant les céréales, les figuicrs et les vignes.

Homėre, Strabon, Aristote et Théoplıraste nous signalent aussi des inscctes nuisibles aux forêts, à la vigne, à l'aristoloche, cle.

Parmi les latins, Columelle, Gicéron, P'aute, Calon et Pline commentent, copient, cxagèrent ou complètcnt ce qu'avaient écrit les Grees sur les vers, les chenilles et les insectes nuisibles. Au moyen àge enfin, les faits de celte nature qui nous ont été conservés por lcs écrivains de celte époque, ne sont, en gćnéral, que la rejuroduction de ceux de l'antiquité, ou, s'ils sont nouveaux, on les trouve empreints des idćcs superstitieuses du temps, ct lous accusent la plıs complètc ignorance entomologique.

les cultivateurs quil avaicnt ainsi à subir les ravages causis

' Voyez Walkenaer, Annales de la Socièlé entomologique de France, année 183:;, page 687 el suirantes. 
frar les insectes, lenrs chenilles ou leurs larves, soit d'une manière permanente, soit à des époques déterminées, durent nécessairement rechercher les moyens propres à ćviter ces dégâts ou à se débarıasser de ces parasites incommodes.

Aisssitrouvons-nous déjà, dans les Géoponiques, des indications pour éviter la formation des petits vers qui rongent la vigne et mangent ses bourgeons. Columelle conseille l'emploi du sang d'ours ou de l'huile cuite pom enduire le tronc des arbres afin d'en éloigner les insectes, et l'usag'e de l'ail ou de la peau de castor pour frotter les instruments arec lesquels on doit tailler les arbres.

Certainement ces moyens sont peu efficaces et surtout peu praticables de nos jours; mais toujour's est-il qu'ils sont beaucoup plus ralionncls que ceux dont le moyen âge nous a conservé la recelte. Les réquisiloires et les exorcismes sont en effet les seuls remédes employćs, et encore, pour comble d'erreur, ces peines ne sont applicables qu'aux vilaines bestes et non aux individus qui auraient pu ou dù chercher à les détruire et qui négligeaient de le faire.

Chorier, dans son hisloire du Dauphiné, raconte qu'au commencement dı seizième siècle, le procureur général lit. un réquisitoire pour enjoindre aux chenilles qui s'útaient considélablement multiplićes " de déguerpir el de vider les lieux. " En 1543, les chenilles ayant causé de garands donmages dans. le midi de la France, la municipalité de Grenoble, sur la proposition de l'un de ses membres, prenait une décision «tendant à prier Monsieur l'official de vouloir exeommunier lesdites bestes (les chenilles), el de procéder eontre elles par voie de censure, pour obvier aux dommages qu'clles faistieut journellement et qu'clles feruient à l'avenir. " "

Pourquoi donc maintenant nous étonner de tant de naïveté et d'une pareille ignorance, quand nos campagnards se laissent encore exploiter par des individus dont les grimaces et les contorsions ont la vertu de guérir lem vache, do chasser

1 Voy. Themis, 1. 1, page 197. 
leurs rats ou de désensorceler leur troupeau, et cela en France, au dix-neuvième sic̀cle ct dans des localités traverscies chaque jour par la locomotive el l'étincelle électrique. Ne faut-il pas chercher l'cxplication de pareilles anonalies daus un vice inhérent à tous les peuples et à toutes les époques, celui de l'insouciance générale pour les calamités quelconques, lorsqu'clles sont passées ou conjurées. Qu'une iuvasion de la Pyrale détruise les espérances de nos vignerons, que les Scolytes fassent mourir des milliers d'arbres de nos forêts, que le Chlorops ou la Cécydomyie compromettent les récoltes de toute une contrée, alors tout le monde s'ćmeut, on aecuse la science d'impuissance ct le gouvernement de mauvais vouloir, et l'on s'empresse d'accol'der confiance à tous les empiriques qui préconisent des remèdes insuffisants ou inapplicables. Puis la crise éloignće, le mal est vite oublić et tout le monde passe des craintes les plus exagér'ées à la sécurité la plus coupable.

Heureusement qu’à travers ces erreurs ct ces défauts, la science entomologique n'a pas ralenti sa marchic, et que, grâce aux travaux des Réaumur, des Lyonnet, des Degéer, des Geoffroy, des Audouin, des Erichson, des Mlacquart (pour ne parler que de ccux qui ne sont plus), on peut dire que maintenant déjà nous connaissons le plus grand nombre des cnnemis que nous avons à combattre. La science en a tracé les mœur's, les ruses, les instincts, les labitudes, et, dans la plupart des cas, la question serait complètement résolue si les frais de destruction n'égalaient ou ne dépassaient les produits à sauver. On accordera volontiers qu'à ce point de vue la solution ne dépend plus seulement de l'entomologiste, et l'on peut même assurer que si les hommes pratiques étaient en général moins étrangers à tout ce que l'on connaît sur les habitudes des insectes, ils auraient déjà doté l'agriculture de procédés ćconomiques propres à éloigner bien des causes de destruction en introduisant des espèces ou des variétés à l'abri des altaques de certains insectes, ou en modifiant les procédes de culture en usage dans certaines localités. 
Cçendant une question se présente à l'esprit, et son importance mérite de fixer notre attention. La science seule suffitelle pour conduircaux résultats qu'on veut atteindre? et l'litat lui-mêmen'a-t-il pas une mission à remplir? enfin, dans quelles limites son intervention doit-elle être réclaméc? Pour arriver à jeter quelque lumière sur cette partie du problème, il est bon d'examiner les tentatives faites 'par le gouvernement pour eneourager ou réeompenser les travaux d'entomologie appliquée, et l'état de la législation coneernant la destruetion des animaux nuisibles, l'échenillage, ete.

La France, qui a tant de titres pour revendiquer l'honneur de marcher à la tête des nations, est malheureusement fort cu retard sous le rapport des iustitutions agricoles et de la plupart de celles qui s'y rattachent. Nous n'avons pas de chaire où l'on enseigne, comme en Allemagne, l'entomologie forestière et appliquée, et c'est à peine si nous avons eu quelques missions scientifiques pour ćtudier chez nous les insectes qui nous rongent, tandis que l'on prodigue relativement les expéditions lointaines pour explorer les contrées nouvelles et en rapporter les produetions '.

Les missions confićes, en 1836, à Audouin pour étudier la pyrale de la vigne, en 1846 et 1847, à M. Guérin-Méneville pour étudier les insectes qui ravageaient les oliviers et les cérćales du midi de la Franec, ont cependant produit d'assez beaux résultats pour engager le gouvernement à user plus sonvent du savoir et du dévouement de plusieurs entomologistes dont les travaux sont depuis longtemps dirigés vers la solution pratique des questions de cette nature.

Fournel, dans son Trailé du voisinage (tome 1, page 505), eite l'arrêt dı Parlement de Paris, du 4 füurier 1752, comme ayant introdnit en France l'obligation légale de dćtruire les insectes nuisibles. Mais eomme les mesures prescrites n'avaient ćté suggérées que par l'abondante quantitć de chenilles qui,

1 Il ne faut pas pour cela que l'on me préte l'idéc de combaltre ici le ròle séricux a si souvent ulile des expédilions scientifiques. - Ie ne fais que comparer. 
en 1731, avaient ravagé une partic du royaume, il s'ensuivit que les dispositions de cel arrêt du Parlement de Paris ne furent que relatives à la destruction des larves de lépidoptères. Cet arrêt, en effet, "prononce une amende de 30 livres, ou autre plus grande s'il y échet, contre toute personne qui n'aura pas échenillé, et ordonne que les bourses et toiles seront tirées des haies, arbres ou buissons, et qu'elles seront sur-le-champ brûlées dans un lieu de la eampagne où il n'y aura aucun danger de communication du feu'."

Je rois à l'obligeance de notre lıonorable collègue, M. Victor Simon, conseiller à la Cour inpériale de Metz, la communication d'un arrêt dı Parlement de Metz, en date dı 24 janvier 1752, qui ordonne \& aux maires et habitants de claque village du ressort, de couper toutes les branches sur lesquelles se trouvent des nids de chenilles, et de les brûler avant le 15 mars. "Cet arrêt antéricur de quinze jours environ a celui du Parlement de Paris cité par Fournel, fait done remonter au Parlement de Netz l'honneur d'avoir pris l'initiative de dispositions légales pour faire écheniller. Il est probable que c'est encore l'abondance des chenilles dans le pays messin, en 1731 , qui aura motivé cet arrêt, el c'est pour cela qu'il n'y est question que de ces animaux.

Aux prescriptions de ḷ'arrêt du 4 février 1732, il faut ajouter les règlements partieulier's que firent les intendants de provinees pour en assurer l'exécution et dont plusieurs furent transformés en arrêts du Parlement, notamment celui du 2 mars 1738, de M. l'intendant de Paris " pour faire ćchenillel" les lıaies, jardins et lıéritages daus l'élenduc des paroisses de la Généralité de Paris". "

L'article premier dı titre XVIII du Code de police pour les villes et faubourgs de Naney, en date du $2 /$ décembre 1768, et lomologué le 4 janvier 1769 par arrêt de la cour

1 Voyez le mol Chonille, p. 159 ) du Dictionnaire ou Traiti de police génerale, par Edm. de la Poie de Fréminville.

${ }^{2}$ Code rural, lome 2, page $47 \%$, éllilion de 1762. 
sonveraine de Lorraine et du Barrois, prescrit l'échenillage en prowonçant " une amende de cini sols par nid pour la première fois, 3 livres pour la seconte et 10 livres pour la troisième, applicable au domaine du Roy."

Dans la loi du 28 septembre 1791 (section IV, art. 20), l'asscmblèc constituante reenmmandait aux corps administratifs " d'eneourager les habitants des campagnes par des récompenses, et suivant les localités, à la destruction des animaux malfaisants qui peuvent ravager les troupeaux, ainsi qu'à la destruction des animaux cl des insectes qui peuvent nuive aux récoltes."

Ici đéjà la question fait un grand pas, puisqu'clle n'est plus applicable aux chenilles seulement, mais à tous les insectes nuisibles; malheureusement il n'y avait ni obligation de le faire, ni sanction pénale pour les négligents, et ces sages instructions, comme beaucoup d'autres, sont restées à l'état de letlres mortes dans les eartons des administrations départementales. ${ }^{\prime}$

Sous le Directoire, l'arrèt du Parlement de 1732 fut mo-

1 M. Clabert, notre obligeaul secrẻlaire, possede, llans sa colleclion de nanuscrits, une piece provenant de la billiollièque du comte Emery, et qui conlient un règlement rendu le 22 fírrier 1658 , sisne par Abrahan Fabert, alors maitreéchevin de Helz, conformémenl à un avis dı grand conseil, et qui enjoint aux gens des campagnes, aux euvirons de la ville et proclıe d'icelle, on placés sons sa juridiction, de déıruire par le feu nu autres mnyens, les nisls et amas de chenilles et autres insccles nuisibles se trouranl sur les arbres fruiliers el autres espices, et dans le plus bref délai. \& Ce faisant ainsi, mentionue le règlement ılont il s'agìi, on fera devoir; car, on y a été ci-devant contrainct par précédcntes ordonnances, ct on en relirera grand proft."

Ce document esı remarquable en ee qu'il y est dejjä fait menliou des insectes nuisibles aulves que les chenilles.

Celte eirconslance me fit soupçonner l'exislence de quelque naturaliste parmi les Treize ou parmi les Conseillers de la cilé, et en effel, parmi ces derniers ligurait, eu 1638, un nomní Geoffroy que dom Sćbastien Florel cite comme ualuraliste dans ses annales manuserites. Je crois liu'on peul, en loule juslice, lui altribuer l'lonuenr d'avoir introduil les mols, el autres insectes, dans l'arrèt de règlemenl donl il s'agil. Ce Geoffroy sernil-il parent ascendant de l'illustre auteur de l'histoire tes insecles des environs de Paris? 
difié el trimsforme en loi le 20 venlise an IV. Comme les arrêtés yni lui ont servi de base, celte loi ne parle que de l'obligation d'éclieniller, du commencement de clacjue année (23 septembre) au fer venlòse (22 févier), et prononce une amende qui ne pourra ctre moindre de trois journées de travail el plus forle de dix (arlicles 1 el 6). L'article 2 ordonne, sous les mêmes peines, de brûler les bourses ct loiles qui seront tirces des arbres. L'article 3 prescrit aux administratem's des départements (les préfets) de faire écheniller, dans le délai précité, les arbres étant sur les domaines nationaux non affermés. L'article \& charge les maires de surveiller l'exécution de la loi, les dèclare responsables des négligences qui seront déconvertes, el\%. Enfin, l'article 8 preserit aux maires des communes de publicr charne annéce celle loi avant le 1 er pluviòse ( 22 janvier).

Il est cerlain que si, à l'époque oú furent volées ees disposilions, on cût consulté des loonmes spécianx, on cùt pu faire beancoup nienx, et que l'on cùt rendu obligatoire la destruction de lous les inseeles qui allinfuent nos cultures ou nos provisions, soit à l'état de láre ou de mỵphe, soit à l'état d'insecte parfait. Nous verrons plus loin de puelles institutions nous aurions hesoin pour rendre complete el effieace une nouvelle loi sur la malière, el surtout pour permettre anx administrations locales de prescrio les inesures nécessaires en temps utile et opportun.

$A$ eclle loi du 26 venkise an $1 i$, il faut ajonler l'article $471, n^{\circ} 8$, du Code pénal, "jui prononce " une amende de $1 \mathrm{f}$. a 5 fi. contre cenx qui auraient négligé d'écheniller dans les campagnes ou jardins oir ee soin est preserit par la loi et les règlements. Belle iisposition nouvelle du Code me eliange en rien l'économic de la loi de l'an IV, mais comme elle modifie la pénaliti, elle clıange anssi la juridiction. Enfin nne circulaire dn ministre des finanees, du 11 avril 1821, disfiense les forêts el les lisières d'ieelles de l'opération de l'échenillage, en se fontant sur re que "Ia loi dn 26 ventose ne. 
pouvait pas plus s'appliquer aux forcts yue les dispositions du Code civil sur les élagages, et que d'ailleurs la ilépense qui en résulterait serait ćnorme, quand mème on se bornerait aux lisières des bois; que si l'échenil!ıge n'avait licu que sul' les lisières, il produirait peu d'effets, parce que les insectes placés sur ies taillis de l'intéricur, gagnant de proclıe en proche, finiraient par atteindre les arlores qui en auraient été purgés. "'

Tel est maintenant l'ensemble de la législation fıançaise sur la destruction des insectes nuisibles et à laquelle on reproche surtout:

10 De n'ètre applicable qu'aux chenilles, tandis qu'elle laisse en dehor's une foule d'autres larves ou d'inscetes également nuisibles;

20 De n'être obligatoirc que jusqu'au 22 févricr de chaque année ct, par conséquent, d'épargner une foule de chenilles mineuses et autres, dont l'apparition n'a lieu que plus tard, et dont les ravages sont beaucoup plus considérables;

$3^{0}$ Enfin de confier le soin de son exécution aux officiers de l'ètat civil, qui sont responsables des négligences; et la constatation des contraventions aux gardes cliampètres et ì la gendarmeric, fonctionnaires dont les connaissances sont peu en rapport avec de semblalles attributions.

Quoiqu'il cu soit, ct quel que soit le degré d'imprortance que l'on attache à ces objections, ce qui doit le plus étonner, ce sont moins les dispositions incomplètes et insuffisantes de la loi, que la négligence avec laquelle l'ćchenillagre est pratiqué dans beaucoup de localitćs, et aussi par certaines administrations sur les domaines dont elles ont la jouissance, lesquelles fondent leur incrtie sur une interprétation trop ètendue de la circulaire ministérielle du 11 avril 1821.

En terminant l'historique qui précède, je dois encol'e mentionner deux tentatives faites pour ćlendre ou conpléter la loi du 26 ventòse an IV.

I Voy. Traile général des caux et forèls, fre parlie, 1. 909, tome II. 
En 1839, M. Martin (du Nord), alors ministre de l'agriculture et du eommerce, a présenté, à la chambre des pairs, un projet de loi qui n'a pas abouti, lepnel d'ailleurs était eneore ineomplet, mais dont les dispositions étaient applicables à tous les insectes nuisibles; ec projet autorisait aussi les préfets à prendre les mesures qu'ils croiraient utiles pour la destruetion des inseetes nuisibles, et cela sans désignation de temps ni d'espèces'.

En 1849, à l'époque où les propositions de toutes sortes abondaient à la tribune de l'Assemblée constituante, un projet de loi, dù à l'initiative a'un représentant dévoué depuis longtemps aux intérêts de l'agriculture, M. Richard (du Cantal), n'a pas eu de sort plus lıeureux que celui de M. Martin (dı Nord). Il renfermait cependant des dispositions plus complètes et dont l'adoption devait certainement faire faire un grand pas à eette importante question ${ }^{2}$.

$\Lambda$ vant d'aborder l'examen des moyens qui peuvent amener la solution de plusieur's problèmes d'entomologie pratique, et d'indiquer ce que, collectivement ou individuellement, nous pouvons faire pour eoopérel effieaeement à eette ouvre d'intêrêt général, il est bơn de fixer son attention sur une question souvent controversée, résolue différemment par des lommes très-eompétents et par plusieurs compagnies savantes.

Une loi sur l'échenillage est-elle utile, nécessaire, possible, et ectte opération elle-mème n'est-elle pas souvent nuisible?

L’affirmative et la négative ont été tour à tour exposées et soutenues par des arguments fort justes. Il n'est pas inutile d'en rappeler les prineipaux, d'eı examiner' la valcur', de faire connaître les objeetions par lesquelles on y a répondu, afin de permettre à ceux qui n'ont pas de parti pris, d'asseoir leur opinion et de formuler leur jugement.

\footnotetext{
- Voyez, pour l'exposé des molifs, le Monileur de 1859, page 26, ct pour le projet de loi, le Moniteur de 1859, page 29.

a Voyez, pour l'exposé des motifs el les articles du projel, la liceuc zoologique de Guiriu Menneville, fivrier 1830.
} 
Aux oljjcetions présentées plus laut contre la loi du 26 ventôsc an IV, les adversaires de tout ćehenillage, légal ou non, invoquent cncorc la eirculaire ministérielle adressée, en 1821, à l'adıninistration des forêts, laquclle dispense les propriétaires de bois de fairc éehcniller. Il y a là, sclon eux, une injustiec flagrante qui eonsistc à foreer l'ćehenillage l'un jardin eontenant quclques arbres, tandis que, dans son voisinage, le proprićtaire d'une forêt scra dispensé de cctte opération, inême sur la lisièrc, ses arbres fusscnt-ils rongés par les elicnilles.

$\Lambda$ ees arguments, les partisans de l'échenillage répondent d'abord qu'il ne faut pas conclure qu'une pratiquc soit absolument mauvaise parec que la loi qui la réglemente est mal conçue, mal appliquéc ou ineomplète. T'ous reconnaissent que des dispositions légales nouvelles sont néeessaires, en ajoutant. toutefois quc si l'on n’obticnt pas tous les résultats désirables de-celles en vigueur, c'est parcc quc très-sonvent elles ne sont pas exéeutées. Quant à la eirculaire que l'on invoque, on peut y répondre en disant que lc nombre des espèees de clienilles polypliages n'est pas si eonsidérable; - que l'essenee même des forêts s'opposera souvent à l'iurasion des ehenilles provenant de eelles-ei dans les vergers voisins; - que, dans beaucoup de cas, le contraire aura plutòt licu, parce qu'clles trouveront plus facilement à se nourrit daus un bois où les arbres sont nombreux et rapprochés, tandis que, dans un jardin, ees arbres sont plus éloignés et ell nombre limité. - que dans les forèts les dégàts ordinaires sont de peu d'intportanec, puisqu'en général on n'y èlève des arbres que pour le bois, taudis que dans les vergers il n'est pas néccssaire qu'une espèec soit abondante pour faire manqucr la rćcolte des fruits ct par conséquent eauser un plus grand dommage; - que dans les cas où une espéee devient assez. multipliée pour ravager unc forèt, les dégâts ne sont que momentanés et nc durent que deux ou trois ans, après lesquels les insectes disparaissent d'cux-ınĉmes souveıt sans eauses connues; - $\rightarrow$ que 
dans les jardins, au contraire, les chenilles peuvent et ont souvent des raisons pour émigrer et aller se jeter sur les proprićtés voisines; le papillon produit dans celle-ei ira faire sa ponte plus loin, et, de proche en proche, toutes les plantations de la contrée pourront être infestées; - qu'entin un travail de quelques heures suffit souvent dans un jardin pour détruire beaucoup de chenilles et, par conséquent, sauver la récolte des fruits, tandis que, pour éeonomiser une faible perte dans la production du bois, il faudrait faire des dépenses considérables pour anéanlir les ehenilles d'une, forêt. Les adversaires des lois sur l'échenillage ajoutent encore que dans les forêts ee sont souvent les seolytes qui eausent le plus de ravages, et que la loi est impuissante pour les atteindre; celle objection est plus spécieuse que réelle, elle prouve seulement que la législation est à refaire, mais elle ne prouve pas que l'on ne puisse atteindre le propriétaire qui ne fait pas abattre et enlever immédiatement les arbres attaqués par ces xyloplages.

Un reproche souvent adressé anx méthodes d'échenillage et par conséquent à leur emploi, est l'impossibilité de pratiøuer eetle opération d'une manière complète et générale. On ne saurait espérer qu'un pareil résultat puisse jamais être atteint; mais de ee que l'on ne peut détruire tous les loups d'une contrée, faut-il en conclure qu'il ne faille plus les chasser? Et de ee qu'une loi ne peut éviter tout le mal qu'elle se propose de détruire, faut-il pour cela en conclure qu'il n'y a rien ì faire et que la répression est inutile? Parni les adversaires les plus déelarés de l'ćehenillage légal, beanconp conviennent d'ailleur's que celte opération bien faite est trèssouvent utile.

Une objection beaueoup plus sérieuse faite aux partisans de l'échenillage est fondée sur ce que l'intérêt bien entendu des propriétiires on des fermiers suffira toujours pour les contraindre à détruire les insectes qui leur porteront préjudice, et qu'en définitive les pays qui passent à juste titre pour être les plus avancés en agriculture, tels que la Belgique, l'An- 
gleter're et les L̈tats-Unis, sont aussi cenx oủ il n'existe aucune disposition légale concermant l'échenillage.

Il faut bien peu connaître la tenacité avec laquelle nos cultivnteurs, en général, persistent dans la pratique de méthodes les plus contraires à leur's intérêts, et leur insouciance pour tous les maux qui ne les frappent que partiellement, pour croire qu'on les amènera facilement à faire ce que préconisent les bès Messieurs de lé ville. Depuis conbien d'années ne leur répète-t-on pas, sous toutes les formes et sur tous les tons, que la manière dont ils aménagent leur's fumiers est des plus mauvaises et qu'ils en perdent eonstamment les produits les plus féeondants! Allez dans nos villages et vous verrez si les eanpagnards se convertissent facilement à ees théories, dont l'évidence est eependant bien loin d'être contestée par bcaucoup d'entre eux.

Si l'Angleterre et les États de l'Union sont plus avaneés que nous en agriculture, e'est que ees pays ont des institutions qui nous manquent, et uon paree qu'ils n'ont pas de législation en matière d'échenillage. C'est surtont parce que leurs habitants, plus éclairés et moins routiniers que les nòtres, mettent constamment à profit les indications fournies par la scicnee.

D'ailleur's, dans les pays eités par nos adversaires, on $\mathrm{y}$ pratique partout les procédés divcrs indiqués pour détruire les insectes nuisibles, tels que l'échenillage, les changements Ic culturc, les fumures spéciales, ete., et celi sans que les labitants y soient contraints par la loi, mais simplement paree qu’ils sont plus éelairés que chez nous et qu'ils savent déjenser utilement leur argent et supporter plus de peines pour récolter davantage.

Enfin le gland argument des adversaires de l'éclienillage at de tonte destruetion des insectes nuisibles, est fondé sur ce uque, dans la nature, toutes les espèces sont maintenues dans un état eonstant d'équilibre, qu'aucune d'elles ne saurait - rester longtemps dominante, et que les invasions les plus 
redoutables finissent par disparaître d'elles-mêmes au bout de deux ou trois ans.

Lc parasitisme semble en effet une loi entomologique assez. généralc, et l'on connait des chenilles et des larves qui servent de berceau et de nomrilure à plusieurs espèces. Hais de ce que les Iclıneumons on les Tachines, pour opérer leur évolution, détruisent une grande quantité dc chenilles, faut-il pour ccla ne s'en rapporter qu'à leurs instincts et à leur multiplication pour détruirc les clienilles ou les larves que nous pouvons atteindre? Si, fort heureusement, les grands dégâts causés dans les forêts par ees animaux ne durent que quelques années, il n'en est pas de même de ces dégâts partiels, incessants que nous causent annuellement l'Altise dans nos colzas, l'llispe dans les luzernes du midi, le Coupe-Bourgeon dans nos vergers, l'Alueite darrs nos blés, le Charançon dans nos greniers, et eela en dépit des parasites qui attaquent la larve de quelques-unes de ces espèees?

Peut-on admettre que lc nombre des ehenilles ou des larves allant en augmentant, celui des parasites suive tonjours la même proportion, et que les générations des unes et des autres soient concordantes? N'est-il pas probable que de grands dégàts soient déjà accomplis avant que le parasite sur lequel on compte pour les arrêter n'ait fait son apparition? Faut-il enfin, pour se débarrasser des chenilles, les laisser manger toutes nos récoltes afin d'avoir le plaisir de les anéantir en les faisant mourir de faim, ou donner aux parasites le temps d'apparaitre et de se multiplier en quantité suffisante. Je erois qu'en général on ne saurait compter avee raison sur le secours des lehneumons ou autres parasites que dans les cas où plusieurs générations peuvent avoir lieu pendant l'évolution normale des elienilles, nymplies ou larves destruetriees.

Quelle que soit la part d'influenee que l'on doit aecorder à ees auxiliaires naturels pour la destruction des espèees nuisibles, on ne saurait admettre, avee $\mathbf{M}$. Amyot, que l'éehenillage détruise les parasites en plus grande proportion que les che- 
nilles, et que, par conséquent, cette opération soit plus nuisible qu'utile.'

Celte ćtrange opinion, émise par un entomologiste séricux et trc̀s-instruit, n'empêchc pas M. Amyot d'ajouter un peu plus loin que l'échcnillage bien fait profite à tout le monde. S'il arrivc souvent que, dans les annćes abondantes en chenilles, on en trouve, sur trois d'entre elles, deux qui sont ichneumonées, on ne saurait admeltre que cette proportion soit applicable ̀̇ la totalité dı nombre, parce que celles qui sont rencontrées par les observateurs, sont précisémént celles qui, étant plus en évidcnce, sont par cela mème plus exposées aux altaques des hyménoptères ou diptères parasites.

Enfin, commc il faut que Je Français rie de tout, même des choscs les plus sćrieuses, je citerai cucorc le syllogisme fait à propos du sujet qui nous occupe: Plus il y a de chenilles, plus il y a d'ichneumons; or, plus il y a d'ichneumons, moins il y a dc chenilles, done plus il y a de chenilles. moins il y a de chenilles! Raisonnement magnifique et qui peut d'ailleurs être employé avcc le même succès par les champions des deux camps.

la mouche de Hesse (Cecidomyia destructor, Say.) ne s'est. elle pas répandue dans toute l'Amérique du Nord, inalgré le Céroplion qui altaque sa larve en si grande quantilé que souvent peu de Cécidomyies éclosent? ${ }^{2}$ Le charançon du blé (Silophilus granarius, Sch.), qui est si préjudiciable à nos céréales emmagasinces, en continue-t-il moins ses ravages malgré le Ptćromalc qui cn attaque la larve et dont la quantité est quelquefois telle que les tas de blć sont couverts par ce diptère. parasite ${ }^{3}$ Le parasitisme ne fait donc que limiter le développenıent de l'espèce nuisible, mais il faut reconnaitrc que,

' (Voyez Annales de lu Suciélé cntomologique de France, anıée 1851, page xxxvi du bulletin, et la Revue et Magasin de zoologie, de Guériu-Henaevilte, annẹc 1850, 0 "de févricr.)

2 Nortlinger, Die klcine Feinde, etc, page \$51.

3 Goureau, Annales de le Société entomologique de France, page xxxix du bullelin, année 1851 . 
dans la plupart des eas il est impuissant pour empêclier le mal de se produire. D'aillcurs M. Amyot et ses partisnns sont loin d'avoir démontré que toutes les espèces que nous avons intérêt à détruire soient attaquées par des parasites.

Aux nombreuses raisons déjà énumérécs dans ee qui prééde pour démontrer la nécessité d'unc loi sur la ácstruction des insectes nuisibles, les partisans et les défenseurs de l'échenillage ajoutent encore: que les espèces nuisibles n'envahissent pas tout à coup les cultures d'une contrée ou tous les arbres d'une forêt; mais que, pendant plusieurs années, clles sc montrent d'abord d'unc manière isolée, qu'ensuite elles s'étendent de proclie en proche, jusqu'à ce que des circonstances almosphériques ou autres, qui nous échappent, viennent favoriser une multiplication prodigicuse contre laquelle nous avons vu le parasitisme impuissant ou presque toujours insuffisant; que, par conséquent, si l'on attaquait vigoureusement le mal dès son apparition, on éviterait souvent ees invasions dévastatrices, ou au moins on en diminuerait considérablement l'étendue en surface ou en inteusité.

Ils ajoutent encore que si l'on foree un industriel, un propriétaire à exécuter certaines mesures de police dans l'intérêt de la santé et de la salubrité publiques, on peut aussi trèsbien foreer un cultivateur ou jardinier à écheniller afin d'empêcher les insectes qui le rolıgent de eauser préjudiee à ses voisins, et peut-être plus tard à compromettre, par son ineuric ou sa mauvaise volonté, la fortune de tout un canton.

Qu'enfin l'échenillage est reconnu utile, quand on veut bien le pratiquer convenablement, opportunément et aussi souvent qu'il est néeessaire. Que, par conséquent, il ne faut pas demander l'abrogation de la loi du 26 ventôse an IV, mais demander qu'on y introduise des dispositions nouvelles, à la hauteur des connaissanees entomologiques de l'époque. Qu'on veille à son exécution générale, qu'on en confie la snrveillance à des hommes spéciaux, et que les préfets soient éclairés dans la rédaction et la publication de leur's arrêtés coneernant la 
natière, par des eonseils analogues à eeux dont ils prennent l'avis en ce qui touche l'hygiène et la salubrité publiques.

Lin résumé, puisque, malgré les lois d'équilibre qui semblent limiter les proportions relatives de ecrtaines espèees, nous avons néanmoins à en subir trop souvent les atteintes, il faut bien que nous fassions nos cflorts pour réduire le dommage à sa moindre valeur. Puisque l'ignorance, la paresse, l'ineurie ou le mauvais vouloir de quelques hommes, peuvent, dans certains cas, contribuer au développement des chenilles, des larves ou autres inseetes nuisibles, il faut bien demander au législateur des armes pour combattre ees ennemis de nos richesses agricoles et vaincre la résistance de eeux qui cn favorisent la multiplication.

Que l'on dise si l'on veut que l'éelıenillage, tel qu'il est organisé, est insuffisant, inutile, absurde même dans certains cas, je lc veux bien. Mais dire que, pratiqué tant bien que mal comme il l'est aujourd'hui dans la plupart des localités, il ne donne aucun résultat et que plutôt il favorise le mal qu'il veut détruire, c'est aller beaucoup trop loin, et il n'est pas rationnel de demander la suppression d'une loi, paree qu'clle est incomplète et mal appliquée. Exiger de l'homme la perfection dans ses ouvres, n'est-ce pas condamner l'humanité au travail de Sisyphe et laisser toutes les questions sans solutions, paree que rarement celles-ci sont à l'abri de la critique?

Pour plaeer lesinstitutions agricoles de notre pays à la hauteur de celles des autres États, il ne suffit donc pas au gouvernement d'encourager les reboisements, la mise cn culture de terrains improductifs ou le dessèehement des marais, il faut encore que les connaissances en histoire naturelle soient plus répandues, ct que l'enseignement de l'cntomologie soit mis à la portice de nos jardiniers et de uos eultivateurs. Il faut surtout qu'il récompense, honorifiłuement ou pécuniairement, les naturalistes ou les agronomes qui feront connaitre l'histoire de nos espèces nuisibles, les moyens ćconomiques et praticables de les détruirc ou de les éviter; il faut aussi qu'il soutienne et 
seconde, par des subventions annuelles, l'initiative déjà prise par plusieurs sociétés savantes ou comices agricoles pour favoriser et populariser les recherches de cette nature; que chaque fois enfin qu'une espèce deviendra compromeltante pour les produits agricoles d'une contrée, des honınes spéciaux, dévoués et instruits, soient envoyés sur les lieux, et nul doute que du contact des spéculations théoriques avec le bon sens et l'esprit d'observation de certains cultivateurs, il ne sorte bientôt des procédés rationuels pour éviler ou conjurer les dommages qui nous menacent trop souvent.

On sait depuis longtemps qu'une végétalion vigoureuse, une culture bien faite, certains procédés de culture, de clıaulage et de fumure, peuvent préserver, souvent dans des limiles fort remarquables, les plantes ou les arbres des atteintes on de l'invasion des insectes; et, à ce stujel, je dois ajouter que l'on a beaucoup disculé sur la question de savoir si les végétaux ne sont attaqués, par les insectes, que parce qu'ils sont en état de souffrance, ou si ce sont les animaux qui, s'attaquant d'abord à un arbre ou à une plante saine, l'appaurrissent, en font languir la végétation et permettent ainsi le développeınent anormal du nombre des individus ou l'invasion ultérieure de nouveaux parasites. Nous aurons sans doute à revenir sur ce sujel important et qui intéresse surtout le reboisement des terrains et l'aménagement des forêts.

Ce n'est pas seulement au point de vue des progrès du jardinage que l'introduction l'espèces ou de variétés précoces ou tardives peut avoir de l'importance, mais encore dans la diminution, l'éloignement ou la destruction des insectes nuisibles. Dans la culture d'une espèce hâtive, on donne souvent à la plante le temps nécessaire pour acquérir assez de force et de vigueur pour r'ésister aux atteintes de quelques insectes, ou rendre impossibles les attaques de certains parasites; les espèces en retard, au contraire, ne commençant leur végétation qu'après la ponte ou après l'ćclosion des œufs, peuvent ainsi empêcher le développernent de plusieur's espèces ou d'un 
trop grand nombre d'individus en plaçant eeux-ei dans des conditions anormales.

Une eirconstanee qui bien souvent amène des méeomptes et eausc bien des tentatives inutiles, e'est que ee ne sont pas partout les mêmes espèees qui attaquent les arbres de nos vergers, de nos forêts, ou les plantes de nos jardins et nos eéréales. Ainsi, les oliviers sont ravagés par la Tinea aleella dans le département du Gard, tandis que dans le Var et dans l'Ilérault eelte espèee est à peine connue et que la récolte des olives y est souvent eompromise par le Dacus olece. L'IIyponomeula padella est souvent si abondante dans le midi de la Franee, qu'en général, sur trois récoltes des pommiers, elle en détruit deux; en Normandie, en Bretagne, dans le nord et en Belgique, ces mêmes arbres sont ravagés par les pucerons (Aphis laniger), et en $185 \mathrm{I}$, dans le département de la Moselle, la plupart des pommiers étaient attaqués par la Geomelra pomonaria. Il est inutile de multiplier la eitation de faits analogues pour montrer la néeessité de bien préeiser l'espẻee à laquelle on vent appliquer tel ou tel proeédé de destruetion, paree que les moyens reeonnus effieaces dans eertains lieux pourront ou devront néeessairement éehouer ailleurs si l'on n'a pas affaire à la mème espèee, ou si, étant la même, elle se trouve dans une autre période de son développement. Il est done évident que, dans la plupart des eas, les mesures à prendre sont essentiellement loeales, et que les autorités ne saturaient en général les preserire eonvenablement sans prendre l'avis des hommes eompétents.

Enfin, en terminant ees eonsidérations, je dois eneore signaler une erreur assez généralement répandue, laquelle consiste à faire eroire que les hivers rigourcux font périr beaueoup d'inseetes.

La nature prévoyante a donné à ehaque individu l'instinet nér,essaire à la eonservation de son espèee, et eeux qui auraient trop à soulfrir d'un abaissement eonsidérable de température, savent bien ehereher d'avance un abri eontre eette 
éventualité et placer leurs pontes dans les conditions les plus favorables. On sait d'ailleur's que les oufs, les larres, les nymphes ou les insectes parfaits, peuvent supporter, saus mourir pour celle cause, un froid progressif de 25 ou $30^{\circ}$ au-dessous de zéro; on sait aussi que les hivers sees et rigonreux sont an contraire, en général, suivis d'étés abondants en iuscetes.

Mais ce qui ne peut nuire à ces aninaux ou à leurs larves dans les conditions oì la nature les condanme à passer l'hiver, n'agit plus de même quand quelques beaux jours de printemps les ont fait sortir de lemr abri, ou que leurs larves ont déjà recommencé à parcourir les diverses phases de leurs métamorphoses. Alors les gelées tardives, les bourrasques, les pluies froides ou continuclles en font périr de grandes quantités, et si ces intempéries nuisent quelquefois à nos cultures, elles nous préservent parfois en même temps de la multiplication dangerense d'un bon nombre d'insectes.

De tout ce qui précède, on est en droit de conclure que ni le parasitisme, ni les intempéries des saisons, ni en général aucun des moyens qque la nature emploie pour maintenir l'équilibre dans la proportionnalité des ètres de la création, ne sont suffisants pour nous mettre à l'abri des ravages causćs par plusieurs d'entre eux, et que, par conséquent, il faut surtout ici faire l'application du précepte que j'ai pris pour devise: Aide-toi, leciel t'aidera.

Malgré son insuffisance, trop souvent réelle, l'échenillage n'en rend pas moins de grands services dans une foule de circonstances, et il est urgent qu'il soit pratiqué par des hommes moins ignorants en entomologic.

En attendant que l'Etat ait pris les mesures utiles pour le rendre plus général, phus complet et surtout plus applicable aux circonstances variées que présente la vic des insectes nuisibles, il faut que, dans la limite de ses attributions, notre Société encourage et récompense les tentatives faites pour atteindre ce but. Que chacun de ses membres mette à profit les circoustarces spéciales dans lesquelles il se trouve placé 
pour contribuer par ses observations et ses recherches à faire l'histoire entomologique de notre contrée ct de nos cultures.

Confiant dans l'aceucil bienveillant que j'ai recu des membres de la Société d'horticulture de la Moselle, j'ose espérer qu'ils voudront bien ouvrir les colonnes de leur publication trimestrielle aux observations inédites, inconnues en Franec ou peu répandues dans les ouvrages d'agriculture, quej'aurai l'honneur de leur' communiquer dans leur's rénnions.

Privé, par les devoirs que m'impose ma profession, des loisirs nécessaires pour suivre au dehors toutes les phases de la vie de ces petits êtres, je fais appel à la complaisance de tous les membres de la Société et plus particulièrement à eclle des membres collaborateurs dont les travaux journaliers sont plus propices à ecs sortes d'observations.

$J_{c}$ mets a leur disposition tous mes instants disponibles et mes faibles connaissances en histoire naturelle, pour lcur fournir les renseignements en mon pouvoir sur les insectes dont ils auront à souffrir les atteintes. Que chacun apporte, à celte œuvre d'intérêt commun, sa part d'observation et de dévoucunent, ct nous ne tarderons pas à voir disparaitre cette étrange anomalie des difficultés industrielles eonsidérables continucllement vaincues, tandis que nous restons impuissants contre les ravages d'un petit insecte ou d'un animaleule microseopique. Singulier contraste, destiné sans doutc à nous rappeler constamment les ressources que nous offre la science d'une part, et de l'autre notre faiblesse el notre impuissance! 
194.

$x^{2}+x^{-2}$

1

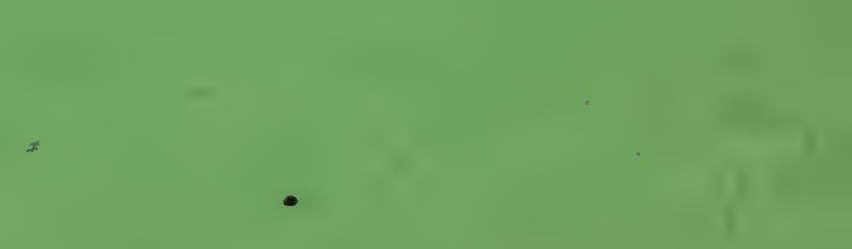




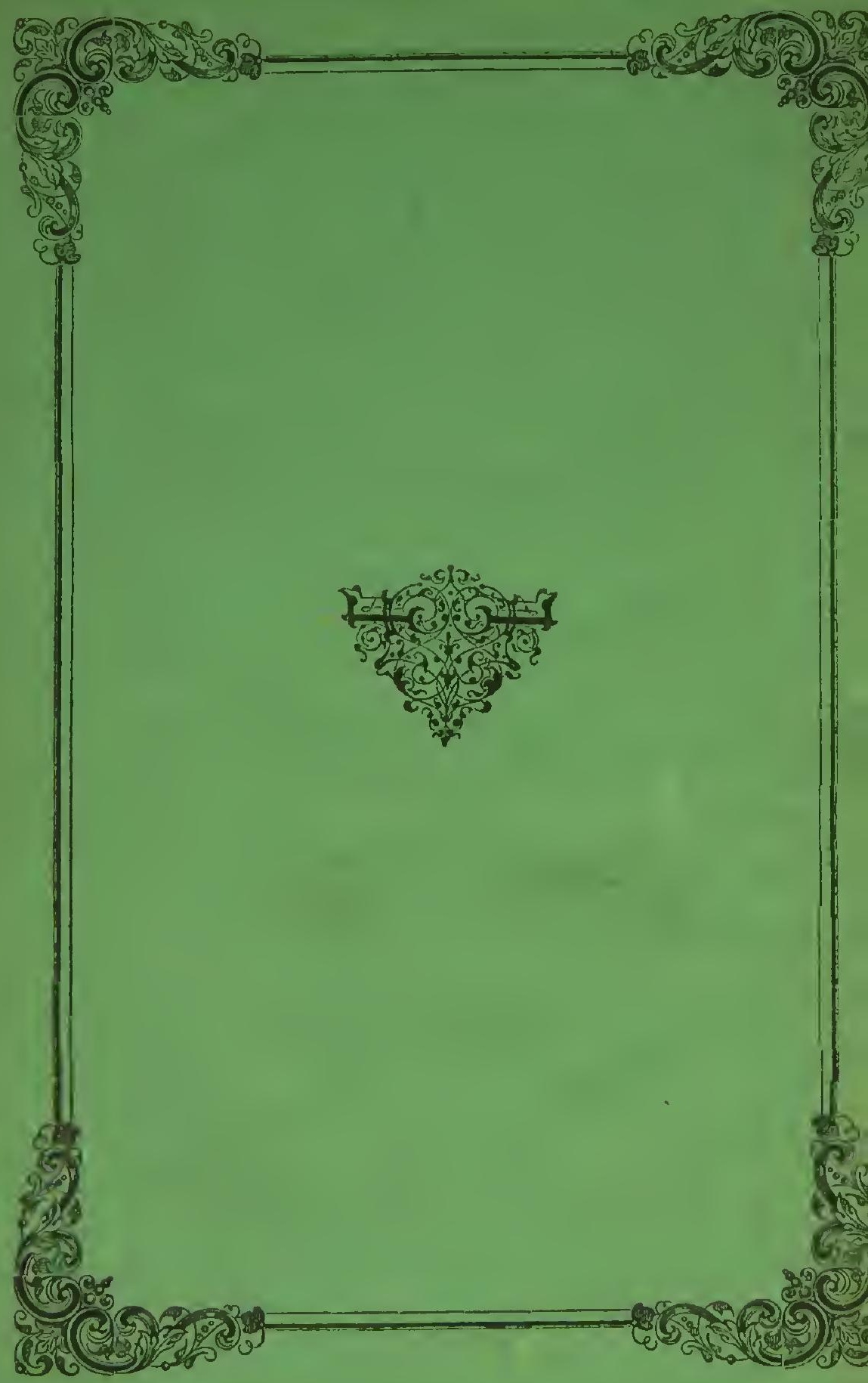



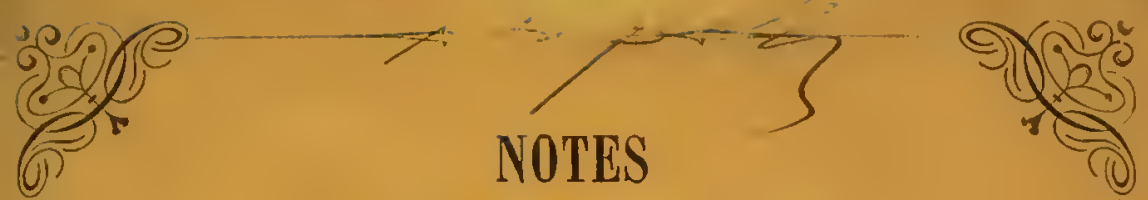

POUR SERVIR A L'HISTOIRE

DES

\section{INSECTES NUISIBIES}

\section{A L'AGRICULTURE}

DANS LE DÉPARTEMENT DE LA MOSELLE,

\section{PAR \\ J.-B. GÉHIN,}

Pharmacien de le classe, - Membre du Jury médical de la Moselle, - Secrétaire du Conseil central d'lyygiène du département, - Membre de la Société entomologique de France, - des Sociétés d'llistoire naturelle et de Médecine de Melz, - Correspondant des Sociétés linnéennes de Lyon, -

1) Histoire uaturelle de Luxembourg, - Entomologique de Stettin, etc., ete.

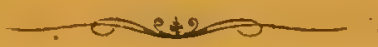

$\mathrm{N}^{0} 2$.

Insectes qui attaquent les blés.

Mémolro lo a la Soolété d'bortloulture de la Moselle, lo 3 aodt 1856.

METZ.

IMPRIYERIE F. BLANG, RUE DU PALAIS.

1857.
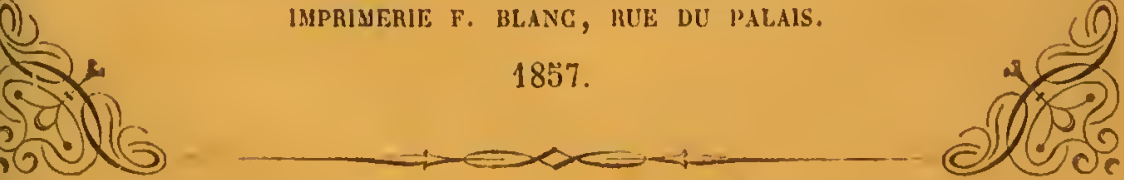



\title{
NOTES
}

\author{
POUR SERVIR A L'HISTOIRE
}

DRS

\section{INSECTES NUISIBLES}

\section{A L'AGRICULTURE}

\author{
DANS LE DÉPARTEMENT DE LA MOSELLE,
}

\section{PAA \\ J.-B. GÉHIN,}

Pharmacien de le classe, - Membre du Jury médical de la MIoselle, - Secrétaire

du Conseil central d'hygiène du département, - Membre de la Société

entomologique de France, - des Sociétés d'Histoire naturelle et de Mrédecine de Metz, - Correspondant des Sociétés linnéennes de Lyon, -

d'Histoire naturelle de Luxembourg, - Entomologique de Stettin, etc., etc.

\section{$N^{0} 2$.}

Insectes qui attaquent les blés.

Mémoire la a la Soolété d'hortloultare de la Moselle, le 3 août 1856.

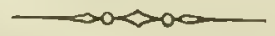

METZ.

IMPRIMERIE F. BLANG, RUE DU PALAIS.

1857. 


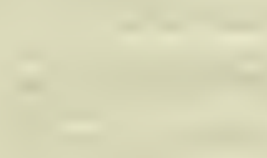




\title{
NOTES
}

POUR SERVIR A LIISTOIRE

DES

\section{INSECTES NUISIBLES}

\section{A L'AGRICULTURE}

\author{
DANS LE DÉPARTEMENT DE LA MOSELLE.
}

INSECTES NUISIBLES AUX BLÉS.

Depuis quelques années, l'insuffisance des récoltes de blé a vivement préoccupé le gouvernement, les administrations locales et les savants qui s'occupent plus spécialement d'agriculture ou des sciences qui s'y rattachent.

Dans un grand nombre de départements, on a pu remarquer des blés forts, bien venus, vigoureux, présentant toutes les apparences d'une récolte riche et abondante, et qui, cependant, laissaient, après la moisson, un déficit plus ou moins sensible darıs le rendement; quelquefois même le battage ne produisait que quelques grains, pelits, maigres ou mal tournés, cratis, enfin, pour me servir de l'expression consacrée.

On comprend aisément que cette richcsse apparente et une réalité aussi fàcheuse, aient dũ causer bien des mécomptes, et qu'elles aient surtout porté le peuple à ajouter foi à tous les bruits absurdes répandus dans les masses sur le commerce des grains, les accaparements, etc., etc.

Cherclıer les causes de ces vicissitudes agricoles, les faire connaitre 
et les discuter, propager les moyens do les éviter, proposer ceux qui paraissent devoir Ics conjurer ou lcs amoindrir, telles ont été les justes préoccupations de toutcs les personnes qui sc livecnt aux études des scicnces naturelles appliquées à l'agriculture. Aux influences douteuscs attribuées aux coups de soleil, à la lune rousse. aux forĉts ou aux cultures voisines, etc., il en est d'autres qui, bien que mal définies ou imparfaitement connucs, peuvent çtre attribuées aux intempéries fréquentes qui frappent notre climal, à l'époquc de l'épiage, de la floraison. etc. Malheureusement ccs diverses causcs. dont on nc saurait nier l'influence dans bicn des cas, sont impossibles à prévoir et trop au-dessus de notre sphère daction pour que nous puissions les éviter. Cctte impossibilité. à laquelle nous ne pouvons nous soustraire, sert d'explication aux ignorants et aux crédules. et couvre la coupable inaction du plus grand nombre.

A ces fầcheuses éventualités, vienncnt encore s'ajouter lcs maladies qui attaquent nos cultures les plus précieuses : tclles que ces végétations cryptogamiqucs, dont l'envahissant mycelium couvrc de rouille le chaume de nos blés, du charbon ses épis, ou ses grains de la carie.

Quelque désastreuses que soient souvent ces invasions de champignons microscopiques, clles sont loin d'attcindre, par leur durée et leur persistance, les dégâts de toutc naturc que les insectcs produisent dans nos cultures. Chaque plante en nourrit qui lui sont propres, el souvent chaque partie de la plante en alimente une espece particulièrc. La petitesse du plus grand nombre, leur prodigieuse fécondité, la difficulté de les atteindre, le cosmopolitisme de plusieurs espèces, sont autant d'éléments qui rendent plus redoutables ces maudites petiles bêtes. à la destruction desquelles nous avons vu concourir, souvent sans résultats, les efforts de l'lomme, les intempéries et le parasitisıne.

C'est à faire l'histoire locale d'un de ces hôtes malfaisants que cette Notice cst consacrée. La plante à laquelle il s'attaque joue un trop grand rôle dans l'économie des sociétés modernes, pour que les faits que j’ai à signalcr n'attirent pas l'attention sérieuse de l'administration. des cultivateurs et de toutes les personnes qui se préoccupent du bien-être public et de l'alimentation générale.

Mais avant d'aborder l'histoire de l'insecte qui nous cause un déficit notable dans nos récoltes de blé. je crois qu'il n'est pas sans 
intérẻt de présenter la liste de toutes les espèces qui, jusqu'iei, ont été signalées comne nuisibles aux blés, afin de les faire connaitre a tous les intéressés, ct de provoquer de nouvelles recherches sur un sujet négligé trop longtcmps dans le département de la Moselle.

Inseetes nuisibles aux bles sur pied. avec leur synongmic et queldues indieations sur leurs mones.

\section{COLÉOPTÈRES.}

10 Calatous latous, DeJEan.

Carabus latus, Linké. - Carabus favipes, Pavkul. - Carabus cisteloides, Illiger. - Harpalus latus, Gyldenhal. - Calathe.

D'après quelques naturalistes allemands, la larve de ce carabique vivrait au pied des tiges de blé. Les dégâts causés par cet insecte sont peu considérables.

\section{Zabrus gibbus, Dejean.}

Ilarpalus gibbus, Gyul. - Carabus tenebrosus, FAB. - Carabus madidus, Ouıv. - Zabre bossu. - Getreïdelaufkofer des Allemands.

Cette espèce est assez abondante dans certaines années et plus particulièrement dans les terrains secs; sa larve est, d'après les allemands, herbivore, nocturne et mange les jeunes épis selon les uns, les jeunes pousses de blé selon les autrês. Enfin. selon quelques entomologistes, pendant le jour elle s"enfoncerait dans la terre près des racines, dont elle vivrait exclusivement. Des mœurs si differentes, selon les circonstances, sont peu probablcs, et il est permis de supposer que l'on a confondu entr'elles plusieurs espèces de la grande tribu des feroniens.

\section{Agriotes segetis, Des.}

Elater segetis, Gylu. - Elater lineatus, Lins. - Elater striatus, Fabr. - Taupin des Moissons. - Maréchal. - Saatschnelkofer en allemand.

Cet insecte, malheureusement assez commur partout, a une 
larve jaune, vivant aux dépens des racines de plusieurs plantes et plus particulièrement de l'avoine et du blé; elle cause souvent des dégâts très-considérables dans les champs où l'on cultive ces céréales, en faisant périr tous les plants qu'elle attaque*.

40 Ptinus crenatus, FABr.

Cet insecle n'a pas encore été observé dans notre département: sa larve vil aux dépens des jeunes tiges de blé, mais elle $n$ 'y cause pas grands dommages.

\section{$5^{\circ}$ Melolontha vulgaris, FABR.}

Searabcus vulgaris, Otiv. - Searabcus Melolontha, Linn. - Melolontha majalis, MoLL. - IIanneton, - IIarlo, - Meuri, Man, - Ver blane, - Meikofer, - Ingerling des Allemands.

La larve de cc coléoptère, trop bien connue de tous les cultivatcurs, vit aux dépens des racines de beaucoup de plantes: ellc a déjà été l'objet d'un grand nombre d'observations, et les moyens les plus divers ont été proposés pour s'en débarrasser: malheurcusement, jusquu'ici, la question est encore à résoudre pratiquement.

\section{$6^{\circ}$ Amphimallon ruficornis, Latreille.}

Melolontha rufieornis, FABn. - Melolontha marginata, IIERB's. Seurabous paganus, OLıv. - Searabcus castaneus, Henbst. Rhyzotrogus paganus, Dejean.

La larve de ce petit melolonthide vit aussi dans lit terre et aux dépens des racincs des céréales, surtout dans les terrains

* M. Dagonnet (Annales de la Sociéd d'agriculture de la Mame 1841) indique eneore les larves des Agriotes gilvellus, Fan., et Gallieus, Dés., comme attaquant les jieds du froment.

A propos des mcours des larves des Elaterides, M. Ed Perris (Annales

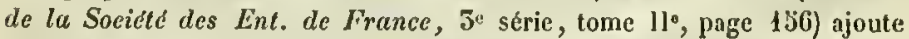
eependant: "Je ne renonee pourtant pas à penser que les larves d'Elaterides, qui semblent exelusivemeut herbivores, sont earnivores dans l'oeeasion; peut-être même leur arrive-t-il quelquefois de n'attaquer les éréales que pour dévorer les larves de Chlorops ou autres qui s'y logent.

Ces citations prouvent eombien il y a de faits nouveaux el intéressants à observer dans les labitudes de ees inseetes. 
secs et sablonneux, où elle est quelquefois assez abondante pour y causer des pertes considérables.

\section{$7^{\circ}$ Anisoplia fruticola, FABR.}

Mclolontha campestris, FanR. - Melolontha segctum, Herbst. Getreidelaubkofer des Allemands.

Ce petit hanneton, très-rare en France, vit aux dépens des racines du blé dans les parties méridionales de l'Europe.

\section{Anisoplia agricola, Mulst.}

Mclolontha agricola, FAsR. - Scarabcus villosus, FABR. - Melolontha fruticola, WALEEN. - Melolontha graminicola, LATR. Feldlaubkofer des Allemands.

Souvent très-abondants dans nos moissons, on voit ces insecles groupés en paquets de quatre. six, huit ou douze individus, le long du chaume des blés, dont ils mangent les jeunes feuilles; la larve vit dans !a terre aux dépens des racines de plusieurs céréales. Dans notre département celte espèce est assez rare. elle est plus méridionale.

\section{Phyllopertha horticola, MuLs'.}

Scarabœus horticolus, Lis. - Melolontha horticola, FAB. - Anisoplia horticola, Latr. - Scarabœus viridicollis, DEgéER. Scarabous ustulatipennis, Vula. - Anomala horticola, Bunm. Gardenlaubkofer en allemand.

Cette espèce se trouve dans nos moissons au moment de la floraison du blé; sa larve vit aussi aux dépens des racines de plusieurs autres plantes.

$10^{\circ}$ Cerandria cornuta, Dejean, catal.

Trogosita cornuta, Fadr. - Phaleria cornuta, LATREHLE. - Uloma cornuta, Guśris.

Dans l'article. sur les insectes nuisibles, de l'Encyclopédie moderne, M. Guérin signale cette espèce comme nuisible aux blés, inais sans entrer dans aucun détail sur ses habitudes. 
Cet insecte est d'ailleur's extrèmement rare dans notre département.

11. Calamobius gracilis, GuÉRIN.

Sapcrda marginclla, FABR. - Agapanthia marginclla, SEnville. - Saperda gracilis, Creutzer. - Cerambix gracilis, Nondungen:

- En français Aiguillon, - En allemand Getreidebckehen.

La larve de ee petit longicorne. essentiellement méridional jusqu'à présent, vit dans lintérieur du chaume du blé, qu'elle pareourt dans toute sa longueur, depuis l'épi jusqu'au collet de la raeine oủ elle passe l'hiver. C'est, d'après MI. Guérin Menneville. a eet inseete que l'on doit rappörter les pertes considérables occasionnées, en 1846. dans les blés des environs de Barbezicux, département de la Charente.

120 Lema cyanella, Fabricius.

Crioceris cyanella, FaвR. - Auchenia cyanella, Marst. - Chrysomcla eyanella, Lis. - Cryptocephalus eyancllus, Lisn. Criocere tout ulcu, Geofrnoy. - Getreide houhchon en allemand.

Cette petite ehrysoméline est assez conmune dans nos moissons, aux mois de juin et de juillet; M. Nordlinger la signale comme nuisible aux blés, mais sans faire connaître ses mœeurs. Labondance avee laquelle on reneontre quelquefois eet insecte mérite de fixer l'attention des entomologistes et des agrieulteurs.

\section{$13^{\circ}$ Lema melanopa, FABR.}

Crioceris melanopa, Fabr. - Crioceris hordei, Founcr. - Chrysomela melanopa, Lin. - Cryptocephalus melanopus, Linś́. - Auelenia melanopa, Mansn. - Lema cyanipennis, Dufto.-Criocèrc bleu di eorselet rouge, Founcnoy.

Espèce peu commune dans notre département, on la rencontre dans les graminćes. M. Nordlinger la signale comme nuisible aux blés, et Réaumur dit que sa larve vit aux dépens de l’orge et de l'avoine dont elle mange les jeunes feuilles. 


\section{YMRNOP'L̈LES.}

$14^{\circ}$ Cephus pygmcus, Fabr.

Banchus spinipes, PAnzer. - Sirex pygmaus, CoQ. - Truchclus pygmous, Jurine. - Getrcide halwespe des Allemands.

Cette petite tenthrèdide, très-répandue dans plusieurs parties de la Francc, est heureusement encore inconnue dans notre département. Sa larve, ou fausse chenille, vit dans l'intérieur du chaumc, près du collet dc la racine; en rongeant les parties internes de la tiggc elle cn affaiblit l'épaisseur et la fait casser au moindre vent. Il serait bon, toutefois, de surveiller les blés versés et de s'assurer si cc phénomène assez fréquent n'aurait pas pour principale cause la présence de cet hyménoptère.

\section{IIÉMIPTL̀丶RES.}

$15^{\circ}$ Miris tritici, GuéniN.

Espèce désignée par M. Guérin *, comme vivant aux dépens des blés, mais cct auteur ne donne aucune description de l'insecte, ni aucune indication sur ses habitudes.

$16^{\circ}$ Miris dolabratus, FABR.

Miris latcralis, Latn. - Cimex dolabratus, Linn.

Cette espèce de punaise, de forme très-alongée, vit sur les épis et les chaumes du blé, dont elle suce la sève: j'en ai trouvé un assez grand nombre dans lcs champs lumides de Borny et de Vallicies.

$17^{\circ}$ Aphis granaria, Cuntis.

Co puceron, dont la femclle était assez commune dans les champs de blé de toutes les parties du département quc j'ai

* Encyclop. mod., page 275, tome xvm. 
visitées, en 1856, au mois de juillet, inplante son suçoir sur le rachis des épis, ou sur le pédoneule des épillets et en absorbe la sève. Si les pueerons étaient très-abondants ils ne laisseraient pas de causer un assez grand dommage aux blés ; inais il parait que cela n'arrive que très-rarement, et seulement dans des eirconscriptions fort restreintes.

$18^{\circ}$ Aphis avenæ, FABR.

Puceron des avoines, - Ilafer blatt lauts des Allemands.

Ce pueeron, aussi remarquable par sa forme globuleuse que par sa eouleur insolite, se reneontre sur un grand nombre de graminées, aux mois de juin et de juillet. Comme le préeédent. on le trouve dans les épis, mais je ne l'ai jamais trouvé qu'en petit nombre sur chaeun d'eux; il paraît eependant plus répandu que le préeédent.

\section{THYSANOP'TERES.}

\section{Thrips cerealium, Burmeister.}

Thrips physipus, Kınву. - Limnothrips cercalium, HaLIday. Thrips obscura, MuLden. - Getreide blasen fus des Allemands.

Ce singulier insecte, dont la elassifieation a longtemps embarrassé les naturalistes, est assez eommun dans les blés de la plaine de Thionville et dans eeux de la vallée de la Seille. mais il n'est abondant nulle part; dans le pays-haut on le reneontre rarement.

A l'état de nymphe blanche, jaune, ou rouge vermeil, il vit entre le grain et ses enveloppes florales, et très-souvent au milieu des larves de la eéeidomyie du froment.

Plusieurs auteurs le signalent eomme trìs-nuisible, mais l'organisation de ses mâehoires et celle de ses mandibules semble eontredire la manière de vivre qu'on lui prête. Cette eirconstanee me paraît devoir néeessiter de nouvelles reeherehes sur ses habitudes. 


\section{LÉPIDOPTÈRES.}

20. Agrotis segetum, OCHSENB.

Noctua segetum, Uuns. - Noctua segetis, Henbst. - Noctua cubicularis, Fabr. - Ceradrina segetis, Guéné. - Noctua sordida, Scuifrex. - La Moissonneuse. - Wintersaaltaule. - Erd raupe des Allemands.

La chenille de ce pelit papillon vit aux dépens des jeunes plantes de blé; clle a quelquefois causé de très-grands dommages en Allemagne.

\section{Agrotis exclamationis, OcIISENB.}

Noctua tritici, Lins. - Noctua vitta, Hunner. - Noctua crassa, Hunner. - Noctua exclamationis, Fabr. - Bombyx cxclamationis, Esp. - La double tachc.

La chenille de cette noctuelle vit, comme celle de la précédente, aux dépens des feuilles de plusieurs espèces de graminées et plus particulièrement de celles du blé.

220 Polia ochroleuca, Borsdural.

Noctua ochroleuca, Hubnen. - Xanthia ochroleuca, Ocrsen. Noctua flamma, Fabr. - Ilarus ochrolencus, Borsd. - Die weizen gelbe aule des Allemands.

M. Nordlinger (Die Kleine feinde, etc.), signale cettc petite noctuclle comme étant très-nuisible aux blés, mais ce naturaliste ne donne pas les détails nécessaires pour fairc connaitre ses mours et ses habitudes.

\section{Pyralis frumentalis, LinnÉ.}

Phalena repandata, Fabr. - Pyrale du froment, Devilux. Scopula frumentalis, Duponcurc. - Pyralis repandalis, Ilubs, - Saatzunsler en allemand.

C'est encore dans l'ouvrage de M. Nordlinger que cette espèce est signalée conme nuisible au froment, mais l'auteur ne nous 
apprend rien sur les habitudes qui sont particulicres à cette 'lineide.

\section{DIPTÈRES.}

\section{Cecidomyia destructor, SAY.}

Cicidomyie. - Ilessian $\cap y$ des Anglais et des Américains. - Mouche de Ilesse. - Ilessen fiege. - Weisen verwuste des Allomands.

Cette tipulaire gallicole serait, au dire des Américains, une importation faite par les Hessois, dans le siècle dernier. Si cettc assertion est exacte, il faut convenir que la mouche de Hesse a tronvé aux États-Unis des conditions bien favorables à son développement; car, en peu d'années, elle s'y est multipliée en telle quantité que c'est par centaines de millions de dollards que l'on évalue les pertes causécs par cette petite mouche.

D’après Kollar*, la Cecidomyia destructor aurait été observée par lui cn Hongrie. Cet insecte est jaune avec des poils noirs sur le corps, les ailes noirâtres, sur lesquelles la couleur jaune du corps s'étend quelquefois. les antennes blancliatres, elc. Ceet insecte, qui n'a été décrit qu’en 1818, par Say, vil à l'étal de larve sur les tiges $d u$ blé, qu'il affaiblit, et au moindre vent ces tiges se couchent en s'entremêlant les unes dans les autres. Rien de ce genre ne s'est encore observé en Francc, en Belgique, en Angleterre, ni dans une grande partie de l'Allemagne.

\section{$25^{\circ}$ Cecidomyia flava, Meigen.}

Cette espèce semblable, pour la forme et la couleur, à la Cecidomyia tritici de Latreille, a des mœur's analogues à celles de la Cecidomyia destructor des Américains. Sa larve vit aussi sur la tige du froment, y parcourt ses métamorphoses et, selon M. Viennerst, ne fait jamais périr la plante.

J’ai recherché celle cécidomyie sur un grand nombre de pieds

- v. Nordlinger, Loc. cil. 
de blé, pendant le mois de juillet, et je n’ai pu la reneontrer dans nos moissons.

$26^{\circ}$ Cecidomgia tritici, Latreille.

T'iputu tritici, Kınв. - Cécidomyie du froment, - Weizen mücke des Allemands.

Cette especee, déerite parfaitement par MM. Nordlinger et Bazin, differe essentiellement de la deseription rapportée plus haut et donnée par Kollar, pour la Cecidomyia destructor de Say, dont les mœurs sont également différentes. D'après Say. Kollar et Aza Fiseht, la larve de la eéeidomyie amérieaine est blanchâtre, elle passe l'hiver sur la tige, et c'est au printemps qu'elle commel ses ravages.

Kollar et Say sont d'aceord pour rapporter à la mêne espèec les ravages oecasionnés en Amérique el dans l'Altenbourg Hongrois. Cependant la deseription de Kollar, qui a vu et observé l'insecte en Hongrie, ne peut s'appliquer à l'inseete déeril par Latreille, sous le noin de Cecidomyia tritici.

Les individus eomparés par N. Lueas, à la Cecidomyia tritici, Latr. , que lui a envoyé M. Bazin, ne sont done pas semblables à la céeidomyie déerite par Kollar. Ces motifs me paraissent établir eomplétement la différenee qu'il y a entre la Cecidomyia destructor, Say, el la Cecidomyia tritici, Latr. Celle-ei a d'ailleurs des mours qui justifient mon opinion. En effet. elle pond sur l'épi, avant la floraison, sa larve est jaune et la nymphe passe l'hiver dans la terre.

C'est à la ééeydomỵie du froment qua $\mathrm{M}$. Bazin rapporte les eauses du défieil de notre deruière réeolte.

\section{$27^{\circ}$ Chlorops lineata, Macquart.}

Musca tineala, Fabr. - Musca punitionis, Guems. - Oscinis lineata, Latr. - Chtorops cerceris, Meigen. - Rogenfliege des Allemands.

Cette pelite mouehe, remarquable par ses yeux verts, ainsi que l'indique le nom générique, est plus répandue dans lo midi de la France que dans les autres départements. J'ai eependant 
trouvé un assez bon nombre d'individus de cette espèce dans un champ près de Vallières.

Elle est d'autant plus redoutable qu'elle a deux générations par année: les individus éclos en mai ou en juin pondent sur la tige du blé, vivent aux dépens de eelle-ei dans les parties recouvertes par les gaînes des feuilles, et font ainsi avorter le grain ou ne lui laissent prendre que peu de nourriture. Ces premiẻres larves subissent leur métanıorphose dans le bas de la tige et Éclosent en septembre ou en octobre, et bientôt ces nouvelles femelles vont déposer leurs œufs sur les jeunes tiges du blé nouvellement levé; les larves qui en éclosent s'y développent à leurs dépens, passent l'hiver engourdies, et, au mois de mai suivant produisent de nouveaux insectes.

28० Chlorops lœeta, WaGa.

$29^{\circ}$ Chlorops Herpini, GuÉrin.

30" Chlorops glabra, Meigen.

$31^{\circ}$ Chlorops frit, Curtis.

Musca frit, LINN. - Oscinis frit, FALLEN. - Mouche frit fritfiege, des Allemands.

32. Chlorops toniopus, Meigen.

Oscinis devastator, Cunrss. - Tephrits hordei, MacQ.

$35^{\circ}$ Agromyza fuscipes, Macouart.

Toutes ces especces de museides, et probablement encore beaucoup d'autres mal définies et mal observées, ont été souvent confondues entr'elles. Il est probable que chaque contréc avait. dans l'origine, son especee particulière, mais que peu à peu, par les changements de semenees, les transports de fourrages, etc.. ces différents insectes se sont répandus, et que c'est ainsi que l'on peut expliquer comment la même localité est tantôt ravagée par une espèce, tantôt par une autre.

Il est aussi bon de faire remarquer que la mêne espece de 
clilorops attaque à la fois plusicurs sortes de céréales : ainsi le Chlorops frit ravage plus particulièrement l'orge de la Suède et le blé barbu du nord; le Chlorops locta. les blés de la $\mathrm{P}_{0-}$ $\operatorname{logne}$, ete.

En résumé, il y a une riche moisson de faits nouveaux et très-intéressants à recueillir dans l'étude de ces petits mouclerons.

Bien que cette listc soit déjà longue, il est évident, pour les entomologistes, qu'elle est loin de représenter la nomenclature de tous les insectes qui vivent aux dépens du froment sur pied.

$\mathrm{Si}$, parmi les ennemis de celle utile graminée, nous trouvons des colćoptères qui semblent avoir abandonné les mœurs carnassières pour en manger les épis, ne devons-nous pas nous étonner de ne voir prendre part à cette curéc ni Curculionite ni un plus grand nombre de Mierolépidoptères.

Une plantc cultivée dans tous les pays el dés la plus hautc alltiquité doil ecrtaincment servir de pâture à un plus grand nombre d'insectes, et il est évident pour moi que plusieurs maladies du blé, attribuécs jusqu'ici à des influcnees atmosphériques, n’ont pas d'autre causc que la présence de quelque larve, ou de quelqu'inscete parfait. attaquant l'un des organes de la plante.

On répète souvent que tout dégénère et que nos cultures sont attaquées par des inaladics ou des inscetes inconnus jusqu'ici : les explications les plus diverses et les plus hasardées ont été données sur ce phénornène facile à comprendre pour les naturalistes.

"Dans les grandes réunions d'homme, disait M. Guérin, en 1851 ", les lois naturelles sont continuellement violées. Nous devons lutter contre les tendances qui ont pour but d'établir une juste répartition des êtres, ear nous avons besoin d'en multiplicr quelques-uns outre mesure, pour nous nourrir, nous vêtir, etc. $\gg . .$. " Les insectes se développent toujours en raison des subsistances qui sont à leur dis-

- Congrès central d'agriculture, session de 1851 . 
position; ils sont plus nombreux, et par eonséquent plus dangereux, dans les pays de grande culture.

A ces raisons il faut encore cn ajouter une non moins importante. et qui atteint chaque jour de plus grandes proportions. Combien de nouveaux insectes introduits dans nos serres, nos jardins, nos champs et nos forêts par les plantes nouvelles que l'on y apporte des pays éloignés, et dont le climat se rapproche plus ou moins du nôtre, ce qui permet à ces nouveaux hôtes d'y vivre et souvent de s'y propager en se fixant sur les espéces congénéres propres au pays.

Après avoir subi les intempéries de toutes sortes, résisté à la rapacité des nombreux ennemis que je viens d'énumérer el de ceux qui nous sont eneore inconnus, le blé est enfin rentré dans nos greniers, ou prend place dans nos magasins après avoir été converti en farine ou en biscuit. Alors une nouvelle légion d'insectes destructeurs va fondre sur lui, et ces nouveaux ennemis détruiront quelquefois en peu de temps tout un approvisionnement.

J'ai également dressé la liste de toutes les espèces qui appartiennent à cette eatégorie, en y ajoutant quelques notes sur la nature des dégâts qui,elles occasionnent.

Insectes nuisibles nux graing ou aux farines cmmagnaines, avee leur synonymic et quelques indicatons

sur leurg mours.

\section{COLÉOPTÈrES.}

$34^{\circ}$ Sitophilus granarius, Schoenner.

Curculio granarius, Lins. - Curculio segetis, Lins. - Rhyncophorus granarius, IIERBst. - Calandra granaria, FADR. - Calandre, - Charancon du ble, - Getreider Rüssel, -Korn kefer, - Korn wurm, des Allemands.

Cet insecte est malheureusement trop connu; des pertes incalculables sont annuellement causées par lui. Bien des procédés ont été inventés pour s'en débarrasser; celui qui consiste à renfermer le hlé dans des greniers mobiles, semble 
avoir eu le plus de suceès jusqu’à présent. A la manutention de Paris, on fait en ee moment des expériences pour détruire ce charançonite. Ces expérienees eonsistent à renfermer le blé dans de grands cylindres en tôle, oủ l'on fait arriver du gaz azote pour remplacer l'air et asphixier ainsi l'insecte et les larves. par leur séjour prolongé dans un milieu privé d'oxigène.

\section{$35^{\circ}$ Sitophilus oryzœ, ScHoenher.}

Curculio oryza, Liss. - Curculio frugilegus, Degeen. - Curculio granarius, Stroes. - Rhynchophorus aryzo, Hernst. - Calandra oryzณ, FABn. - Calandre du Riz. - Rcinwurm, Reisskâfer des Allemands.

Celle espèce, roisine de la préeédente, en compagnie de laquelle on la reneontre souvent, en a les mours et les babitudes, et les moyens de destruetion employés pour la première lui sont également applieables.

\section{$366^{\circ}$ Trogosita caraboides, OLIYLR ".}

Tencbrio nuuritanicus, Lis. - Platyeerus mauritanicus, Georfn.Tenebrio caraboides, Fån. - Trogosile. - Cadelle. - Brodkofor des Allemands.

La larve de eet insecte vit dans la farine, le pain ou le biscuit eonscrvé dans les magasins de l'armée ou de la marine, et y eause, dit-on, quelquefois des pertes sensibles.

- Dans son beau travail sur les Insectes du Pin maritime, M. Ed. Perris dit: " J'ai la certitude que la larve du $T$. Coerulca est carnassière, ear elle fait un grand earnage des larves lignivores au milieu desquelles elle vit, et je l'ai vu souvent, dans les boeaux où je l'élevais, mettre en pièees les larves de longicornes et autres que j'y introdtuisais. „- " Q Quant à la larve du 'I'. Mauritanica, qui, sous le nom de Cadolle, est généralement maudite comme très-préjudiciable aux graiıs, j’ai la convietion, par analogie et sans l'avoir constaté personnellenent, qu'elle est indignement ealomniće et qu'elle ne se trouve dans les céréales que pour détruile les larves de Calandre et les chenilles d'Alueite qui en sont le véritable flèau. „ 
370 Temnochila corulea, Linouson".

Trogosita corulea, Ouv. - Trogosila virescons, Rosss. - Trogosile bleue. - Blane Brod kofer.

Espéce tout-à-fait méridionale, ayant les mœurs et les habitudes de la précédente. mais elle est beaucoup moins répandue.

58" Tenebrio molitor, LINNÉ.

Tenebrion. - Ver blane de la farine. - Mehl hicfer. - Mehlwurm.

Cet insecte, dont la larve est bien connue des meuniers et des boulangers, vit dans la farine. le seul nioyen de s'en débarrassor consiste à bluter les farines où clle se trouve.

\section{LÉPIDOPTL̀RES.}

59० Asopia farinalis, Treist.

Pyralis farinalis, Lix. - Mehlzunzler des Allemands. - Phalena farinalis, Fabr. - Bolys farinalis, Latrenle.

La chenille de ce petit papillon vit dans la farine el s'y trouve quelquefois en grande quantité.

$40^{\circ}$ Butalis cerealella, Gú̉rin.

Tinea cercalella, Treits. - OEcophora cercalclla, Latreille. Alucite. - Alucita ecrealella, Encyclop.

Ce Microlépidoptère, dont la clınille vit dans l'intérieur des grains de blé, a causé de véritables disettes en dininuant de 80 p. $\%$ les récoltes de certaines années.

La ponte se faisant sur le grain presque mûr, le meilleur moyen eultural de s'en préserver c'est de couper les blés avant leur maturité et de les conserver en moyette.

* Yoir la note de la page 17. 


\section{$41^{\circ}$ OEcophora granella, Duponcuec.}

Tinea granclla, Iıx. - Anacumpsts granella, Sнер.- Teigne des blés. - Korn motte, - Weizckornwurm des Allenands.

La clicnille de ce petit papillon , lie ensemble plusieurs grains pour s'en faire une sorte de eoque. Après en avoir mangé plusicurs, elle s'enferne dans l'un d'eux ou se retire sur' les murs et sur les poutres des magasins pour y achever ses transformations el se changer en papillon au printemps suivant.

Le tarare brise insecte, de nolle compatriote M. Herpin, a ćté inventé pour se préserver des ravages de celte Tinéide.

D'aprẻs ce qui préeède on voit que le blé a le triste privilége de nourrir une grande quantité d'inseeles, et que ee n'est pas trop du coneours de tous pour arriver à sauver de leur's dégàts la quantité nćcessaire à l'alimentation publique.

Faire connaître les liabitudes de l'un de ees parasites et proposer' les moyens de le dél'uire ou d'en diminuer les ravages, tel est le but que vient d'atteindre un agronoıne et naturaliste distingué du département de l'Yonne, en publiant une brochure très-l'emarquable intitulée: Notice sur un inseete qui a eausé les plus grands ruvutges dans nos dernières récolles de blé sur pied.

Le titre seul, eomme on le voil, suffirait pour appeler l'attention de toutes les personnes qui s'oceupent d'entomologie appliquée et de tous les cultivateui's intéressés.

Le parasite dont parle MI. Bazin, dans sa broehure, n'est pas nouveau pour la seirnee entomologique, et il figure sur nos Catalogues depuis longtemps sous te nom de Ceeidomyia tritiei de Latreille.

C'est un petit inseete diptère de la tribu des tipulaires, ou, pour parler vulyairement, e'est un petil moucheron jaunlitl'e, ayant deu. longues ailes transparentes et une taille d'environ deux millimétres de longueur sur quatre ou einq d'envergure.

C"est à ec chétif insecte que M. Bazin altribue, avec raison, sclon noi, 
les diminutions trouvées da!s nos récoltes depuis plusicurs annérs. - Déerirc eet insecte à-peu-près ineonnu, rappeler les discttes qu'il a occasionnées dans d'autres pays, discuter les moyens enployés pour s'en préserver, annoneer' sa disparition probable, ou au moins son cantonncment dans des cspaces restreints, tout en ayant la erainte de le voir paraitre de nouveau aprés quelques années, aussi envahissant, aussi redoutable que dans lc présent, " roila lc but que s'est proposé M. Bazin, el je nıe hâte d'ajouter qu'il a traité son sujel avec autant d'it-propos que de talent.

Les dégâts eausés par la Céeidomyie du froment, dans la terre de Fumerault (Yonne, arrond" de Senlis), n'étaient probablement pas limités à cette localité, et les défieits aceusés dans nos récoltes, depuis quelques années, pouraient aussi, dans notre départenent, avoir la même origine.

Constater de pareils faits, éveiller l'altention des autorités du département, des Comices agrieoles, des cultivateurs, etc.. m'ont paru des questions importantes, et vers la fin du mois de juin dernier je me mettais à l'œuvre. Dès le premier jour javais la doulcur de eonstater dans nos blés la présence de milliers de eéeidomyies, les unes roltigeant au-dessus des épis cn fleurs, les autres confiant leur infernale progéniture aux embryons du grain.

Dans les épis défleuris, entre la glume et l'ovaire, j'ai trouvé un. deux, trois, quatre...., quinze et jusqu'à vingt-einq petits vers d'un jaune eitron, suçant la sève, au moment où elle arrive pour grossir le grain.

Pour eclui qui a lu la brochure de M. Bazin, et pour des yeux aussi exercés que ccux d'un entomologiste, il est évident que les blés de nos environs sont aussi attaqués par la cécidomyie; et que. si les indications du naturaliste de Fumerault sont justes, il faut aussi attribuer le défieit de nos réeoltes à ectte pelite mouelıe, ou plutôt à ee petit ver jaune. et chereher à appliquer chez nous les moyens employés ailleurs pour en faire ecsser les ravages.

Dans un travail, que la Société d'horticulture a imprimé dans son Bulletin, j’ai insisté sur la néeessité de bien spéeifier les espèces qui attaquent une plante, quand on veut leur appliquer les procédés de destruetion employés dans d'autres loealités.

Si le doute n'est plus permis sur la présence d'une eéeidomyie 
dans nos blés. l'exanien de la planche qui la représente, la couleur de la larve, la description qui accompagne cette planche. suffisent pour faire naître des doutes sur l'identité spécifique de cet insecte avec la Cecidomyia tritici de Latreille.

Celle-ci, en effet, a une tarierc plus longue, lc corps plus convexe. le premier articlc des antennes de la femelle plus long que l'arlicle correspondant des cécidomyies de nos blés, enfin les ailes de celle-ci sont fortement irisées. Tous ces caracterrcs ne pouvaient avoir éclıappé à M. Bazin, et, l'époque différentc à laquelle les femelles dc l'une et de l'autre espéce opèrent leur ponte, me firent conclure à la différence spécifiquc de ccs deux insectes.

Pour plus de certitude, jai adressé à $\mathrm{M}$. Bazin un certain nombre des insectes pris par moi dans nos blés, ainsi que les larves qui mangent le grain, et voici la réponsc qu'il m'a fait l'honneur de m'adresser: - J'ai reçu votre cécidomyic femelle (et le inâle probablement) arec les jeunes larves et les parasites. Cंc sont précisément les mêmes insectes quc j'ai recueillis au Ménil-Saint-Firmin. département de l'Oise. pendant ces derniers jours et que je recueille ici à mon arrirée. Mais ni celte eécidomyie, ni ce parasite ne sont les mêmes insectes que j’ai rencontrés antérieurement très-abondamment.

La cécidomyie recueillie dans les environs de Metz n'est done pas la Cecydomyia tritici Latreille. Ce ne peut être non plus la $C e-$ cidomyia flava de Meigen, dont clle n'a pas les mœurs; ses habitudes de pondre sur l'épi en fleur l'éloignent également de la Cecidomyia destruclor de Say, dont elle diffère aussi d'aprés la description de. Kollar, trouvéc exacte par Aza-Fritch lui-même.

Nalgré mes recherches. je n’ai pu, jusqu'ici, la rapporter à aucunc espćce décritc du genre Cécidomyie, auquel elle appartient cependant. et, pour évilcr tout équivoque dans ce qui suil, je la désignerai sous le nom de Cecidomyia mosellana, prêt à faire le sacrifice de ce nom à cclui qui l'aura déjà décrite on qui lc fera d'une inanière plus complètc que moi ct plus en rapport avec les exigences de l'entomologie.

A la fin du mois de juin, un grand nombre de larves peuplaient déjå les épillets des blés de Borny, de Plappeville, etc., et on doit en conclure que ce n'était plus que quelques femelles allardées 
qui avaient été capturies, et que le plus gros de la ponte avait eu lícu huit ou dix jours auparavant, e’est-a-dire au moment de la floraison.

A cette différence près, la ponte de la cécidomyic de la Moselle se fait aussi le soir au coucher du soleil, et, pour déposer ses neufs, la femelle introduit sa tarière entre les enveloppes florales, de maničre à placel' les jeunes larves qui écloront à proximité de l'ovaire dont clles doirent absorber la séve. Ces larves sont apodes, comme celles des dipteres, et ressemblent à de petits vers jaunes formés de plusicurs anncaus. Si on n'en rencontre que quelques-unes dans la même fleur, et c'est le cas le plus général, les grains sont seulement réduits du quart, du tiers ou de la moilié de leur grandeur naturelle et produisent ainsi un rendement effectif moindre et relativement de plus petite valeur commerciale, puisque tous ecs grains passent au petit blé, lors du criblage. Si, au contraire, il se trouve dans la fleur une plus grande quantité de ces larves, comme je l'ai souvent observé, les grains avortent complétement et occasionnent au batlage, un déficit dautant plus considérable qu'il y a un plus grand nombre d'éfillets dans ces fácheuses conditions.

Les petils vers jaunes qui eausent un parcil et si déplorable résultat, ont à peinc deux millimétres de longueur sur un deni-millimètre de large, lor'sqüils sont arrivés à leur complet développement; ils sont alors d'une coulcur un peu plus foncée, tirant sur l'orange.

Leur présence dans les épis est souvent décélée par une tache jaunâtre placée sous la glume externe et d'autant plus apparente qu'il y a plus de larves dans l'épillet. D'apres MI. Bazin, cette tache serait placéc a la base de la glume, mais trés-souvent je l'ai observéc au sommet de celle-ci; il arrive aussi assez fréquemment que les épillets renferment des ver's jaunes sans que la glume soit maculéc. Ce caractere riest done pas exclusif (au moins pour la Cécidomyic moscllane), bien que toutefois son cxistence soit l'indice eertain de la présence des larves de cécidomyie. Ce sont ces épillets taclıés de fauve que les cullivateurs appellent des grains choqués, et nous en arons surpris plusicurs, en leur montrant le petit ver jaune occupant la place d'un grain qu'ils croyaient detruit par le soleil.

A mesure que le inois de juillet avance, on rencontre de moins ('n 
moins de larves dans les épis, el, vers le 20, c'est à peine si l'on ea rencontre eneore quelques-unes dans les blés les plus fortement attaqués. Nous verrons plus tard ce qu'elles sont devenucs, et par quelle adroite manœurre elles abandonnent l'épi qui les à nourrics.

Constater l'exisicnec du nal ne suffit pas; il est surtout trés-utile d'en évaluer l'intensité, de préroir ses résultats et de rechercher quelles proportions il peut prendre à l'avenir. Devons-nous eraindre unc aggravation, ou, au contraire, pouvons-nous espérer une diminution dans les dommages que nous subissons; e'est ce que je rais cxaminer maintenant.

Profitant de l'obligeauce de plusieurs personnes et de la tournće que j'ai faite, comme membre du jury de médecine, dans une partie du département, jai pu, depuis la fin de juin jusqu'au 20 juillct, faire le dépouillenent de plus de 500 épis de blé pris au hasard dans plus de 200 sillons différents et provenant des 52 communes suivantes : Ars-sur-Mosclle, Aumetz, Borny, Boulay, Bouren, Bouzonville, Burtoncourt, Colombey, Conflans, Fontoy, Freistroff, Gondreville, Gravelotte, Grimont, Grigy, lIayange, Jarny, Longuyon, Magny. Mcrcy-lc-Bas, Mézic̀res. Mondelange. Montigny, Montoy-Flanvillc, Novéant, Hichemont, Talange, Uckange, Vallières, Viller's-latMontagne, Woippy ot Xivry-Circourt.

508 épis provenant des communes que je viens d'indiquer ont été dépouillés très-attentivement, et, sur 259 d'entr'cux, j’ai pu constạter la présence du petit ver jaune. C'est donc une proportion de 83 épis p. \% qui sont attaqués. Ce rapport, déjả fort alarmant, le devient encore davantage quand on fait la décomposition des chiffres quile représentent. En effel, sur 49 épis signalés comme n’ayant pas été attcints par la cécidomyic, 2 étaient malades et cnvalis par le charbon, 4. appartenaient à une variété tomenteuse, 11 à des varićtés barbues, et enfin 32 étaient des épis chétifs, portés par des claumes rabougris ct ne renfermaient que des grains desséclıés: comme si la cécidomyie prévoyante nc voulait confier sa progéniture qu'i des plantes vigourcuses, capables de fournir aux jeunes larves une nourriture abondante. 11 est a remarquer aussi que bou nombre de ces épis ne sont excmpts de larres que parce qu'ils sont plus courts, l'insecte ayant plus aisé de pondre sur les épis plus ćlerés.

En faisant unc décomposition scmblable sur le chiffre de 2099 qui 
représente le nombre des épis attaqués, on en trouve 59 dans lesquels les larves sont très-abondantes, et dont le nombre des grains avortés dépasse le dixième de la totalité de eeux portés par l'épi ; 220 sont attaqués dans une moindre proportion, mais qui eependant dépasse en moyenne le vingtième du nombre des grains que l'épi aurait pu fournir. Enfin, sur ees 259 épis, il ne s'en trourait que 2 appartenant à des variétés barbues et 12 à des variétés tomenteuses.

En poussant ee dépouillement plus loin, et en prenant 12 épis parmi ceux qui sont le plus fortement attaqués ", j'ai trouvé que ees 12 épis, qui renfermaient 480 grains de blé féeondés, en contenaient 46 complétement avortés, et 58 réduits à des proportions souvent fort petites des grains non attaqués. C'est done 46 grains, plus 58 demi-grains ou 75 grains perdus sur ees 12 épis, et, par conséquent, un défieit total de $15 \% \mathrm{p} . \%$ environ pour les blés fortement attaqués. Ileureusement que dans ce moinent, du moins, eotte proportion ne s'étend qu'à 59 épis sur 508 , ou environ 13 p. \% de la totalité des blés provenant des eommunes indiquées. Tandis que la proportion des épis non attaqués est de $15 \mathrm{p} . \%$ seulement. et que la perte s'élève à plus du vingtième sur le reste des épis, c'està-dire sur $72 \mathrm{p} \%$ d'entr'eux.

Or, dans le département de la Hoselle, on exploite pour la eulture du froment, environ 86 mille heelares de terres qui rendent en moyenne 15 heetolitres par heetare. C'est done une produetion annuelle de 1 million 290 mille heetolitres de blé.

Si sur tous les points du département. les blés étaient aussi fortement attaqués qqu'à Gondreville, à Ar's-sur-Moselle, à Plappeville, la perte totale s'élèverait a près de 200 mille heetolitres, représentant une sommo de 4 millions de franes, au prix ordinaire de 20 Iranes l'hectolitre, et de plus de sept millions de francs au prix de ees dernieres années.

Si, au contraire, tous les blés n'étaient que faiblement attaqués. te qui probablement n'est déjá plus vrai, la perte totale serait encore de 5 p. $\%$ de la produetion annuelle, soit près de 65 mille hee-

\footnotetext{
- Six de l'appeville, trois de Gondreville et trois d’Ars-sur-Moselle.
} 
tolitres, représentant une perte moyenne de 1 million 300 mille francs, et plus de deux millions en suivant les dernières mercuriales.

Èn réalité. la perte totale doit ĉtre calculée d'après les proportions relatives du nombre des épis plus ou moins atteints par la cécidomyie. Nous aurons donc un déficit de 25 mille hectolitres d'une part, 45 mille de l'autre; en tout 70 mille hectolitres pour le delicil de 1856.

Cette quantité représentc l'alimentation de la ville de Metz pendant deux années, et unc perta cn numérairc de plus de deux millions d'après lc prix du blé sur les marchés de 1856. Je suis convaincu que ce cliffre représcnte à peine l'évaluation de notre perte. et jai la crainte de la roir s'augmenter encore, si l'ou ne se hâte d'apporter des obstacles à la propagation de cettc maudite cécidomyie. Selon moi, tout le pays-haut ne fait que subir les premières atteintes du mal, et la question qui m'occupe, demande ine promptc solution, si on ne veut jas avoir à déplorer de plus grands désastres.

La néccssité de ce que j'avance a été bien comprise par M. le Ministre des travaux publics et de l'agriculture, aussi vient-il de confier à M. Bazin, de Fumcrault, une mission au sujet dc laquclle celui-ci in'écrivait :

- Votre lettre m'est remisc au moment oủ j'arrive de Bourgogne. après avoir parcouru une grande partie de la France pour étudier les ravages de la cécidomyie. J'ai acquis la certitude que cet insecte, éminemment destructeur, est néanmoins complétement inconnu ou tout au plus entrevu quclquefois à l'état de larve, quoiqu'il se rencontre presque parlout, el que sur quelques points, du midi ou du nord, où jai pu séjourner quelque temps pour en apprécier le nombre, il occasionne cette année dcs dégâts trẻs-sensiblcs. En présence de cctte sécurité coniplète de la part des cultivateurs, il m'a fallu entrer en relation avec les entomologistes, leur montrer dans les champs de blé la cécidomyie pondant sur les épis, les pricr d'étudier lcurs nocurs, de donner connaissance de leurs obscrvations aux sociétés agricoles, dans l'espoir que l’on se préoccuperait cnfin d'un cnncmi qui est partout ct qui n'est vu nulle part. Il va done y avoir cetle année, cl il y aura encore plus l'année proclıainc, je 
l espère. des observations séricuses faites sur ee sujet, sur lous les points de la France. "

Le département de la Moselle, envahi, eomme tant d'autres, par la ećeidomyie, ne saurait rester en arrière dans eette eroisade générale contre un ennenji commun, et j'ose espérer que la Société d'hortieulture fera tous ses efforts pour appeler l'attention de l'autoritć et eelle des hommes eompétents sur une question aussi inportante.

J'ai eonsulté plusieurs eultivateurs sur l'existenee des petits vers jaunes dans les épillets. Quelques-uns avaient déjả observé ees ver's. dès 185\%, dans les eommunes de Herny, d'Arriance, de Many, ete. ou ils élaient moins abondants que cetle année, mais sans pour eela y attacher d'importanee, et sans se douter de la nature du mal qui envahissait leur's moissons. D'autres cultivateurs, et éćtait le plus grand nombre, ignoraient complétement l'existenee de ee fléau. Enfin, d'autres ont attribuć les taehes observées sur les épillets, aux brouillards, aux derniers froids et aux coups de soleil. Pour ecux-ei, la présence de ces larves était la conséquenee toute naturelle de l'altération survenue dans le tissus des enveloppes du blé pendant le mauvais temps. Malgré mes raisonnements, je reste convaineu que je ne les ai pas persuadé el que plusieurs se moquent eneore de mes petiles mouches mangeant leurs quartes de blé. Dans le payshaut, ils n'ont pas eu á souffirir de déficit dans leurs récoltes de ees dernières années; ils ne eonnaissent pas eneore le petit ver que je leur ai inontré et dont ils semblent fort peu se soucier. Puissent-ils toujours rester aussi confiants et conserver longtemps la presqu'inınunilé dont ils jouissent eette année?

Après avoir démontré la présence de la céeidomyie dans nos moissons, aprés avoir évalué les pertes qu'elle y oeeasionne, il est temps d'aborder la question principale et d'examiner les moyens proposés ou à proposer pour s'en débarrasser.

Suirant la loi générale, que j'ai déjà cu oceasion de rappeler ailleurs". le développement prodigieux de cette Tipulaire a dû aussi favoriser considérablement la multiplication de ses ennemis naturels.

* Introduction à l'IIistoire des Insectes muisibles, dèns lo déparlement de la Moselle. 
Le parasitisme, enfin, est le premier secours envogé par la lroviJence pour limiter la fécondité extraordinaire de cette cécidomyic.

En examinant les épis de blé, on remarque sur presque tous un petit insecte noir, à quatre ailes transparentes, qui semble se promener avee inquiélude sur le raclis de l'épi ou sur le pédoneule des épillets. Ce pelit moueheron a environ un millinètıe et deni de longueur; il appartient à la grande famille des jelıneumons et au genic Platygaster des entomologistes.

Le nombre des parasites que l'on rencontre dans le même épi, est trés-variable, ordinairement deux, trois ou quatre; M. Bazin les a sus quelquefois il plus d'une douzaine. Tres-rarement je les ai aperçus sur des épis dépourvus de larves de é́cidomyie.

D'après $\mathrm{N}$. Bazin, le Platygaster qu'il a rencontré en compagnie de la Cecidomyia tritici, pond ses ouls à côté de ceux de celte dernière. Je n'ai pu voir le Platygaster de nos moissons pondant sur les épis. Mais ce que jai vu, e'est que, sur mon bureau, des plalygaster ont pondu sur des vers jaunes, et l'un d'cux y avait mêne tellement enfoncé sa tarière qu'il a traîné la larve à sa suite à unc distance de plusieurs centimétres avant de pouvoir la dégager.

En regardant attentivement, on ne tarde pas à reconnaitre parmi ces parasites deux espęces bien distinctes, l'une, beaucoup plus abondante que l'autre: e'est le Plalygaster punctiger de Nees (Inoslemma punctiger, Walker), l'autı'c, beaucoup plus curieuse et plus variée de coulcur, e'est le Platygaster sculellaris, Necs. Enfin, M. Goureau, dont le nom se reneontre loujours quand il est question d'entomologie appliquée, m’a adiessé les observations suivantes: a Les plus beaux froments, dans les environs de mon village (Sautigny, daus le département de l'Yonne), sont ecux qui m’ont paru le plus attaqué; les parasites jouent leur rôle et sont en grand nombre, mais il me semble qu'ils n'ont pas cncore pris le dessus complétement. Le principal d'entr'cux, le plus nombreux, est un tris-petit Oxyurien du genre Platygaster, appelé Platygaster inserens par Curtis: il est entiérement noir. Chaque platygaster coutte la vie a une cécidonyic, et il en faut une multitude infinie pour arrèter le developpement de ees dernières. Il existe un autre parasite plus expéditil. mais beaucoup moins ahondant, qui contribue pour sa part a protéger nos moissous. Ciest un asse\% grand iclmenmonien du genre 
Coleocenter, dont jignore le nom dans ec inoment; la femelle pond scs œufs dans les épillcts remplis de larves de cycidonyics ct sa larve consomme une grande quantité de celles de ce diptère. n

Ainsi, partout oủ la cécidonyie cxerce sa funcste influcnce, partout le parasitisme se fait sentir. Ici, une cspèce pondant sur les larves: là, à côté de l'ouf qui doit la produire: ailleurs, peut-être. d'une autre manière encore; mais partout cn produisant une larve qui vivra aux dépens de cclic de la cćcidomyie; de sorte que l'annéc suivante il éclorera un platygaster au lieu dc la nuisible tipulaire. Si donc les parasites sont abondants, on peut espérer que beaucoup de larves de cécidomyies avorteront et que leur accroissement cessera d'avoir licu. Mais si, au contrairc, le nombre des parasites est trẻs-rcstreint, ne doit-on pas en concluro que le nombre des cécidomyies ira en augmentant? c'est ce que les cultivateurs du payshaut ne tarderont pas à apprendre. car c'est à pcine si j'ai rcncontré les Plalygasler sculellaris, punctigẹ ou inserens dans les épis de cette partic du départcment; cependant un bon nombre des épillets contenaient des larves de cécidomyies.

Je crois avoir démontré que le sccours naturel du parasitisme ne suffit pas pour détruire les insectes nuisiblcs, et que, dans tous les cas. il ne peut empêclıer leur invasion dans une contréc. M]. Bazin, dans sa brochure, semble être plus confiant que moi dans ces auxiliaircs providentiels. J'ai cru devoir lui communiquer mes doutes à ce sujct at voici sa réponse: "Les croyants, tels que nous, ne peuvent pas exprimer toutes leurs appréhensions de crainte de scmer le découragement au milieu des liabitants des campagnes, déja si maltraités par les dernières récoltes. Je ne puis parler des ravages des cécidomyics qu'en exaltant bien haut les services rendus par les parasites ct en les faisant meilleurs qu'ils ne sont. o Ceci ine parait péremptoire el suffira, je pense. pour prouver à tout le mande qu'il est enfin temps de sortir de l'aveugle confiance avec laquclle on semble compter sur la disparition spontanée d'un mal dont on ignorait l'origine, et qui s'cst cependant développé, accru ou propagé. malgré l"existence de plusicurs espèces destinées par la nature à en diminucr le nombre.

C'est donc à l'homme qu'il appartient de combattre cet cnneni de nos froucnts: c'cst sur son travail qu'il peut désormais eonıpter 
pour anćantir ce clıélif inscete ou limiter les dégàts quiil nous cause.

Mais comment atteindrc ce faible movclıeron, qui échappe presque à nos yeux, ct auqquel la bonlé divine a donné un admirable instinet et a appris la botaniçuc pour découvir au loin un nouveau champ de blé . l'organograplie pour y découvrir la place de l'ovaire, et la physiologie pour y opérer sa ponte au moment propice, afin que la jeune larve Éclose à l'époquc où une sève riche ct succulente arrive cn abondance priur forner le grain!

Quels moyens culturaux " employer? Quels sont ceux que la théorie indique et. parmi ceux-ci, quels sont ceux que l'expérience a consacrés?

Dans le mémoire que j'ai déjà eu occasion de rappeler, je disais que le clıangement de culture, l'introduclion de variétés nouvelles, pouvait souvent amener, saus grandes dépenses, des résultats trèssatisfaisants dans la destruction des insectes nuisibles. II a été dit plus haut que, sur 508 épis, il ne s'en était trouvé que 2, appartenant à une variété barbue, qui avaient élé atteints par la cécidomyie, landis que 13 cn étaient exempts. Les variétés à bales ct à glumes tomenteuses étaicnt aussi plus épargnées. Toutes choses égales d'ailleurs, il y aurait peut-être avantagc à ne cultiver, pendant un certain tenıps. que des froments à épis velus ou barbus, afin d’arrêter les progrẻs de la cécidomyie.

Celte especce d'immunité dont semblent jouir les variétés de blé

- M. Guéril propose la dẻnomination de moyens culluraux pour tous les proeédés de destruetion des insectes nuisibles, basès sur la connaissance exacte de l'organisation, et surlout des mœurs de ces animaux, trouvés principalement dans quelque pratique d'agrieulture, qu'il est possible et facile d'appliquer dans la grande culture, et dont le prix de revicnt n'est pas hors de proportion avee la valeur de la récolte qu'il s'agit de préserver.

Les moyens horlicoles sont ceux d'un emploi long, difficile, continu, et dont il n'est possible d'user que sur une échelle très-restreinte et dans les jardius.

(Mémoire sur les moyens de se piescrver des altaques de l'alueite, par Mi. Guérin Menneville, lu à l'Académie des Seiences, le 8 décembrc 1851.) 
que je viens d'indiquer, se comprend aisément. En eflet, les longues barbes dont sont aristées les glumes s'opposent à l'approclıc de la cécidomyie, dout les ailes de gaze seraient déchirées par les dents de ces longues arêtes. La villosité qui recourre les enveloppes florales des especes tomenteuses, est aussi un óbstacle à l'introduetion de la tariére des femelles; car ees tarières sont si fragiles que souvent elles restent engagées dans le tissu des enveloppes des épillets ordinaires.

D'autre part. l'étude comparative des variétés cultivées dans une contrée aménerait peut-être la découverte d'espièces ou de variétés plus souvent attaquées, et, par conséquent, leur proscription dans nos cultures? Dans tous les cas, ees recherches sont trés-utiles à tenter, mais, il ne faut pas se le dissimuler, elles sont entièrement locales: on doit s'altendre à rencontrer ici unc variété privilégiée. tandis qu'ailleurs cette variété scra plus attaquée, et vice versà.

Nous avons dit que la cécidomyie de la Moselle opérait sa ponte vers la fiu du mois de juin, pendant la floraison. Semer plus tôt ou plus tard, cultiver des variétés précoces ou tardives, de manière à avancer on à retarder la sorlie des étamines, et troubler ainsi l'insecte dans le moment d'opérer sa ponte, en ne lui présentant que des épis trop jeunes ou des grains déja formés. ne paraissent pas des moyens faciles à emplojer et capables de produire de grands résultats pendant quelques années.

Un écart de quinze jours cn avant ou en arrière de l'époque de la semaille (la saint Remy). est d'ailleurs tout ce que l'on peut espérer dans ce sens, parce qu'il faut que le blé ait une ectaine forec pour passer l'hiver, et que l'on doit aussi faire en sorte qu'il ne soit pas trop arancé quand les premiers froids se font sentir.

On sait aussi qu'un retardement de quinze ou vingt jour's dans l'époque de la semaille ne produit pas une aussi grande différence au moment de l'épiage ou de la floraison. L'expérienee semble d'ailleurs condamner cette méthode: et, si certains terrains ou quelques valiétés de blé permettent de l'employer on ne saurait. en général. en tirer parti pour la destruction de la cécidonyie. J'ajoutcrai encorę que cet insecte n'éclosant jamais dans le licu oú il doit pondre, il est obligé d'úmigrer pour aller chercher d'autres champs. Ces petites mouclies sauront done loujours trourel des froments dans un flal 
convenable de floraison, celle-ci ne se faisant pas à la fois dans tout un eanton, de même que toutes les cécidonyies n'éclosent pas et ne pondent pas en mème temps.

Si toutes ces raisons sont insuffisantes pour démontrer combien il faut peu compter sur l'efficacité d'un changement à produire dans l'époque de la floraison ou de l'épiage, jajouterai enfin que les animaux supérieurs, que l'on change de climat, ne tardent pas a meltre l'époque de leur parturition en harmonie avec les nouvelles conditions climatologiques ou on les a placés. Je suis convaincu qu'il en serail ainsi avec les cécidomyies, et qu'il faudrait varier continuellement les époques d'ensemencement pour n'obtenir qu'un résultat de peu d'importance. Dans notre climat, ou l'on rencontre des terres de nature aussi variée. rien de génciral ne saurait d'ailleur's être entrepris avec succès.

C'est done l'insecte lui-même que nous devons chercher ì détruire, et nous allons examiner successivement ses divers états, afin de trouver celui sur lequel il nous scra plus facile d'agir avec succès.

Le moment où les étamines commencent à sortir des enveloppes florales, est, je crois, pour la cécidomyie mosellane, celui de la plus grande activité parmi ces insectes. Pendant le jour cette mouelie so cache probablement sous les feuilles ou au bas des tiges. mais si le cicl se courre pendant la journée, et le soir, au couelier du soleil, on les voit par centaines voltiger au-dessus des épis. Ést-ce pendant ce temps que s'opère le rapprochement des sexes? écst ce que je ne saurais dire: mais il est permis de le supposer, puisque lon rencontre constamment des mâles, peu nombreux du reste, avec les femelles que l'on capture.

Quoi qu'il en soit. les manouvres continuelles de ces moucherons ont surtout pour but eclui de reconnaitre les eppis et de leur conficr les oufs, dont le nombre me parait très-variable pour chaque femelle; mais qui cependant parail assez considérable, puisque la même lemelle pond successivement sur plusieurs épis dans chacun desquels elle laisse deux, trois, quatre. cinq ou six oufs. Quand il y en a davantage, dit M. Bazin (pour la cécidomyie du froment), c'est que plusieurs femelles ont pondu sur le même épi. C'est à ce moment de la journée que l'agronome de l'umerault conscille de cliasser ces insectes, en se servant, pour cette opération, d'un filet analogue 
á celui des entomologistes. Cette opération doit se pratiquer dès le début de l'apparition des ééeidomyies, ear eelles-ei ne vivant pas longtemps, la eapture d'une femelle dont l'ovaire est déjả vidé ne servirait à rien.

J'avais eonçu des doutes sérieux sur l'effit:aeité de eetle ehasse et sur la diffieulté de la pratiquer, pendant plusieurs jours, sur des eentaines d'heetares eultivés en froment. Voiei ee que M. Bazin m'a fait l'honneur de répondre aux objections que je lui avais adressées:

- Quant au mode de destruetion de la eéeidomyie du froment, je ne partage pas vos antipathies pour le filet des entomologistes modifié. alongé de maniẻre à embrasser de grands espaees à la fois. Les eéeidomyies sont dans les pièees de blé dès avant l'épiage, tous les soirs, un peu avant le eouelier du soleil, elles voltigent pour s'assurer si les épis eommeneent à paraitre. Les premiers épis qui sortent sont done envahis el abimés de larves. Par un beau temps toutes les eceidomyies sont en mouvement le soir. En ayant égard à ees eireonstanees j’en ai reeueilli et détruil, par le simple proeédé que j’indique. sur un lieetare de terre, une quantité beaueoup plus eonsidérable que celle sur laquelle je eomptais. Dans les terres eultivées en planehe. l'emploi de eette méthode est surtout faeile. On ne nuit pas à la floraison ", puisque dès que les étamines paraissent la eéeidoniyie du froment eesse de pondre. Cette ehasse demande peu de temps, elle ne dure que quelques jours pour ehaque pièee de blé et prend seulement les soirées de ees journées. On peut répéter eetle épreuve plısieurs jours de suite dans le même ehamp et arriver à des résultats étonnants. J'ai erains d'effrayer les liabitants des eampagnes par ees manœuvres insolites, autrement je serais arrivé à une expérienee plus complète. J'atteins, quoi qu'il en soit, des ehiffres eneourageants pour de nouveaux essais, l'année proehaine, de ee mode de destruetion. Instruments peu eoûteux, faeiles à faire, faeiles à manier. sans qu'il soit néeessaire ni de force ni d'adresse. Mode peu dispendieux, puisqu'il embrasse un délai très-eourt et seulement quelques instants de quelques journées. Mode très-avantageux, du reste. en ee sens quion détruit les eéeidomyies sans détruire un grand

* Ceci pourrait ne pas ètre vrai pour notre cicidomyie. 
nombre de leurs parasites, qui, pour la plupart, ne paraissent que quelques jours après. On touche du doigt le résultat que l'on obtient. puisque les cécidomyies détruites. on les voit et on les compte.

- Je prends soin de mettre en relief lcs avantages de cc procédé. non pour vous détourner d'en patroner d'autres, mais pour vous dissuader simplement de trop de défiance pour les avantagcs de celui-ci. Quant aux autres, il serait bien important que leur emploi soit encouragé. L'impulsion que vous voulez donner autour de vous à ces essais curatifs, aura du retentissement et des imitateurs. On arrivera ainsi évidemment à une solution quelconque. Le plus grand empêchement est que la conviction n'est pas venue touchant la présence et les rarages de la cécidomyie du froment. :

Commc on le voit, en ayant égard à la différence dans l'apparition des deux inscetes, les tentatives faites à Fumerault sont encoutrageantes, et il serait très-utile de faire dc nouvelles expériences dans nos champs, lor's dc la floraison, en 1857.

Si la chassc n'a pas eu lieu ct si la cécidonyie a eu le temps de conficr ses œufs aux épis de froment, nous ne pouvons avoir aucun espoir de les détruire non plus que lcs larres qui doivent en éclore.

Si le temps est chaud et liumide, cettc éclosion se fait rapidement; peu d'œufs avortent, et les jeunes larves sont prêtes à sucer le liquide qui doit affuer dans l'ovaire après la fécondation. Si , au contraire. le temps est froid, humide, l'éclosion est lente, difficile, et plusieurs oufs ne donnent pas de larves.

A l'état d'œuf́s, qucls moyens cmployer pour les détruire? Comment seulement constater la présence de germes dont lc diamètre n'atteint pas un centième de millimètre? Pcndant toute la vie de la larve que faire pour la détruirc sans nuiro à la plante, comment en débarrasser l'intérieur des épillets? Nous devons, pendant loute celte période, rester spectateurs impuissants à combattre le inal et assister sans défenso à la destruction du grain qui forme la partie principale de notre subsistance. On peut seulement, dit II. Bazin, constatcr la présence des larves cl apprécier les pertes qui en resultcront.

Le petit ver jaune. qui sort de l'œuf, est d'abord peu coloré. sa coulcur ne tirc sur l'orange qu'au moment où la larve est arrivée à toute sa grandeur. 
Comme toutes les larves de diptères, celle de la cécidomyie n’a ni mandibulcs, ni mâchoires, elle ne peut donc absorber qu'unc nourriture liquide, et, conme je l'ai déjà dit, c'est la sère destinée à former le grain qui lui fournit les éléments nécessaires à sa subsistance.

Une régétation vigoureuse, favorisée par la variété de froment que l'on cultive ou par des engrais abondants, un temps chaud et sec vont pousser la croissance du grain avec vigueur, et bientôt arrivé au terme de son développement, son épiderme prendra de la consistance. et les jeures larves périront d'inanition au milieu d'une nourriture trop' substantielle. Dans les années froides et humides, ou plutôt encore lorsque la végétation scra languissante, les sucs resteront longtemps liquides et les larves auront toujours la nourriture suffisante pour accomplir toute leur évolution, il n'y aura alors que cclles qui seront échneumonées qui ne produiront pas de céeidomyie l’année suivante.

C'est ainsi que souvent les variations de température, et en général tous les phénomẻnes métćorologiques, influent sur les productions de nos cultures, sans cependant agir d'une manicre directe, comme on le croit toujours, sur les végétaux destinés à nos besoins.

Si on examine les épis de blé dix ou quinze jours après l'éclosion des œufs ou vers le milieu de juillet, dans les blés précoces comme ceux de la plaine de Thionville, vers la fin dn mois pour ceux du pays-haut, c'est à pcine si l'on y rencontre quelques larves. Cellcs-ci nic sont plus d'un jaune citron, mais d'une couleur orangée, elles sont amincies par les deux extrémités, et presque sans mouvements. Cependant, dans ces épis, des grains avortés d'autres mal tournés ou réduits au liers ou au quart de leur taille normale, sufisent pour indiquer le séjour d'un plus grand nonbrc de larves. Celles-ci ont donc abandonné leur berecau et sont allées. comme cellcs de la cécidomyic du froment, se cacher dans la terre ou à la basc du chaume. s'y abriter et y passel' ainsi l'hiver dans une sorte d'engourdissement counu sous le nom d'état dormant.

$\mathrm{Si}$ on prend un de ces petits vers jaunes et quon le plaee sur la main, sur le papicr, etc., il ne tarde pas à rapprocher ses dcux extrúmités et a sc lancer dans l'espace en détendant r'apidement l'arc ainsi formé. C'est sans doute par une manœusre de cettc sorte que 
Ia eécilomyie de la Moselle quitte les épis ou elle a acquit tout son accroissement. Privé de pieds, devenus inutiles parce que la larve prend sa nourriture à mesure que la force de végétation la fait affiuer dans la graine, ee ver a reçu de la nature le mnyen d'accomplir rapidement un grand voyage, et bien plus surement qu'avee le secours des six petites pattes dont sont pourvues beaucoup d'autres larves.

C'est dans sa nourellc demeure que ee petil ver peut être facilement attaqué, n’étant plus protégé par les enveloppes de l'épillet ni pourvu des longues ailes qui plus tard lui permettront d'émigrer et d'échapper à nos poursuites.

Ces larves ainsi réfugiées à la base du ehaume, ou enfoncées de deux a trois eentimètres dans le sol, sont-elles suffisamment protégées et faut-il compter sur les pluies ou sur les gelées pour en faire périr un ecrtain nombre? J'ai dit dans l'introduction du trava!l que $j$ 'entreprends sur les insectes nuisibles, quels résultats ont les intempéries et les froids les plus intenses sur la reproduction ou la multiplieation de ces petits animaux",

II. Bazin , pas plus que les entomologistes sérieux, ne fait grand cas de ces ressourees, et il conseille un labourage profond immédiatement après la moisson. Je ne saurais nier eomplétement l'effieacité de ce moyen; mais je crois qu'un labourage de dix, quinze ou mème vingt eentimètres de profondeur, permettra toujours à l'air de pénétrer dans le sol en quantité suffisante pour entretenir la vie dans une larve de deux millimètres de longueur. Combien d'autres larves d'ailleur's qui vivent dans la terre à une plus grande distaneo de la surface, et qui cependant sont plus grosses et ne sont pas engourdies. Les autres moyens que j’ai à examiner n'empêcheront pas d'employer celui-ci plus tard, et je crois que le résultat final sera le mòme, que l'cnfouisscment de la larve ait lieu tout de suite après la moisson ou un peu plus tard.

Le procédé sur lequel je fonde le plus d'espoir, tant par son effi-

- Cependant il est permis de supposer que la nature du sol n'est pas eomplétement sans influenec; ne serait-il pas très-intéressant de faire des recherches, sur cette aetion, dans les terrains qui eouvrent nolre département (Marnes irisécs, Musehelealk, Oolithe, Lias, ete.). 
cacité réelle que par la facilité de son emploi et le peu de dépense qu'il occasionnera, consiste dans la pratique d'un sartage à feu courant, fait dans les champs de blé immédiatement après la moisson. Pour faciliter la communication du feu et augmenter sa durée. il faudrait couper haut, passer le rouleau après la moisson ou répandre un peu de menue paille sur le champ avant d'y mettre le feu.

Cette méthode. dont l'expérience est facile à faire, a en outre l'avantage de détruire les autres larves d'insectes". nuisibles, tcls que les Chlorops, les Calamobins gracilis, les Cephus pygmoeus, etc. Malheureusement elle a aussi l'inconvénient de ne pouvoir être pratiquée dans les localilés où on a l'habitude de semer du trètle dans les chainps de blé.

L'arrosage, avec un liquide contenant en dissolution uno substance capable de faire périr les larves sans laisscr dans le sol de traccs nuisibles comme cela a lieu avec les chaulages à l'arsenic. ne me paraît pas un moyen cultural profitable, à cause du matériel que cette méthode exigerait cl surtout de la grande quantité de liquide qu'il faudrait employer. On pourrait cependant faire servir à cet usage bien des résidus industriels ordinairement perdus, tels que. par exemple, les eaux du gaz, celles de savonneries, celles des fonderies de suif par le procédé Darcet, du dégraissage des laines, ctc., qui, chargés de matières grasses ou d'huiles cssentielles, sont très-nuisibles aux insectes. En employant des scls de fer, de zinc, etc., et en les faisant dissoudre dans les purins destinés à fumer on attcindrait deux buts à la fois. Enfin on pourrait répandre ces substances sur le sol à l'état pulvérulent ct charger les eaux pluviales de les dissoudre et de les conduire dans la terre.

Dans ces derniers temps, M. Thénard fils a conseillé, pour détruire les insectes qui atlaquent la vigne, l'emploi des tourteaux de colza ou de toute autrc graine de crucifëre. En répandant de pareilles matièrcs sur les terrains où l'on aurait cultivé du blé atleint par la cécidomyie, leur contact avec l'humidité du sol ou avcc les eaux de la pluie, produirait des huiles essentielles dont l'action est extrêmement funeste aux insectes ct à leurs larves.

kien n'empêche d'aillcurs d'avoir recours aux labourages pro- 
fonds dont M. Bazin conseille l'emploi, après que l'on aura fait usage des procédés que jindique.

Dans les questions de la nature de celle qui m'occupe, on est obligé souvent de marcher sans guide, et le hasard peut conduire à une solution plus complète et plus rapide que celle que la théorie nous indique.

Une chose trés-importante et qu'il ne faut pas perdrc de yue. c'est que l'on ne saurait arriver á un résultat satisfaisant que par la pratique des moyens reconnus bons sur une vaste échelle. tel que sur tout un canton à la fois. Tous les efforts individuels ne peuvent amener que le découragement: tous les sacrifices faits par un cultivateur intelligent seront complétement perdus, s'il reste seul, dans son canton, à lutter contre la cécidomyie. A quoi, en effet, peut aboutir l'emploi dc la méthode la meilleure possible. si à côté du clamp quil cultive il se trouvait du blé cécidomyié l'année précédente et que l'on ait rien fait pour y détruire ces insectes.

Dans l'Amérique du Nord, une espèce du même genre. la Cecidomyia desiructor, voyagc quelquefois en essaims si considérables. qu'ils forment comme des nuages. Les chaînes de montagnes lcs plus hautcs. les fleuves les plus larges, ne les arrêtent pas dans leur migration. L'instinct de la reproduction ferait sans doute entreprendrc de parcils voyagcs à notre cécidomyie, et les pays où l'on pratiquerait fructueuscment la chasse à ces insectes, ne tarderaient pas à ĉtre cnvahis par celles qui viendraient des contrées voisines, si pendant quelques années on ralcntissait son activité.

Mais alors. si des mesures d'ensemble sont reconnues indispensablcs, comment en obtenir l'application? et, si pour arriver à ces résultats, il faut faire intervenir l'autorité administrative, n'y a-t-il pas à craindre des cntraves ou des prescriptions peu compatibles arec nos mceurs, nos institutions et surtout nos pratiques agricoles? Ces questions sont sérieuses et méritent de fixer l'attention des hommes plus compétents que moi cn pareille matière.

Maintenant mon devoir est accompli. J'ai signalé le mal qui atteint nos cultures: j’ai suivi l'insecte dans toutes les phascs de son existence, et $j$ 'ai exposé. avec conscience et bonne foi, tous les procédés qui pcurent être employés pour conjurer de plus grands désastres.

C'cst à l'expérience à prononcer, car, en agriculture, moins 
qu'ailleurs, on ne peut déclarer bonnes que les méthodes qui ont été consacrées par elle. Toujours on doit se défier des spéculations de cabinet ou des conséquences d'une théorie trop absolue. C'est pour ne pas avoir toujours bien compris ces choses que la majorité des cultivateurs est en délicatesse avec la science. Que de fois. en effet. les faiseurs et les deni-savants n'ont-ils pas poussé les agronomes à des tentalives inutiles ou onéreuses. ct amené ainsi. cliez les uns le découragement. la haine pour toute innovation chez les autres, et la défiance cliez presque tous.

Tous les procédés indiqués, et ceux qui surgiront sans doute á la suite, ont leurs avantages et leurs inconvénients. Ce n'est que par leur application que l'on pourra faire le choix des meilleurs, les perfectionner et en répandre l'emploi. C'est aux Sociélés d'horticulture. aux Comices agricoles et surtout à l'autorité départementale. qüil convient de prendre l'initiative de ces expériences.

Que chacun se melle donc à l'œurre, et il arrivera un jour où nos efforts seront récompensés, par l'augmentation de la production du sol et par l'affranchissement du tribut des millions d'hectolitres de blé que la France est obligée d'acheter à l'étranger pour sa subsistance. 




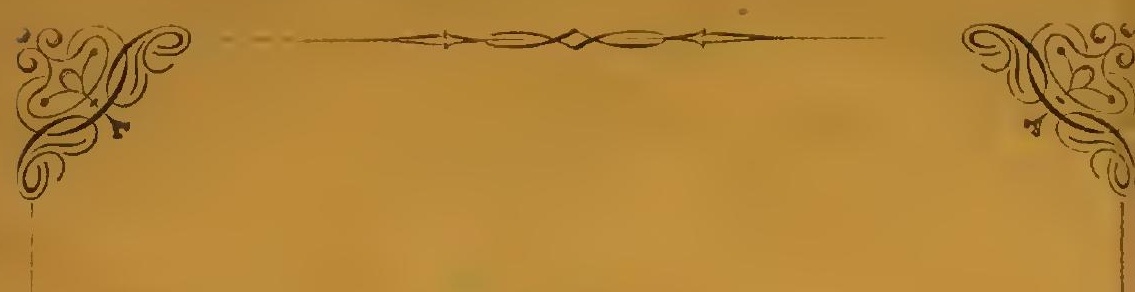

\section{EN VENTE:}

Notes pour servir à l'histoirc des inscctes nuisibles à l'agriculture.

à I'horticulture et á la sylviculturc, daus le département de la Iloselle, par J.-B. GÉHIN.

No 1. - Introduction. - Brochure in- $8^{\circ}$.

No 2. - Inscctes qui attaquent lcs blés. - lirochurc in- $8^{\circ}$.

No $\bar{j}$ (sous presse). - Inscctes parasites du poirier (Coléoptères).

Catalogue des Coléoptères de sa collection: Cicindéliens, - Dytisciens, - Gyriniens. - Dcux brochures in- $8^{\circ}$.

Ciatalog̣ue des Coccinelles observées dans le léparicnent de la Moselle, avec des indications sur leurs mours, les lifux ou elles se trouvent, etc. - Brochure in- $8^{\circ}$.
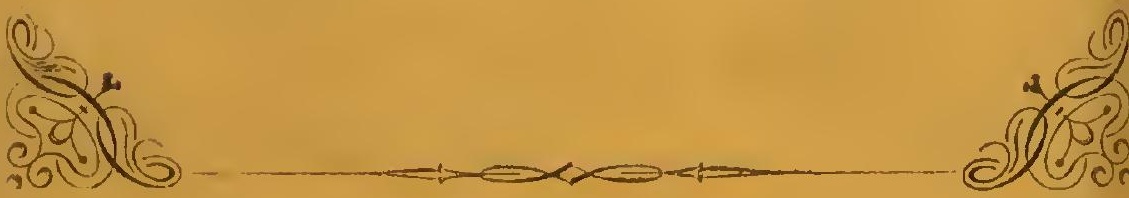
3nk $p^{2}=$

Kehim, macteo niane 
WPHIBIA AND REPTILES

GADOW

* $\quad *$

$\infty$ 


\section{NóTHes}

POUR SERVIR A L'IISTOIRE

\section{DES INSECTES NUISIBLES}

DANS LE

DEPARTEMENT DE IA MOSELEE،

warroum

INSECTES QUI VIVENT SUR LE POIRLER.

urounarm

\section{PREMIERE PARTIE.}

\section{COLÉOPTÈRES.}

Par J. - B. Géhin, Puarmacien,

Membre de la Société d'Ilistoire baturelle de la Moselle.

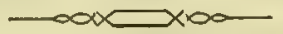

Au mois de septembre 1855 , la Société d'horticulture dı département de la Moselle me faisait l'honneur de me consulter sur l'origine et la nature d'une sorte de galle, qui avait envahi les feuilles des Poiriers d'un grand nombre de jardins de Metz et des autres parties du département.

Étant, jusqu'alors, resté étranger à ces sortes de recherehes, et, trompé par l'apparence toute partieulière de ees cxeroissanees, j'en attribuai le développement au travail d'un insecte, et je considérai leur présence, connme la conséquence des piquires faites par les femelles, pour dèposer leurs cufs dans le parenchyme de la feuille, et préparer ainsi les aliments néeessaires à la subsistanee de la jeune larve.

Dans le rapport que jindressai a ce sujet à la Sociéte

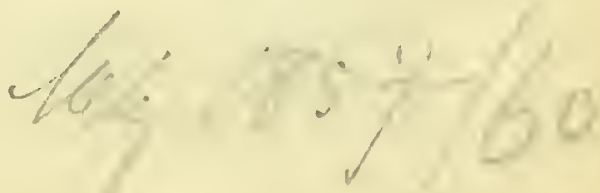


d'horticulture, tout en reconnaissant que ces végétations parasites étaient ducs au développencnt de l'OEcidium cancellatum, de Persoon, j’avais eneore fait des réserves sur l'origine de ee Cryptogame. Malgré la vicille expérience du naturaliste de Saint-Scver, mais entrainé par une grantle ressemblance avee certaines galles, par des iclées préconçues, et aussi par des doutcs exprimés par des entomologistes d'un grand mérite, je croyais pouvoir découvrir la trace de quelque inseetc, lor's de l'apparition du parasite des feuilles du Poirier. Aujourd'lıui, le doutc n'est plus permis, ct, gràce à l'obligeanec de plusieurs propriétaires des environs, j’ai pu, en 1856 , suivre le développement de l'ODeidinm cancellatum, depuis le moment où il apparait sur la feuille, jusquau moment où ses sporules se dispcrsent pour l'eproduire, l'annce suivante, de nouvelles galles, sur les nouvelles feuilles du Poirier.

Comment lc Myeellium de ec parasite pénètre-t-il toutes les parties de l'arbre, pour ne développer ses eonecptacles que sur les parties vertes ou licrbaeées ıc la plante? C'est là une mystéricusc reproduction pou. les Gryptogames épiphytiques qui est eneore entouréc de beaueoup d'obseurité, et les travaux des mycolognes les plus distingués n’ont pas eneore élucidé la marche de ectte singulière végétation. A ce sujet, jc me lıả c de faire une rectification importantc sur les moyens que jai indiqués pour la destruetion de ees Champignons. Celui que je eonsidère eomme lc seul pratieable et le scul efficace, d'après les cxpéricnces faites en 1853 et 1836 , ècst la combustion de toutes les feuilles atteintes. On aura soin de les enlever avant la maturité des sporules, en aoùt, pour la plus grande partie, et après la récolte des fruits, pour le restc.

Pour en finir avee ect OEcidium, je dirai que, malgré toutes mes reeherches, je n'ai trouvé qu'un seul insectc dans les conceptacles de plusicur's centaines de ces Ciryplogames. 
C'est un petit Staplıylinien, de la tribu des Sténides (le Stenus juno de Gravensh.). Pour ecux qui connaissent les mœurs de ees insectes, il est évident que ce n'est que par hasard qu'il se trouvait là, et qu'on ne saurait lui attribuer aucune part dans la production ou le développement du Champignon parasite des feuilles du Poirier.

Mais avant d'arriver aux conclusions qui précèdent, j'ai dù observer la marelıe du phénoméne, et me convainere quaucun des insectes signalés par les auteurs, comme vivant en parasites sur les Poiriers, ne contribue en rien à la manifestation de l'invasion eryptogamique.

C'est en faisant des reelıerches à ce sujet que je ne tardai pas à reconnaitre, qu'en France du moins, les travaux sur les inseetes nuisibles avaient été singulièrement négligés, et que, depuis les mémoires des grands naturalistes du siècle dernier, les horticulteurs et les entomologistes de notre pays, s'étaient en général fort peu préoceupés de l'étude des mours des insectes nuisibles aux arbres fruitiers, à ceux des forèts, aux plantes utiles ou à celles d'agrément (1).

C'est alors que je formai le projet de réunir les observations recueillies par moi depuis longtemps, et de publicr un essai sur les insectes nuisibles au Poirier. Pour justifier l'opportunité d'un travail le cette nature, je vais eiter et examincr quelques uns des principaux articles publiés en France, depuis une vingtaine d'années, coneernant les insectes nuisibles au Poirier (2).

(1) Il faut toutefois fairo exception pour le travail remarquable et tout a fait hors ligne que publie en ce moment M. Ed. Penris, sur les insectes du Pin maritime. Voyez annales de la Société entomologique de France, annécs $1854,1855,1856$.

(2) Pour avoir unc liste à peu près complètc des travaux spéciaux de cette nalure publiés avant 1836 , voyez: Pencuenon, Bibliographic entomologique, Paris, 1837, 2 vol. in-80. 
1' 1836. - Ann. de la Socicté entomologique de Franee; page LXX du bulletin.

Audouin y signale une larve de Coléoptère (Serricorne), qui se creuse des galeries lans les jeunes branclıes du Poirier.

$2^{\circ}$ 1838. - Mímoire de l'Aeadémic des Seienees et BellesLeltres de Dijon; page 63 .

M. Vallot y public un mémoire sur les fausses galles des fleurs du Pommier et du Poirier. "An printemps, dit M. Vallot, on peut voir, sur les Pommicrs et les fooiriers de nos jardins, des fleurs qui ne s’épanouissent pas; leurs pétales restent unis, présentent une couleur rousse, et forment alors le clou de girofle. ".... et plus loin " $A$ yant suivi le développenent de ecte larve, rangée par le vulgaire parmi celles appelées Mazars (du nom patoi Mazar, mangeur), j'ai obtenu l'insecte à la fin de mai : e'est un insecte appelé Clıarançon brun à écusson blane, par Geolfroy ; - Curculio pomorum, par Linné en 176k; - Cureulio fusens , par Gmèlin, en 1762; - Cureulio seutellaris, dans l'encyclopédic d'llistoire naturelle, tome $\mathrm{V}$, page $\$ 21$; - Cureulio brunnirostris, par Olivier; - Rlıynehœnus pomorum, par Olivier en 1789; - Erirhinus seirrhosus, par Selıcnherr. „

Je n'ai pas vu l'inseete dont veut parter II. Vallot; mais, par ce qu'il dit de ses mours, on doit penser qu'il a voulu déerire l'Anthonomus pomorum de Germar, lequel est en effet le Curculio pomorum, de Linné, mais qui est bien différent du Cureulio fuseus, de Gınélin, ineonnu à Selıonlıerr lui-nème; ce ne peut ètre non plus le Curculio fuseus, de Degéer, car celui-ci appartient au genre Errivhinus, de Sehoenlı., et les insectes de ee genre ont des mours qui ne laissent aucun doute sur leur différenee avec eclui qui a été signalé a l'Aeadémic de: Dijon.

Le Cureulio brumuirostris, d'Olivier, est bien l'Erirhimus 
scimhosus, de Schoenherr, mais ee n'est pas l'Anthonomus pomorum, de Germar, dont M. Vallot a voulu établir la synonymic. Je doute d'ailleurs que l'Erirhinus, dont il est question, vive sur les arbres fruitiers, ear, outre que je ne l'y ai jamais rencontré, Gyllenlıal, qui connaissait bicı les mœurs des insectes de son pays, dit positivement, à propos de ce Clıaranẹon : "habilat locis humentibus, " indieation qui s'iccorde parfaitement avec ec que l'on sait des mours de eet insecte et de celles de ses congénères Sparganii, Festuca, Caricis, Nerois, Palustris, ctc., ctc., qui vivent en effet surles plantes aquatiques ou marćcageuses.

$5^{\circ}$ 1838. - Encyclopédic d'histoire nalurelle; tome VII, page 311 .

On y lit: "Il y a un Charançon, dont la larve habite dans Ies fleurs du Poirier, qui les empèche de s'épanouir el de donner des fruits."

II est probable que e'est encore de l'Anthonomus pomortum dont il est question; mais de quelle manière eette larve arrive-t-elle dans les boutons à fleurs? Quel est le inoyen de la détruire ou d'en diminuer le nombre... ete.? - Telles sont les questions dont la solution importe plus particulièrement à l'arboriculteur, el dont il n'est pas fait mention daus l'article de l'Encyclopédic.

4' 2839. - Dielionnaire pilloresque d' Iistoire naturclle, de M. Guérin Menneville; tome VIII, page 195, article Pounen.

Comme insectes particulièrement nuisibles à cet arbre, on: cite seulement le Tigre ou Puecron des Poiricrs (Tingis pyri, des entomologistes) et la Punaise à fraise antique de Geoffroy.

Au tome IX, page 507, du mẻme ouvrage, el à l'article TenTunk̀bides, on cite eneore, comıne étant nuisibles aux Poiniers, la larve ou fausse Cienille de l'Allantus OEthiops, Je Pabrieius; e'est le Ver limace decrit par Degéer dans ses némoires. 
$5^{0}$ 1839. - Nulsant; IIstoirc naturelle des Coliopteres de France; $1^{\text {re }}$ partic, Longicornes.

Dans cette monographic, M. Mulsant indique plusicurs espèces dont les larves vivent daus le Poiricr, telles que: Polyopsia prousta et Phylacia nigricornis, de Linné, et d'autres qui habitent dans les arbres fruiticrs en générar; mais l'autcur n'entre dans aucuns détails sur leurs mour's, la uature des dommages qu'clles ocensionnent, ete., ctc.

$6^{\circ}$ 1840. - Suites à Buffon; édit. Dumẻril.

M. E. Blanchard, qui a fait l'histoire des insectes compris dans ect ouvrage, y signale quelques espèces comme vivant sur le Poiricr, et plus particulièrement : Psylla pyri de Burmeister (Chermes pyri, de Linné) qui se trouve dans la plus grande partic de l'Europe.

$7^{0}$ 1844. - Annales de la Société cnlomologique de France; tome II, 2 séric, page $4 \overline{5} 1$.

Dans ce volume, M. Goureau donne des détails fort intéressants sur la larve de la Saperda scalaris, qu'il a trouvé sur un trone de Poiricr.

M. Gourcau ajoutc cncore : "Lc '̉ mai 1853, ayant soulevé un grand fragment d'écorce à un Poiricr abattu depuis plusicurs mois, je vis une multitude d'insectes de différentes espéces: il y avait en abondance des Ditoma erenata; Sylvanus unidentatus; Platypus eylindrus; Ilololepta depressa, et en outre, un nombre presqu'infini de larves et de clirysalides d'unc espèce de Seolylus d'une taille intermédiaite entre le Destructor et l'Intricatus. On y distinguait aussi des larves et des chrysalides d'une dimension beaucoup plus grande, appartenant au Leiopus nebulosus et à la Saperdu sealaris, qui se tenaient sous l'écorce inème, ou enfoncées dans l'aubier. Je ne dois pas omettre de dire quau milieu de ecte multitude pacifique, se trouvaieut plusieurs larves d'Elater, d'une 
lumeur moins douce, vivant à discrétion au seill de la plus grande abondance, et dévorant celles de ces larves qui lcur convenaient."

$8^{0}$ 1845. - IIisloire des insecles, par NI. Emile Blanchard.

Dans eet ouvrage, on trouve une grande quantité de détails sur les mœurs de beaucoup d'insectes de tous les ordres. Voici eependant tout ce quil contient, relativement à ceux qui vivent sur le Poirier :

Tome I, page 58. "Le genre Ynosiemma, renferme parlieulièrement une espéce (Ynostemma Boscii, Lepelletier), qui a été signalée comme vivant sur les Poiriers ; mais il nous parait plus probable que eet Himénoptere, venait attaquer d'autres insectes habitant ees arbres fruitiers."

Page 190. "On a décrit plusicurs larves de Lydas; quelques-unes d'entre elles vivent sur le Poirier, telle est la Lyda des forcts (Lyda sylvalica, Fabr.)."

Tome Il, page 80. "Une espèce voisine (Agrilus pyri, Blanclıard) passe ses premicrs élats sur les branclıcs du Poirier."

Page 86. " M. Waterhouse a fait connaitre la larre du Dasyle serricorne (Dasyles serricornis, Parreys); clle vit sur le Poirier."

Page 122. "L'Anthonome des Polmniers (Anthonomus pomorum, Selı.) attaque les flcurs, et quelquefois les fruits, des Pommicrs et des Poiriers."

Page 408. "Le type du genre Carpocapsa (la Carpocupsa pomonana, Linn.) est très-nuisible aux fruits, sa clıenille vivant dans l'intéricur des poires et des pommes. "

Page 410. "L'IIyponomeula padella $\left(F_{\text {. }}\right)$, dévaste de la mème manic̀re que l'Iyponomeula evonymella (Linn.), les Poirier's et les Pommiers. "

$9^{\circ}$ 1846. - De le laille du Poirier el du Pommier en fuscau, par Clıoppiu (Bar-lc-Duc, 1846).

Au Chap. IV, l'auteur cite conme animaux nuisibles aux 
Poiriers: $1^{\circ}$ les Pucerons ; $2^{\circ}$ les Punaises grosses et petites; $3^{0}$ diverses especes de Clicnilles; $4^{\circ}$ enfin des petits Vers.

10 1848. - Cours de Zoologie Forestière, par Augustc Mathicu, professenr à l'école impériale forestière de Nancy.

On trouve, dans eet ouvrage, les renseignements suivants, coneeruant les inseetes du Poirier :

Tome II, page 15. "Les Sinodendres vivent, à l'état de larve, dans les Clıènes et les Poiriers. "

Page 94. a Les Anthonomes hivernent à l'état parfait, sous les ćeorces et au piel des arbres; au pritltemps, ils se trouvent sur les Pommiers, les Poiriers, suivant les espèces. „

Page 204. " La Vanesse grande tortue, est quelquefois assez nuisible en rongeant les feuilles des Ormes, eelles des Poiriers, ete.

Page 219. "La Chenille du Bombyx livrée (Bombyx neustria, Lin.) attaque tous les arbres fruitiers, mais partieuliirement les Pommiers, Ies Poiriers , ete."

Page 230̈. "Les Chenilles de ees teignes (Tinea padella, Lin.; Cognatella, Hubner, ete.) vivent sur différents végétaux : le Fusain, le Cerisicr, le Poirier, ete. ".... " elles y forment des sociétés quelquefois tellement nombreuses, que l'arbre parait enveloppé d'une seulo toile, et est dépouillé de toutes ses feuilles."

$11^{\circ}$ 1851. - Dubreuil; Cours élémentaire dhorticulture.

Le savant professeur d'arboriculture signale, eonnme nuisibles aux arbres à fruits, à pépins : "le Tigre, le Ilanneton, Ies Clıarançons de plusieurs espèees, dont nous citerons particulièrment deux, l'un gris, l'autre vert, connus sous les noms de Liselle el de Coupe-bourgeon...., diverses Chenilles, partieulièrement la Chenille (sie) d'une Teutlıridide...., d'autres Vers, eonıus des jarliniers, sous les noms de Vers, Verdelets, ete., et qui rongent les sommités des bourgeons." 
Enfin, M. Dubreuil cite encore a les Peree-oreilles, les Fourmis, les Guêpes, les Frelons, les Puecrons, la Pomonelle, et plusieurs Papillons."

$12^{\circ}$ 1851. - Maequart; Annales de la Soeièté des Seiences el Arts de Lille. Cutalogue des Inscetes nuisibles aux arbres pruitiers.

Dans ee travail, l'illustre diptérologiste de Lille, signale un grand nombre d'especees de tous les ordres, comme vivant sur le Poirier. Aux espèees déjả mentionnées darıs les ouvrages que je viens de eiter, il faut eneore ajouter les suivantes: Capnodis tenebrieosa (Fabr.); - Agrilus viridis (Fabr.);-IIololepta depressa (Fabr.);-Osmoderma eremita (Fabr.); - Mecinus pyrasicr (Herbst.); - Phyllobius calearalus (Fabr.); - Bitoma erenata (Fabr.); - Platypus cyliudrus(Fabr.);-Sylvanus unidcntatus (Fabr.); -Leiopus ncbulosus (Fabr.); - Pamphilus pyri (Fabr.); - Aphis pyri (Fonseol.); - Capsus pyri (Lin.); - Phylocoris magnicornis (Fabr.); - Aradia lubrieipeda (Fabr.); Bombyx quercus (Fabr.); - Eriogaster evcria (Fabr.); Lophopteryx camclina(Lin.); - IIymcria pennaria(Lin.) ; - Melanthia fuctuaria (Boișl.); - Glyphypterix bergastrclla (Fabr.); - Eluehista serratella (Fabr.); - Tinea angustclla (Costa.); - Tinca einclella (Fabr.); - enfin, Che manophila gclatella (Lin.). - Les renseignements fournis par Maequart, sont, du reste, extrèmement courts, et portent plutôt sur l'habitat des inseetes ou eclui de leurs larves, que sur leurs.mours ; et les moyens de les détruire y sont d'ailleurs complètement négligés.

15० 1855. - Cour's complet d'Agrieulture, par une so. ciété de savants; ouvrage en voie de publication.

On trouve au tome $X V$, page 11, à l'artiele Poirier, et eomme insectes nuisilhles ì eet arbre, les espèces suivantes : " le Tigre; 
un Charançon qui, au printemps, dévore les bourgcons; I'Attelabe alliairc (Rynthites eonicus, Illig.), qui coupe les pétioles des feuilles naissantes; enfin diverses Chenilles mangent les feuilles, et d'autres insectes les fruits."

A cette liste, on peut cncore ajouter les ouvrages spéciaux publiés, sur les Lépidoptères, par MM. Duponchel, Godarl, Boisduval, etc., etc., et dans lesquels on trouve une foule d'indications, concernant les mœurs des Chenilles qui vivent sur les arbres fruitiers en général, ou sur le Poirier cxelusivement. Enfin, quoique non publié cn France, mais parec que ce travail est écrit en français et dans une localité voisine de notre département, je dois surtout signaler un Cataloguc très-intércssant, de tous les végétaux naturcls et cultivés dans les environs de Trèves, avec la liste correspondante de toutes les espèces de Chenilles qui vivent sur eliacun d'eux (1). Je reviendrai d'ailleurs sur ces divers ouvrages lorsque je ferail'listoirc des Lépidoptères, parce que les insectes de cet ordre, qui vivent sur le Poiricr, sont de tous, les plus nombrcux, et ceux dont on remarque plus particulic̀rement la multiplication dans certaines années.

D'après les eitations qui préeèdent, il est facilc de voir combien, en général, les renseignements sont incomplets, disséminés dans une foule d'ouvrages peu répandus, ou noyés dans des descriptions seientifiques, où les intéressés ne sauraient aller les découvrir. D’autre part, les détails donnés par les entomologistes sont trop courts, trop superficicls, ct on voit que, presque toujours, ils ne sont fournis que dans le but de fuciliter la recherehe des espèces dont il est question, mais non d'éclairer le lectcur sur la nature de leurs

(1) Bulletin de la Société des Scienees naturelles rle Luxembourg; annẻe 1854 , page 106, par M. Hymmen de Trèves. 
travaux. Les indications fournies par les horticulteurs, sont, au contraire, remplies de fautes synonymiques, et manquent ordinairement des détails nécessaires pour faire reconnaitre l'insecte dont il est question. Les uns et les autres, enfin, passent sous silence la partic pratique de la clıose, l'indication des moyens employés pour liıniter les dégàts, ou ceux à employer pour amoindrir lc dommage que nous eausent ces petits animaux.

Les immortels travaux de Réaumur ont pendant longtemps rendu inutiles de nouvelles études sur les mœurs tles insectes, alor's surtout que l'anatomie, la elassification et le catalogue de nos richesses étaient encore à faire. Mais, si, sous ee rapport, l'Entomologie française n'a rien à crivier à la seience des autres pays, nous devons eependant reconnaitre qu'en Angleterre et en Allemagne, surtout, l'Entomologie pratique et appliquée y ont aequis un développement à la lıuteur des progrès accomplis, dans ces deux pays, en agriculture et en horticulture.

Chez nous, d'ailleurs, les nécessités de la vie et le peu de eas que semble fairc le public pour ces sortes de travaux, en éloignent bien du monde; beaucoup d'entomologistes possèdent de précieux documents sur la vie et les métamorploses d'une foule d'insectes, mais ils négligent de les publier, dans la erainte, hélas! peut-ètre trop réclle, de ne pas ćtre écoutés, et de donner des conseils inutiles. Malgré cette sorte d'indifférence, on finira, tòt ou tard, par recommaitre son erreur; car si l'on parvient un jour à donter des moyens récllement infaillibles et pratieables pour se débarrasser d'un insectc nuisible, ee sera bien certainement a l'Entomologie qu'on le dlevra, parce qu'avant tout, il faut connaitre à fond les mours de l'ennemi que l'on veut combattre, et qu'il n'y a qu'un ertomologiste qui soit en état de les découvrir et de les apprécier convenablement. 
En Allemagne, il est loin d'en ètre ainsi, et il suffit de citcr lesouvrages de Bouché (1); le Freyer (2); de Ratzburg (5); de Schmitberger (4); cnfin de Noerdlinger (5), pour justifier ec que je disais tout à l'heure, des progrès accomplis dans ec pays par l'Entomologic appliquée, progrès intimement liés lu reste, à ceux de l'agriculture.

Mais c'est à peine si ees travaux, malgré leur mérite scientifique incontestable, sont comnus en France par les entomologistes, et l'on peut affirmer qu’ils y sont complëtement inconuus aux horticulteurs. D’ailleurs, plusicurs de ees publications présentent également les imperfections signalées plus haut, et M. Noerdlinger, qui peut à bon droit passer pour le plus heureux de tous ecux cqui ont traité ec sujet, est loin de comprendre, dans son ouvrage, toutes les espèces qui vivent sur le Poirier. Cet auteur, en effet, qui a le méritc d'avoir fait son travail au double point de vae de la science et de la pratique, ne mentionne que 70 espèces environ, comme étant nuisibles au Poirier, tandis que, d'après mes recherches bibliographiques, ma correspondance avec les entomologistes du premicr mérite, et mes observations personnclles, j’ai pu cataloguer plus de 1300 espèces d'insectes de tous les ordres, comme vivant aux dèpens du Poirier. C'est done plus de deux fois autant d'espèces que n'en signale le professeur de Stutgard, et le nombre devra encore en étre augmente, quand des recherches suivies auront été fuites it la fois, par les jardinier's et les entomologistes.

(1) Naturgeschicht Jer Schedlichen und etce; in-8 $8^{\circ}$, Berlin 1833.

(2) Dic Schxdlichen Schmottcrling, clc.; Augsbourg, 1837 ,

(3) Die Forst insccten, etc,; Bonn., 1839.

(4) Betreigt zur obst baume zuch und zur Naturgeschicht, etc.; Lintz, 1821 1824-1827-1828-1831.

(5)Die klein Feinde der Landwirschaft, cte. ; Stuttgard, 1855. 
Licrire l'histoire de toutes ces espèces, faire connaitre la nature et l'élenclue du mal qu'clles peuvent nous causer, exposer les moyens de les arrêter ou de les détruire, est unc táche, pour l'accomplissement de laquelle il faut de longues anncies, et surtout des connaissances entomologiques et horticoles plus étendues que les miennes. Cependant, et malgré mon insuffisariec, je crois pouvoir encore c̀tre de quelqu'utilité en publiant le résumé de tout ce qui a été écrit à ce sujet, et en ajoutant mes observations personnelles, à cet inventaire, aussi complet que possible, de nos connaissances dans cettc partic de l'Entomologic pratique.

Conme il est facile de le penser, toutes les especes d'insectes signalćes comme étant nuisibles aux Poiriers, ne le sont pas au même dégré, ni de la mème maniẹ̀re ; aussi les détails que je me propose de donner sur quelques insectes, seront-ils étendus en raison de l'importance de l'organe attaqué, de la fréquence de cette circonstance, ou de l'abondance des individus qui les produisent. Dautre part, voulant surtout donner un cachet d'intérét local à mon travail, je traiterai avee moins de détrils, l'histoire des espèces qui n'ont pas encore été signalées dans nos contrces, mais qui font partic d'une faune plus méridionale, ou qui apparticnnent à un autre continent.

A tous les áges et dans toutes les phases de sa végétation, le Poirier est la proie des insectes nuisibles. Le Melolontha vulgaris, en attaque les racines; les Rhynchiles auralus, bacclus, cuprouts, etc., en attaquent les jeunes bourgeons; les Anthonomus pomorum, pyri, etc., en percent les boutons à fleur's; la Carpocapsa pomonana, en perfore les jeunes fruits; les Polydrosus, les Phyllobius, et unc foule de Chenilles, la fausse Chenille de l'Allantus othtiops, en mangent les feuilles pendant le jour; les Otiorlhynchus picipes et 
raueus, pendant la nuit; la Formica rufa, la Vespa vulgaris, entament ses fruits quand ils sont múrs. Sous les écorces des vicux sujets, ou dans les trous de ceux qui sont cariès, vivent les Sinodendion cylindricum, la Saperda scalaris, tandis que les jeunes pousses sont envahies par les Pucerons, les Chermès et les Psylles, qui en absorbent la sc̀ve à mesure qu'clle y aflluc. Ce sont là de véritables insectes nuisibles, et ecux dont il importe le plus de connaitre les moeurs et les habitudes.

Mais à còté de tous ecs insectes, la providence en a placé d'autres qui sont destinés à en limiter le nombre, soit en déposant leurs œufs sur leurs larves ou sur leurs Chenilles, soit en produisant des larves ou des insectes earnassicrs, dont la voracité amène la suppression d'un grand nombre des premicrs. Ces insectes sont done, en quelque sorte, les protceteurs de nos arbres fruiticrs, et lcur histoire est aussi utile à connaitre que celles des espèces nuisibles auxquelles ils font la guerre. Mais, pour ne pas étendre trop ec travail, je ne parlerai des insectes de cette catégoric, que lorsqu'ils auront été signalés par des autcurs qui se sont trompès sur leurs mours, ou quand il y aura encore quelques doutes sur leur manic̀re de vivre à différents états.

Enfin, quand les arbres sont mal soignés, que leur écorec se couvre de mousse et de lichens; quand ils sont malades ou vieux, et que leur trone sc caric, une foule dinsectes de tous les ordres y viennent ehereher un refuge contre les intcmpérics, durant l'été, ou contre les rigucurs du froid, durant l'hiver; d'autres, au contrairc, viennent faire la cliasse à ccux qui s'y trouvent, qu'ils soient nuisibles ou protecteurs; quelquefois, aussi, des femelles viennent pondre leurs œufs dans les abris que leur offrent ces trones malades ou mal entretenus, 0 n comprend sans peine que l'histoire des insectes de cette eatégoric 
ne doit pas figurer dans un travail du genre de eelui-ci; et que la vie d'un Aphodius trouvé sous l'ceoree d'un Poirier', est aussi independante de la vie de eet arbre, que eelle de labeille qui vient sur la fleur pour en butiner le pollen (1).

Certaines esprees sont malfiisantes dans plusieurs périodes de leur's transformations; ainsi les Eccoptogaster pruni ct rugulosus, ereusent des galeries dans le bois à l'état de larve et a l'ètat d'inseetes parfaits; le Hanneton mange les feuilles de l'arbre, tandis que sa larve en ronge les raeines. Les Fourmis, les Forficules, les Guépes, ne sont nuisibles qüi l'état parfait, ct les Lépirloptéres, quà l'état de Chenilles; d'autres inseetes, au eontraire, ne sont nuisibles que pour proeurer í leur progéniture les aliments nécessaires à leur dévéloppement, lorsqu'arrivera le temps de l'éclosion des aufs. II est done important de faire eonnaitre, autant que possible, les diverses pliases des métamorphoses de ees petits inscetes nuisibles, et surtout de bieı préciser l'état sous lequel ils sont le plus à redouter.

Pour beaucoup de personnes, les renseignements préeis semblent inutiles ou superflus; e'est à ce défaut d'exactiturle qu'il faut attribuer les indications vagues et générales, fournies par plusicurs auteurs qui se contentent de dire: ectle espèee vit sur les arbres; se trouve sur les arbres fruitiers; ou bien, dans les vergers, dans les jardins, ete., etc. Jai négligé tous les renseignements de eette nature, et n'ai eompris sur ma liste que les espèces observées par moi sur le Poirier, ou signalćes par des indieations préeises des auteurs compétents.

Malgrẻ eette réserve, plusicurs espéees semblent eneore

(1) Bien entendu qu'ici je ne fais qu'envisager la question an point de vue entomologique, et que je n'enlends pas m'oecuper de l'influence que doit avoir le pollen de diverses fleurs d'arbres d'espéces voisines, sur uno variélé particulière, la conservation de ses quualités, ete. 
devoir être rangées dans la troisième des eatégories que jai établies plus lıaut, mais j'ai voulu respecter l'autorité de noms célèbres, en laissant, à ees auteurs, la responsabilité des faits qu'ils signalent, et en mie réservant de faire connaitre mes doutes, quand je parlerai de ees insectes.

C'est particulièrement pour les espèces qui vivent dans le trone des vieux arbres et dans la vermoulure de leui cavité, que l'on peut concevoir le plus d'incertitude sur la nature des clommages qu'elles ocensionnent. En effet, si les larves ou les iusectes vivent de la substance mème du terreau, il est évident que l'arbre ne saurait souffrir de leur présence; mais si ces larves on ces insectes, déchirent le bois avec leurs mandibules pour en extraire le sue, ou pour y attirer la sève, il est certain qu'ils devront eauser un dommage considérable aux sujets aux dépends desquels ils se nourrissent (1).

J'ai déjả eu oceasion de faire remarquer combien ćtaient incomplets les renseignements fournis par les entomologistes,

(1) Le terreau (ulmine, acide ulmique etc., dcs climistes) se furme comme on sait, par un phénomène d'érémacausie ou de combustion lente, par la combinaison de l'oxigène de lair, avec les écéments combustibles du bois (Carbone et Ilydrogène), et de laquelle combinaison il résultc particulièreinent de l'eau et de l'acide carbonique. Les larves ou les insectes qui vivent dans le terreau, sont donc continuellement plongés dans une atmosphère de ce gaz, et cela quelquefois pendant plusieurs années. Il y a, dans ces conditions particulières a la vie de ces petits animaux, un phénomène pliysiologique, qui ne semble pas encore avoir été étudié par les naturalistes, et duquel il paraît résulter que l'acide carbonique n'agit pas sur les insectes, comme sur les animaux des classes plus élevées, que même, il tue par absorption.

La nutrition des insectes au inoyen dlu terreau est d'ailleurs facile à expliquer, par l'action dissolvante propres aux liqueurs alcalines qu'ils excrétent par la bouche, et qui transforment ainsi l'acide ulmique du terreau, en ulmate soluble et assinilable. Il est très-remarquable de voir la même substance servir à la nutrition des animaux et des végétaus, par suite d'une méme combinaison dont elle forme l'ćlément électro-positif. 
et d'autre part, combien étaient incxactes ou insuffisantes, les indieations synonymiques et les deseriptions données par les lıorticulteurs. Jai chereiıé à éviter ec double défaut; et, sans faire ici un travail monographique, j’ai donné la synonymic la plus complète et la plus exacte possible, en prenant pour guiles MM. Mulsant, pour les Lamellicornes et les Longieornes ; Sclıonlıerr, pour les Curculionites; Gory ou Ratzburg, pour les Sternoxes; Blanchard ou F'onseolombe, pour les Aphidiens ; Macquard, pour les Diptères; Boisduval ou Duponelıcl, pour les Lipidoptères, ete., cte. IIes deseriptions seront anssi courtes que possible, et, eependant, suffisantes pour faire reconnaitre l'insecte, et bien préciser l'espéce dont je veux parler.

Souvent il arrive que plusicurs espèes d'un mème genre ont des mœurs, si non semblables, au moins fort analogues: e'est ce qui a souvent amenć bien de la confusion, et a fait attribuer à un seul insecte, les mœur's de plusicurs de ses congénères. G'est dans le but d'éviter ectte erreur que j’ai cru devoir donner les earactères génériques de toutes les espèces que je déeris, el, à la suitc de ces deseriptions, présenter un ensemble de tout ee qui est commun aux espices d'un mème genre.

Un mot, maintenant, sur l'arbre dout je me propose de faire l'histoire entomologique; sur les diverses conditions dans lesquelles il se trouve daus notre département, et sur les prineipales variétés qui y sont plus partieulièrement eultivées.

Lc Poirier (Pyrus Communis. L.) est un arhre d'une importanee trés-considérable dans eertaines contrées de la Franec, où on cultive surtout les variétés dont les fruits servent à la fabrication du cidre. Dans le département de la Moselle, où la vigne est eultivéc dans une assez grande étendue de territoire, la boisson si chère aux Normands̀ n'est consomméc 
quedans la partic allemande de l'arrondissement de Thionville, et dans presque loute l'étendue de eclui de Sarreguemines. Il y a 25 ou 50 ans, on y cultivait a lors, dans une plus grande proportion qu'aujourd'lui, l'espèce sauvage et quelques-unes Je ses variélés. Je n'ai prl connastre les raisons qui avaient amené l'abandon de eelle production, dont on retrouve cneore des traees dans les nombreux Poiricrs disséminés dans les autres eultures de cette partie de la Noselle, d'où ils ne tardcront pas à disparaitre, à canse de leur vétusté ct du peu de soins dont ils sont l'ohjet.

Dans les environs de Metz, e'est surtout eomme arbre à fruit de table qu'on y cultive le Poirier; $\mathfrak{e l}$, quoique restreint ì eet usage, il oeeupe eneore une place importante dans nos eultures; ct le nombre des variétés qu'on y élève, llépasse certainement la eentaine.

C'est prineipalement sur les étes de la rive gauehe de la Moselle, entre Metz et llayange, et plus spéeialement à Smécourl, Fèves, Marange, Sylvange, Rombas, ete., que le Poirier est cultivé en plus grande quantité, et ce sout les produits de ces loralités qui alimentent les mareliés de Metz.

A l'ćtat sauvage, le Poirier existe dans toutes les forèts du département, et e'est l’ả que l'on va cliercher les sujets sü lesquels on greffe les variétés cultivées. Dans le Luxembourg et la Prusse Rliénanc, cet arbre y cst mème assez abondant pour qu'on en recueille les fruits qui servent ensuite a fabriquer une eau-de-vie de poire, assez estimée daus ces contrées.

Le Poirier est assez rustique, et il eroit bien dans tous les. terrains de la couche cultivable du département; dans les terres argileuses fortes, il est génèralement plus vigoureux que dans les terrains ealeaires. En somme, il parait que, dans notre elimat, ee sont les terrains argilo-calcuires qui lui conviennent le inicux. 
Depuis plusieurs annécs, une maladic particuliẻre sévit sur un grand nombre de variétés aneiennes; malgré toutes les recherehes des lıortieulteurs et des cntomologistes, la cause de cette maladie reste eneore inconnue, et ses progrès eonstants menacent de stérilité tous les Poiriers de nos environs. J'ai eu beau en explorer un grand nombre et des plus malades, je n'ai pu y découvirir aucune espèee d'insecte assez fréquente el assez abondante, pour qu'on puit raisonnablement lui attribuer une part queleonque dans la propagation de ectle sorte de brülure.

Jusqu’a présent, il ne me semble pas que telle variété soit, plutót que telle autie, plus souvent ou plus fortement attaquée par les insectes; mais il me parait que le mode de culture inllue, au eontraire, considérablement sur lcur propagation et leur multiplication.

Dans les jardins, ees arbres sont en général l'objet de soins plus constants : les visitant souvent, on s'aperȩoit vite de l'attaque des insectes, et on se hate d'y porter reméde. Par la taille et par l'ébourgeonnement, on supprime beaucoup de jeunes branelıes, et on enlève ainsi les aliments de bon nombre d'espèces qui vivent dans l'iltéricur de ees organes, tels que Agrilus, Polyopsia, etc.; enfin, la propreté dans laquelle les jardiniers soigneux entretiennent le trone des arbres, eontribue aussi à produire ces résultats, ear en raclant la mousse et les lichens, en bouchant les erevasses, ete., ils enlèvent aux inscetes ou à leurs larves, l'abri que ceux-ci trouvent sur des arbres mal cntretcnus, lorsque les intempéries les forcent à quitter les fleurs, les feuilles, les botrgeons ou les fruits sur lesquels ils exercent leurs ravages.

La forme que l'on donne aux arbres ne parait pas uon plus ctre sans influence; ainsi, leTigre (Tingis pyri) et le ver Limace (Allanlus whiops) altaquent de préférence les Poiriers 
eultivés en espalier. Les Chenilles des Bombyx dispar et Chrysorrhoa les ípargnent, au eontraire, et se rencontrent plus ordinairement sur les liauts-vents. L'Apion pomonœ et l'Anthonomus pomorum paraissent préférer les arbres élcvés en quenouille ou cu cordons. En général, les hauts-vents sont plus exposés que les autres aux ravages des insectes, soit à eause des soirs qui leur manquent, soit par leur agglomération sur une grande étendue de terrain, quand ils sont l'objet d'une eulture spéeiale, comme par exemple, sur les eòtes de la rive gauche de la Moselle, soit enfin par leur voisinage avee d'autres eultures analogues. C'est surtout dans ees derniers eas que l'on reneontre ces invasions si désastrcuses des Chenilles de plusieurs espéces du genre Bombyx, eomme on a eu occasion de l'observer quelquefois dans les vergers de Lorry, de Plappeville, ete., ete.

Combien dc fois nai-je pas cu occasion de reeonnaitre la vérité de ee qui précède, dans une chasse de plusieurs heures faite dans des jardins bien entretenus: e'cst à peine si je rencontrais quelques rares individus d'un petit nombre d'espèees d'inseetes nuisibles, tandis qu'il me suffisait souvent de quelques instants pour constater, dans les vergers mal soignćs, la présence de 10 à 13 espéees d'insectes exeręant, souvent en nombre considćrable, leur ravage sur les arbres.

La nature du sujet sur lequel on greffe, ne me parait pas non plus ètre sans influenee, relativement aux attaques des inseetes. Ainsi, on a remarqué que le Ilanncton nuit beaucoup aux arbres greffés sur frane, tandis qu'il semble, au eontraire, ménager ecux qui sont greflés sur Coignassier. La nature du terrain y est aussi très-probablenent pour quelque elıose; mais, malgré mes recherelıes dans le but d'éelairer eette question, je ne puis rien conclure en ee moment.

A part ces particularités, on peut dire que tous les Poiricrs, 
jounes ou vieux, petits ou grands, espaliers ou hauts-vents, sauvages ou cultivés; sont altaqués par des insectes, mais qu'il y a des circonstanees particulières qui les disposent tous à l'étre d'avantage ou plus profondément. Leur état de vigucur ou de maladic est, pour les liorticultcurs, la cause qui, sous ce rapport, a le plus d'influence sur le développement des insectes nuisibles. Beaucoup d'entomologistes, au contraire, prétendent que les insectes attaquent d'abord les arbres sains, ct que la maladic dont ils sont ensuite atteints, n'est que la conséquence de l'invasion de ees parasites. Il y a, je crois, cxagération de part et d'autre, et, tout en reconnaissant que des arbres sains et vigoureux sont quelquefois attaqués par les insectes, il ne faut pas croire que leur maladic n'ait que ces petits animaux pour cause déterminante. Il cst bien entendu, qu'il ne saurait étre question iei de quelques rares individus, mais que dans la discussion présente, je ne parle que des grandes invasions de Bombyx, de Pliyllobius, d'Allantus oethiops, etc. Les horticulteurs ne voient souvent que le résultat final; et, de ee que des arbres malades et languissants sont envahis par les Capsus pyri, Psylla pyrisuga, etc., il ne faut pas en conclure que ee soient ces liémiptères qui ont déterminé leur état maladif. Linsi, la maladic toutc spéciale dont sont atteints presque tous les arbres fruiticr's d'aneiennes espèces, et plus particulicrement ccux des cótes de Marange, de Sinćcourt, de Fíves, de Rombas, ete., jointe à la vétusté d'un grand nombre de sujets, nous autorisent à prédire, que, d'ici à quelques années, il y aura un développement considérable d'insectes, si l'on n'exécute le vœu souvent exprimé par les liommes compétents, lequel consiste à rajeunir par des greffes nouvelles, la plus grande partic des anciennes espèces cultivécs dans ees localités. Si, comme il arrive trop souvent, on néglige les avertissentents donnés par des prati- 
ciens expérimentés et eonseieneieux, et que mes prévisions vienuent mallıeureusement à se réaliser, on ne manquera pas Ic mettre sur le eompte des inseetes, les aceidents qui doivent nécessairement se manifester avee ou sans leur coneours, mais dont ils ne feront que hàter l'apparition ou augmeuter l'intensité.

L'époque à laquelle apparaissent eertaines espèces d'inseetes est aussi très-importaute à eonsidérer, soit au point de vuc cles dommages qu'elles peuvent eauser, soit à eelui des moyens à mettre en usage pour les combattre. Ainsi, par exemple, depuis plusicurs années, le Ver limaec a fait une invasion générale sur tous les Poiriers, partieulièrement sur ceux qui sont cultivés en espalier, dans le norl-cst du départcment de la Moselle; et ecpendant, les dommages qu'il y a causés, sont presqu'insignifiants, parec que son apparition n'a lieu qu'en aoùt ou cn septembre, et qừ̀ ectlc époque de l'annéc, les fruits ćtant presque nùrs, la suppression d'un plus ou moins grand nonbre de feuilles est indifférente à la végétation de l'arbre. En 183̋, au contraire, les Poiriers des environs de Vitryle-Français ont souffert considérablement, paree que ectte fausse Clıcnille y a fait son invasion dès le mois de juin, alors que la plante a besoir de tous ses organes respiratoires.

Voilà, en résumé, dans quelles eouditions se trouve mainteuant le Poirier eultivé dans le dẻpartement, et sur lequel nous allons faire, de la base au sommet, une ehasse entomologique, fruetucuse à toutes les périodes de sa végétation. Toujours nuisibles et souvent désastrenx, les insectes qui vivent sur eef arbre doivent eonstamment tenir le jardinier en éveil, et les ruses dont ils font usage épuisent souvent toutes les ressourees le l'lortieulteur pour arriver à les combattre avee efficaeitć. Eulevement des mousses et des licliens qui salissent le trone eles arbres; abattages de ceux-ei quand ils sont vieux, earićs ou 
malades; badigconnage à la chaux ou avec des caux du gaz; insufllation de cendres fines ou de cliaux vive; arrosages avec des caux salécs, alcalines, ou des décoctions de tabac ; fumigations sulfureuses ou narcotiques: tels sont les moyens généraux mis cı usage pour se débarrasser de ces parasites incom.modes. Je reviendrai, d’ailleurs, particulièrement sur ecs diverses méthodes, en faisant l'listoire de chaque insecte (1).

Pour exécuter le travail que j'cntreprends, la marche qui scmble la plus naturclle à suivrc, c'est celle qui consiste à examiner la plante suecessivement dans les phases diverses et successives de sa végétation, et à faire l'lıistoire des insectes à mesure qu'ils viennent en attaquer une partie; ou bien encore, à prendre les divers organes du végétal, et à indiquer"quelles sont les especes qui leur portent préjudicc. Mais, bien qu'un grand nonbre d'insectes aient des habitudes bien caractériscies, il en est aussi beaucoup qui sont d'une humcur plus vagabonde et qui ont des appétits moins constants; d'autres ont deux génératious dans l'annéc; ceux-ci attaquent indiffér cmment toutes les partics de la plante; ceux-lá, au contraire, ne touclıent à un organe que parec qu'un autre fait défaut, ou que déja ils l'ont fait disparaitre.

Quel que soit d'ailleurs l'ordre que l'on adoptera, il offrira des avantages et des inconvénicuts, selon qu'on l'examinera au point de vue de la pratique ou à eclui de la scienec. $\Lambda$ près bien des tàtonnements, j’ai fini par me déterminer à suivre li elassification des naturalistes, me réservant de donner ensuite

(1) Outre ees moyens artificiels de détruire ou d'empêeher le développenent des insoctes, les jardiniers reçoivent un secours eonstant el souvent très-effieace de la part des Chauves-Souris, Hérissons, Musaraignes, Oiseaux d'un grand nombre d'espèees, Lézards, Araignées, Carabes, ete., qu'ils devront s'attacher à protéger, au lieu de s'acharner ì leur destruction, comme on le fait génémalemen:. 
des tableaux qui poarront conduire à la détermination des espèces, soit que l'on possède l'insecte lui-nıème, soit que l'on eonnaisse la nature des dégâts qu'il occasionne.

Jai dit dans le commeneement que le nombre des espèces d'inseetes qui attaquent le Poirier s’élève à plus de 130 . Fairce un parcil travail d'ensemble était au-dessus de mes for'es: tenant, autant que possible, à vérifier les faits que j'uvance, et, saehant que tout est à faire dans certains ordres, j'aurais été obligé d'cn ajourner eneore longtemps la publication, si je n'avais pu diviser ma tiehe $\mathrm{cn}$ trois parties, dont ehacunc comprendra cnviron anc einquantaine d'espéces ; ectte division aura aussi l'avantage de licr entre eux les insectes qui ont le plus d'unalogic.

La première partie, que je publie aujourd'hui, comprend l'ordre des Coltopténes. La seconde, pour laquelle j'ai dléjà de nombreux matériaux, dont la plupart sont creore inédits, comprendra les Orthoptéres, Ics Hémptéres, les Tuysanoptènes, les Ityméxoptènes et les Dirténes; cnfin, la troisième partie ne r'enfermera, non plus, qu'un scul ordre, celui des LípidopTÈnes, dont les Larves ou Chenilles sont en général les animaux les plus nuisibles à nos eultures.

Loin de moi, eependant, la prétention d'avoir indiqué tous les parasites du Poirier: aussi, cst-il probable qa'un supplément général devicudra nécessairc, soil pour y ajoater des espèces nouvelles qui peavent m'avoir échappé jusqu'à ce jour, soit pour y consigner les observations que je sollieite de la part de tous les jardiniers, et de tous les entomologistes qui comprennent l'importanee du sujet. Ainsi que je l'ai dit en commençant, je n’ai voulu faire qu'un résumé de tout ce qui avait été écrit jusqu'iei, en y ajoutant le résultat de mes observations personnclles; et, en ouvrant la voic pour préparer l'histoirc complète des insectes nuisibles à un arbre très-répandu et dont les fruits 
tiennent une place importante daus l'alimentation, j'ai surtout eherché à étre clair, vrai et utile.

Avant de terminer ces préliminaires, un peu longgs peut-ètl'e, je dois adresser des remereiments aux personnes qui ont bien voulu m'aider de leurs conscils et mettre à ma disposition les matériaux qu'elles possédaient conecrnant les insectes qui vont m'oceuper. Ce sont plus particulièrement M.I. Léon Dufour, de Saint-Sever; Ed. Perris, de Mont-de-Marsan ; Colonel Goureau, Émile Blanchard, Doeteur Sichel, Annot et Clıcvrolat, de Paris; Mathieu, de Nancy; Dutreux, de Luxembourg; Bellevoie, Guillemard, Fridriey et Thomas, de Metz, ete..... C'est aux bons avis des uns et à la complaisance des autres, que je devrai d'avoir pu mener à fin, l'essai que j’ai l'honneur de prísenter à la Société d'Histoire naturelle de Metz.

Metz, 20 Novembre 1836.

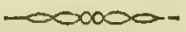

LISTR des Lspices de Coléoptères qui vivent sur le Poirier.

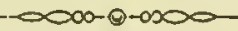
SCARABÉIENS. Oswodenma enemita (L.). La larve vit dans le bois. Melolontila vulganis (F.). La larve vit dans la terre et l'insecte mange les feuilles.

LUCANIENS. SINODENDNON CYLINDMICUM (L.). La larve vit dans le bois. HISTÉRIENS. Platrsoma depressum (Fabr.) / Ces insectes vivent TROGOSSITIENS. Turmalus undatus (Latr.). sous les ceorees; COLYDIENS. - Ditoma cRENata (Ilerbst.). CUCUJIENS. SILvanus unidentatus (Fabr.) ils paraissent plutòt étre proteeteurs que nuisibles.

MALACHODERMES. DAsytes senmconsis (Parr.). La larve vit dans les jeunes branclies. 
BUPRESTIENS. CapNodis TExeBnicosa (Fabr.). Espèce méridionale. Sa larve vil dans le bois.

Agmues vinis (Gernar). La larve vit sols les écorces.

Id. mri (Blanchard). Eş̣èce non déterminée.

CURCULIONIENS. BrachytaRsus virius (Fabr.). L'insecte se trouve sous les écorces.

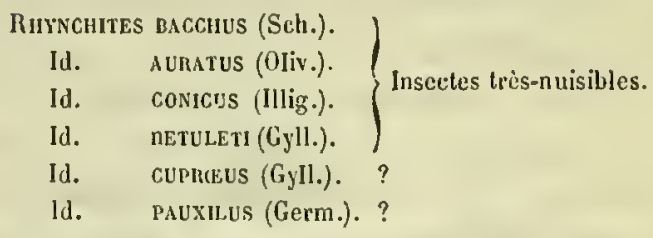

Apion pasone (Germar.). Nuisible.

Polydrosus sericeus (GyIl.). L'ins. mange les feuilles et les bourgeons.

Pirylodius calcaratus (Fabr.). Id. Id.

Id. pyr (L.) L'ins, mange Ies feuill. Très-nuisible.

Id. Arcentatus (L.). L'ins. mange Ies fcuill.

Id. vespentinus (L.). Id. Id.

Id. obloxcus (Fabr.). L'ins. mange les feuill. Très-nuisible.

Id. UNIForms (Marsh.). L'ins. mange Ies feuill. Pentetus criseus (0liv.). L'insecte est très-nuisible. Il mange surtout les jeunes bourgeons.

Otmonirncuus raucus (Fabr.). Mango les feuilles pendant Ia nuit.

Id. picipes (Fabr.). Id. Id.

Magdalis cerasi (Germ.). L'inscete mange la face supérieure des feuilles.
1d. Pnusi (Fabr.).
Id.
Id,

Anthoxomus postonum (Germ.). La larve vit dans les boutons à fleurs. Très-nuisible. 
Axthonomus ulai (Steph.). ?

Id. pyo $\left(\mathrm{Ch}^{\prime}\right)$. Vit dans la fleur à l'état de larve.

Mecinus pyinaster (Ilerbst.). L'insecte vit sous les ecorces.

BOStRIChIENS. Scolytus destructor (L.). La larve et l'insecte creusent des galeries dans le bois.

Id. PRUNI (Ratreb.).

Id. Ií.

Id. Ruculosus (Ratzeb.). La larve et l'insecte creusent des galeries dans les jeunes hranches.

Platypus crlindrus (Fabr.), Id. Id.

CERAMBy yelens. Clutus arguatus (F.).

La larve vit sous les écorces et dans le bois.

Lejopus nebulosus (L.). La larve vit sous les corces.

Id. Id.

SAPERDA SCALATIS (L.).

Id. CANDida (Fabr.).

Espèce américaine; elle vit dansle bois à l'ẻtat de larve.

Polyopsia preusta (L.). La larve vit dans le bois. Phitecia nigriconnis (Fabr.) Id. Id.

CHRYSOMELIENS. LUPERUS FLAVIPES (L.). L'ins. ronge les feuilles. COCCINELLIENS. IDALIA BipunctatA. (L.).

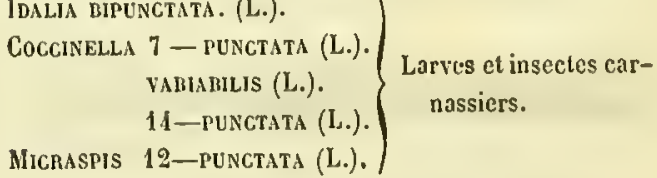

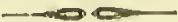




\section{PREMIERE PARTIE.}

I. OsmodERMA, Enoyclopédio (1).

Lacordaire; Génèr. des Ins. Coléoptères; tome 3, page 557.

Ilanches postéricures contiguës; màchoires à lobe externe corné; écusson triangulaire; tête des inâles avec dettx petits tubercules; élytres larges, parallèles et planes en dessus ; pattes longues, jambes antéricures tridentées, les postéricures bidentècs; pygidium bombé, très-grand, surtout chez les mâles.

Les Osmoderma sont de grands et beaux insectes, de couleur foncée et bronzéc, à démarche lente et lourdc, volant le soir, vivant, ainsi que leurs larves, dans le bois vermoulu des vicux trones d'arbrcs. On n'cn connait que quelques espèces, propres à l'Europe et à l'Amérique du Nord. Ces insectes sont remarquables par l'odeur forte qu'ils répandent, et qui rappclic celle de l'abricot ou du cuir de Russiè.

\section{1. ogmoderma eagmita (Linné).}

Mulsant; Colèoptères de France. Lamellicornes; jage 526.

Synonymic: Scarabous eremita (Lin.); - Celonia eremita (Fabr.); - Trichius eremita (Fabr.); - Gymnodus eremila (Kirby.); - Melolontha eremila (Ilcrbst.); - Searabœus enriaeeus (Degier); - Celonia eremitiea (Knoch.); - Trichie ermite, - Prunier, - Pique-prune des jardinicrs; - Eremitscharkafer des Allemands.

D'environ $0^{\mathrm{m}}, 03$ de longueur; d'un brun noir foncé, à reflets plus ou moins bronzés, selon les individus, ou plutòt

(1) Synonymic: Scanaboeds (Lin.); - Triciur grmodi (Kirby.); - Gymodus (Kirby.); - Thichus (Fabr.); - Cetonia (Knoch.). 
selon les localités; chaperon carré et fortement rebordé tout autour ; feuillets des antennes eourts et arrondis; corselet arrondi, hicarc̀nć longitudinalement au milieu; écusson atlongć, triangulaire; pattes courtes et robustes.

Maequart indique la Triehic ermite comme vivant sur le Poiricr; M. Nordlinger la signale comme attaquant le Pomunier; Ratzeburg et la pluparl des auteurs, eomme vivanl dans l'intérieur des llêtres, des Peupliers, et surtout des Saules. C'est, en effet, dans les vieux arbres de cette dernière espèce que, dans notre département, on reneontre la Triehic ermite et sa larve. Celle-ei est quelquefois assez abondante dans les Saules de Vallières, de Grimont, ete.; mais, quoique j'aic exploré un grand nombre de vicux Poiriers eariés, je ne l'y ai jamais reneontrée.

La larve de la Triehie ermite a ćtć déerile et figurée pour la première fois, par Drumpelman, en 1811. Elle est blanelıe, arquée comme le sont toutes eclles des Lamellicornes; tête ćeailleuse, jaune; eorps blaue, renflé à l'extrémité. La peau est trausparente, et laisse apereevoir le eanal intestinal rempli de matière noirc, ce qui donne une teinte grise à la larve, daus les parties eorrespondautes. Elle vit ordinairement trois années a vaut de se transformer en nymphe. Les larves àgées d’une année ont de 20 à 23 millimètres de longueur; celles de deux ans, de 35 à 40 millimétres. Je n'ai pas vu la nymphe, mais jai trouvé, en juin, des iudividus nouvelIement ćelos.

Ce n'est qu'accidentellemeut qu'on a pu la reneoutrer sur le Poirier et sur le Pommier dont le bois très-dur résiste plus facilement à l'aetion des màehoires, que eelui des Sanles ou des Peupliers.

En ayant soin de boueher soigneusement toutes les erevasses des vieux arbres, on évitera les atteintes de la Trichic.

II. MELOLONXHA, Fabricius (1).

Lacordaire; Gènèr. des Insectes Coléopteres; tome 3, page 295.

Antennes de 10 artieles, les 7 derniers dans les màles, les

(1) Synonymie: Scrnabeus (Linné). 
6 derniers ehez les femelles, formant la massue; labre bilolé; ehaperon carré et rebordé; prothorax transversal; ćlytres plus ou moins allongées, parallèles; jambes antérieures dentées et éperonnées dans les deux sexes; pygidium perpendiculaire, souvent prolongé dans l'un ou dans les deux sexes.

Inseetes de grande taille, propres à l'lỉurope, à l'Asic et aux Iles Plilippines; vivau, à l'état parfait, du fenillage des arbres, qu'ils dépouillenı quelquefois complètenient en fort peu de temps; mais e'est surtout à l'état de larve qu'ils commettent le plus de dommage, cu rongeant les racines de toutes les espéces de plautes.

2. MEzozoкTнa VULGaRis (Fabr.).

Hulsant; Coléopt. de France; Lomellicornes; page 411.

Synonymic: Searabaus melolontha (Lin.); - Melolontha mayalis (Molli); - IIanneton; - Neuri; - Ilarlo; Meikafer; - Ingerling des Allemands.

La Larve: Ver blanc; - Mans, ete.

Ces insectes, répandus dans toute l'Europe, sont malheureusement trop connus pour qu'il soit nécessairc d'cul donner une description. On sail, en effet, (qu'en certaines années, on lcs roit apparaitre par myriades en mai ou en juin, et qualors ils dévorent, en peu de temps, toutes les fevilles des arbres d'un verger. Quels que soicnt les ravages causés par l'inseete parfait, ils ne sont pas encorc aussi considérables que ceux qui sonl produils par les larves que les cultivateurs et les jardiniers nomment Ver's blancs.

La larve et les mocurs de ect inseete ont déjà été déerites bien des fois, et les détails les plus intéressants de leur listoire, sont reproduils dans tous les ouvrages d'horticulturc. On a égalenent éerit bien des artieles de journaux et bieu des brochures sur les moyens à enployer pour se débarrasser de ces lótes ineommodes. Mais, jusqu’à ec jour, tous ees moyens tant vantés el prétendus infaillibles, sout, ou impralieables en grand, ou l1.op dispendieux; et il esl pro- 
bable qu'on n'arrivera à quelque elıose de rationnel et de satisfaisant, "gu'en utilisant industricllement ces animaux, afin de couvrir une partie des frais que nécessite leur récoltc. C'est surtout à la elimie organique qu'il appartient de faire des reelecreles sur la composition du corps de ecs insectes, et de voir si l'on ne peut pas en tirer quelque prineipe parliculier, ou utiliser ceux de ees prineipes que l'on connait déji (1).

III. SINODENDRON, Helwig (?').

Lacordaire; Gènèr. Ins. Coleoptères; tome III, page 13.

Antennes plus courtes que le corselet; corps cylindrique; jambes antéricures dentécs; antennes en massuc de 5 articles; mandibules très-courtes; ćcusson large, en triangle curviligne; pattes courtes; les malles ont sur la téte tune corne, droitc à la base, un peu recourbéc en arrière au sommet; les femelles n'ont qu'un faible tubcreule placé ì pen de distance du bord antéricur du chaperon.

Insectes noirs, de faible taille, propres à l'Europe et à l'Amérique du nord, vivant, eomme la plupart des Lueanides, dans le bois vermoulu.

(1) Pour donner une idée de la valeur de beaucoup des articles publićs sur le Ilanneton, je une borne a citer celui-ei, que je copie dans un journal d'horticulture édité en Belgique (1857, No de février): "Pour procéder ivantageusement à la destruction du IIanueton, il fant étudier ses meurs et le suive dans ses métamorphoses. "Clest certainement très-vrai, mais roici comuncnt l'auteur continue: "Le Hanneton des Poumiers, Melolontha mali, est très-petit, d'une eouleur marron très-fonec; il dévore les fleurs d'une grande partie des arbres de la fanille des Rosaejes, ainsi que les jeunes fruits, no tamment ceux du Pommier, etc, etc. "Ceci est très-joli et mérite la réponse que l'on prète à Cuvier à propos de la définition du mot Éerevisse, car il n'ỵ a pas de Melolontha mali, et l'insecte dont il est question est un Charansonite du genre Phyllobius! Fiez-vous aux procedós que l'auteur va vous proposer, ot qui sont basés sur la comaissance exicte des mneurs du Itmneton!!

(2) Synonymic: Lugxipekd (Fabr.); - Scanturets (Lin.). 
3. SINODENDAON CYIXNAICUM (Eabr.).

Mulsant; Coleopt. de Franee; Lamellicornes; page 601.

Synonymic: Scarabaus cylindricus (lin.); - Lueanus cylindricus(Laich.); - Ligniperde cylindrica (Fabr.); Sinodendre.

Long d'environ douze millimetres; cylindrique; d'un noir luisant; eorsclet marqué de poimts enfonés, avec une ligne longitudinale lisse au milicu, et une saillie en avant; élyıres striécs, fortement ponetuées, $5^{\mathrm{e}}$ et $\dddot{\partial}^{\mathrm{e}}$ stries fortement relevécs antéricurement, presque effacécs postéricurement.

Cet insecte, très-rare dans notre département, est indiqué par les auteurs eomme vivant aux dépens des Hêtres et des Frênes. Macquart l'indique comme élant nuisible au Poirier; el c'est en effet sur cet arbre qu'il a ćlé rencontré dans la Moselle. M. Mathien, dans son Cours d'Entomologic forcstière, le signalc aussi comme nuisible à ect arbre.

La larve a été décrite pour la première fois et figuréc en $\mathbf{1 8 3 9}$, par M. Westwood (Iutrod. the modern. class.; t. I, fig. 18). 1'après M. Mnlsant (Col. de Fr.; p. 600), elle est d'un blane eendré arec la têtc jaune ct écailleuse ct le corps arqué. Comme on roil, clle se ripproche des autres larres de Lamellicornes.

Elle vit, eomme la Trichie crmite, dans les parties morles et cariécs des vicux Poiricrs.

IV. PIATYSOMA, Leach. (1).

Lacordaire; Géner. Ins. Col.; tome II, page 255.

Corps en ovale allongé ou cylindrique; tủte rétractilé; antennes insérées dans unc fossette cntre les yeux et les mandibules, de 11 articles, dont les 4 derniers forment

(1) Sṭnonymie : Ilisten (Fabr.); - Ilololeprn (Paykul.). 
une massue ovale, compriméc, et couverte d'un duvet soyeux; corselet earré ou transversal; écusson petit et triangulaire; élytres plus ou moins allongées, parallèles; pattes assez longues, avec une seule rangée de dents au cóté interne; janbes bi-èpineuses ; tarses de $\mathbf{b}$ artieles dont le dernier est aussi long que les 4 autres réunis.

Ge genre, eomposé d'cnviron 25 espèces, a des représentants dans toncs les parties du monde; 6 seulemeut appartienneut a l'Europe.

Jusqu'a ces derniers tcmps, les mocurs de ces inscctes et de leurs larves étaient inconnues : c'est M. Perris qui, le premier, a fait comaitre la larve Platysoma obtongum. Cette larve vil sous Ics écorces : ellc est caruassière. C'est aussi lá qu'on reneontre les insecles parfairs.

\section{PIATXBOMa dEPRESSUM (Fabr.).}

De Marseul; An. de la Soc. ent. de France; 1851, page 218.

Synonymic: IIister depressum (Fabr.); - IIololepla depressu (Payk) (Vur. A.); -IIololepla deplanala (Payk.).

Longueur $0^{\mathrm{m}}, 004$, largeur $0^{\mathrm{m}}, 002$; noir, luisant, ovale oblong, déprimé; antennes brunes; corselet plus large que long, échancré en avant, bords latéraux obtus et pointillés; ćcusson petil; ćlytres allongées, un peu rétrécies en arrière; pygidium ponetuć, non rebordé; pattes couleur de poix, janbes antéricures quadridentées.

Cet insecte est rare dans nos cnvirons; il rit sous les ceorees, et c'cst sous eelle d'un vicux Poirier que, M. Gourean l'a rencontré. On ne connatit rien aulre chose sur ses habitudes, ct eelles de sal larve sont tout à fait inconnues. Mais, d'après ee que nous savons maintenant du $M l$. oblongum, il est permis de supposer que rinsectc, ainsi que la lare, sont carnassiers, et que, par consci- 
qquent, l'insecte dont il cst question, doil ćlre elassé parmi ceus qui souı plutòl utiles que nuisibles.

V. THYMaLus, Dufstchmidt (1).

Lacordaire; Gener. des Ins. Col.; tome III, page 350 .

Menton petil; lobe des mảelıoires corné; mandibules eourtes, robustes, en partic recouvertes par le labre; tite enticrement cachée sous le corsclet; yeux gros; antennes de 11 artieles, le $1^{\text {er }}$ assez long, les $9^{\mathrm{e}} 10^{\mathrm{e}}$ et $11^{\mathrm{H}}$ formant une massuc allongéc; un sillon bien marqué, sous les yeux, pour loger les antennes; corselet transversal; éeusson en triangle curviligne; ćlyt'es r'ebordées; pattes courtes et robustes.

Insecıcs Irès-rcinarquables par leur faciés coceinclloïde; on n'en conuail qqu’une espéce européenne el deux américaines: toutcs trois sont propres aux eontrées froides et montagneuses.

5. Thxmalus cimbatug (Fabr.).

Laporle; Hisloire naluelle des Col., tome II, page 8.

Synonymic: Pellis limbalus (Fabr.); - Pellis brunneus (Payk.); - Casside limbala (L.).

Long de 7 millimètres et large de 4; corps d'un brun bronzé assez brillant; ḋlytres avec des stries assez irrégulicres de points enfoncés; bord cxtérieur d'un brun rougeâtre ; pattes ferrugineuses; eorps souvent enticiement recouvert d'unc eflloresecnee blanchatere, qui se renouvelle quand on l'enlève pendant la vie de linseete.

La larve du Thymalus limbatus a été décrite par MM. Chapuis et Candèze (Calalogue des larves de Coléoplères, dans les Mćmoires de la Soeićtć des Seicnees de Liège ; anućc 1853, page 407).

(1) Synonymic: Pelts (Fabr.); - C.Issıu. (Lin.); - Ası (Oliv.). 
Cette larse, longue de 9 à 10 millimètres, est d'un bleu hyalin, sauf la tête qui est d'un jaune sale; l'éeusson prothoracique et celui du dernier segment sont d'un brun brunatre, ainsi que deux sérics de petites dents latérales arrondies, disposées au bord interne et de claque cóté des anneaux dorsaux, depuis le mésom thorax jusquà l'avant-dernier, ou le dernier segment abdominal. Le corps est un peu déprimé, contracté ct comme festonné latéralemeut par la préscnce des bourrelets des arçaux; il est recouvert de quelques poils blanchatres, mous ct flexueux, entremêlés de poils plus eourts.

Ces larves ont étć trouvées, par les entomologistes liégcois, au mois de janvier, sous les écorces d'un Poirier sauvage. Elles paraissent se nourrir exclusivement de substance ligneuse. Elles se sont transformées en nymplics au mois d'arril suivant; ces nymphes sout parsemées de quelques poils, plus courts que ccux de la larve, el leur abdomen est tcrniné par deux pctites pointes aiguës.

Au mois de novembre 1835, j’ai trouvé également, sous l'ćeorce d'un Poirier abattu à Vallières, 4 larves dont la description se rapporte assez à celle qui précède (moins la taillc ccpendant). Je n’ai pu les élever, ni déterminer à quellc cspèce de Coléoptères elles appartiennent. Cellcs qui me restent, ayant été eonservées dans l'alcool, ne me permettent pas de conelure à leur identité parfaite avec eelles du Thymalus limbatus.

Quoiqu'il en soit, il parait que l'insecte dont il s'agit, doit ĉre compris dans le nombre de ecux qui sont nuisibles au Poirier, au moins à l'élat de larve; ear on ue eonnait pas les mocurs de l'iuseete parfait, extrềnement rare dans notre pays.

\section{vI. DrTomA, rlliger (1).}

Lacordaire; Gener. Ins. Coleopt.; tome 11, page 363.

Menton earré; lobe interne des mảchoires petit et cilić au

(1) Synonymie : Brtom (Herbst.); - Lrctus (Fabr.); - le's (Olivier); Honotoma (Panzer); - Sxachira (Ilelwig.). 
bout, lobe externe plus grand et cilie au dedans; dernier article des palpes labiaux grand; antennes de 11 articles, dont les deux derniers forment une massue; yeux saillants; élytres allongćes ; pattes courtes, jambes proportionnellement assez longues, tarses de 4 articles.

Insectes de petite taille, vivant sous les éeorees, ou dans le bois mort; propres à l'Ĺurope, à l'A mérique et aux Iles-Marquises.

\section{Ditoma chenata (Herbst.).}

Erichsun; Naturgesch. Der Insect. der Deutschland; page 266.

Synonymic: Biloma cronala (Herbst.); - Lyclus crenela (Fabr.); - Ips crenala (Oliv.); - Monoloma crenata (Panzer) ; - Synchila crena la (Helwig.) ; - Lyclus rufipennis (Fabr.) (Var. B.); - Ips picipes (Oliv.) (Var. A.).

Longueur de 5 à / millimètres; corps noir, 2 cótcs relevées de chaqque còté du eorselet; élytres ponctuécs et striécs, à intervalles alternativement élevés avec unc taclıe fauve strr clracunc. Ces taches sont plus ou moins grandes, et, quand clles s'étendent sur toutc l'élytre, clles produisent la Var. A.; quelquefois aussi l'insecte est entièrement d'un brun tcstacé: c'est alors la Var. B. Dans le type comme dans ses variétés, les jambes et les tarses sont rougcatres.

Ces petits inseetes, extrêmement répandus, se reneontrent souvent sous les écorees d'un grand nombre d'arbres, particulic̀rement sous eclles de l'Orme et du llêtre; rarement sous eelles des vieux Poiriers. Maequart et M. Goureau, ainsi que moi, les y avons eependant trourćs. MI. Nordlinger n'en parle pas dans son ouvrage.

D'après Mr. Rouget (1), l'inseete parfait vole le soir, et se rencontre, à eet état, du printemps à l'automne.

(1) Catalogue des Insectes de la Còte-d'Or, no 512 ; Dijon, 1856. 
M. Perris a douné une description complète de la larve du Ditoma cremala dans son Hlistoire des insectes du Pin matitime. Cette larve est linéairc, déprimée, d'environ 6 mill. de long, et d'un blanc lavé de roussảtrc. Sclon eet entomologiste, clle vit plus ordinairement sous l'ćeorcc des Chènes, dans les nids du Tomicus fuscus. Elle se troure aussi, mais moins souvent, sous l'écorce des Pins, principalement dans les nids de Tomicus laricis, dont elle dévore les larves el les nymphes. Celte larve est donc carnassière, et, par conséqquent, doit être classéc parmi les insectes utiles. Je n’ai jamais trouvé de galeries de Tomicus sur les trones de Poirier où je rencontrais le Diloma crenala, lequel pouvait, d'aillenrs, n'c̀tre là que pour rechercher les galeries d'autres Xylophages afin d'y déposer ses œufs.

\section{vII. SIIVANUS, Latreille (1).}

Lacordairc; Gènèr. des Insectes Coléoptères; tome II, page $\$ 15$.

Menton transversal; lobes des màchoires courts, l'interne cilié, l'extcrne barbu; mandibules courtes; tète allongéc en avant, retrécic en arrière; antennes assez longues, de 11 articles, dont les trois derniers forment une massue un peu allongée el serréc; élytres longucs, parnllèles; pattes médiocres, tarse de 5 articles, le dernicr long, les 5 premicrs velus cndessous.

Ces insectes sont de petite taille et ont des habitudes assez varićes: les uns habitent sous les écorces; d'autres vivent dans le bois mort; quelquefois aussi on les reneontre sur les plantes herbacées. Plusieurs vivent dans les céréales emmagasinées ou dans divers produits coloniaux, an moyen desquels certaines espèes cxotiques ont ćté introduites en Lurope, oì elles s'y sont acclimatécs.

(1) Synonynie : Uenuestes (Linu.); - Cowdumę(Fabr.); - Ips (0liv.); - Lrctus (Kugelon); - Lewtes (Dnftsch.); - Cnrrtornigus (Walt.). 


\section{SIIVANUB oxIDENTatus (Fabr.).}

Erichson Naturgesch. Der Insect. der Deutschland; page 338.

Synonymic: Dermestes unidentatus (Fab.); - Colydium unidentatum (Payk.); - Lyelus unidentatns (Kugelm.); -Ipsunidentuta(Oliv.); - Leptus unidentata (Duftsch); - Colydium planum (Herbst.); - Silvain.

Long de 5 à 4 millim., ferrugineux, linćaire, déprimé, un peu pubeseent; tète dentieuléc de ehaque cóté près des yeux ; corselet allongé, ćtroit ì la base, angles antérieurs sous-épineux, les postéricurs denticulés; élytres ponetuées et strièes.

Cet insectc est très-commun, et, comme le précédent, se trouve sous les écorees d'un grand nombre d'arbres (Charme, Clıênc, Hêtre, Noycr... etc.). Macquart, ainsi que M. Gourcau, l'ont trouvé sous l'ćcorce d'un Poiricr; mais l'on ne counait ricn autre chose sur lcs lıabitudes de l'inscetc parfait.

M. Perris (Loc. cit.) en a dćcrit la larve. Ellc est longue d'cnviron 5 millimètres, ct d'un blanc jaunàtrc, avec la tête et lc prothorax plus foncés. Le corps cst aplati et un peu rétréci cn arrièrc. \& Cette larre, dit M. Perris, est à peu près cosmopolite; clle court çà et là avec bcaucoup de vivacité, ct sc cache promptement quand on l'expose à la lumière qui l'offusque. »Selon MII. Coquerel et Wcstwood, les larves d'unc cspice voisinc, lc Sylvanus bidentatus se nourrisscut de substanecs végétales ou de sucre; mais, d'après Blisson ct M. Perris, ces larres sont réellement carnassières ct, comme celles dn $S$. unidentatus, elles vivent de larves, de nymphics ou des dépouilles d'autres insectes, et, subsidiaircment, des exeréments des larves qui ont vécu avec clles.

Quoicqu'il ne soit pas rare dans motre départcinent, je n'ai pas encorc rencontré cet insectc sous les écorecs du Poirier. D'après ce qui precède, il doit, comme le Ditoma crenata, ĉtre clasșć parmi ceux qu'il scrait utile de conserver. 
VIII. DASYTES, Raykul (1).

Lacordaitc; Gènér. des Ins. Coléoplères; tome IV, page 100.

Menton transversal; màchoires bilobées; dernier artiele des palpes tronqué; mandibules larges; labre saillant; tćte courte; antennes de longucur variable, souvent dentées en scic à partir du $3^{\circ}$ ou $4^{\circ}$ article; ycux saillants ; élytres allongćes, à peine plus larges que le prothorax; pattes grèles; tarses de 3 articles; corps plus ou moins velu.

Ce genre, qui comprend un très-grand nombre d'espèces (plus de 150), se compose de pelits insectes à eorps assez mon, d'une conleur souvent métallique, et fréquentant, en général, les fleurs à l'état parfait. On ne connait eneore que la liorve de deux espèees de Dasytcs (D. serricornis el flavipes); et, d'aprìs ec que dit M. Perris de eelle dn D. favipes, il est à présumer que ecs larves sont carnassières, eomme celles des espéecs qui précèdent: c'étaiı, d'ailleurs, le sentiment de Latreille.

\section{DASTTES SERRYCORIKS (Parreys).}

Malnrẻ mes recherehes, je n'ai pu me procurer le muméro du journal l'Isis (annce 1854) dans lecpucl se trouve la description du Dasyte scricornc.

J'insecte lui-mêne, rare dans les collections, n'a pu non plus m’çtre eommuniqué. Sa larve cst indiquée, par M. Waterhonse, comene vivant dans les branches du Poirier. Selon cet auteur, ectte larve est verdàtre, marquéc de taches d'un vert plus foncé; elle est allongéc, pubesecnte et élargic postéricurement, avec l'extrémitć munie de denx pointes aiguës.

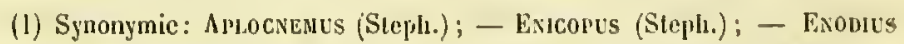
(Casteln.); - Divales (Cast.); - Davacea (Cast.); - Derm.stoma (Mlotsch.); Anthoxenus (Motsch.); - Lasies (Motsch.); - Mikropogon. (Motsch); Cosmoconus (Kuster); - Psllotma (Kuster); - Memaesies (L.); - Ilispis

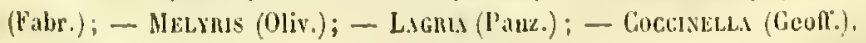


M. Perris dit positivement que la larve du Dasytes flavipes vit de larves du Tomieus bidens, sauf à se nourril des matières excrémentiticlles déposées dans les galeries de ce Xylophage, lorsqu'elle ne trourc plus à satisfaire ses appétits carnassiers. L'autcur ijoute cucorc, qu'ả l'état parfait, les Dasytes lui paraissent avoir les mêmes habitudes que les Malachies, et que, par conséquent, ils sont carvassier's ou plutót Staminiphages. Je viens d'aroir occasion de vérifier l'exactilude des prévisions de l'habile entomologiste de Mont-deMarsan: Le 24 Mai 1857, j'ai vu des Dasytes plambous manger, en compagnie des Malachius acneus et bipustullatus, les étanines du seigle en fleur; et, pour mettre ce fait hors de doute, j'ai renferuć plusieurs Dasytes dans un tube avec des étamines de seigle. Ces insectes n'ont pas tardé à se repaitrede ces organcs; et, au bout de quelques heures, les étamines avaient complètement disparu.

L'inscete signalé par l'auteur anglais comme étant nuisible au Poirier est done un insecte protecteur qu'il importe de conserver, au moins à l'état de larre.

IX. CAPNODIS, Eschscholtz (t).

Lacord.; Gènér. Insec. Colèopt.; tome IV, page 31.

Labic transversal; tétc plane; antennes courtes de 11 artieles, les 5 premiers courts, les 7 derniers plus ou moins dilatés ; yeux grands, non saillants ; prochorax transversal, avec une fosselte profonde en arrière; écusson petit; élytres rétrécies dans leur tiers postéricur; pattes robustes, tarses de 5 articles, les 3 premiers égaux, le $4^{\mathrm{e}}$ très fortement bilobé et embrassant le dernier; corps robuste et peu convexe.

Insectes d'une forte taille, d'un faciès tout particulier, de coulenr noire ou bronzéc obscure, arce des taches blanches irrégulières sur le corselet, ou des plaques euivreuses sous les anneaux de

(1) Synonymic: Anguta (Gistl.) ; - L.tripalds (Sol.); - Burnestis (Laporte). 
rabdomen. Toutes les espèces, à l'exeeption d'une seule, sont propres aux pays qui bordent la Méditerranéc.

9. CAPNODIB TENEBRICOSA (Fabr.).

Laporte et Gury; Itist. nat. des Ins. Col. Buprest.; tome II, page 9

Synonymic: Buprestis tencbrionis (Rossi non L.).

De 15 à 20 millimètres de longueur ; d'un bronzé obseur, quelquefois noir' téte et corselet fortement et irrégulièrement ponctués; élytres avee des strics longitudinales de points enfoneés et des taclıes bronzées nombreuses disposées sans ordre et formées par un duvet court, soycux, placé sur des groupes de points enfoncés; dessous du corps noir parsemé de gros points enfonećs et dorés.

Ce n'est que d'après l'autorité de Macquart que j’ai placé ect inseete parmi eeux qui vivent sur le Poirier. Essentiellement méridional, il ne s'est jamais égaré jusqu’à venir dans notre département. Sa larve n’a pas eneore été décrite, bien qu'elle soit assez eommune dans le midi de la Franee, oì notre collègue, M. Capiomont, l'a observée dans le trone des vieux arbres frnitiers, aux dépens desquels elle vir.

X. AGRIIUS, Megerle (1).

Lacordaire; Géner. Ins. Col.; lome 1V, prage 83.

Tète courte; antennes de 11 articles, les $2^{\circ}$ et $5^{\mathfrak{e}}$ égaux, les suivants plus ou moins dentés; yeux assez grands et peu saillants; prothorax transversal; élytres allongées et presque toujours débordées par l'abdomen vers le milieu de leur longueur; tarses grêles, de 3 articles, dont le premier est trèslong.

(I) Synonynie: Iburests (I.) ; - Imomptosom (lap.); - Conced (Irap.). 
Ce genre renferme un grand nombre de petites espèces de eouleur bleue, verte on inétallique, avec des taelıes latérales blanelâtr'cs. Les fenclles pondent leur's oufs sur lcs arbres malades, morts ou en bon état, mais toujours dans les parties recouvertes par l'éeorce (1). Les larves sont blanclics ou roses, apodes, r'enflées antéricurenent, ee qui leur donne la forme d'une sorte de pilon. Eilles sont lignivores, et creusent, suivant les espèces, dans le bois ou dans l'aubier, des galeries tortueuses qu'elles remplissent de vermoulure et d'excréments disposés par petites couches formant des ares concentriques dont l'ouverture est tournée du cóté de la larve. Cette disposition singulière est earactéristiçue pour les larves d' $\boldsymbol{A}$ grilus. « Cet arrangeunent symétrique, dit M. Perris, auquel j’emprunte tous ces détails, a, pour eause première, les dimensions de la galerie, qui sont hors de proportion avec l'abdomen de la larve. Celle-ci, à cause du volume de la partic antćricure du corps, est obligće de donner à sa galeric une largeur telle, que la partic postérieure y exćeute librement des mouvenents de va-et-vient, qui ont pour résultat naturel de disposer en arc les matières rejetées en arrière. D'un autre cóté, toujours par suite des dimensions de la galerie, la larve, afin d'avoir' des points d'appui, est obligée de replicr sur elle-même la partie postéricure de son corps; ordinairement même, on la rencontre dans cette atlitude, qui lui permet d'agir contre les parois pour se pousser en avant; unais, dans eet état, l'abdomen forme un are qui, appuyant du cóté conrcse sur les détritus, détermine la coneavité des couclies successives. »

Elles offreut, eomme celles des autres buprestes, une particularité très-remarquable: c'est la présence d'un treizième segment, non compris la tête, tandis que l'on n'en compte que douze dans les larves de toutes les autres familles de l'ordre des Coléoptères.

La durée de la rie des larves d'Agrilus est d'une année; et on reconnait facilement les trous de sortie de l'inseete parfait, par la forme particulière à ces ouvertures, forme qui est celle d'une

(1) Mathicu; Cours de Zcologie foresliere; Nancy, 1818. 
bouche de four et par lacpuelle s'éelıappe l'insecte, le dos tourné rers la partic plane, et par conséquent le venıre en l'air.

L'inseete parfait ne se montre que dans les chaudes et belles journées de l'été, et semble ne ponvoir faire usage de ses ailes que sous l'action d'un ardent soleil. Aussi, le nombre des espèces méridionales et tropicales est-il plus considérable que celui de nos contrées tempérées. Les individus d'une même espèce se multiplient quelquefois d'une manière prodigicuse, et vont, dans certains cas, jusqu'ả causer la mort des arbres sur lesquels ils se développent.

\section{AGRILUS VIRIDIS (Germar.).}

Laporte et Gory; IIst. des Bupr.; tome II, page 48.

Synonymic: Buprestis viridis (variélés) (linn.); - Buprestis clongalus (Herbst.); Buprestis rosana (Scopoli.); - Buprestis lincaris (Schranck.); - Le Richard vert allongi (Geoffroy).

Cet insecte varic beaucoup pour la taille (de 53 à 10 millimètres) et, pour la couleur, du vert clair au vert bleusitre ou bronzi; antennes proportionnellement plus courtes que dans les autres espèces; corselet échaneré en avant, bilobé en arric̀re, avec des stries transversales sinucuses, et deux inupressions sur le disque; écusson pointu en arrière ; élytres allongées, sinuées latéralement, finement rugueuses, avee une forte impression à la base; dessous du eorps parsemé de petites taches blanches.

Sans être bien rare dans notre départenent, eet Aogrilus ne s'y rencontre pas souvent en grande quantité. Jamais je ne l'ai trouvé sur les Poiriers élevés en quenouille ou en espalicr, tnais sculement daus les vergers ou l'on renicontre également d'autres arbres fruiliers. Macepuart l'indique positivement eomme l'un des parasites du Poiricr, mais sans y ajouter d'observations particulieres. Necrl- 
linger a constaté, sur des Poiriers, des trous de sortic ayant la forme que nous avons signalée plus haut, eomme propres aux insectes de cette famille, et qu'il attribue aussi à une espèce d' $/$ grilus, mais sans entrer dans plus de détails et sansindiquer ả laquelle des espèces de ce genre il faut la rapporter.

En 1856, Audouin a publié unc note (1) sur une larve d'insecte qui se creuse des galeries dans les tiges du Poirier. Ciest à la présence de ees galeries qu’il attribue la formation des erévasses que l'on observe souvent sur le trone des arbres, et que les jardiniers attribuent, bien à tort, à la nature du sol ou à la rigueur des hivers.

En enlevant l'écorce, au-dessus de ces fissures, on trouve des sillons creusés par les larves aux dépens de l'aubier et de l'ćcorce. Sur dessujets de 1 inètre de hauteur, quelgues-unes de ces galerics avaient alteint la longueur de 0,60 centimètres, sans compter les sinuosités. Ces galeries, ou sillons, se dirigetient toutes vers la racine de l'arbre, et partaient d'un point correspondant à une taille de I'année précédente; et les oufs avaient été déposés entre l'écorce et le bois de cette surface de taille. Audouin rapporte cette larve a celle d'un Coléoptíre serricorne, mais sans en préciscr davantage la famille ou le geure.

En 1857, MI. Aubé a décrit (2) la larve de l'Agrilus viridis, qu'il a trouvée vivant en socićté dans l'ćcorce et le bois des bouleaux du Bois de Boulogne près de Paris. Celtc larve se creuse des galeries tortucuses, dirigées en tons sens, et dont la largeur et la profondeur varient a vec l'accroissement; arrivées au moment de se transformer en nymplies, elles se creusent dans le bois unc pctitc cavité, d'où l'insccte s'échappe en perforant l'ćcorce, et en y laissant une ouverture en bouche de four.

Selon M. Aubć, l'insecte indiqué par Audouin, comme virant sur le Poirier, serait aussi l'Agrilus viridis. Cependant la manière de pondre á l'extrémitć des branches et la forme des galeries ne

(1) An. de la Société entomolog. de France; page 70 du Bulletin.

(2) An, de la Sociétí entomolog de Fr,; tome VI, page 191. 
paraissent pas autoriser ce rapproehement. De nouvelles observations deviennent donc nécessaires pour décider, d'une manière exacte, quelle est l'espèce que cet entomologiste a rencontrée sur le Poiricr.

En général, conıme une sève abondaute empêche les inscetes de vivre cutrc le bois el l'éeorec, et que c'est lá que se développent surtout les larves des Agrilus, on comprend combien il importe de n'avoir que des sujets vigourcux, et de boucher toutes les crévasses ou entailles qui se trouvent sur leurs troues.

En arrosant le pied des Peupliers attaqués par la Chenille du Cossus ligniperda, avee une solution faite d'unc partic de sulfatc de cuivre pour mille parties d'eau, on détruit ecs insectes, ou on leur fait abandonner l'arbre : il est probable qu'un pareil moyen, cmployé dans les verget's, pourrait débarrasser beaucoup d'arbres des insectes lignirores qui en détruisent le trouc. Je fais en ce montent des expérienees sur l'efficacité de ee procédé, et j'aurai plus tard loecasion d'en consigucr les résultats dans la suite de ec travail.

\section{AGRILUS PYAI (Blancliard).}

Synonymic: Agrilus viridis, Var.? (Ghev'.).

Dans l'listoire des insectes, M. Blanchard dit qu'une espèce d'Agrilus passe ses premières annécs dans les branclıes du Poiricr; et il ajoute que sa larve et sa nymphe ressemblent bien eomplètement à celles de l'Agrilus viridis.

L'iuscete dont parle M. Blanchard, et qu'il désigne sous lc nom d'Agrilus pyri, est celui dont Audouin a trouvé la larve eu 1856, et que M. Aubé a rapporté à l'Agrilus viridis.

Lin 1856, M. Blanchard, à qui j'arais demandé la description de l'Agrilıs pyri, me répondait: « Quant à cette cspéce, elle cst très-roisine de l'Mgrilıs viridis, et je vais recherelıcr les individus trouvés par Audouin, ear je suis loin d'étre sûr que cctte cspèce n'ait pas été décritc sons un antre nom »... Après aroir cité un passage de Westwood, relalivement à cet 
inseete (1), mon honorable eorrespondant ajoute: «Depuis, il n'y a rien eu de publić sur eet inseete, que ec que j'en ai dit, en 1845, dans mon Ilistoire des inseetes. 》

De ec qui préeède, il résulte évidemment que l’Agrilus pyri n'a pas eneore été déerit; que ee n'est probablenent que l'une des nombreuses variélés de l'Agrilus viridis (L.), et que l'on est encore loin d'ètre fixé sur les noms et le nombre des espèees de ec genre qui, à l'état de larve, vivent sur le loirier

\section{BRACHYTARSUS, Schœnherr (2).}

Schœnherr; Synonym. Curculionid.; tome 1, page 170.

Antennes eourtes, presque droites, inserrées sur le milieu lu rostre, el logies dans une eavité transversale; massue ovale et eomprimće; rostre eourt, large et ineliné; yeux grands et latéraux; corselet eourt, avec les angles postérieurs acuminés; ćlytres artondies à l'extrémitć; jambes entières, tarses eourts.

Insecte de petite taille, de eouleur sombre et variće de gris ou de blane; propres aux contrées tempérées des deux eontinents. Les larves de Brachytarsus présentent une partieularité anatomique assez remarquable dans la famille des Curculionites : e'est la présenee de pattes rudimentaires. Mais elles offrent eneore, dans leurs lıabitudes, une anomalie plus singulière. On sait, en effet, que toutes les larves connues de Cureulionites vivent de substanees végétales à divers états. Les larves de Branelyytarsus sont au eontraire earnassières, ct earnassièr'es d'une manière toute partieulière: d'après Friselı, Latrcille, Dalınan, MM. Vallot,

(1) " M. Audouin has álso discodered the larve of another speciers of Agrilis, burrowing in the wood of the pear. " - (Ml. Anduuin a aussi décrit une. larve d'une espèce du genre Agrilus, et qui vit sur les branches du Poiricr.) Vestwood; Introduction te the moderne classification of insectes; 1837.

(2) Synonymie : Panorus (Megerlc); - Axthnaus (Gyll.); - Bnucnus (Fabr.); - Macrocephalus (Oliv.); - Cunculio (Payk). 
Ratzburg et Limucs, c'cst dans les coques des Coehenilles et des Chermès, quc ces larves vont chercher leur nourriture. Il reste encore cependant bien des points de leur histoire á éclaircir; et e'est en vain que j'ai exploré un grand nombre de coques du Chermès de l'Orme ct du Poirier, ear je n'y ai jamais rencontré unc Jarve ou un insecte appartcnant au genre Brachytarsus.

\section{BRAGHYTARSUS VARIUS (Fabre).}

Schœenherr; Synonym. Curculionid; tome I, page 171.

Synonymie: Anthribus varius (Fabr.); - Bruchus varius (Lin.); - Macrocephalus varius (Oliv.); - Anthribus varicgatus (Fourer.); - Bruchus capsularius (Seriba); Bruchus clalkralus (IIerbst.).

Longueur : 5 millimètres; d'un noir terne; eorselet avee une ligne longitudinale grise; élytres parsemées de petites taehes grises et soyeuses.

Sur un Poirier laat-vent d'un jardin de Quculcu, M. Bellevoi a trouvé, en 1854 ct en 1855 , un grand nombre d'individus de cctte cspèce dc Curculionitc. Ils s'étaient réfugiés sous l'ćcorcc et dans ses crévasses, en eompagnie d'une grande quantité d' $\boldsymbol{A} n$ thonomus pomorum. Les uns et les autres y étaieut probablement venus eherclicr un abri contre la pluie.

Lin 1856 et cn 1857 , le mềmc arbre nous a fourni plusieurs Brachytarsus varius; mais à toutcs les ćpoques de l'annéc, ils se trouvaient sous les écorces; ct, malgré nos recherclics, le Poiricr, sur lcquel ils habitaicnt, ne nous a offcrt aucun Chermès ni aucun débris de cet inscctc, qui ait pu nous faire supposcr que des larves de Bracliytarsus s'y soient dércloppécs.

Une chose non moins remarquable, c'est que je n'ai pas rencontré un seul de ces inscctes sous les écorces de Poiricrs, ct que je n'ai pu décourrir ce que pouvaicnt fairc ccux que je reneontrais sous les écorces du Poiricr dc Quculeu.

Si réellement les Braelyytarsus, ou au moins Icurs larres, sont 
Coceiphages, ils seraient les premiers Curculionites connus que dut neénager l'horticultcur

La larve de cet insecte a été déeritı, pour la première fois, par Dalman, en 1824; Ratzburg, l'a fait figurer dans son ourrage en 1857, et depuis, en 1848 , M. Nocrdlinger en a complété la desrription dans le journal de la Société entomologique de Stettin.

XII. RHYNCHITEs, Herbst. (1)

Sehœnl.; Synonym. Curculionid.; tome 1, page 210.

Antennes de 10 artieles, dont les 5 derniers forment la massue; trompe allongéc, élargic à son extrémité; palpes courts; corps rétréci en avant; corcelet conique, quelquefois épineux latéralement dans l'un des sexes; abdomen earré, arrondi et découvert postéricurement; pattes éperonnées, avant-dernicr article des tarses bifide.

Les Rhynehites sont en général assez petits, ornés de couleurs brillantes et métalliques; on les rencontre daus toutes les partics du inonde. L'Europe en reuferme ecpendant le plus grand nombre d'espéecs, probablenent parec qu'ils $y$ ont été micux olsservés. Tous sont plus ou moins nuisibles, et méritent de fixer tout particulièrement l'attention des liorticulteurs. Les femelles, surtout au moment de la ponte, excrcent unc industric fort curieusc sans doute, mais souvent fatale aux jcuncs pousses, aux loutons a fruits ou aux feuilles, selon qu'elles confient leurs œufs à l'un ou à l'autrc de ces organes; la larve qui doit en ćclore devant puiser sa nourriture dans la moelle du jeune bois, dans le parenchyme du jeune fruit, ou dans le tissus de la feuille à demi-fanée.

Cette opération, si simple cn apparenec, de déposer un œư sur un bourgeon, sur une flcur ou sur un fruit, est ccpendant, clicz Ics Rlyynchites, accompagnéc de tant de soins, de travaux si péni-

(1) Synonymie : Involvulus (Schranck); - Cunculio (Lin); - Attelanus (Fabr.); - Ruinomacen (Laich.). 
bles et si variés, suivant les espèees, que l'histoire de ees inseetes semble un roman de la nature, et que ec n'est pas trop de ses observations personuelles et du témoigrnage des entomologistes les plus sćrieux, pour en comprendre les détails et en admirer l'harmonie.

Dans eertaines années, on a vu la récolıc des arbres fruiticrs de toute unc contréc eutièrement eompromise, par le développement considérable de plusieurs espèces. Citer les noms de Lisetle, Biche, Coupc-bourgeon, Perce-pomme, ele., que quelques-uus de ees insectes portent dans eertains pays, n'est-ec pas signaler aux jardiniers des ennemis bien connus.

On connait les larves de 6 on 7 espèces du genre Rhynchites. $\mathrm{Si}$, sons le rapport anatomique, elles présentent peu de différence entre elles, il n'en est plus de mème, sous le rapport de leurs habitudes, qui, en effet, semblent varier pour chaeune d'elle.

13. RHYNGHTES Bacchus (Lin.).

Schœenherr; Synonym. Cureul.; tome 1, page 249.

Synonymic: Curculio bacchus (Lin.); - Atlclabus bacchus (Fabr.); - Rhinomacer bacchus (Laich); - Involvulus bacchus (Schrauck); - Rhynchitcs lutcus (Gernar); Curculio purpurcus (Degéer); - Becmare (Geoffroy); - Perce-pomme des Jacdinicrs; - Apfellescher des Allemands.

Long de $0^{\text {m }}, 008$ a $0^{m}, 010$; d'un beau rouge dorí, à teflets verdàtres; corps mon, légèreınent soyeux; rostre plus long que le corselet, grèle et entierement d'un noir violacé; tète coutte, yeux saillants; corselet mutique dans les deux sexes, mais plus globuleux chez la femelle.

Cet insecte n'est pas rate en Franee: on le tronve an premicr printemps, et quelquefois en abondance, sur les Pommiers et les Poiriers en fleurs. Il prend sa nourritne dans be sne, diuns lit sćve ou dans la inoclle des jeunes pousses. Pour se procurer 
J'un ou l'autrc, il perce, arec sa trompe, de nombreux trous sur les jeunes branches, qu'il affaiblit ainsi, et que le moindre vent suffit alors pour séparer en partic du trone, auquel elles restent souvent suspendues, conslituant ainsi ec que les jardiniers désignent sous le nom de brindilles. Quelques entomologistes pensent, eependant, que ect insecte n'opère eesperforations que dans le but de reehercher une place convenable pour y déposer un ocuf; dans ce cas, comme il n'y a que la femelle chargée de ce travail, il n'y a, par conséquent, que ee sexe qui soit nuisible. Contrairemeut à cet avis, je dois dire que l'on trouve des Rhynchites bacchus perforant des feuilles et des pétioles bien avant l'époque de la ponte, et que c'est bien eertainement pour y puiser leur nourriture qu'ils opèrent ecs mutilations.

La ponte a lieu dans le courant du mois de juin, vers la SaiutJean, dans les annécs ordinaires; beaucoup plus tòt, quand le mois de mai a été favorable à la végćtation: dans tous les cas, e'est après le nouage des fruits que celte opération se fait. La femelle, à l'aide de sa trompe, peree sur les petites poires, un trou de 3 à 4 millimètres de profondeur, qu'elle élargit un peu dans le bas. Elle so retourne et dépose un œuf blanchàtre qu'clle pousse au fond dn trou avee son rostre. Cet organe lui sert aussi pour reboucher en partie l'ouverture qu'elle vient de pratiquer; et, pour la fermer eomplètement, elle y dépose une matière glutineuse qu'elle lisse cusuite arec son abdomen; tout ce travail s'opère en moins d'une heure, dont le premier quart est ensployé à la perforation du trou.

En général, une femelle ne confie qu'un seul œuf à ehacque fruit; eependant, ou en trouve quelquefois un deuxième à eóté du prenier, mais dans un trou séparé; quelquefois aussi, et seulement dans des eas très-rares, on trouve un troisième ouf et même un quatlic̀me, placés l'un près de l'autre dans une autre partic du fruit. Dans ee cas, il est naturel de supposer que ees derniers soient l'œurre d'une autre femelle qui n’a pas vu que déjà la jeune poire avait reçu un dépót semblable.

Dans certaines années, on reneontre un graud nombre de poirettes qui deviennent noites à la surfaee, molles à l'intérieur, 
et sur lesquelles se trouvent un ou plusienrs trous pratiqués par un Rhynehites; mais, si l'on examine l'intérieur de ecs trous non bouchés, on n'y rencontre jamais d'œufs, parce que, probablement, la femelle a senti que déjà la partie charmue du fruit était en décomposilion, et qu'elle ne pourrait servir aux développements ultérieurs de la larre. En 1857, cette maladie était assez commune sur les fruits des Poiricrs en quenouilles de certains jardins de Vallières, de Plantières et de Vaux; en ouvrant les poires ainsi affectées, on découvrait, dans quelques-unes, de 18 a 20 larves apodes et jaunàtres, an milieu d'une pulpe noiratre. Contrairement a l'opinion émise par plusieurs horticulteurs, je puis assurer que ees larves n'appartiennent pas aux Rlyynchites, et qu'elles sont celles d'un inscete dont l'histoire sera éerite dans unc autre partie de ce travail.

Au bout d'un temps plus ou moins long, selon la température, mais qui, en général, ne dépasse pas une semaine, il éclòt une petite larve apode, d'un blane rosé, molle, courte, composée de 12 anneaux peu distincts, avec la tête noire et écailleuse. Cette larre commenee immédiatenent à ereuser une galerie qui va jusqu'à l'endocarpe, et qu'clle continue ensuite jusqu'à ce qu'elle arrive à percer une seconde ouverture de l'autre cóté du fruit. Dans quel but s'accouplit ce double travail? est-ce pour que la larve puisse recevoir l'air extéricur, ou donner une issue ả ses excréments? ces deux suppositions sont également admissibles; et, pour mon compte, je pense que e'est pour atteindre ce double but, que la larve opère cette perforalion, ear je n'ai jamais trouvé d'exeréments que dans la branclse de la galerie qui correspond au trou pratiqué par la femelle. L'enduit glutineux que celle-ci dépose à l'entrée du trou, afin de mettre sa progéniture a l'abri des intenupéries et des attaques d'autres insectes, étant trop dur pour les faibles mandibules de la jeune larve, il s'en suit que eelle-ei ne peut pas l'entamer et donner ainsi passage aux insectes carnassiers ou aux Iclıneumous parasites; il est probable aussi que l'ueuf est toujours disposé de telle sorte que, lors de son éclosion, la larve n'a qu’à ereuser devant elle pour trouver sa nouriture. 
$\Lambda$ bout de quelques jontrs s'opère une première ıne; et $\mathbf{3}$ it I semaines plus tard, la larve a aequis tout son déreloppement. Alors elle abandonne le fruil, dont elle détermine presque toujuurs la eluute par sa présenee, et va s'enfuneer dans la terre ou elle se transforme en nymple et attend, dans eet état, le printemps suirant, oủ elle éelòt á l'époque de la floraison des Puiriers et des Pominiers.

C'est en effet sur ees deux arbres qu'on reneontre l'inseete le plus ordinairement. Selon Walkenaer, il se trouve aussi sur la vigne; mais il a bien eertainement eommis une erreur, et le savant orientaliste a confondu eet inseete avec le $R h$. betuleti, ou avec le $R h$. Conicus. Quelquefois aussi il attaque les jeunes eerises et les jeunes prunes, mais il parait que cela n'arrive que quand elles sont trèsabondantes, ou que les poires et les pommes font défaut. Je n'ai trouvé aueun renseignement qui puisse m'autoriser à eroire que eet insecte soit fréquent dans notre département, uu qu'il y ait causć des dominages considérables. Cependant, eomme les observations de ee genre ont été fort mal faites jusqu'iei, et que souvent on se plaint que les poires tombent pendant le mois de juin, il est bon de visiter fréçuemment ees arbres et de détruire aree soin tous les fruits perforés qu'on y reneontre.

Quant à l'insecte lui-même, il passe ordinairement la nuit sur les feuilles de l'arbre; il sera done très-utile de secouer ceux-ei le matin, au-dessus d'une toile, afin d'en faire tomber les individus qui l'habitent, et de pouroir alor's facilement les détruire.

14. AhYNahites atRatus (Scopoli).

Schocnherr; Synonym. Curculionid.; tome 1, page 219.

Synonyınic: Curculio auratus (Scopoli); - Atelabus bacchus (Olivier); - Allclabus auralus (Payk); - Rhynchites rubens (Mégetle; Dej. catal.); - Curculio aurifer (Oliv.); Altelabe doró.

Cette espèce est voisine de la précédente, et s'en distingue 
surlout par sa trompe proportionnellemenl plus eourte et plus grosse, et dont plus de la moilié esı d'un bcau pourpre doré, tandis qu'clle est unieolore dans le $R h$. bacchus.

En général, ecl insecte est rare dans le départemenl de la Moselle, je ne l'y ai jauais reneonlré sur le Poirier. Selon Ralzeburg, il a des labitudes à peu près semblables à celles que nous venons de faire connailre pour le $R h$. bacchus. M. Nordlinger l'indique comme vivam également sur le Poiricr et le Pommicr.

On n'en connait ui la larve ni les mours, el on ne sait si sa femelle pond sur les jeunes fruils, comme le $R h$. bacchus, ou sur les jeunes pousses, comme nous allons le voir pour le $R$ h. conicus. Il csı probable que eelle opération offre quelque parlicularité intéressante a observer, mais la rareté relative de l'insecte, fail quion sera peut-êlre longtemps avant de connaitre toutes les phases de son histoirc.

\section{RHYNCHITES coNIcus (Illiger).}

Schonherr; Synonym. Curculionid.; tome I, page 231.

Synonymie: Rhynchiles conicus (Illiger); - Allelabus alliuria(Fabr.);-Curculio alliario (Oliv.); - Involvu lus alliario (Schranck); - Curculio corulcus (Fourcr.) ; Curculio nanus (Marsh.); - Altelabus pubescens (Rossi); - Curculio icosandrio (Scopoli); - Rhinomueer (Geof.); - Beche, - Liscıte, - Coupe-bourgeon de nos jardinier's;

- Zwcigabstescher des allemands.

Cet insecte est beaucoup plus petit que les précédents; il est aussi proportionnellement plus court et plus velu; d'in beau bleu d'acier brillant, avec les tarses et les antennes noires; élytres profondément striées ponetućes.

On le reneontre an mois de mai sur tous les arbres fruitiers, ct aueune de leurs variétés ue m'en a paru cxempte. Souveut on le trouve aceouplé, et le nombre des inclividus en est quelquefois vraiment prodigicux. Il y a quelques anućcs que, sur ü jeutr 
Poirier de Vallières, j’ai pu en reeueillir un assez grand nombre pour remplir, en quelques instants, une boite de la contenance d'un centilitre et demi.

Cet insecte se nourrit du suc des jeunes bourgeons et des jeunes feuilles, dont il attaque aussi quelquefois le parenclyyme; quand il est abondant, on le rencontre surtout dans la fleur, dont il coupe ou perfore tous les organcs. Mais ce sont plus particulièrement les femellcs qui, au moment de la ponte, causent les plus grands dommages aux Poiriers, en coupant une grande quantité de jeunes pousses, pour y déposer leur's oufs. Voici d'après les auteurs allemands, et d'après ce que j'ai vu moi-même, comment s'accomplit ce travail:

Au printemps, au momeut où les arbres à fruits commencent à pousser, et presqu'aussitót après l'accouplement, la femelle procède d'abord au choix d'une jeune pousse de dimension variable, mais toujours tendre, verte et non encore ligncusc. Souvent ce n'est qu'après avoir essayé de couper deux ou trois pousses, qu'elle en trouve une à sa conveṇance. Alors, sur le eơté de eette jeune branclıe qui fait facc au trone de l'arbre, et à quelque distance de son insersion, l'inscete fait, arce ses mandibules, une incision oblique de la largeur de la trompe; puis, se dirigeant vers l'extrémité de la pousse, il perce, non loin de l'incision qu'il vient de faire, et sur le còté interne de la branche, un trou qu'il creuse jusqu'à la moelle. La femelle se rctourne, dépose un ouf au fond, et l'y arrange convenablement arec sa trompe. Comme elle ne bouche pas l'ouverture dn trou, il est probable qu'clle fixe l'œuf au fond, au moyen de quelque matière glutincuse qu'elle secrìte par la bouche; toujours est-il, qu'il est difficile de détacher l'ouf ainsi déposé an fond du trou. Cette première partie du travail dure environ une heure: après son accomplissement, la femelle, sans se reposer, l'ctourne à l'incision, l'agrandit en rongeant alternativement des deux còtés, et en enlevant toute la partic supérieure de cette partie du pétiole; clle continue ainsi à creuser jusqu'à ce que, par son propre poids, la pousse tombe, et ue reste plus suspendue a l'arbre que par la partic 
corticale exterue. Ce sont ees branclies pendautes qui constituent réellement les brindilles de nos jardiniers, plutòt que celles qui sont aceidentellenent produites par le $R h$. bacchus.

Quclquefois l'insectc quilte son travail pour aller à l'extrémitć de la branche, soit pour s'y reposer, soit parec que, trouvant le temps long, il craint de s'êtrc trompé, et veut s'assurcr que c'est bieı a une jeunc pousse qu'il a confié un œuf. On ne saurait admettre, comme quelques observatcurs l'ont avané, que celle mancuvre a pour but de hàter la elute de la brindille par le poids de l'insectc. Il est évident, dit avee raison Selmithberger, qu'un coup de mandibules aurait plus de suecès.

Cetle sceonde partie du travail dure environ une heure et demic, après quoi, l'insecte se repose pendant quelques instants sur une feuille, qu'il pique ģi et là à la surface, dans un but asscz difficile a comprendre, ear on ne peut supposer que ee soit pour sa nourriture, vu le peu qu'il en prend. $\boldsymbol{A} u$ bout d'unc heure environ, la femclle recommence son travail de ponte en perçant, sur la pousse pendante, un trou à cótć du premier, et dans lequel cllc dépose aussi un ouf, et elle continue ainsi, en proportionnant le nombre de trous à la longueur de la brindille. J'ai rarement compté plus de quatre trous de poutc, et jantais plus de six, bien que, cependant, la longueur de la pousse où ils se trouvaient, cút aussi facilement permis d'en placer davantage.

Quclqu'opiniàtre que soit le travail d'une femellc, elle fait rarenent plus de deux coupes par jour; si la nuit vient la surprendre, elle interrompt son travail et se retire sous une feuille voisine; le lendemain, elle reprend la perforation du trou, ou l'ineision de la branehe qu'clle avait été obligée d'abandonner; souvent aussi le froid, la pluie ou le vent vient déranger le Rhynchites dans l'exćcution de ectte singulière industrie.

$\Lambda u$ bout de 8 jours, si le temps est favorable, il ćelòt une petite larre blanche, avec la tête d'un brun noiritre, apode comme toutes eclles du mềne geure; elle a l'abdomen garni de petits manclons eonstamment lubriliés par une humeur visqueuse. C'ctto larye se nourrit de la moelle de la jeune pousse a demi-fanée; 
clle clange de pea plusieurs fois, et 5 à 4 semaines plus tard, clle a altcint toute sa croissance. Alors elle quitte l'arbre, s'enfonce daus la terre, à une profondeur de 3 à 6 eentiuctres, $y$ passe l'hiver á l'état de nymphe, et ćclòt au printemps suivant.

En général la ponte du Coupe-bourgeon a lieu en mai ou en juin, toujours uu peu plus tót sur le Poiricr que sur le Pommicr, dont l'insecte n'attaque les jeunes pousses que lorsque eclles-ci ont perdu la plus grande partie du durce cotonneux qui les recouvre dans leur jeunesse. $\boldsymbol{A}$ partir de juillet, le nombre des brindilles nouvellement coupées va de plus en plus en diminuant, et, au mois de septembre, on n'en rencontre plus.

Le Rhynchiles conicus attaque un grand nombre d'arbres; mais il coupe de préférence les jeunes grefles, paree qu'elles sont engénéral plus tendres que les autres bourgeons. Richter eite des années ou il perdit les, neuf dixièmes de ses greffes par suite des ravages de ect insecte; Sclmitlıberger dit qu'il aurait perdu la totalité de ses gręffes dans certaines annćes, s'il ne lui avait fait une chasse continuelle. Ce Charançon n'attaque souvent qu'un cil de la greffe; quelquefois deux, et rarement trois.

On comprend aisément quelle influcnce doit avoir la température du printemps sur le développement et la propagation de eet insectc. En effet, un temps sec et chaud fait rapidement dessecher les brindilles; alors leur bois se contracte et compriune Ies oufs ou les larves qui s'y trouvent, ec qui les fait avorter. Un vent violent, en les faisant se délacher de l'arbre, contribue eneore ả hảter cette disseccation, et, par consćquent, à faire périr un grand nombre d'individus. Aussi, je recommande tout particulièrement aux jardinicrs de ne pas sc eontenter, comme ils Ic font trop souvent, d'enlever de dessus leurs arbres les brindilles qu'ils y rencontrent. Il faut les réunir et les brủler, afin d'assurer la destruction de tons les parasites qu'elles renferment.

En secouant les arbres, le inatin et pendant le jour, au-dessus d'une toile, on y fera tomber beancolip des insectes qui s'y trourent, et on pourra fitcilement les faire périr. 
C'est, sans contredit, l'unc des espèees d'insectes les plus nuisibles aux arbres fruilier's, el celle dont il importe le plus de se débarrasser, surtout dans les pépinières oil l'on élère des jeunes sujets. Dans les jardins, ils atlaquent de préférence les jeunes arbres, probablement paree que leurs pousses sonı plus tendres.

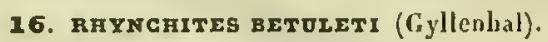

Sehmenterr.; Synonym. Curculionid.; tome 1, page 222.

Synonymic: Altclabus betuleti (Fabr); - Cureulio betulos (L.); - Rhynechites bctuloe (Oliv.); - Altclabus betuloe. (01.); - Rhinomacer bctuloe (Laichart.); - Altclabus populi (Payk.); - Gurculio populi (Payk.); - Rhinomacer viridis (Fourer.); - Rhinomacer alni (Muller); - hvolvulus alni (Schranck); - Curculio bctula, Vur. violacca (Donovan.); - Curculio nilcns (Marsh.); - Curculio violaccus (Scopoli); - Rhinomaccr bispinus (Muller); Curculio bispinus (L.); - Rhinomucer unispinus (Huller); - Rhinomaecr incrmis (Muller); - Cureulio incrmis (L.); - Allelabe du boulcau; - Coupcur de raisin; Rebenstecher, des allemands.

Corps d'un beau vert brillant en dessus, d'un vert doré en dessous; trompe et pattes de ectle dernière couleur; la femelle ayant de chaque cóté du eorselet une épine droite, longue et aiguë (1).

$\mathbf{A}$ différentes époques, ect insecte a attiré l’attention des cultirateurs, el plus partieulièrement eclle des vignerons, a cause des ravages extraordinaircs qu'il a causés dans certains rignobles. Souvent

(1) Relativement au sexe qui a le eorselet épineux, il existe un désaccord extraordinaire entre les entomologistes. Linné dit positivenent que e'est te mîle qui est dans ee eas; Nordlinger, qui a vu des individus aecouplés, dı également que éest celui qui est dessus, et par consépuent te mâle, qui a le 
il a été eonfondu arec le Rhynehites bacelıus (Latreille lui-même at commis cette erreur, et, par son autorité scientifique, a cntrainé eelle de beaucoup d'entomologistes), ct, à cause de sa ressemblance avec le $R$. populi, plusicurs auteurs allemands sont allés jusqu’à prendre eelui-ei pour des jeunes du Conpeur de Raisin.

C'est ordinairement en juin que l'on rencontre le $R$. betuleti en plus grande abondance; mais l'on en trouve eneore en juillet, en aoùt, et mème en octobre d.ıns certaines années. En général, c'est à la vigne qu'il eause le plus de dommage. Les arbres qui en sont ensuitc plus particulièrementaltaqués sont: le Hêtre, le Peuplier, le Tremble, le Tịlleul, les Saules, YAlizier, le Bonleau et le Noisettier; rarement on le trouve sur les Poiriers ou les Coiguassiers. Enfin Walther dit l'avoir reucontré sur le Pommier, ce que M. Nordlinger déclare impossible.

Rarement je l'ai vu sur le Poirier, et chaque fois que eettc eirconstanec a cu lieu, j’ai toujours observé que c’était dans des jardins voisins des vignes. On peut done supposer que les $\boldsymbol{R}$. Betuleli qui s'y rencontraient, étaient des enfants perdus de l'espèec, et aecidentellement éloignés de leur véritable habitat.

Malgré tout l'intérêt local que pourrait avoir l'histoire complète de l'inseete en question, je me bornerai à donner quelques détails particuliers à sa manière de virre, quand il s'altaque au Poi-

corselet muni d'une épine de chaque côté; Panzer, el d'après lui, Schuenherr el Ratzburg, disent au contraire que c'est la femelle. Ilelwig a IrJuvé des måles non épineux, d'autres quil'ćtaient, el des femelles qui l'étaient également. J'ai aussi rencontré des femclles dont le corselet est épineux, et je n'ai jamais vu de màle ayant ce caractère. Un fait certain, c'est que dans les $l$. bacchus et populi, ce sont toujours les femelles qui présentent ces sppendices prothoraciques: il est donc probale qu'il en est ainsi relativement ì l'insecle qui m'occupe, el que, dans la majorití des cas, ce sont les assertions de Schoenherr et de Ratzburg qui sont les plus exactes.

MI. Nordlinger reproche aussi à Schonherr d'avoir dit, dans sa description, que le corps de cet insecte est viridi sericeus, tandis que l'insecte est loin d'être velu. J'en denande bien pardon à M. Nerdlinger, mais pour des entomologistes, ce n'est pas velu que Sehonherr a voulı dire, mais bien vert snyeux, ce qui est hien different et parfaiternent exacte pour le Rh. betuleti. 
rier. Ces renseignements seront empruntés à l'ouviage de $\mathbf{M}$. Nordlinger, ear je n'ai jamais vu travailler le Coupeur de Raisiı ailleurs que dans nos vignes.

Au mois de juin, au moment de la ponte, le Rhynchiles betul'ti recherche les pousses encore séveuses du Poirier, et, à environ unc longueur du doigt de lcur extrémité, il fait, avee sa trompe, une cotaille transversale, de manière à faire pendre le bourgeon terminal, mais sans le séparer eomplètement; les feuilles de ce rameau, ainsi incisécs, ne recevant plıs qu'une faible nourriture, ne tardent pas à se faner, et l'insecte oblient ainsi une alimentation plus molle et plus a sa convenanec, pour fabriquer l'étui dans lequel sa femelle opère la ponte. Pour prendre eette nourriture, l'insecte racle la surface supérieure de la feuille, en enlève ainsi tout l'épiderme et le parenclyyme, et ne laisse que l'épiderme inférieur. C'est en ligne droite qu'il pratique ectle manœure dont il laisse souvent une trace entte ehaque nervure. D'après Breieher, qui a nourri des Rlıynelites longtemps en eaptivté, il parait que linsecte attaque aussi les grandes feuilles, dont il coupe d'abord une partie du pétiole pour les faire funer, mais eet auteur ajoute qu'il n'cmploie ce moyen que quand il n'y a plus de pousses a eouper, et que les autres ressourees lui font défaut.

Laccouplement se fait en juin; et, selon Bruschel, les màles restent constants el n'abandonnent pas leur femelle, même après que eelles-ci ont pondu. Pour opéser celte ponte, on sait que la femelle enroule les feuilles de la Vigue en folme de eigare, en se scrvant pour eela de son bee et de ses pattes. Quand elle poud sur le Poirier, dont les feuilles sont plus lisses et beaucoup plus petites, son travail est plus long et plus pénible; elle emploie alors de 15 à 18 feuilles pour la eonfection de son rouleau. Sur le Coiguassier, dont les feuilles sont un peu plus grandes et un peu velues, elle n'en prend que 4 ou 3 . La couversion d'un extrémité de rameau on d'une grande feuille en brindille, n'a pas seulement pour but de préparer à l'insecte une nourriture plus tendre, mais aussi, et principalement, de faire faner les feuilles et de faciliter leur enroulement; et eela est si vrai, que, si 
la section d'une branclse est trop longue, si elle est interrompue par le mauyais temps, ou si elle reste sans résultal, l'insecte fait alors une entaille à la base du pétiole de toutes les feuilles qu'il veut enrouler afiu de hàter leur étiolennent. C'cst eneore le procédé qu'il emploic, quand il veut emprunter, à un autre bourgeon, les feuilles qui manquent sur celui arec lequel il a commeneć son étui.

On sait que les Chenilles qui ont l'habitude d'enrouler les feuilles pour se mettre à l'abri, se servent, pour ectte opération et pour maintenir la courbure de ces feuilles, de la soic qu'elles tirent de leur filière: les Ihhynchites n'ont pas eette ressource, aussi ont-ils soin de faire fancr d'abord les fevilles, pour leur faire perdre lenr élasticité, et les maintenir facilement dans la disposition eonrenable. Sclon les auteurs, ees feuilles scraient complètement fixécs au moyen d'une sorte d'enduit gommeux sécrété par l'insecte qu'il fait sortir par la bouthe. M. Nordlinger dit ne janais avoir trouvé de traces de cette substance.

Quand un roulcau est terminé, la femelle y perce un tron, pond un couf à l'ouverture, et l'y enfonce ensuite avec sa trompe, sauns l'enduire d'une matière collante destinée à le fixer dans l'intérieur du rouleau. Après avoir disposé 5 ou 6 œufs dans autant de trous différents, et sur le mème étui, la feunelle passe a la confection d'une autre brindille et d'un autre roulcau. Ces aeufs sont blanclaâtres et de la grosseur d'un grain de millet; du luitième au douzième jour après la ponte, selon l'état de la température, ils éelosent et donnent naissance à de petites larves apodes, blanchàtres, avec la tête rougeâtre el une raie noire lougitudinale sur le dos.

On ne sait pas si le séjour des roulcaux sur l'arbre est utile au développement de la larve; on ne sait pas non plus quelle est, sur ces larres, l'influence de la pluie, du soleil, de la roséc, ete. Toujours est-il qu'elles se ereusent des galeries dans le roulleau, et qu'elles se nourrissent de la substanee des feuilles qui le eomposent. On n'est pas d'accord non plus sur le nombre de mues qu'elles y subissent, ni sur le temps qu'il leur faut pour acheverleur croissance. Sclon quelques auteuts, celte duréc est de 
5 semaines; selon d'autres, elle est de 5 semaines. Pour ecux qui ont élevé des Chenilles ou des larves pliythophages, ils savent combien eette duréc est variable et dépend de la quantité de nourriture, de la température du milieu où elles vivent, etc. Je pense qu'il en est de même pour les larves des Rhynelites, et que ceux qui dunnent le ehiffre de 21 jours, peuvent avoir aussi bien observé que cenx qui assignent une durée de 35 jours à la vie de ees larves. Quoiqu'il en soit, au bout de cette phase de leur métamorphose, elles pereent le rouleau el vontse réfugier dans la terre, où elles se fabriquent une eoque et en enduisent l'intéricur d'une substance gommo-résineuse. $\Lambda$ u bout de quelques jours, elles s'y transforment en nymplıes; environ trois semaines après, l’insecte est tout formé, et n'attend que la pluic pénétrante de l'arrière saison, pour sortir de sa retraite. C'est alors qu'on en voit des individus sur les feuilles des arbres qu'ils continuent à ronger, ju`qu'à ee que les premiers froids les obligent à elıercher un abri pour passer l"hiver.

Il arrive quelquefois que les Bhynehites qui éclosent en juillet ou en août s'accouplent à cette époque, ce qui produit la sceonde génération de l'aunce. Mais on ne sait pas si les femelles opèrent ectte deuxième ponte sur des paquets de feuilles enroulées, ou si, comme quelques autcurs le prétendent, les larves de eette génération a ceidentelle vivent à déeouvert sur les feuilles des arbres, ou enfin, si elles y ereusent des galeries eomme certaines Chenilles mineuses? En général, la plus grande partic des insectes qui éclosent ne s'aceouplent pas, et aux premiers froids, ils se eachent dans les crérasses des arbres, dans les fentes de l'écoree, sous les mousses et les lichens, daus les fissures des tuteurs, etc., ete, pour n'en sortir qu'au printemps suivant, au moment où les arbres commencent à pousser. Les individus de la seconde génération, quand celle-ci a lieu, passent au contraire l'hiver dans la terre, renfermés dans leurs coques, et u'en sortent qu'aux premiers beaux jours.

Comme je l'ai dit plus haut, c'est surtuut à la Viguc, que le Cuupeur de Raisin eause le plus de domnages; cependaut, les autres arbres fruitiers sont Join de jouir d'une inmunité complète, 
at il scrait certaincment curieux de savoirà quelles circonstances climatologiques on doit atribuer la préférence qu'il parait donner, a certaincs époques, aux ycrgers ou aux vignes, quand ces deux éléments se trourcut également à sa portéc, comme ecla a lien dans le département de lia Moselle (1).

Outre les procédés de destruction indigués plus haut, pour les Rhynehites bacchus et conicus, it faut aroir soin de réunir tous les rouleaux et de les brüler.

Quand il cst cxtrènement abondaut, on a conseillé l'arrosage, des plantations envalies, avee des solutions de sel de saturne (acétate ncutre de plomb), le sublimé eorrosif (dentochlorurc de mercure), les eaux acidulécs, etc., etc. Mitis, ontre que ces moyens n'ont pis réussi, et qu'ils sout unêrne dangercux, ces substanees pcuvent nuire beaucoup plus a la plante que l'iıscete lui-même. Ia solution de sulfale de cuivre, lont il a été question à propos de l'Agrilus viridis, reussirait-elle mienx?

Secouer les arbres dans le moment de la ponte, de l'accouplement ou de la confection des rouleaux, est une opération assez difficile à mettre à exécntion sur une large échellc, mais e'est encore le scul moyen qui puisse raisonuablement être conscillé.

\section{RHYNCHITES GUPROEUS (Gyllenlial).}

Sclinenherr; Symonym. Curculionid.; tom. 1, page 21.

Synonymic: Altelabus cuprous (Fabr.); - Cureulio euprous (L.); - Rhymelites punetalus (Herbst.); - Allelubus

(1) Pour donner une idéc de la proportion que, dans certains cas, peut prendre la multiplication de cel insecte, je cilerai les deux faits suivants qui se sont passés, au siècle dernier, dans des provinces voisines de la nòtre: Fn 1750, les vignes de Landall étaient complétement dépouillées de leurs feuilles, nvant la fin du mois de juin, et le bois tellement endommagé par le $R$. betuleli, que la récolte de l'année suivaute fut réduite an trente-cinquième. En 1756 , le $R$. betuleti a étú trouvé à Roth (grand-duché de Bade), en si grande quantité, que lon a pu en ramasser quatre hectolitres en un seul jour, et que les neuf diriemes le la recolte furent perdus ( V. Norllinger; l.oc. cit. ; page 130 ). 
ancus (Latr.); - Involvulus melallicus (Schranck.), Var. B.; - Rhynehites cupreeus (Oliv.); - Curculio murpureus (Lin.); - Allelabus mupureus (Fab.); - Apion murpureus (Herbst. ); - Perec-Prune, des jardiniers; Plaumenborer, des Allemands.

Insecte de la taille du $R$. conicus, et par conséquent beaucoup plus petit que le $R$. baechus, dont il a la coloration métallirque, couverte d'un très-léger duvet grisitre; dessous du eorps de coulcur plus obsenre ; élytres presque carrées, planes et lortement ponetuées strices.

Cet inseete est très-rare dans le département de la Moselle : je no l'y ai trouvé qu'une fois sur un Cerisier. D'après M. Noerdlinger, c'est à cette espèce qu'il faudrait rapporter des ocufs trourés par cet auteur, au mois de juin 1831, sur les pousses d'uu jeune Poirier.

Ces branches neétaient pas séparées de l'arbre par une coupe partielle comme celles auxquelles le $R$. conicus confie ses ocufs. Les trous qui renfermaient des oufs de Rlyynchites élaient disposés en spirale et non en ligne droite.

D'autre part, on sait parfaitement que le Rh. cuprecus a l'habitude de pondre ses ocufs sur les jeunes prunes ou sur les jeunes cerises, et qu'il fait ensuite une incision sur le pédoncule de ces fruits, et jamais sur les pousses de l'arbre; il y a done tà une contradiction évidente entre ees deux manières d'assurer l'arenir de leur progéniture, et on ne peut raisonnablement les attribuer toutes les deux à la neème espèce d'insecte, laquelle modifierait d'une manière aussi radicale ses lıabitudes, quand les prunes on les cerises viennent à faire défaut.

Dans une lettre que m'écrivait mon sarant ami M. Clıevrolat, en octobre 1855 , il me signalait le Rhynchites minutus G. (1)

(1) MuYchites minutus (Germar).

Schoenherr; Synonym. Curculionid,; tome 1, page

Synonymie: Rhynchites arcuatus, (Dej., Catal.); - Rhynchites viridis (Mégerle).

Ovale, d'un vert foncé et blanchatre, pubescent un peu brillant; corselet 
eomme vivant plus particulièrenent sur les Pommiers et sur les Poiriers. J'ai depuis, en effet, trouvé ect insecte sur de jeunes Pomniers élevés en espaliers, près de nombreux Poiriers; mais je n'ai pu trouver de trace de leurs travaux. Malgré toutes les probabilités, je ne puis donner, avee eertitude, eet insecte comme étant celui dont le professcur de Stuttgard décrit la manière de pondre. Je dois encore, à l'appui de cetle dernière manière de voir, faire remarquer que le $R$. cuprceus est beaucoup plus grand que le $R$. minutus, et que M. Nordlinger dit positivement que les oufs qu'il a trourés sont de la grosscur de eeux qui sont pondus par le $\boldsymbol{R}$. conicus, dont la taille se rapproche le plus de celle du Rh. minutus.

\section{RRYNGRITES PAUXILUS (Germar.).}

Schœnlerr; Synonym. Gurculionil,; tome I, page 932.

Synonymie: Curculio alliaria (Rossi.); - Attelabus coruleus (Fabr.); - Rleynchites germanicus (Ilerbst.); - Involvulus sulcidorsum (Schranck).

D'un bleu foneé; plus petit et plus velu que le $R h$. conieus; yeux proéminents; eorselet cylindrique, canaliculé au milieu, et fortement ponetué; élytres strices, portuctes, avec les intervalles de strics étroits et eonvexes

Cette espèce est très-eommune daus nos environs, sur les Poiricrs et les Pommiers, pendant leur fluraison. Quoique je n'aie pn faire des observations complètes sur la manière de pondre de ect inscete, je n'hesitc pas, d'après ec que j'en ai ru, a lui attribuer ee que Schmithberger rapporte d'un Rhynchites plus petit que le R. conicus. D'aprìs ect auteur, la femelle de cette espèce inennnue, pond ses cufs sur les pétioles des feuilles, après les aroir en partic coupés, en y pratịuant un culaille à droilc

fortement ponctué; élytres profondément strićes el poncluces, avee les intervalles lisses. 
't à gauche, et non en dessus comme le tais le $R$. conicus, quand, par lızird, les jeunes pousses lui funt défant, et qu’il est réduit à pondre sur les pétiules des feuilles. M. Noerdlinger dit aussi aruil trouvé, au commencement de juillet, des jeunes larres pruvenant d'œufs pondus dans les conditions qui vienuent d'ètre indiquées, et vivant à décourert sur le dus de la feuille, à la base du pétiole. Ne serait-ec pas ces jeunes larres que l'on aurait à tort rapportées à la seconde génération du Rh. betuleti? Car, rout en admettant la possibilisé de cette seconde génération, n'est-il pas difficile de comprendre une aussi grande différence dans les mours des larves d'une même espece mais provenant de deux pontes suecessiyes?

Malgré deux tentatives infructueuses, je u'ai cneore pu élever les petites larves que j’attribue à $R \boldsymbol{h}$. pauxilus, et résoudre cumplètement la question qui m'occupe : j'espère ètre plus licureux en 1857. Toujours est-il que les larves en questiun, qui me paraissent bien appartenir à us Curculionite, ne ressembleut en ricn à celles de Rhynchites que l’ou a déjá fait connaitre.

I.es moyeus de destruction indiqués précédemment pour les es pèces congénères, peuvent ètre appliqués à cet insecte. Quant aux larves qu'un lui attribue, un soufflage à la clıax ou aux cendres de bois lesfait rapidenent disparaitre; pour dés'uire les oufs, il faudia brùler toutes les feuilles isolées pendantes par une partic de leur pétiule.

XIII. APION. Herbst. (1).

Schonherr; Synomym. Curcul.; tome 1, page 249.

Antennes médioeres, insérées ì la base ou au milicu du rostre, les trots dernières en massue; lète enfoneće dans le corselet; trompe longue, filitorme, droite out plus ou moins

(1) Synonymie: Apus (Bilberg); - ATtecabus (Fabr.); - 0xrstom (Uuméril); - limsosscen( Laicharting); -Cunculo (Linnó). 
arquice; clytres ovales, tris-convexes, recouvrant complètetheilt I'abdomen.

Inseetes de très-petite taille, de eouleur rouge, bleue ou métallique, quelquefois reeouverts d'un duret grisàtre; les espèces sont très-nombreuses (plus de 200 sont déerites dirns les auteurs), et beaucoup d'entre elles se font remarquer par le nombre prodigieux des individus; ils vivent en société sur diverses cspèces de plantes, auxquelles ils carsent souvent de grands dommages, par leur étonnante féeondité.

On a déerit les larves d'environ dix espèces d'Apion; malgré ee petit nombre, relativement à la riehesse du genre, on a observé, pour plusieurs d'entre elles, les mœurs les plus disparates. $\Lambda$ insi, les unes virent dansles graines de légumineuses; d'autres habitent des galles, à la manière de certaines Hyuénoptères; enfin, quelquesunes creusent des galeries dans les jeunes tiges de rertains arbres, cte. On a également fait eonnaitre plusieurs Iehneumons qui vivent en parasites sur quelques-unes d'entre elles.

Les $\Delta$ pions qui vivent sur les arbres fruitiers, leur sont partieulièrement nuisibles; les femelles de ces inseetes perforent les jeunes tiges, les ovaires ou les bourgeons, pour déposer leurs œuf's dans les trous pratiqués par des procédés non eneore bien observés; les larves qui en éelosent se nourrissent de la moelle ou des organes embryonnaires de la fleur, ou des feuilles, selon qu'ellesvivent aux dépens de l'une ou de l'autre de ees partics de la plante.

\section{ApION POMONOE (GeImar).}

Schœenlıerr.; Synonym. Curcul.; tome I, page 250.

Synonymic : Attclabus pomonœ (Fab.); - Apron cœrulesccns (Kirby); - Curculio corulcscens (Marsiram); - Attclabus cyancus (Panzer); - Curculio glabcr, var. B. (Marslram); - Obstspitzmaüchcn, des Allemands.

Long de 4 à 5 millimètres; noir; trompe effiléc en aléne; 
corsclet poncuic, canaliculé postérieurement; élytres presque ovales, gibbeuses, bleues, avec des stries ponctuées.

En avril et en mai, celle espèce parait en trẻs-orande abondance sur les fleurs des arbres fruitiers, qui en sont quelquefois littéralement couvertes. En 1844, j’ai pu, dans un verger de Woippy, en réeolter une quantité suflisante pour en remplir un flacon de la contenanee de 6 centilitres.

Litceouplement se fait au moment de leur apparition la plus abondante : les mitles ne tardent pas a disparaitre, et les femelles perforent, avee leur trompe, les parties interues de la fleur, non pour s'en nourrir, comene le dit Bouehé el comme semble le croire M. Noerdlinger, mais pour y déposer leur poute.

Je n’âi pu eneore savoir si une femelle eonfie plusieurs ocufs au mème trou, ou si les nombreuses perforations que j’ai sourent observées sur la mème fleur, sont l'ouvrage d'autaut de fentelles différentes. La larve n'a pas encore été décrite; mais j’ai en ee: moment des jeunes poires et des jeunes pommes qui renferment des larves que jai tout lieu de eroire appartenir à un $\Lambda$ pion, et que j'espère pouvoir faire connaitre plus tard, quand elles auront subi toutes leurs métamorphoses.

D'après les anteur's allemands, il parail que l'insecte parfait éclót en aoút, en septembre, et quelpuefois inéme en oetobre. Ce qu'il y a de eertain, e'est que l'Apion pomonx, dont on ne retrouve plus un seul individu fin de juin ni en juillet, se rencontre de uonveau, en aout el en septembre, sur les feuilles des Poiriers et des Pommiers, et cu'alors il se nourrit, dit-on, du sue moelleux des I'ueerons (Blatlaüs), qui habitcut les feuilles de ees arbres fruiliers. Aux premiers froids, ees insectes se retirent dans les crerasses de l'éeoree, sous les mousses, cte., pour y être à l'abri pendant l'hiver; et au printemps, ils reparaissent aux premiers beaux jours.

LiApion pomonx at-il une seconde génćralion daus l'auncé? Je ne puis résoudre complétement cette qusstion, mais je dois fatre observer que les individus que l'on rencoutie pendant la 
fin de l'été, sont loin d'être, par leur nombre, en rapport avec ceux que l'on trouve souvent par uilliers au printemps suivant. Si cutte seconde génération n'a pas lieu, il faut alors admettre que les individus qui apparaissent en été, sont des produits des premières pontes, et que les insectes formés en aout ou en septembre, trompés par la température de celte époque de l'annćc, sorlent de lcur eoque avant le plus grand nombre des individus qui sont alors moins avané́s et qui doivent passer l'hiver dans le milicu oủ ils ont subi leur dernière transformation.

Pour détruire les Apions, il faut, le matin surtout, seeoner les arbres au-dessus d'un linge, et briler les insectes qui y tombent.

XIV. POIYDRosus. Schonberr (1).

Schœenherr.; Synonym. Curculionid.; tone II, page 131.

Antenmes tenues, assez longues, avec le seape en massuc, les 2 premiers atticles du funieule assez longs, les autres cour's, massue ovale et allongéc; ycux assez saillants ; corselet petit, un peu rétréci en avant; élytres ovales et allongécs avec les épaules bien marquées; jambes presquégales, euisses inttiques ou dentécs, jambes épineuses.

Petits insectes couverts d'écailles soyeuses, ordinairement d'un vert tendre ou d'un vert doré, quelquefois bruns, maculés de gris el de blanes. L'Europe tempérée semble être leur patrie de prédilection: l'Asic et l'Amérique en nourrissent cependant quelques espèees. Tous vivent sur les plantes et s'y multiplient quelquefois en grande quantité. On n'a eueore observé les larves que d'une seule cspèce, $P$. Cervinus (L.) (2), mais leur histoire est loin d'être complète, car, outre qu'on ne saclıc rien de leur organisation inté-

(1) Synonymie: Polydnusus (Germar); - Dascillus (Mégerle); - Munanus (Mégerle); - Cunculo (Degéer); - Priyllerastes (Stéven).

(2) Mir. Chapus et Candeze (Catalogue des larves de Coleoptères), comnettent une erreur en signalantau genre I'olydrosus (F.) la larve du P. oblongus décrite par Kollar en 1837 (Naturges; der Schodlieh; page 259). ("'est du Plyyllobius oblongus (L.) dont il est juestion, et la citation faite à la page 5.10 
rieure, on n'est pas d'accord sur leur genle de nourriture : ainsi, MM. Kollar et Ratzburg les croicut Phytoploges, tandis que, selon Bouché, celle du P. Cervinus est phyllophage, el ne s'enfonce dans la terre que pour y subir ses inćtinnorphoses.

20. Poutdrostes sericeds (Gyllenhal).

Schoenhert.; Synonym. Curculionid.; tome 11, page 1.18.

Synonymic: Curculio sericeus (Gyll.); - Polydrosus smaragdinus (Dej.); - Dascillus smaragdinus (Hligerle);Polydrosus squammosus (Gicrm.); - Curculio splendideus (Herbst.); - Dascillus malachiticus (Knoch.); - Curculio formosus (L.); - Golsdchneiden, - Graurusseler kicfer des Allemands.

Oblong, noir, mais couvert d'écailles soyeuses d'un beat vert tendre; antennes et pattes d'un jaune testacé.

Long d'cnviton $0^{\mathrm{m}}, 004$; ce charmant pelit insecte est assez. commun au mois de juin sur nn grand nombre d'arbres. Les individus frais ont tout le corps d'un beau vert, mais au bout de quelıues jours, les femelles, surtout, perdent unc partie des écailles qui less recourrent, et une plus ou moins grande partie de leur eorps parait noire.

Lanologic qui existe entre les diverses espéces du genere l'olydrosus et quelques-unes de cclles du genre Phyllobius, a dû faire confondre les mours de la plupart de ces insectes, surtout par les horticulteurs, qui sont peu aptes, en général, à distinguer les différenees spécifiques de ces Curculioniles.

En 1856, j’ai ru, au Sablon, un Polydrosus sericeus perforant un bouton de Poiricr (var. Saint-Germain). Il est probable que l'altention que j'apportais à son travail l'importunait, car il l'a abandonné, el je n’ai pu roir dans quel but il l'arait commenec.

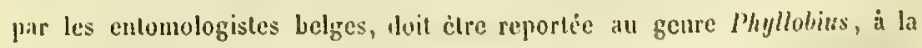
page 519 du aime ourrage. 
Cet individu était une femelle dont, en ouvrant l'alsdomen, j’ai retrouvé les ocufs.

D'après II gegelsehwiller, unc espèce voisine, le $P$. Mali, pond ses oufs dans une petite eavité des boutons à fleurs. La femelle pratique ce trou aree sa trompe; quelquefois aussi elle perfore les jeunes pousses ou les boutons a feuilles. Ces wufs sont an nounbre de un ou deux seulement pour chaque ouverture. Cet observateur ajoute que la ponte se fait, sans donte, pendant la nuit; ear, peudant le jour, l'iusecte role çá ct là avec beaucoup d'agilité. La larve éclòt au bout de lmit jours, entre plus avant dans le bouton, et ronge une partic des organes qu'il reuferme, mais pas toujours couplètement, de sorte que sourent la fleur peut eneore s'épanouir et donner un fruit qui, dans ee cas, est petit, maigre, mal tourné et dur. Arrivée au moment de se transformer en nymphe, la larve peree une galeric qui va jusqu’an pédoneule du fruil; ecluici tombe, at la larve s'enfonce dans la terre, ou elle acheve de s'y métamorphoser en un insecte qui passe l'hiver dans le sol, et d'oủ il sort all printemps.

Cette nanière de pondre, en perforant des boutons ì fleurs ou à feuilles, est eertainement très-remarquable ehez un insecte qui a tune trompe aussi courte que celle des Polydrosus. Aussi, j’ai d'abord eru qu'on avait eonfondu ce Charançun aree quelques autres à rostre plus effilć, jusqu’au moment oủ j’ai ru moi-mème, uı P. sericeus perforer un bonton de Poiricr. Est-ec tonjonrs à celtc espèce qu'il faut attribuer la cluute, souvent considérable, de jeunes poirctles? Dans ces fruits ainsi tombés, on obserre ordinairement une larveblanchiale et apode. Je cherehe, depuis plusicurs annćes, a suivre les transformations de celte larve, sans y parvenir.

Ainsi que je l'ai déja dit, le Polydrosus sericeus vit sur un grand nounbre d'arbres, même sur des conifères. On ne sait eomment il opère sa ponte dans ees nouvelles conditions. Les individus qu'on rencontre sur ecs arbres résineus, ćlant proportionnellement toujours plus petits, plus élroits, el d'un beau vert tendre uniforme, il est permis de supposer antils constituent une espece différente. 
Dans les jardins, on aura soin de ramasser et de brúler, ou au unoins de porler au funicr, toules les brindilles, les fenilles sèches el pendantes, les fruits tombés, ete, puisque nous voyons que, daus la naajorilé des eas, ces parties séparćes de l'arbre renferment les larves ou les aufs d'insecles plus ou moins nuisibles.

XV. PHYILOBIUS. Schønherr (I).

Sehœnlierr ; Synonym. Curculionid.; tome 11, page 131 .

Antemnes longues, les 2 premicrs articles du funicule allongés; massuc ovale, allongéc, pointue; rostre court et épais; yeux saillants; corselet retréci en avant; élytres ollongues; épaules efficécs; corps ailé, un peu mou; pattes longues.

Les Phyllobies ont le corps brun ou courert de jolies écailles vertes, soycuses, dorécs ou bleuàlres. Ils cont propres à l'kurope (2), d'où on en a déjả déerit plus de 73 espèces; le nombre des individus en cst quelquefois très-considérable. Ainsi que leur nom l'indique, ils sont phyllophages. C'est sur les feuilles, el ordinairement à leur face inférieure, quand il fail trop chand, sur les jeunes bourgeons ou sur les jeunes greffes, qu'on les Irouve le plus souvent. Ces insectes mangent les feuilles dans toule leur épaisscur, el non en les raclant, comme le font les Magdalis, ainsi que Ic dit M. Nordlinger, en parlant de ees derniers.

Quoique ces insectes soienl très-communs, très-abondauts, et qu'ils aient été souvent observés, on ue connait cucore rien de l'histoire de leurs larves. Les autcurs sont loin d'être d'aceord sur lcurs habitudes, leur unanière de pondre, cte. C'est en juin qu’on les trouve accouplés pendant très-longlemps à la face inféricure

(1) Synonymie : Polvonusus (Jcjean); - Privl.erastes (Sch.); - Cimrsis (Mégerle.), - Dascillus (Mégerle); - Munsnus (Mégerle); - Cunculio (Liin.).

(2) Le l'h, toeniatus (Say.) n'appartient pas au geme dans lequel Schenhere l'avait placé. M. Melsheim le place dans le genre Apunastes. (Catalogue of the elescribed Coleuntera of llic United States, ele; Washington, 1855.) 
des feuilles de beaueoup de plantes, et les feunclles restent longrenps avant de disparaitre. Malgré la grande abondance des Phyllobius en 1857, je n'ai pu découvrir, cette année, leur manière de pondre, ni savoir si leurs larres sont mineuses, ou si elles vivent dans des paquets de feuilles qu'elles agglomèrent entre elies, comme l'avaucent certains autenrs. Mais, comme je l'ai déjả fait observer en plusicurs oecasions, les espèces de ce genre ont dú être confondues entre elles, et on a probablement attribué à une espèce quelque particularité qui n’apparticut qu'à un insecte voisin. Les Plıyllobies ont des earaetères distinetifs souvent diffieiles à eonstater, et le grand nombre de variétés que présentent ecrtaines especes, sont autant de causes qui ont pu produire ce résultat.

\section{PHYzZOBIUS GazGaRATUS (Fabre).}

Schoulerr; Synonym. Cureulionid.; tome II, page 435.

Synonymic: Curculio calcuralus (Fabr.); - Curculio pyri (Illiger); - Polydrosus pyri (Déj.); - Polydrosus scopoli (Dalm.); - Curculio glaucus (Seopoli); - Curculio carniolicus (Scopoli).

Longueur 5 a 7 millimcitres; oblong, noir, un peu velu, couvert de petites taches soyeuses d'un gris eendré; antennes et pattes rougeâtres; corselet légèrement comprimé; écusson arrondi en arriere.

La couleur des taches est très-variable; souvent elles sont grises; quelquefois elles sont d'un beau rouge euivreux et doré, vertes ou verdatres; enfiu, eliez quelques individus, surtout sur les deraiers que l'on rencontre, elles manquent sur une plus ou moins grande étendue. La couleur des pattes est également trèsvariable, et l'on trouve tous les passages du jaune testacé clair au rouge ferrugincux plus ou moins foncé, et jusqu'au noir. Les autennes sont ordinaircment de la couleur des pattes, et prósenteut, sous ce rapport, aussi peu de fixité que ces organes. 
Le Ph. ealcaratus est, dans nos euvirons, eclui de toutes les espèees de ee genre que l'on reneontre le plus rareinent sur les arbres fruiliers. C'est ordinairement sur les Poiriers en espaliers qu'on en trouve quelques individus, qui en rongent les feuilles, surtout pendant la nuit. Ce sont là les seuls renseignements exacts que j’ai pu recueillir de la part de quelques jardiniers; car on sait qu'en général, on ne peut espérer des observations bien faites de la plupart d'entre eux.

22. PHYIXOBIOS PYRI (Linne).

Schonherr; Synonym. Curculionil.; tome II, page 437.

Synonymic: Curculio pyri (L.); - Curculio aruginosus (Payk); - Curculio argentatus (Laich.); - Curculio cosiuts (Marsh.); - Curculio alncli (lllig.); - Curculio pyri; var. (Payk.) ; - Curculio cnidcs (Marsh.); - Curculio ribesii (Brem.); - Curculio urtice (Degéer); Curculio prasinus (Oliv.).

Long de 6 millimétres; voisin du précédent; oblong, noir, couvert d'écailles soyetıses, variant du vert tendre au verdàtre; antennes et pattes d'un brun ferrngincux; corselet court, fortcment rétréci en avant; écusson pointu en arrière : ec dernicr caractère permet toujours de listinguer ecte espéce de la précédente.

Cet inseete est très commun sur les Poiriers et plusicurs autres arbres fruitiers de la uectune famille.

Au mois de juillet 1856 , j’ai trouvé, sur un jeune Poirier sur lequel j’arais observé un grand nombre de P/h. pyri quelques jours auparavant, plusieurs feuilles minées par des larves de Curculionites. Ces larves étaient blanehatres, avaient environ 3 millimètres de longueur, el elles pourraient bien être eclles de ce Phyllobius. Malleureusenent, je n'ai pu suivre ees larves dans toutes leurs trauformations; et, 10 jours plus ıard, quand je suis retourné pour les observer de noureau, elles araicut entièrement 
disparu: il est probable qu'elles s'étaient réfugićes en terre pour y achever leur transfor:nation.

23. Phyllobids argentatus (Linné).

Sthocherr; Synonym. Curculionil. ; tome 11, page $4 \$ 7$.

Synonymic: Curculio argculatus (Lin.); - Curculio sericcus (Seriba); - Var. Curculio arborator (Ilerbst.); Silber yraurinssler des allemands.

Un peu plus petit que les préeédlents; oblong, noir, couvert d'écailles d'un vert argenté, d'un cendré verdatre ou d'un bleu verdatre, parseme en outre de quelques poils raides, rares et disposés en séries longitudinales plus fournies vers les extrémités des élytres; anteunes un peu épaisses; jambes et tarses jaunátres ou variant, comme les antennes, de cette couleur au roux ferrugineux.

Cette espece, dont la forme est étroite et allongée, diffère notablement des deux préeédentes avec lesquelles elle n'aurai pas dì être confondue par un si grand nombre d'horticulteurs. C'est d'ailleurs avee ces deux espècés que le Phyllobius argentaus se rencontre souvent sur le meme arbre, et je ne connais de son listoire, aucune particularité remarquable, ni qui ail élé obserrée.

24. PHYLLOBIUS OBLONGUB (Linné).

Schœnherr; Synonym. Curculionid, tome II, page $4 \$ 8$.

Synonymic: Curculio oblongus (L.); - Curculio floricole (IIerlsst.); - Curculio pruni (Seopoli); - Curculio querncus (Fourer.); - Curculio fuscus (Laieh.); - Curculio rufesccns (Marilı.); - Curculio varians (Besser); - Braun grünrussler des allemands.

Insecte tris-varial)le pour la taille et la coulcur; allongé, noir, hronn ou ferrigineux; couvert d'une pubesence grise 
plus out moins serrée; antennes et pattes rousses ; élytres de couleur peu eonstante: quelquefois elles sont unicolores, d'atttres fois elles sont faiblement bordées d'une eouleur plus foncé; elıez quelques individus, enfin, celle bordure est beaucoup plus large.

Cet insecte est exeessirement commun sur une grande quantité d'arbres d'esseuces très-rariées. Dans les rergers, vers la fin de mai et le eommeneement de juin, il y est quelquefois si abondant, que les feuilles des Pommiers, des Poiriers, des Cerisiers surtout, en sont à moitić dévorées. En 1857, les Phyllobies étaientsi multipliés qu'on les reneontrait sur toutes sortes de plautes, et que les jeunes cerises elles-mêmes, en ćtuient attaquées. Quand les Phyllobies font leur apparition de bonne heure, ils commencent à manger les jeunes bourgeons, surtout ecux des jeunes greffes qui sont plus tendres, et iju'ils paraissent affeetionner d'une maniẻre tonte partieulière; quand la régétation est languissante, ils font un mal considérable, paree qu'alors ils attaquent jusqu'au cour des bourgeons, au point que le plus grand nombre ne peut plus se développer.

Selmmithberger dil, qu'en 1831,1834 et 1853 , il a eu de la peine à sauver les jeunes Poiriers de ses pépinières.

Il cst vraiment extraordinaire que li manière de pondre, et, en général, toutes les phases de la vie d'un insecte aussi commun, soient encore presque complètement ignorées. Malgré le grand nombre d'individus accouplés que j'ai conservés en eaptivité, eette annce, je u’ai pu découvrir des oufs de la femclle sur les feuilles que je leur dounais à nanger. Schmithberger dit que eette femelle pond dans la terre et que la larve vit aux dépens des racines des graminées (1).

(I) En 1857, année où les $P h$. oblongus étaient excessivement communs, jen ai trouve un grand nombre sur des elıumes et des cjpis de seigle, mais je dois ajouter que les ehamps plantés de eette céréale étaient environnés de nombreux arbres fruitiers, quil avait fait heaueoup de vent les jours précédents, et que sur une foule d'autres plantes on trouvait tes traces du passage the nombreux Phỵllohius. 
D'après M. Nocrdlinger (Entom. zeit.; Stcttin, 1848, page 252), la larve du Phoblongus réunit en paquet les feuilles du Populus canadensis, pour $y$ faire sa ponte à la manière du Rhynchites betuleli. En 1855, l'auteur dit ciu'il croit avoir commis unc crreur, et que les Pho oblongus qu'il a rencontrés dans des roulcaux formés par le Rhynchites populi, ne se trouvaicnt là que parce que les jeunes feuilles à demi-fanécs, qui le composaient, étaicnt plus tendres et plus convenables pour sa nourriture.

Je sais par cxpóricnce que lc $\mathrm{Ph}$. oblongus est peu diffieilc pour le ehoix de ses aliments, ct qu'il se fourrc partout, pour sc les procurer, quand ils lui manquent, que la nécessité l'y oblige, ou qu'il croit en trourcr de meilleurs: il n'y a done rien d'étonnant si M. Nordliuger l'a rencontré dans un rouleau de Rhynchites. Mais je puis affirmer que ce n'cst pas là l'industric cmployéc par les femelles pour assurer la subsistance de leur progéniture.

Dans les années ordinaires, c'est surtout dans les vergers lumides que l'on rencontrc le plus communément ect iusecte, auquel on fera la gucrre avec succès, en sccouant les arbres dès lc matin (car l'insecte vole très-bien) au-dessus de toiles tendues sur le sol. Dans les pépinières, on a conscillé, à tort, selon moi, l'usage d'un cornct de papicr pour cn recouvrir les greffes que l'on veut préserrer: outre que ce cornet ne préserve que le haut de la greffe, et qu'il latisse une issue pâr le bas, par où l'insecte peut fort bien s'introduire, il a encore l'inconvénicnt de soustraire ecte grefe à l'action des rayons lumineux, et, par consćquent, de nuire à son dércloppement.

25. PBYLIOBIDG VEEPERTINUB (Fabre.).

Schonherr; Synonym. Curcul.; tome II, page 453.

Synonymic: Curculio vesporlinus (Fabr.); - Curculio mali (Gyll.); - Curculio fulvipes (Fabr.); - Curculio padi (Oliv.); - Curculio rufipcs (Bond.); - Curculio érythropus (Lin.); - Polydrosus mali (Dij.); - Curculio aruginosus (Liegl.); - Curculio var. (Panz.). 
Longuetr "y à 6 millimètres; oblong, noil, couvert d'écailles grises, brunes ou d'un cuivreux doré; antennes et pattes d'un jaunc testacé, ou d'un brun plus ou moins fonce; cuisses marquécs d'une taclie noirc plus ou moins grande, nulle dans quelques individus; corselet beaucoup plus étroit que les élytıcs; écusson grand clıcz la pluparı.

Le Phyllobius vespertinus sc trouve dans les mèmes circoustances quc lcs Ph. argentatus et prri. On ne connait ricn sur leur maniìre de vivrc.

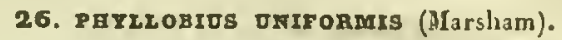

Schonherr; Synonym. Curcul.; tome II, page 458.

Synonymic: Curculio uniformis (Marsh.) ; - Curculio parvulus (Gyll.); - Curculio fulvipes (Pagk.); - Curculio argentatus (Bonsd.); - Curculio viridi areis (Laich.); - Curculio forlicornis (Koch); - Curculio fulvipes var. B. (Payk.).

Court, noir et couvert d'écailles d'tn vert pàle, d'un vert blettilte, d'un bleu plombé ott d'un cendré jaunattre opaque; antennes bruncs, un pcu épaisses; jambes ct tarses d'un testacé clair, on ferrugincux plus ou moins obscur; cuisses couvertes d'écailles verdàtres.

Gctle espéce est beaucoup plus rarc que les précédentes. En 1855 , j’en ai ccpendant trouvé unc quarantaine sur un petit Poiricr élevé en cordon; à la fin du mois de juin, il y avait encore beaucoup d'individus accouplés. Cícst lá tout ce que je sais de particulier sur les habiludes de ect insecte.

XVI. PEITTELos, Germar (1).

Schonherr; Synonym. Curcul.; tome 11, page 511.

Antennncs assez longucs; scapc courbe et plus long que la

(1) Sjnonymie : 0nus (Dej.); - Pacurgaster (Sturm.); - Centmicnemes (Stév.); - Cunculio (Oliv.). 
tète; massue presque ovale et acuminćc; rostre aussi long mais plus ćtroit que la tétc; corselet court et rétréci en avant; ésusson caclıc ; ćlytres ovales, élargies aux épaules; corps privé d'ailes.

Insectcs de petilc taille, couver's d'écailles grisiltres, propres à l'Europc centralc, où ils vivent sur les arbres, dont ils ınangent les bourgeons et les feuilles, comme les Plyyllobies. Les larves dc Peritelus sont complètement inconnues, et l'ou ne connait pas grand chose sur lcs mours des insectes eux-mèmes.

27. FaRITEzUg GRISEUg (Olivier).

Schœnherr.; Synonym. Curcul.; tome II, page 512.

Synonymic: Curculio griscus (Oliv.); - Peritchus sphac. roides (Germ.); - Pachygasler griseus (Dej.); - Curculio inquinatus (Yllig.).

Ovale, oblong; couvert d'écailles soyeuses, courtes, trèssetrées, ct d'un gris cendré ou jaunitre en dessous; antennes et pattes couleur de poix, pubesecntes; une petite ligne longitudinale cnfonećc sur la tète; élytres trẻs-finement striées, ponetuées.

Insecie de la taille du Ph. oblongas; très-commun sur les arbres fruitiers ct particulic̀rement sur les Poiriers et les Pounmicrs. C'cst au mois de juin qu'il apparait en plus grand nombre et quclquefois en quantité considćrable. C'est surlout aux jcuncs pousscs cl aux greffes qu'il cause le plus de dommages.

Il parail que ecs insectes ont des habiludes analogues à celles des Plıyllobies, mais leur histoire est cncore à fairc. Pour les détruire, ou doit employer les mèmes procédés que ecux que nous avons indiqués plus liaul, $\mathrm{cl}$ comue ils sont aptères, ont pourra sccoucr les arbres à tous les instants de la journéc, sans craindre de les voir s'échapper. Quelques indications que je possc̀de sur ces insecles, me portent à croire qqu'ils sont nocturncs. Pendant le jour, 
on les trouve sourent immobiles daus l'aisselle des fenilles ou des rameaux; ils sont cependant très-voraces et c'est le plus ordinairement le matin que les jardiniers s'aperģoivent de leurs dégáts.

\section{OTIORHYNCHUS, Germar (1).}

Schonherr; Synonym. Curcul.; tome II, prage 551.

Antennes assez longues; scape plus ou moins renflè à l'extrémité, dépassant les yeux; massue oblongue; trompe plus longuc que la tète, ct renfléc à l'extrémité; fossctles antennaires courtes et larges; yeux arrondis; éeusson petit; élytres plus larges que le corselel, convexes; corps dur et aptère.

Les Otiorliynches, remarquables par la forine globuleuse de leur abdomen, sont ordinairement de couleur brune, noire ou obscure, leur corps est souvent recourcrt d'une pubesence grise ou jaunàtre; deux ou trois espèces seulement ont des tiches métalliques; la taille du plus grand nombre est de 10 à 15 milliuétres. Lcur élude est très-difficile : j’en possédais plus de 200 espèces dans ma collection, et le nombre de celles que l'on connait dépasse le double.

Propres aux contrées moutagneuses de l'Europe; l'Asic, l'Afrique et l'Amérique n’en possẻdent que quelques espèces. Certains Otiorliynches (ceux dont il nous importe le plus de connaitre les mocurs) sont uocturnes : pendant le jour, on les trouve immobiles sous les feuilles des plantes, sous les pierres, daus les erérasses de l'écoree, el, pendant la nuit, ils quittent leur retraite pour aller manger les feuilles ou les bourgeons de certaines plantes.

On ne connail d'ailleurs presque rien de l'histoire de ces insectes. Les larres de deux espèces seulement sont connues, celle de l'O . sulcalus (Sch.), décrite par Bouché en 1854 et celle de l'O $O$. alcr (IIerbst), décrite par Ratzburg en 1837. D'après ces auteurs, ces larres ont des pattes rudimentaires, circonstance assez

(1) Synonymie : Bracirnunvs (Latr.); - Lobonurncuus (Mégerle); - PAcingaster (Stéven); - Simo (Mégerle); - Cunculo (Lin.); - Panaphilis (Méger]e). 
rare dans la famille des Curculionites; clles sont rhizophages, accomplissent tontes leurs métanorphoses dans l'intérienr de la terre.

Unc clıose remarquable qui eonfirme ce qui préeède, et que je ne rois eonsignée nulle part, e'est que l'on trouve la même espéce d'Otiorliynelıes, indifféremment sur diverses espèces de plantes, ee qui a ordinairement lieu quand les larres de ces insectes se nourrissent de racines.

\section{OTMORHYNORUS PIOTPES (Fabricius).}

Schonherr; Synonym. Curcul.; tome II, Page 613.

Synonymie: Curculio picipes (Fabr.); - Curculio notalus . (Bonsl.); - Curculio asper (Marsh.); - Curculio granulalus (Herbst.); - Curculio singularis (Sehranek); Curculio squamiger (Marsh.); - Der diekleib rïssler des allemands.

Long de 7 à 8 inillimètres; ovale, oblong; de couleur de poix, avecle corps eouvert d'ćcailles grises tris-serríes; corselet granuleux; élytres sillonnées, avee de très-gros points enfonés dans les intervalles; pattes glabres, de la eouleur du corps, euisses faiblement ilentées.

Insecte très-comnun, anx mois de juin ct de juillel, dans les vergers. En géuéral, il est très-abondant daus les lienx lıumides et eouver'ls. C'est ce qui explique pourquoi on le trouve proportionnellement moins souvent dans les cótes de la rive gauche de la Mosclle, que dans les jardins de St.-Julien, de Vallières et de Grimont, où il en existait une grande quantité en 1849.

Jc l'ai trouvé souveut sur le Poirier, mais tout aussi sourent on le rencontre sur les atutres arbres du visinage; il ne semble done pas avoir une préférenec spéciale pour l'un de nos arbres fruitiers. Selon Kirby et Spence, il mange les jemes bourgcons et plus particulièrcuent eenx des greffes. S'il cn était ainsi, il an- 
rait des habitudes analogues à celles que nous avons indiquées plus haut pour lc Peritelus griseus, ct l'on pourrait lui appliquer les proeédés de destruction qui ont été signalés cn parlant de cet insecte.

\section{9. оттоватснб' Radods (Fabricius).}

Schœnherr; Synonym. Curcul. ; tome 1I, page 614.

Synonymic: Curculio raucus (Fabr.); - Curculio tristis (Bonsl.); - Curculio arenarius (Herbst.); - Var: B. Curculio tristis (Fabr.); - Var P. Curculio fulvus (Fabr.); - Otiorhynchus luctuosus (Latr.).

Espèce voisine de la précédente, noire ; antennes et pattes roulcur de poix; ćlytres profondément striées et ponctuées, carénées postéricurement; couverte d'une pubesecnee courte, grise el varice.

Ccltc cspèce est également assez commune, mais je ne crois pas que, conme la précédente, clle affectionne plus particulièrcment les vergers et les jardius humides. Elle me parait répandue assez uniformément partout. Quant au reste de son histoirc, il n'est pas inienx connu que eelle de l'espèee précédente, et tous les cutomologistes gardent le silence à ce sujet.

ZVIII. MAGDALIS, Germar (1).

Schœnherr; Synonym. Curcul.; tome III, page 263.

Corps allonge; antennes courtes, presque droites; seape court; masstic allongéc, acuminéc; rostre plus ou moins long, mince et arqué; yeux grands et rapprochés; corselet plus ou Inoins rétréci cn avant; écusson triangulaire; élytres allongécs presque cylindriques; pattes courtes.

(1) Synonymie : Tusunopimuus (Schocnh); -Rninodes (Dej. cat.) ; - Mlicdalinus (Germar); - Paates (Stephens); - Rirrcilexus (Fatre); - Ruixs (Olivier); - Cunculo (Liu); - Evo (Germar). 
Inseetes petits et ailés, de couleur noire on rougc, vivant du parenchyme des feuilles qu'ils raclent avee leur rostre. On connait plus de $\mathbf{3 0}$ espèces de Magdalis, de toutes les eontrées du globe. Les espćecs européenncs sont assez voisines l'une de l'autrc, et leur étude est des plus diffieile: on a confondu entre elles plusicurs espèees, ee qui n'a pas peu contribué à embrouiller l'histoire, assez obscure déjà, des inscetes de ee genre.

Les larves sont, $c$ général, aussi mal connues que les inseetes parfaits (1). On sait eependant que plusieurs vivent sous les écorees dlcs arbres fruitiers où elles se creusent des galeries tortueuses, d'autant plus profondes, que le froid est plus intense pendant l'hiver; quelquefois même elles pénètrent jusqu'à la moelle des jeunes branches $\mathrm{ct}$ eausent aiusi un tres-grand dommage aux arbres fruitiers sur lesquels clles se trouvent. J'ai ouvert un nombre cousidérable de jeunes branches mortes, provenant des arbres fruitiers des cótes de Woippy, de Lorry, de Sinćcourt, ete., et je n'y ai reneontré aueune larve de Magdalis, bien que, cependant, quelques individus du Magdalis pruni aient été trouvés sur les ćeorees de ces arbres malades.

L'aecouplement se fait cn mai ; quelques jours après, les femelles dćposent leurs ocufs dans les crcvasses de l'écoree, selon quelques auteurs, et dans des trous qu'elles y pratiquent avee lcur trompe, selon d'autrcs.

30. MAGDAZIS GERABI (Germar).

Schonlı.; Synonym. Curcul,; tome III, page 267.

Synonymic: Curculio ccrasi (Lin.); - Rynchenus ccrasi (Gyll.); - Thamnophilus icrasi (Gyll.); - Rhinodes cerasi (Steph.); - Rhynchenus armeniace (Fabr.); Curculio cerasi (Fabr.); - Magdalis nassala (Germ.); -

(1) M. Perris a donné dernièrement une histoire complète du Magdali curbonarius qui vit à l'état de larve dans l'intérieur du canal médullaire des liranches des Pins. (An. de la Soc. ent. de Fr.; 1856 . page 253). 
Curculio carbonarius (Panzer); - Curculio strialulus (Lin.).

Petit, noir et opaque; corselet court, très-ponetué; élytres granulées avee les intervalles ponctućes; rostre court, eylindrique; cuisses dentées, massue des antennes noires (1).

Cet insecte vit ordinairement sur les Cerisiers; je l'ai aussi trouvé fréquemment sur des Poiriers hauts-vents, mais toujours isolément et en petit nombre. Les entomologistes allemands rapportent qu'en 1750 , il a ravagé tous les vergers de la Suède et particulièrement les Pommiers de ce pays.

Ilerbst dit que la larve de cet insecte est mincuse et qu'elle habite de préférence les feuilles des Cerisiers et celles des Poiriers. Malgré toute la confiance que doit inspirer le nom de ce eélèbre entomologiste, je crois, qu'en celte circonstanee, il a eommis une erreur; car ce que l'on connait des liabitudes des larves des Magdalis violaceus, pruniet carbonarius ne permet guère d'admettre une aussi grande dissemblance dans la structure nécessairement différente des organes buccaux, une manière de pondre également fort distincte, etc.

31. MAGDALIS PRUXI (Fabricius).

Selœenherr; Synonym. Curcul.; tome III, page 274.

Synonymie: Rhynchenus pruni (Fabr.); - Thamnophilus muni (Sch.); - Curculio pruni (Lin.); - Rhinodes ccrasi (Steph.); - Edo prnni (Germar); - Rhina pruni (Oliv.); - Gurculio cerasi (Lin.); - Curculio erylhroccros

(1) M. Nordlinger dit que les antennes de cet inscete sont noires et velues; il y a lii, I sans doute, erreur ou confusion. Le MI. Cerasi n'a pas les antennes velues, mais ce sont celles du Magdalis barhicomis qui Hrésentent 'ce caractère. Celui-ri 'eependant ne se trouve pas sur les Poiriers, et je ne l'ai jamais rencontré qu'en battant les haies. 
(Ilerbst.); - Curculio incognilus (Herbst.); - Curculia ruficornis(Selıranek); -Magdalinus barbicornis (Mígerle);

- Der Pfaumen russel Kofer des allemands.

Noir, un peu terne, de 5 millimètres environ de longueur; antennes droites, plus courtes que le rostre, ferrugineuses; corselet bituberculé; ćlytres allongées, striées et crénclées.

Insecte assez eommun vers la fiu du mois de mai et le eommencerncut de juin. On le trouve ordinairement d'unc manière isoléc, plus souvent sur les Pruniers et les Pommiers que sur les Poiriers. Selon M. Perris (in Litt.), les larres de ce Magdalis doivent vivre dans les jeunes branehes seulcment; e'est sur ce renseignement fourni par mon savaut eollègue que j'ai eu l'idćc de rechercher les traces de eet insecte daus les raneaux malades ou morts des arbres fruiticrs de la rive gauclıc de la Moselle.

Selon M. Nœrdlinger, la larve des Magdalis pruni vit sous l'éeorce, oú elle creuse des galeries, dont la trace peut être snivic a l'extérieur de l'arbrc, par la couleur plus foncéc que prend l'épiderunc dans ces partics. Poul' sortir, après que tontes ses trans. formations sout općrées, l'inscete ereuse un trou dont la forme se rapproelie de celle que nous avons indiquée en parlant des Buprestes du genre $\mathbf{A g r i l e s .}$

On a confondu la larve de l'Allanthus athiops avce eelle du Magd. pruni, non parec qu'elles ont la moindic analogie, mais parec que cette fausse Chenille raele l'épiderınc des feuilles du l'oiricr comme le fait l'insccte avce sa trompe.

Pendant le jour, il se tiemt ordinaircment sous les feuilles; alors, e'est leur surface inféricurc qui est labourée par le rostre de co Rhýuehène; pendant la nuit, au eontraire, c'est de la face supérieure que l'iuscete enlève sa nourriture. Dans les maurais temps ct aux preniers froids, il sc retirc dans les ercvasses, sous les mousses et les lichıns des arbres mal soignés. C'est dans ces retraites qu'on peut souvent cn détruire un grand noinbre, en enployant un badigconnage á la claux, au goudrou du gaz, elc. Si l'on vcut sceouer les arborcs pour en fairc tomber les individus placés sur les feuilles, 


$$
\text { Prealète Pantif. - Colb́optẻnes. }
$$

il faut aroir soin de ne faire ectte opération que le matin, ear, pendant le jour, l’insecte vole très-bien et s’écbappe facilement.

xIX. ANTHONOMUS, Germar (1).

Schocnherr; Synonym. Curculionid.; tome III, page 3.

Antennes longucs et grèles; funicule de 7 articles, dont les 5 derniers sont courts et lenticulaircs; massue ovale, allongée; trompe longue, filiforme et arquéc; corselet trèsétroit en avant, presque conique; éeusson allongé; élytres eonvexes; pattes antérieures plus longues que les autres; cuisses épincuses et dentécs; corps pubescent et ailé.

On connait les mocurs de 5 ou 6 espèces d'Anthonomus: tous vivent à l'état de larve dans l'intérieur des boutons à feuilles ou à fruits de diverses espèces d'arbres de nos jardins (Poiriers, Pommiers, etc.) ou de nos forêts (Mérisiers, Ormes). Comme dans le genre Rhynchites, ce sont les femelles qui, pour assurer la nourriture et un abri convenables à leur progéniture, causcnt la perte d'un grand nombre de boutons, en les perforant avec leurs trompes. Cependant, les dégaits occasionnés par ces insectes, quoique considérables dans certaincs annćes, sont loin d'atteindre la proportion de ceux que l'on attribuc aux $R h$. conicus, Betulas, etc.

A ces nombrcuses analogies entre les habitudes de ces insectes et celles des Anthonomus, je dois encore ajouter que ceux-ei vivent somvent sur plusieurs sortes d'arbres sans avoir l'air d'en préférer aucun.

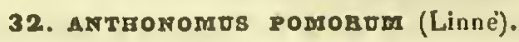

Schonherr; Synonym. Curcul.; tome 1II, page 340.

Synonymie: Rynchamus pomorum (Fabr.) - Curculio pomorum (Linné); - Anthonomus clavalus ('Ziegl.); Anthonomus incurvus? (Stephens); - Coupe-bourgeon;

(1) Synonymie: Cunculio (Linne); - Rnrxchaxus (Fabr). 
- Brileur ; - Ap/elbluthentescher, - Rothenwerden des Allemands.

Long de 5 à 6 millimètres; d'un brun rouge; éeusson blane; trompe aussi longue que la tête et le corselet réunis; corps entièrement couvert d'un duvet très-cotrrt, très-fin, visible sculement a la loupe, de coulcur beaucoup plus claire dans le liers postérieur des élytres, et formant une ligne plus obseure tout autour de cette tache.

Cet insecte est très-comnun dans toute l'Europe septentrionale ct tempérée. C'est particulièrement aux Pommiers et aux Mérisiers qu'il canse le plus de nal; il ne se répand sul les Poiriers que quand les autres arbres fout défaut.

Il passe l'liver sous les feuilles mortes, sous les pierres, sous la mousse on dans les crévasses de l'écor'ec, et même dans les fissures de l'aubicr, dans une sorte d'cngourdissement dont il sort aux premiers rayons du soleil de mars ou d'avril ; c'est alors qu'il se répand sur les arbres fruitiers hants-yents, quenouilles ou espa liers, pour y opérer l'accouplement qui ne tarde pas à avoir licu. L'inseete n'effectuc pas celte invasion uniquement, comme le dit Selımidberger, en grimpant le long des trones et des branches, mais bien aussi, et surtout, selon moi, en foisant usage de ses ailes, qui sont très-dévcloppćes.

Quand on l'inquiète, il sc laisse tomber, applique sa trompe contre sa poitrine, étend et eroise ses longues pattes antéricures, ct, immobile dans cette position, il contrefait le mort jusqu'à ec qu'il croit le danger passć.

I'aceouplement se fait dans les premiers jours du printemps; et, deux à trois jours plus tard (aussitót que les boutons commeneent à pousser), les femelles procèdent au travail de la poutc. Pour cela, clles commeneent par faire choix d'un bouton conrenable. Cette opération est souvent fort longue, ear il arrive quelquefois qu' elles commencent à perforer 5 à 6 boutons avant d'eu trouver un á leur convenance pour y déposer un ocuf. Ou comprend combicu ees tattonucments doivent coutribuer à augmenter le inal 
que causerait sculement la ponte, s'il n'y avait pas plus de boutons attaqués que d'œuf́s daus l'ovaire; et, surtout, si une même femelle confrait indifféremment ses ocufs aux boutons a fruits et aux boutons à feuilles, circonstance qui n'arrive qu'aceidentellement, soit par maladresse de l'iuscete, soit par le défaut d'un nombre suffisant de boutons a fleurs. Quand la femelle ereuse un bouton, et qu'elle n'y rencontre pas d'étamines, elle l'abandonne alors, et les folioles qui s'épanouissent plus tard, conservent des trous ronds de 1 à 2 millimètres de diamètre, et qui sont les indiees du travail d'une femelle d'Anthonomus.

Arrivé avec sa trompe aux organes floraux, en traversant le calice et la carolle non encore épanouis, l'insecte dépose un acuf d'environ 1/2 millimètre de diamètre, de forme ellipsoïde, d'un blane sale et pointu aux deux extrémités. Avec sa trompc, la femelle pousse l'œuf qu'elle vient de pondre plus loin, au milieu des étamines ou on peut le trouver en ouvrant le bouton. Cette première partic du travail dure environ trois quarts d'heure.

Il arrive quelquefois que l'inscete, bien qu'ayant rencontré un bouton a fruit, abandonnc son travail, soit parce qu’il trouve le temps long, soit pour d'autres eauses assez difficiles à comprendre. Ces nourcaux tâtonnements viennent encore augmenter les causes d'avortement; quelquefois aussi, il arrive que la trompe va trop loin, et que l'ouf est introduit dans l'ovaire de la fleur; alor's cclle-ci est perdue: clle tombe ou se dessèche, et l'ouf n'éclòt pas, ou la larve qui en est sortic, meurt et ne clonne pas d'insecte. Quelques auteurs prétendent que les boutons perforés et abandonnés par l'insecte, ne sont pas pereés par maladresse, mais pour qu'il puisse y prendrc sa nourriture.

Coinme l'Authonomus ne dépose qu'un seul œuf dans chaque bouton, ou comprend fort bien qu'il ne faille pas un grand nombre de femelles sur le même sujet pour y causer beaucoup de mal. Si le temps est favorable, et que la floraison marehe rapidement, l'insecte n'a pas le temps d'y opérer toute sa ponte, et il quilte l'arbre pour clicrclıer un sujet moins précoce, ou une espèce moins nàtive; car l'ocuf, déposé trop tard, ne nuit plus aux organes de 
la fleur. L'épanouisscment de celle-ci a lieu malgré la perforaison, et la jeune larve, mise à découvert, ne tarde pas à périr.

Les Pommiers sont de préférence choisis par l'Anthonomus pour deux raisons faciles à compreadre : la première, e’cst que l'épanouissement de la fleur du Poirier se fait plus rapidement que celle des Pommiers, et que la larve n'a pas le terops d'y accomplir toute son évolution; la seconde, e'est qu'eu général le temps de la floraison dure plus longtemps que celle du Poirier, et que la femelle trouve le temps nécessaire pour déposer tous ses cufs. L’équilibre entre les deux arbres ne s'ćtablit que rareurent et par suite des intempéries de l'atmosphère, par la vature du terrain, ou la mauraisc constitution de l'arbre.

Si, peudant le travail de la ponte, il pleut ou s'il fait froid, les insectes se retirent dans les abris que leur présentent l'écorce ou les tuteurs, et c'est là qu'on peut leur faire la chasse avec succès, ear ils s'y trourent quelquefois en graud nombre.

Sclon Schmidberger, l'éelosion de l'veuf a lieu vers le cinquième ou le sixième jour. H. Nordlinger dit qu'clle n'a lieu, au contraire, 'pue vers le huitième jour, et encorc faut-il pour cela un temps favorable. Pendant le beau printemps de 18:37, l'éclosion a eu lieu du sixième au neuvième jour.

La jeunc larve se nourrit des élamines, des pistils, et quelquefois même de l'ovaire. Par suite de la destruction des organes de la reproduction, la fécondation de la plante n'a pas lieu, les fleut's se flétrissent avaut l'épanouissement du calice et de la corolle; les pétales se dessèchent et forment une sortc de voúte de couleur feuillc mortc, qui protége la larre contrc les intempérics, ou contre l'ardeur du soleil. C'cst cette coloration particulière, souvent rougeitre, des boutous arorlés qui a fait dire aux jardiniers que les fleurs étaicnt brúlées par le soleil, la lune rousse, la gelćc, la mauraise sève de l'arbre, ete. Ces boutons roussis trahissent facilement la présencc de l'insecte, et on retrouve eneore sur leur cnveloppe le trou par lequel la femelle a introduit son couf.

En 3 a $/ 4$ scmaines, selon l'état de la saison, la larre a acquis 
lout son aecroissement; elle est alors longue de 6 a 9 millimètres, un pen pointuc aux deux extrémités; la tête est petite arec deux taclues brunes on noires réunies en are sur le derrière; les anneaux sont peu apparents, ct la peau est si mince que l'on voit, au travers, l'intestin d'uu bean rouge, causé par la couleur des anthères qu'elle a dévorées; tout le corps de la larve est lisse, avec quelques poils derrièré la tête, et une sorte de crète mamelonnéc le long du dos.

Du 15 au 30 mai, o: trouve des nymphes d'un jaune pałle, et sur lesquelles la place des yeux est fort apparentc. Après être restés sons cette forme pendant huit à dix jours, les insectes conmencent á éclore, mais ne sortent pas immédiatement de l’abri qui les a protégés jusqu'alors : ils semblent attendre pour cela que la corolle, complètement desséchée, commence à se détaclacr du calice pour leur livrer passage. Qnelquefois aussi, ils percent un trou dans la corolle, et sortent par cettc ourerture. L'évolution complète dure environ einq à six scmaines, à partir de l'accouplcment, et du 15 arril aux premicrs jours de juin dans les annécs ordinaires.

On rencontre les Antlonomns snr plusicurs arbres de nos jardins et de nos forêts; en juin et en juillet ils sont très-abondants; mais, à partir de ectte éporque, leur nombre ra en diminuant à mesurc que la saison avance; et, soit par accidents, ou par lc fuit du ravige de lcurs ennemis (oiscaux, insectes carnassiers, etc.), il n'en reste plus, à l'automnc, que quelques individus destinés à la propagation de l'espéce au printenps suivant.

En général, on exagèe les dommages causés par l'Anthonomus pomorum; dans les années ou la floraison est belle: on croit que tout est perdu parec qu'nn grand nombre de fleurs se trouvent perforées par ces inscctes; ét, cependant, connme en 1837, il en reste cncore assez pour donner l'espérance d'une récolte rclativement satisfaisante; on peut même dire que, dans certaines années, cette destruction particlle d'un certain nombre de fleurs est mème nécessaire pour empêcher les arbres de succomber sous le poids de leurs fruits. Son inflnence est toutefois très-remarqunable sur les sujets ual taillés, on paurres en boutons à fruit. C'est plus 
particulic̀rement encore dans les années oú la floraison est maigre et languissante, dans les vergers liumides et froids, dans les terrains stériles que l'on remarque davantage l'étendue des dommages qu'il cxeree, parce qu'alors la floraison marchant avec lenteur, l'insecte peut détruire un plus grand nombre de boutons. Si le printemps est beau, la végétation marche plus rapidcment, et beaucoup de boutous s'épanouissent avant que l'inscete ait en lc temps de les attaquer. Il scmble eependant qu'il ait la conscicnce que beancoup de flcurs vont lui échapper, ear il redouble d'aetivité, et n'interrompt son travail que pendant l'ardeur du soleil du milicu du jour.

Unc fois la ponte aecomplic, tout ralentissement dans la végćtation, concordant surtout avec un tcinps clair et elıaud dans la journéc, est favorable au développenent de la larve, qui aequiert alors la force nécessaire pour ponvoir détruire les organcs intéricurs de la fleur el en empêcher l'ćpanouissement. C'est lá surtout l'explieation de l'influence que l'on prêtc à la lunc rousse sur la floraison des Poiriers et des l'ommicis. Si, en eflet, eette lune n'est pas eachéc par les nuages, le rayonnement de la nuit abaisse la température et arrête la végétation des arbres, tandis que l'insecte cst toujours a l'abri du froid sous les enveloppes de la fleur, et que, pendant le jour, les rayons du soleil augmentent la chaleur de sa logette sans dessécher la larve qui s'y trouve.

Souvent les moineaux, les pinsons, ete., brisent le bouton desséché et mangent la larve qui l'habite. M. Nœrdlinger dit aussi que ees larves sont quelquefois altaquées par un petit Ichneumon parasitc. J'ai ru, en cffet, róder sur les boutons avortés d'un Pommier, un petit insecte que jc n'ai pu encore déterminer, mais qui appartient à cette famille.

Pour mettre plus sủrenent les arbres fruitiers à l'abri des atteintes de l'Anthonomus, il faut fitire un clioix d'espèces poussant tard, fleurissant rapidement, et ayant les houtons durs et serrés.

Sclimidberger eonscille d'entourer la base des arbres d'unc ceinture de goudron, pour einpêelıer les insectes d'y aller faire leur ponte. Nous aurons plus tard l'oecasion de conseiller aussi l'em- 
ploi de ee moyeu pour se préserver d'autres petits ennemis; mais pour l'Anthowomus, ce proeédé serail d'une fixble ressouree, car l'insecte vole parfaitement, et eeux que l'on reneoutre grimpant sur les arbres, sont probablenent ceux qui ont passé l'liver dans quelque erévasse ou sous quelque mousse.

En secouant les branehes au dessus d'une toile, on en détruira un certain nombre, surtout si celte opération est faite par un temps froid, parce qu'alors l'insecte vole plus diffieilement. Cette pratique, pour être fructucuse, deınande à ćtre renouvelée souvent: mais, alors, le dommage que l'on veut éviter, est-il en rapport arec le mal que l'on se donne, et n'est-il pas préférable d'employer son temps d'une inanière plus produetive?

Frisch dit qu'il faut tailler les arbres, et fumer les sujets faibles et languissants, de maniẻre à pousser la végétation et à favorisel l'épanouissement de la fleur et le développement des bourgeons. Cette méthode eonseillée par Frisch, ne saurait produire aueun résultat, attendu que tous les jardiniers soigneux la pratiquent, ce qui n'empêclıe pas les Anthouomus de se propager cliez eux.

Enfin, le badigeonnage à la clitux, qui a été aussi préconisé, ue signifie absolument rien dans ee eas-ci, car ce proeédé de destruction n'a rien de commun avee les mœurs de notre iuseete.

Le seul moyen vraimeut efficace, eonsiste à écraser, cutre les doights, tous les boutons roussis, de manic̀re à éeraser la larre ellemc̀me, ou à la mettre à déeouvert, ce qui lui est également funeste. Malleureusement, ce procédé ne peut s'appliquer qu’aux petits arbres et ne peut ĉtre praticable en grand.

\section{ANтhonomos ormi (Gyllenhal).}

Sclionherr; Synonym. Curcul,; tome III, page 338.

Synonymic: Anthonomns ulmi (Stephens); - Rhychremus ulmi (Gyll.); - Curculio ulmi (Degécr); - Anthonomus pediculavius (Germar); - Rhynchanus avarns (Fabr.); - Curculio cuvarus (Fabr.);-Amhlhonomusravarus (Sturn.); 
- Anthonomns clavalus (Dej., Catal.); - Curculio bavarus (Schranck); - Curenlio tricolor (Oliv.); - Anthonomus pyri (Kollar).

Var. B.: Anthonomus fascialus (Stephens); - Curculio fasciatus (Narsham).

Var. P.: Anthonomus pomonce (Germar); - Rhynchcenus avarus (Olivier); - Cureulio pedienlarins (Linné); Anthonomus pedicularius (Stephens).

Plus petit, et d'un rouge plus vif que l'Anthonomus pomorum (L.). dont il est du reste très-voisin; téte et poitrine d'un brun coulcur de poix; trompe mince, allongée ct arquéc; une ligne légère et ınćdiane sur le corselel; ćcusson et une bande transversale postéricure oblique, de coulcur blanchitre, sur chaque ćlytre.

Comme l'espèce précédente, elle se rencontre sur un grand nombre d'arbres de nos jardins et de nos forêts. Il parait que Kollar arait eu occasion de l'observer sur le Poirier, car, la croyant nouvelle, il l'a décrite sous le nom d'Anthonomus pyri (I). Selon lui, la femelle de l'Anthonomus ulmi pond ses oufs dans les boutons; mais contrairement à ce que nous avous ru pour l'Anthonomus pomorum, qui ne confie jamais ses œufs qu'aux boulons à fruils, eet insecle pondrait indifféremment sur les bourgeons de toute nature. On ue sait s'il en cst véritablement ainsi, car, jusqu'à présent, on ne parait pas aroir vérifié l'asserıion de Kollar, et dans notre loealilé, bien qu'on rencontre assez souvent celte espèce, je ne l'ai jamais trouvée sur les Poiriers ni sur les Pommiers.

34. ANTroxomos PYAI (Clicvrolal).

Revue zoologique de M. Guérin; année 1841. page 145.

Synonymic: Anthonomus ulmi, Var. B. (Schonherr); Rhynchenus ulmi (Gyllenhal).

(1) Schodlichen der Insceten, cte.; Vienne, 1837. 
Corps noir ou d'un brun fonce; tite convexe, avec une ligne longitudinale blanclıc; trompe arquic elıez les máles, droite clic\% les femelles; base des antennes de couteur plus claire que le funicule ; corselet arrondi, tur peu rétréci en avant, rouge, rugueux, avec une ligne longitudinale błanche au milicu; écusson blanc; élytres d'un rouge obsetur, avec une bande transversale blanche, élargic en dehor's et interrompuc à la suture; pattes de coulent ferrtigincuse, annelies de noir.

M. Clievrolat ajoutc encore à cette description : « l'Anthonomus ulmi de Selı. cst plus petit, plus étroit; il a la trontpe rouge, mais moins sillonnée, son corselet est tellement velu, que l'on ne peut aperccroir qu'arec peine sa ponctuation; les élytres sont moins renflécs postérieurement, et la bande transversale est moins étendıe » .... « Cette espèce, confondue, par Gyllenhal ct Schrauck, arce l'Anthonomus ulmi (Sch.), comme n'en formant qu'une rarićté, rit exclusivenent sur le Poirier'; notre beau-frèrc Cosnard, l'a prise en septembre sous les écorces de cet arbre. $\downarrow$

En 1856, M. Chevrolat m'écrivait que depuis 1844, il u’avait jamais reneontré l'Anthonomns pyri ailleurs que sous les écorces ou sous Ies lichens des Poiriers. La larve, ses mocurs, ainsi que celles de l'insecte lui-même, sont d'ailleurs complètennent ineounues; ct, bien qu'aux mois d'avril 1856 et 1857 , j’aie rencontré quelques rares individus de celte espèce ('́galement sur le Poirier), je n'ai encore pu trouver une femelle occupée à faire sa ponte.

L'analogie spécifique, qui existe entre ces trois espèces d' $A n$ thonomus ('Pomorum, Ulmi, Pyri), a sans doute fait confondre leurs liabiludes par desobservateurs moins scrupuleux que les Entomologistes sur la valeur et la nature des caractères distinctifs, comme le sont en général les liorticultcurs. Il est possible aussi que ces iusectes aient des mours semblables entre elles, mais qu'ils affectionnent cliacun une cspèce d'arbre particulier, ou bien qu'ayant des mocurs dissemblables, ils puissent vivre à la fois sur lc mềne arbre: ce qui, dans l'um ou l'autre cas, aura amené la eonfusion cles espèces et celle de leurs liabitudes. 
XX. axc1NUS, Germar (1).

Sclinenherr; Syn. Curcul.; tome IV, page 776.

Antennes courtes et minces; massue composée de 4 articles Irès-rapprochés; rostre long, tenu et arqué; ycux latéraux; corsclet carré; ćlytres trìs-allongées; anus en partic découvert; pattes conrtes, toutes les jambes apicalement éperonnées.

Insecte de petite taille, ayant le corps allongé, étroit, un peu pubesccut et ailé. Ce genre est peu nombreux cn espèces : toutcs sont propres à l'Europe et à l'Algérie. On ne connail encore la larve quc d'unc seule espèce (MI. Collaris, Germ.); elle vit dans les hampes du Plantago marilima. Les autres cspèees connucs sc trouvent sous les écorces de différents arbres, mais on ne sait ricn de plus de l'histoire de leur métanorphose.

35. MEcivus PYRASTER (Herbst.).

Schonherr; Syn. Curcul.; tome 1V, page 777.

Synonymic: Curculio pyraster (IIcrbst.) - Rhynchanus scmi-cylindricus (Gyll.); - Curculio pyrastri (Stepl.); - Macipus pyraster (Stéven.) ; - Curculio ccrasi (Payk); - Var. B. Curculio hanorthoidalis (IJerlsst.); - Neptaphilus pyrastce (Mègerle); - Curculio denigratus (L.). Noir, allongé, plus court clıez le mile; couvert d'un duvet ecndré; trompe très-allongẻe, fortement arquéc; corselet ponctuć ; élytres strièes et ponctuées; cuisses armées d'une petite dent.

Cet inscete a un faciès tout particulier, qui rappclle celui de la Calandre du blé (Silophilus granarius), trop bien connue de tout le mondc.

(1) Sỵnonymie: Rurxchexus (Cyll.); - Neptaruluos (Mégerle); - M cipes (Stéven); - Cuiculio (llerlsst.). 
On rencontre les Mecinus sous les écorees d'un grand nombre d'arbres. Macquart indiquc cettc espèce comme ltabitant plus particulièrement le Poirier. M. Perris dit que positivement la larve de cet iusccte vil dans le bois du Poirier. J'ai, en effet, trouvé le Mrecinus pyraster, plusieurs fois sur les Poiriers malades, dont il a été déjả souvent question dans ce mémoire; mais je n’ai eneoro pu en déeouvrir la larve, ni observer les habitudes de l'inseete parfait.

XXI. ScoLYTUS, Geofroy (1).

Blanchard; Hist. naturelle des Inscetes, tome II, page 129.

Autennes de 11 articles, les 5 derniers en massue ovoïde et articulée, les 6 préećdents en eòne allongé; jambes terminées par ın onglet; avant dernier article des tarses bilobé; tčtc arrondie, enfoncéc dans le corselet.

Les Scolytes sont mallieureusement trop connus, et pour faire leur histoire complète, il faudrait sortir du cadre que comporte ce travail. Le nombre des espèces de ce genre n'est pas considćrable, mais celui des individus est quelquefois tel, que c'est par millicrs qu'il faut compter les arbres qu'ils font périr ehaque année. L'Europe et l'Amérique du nord paraissent jusqu'ici être leur patrie exelusive; mais il est probable que des obscrvations mieux faites, en fcront trouver dans d'autres eontrées.

In général, c'est dans le bois que vivent ces insectcs ì leurs divers élats; et e'est cn ereusant des galeries dans la substance des arbres, qu'ils font un tort considérable à ceux-ci, en donnant des issues par lesquelles la sève s'extravase. Bien que plusicurs espèces aicnt unc prédilection partieulière pour tel ou tcl arbre, elles peurent en général s'aecommoder de l'essenee de plusicurs, et il n'est pas rar'c de rencontrer le Scolytus destructor, par exemple, sur l'Orne, le Chêne, le IIĉtu'c, le Tilleul; le Sc. pruni, sur un grand nombre d'arbres fruiticr's, ete. Sur le mêmc arbre, cer-

(1) Synoymic : Eccontogasten (Herlist.); - Conrorasten (Duftschm); MLESINTS (1)ufstchü). 
taines espèces rivent également dans toutcs ses partics, tandis que d'autres n'habitcut exclusivement que les hranches ou le trone. Janais les Scolytes n’attaquent des arbres résineux. La forme des galerics, très-variable, selon les espèces, est, cu général, assez constante pour chacunc d'clles, et pcrmet très-souvent de recons. naitre l'cspèce qui les habite. C'est ordiuaircment dans les couches du liber et dans celles de l'aubicr que se $\operatorname{logent}$ les Scolytes; cependant, on en reneontre aussi dans l'éeorec. Dans les hivers rigoureux, ceux qui se trouvent sur Ics branclies ou sur les jeuncs sujets, creusent plus profondément leur galeric.

C'est plus particulièrement à propos des ravagcs causés par les Scolytes, que s'est engagée la fameuse et interminable discussion sur la qucstion de sayoir si ce sont les insectes qui rendent les arbres malades, ou s'ils ne font qu'envahir ceux qui sont déjả souffrants. Sclon M. Guérin, ct eet auteur cite des faits nombreux à l'appui de ses assertions, jamais les Seolytes n'attaquent lcs arbres sains. Ratzeburg et ses partisans citent égalennent des faits a l'appui de l'opinion contraire. Saus entrer dans le fond de cette discrussion, jc dois dirc que jusqu'ici je n'ai pas encore rencontré un seul Scolyte sur des Poiriers en bon état, tandis que j'ai trouvé souvent le Scolytus pruni sur des Poiriers vieux, crévassés, couverts de chancres, ou atteints de la brủlurc orgauique dont nous avons parlé plus lıaut. En 1856, il était même assez abondant sur quelques-uns des arbres les plus malades; et il est à craindre que, vu les conditions farorables à son développement, qu'il doit reneontrer dans les arbres des vergers de la rive gauehe de la Mosellc, il n'y acquière cn quelques anuées, des proportions con- sidérables, et clu'il ne contribue ainsi à hiter la perte de ecs productifs cóteaux.

L'accouplcment des Scolytes se fait dans l'intćrieur des galeries ou en dehors de cclles-ci, selon Ics espèecs. La femelle pond ses rufs et les dépose un à un, et isolément, daus de petites cavités où elle les recouvre souvent de débris de bois pour les soustraire il la voracité des larves des Sylvamus, des Ditoma, ete., que l'on reneontre fréquemment après elles. 
Les larres éclosent de ees oufs, au bout d'un temps plus ou moins long, selon les espèees, la températule, etc.: elles sout apodes, et ressemblent assez à celles des Curculionites, dont elles différeut, cependant, par la forme plus allongée de leur tête, et par lc dércloppement de leurs maudibules, ce qui est parfaitement en larnonie avec leur genre de vie. A peine écloses, les jcunes larres coumenceut à se ereuser des galeries, au bout desquelles elles se transforment en nymples, el d'où l'insecte parfait s'échappe en perforant le bois jusqu'a l'extérieur. Ces galeries seeondaires sont diversement inclinées par rapport à la direetion de la galeric priıcipale; mais ee qu'il y a de remarquable, e'est que toutes aboutissent à une partic voisine de l'écoree, de manière à rendre plus facile la sortic de l'insecte, et que jamais elles ne se toucheut, quel que soit leur rapprochement initial. Cies galeries sont faciles a distinguer de eelles que ereusent les femelles pour y déposer lcur's oufs, ear eelles-ci ont un diamètre constant, tandis que daus les itutres, le diauche va en augmentant à mesure que la larre grandit, et, par conséquent, que son travail est plus avancé.

Iat durée de la vie des Seolytes, à l'état de larve, varie, selou les espèees, de quelques mois seulement eliez quelques-mues, de plus d'une annéc clicz d'autres. On a confondu plusicurs espéces eutre elles, el éest ee qui explique la confusion et les contradictions noubreuses que l'on reneontre daus les auteu's, relativement à eette durée; probablement, aussi, que la nature et la température du unilieu ou elles vivent, doivent avoir une ecrtaine influence sur la rapidité plus ou moins greande arec laquelle elles peuvent aceomplir leur aceroisseurent.

La fécondité vraiment prodigicuse des temelles de Scolytes, ne tarderait pas a multiplier ees insectes a tel point, que les atbres en seraient infestés, si la nature u'avait, avee une sagesse infinie, eréé une foule de larves earnassières, qui, virant dans les galeries, font souvent un véritable carnage de ees larves de Iyloplıages; plusieu's Icluneumonides sont égaleırent daus ce cas, et il arrive quelquefois que toute la poute d'une femelle de Scolyte ne produit aueun de ces insectes. 
Comment atteindre et détruire ees petits animaux quand ils sont logés dans l'intérieur d'un arbre, et qu'ils y pratiquent leur funeste industrie? Bien des moyens out été proposés et vantés comme infaillibles pour leur destruction; mais il n'y a récllement que l’abattage de tous les arbres fortement atlaçués, et leur enlèvement immédiat, qui puissent amener des résultats satisfaisants. Quant au Poirier, si, comme j’ai eu occasion de le vérifier jusqu'ici, ecs inseetes n'attaquent que les arbres malades, le remède est tout simple: il suffit de faire cnlever les sujets qui sont le plus gravement compromis, recéper eeux qui le somt moins, et entretenir convenablement ceux qui sont dans de mauraises conditions.

36. scorytus Destaugtor (Linné).

Ratzeburg; Die forst. Insect.; tome 1, page 186.

Synonymic: IIylesinus Seolylus (Fabr.); - Eccoplogasler destruetor (Herbst); - Seolyte destrueteur; - Stutzlorken $K \propto f e r$ des allemands.

Long de 4 à 7 millimètres; corps d'un noir brillant, ponctué; antennes, élytres et pattes d'un brun marron plus ou moins foner,; téte et unc partic du dessous du corps couvertes de poils raides, courts, d'un blanc jaunàtre; élytres avec des strics longitudinales le points, plus ou moins marquées.

Insecte à facies aphodivïde, commun partout. Plusicurs ante urs l'ont signalé comme babitant le Poirier; je ne l'ai jamais reneonIré que sur des trones abattus depuis longtemps et appartenant à eet arbrc. Ces notes étant spéeialemeut rédigées dans le but d'éelairer les arborieulteurs sur la nature des dommages eausés par les inseetes, aux arbres qu'ils eullivent, j’ai omis à dessein l'histoire de tons les insectes qui peuvent se rencontrer sur les vicilles souelics abattues, parce que ee travail m'eủt entrainé trop loin, et que les détails qu'il comporte ne paraissent pas intéresser . directement les horticulteurs. 
37. SCOLYTUB PRURI (Ratzoburg).

Ratzeburg; Die forst, Insect.; tome 1, jage 187.

Synonymic : Scolylus pyri (Ratzeb.) (1); - Eccoplogaster pyri (Ratzeb.) (2); - Scolyte du prunier ; - Der grosse

Stutzlorken Kefer der obstuaumen des allemands.

Long de 4 à 5 millimètres; éljtres un peu rétrécies en arrière; corselet finement ponctué; stries des élytres très-finement pointillées, ce qui lcur donne, sur le dos surtout, un aspeet lisse et brillant; eorps et corselet noirs; élytres brunes; jambes et antennes de couleur plus claire que eelles des élytres.

C'est principalement eette espèce que l'on reneontre sur les arbres fruitiers, et plus ordinaircment sur les Pruniers; je l'ai aussi trouvée sur le Poirier et sur le Pommier.

Laceouplement du Sc. pruni sc fail daus lit galcrie prineipale, dans laquelle on troure ordinairement de 4 à 6 individus: ces galeries sont construites dans le liber et dans l'aubier; celles qui sont habitćcs par des larves, se termineut à l'écorce. Les arbres vienx et souffrants sont les seuls sur lesquels j'ai reneontri ect insecte; M. Nordlinger dit, an contrairc, qu'il habite de préférence lcs jeunes sujets. Je n'ai pu vérifier l'exactiude de ce fait, an moins

(1) Je n'ai pas vu le Sc. pyri de Ratzebura, el n'ai, par conséqueut, pu le comparer au Sc. pruni ou ả ses varictís, donl le nombre est assez considerable, eomme d'ailleurs eela s'observe dans plusieurs espèces du genre Scolytes. M. Noerdlinger n'hésite pas à operer la rúnnion des deux espéces dèerites par M. Ratzeburg.

(2) Depuis la publication de l'ouvage de M. Ratzebury, faite en 1837, il a été déeril, sous te nom de Scolytus pyri, une espèee de l'Amérique du nord qui, bien probablement, u'est pas la méme que celle dont nous parlons. Si le nom donné par M. Ratzehuror dnit rester, eclui de l'auteur américain, M. Peck, devra être ehangć comme faisant double emploi. Voyez: Harris: A treatise of some, of the insecten, of new England. Which are injurions vegetation, efc. ; 1837 Cambrillye (Ŕtat de Massaschusselt de l'Union amiricainc). 
en ce qui concerte le Poirier. Cet auteur ajoute cncore que le domınagc causé par lc Sc. prouni n’cst jamais considérable. Ici cncore, je nc suis pas d'accord avec le savant professeur de Stuttgard, et je pense que les résultats qui sont la conséquence des ravages du Scolytc, sont cn rapport avec Ic nombre des iudividus, la végétation de l'arbre, les soins que celui-ci reçoit, etc. Dans tous les cas, jc puis affirmer que, s'il ne cause pas la mort des arbres, il en arrètc la végétation, et que les sujets qui cn sout atteints, nc donneut que des fruits rares, pctits et dégénérés, au point que l'on nc reconnait plus la variété de l'arbre qui les a produits.

La durée de la vic de la larve n'est pas cucorc connuc; mais elle doit ctre assez longue, si l'on en juge par la dimension des galerics qu'clle sc creuse avant de se transformer en nymplie.

38. BcorYTUS RUGULOBU (Koch).

Ratzeburg; Die forst Insecien; lome 1, page 192.

Synonymie: Eecoptogaster rugulosus (Ratzeburg); - Der klein Sultz borlien Kéfer des allemands.

Long de 2 à 3 millimètres; corselet fortement ponetuć; élytres avee tles stries si fortement ponetuées qu’elles en paraissent rugueuses; corps noir ; clytres brunes; abdomen relevé et un peu èlargi.

Le Scolytus rugulosiss se irencontre ordinairement daus les jeunes branches des Cerisiers, et plus particulièrcment dans celles du Pommier. Je n'ai encore eu l'oceasion de l'observer qu'unc seule fois sur le Poiricr. M. Perris l'a également trouvé sur des jeuncs branches de ect arbre. C'cst peut-être de cettc espèce que M. Nordlinger a voulu parler, quand il dit que le Sc. pruni u'attaque que les jeuncs arbres ou les jeunes branches. La difficulté de creuscr des galcries d'assez grandes dimensions dans de petits troncs, explique pourquoi l'accouplement se fait au dehors. Cependant, il arrive quelquefois que le Sc. rugulosus pénétre daus de gros 
arbres, et alors, on trouve toujours une galerie centrale, plus large, et dans laquelle se fait le rapproehement des sexes. La femelle pond ses cufs à mesure qu'cllc avance dans son travail, el les latres établissent leurs galeries dans l'aubier ou dans le bois, selon l'épaisseur de l'écorec et la température de l'hiver. La ponte se fait ordinaircment au mois de juin; mais elle continue souvent jusqu'en septembre el oetobre. MI. Noerdlinger dit que les larves ne mettent qu'un mois pour accoinplir toutes leurs métamorphoses; tandis que Selınidberger di positivement que cette période dure plus d'une annéc.

XXII. PIATYPUS, Herbst (1)

Blanchard; Ifistoire des Insectes; tome II, page $1 \geqq 9$.

Títe aussi large que lo corselet, cartée en arrière; antenIles courtes, de 11 articles, dont la massue est grande, ovoïde, comprince et non articulće; jambes courtes; tarses de 4 articles.

Insectes de petitc taille, de forme allongée, cylindrique; de couleur sombre; plus spécialcment propre à l'Amérique inter-tropicale. Une seule espèce vịt en Europe. Tous, ainsi que leurs larves, virent dans le bois dans lequel ils se ereusent des galeries.

39. PLATYRUS GXIINDRICUS (Fabr.).

Laporle; IIisl. nalur. des Insect.; tome II, page 372.

Synonymie: Bostrichus cylindrus (Fabt.); - Bostrichus cylindricus (Oliv.); - Platypus cylindricus (Latr.); Cylindra bimaculata (Dufstehm.); - Kernkoltzkofer des allemands.

Cotps d'un brun noiritre, un peu velu, surtout ver's l'extréutité des élytres, long de 3 ì 6 millimètres; auteunes et

(1) Synonymic: Bostucius (Vab.); - Crusph (Duftschm.). 
pattes brunes; tète plane en avant, rugueuse; corselet finement ponctué; élytres ponctuées, striées avec des cótes élcvées, dont la troisième est terminéc par une petitc épine; cuisses dentées; jambes terminées par une épine.

Inseete eommun dans toute l'Europe; e'est ordinairement sur le Iêtre et lo Chêne qu'ou le rencontre. Cependant, j'en ai trouvé, en 1856, plusieurs individus isolés sur des Poiriers sauvages des bois de Bricy et de Confans; et, en 1857 , deux individus sur un Poirier malade d'uu jardin de Vaux.

C'est là tout ee que je sais de partieulier du Platipus cylindrus, quand il attaque les Poiriers. Sur le Chène, on sail qu'il $s^{\prime} y$ creuse des galeries dans le bois sain, et qu'on y rencontre souvent leurs larves plusieurs a la suite les unes des autres. Les galeries de ponte sont pénérrautes ou perpendieulaires à l'axe de l'arbre, ce qui les distingue facilement des galeries de ponte des Seolytes, qui sont longitudinales ou paralléles à l'axe de l'arbre. Quant aux galeries latérales, on sait que les larves des Seolytes $y$ sont toujours solitaires, ce qui n'a pas lieu pour les Platypes.

XxIII. CLYTUS, Fabrioius (1).

Blanchard; Hist. des Insectes; tome II, page 153.

Autennes filiformes, assez épaisses, de moitié plus courtes que le corps; cuisses longues, pen renflices; pattes postéricures beaueoup plus longues que les autres; corsclet rond, convexe, sans épine.

Insectes de 10 à 20 millimètres de longueur, propres à l'Europe, à l'Algérie et à l'Amérique. Leur nom leur vient (2) du bruit qu'ils produisent en frottant leur tête contre le prothorax. Voiei comment

(1) Synonymic : Leptun. (Lin.); - Callidera (Panzer); - Cemandrx (Linué); - I'Latrivotus. (Mulsant); - Anagliptus (Mulsint).'

(2) y>viš, quion entent. 
M. Mulsant nous décrit leur forme et leurs habitudes : « Ces insectes sont généralement remarquables par l'éléganee de leur parure. Les uns, sur lcurs ćlytres de velours jaunc, portent des points ou des bandes d'ébène; plusieurs, sur un fond obscur, montrent des espèees de signes hiéroglyphiques, des lignes courbes ou flexueuses, des chevrons ou des croissants d'argent; les autres, sur leur eorps de jais, semblent chamarrés de galons d'or, eomme nos hommes de cour. Les goủts de ces gracieuses créatures sout en harmonie avec leur beauté. C'est aux fleurs que la plupart vont dernander le peu de nourriture qui leur est nécessaire. Elles rolent des ombelles du panais aux corymbes de la millc-feuille, ou cherchent d'autres fois, sur des planles bumbles, les sues mielleux dont elles sont avides. Lcurs pieds longs et déliés, approprićs à lcur genre de vie, sont des indices de leur légèreté: dans les journćes chaudes, surtout, leur agilité désole souvent la main qui clıerehe à les saisir. 》

Les larres des Clytes sont eneore peu eonnues. M. Perris a déerit celle du $\mathrm{Cl}$. arietis; ct, selon MM. Chapuis et Candèze, I'Entomologie Magazin conticnt la description de celle du Clytus arcualus. Ces larves vivent dans le trone des arbres vicux ou abaltus; clles s'y creusent des galerics entre l'éeorce et l'aubier, et se transforment en nymplies et en insectes daus des loges ereusées dans le bois. C'est ee genre de vic des larres de Clytes qui explique la présenec de ces insectes dans les bücheries, les clianticrs, etc.

40. cittus agotatus, (Fabricius).

Mulsant; Longicornes de France; page 73.

Synonymic: Leptura arcuata (Lin.); - Callidium arcusbtum (Panzer); - Plalynolus arcuctlus (Nulsant).

Dessus dtt corps d'un uoit velouté; corsclet transversal, ayant une bande jaune en avant et une autre de la mème cottleur, interrompues au milieu; éctsson jaune; élytres ayan 
chactine dleux points vers la base, trois bandes arquées sur le disque et une ligne oblique, à l'angle sutural, de la mème couleur.

Au priutemps 1857, j'ai trouvé sur des sonelies de Poiriers nouvellement abattus, un grand nombre de larves que je croyais appartenir à la Saperda scalaris; mais après avoir consulté M. Goureau el Perris, je les ai rapportées à une espèce du genre Clytus.

Ces larves sont longues de 10 à $2 \breve{3}$ millimètres; la tête est plate et ćlargic; les anneaux du eorps sont bien distinets, le $8^{\mathrm{e}}$ et le $9^{\mathrm{e}}$ sont plus étroits que les autres, ce qui donne un facies tout partieulier à ces larves. Le corps est blane, à l'cxception de la partic antéricure du premier anneau qui est plus ou moins jaune; les parties de la bouclie sont d'un brun foncé. Les pattes, au nombre de 6, sont très-courtes et très-ćcartées, surtout les postéricures; les jambes sont coniques et composées de 4 articles; les mandibules sont courtes, fortes et arrondies.

Ces larves creusent des galerics sinuenses entre l'ćcoree et l'aubier, en entamant celui-ci plus profondément que l'éeorcc. Ces galeries sont d'autant plus larges qu'elles s'ćloignent davantage de leur origine; clles sont faiblement creusécs et remplics de vermoulure. Plusieurs larves se trouvent dans une mêne galerie à la suite les uncs des autres: les plus grandes les premieres, ct les plus petites les plus rapprochées de l'origine.

Vers le mois de mars ecs larves quittent les parties superficielles du bois pour s'enfoncer plus avant daus l'intérieur, et s'y crcuscr une loge ellipsoïdale, allongéc, et dont elles bouehent l'ouverture aree de la vermonlure. Ln ce moment les liurves se raecoureissent, deviennent cylindriques, prennent uuc eouleur jaune eitrou, et vers la fin de mai, on en trouve déjà qui sont transformécs en nymphes. Le 10 juin, en ourrant une des buiches que je conservais pour obtenir ces inscetes d'éclosion, j'ai trouvé un Clyılus arcualus. La présence de cet inseete est done venue confirmer les prévisions de M. Perris, qui, en procédant pal exelusion, en était arrivé à conclure que les larves que je lui avais 
communiquées, devaient appartenir au genre Clytus, et peut-ètre au $\mathrm{Cl}$. arcualus.

Le nombre des larves trouvées sur les Poiriers que jai explorés, s'élevait à plus de deux eents: il y en avait de tailles fort différentes, et je ne puis eneore décider si elles appartiennent toutes au Clyte arqué, ou si elles proviennent d'une autre espicee du même genre, ou, enfin, si les prenières qui se sont transformées, apparticuneut à une génération antérieure à celles des autres larves trouvées dans les mẻmes galeries.

Ordinairement le Clytus arcualus vit dans le Chêne. J'ai pris des renseignements, et j'ai appris que les Poiriers sur lesquels j’avais trouré les larves de eet insecte, provenaient des environs de Sierek, et qu'ils avaient été abattus, paree que, depuis 2 ou 3 ans, ils ne donnaient plus de fruits. Non loin de là, aussi, existe une grande forêt de Chênes dans laquelle je me propose de faire, cetle année, une excursion, pour voir si les Clytes y sont nombreux, et ehereher si il n'y aurait pas là un nouvel exemple d'inseetes curalissant les eultures roisines, quand ils deviennent trop nou. breux dans leur milien naturel.

Pour terminer ee que j'ai à dire sur ec Clytus, j’ajouterai que les larves de eet inseete se trouvaient sous l'écorec du trone, sous eelles des grosses branches, et même sous eelles des branehes de 6 centimètres de diamètre seulement.

XXIV. LEIopus, Serville (1):

Mulsant; Col. de France; Longicornes; page 1 to.

Antennes une fois et demic aussi longues que le corps ; eorselet ayant de ehaque eòtć une épine courbéc; épaules earrées; front long et plat; segment oval des mảles caclıć, allongé dans les femelles, taric̀e cachéc pattes antéricures tun

(1) Synonymie : Cenasexx (Lin.); - LaMu (Schmolı.). 
peu plus longues que les autres et sans houppes soycuses aux tarses; corps ailé.

Insecte de taille assez petile pour la famille : nombreux en espèces, deux ou trois seulement habilent l'Europe. Les larves vivent dans le bois et sous les écorces de plusicurs arbres. Elles sont d'ailleurs très-peu connues, et leur histoire est eneore á faire.

\section{LEIOFU8 TEBULOSUS (Linné).}

Mulsant ; Col. de France; Longicornes; page 150.

Syıonymic : Cerambyx nebulosus (Lin.); - Lamia nebulosa (Schoen); - Lamie nébuleuse.

Long de 6 à 10 millimetres; corps couvert d'un duvet cendré; élytres mouchictícs de taclıes plus foncées et groupées de manière à former unc bande transversale, un peu au delà du milieu; antennes et pattes annelées de noir et de blanc.

Cet insecte est assez rare aux environs de Melz. M. Westwood en a fait connaitre la larve, mais non ses habitudes. D'après M. Goureau, cette larre aurail des nocur's semblables à celles de la Saperda scalaris, en compagnie de laquelle il a trouré sa Chrysalide sous les écorces d'un vieux Poirier abattu.

XXV. SAPERDA, Fabricius (1).

Mulsant; Col. de France; Longicontes; parge 185.

Antennes presque aussi longues que le corps, de 11 articles; front aplati; yeux très-ćchancrés, mais non divisés; élytres parallèłes ou un peu rétrécies en arrière; segment oval, échancrés dans les måles; pattes assez longues; jambes intermédiaires échancrées.

(1) Sygonymie : Cenumbrx (Scluranck). 
Insecte d'assez grande taille, courert d'un duvet court, de couleur assez variée sclon les espèces; propre à l'Europe et à l'Amérignie du nord. Les larves des espèces connues virent dans le tronc des arbres de diverses nalures: Ies unes se ereusent des galeries cntre l'écorce et le bois; d'autres labitent l'inlérieur du trone des arbres sains; quelques-unes au contraire subissent leurs mélamorphoses dans les tiges des plantes herbacées. Jusqu'ả présent on a décrit les larves de luit espèces de Longicornos appartenant au genre Saperde.

42. SAPERDA SGALARIS (Limné).

Mulsant; Col. de France; Longicornes; page 188.

Synonymic: Cerambyx scalaris (L.) ; - Saperde portcćchelle;-Der grünlcilerigcbockkœfer des allemands.

Longue de $15^{\text {à }} 20$ millimètres, noire; élytres déprimées, ornées le long de la suture, d'une bande dentelèc, forméc par un duvet jaunc citron, court et très-serré; bords cxtéricurs ayant des points et des traits de même couleur, irrégulièrement distribués.

Ce beau Longicorne, rare pendant quelques années, puis tout à coup assez fréquent dans quelques localités, a été sigualé, comme un parasite du Poirier, par Macquart, M. Goureau , ctc. Ce dernier entomologiste en a fait connaitre la larve en 1844:

« Ces larves habitent sous l'écorce des vieux Poiriers et y vivent de la sève décomposée ou des jeuncs fibres de l'écorce. Jamais clles n'attaquent le bois que quand clles sont jeunes, et c'est toujours dans les couches intéricures de l'écorce qu'elles creusent leurs galeries. Ce n'est qu'au unonent de passer à l'état de nymphe qu'clles entauent l'aubier pour s'y ereuser une loge dans laquelle elles se retirent; pliées en deux, d'abord, elles en agrandissent ensuite l'intérieur et en ferment l'ourerture avec les débris du bois qu'elles ont dérruit avee Icurs mandibules. Ces larres semblent vire trois années arant d'aroir atteint leur développenent. C'ess 
cn avril qu'clics se retirent dans les cellules de l'aubier où cltes se transforment en Clurysalides, vers le commenecment de juillet, et ćclosent vers le 20 du mêntc mois. »

a Il cst probable, dit cncore M. Goureau, que la femelle pond ses aufs dans les crćrasses de l'écorce. Sa larve cst allongéc, fluelte, d'une eoulcur vincuse qui semble résider dans l'intéricur du corps, et surtout dans le tube intestinal. Elle a la tête grande, de forme earrée, et pararaissant pouvoir rentrer daus le prenicr anneau, les mielioires bruncs et fortes, et les antennes courtes. Les mamelons sont rctranchís; le premicr anneau est orné, en dessus, de 2 taches jaunes. La Chrysalide est blauche. Quand l'insectc vient d'éclore les poils sont blancs et ne prennent la eouleur jaune qu'au bout de quelques jours. 》

Selon M. Goureau, il fandrait écorcer tous lcs arbres aballus afin de détruire toutes les larves qui s'y réfugicint.

\section{SAPERDA GANDIDA (Falricius).}

Fabr.; Systema Eleuterat; tome 11, page 319.

Symonymie : Saperda bivillala (Say.); - The apple tree borer des américains.

Ce n’est que pour mémoire que je mentionne ici ect insectc, dont la larve a étć décrite et figurée en 1855, par M. Aza Fitch, d'Albany (État de New-Yorck); ellc vit dans l'intéricur des troncs des Pommicrs, el quelquefois, dans cclui des Poiriers de l'Amérique du nord (1).

\section{Porropsia, Mulsant (2).}

Nulsant; Col. de france; Longicornes; page 190.

Antennes filiformes, un peu plus longues que le eorps, asse\%

(1) Voyez: Firts raport on the Woxious, Brneficial und other hosectes of the states of New-Yorch. Hi. Aza litch 1. m.; Albany, 1855.

(2) Synonimie: Leptuna (Linué); - SAlEnda (Schenh.); - Tetrois (Stepliens); - Axctis (1) j.). 
fortement eilićes en dessous; yeux séparés en deux par la base des antennes, la plus forte partie en dessous; élytres presque parallèles, allongées; pattes eourtes; jambes intermédiaires éclıancrécs.

Insectes d'assez petite taille, propres à l'Europe, et dont les mélamorplıses sont encore ineonnues.

\section{Polropsia pRozUsta (Linné).}

Mulsant; Col. de France; Longicornes; page 190.

Synonymie: Leptura prausla (L.); - Saperda præusta (F.); - Anoelia prousla (Dej.); - Telrops prousta (Stepl..) ; - Der augebraunte bocken kofer des allemands.

Long de 3 à 5 millimétres; tétc noire, eouverte de poils cendrés, et, en dessous, de eils rares et courts; corselet eourt, avec une ligne longitudinale et un sillon transversal hien marqués; élytres ponctuées d'un jaune livide, avee l'extrémité noire; llessous du eorps noir et luisant; pattes testacécs, arec les cuisses postérieures plus ou moins noires.

Quoiqu'assez rćpandu dans toute la France, cet insecte qui parait au printemps, n'a eneore été rencontré, par nıoi, qu'une fois", en 1854, sur un l'oirier. La larve est inconnue, et, selon M. Mulsant, elle doit vivre dans les jeunes pousses de plusieurs arbres, et notamment dans celles iu Cluènc, du Charme et du Poirier.

Comme c'est an printemps qu'il fait son apparition, on comprend combien la taille des arbres doit être préjudiciable à sa multiplication; seulement il faudrait, lorsqu'on pralique cette opération, enlever toutes les branches coupées, les briler immédiatement, et ne pas en faire des fagots que l'on rentre et que l'on conscrve, eomne si l'on voulait assurer l'éclosion des larves et des nymphes qu'elles renferment. 
XXVII. PHYTozCIA, Mulsant (1).

Mulsant; Col. de France; Longicornes; page 199.

Antennes de 11 articles, de la longucur di corps, le pre micr article est renflé, le second plus petit, le troisicme le plus long; corselet court; élytres rétréeics cn arrièrc (au moins elıcz les màles); pattes courtes; jambes intermćdiaires ćchanerées; croclicts des tarses bifides.

Inseetes de taille au dessous de la moyenne, de eouleurs variées, souvent verts ou verdatres. On en eonnait un assez grand nombre d'espèees, les unes européennes, les autres amérieaines; quelques-unes habitent l'Algérie ou l'Asie. Leurs larves sont eneore ineonnues, et les inseetes eux-mêmes ne sont pas très-communs.

45. PHхтоzCIA NIGRICORNIs (Fabricius).

Mulsant; Col. de France; Longicornes; page 208.

Synonymic : Sapcrda nigricornis (Fabr.).

Long de 8 à 11 millimètres; dessus du corps et pattes couverts d'un duvet ardoisé ; corselet ayant trois lignes longitudinales formées par un duvet cendré, celles du inilieu plus longues que celles des eòtés; antennes noires.

Il y a déjà longtemps qu'on a signalé la larve de eet inseete, eomme rivant dans les jeunes branehes du Poirier, mais sans qu'atleun des auteurs qui ont parlé de ees faits, aient rien fait connaitre de partieulier sur l'organisation ou sur les mours de eet inseete.

\section{IUPERUS, Geofroy (2).}

Blanchard; Ilist. natur, des Insectes; tome II, page 190.

Antennes grèles et filiformes, presqu'aussi longues que le

(1) Synonymio: Cerambix (Lin); - Saperda (Fabr.).

(2) Synonymic: Gutusomela (Lin); - Cuocems (Fabr.). 
corps, très-rapprochécs a la base; corselet carré ; ćlytres allongées; pattes de grandeur moyenne; cuisses non renflées, impropres au saul.

Insectes de pelite taille, vivant sur les feuilles d'un grand nombre de plantes; les larves sont á peine connues, et leurs mocurs nc le sont pas davantage; il parait cependant qu'elles sont plyyllophages.

\section{LUPERUS TLatipes (Linné).}

Linné; System. natur.; tome II, page 601.

Synonymie: Luperus flavipes (Geofrr.); - Clerysomele flevipes (Lin.); - Crioeeris /lavipes (Fabr.); - Luperus rufipes (Dejean); - Crioeeris rufipes (Lin.).

La différence qui existe entre les dcux sexes nécessite une description séparée de chacun d'cux :

Male. - Noir; brillant; base des antennes d'un jaune testacé ;

Femelle. - Noire; antennes, pattes et corselet d'un jaune plus ou moins foncé.

D'après M. Chevrolat, les Crioceris rufipes et flavipes ne doivent former qu'unc seule espèce. Ces insectes sont excessivement communs sur les fenilles d'un grand nombre d'arbres fruitiers.

En 1854, les feuilles de plusicurs Poiriers d'un jardin de ValIières en étaient eriblées. Selon Gcoffroy, la larve du Luperus flavipes est assez grosse, courte, de furne ovale, et ayant 6 pattes et la tête éeailleusc. Le reste du corps est d'un blane salc, mou; elle labite de préférence les feuilles de l'0rme.

Selon Sclumidtberger, au contrairc, ces larves sont rhizophages. Quoiqu'il en soit, il dit ne jamais les avoir rencontrées sur les feuilles.

Quand les Lupères sont aboudants, on peut, pour les détruire, fairc un feu de paille monilléc sous les arbres, de manière à les isphyxicr. 


\section{IDALIA, Mulsant (1).}

Mulsant; Col. de France; Sécuripalpes; page 1.4.

Corps glabre; antennes à massuc peu marquéc, découvertes à la base ; repli des élytres sans fossettes ; palpes labiaux grèles; cuisses débordant faiblement les cótés du corps.

lnseetes de petite taille, connus sous les noms de Coccinelles. Le genre Idalia renferme 17 espèces dont plus de la moitié sont étrangères à l'Europe.

Leurs larves et les insectes eux-mêmes sont aphidiphages; ils sont donc utiles aux plantes sur lesquelles on les rencontre, puisqqu'ils font la guerre aux Pucerons qui s'y trouvent.

\section{LDALia BIPUNCTata (Linné).}

Mulsant; Col. de France; Sécuripalpes; page 51.

Synonymic : Coecinella bipunelala (Lin.); - Coccinclle à lleux points rouges (Geoffr.); - Coccinclla dispar (Sch.); - Coccinella quadripusullala (Donov.); - Coccinclla unifasciala (Fabr.); - Coccinella ammulata (Lin.); - Coccinella liestala (Oliv.); - Cocoinella pantherina (L.); - Coccinella oelopunetala (Schoffer); Coceinella sexpustullata (Scriba); - Coccinella varia (Schranck); - Coccinella Iripustullala (Zcltersı); - Coccinelle noive à points rouges (Gcoffr.); - Coccinella cineta (Mluller); - Coecinella quadrimaeulata (Scopoli); - Coccinclla quadripustullala (Scopoli).

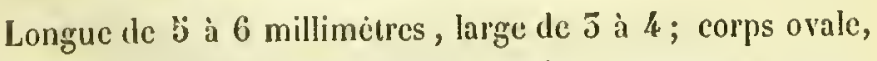
convexe et pointillé; tète inclince, noire, ayant sourent une taclıe blanchitre de chaqque cóté; antennes faures, obscures

(1) Synonymie: Coccinelus (Fabr.). 
au sommet; corselet ćclıncré en avant, ćlargi en arrière; ćcusson triangulaire, petit, noir; ; '́lytres peu convexes, tantòt rouges plus ou moins clair, avec un point diseal, ou des ramifieations uoires; quelquefois les ćlytres sont noires avec des taches rouges, le bord jaune ou rougeàtre, et l'angle lıumeral rouge; dessous du corps noir, presque glalıe; palles noires, les antéricures et les intermédiaires quelquefois rougeàtr'es.

Cet insecte, ordinairement très-eommun, se reneontre pendant une grande partie de l'élé sur les plantes envahies par les Pucerons, dont il fait sa nourriture. Il est eependaul plus abondant daus les vergers que dans les jardins, ınais pas plus partieulièrement sur certaines especees d'arbres que sur d'aulres. La larve a élé déerite la première fois, en 1720 , par Friselı; elle se trouve dans les mêmes lieux que l'insecte parfait, et eomme lui, elle fait la guerre aux Pucerous. Cette larve est allongée, rélrćeie en arriẻre, de couleur ardoisée, avee des taehes noires, disposées longitudinalement sur le ventre.

Au lieu de détruire les Coeeinelles et leurs larves, on devrait, au contrairc, elicreher le moyen d'en propager la raee, pour nous aider à la destruetion des Pucerons, qui eausent beaueoup de mal aux plantes, ainsi que nous le verrons dans la deuxième partic de ce mémoire.

XXX. COCCINELIA, Iinné.

Mulsant; Col. de Franee; Sécuripalpes ; page 71.

Corps glabre; antennes recourertes à la base; yeux peu saillants; euisses dépassant peu le bord des élytres; mésosternum entier; plaques abdlominales courbes, irrigulières et touchant le bord du premicr arceau.

Ge genre, qui a servi de type à la famille entière des Coccirellicns, est bien eonuu de tout le monde; son corps, eonvexe et 
noir, a des élytres ordinairement parées de cliarmantes couleurs, disposćes en capricieuz dessins; jamais elles u'ont, ccpendant, de reflet métallique. Malgré toutes les coupures qu'y a faites M. Mulsaut, ce geurc renferme eneore 35 espèces dont 7 seulement sont curopécuncs. Deux d'entre elles se trouvent, non-seulement rans toute l'Europe et en Afrique, mais ellcs se sont encore propagées en $\Lambda$ mérique.

Tous ees insectes sont aphidiplages; la plupart de leurs larves le sont également: cependant, sclon quelques auteurs, quelquesunes ne le sont pas et vivent au contraire de la partie herbacée de quclques plantes. Jai lieu de croire que, dans ecrtains cas du moins, des larves qui sont earnassic̀res pcuvent, pendant quelques jours, rcmplacer leur alimentation ordinaire par des substanecs végétales. J'ai vérifié l'exactitude de ee fait sur une larve de la Coccinella seplempunclala, que j’ai nourrie pendant 4 jours avec de jcunes bourgeons, dont ollc dévorait toute la substance (1).

(1) Dans l'ourrage que nous avons cité plus haut, sur les insectes nuisibles de l'État de New-Yorek, M. Aza Fiteh y donne de très-eurieux détails sur la manière dont, en $\Lambda$ mériquue, les larves des Coecinelles font la ehasse aux. Puecons: "Les coufs mûrissent en peu de jours, dit cet entomologiste, et il en sort une larve blanclie, à corps minee, termine en pointe extérieurentent, et armé, en avant, par sis petits pieds. Elle court de tous cỏtés avee animation; at si elle vient à reneontrer un Pueeron, quelque gros qu'il soit, le petit héros, à peine âtzé de quelques mimutes, le saisit hardiment, et eelni-ei, comme un poltron, ne lui oppose d'autre résistance que quelques mouvenents pour le renverser. Mais le petit assaillant se eramponne à son corgs, l'cmpeche d'avaneer d'un pas, et se servant pour armes de ses membres antérienrs, il détache le Pueeron de la feuille et dévore son eorps à son aise, en ne laissant que l'enveluppe. En grossissant, les eỏtés, et dans quelques espèees, toute la surface du corps de la larve, se eouvrent de taelies rougres ou jaunes, de points élevés ou de tubercules disposés longitudinalement. Ces petites eréatures sont d'une très-grande voracité, eourent sans eesse sur les feuilles et les branches a la recherehe de leur proie, et consomrnent des centaines de Pucerons. Dans le eourant de 2 ou 3 semaines, elles atteignent la longueur d'un quart de pouce anglais ( $=5$ à 6 millimètres); alors elles se fixent, par leur extrémité postćrieure, à une feuille, à une lranelıe ou à un trone d'arbre, et se penchant la tète en arrière, sa pean se fend jusqu'au milien 
En général les larvcs carnassic̀res, qui apparliennent aux Coccinelles, sont agiles et n'ont sur Ic eorps que des tubercules arrondies, ou des renflements inégaux. Les larves qui sont phytophages sont, au contraire, plus lentcs dans leurs dćmarches ct restent toute lcur vie sur la plante qui les a vues naitre; lcur corps est hérissé d'épines ou de namelons pointus sur les anneaux dorsaux el thoraeiques.

48. coccinglia sertemfunctata (Linné).

Mulsant; Col. de Franee; Sécuripalpes; page 79.

Synonymic: Coccinclla seplemmaculalu (Tigny); - Coccinella divaricala (Oliv.); - Coccincllc rouge i 7 points noirs (Gcoffroy); - Coccinellc ; - Agathe ; - Narichal ; Bétc du bon Dicu; - Marien kw/er sicbenpunctiri des allemands; - Crapecuex de dame des anglais.

Longues de 5 à 8 millimètres; larges de 4 à 7 ; corps hèmisphérique; corsclet noir, avec une taehe quadrangulaite aux angles antéricurs; ćlytres rouges, pâles sur le pourtour de l'ćcusson (cclui-ci noir), et ayant sur chacune trois points noir's.

Cet insecte cst très-eommun, et c'est plus partieulièrement à lui quc l'on a donné tous les noms vulgaires par lesquels on distinguc les Coccinelles cn général.

Répandue dans toute la Franec, on la rcncontre sur toute sortc de végétaux, sur lesqucls clle est toujours à la reeherehe des Pucerons. Contrairement à cc que l'on observe dans un grand nombre d'espices de la fanille des Coccinelles, les couleurs de

du dos, et la nymphe sort avee sa peau unie, mais reste protégée en dessons el sur les côtés, par les débris de la vieille peau de la larve. Dans qquelques especes (fail que je ne trouve mentionne dins aucun autenr), la peau de la larve est entierement rejetté, el les débris desséchés, restent fixés i l'extrémite de la uyouhe. 
celle-ci sont très-constantcs. Sa larve est bien connue, clle a été déerite pal un grand nombre d'entomologistes, et pour la première fois par Gredart, en 1700. Longue d'environ 12 à 15 millimètres, quand elle cst arrivće au moment de sa transformation; clle est de couleur ardoisée, tirant plus ou moins sur lc bleuàtrc, avec 6 petites pattes, et les anncaux thoraciques courerts de plaques épineuses. Deux taclıcs jauncs surlc premicr anncau; des taclies d'un jaune pále sur le quatrième et le septième; les autres ont des taclics noires. La nymplic est de couleur orange, arec 2 rangécs de petites taches noircs.

La Coccinelle à 7 points a, ainsi que sa larve, des habitudes aphidiplıages bicu conuues, et malgré l’inconvénicnt, signalć plus haut, de la voir, dans quelques ças extrĉmes, s'attaquer aux bourgeons, on doit la considérer comme unc espècc fort utile, dont on doit conserver les individus que l'on rencontre.

49. coccinelia vagiabizis (Linnè).

Mulsant; Colèopt. de France; Sécuripalpes ; page 95.

Synonymic: Coeeinella qualuordecimpunetata (Muller); Coccinelle rouge, $\dot{a} 11$ points et eorselet jaume (Geoffroy); - Coccinelle noire à 10 points jaunes (Gcoffroy) (1).

Ovale, longuc de 5 à $5 \mathrm{~b}$ millimètres, large de 2 à 3 millimètres et demi; élytres ayant à l'extrémité une ligne transversale élevéc; tepli des élytres ineliné; ćlytres d'un ecndré plus out moins foneé, sans taches, ott ayant chacune tun, deux, trois, quatre, cinq ou six points noirs; ecs taches noires sont quelquefois isolées, d'autres fois elles sont diversement liées entre elles par un trait, de manière à former des bandes transversales, des lignes, des bordures, etc. Enfin, chez quelques

(1) La synonymie des variétés de cette especce est tcllement longue, que je ne veux pas la reproduire entièrementici. (Voyez: Catulogue des Coccinelles observèes dans le département de la Mosclle, par J.-B. Géhin; Metz, 1855.) 
individus, la couletrr noire envahi la surface de l'élytre. Pattes en grande partic d'un jaune livide.

Cette cspèec, exeessivcmenl commune dans toutc l'Europc, cst tcllement variable, que l'étude de l'Entomologie serait impossible, si dans claque famillc, on rencontrait aussi pcu de fixité quc dans celle-ci, parmi les caractèrcs spécifiques d'une espèce seulcment.

Cette Coccinellc cst aphidiphage. M. Mulsant fait remarquer unc chose, que nous avons déjà sigualée nous-même dans le cours de cc mémoire; c'cst quc, bicn que l'inscete soit répandu, sa larve n'a pas encorc été décrite, ct que l'on nc sait rien sur scs labitudes.

50. CocGINELIA QUATUORDEGIMPUKCTATA (Linné).

Mulsant; Col. de France; Securipalpes; page 152.

Synonymic: Coecinella tessulala (Scopoli); - Coecinclla tetragonala (Laichart); - Coeeinella conglomerala (Ilcrbst); - Coceinella conglobala (Illiger); - Coecinella quatordecimmaeulata (Fabr.); - Coeeinella tesselata (Schncider); - Coccinella duodccimpustullata (Fabr.); - Coccinella fimbriala (Jutz); - Coccinella dentala (Cant.); - Coccinclla leucoeephala (Zsch.); Coccinella bissexpuslullata (Fabr.);-Propylea qualuordccimpunctata (1ll.); -Coccinellc à l'éehiquier (Gcoflroy).

Longue de 3 a 6 millimètres, large de 2 a 4 ; ovale ; ćchancrure du bord antéricur du corselet sinucusc, celui-ci cntièrement jaunc, ou sur les còtés seulement; élytres jaunes, avec 7 points noirs et quadrangulaires ; quelquefois elles sont noires, et ornées de taches jaunes; base des cuisses, jambes, tarses et taches sur le ventre d'un jaune très-pâle.

Insectcs variant aussi pour la couleur; ćgalement communs sur les Poiricrs et la plupart des autres arbres fruitiers, dontils mangent les Pucerons. La larve a été décrite, en 1720, par Frisch : cllc cst grise, et de tubcrculcs noirs, couycrt et, commcl'inscctc, clle cst aphidiphage. 
XXXI. MIChasPIS, Chevrolat (1).

Mlulsant; Col. de France; Sécuripalpes, page 162.

Mandibules faiblement bidentécs ; màchoires bilobées ; écusson peu apparent; corps presque hémisplıérique; massue des antennes Ironquéc; cuisses au niveau du bord externe des élytres.

Insectes de petile taille, eommuns dans toute l'Etrope; l'Asic en renferme aussi 2 espèces. Ils sont aussi aplidiphages, mais leurs larves sout eucore inconnues

51. MICRASPIS DUODEGMPUNGTATA (Lin.).

Mulsant; Coléplères de France; Sécuripalpes; page 163

Synonymie : Coccinclla sexdecimpunclala (Lin.); - Coccinclla sulurata (Gooze); - Coccinella octodecimpunctata (Fuesly); - Coccinella undccimpunctala (Gmel); - la Coccinelle jaune à suturc, de Geoffroy.

Presque lıémisphérique, jaune en dessus; corselet avec 4 points noirs au milicu, disposés en demi-cercele, et un autre de cliaque cóté ; élytres avec la sulure et 4 points noirs, disposés en série longitudinale, un sur le calus et quatre sur le bord externe; les taches du corselet ainsi que eelles des élytres, sont souvent lićes entre elles par des traits noirs.

En 1857, j'ai va cette petite Coecinelle au milicu des Puecrons du Poirier. Il est done probable qu'elle est aussi aphidiphage. Quant à la larve, ses mœurs et son organisation sont également ineounues.

(1) Synoymie; Coccinella (Lin.). 


\title{
TABLE $\triangle L P H A B E T I Q U E$
}

DES NOMS DES INSECTES CITES

\author{
dans celte première partic.
}

- क्र००28

Agathe.............. 151

Agrilus ............. 55, 77

Agrilus pyri............ 62, 81

- viridis...........62, 79

- viridis............ 80, 81

Allanthus athiops........ 19, 55

Amoiphosoma............. 77

Anaglyptus ............... 138

Anotia jrousta ........... 1.15

Axthoпомus ............. 12I

Anthonomus ararus ......... 127

\begin{tabular}{|c|c|}
\hline & elatatus ..... \\
\hline & fascialus.... \\
\hline & ineurvus.... \\
\hline & pedienlarius. \\
\hline & pedieularius; vat \\
\hline & pomana...... \\
\hline & pontorum. 49, 5 \\
\hline & pyri ..... 49, 63, 1 \\
\hline & $\eta y n \ldots \ldots$ \\
\hline & ulmi... \\
\hline & $\therefore$ \\
\hline & atme; vat. L \\
\hline
\end{tabular}

Anthovenus............. 75

Anthribus variegatus......... 83

- varius........... 83

Apfelbluthentescher.......... 122

Apfeltescher............. 85

Aphodius............... 51

Aphrastes ............... 107

Aplon.................. 101

Apion earuleseens........... 102

- pourono.......55, 62, 102

- purpureus ........... 99

Apius................ 101

Aplocnenus.............. 75

Arganta................ 76

Asila ................. 70

Altelabe doré.............. 88

- du bouleau......... 93

Attelabus auratus........... 88

alliavia ........... 89

- bacehws........ 85, 88

- betwleti............ 93

- betula............ 93

- coruleus.......... 100

- ruprous.......... 98

- cyamens......... 102
Attelabus oneus............ 99

— pomona........... 102

- populi.......... 93

- pubescens......... 89

purpureus......... 99

Angebraunte boelien kiofer . . . . 145

Bêelle............... 89

Becmare.............. 85

Bête du bou Vieu........... 151

Bitoma erenata........... 79

Bombyx dispat............ 56

- elrysorhoa......... 50

Bostrichus cylindrus......... 137

eylindrieus........ 137

Braehyrhinus ........... 115

Bnacivtarsus........... 82

Bracliytarsus varius.......62, 83

Branugriunrussler........... 110

Bruehus eapsularius........ 83

- clallratls,........ 83

- varius........... 83

Brûleur............... 122

Buprestis elougatus......... 79

- linearis............. 79

- rosana........... 79

lenebrionis......... 77

viridis........... 79

Callidium areuatum. ...... 139

Cisprodis ................ 76

Capnorlis tenebricosa......6. 62, 77

Cupusus pyri.............. 57

Carpocalsa ponionana ........ 49

Cassida limbata............ 70

Centrienemus............. 113

Cevambyx nebulosus ........ 142

scalaris........... 1.13

Celonia evemita........... 64

- eremitiea .......... 6.1.

Chrysis................ 107

Clirysomela flavipes.......... 147

Cevitus................. 138

Clytus .arcuatus........ 63, 139

- wrictis............. 139

Coccindula........... 75,149

Coccinclla amumlata......... 1.18

- bipnuctata........ 148

— bisexpustullata..... 153

- dentuta......... 153 
Coccinella disper.......... 148 - duodccimpustullata... 153 - divaricata......... 151 cineta............ 148 conglobala........ 153 canglomerafa ...... 153 fimbriala .......... 153 haslala.......... 148 leucaccplaala ....... 153 octodecimpunctata.... 154 oclopuctala. ..... 148 paulherina......... 148 quadripuslullata. ... 148 quadimaculata...... 148 qualuordecimmaculata 153 quatuordecimpunctata 63

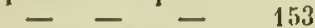
qualuordecimpunelata 152 sulurula.......... 154 sexdlcimpunctula .... 151 septempunitata .. 63. 151 seplemmaculata .... 151 sexpustullata ...... $1 \$ 8$ tessclata ........ 153 tessulata.......... 153 tetiagonala....... 153 Iripustullala ....... 148 undccimpunctatu.... 154 unifasciala......... 148 varia............ 148 variabilis......6.63, 152 Coccinelle ì 2 pains ronges .... 148 — à l'échiquier......... 151 - jume à sufmc....... 1593 - noirc, à points rouges. 148 - noire à 4 poiats rouges. 141 - ronge, i 7 poinis noirs. 151 - routge, a 11 points, ct corselel jaune..... 152 moire, is 10 points jaumes 152

Colydium unidenlatum....... 74

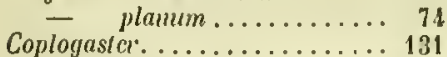
Corcebus................ 77 Cosmiocomus............... 75 Cossus ligniperda........... 81 Coupe-baurgeon.......... 89, 121 Coupeur de raisin ........... 93 Crapeanx de damc........... 151 Crioccris flavipes........... 147

- rufipcs ............ 147 Cryptophagus............. 73 Curculio alliarice........89, 100

$$
\text { - alneti............ } 109
$$$$
\text { - arborator; var........ } 110
$$

Curculio arenarius.......... 117

- argenlatus. . 109, 110, 113

- asper............ 116

- auratus............. 88

- aurifer............ 88

- avarus.......... 127

- bacchus....... 85

- bavarus ........ 128

- betula......... 93

- befule; V. violucen .. 93

- bispinus....... 93

- calcaralis ....... 108

- carbonarias ....... 119

- camiolicus...... 108

- ccrasi..... 118, 119, 130

- cnides........... 109

- ccerulcsecus.. . . . . . 102

- cervileus .... . . 89

- cesius......... 109

- cuprous....... . 98

- denigratus ....... 130

- crythoccios..... 119

- crylltopus ....... . 112

- frscialus.......... 128

- floricola.......... 110

- formosus....... 105

- forticornis......... 113

- fulvipes.........112,113

- fulvipes ; var. B.... 113

- fulvus; vac. P...... 117

- fuscus............. 110

- glaber; var. B..... 102

- glaucus............ 108

- gramulatus.......... 116

- griseus............... 114

- homorrhoïdalis; var. B. 130

- irosandric......... 89

- incognilns ......... 120

- inermis............ 93

- inquinalus........... 111

- mali.............. 112

- namks............ 89

- milens............ 93

- nofatus............... 116

- crupinosus...... 109, 112

- oblongus............ 110

- padi............... 112

- parvulus............. 113

- pedicularius......... 128

- picipes............. 116

- pomorum............. 121

- populi............ 93

- prasims............ 109

- muni.. .......110, 119

- purureus...... 85, 99 
Curenlio pyraster........... 130

- pyrnstri.......... 130

- pyri.......... 108, 109

pyri ; var........ 109

querneus.......... 110

raueus ........... 117

ribesii............ 109

rufeseens ......... 110

ruficormis.......... 120

rufipes.......... 112

sericens........ 105, 110

singularis......... 116

splendideus........ 105

squamiger ......... 116

slrialulus.......... 119

tricolor........... 128

Iristis............ 117

Iristis; var. B...... 117

ulmi........... 127

uniformis.......... 113

urtiece........... 109

varians............ 110

vespertinus......... 112

violuceus.......... 93

viridi areis ......... 113

Cylindra bimaculata........ 137

Danarea................ 75

Daseillus malaehitieus........ 105

smuragdinus........ 105

DasITES............... 75

Dasyles fnvipes. ....... 75, 76

- plambeus............

- serricornis ......61, 75

Dermatoma .............. 75

Dermestes................. 75

unidenlatus....... 74

Dielleib rüssler . .......... 110

Питана................ 71

Ditoma erenata......61, 72, 74

Divales................. 75

Eceoptoyaster destructor...... 131 muni........ 136 pyri ....... 51, 135

- rimglosus.... 51, 136 Edo prumi.............. 119

Enicopus............. 75

Enodius................. 75

Eremilscharkefer........... 64

Formiea rufa............. 50

Golsilehneiden............. 105

Grunrusselerlinefer.......... 105

Grosse stmize bortien hefer der

obst baunen............ 135

Griin leiterige Bockiticfer....... 113

Gymnodus eremilu..........
Jlanneton............. 60

Ilarlo................ 66

Ihispa................. 75

Ilister depressum ........... 69

Inololepta depressa; var. A... 69

deplanata........ 69

IIylesinus Scolylus........... 134

IDALIA................. 148

Idalia bipunetata........63, 148

Ingerling............. 60

Involvulus alliarice......... 89

- almi.............. 93

- brechus............. 85

- melallieus........... 99

- snleidorsum; var. $B . . .100$

Ips erenata............. 72

- pieipes ; var. A......... 72

- unidentata............. 74

Kernholtz Kofer............ 137

K'lein salls borken Kaefer...... 136

Lagria ................. 75

Lamia nebulosa ........... 142

Lamie nébuleuse........... 142

Lasius ................ 75

Latipalpis................ 76

LEIOPUS ............... 141

Leiopus nebulosus........63, 142

Leplnra arenata........... 139

- præusta............ 145

Leplus unidentala........... 74

Ligniperda eylindrica........ 68

Lisetle............... 89

Loborhynehns.......... 115

Lneamus eylindrieus......... 68

Lupenus................. 146

Luperus flavipes........63, 147

Aavipes. . ......... 147

rufipes............ 147

Lyetus crenata............. 72

- rnfipennis; var. B..... 72

- unidentalus.......... 74

Maeipus pyrasler.......... 130

Macroeephalns varius........ 83

Marropogon.......... 75

Magdalinus barbicornis ....... 120

Magdalis ............... 117

Magdalis earbonarius......... 119

- cerasi.......6.62, 118

- nassata............ 118

- pruni ........6 62, 119

violaeens........ 119

Malaehius oners. . . . . . . 76

- bipustullatus...... 76

Mans................. 66

Marechal. ................ 151 
Marienkafer siebcupunetirt..... 151

Mecivus. ............... 130

Mccinus pyraster........63, 130

— collaris............ 130

Beikefer ............. 66

Mtilolontia.............6.65

Melolontha ercmila......... 64

mali

- mayalis............... 66

- vulgaris.....49,61, 66

Mclyris................ 75

Meuri............... 60

Micnispis............... 154

Micraspis duodecinpunctata . 63, 151

Monoloma crenala.......... 72

Muramus.............. 104, 107

Neplaphilus pyruster......... 130

Obstspilzmaüchen........... 102

OsMODER............... 64

Osmoderma eremita ......... 64

Otronixwchus............ 115

Otiolynchus luctuosus . . . . . . 117

- picipes. . 49, 62, 116

Oxystoma raucus. ... 50,62, 117

Puehyyaster griseus.......... 114

Pancs.................. 117

Panaphilis ............. 115

l'aropus. . . . . . . . . . . . 82

Pellis brunneus........... 70

- limbalus............. 70

Perce-pomme............ 85

Pcrce-prune.............. 99

P'eritellus ................. 113

Peritelus griseus ........6. 62, 114

— sphocroïles......... 114

Pistecia................. 146

Pliytoecia nigricornis......663, 146

Pllaumenborer............. 99

I'taumenrusselkcefer. . . . . . 120

Phyllerasles.......... 104, 107

Puyluomus............ 49, 107

Phyllobius argentatus. . . . 62, 110

- calcaratus....69, 108

- calcaratus..... 62, 108

- pyri..........6. 62, 109

- Tonialus......... 107

- uniformis...... 62, 113

- vespertinus. .... 62, 112

Piquc-prune............. 6.

Palymolus arcualus .... . . . 139

Platrieus................ 137

1'latypus cylindricus ...... 36, 137

Platisoma .............. 68

Platysoma ilepressum.....61, 69
Platysoma oblonymm....... 69

Poliorsia........... 55, 144

Poliopsia prousta .......663, 145

Polvnuosus............ 19, 104

l'olydrosus cervinus. . . . . . . 10 105

magdalimus....... 113

mali....... 106, 112

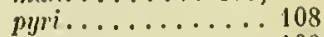

scopoli............ 108

sericeus . . . . 62, 105

smaragdinus...... 105

squammosus...... 105

Polydrusus.......... 104, 107

Propylea quatuordecimpunctala. . 153

Prunier..............61

l’sylla pyrisuga.......... 57

Psilotix............... 75

Hebenstecher. . . . . . . . . . 93

Rhina pruni............ 119

Rhinodes cerasi........ 118, 119

Rhinomacer........... 81, 89

Rhinomacer almi.......... 93

— bacchus......... 85

- bclulo......... 93

- incrnis ........ 93

- unispinus........ 93

viridis......... 93

Runnchites ............. 84

Rynclites areualus......... 99

- auratus....49,62, 88

- bacclius... 49, 62, 85

- betuleti.......62, 93

- bchle......... 93

- conicus.......6.62, 89

- conieus.. ....... 89

- euprous... 49,62, 98

- cuprous........ 99

- germanicus....... 100

- Iuleus.......... 85

- minutus........ 99

- pausilus. . . . . 6. 62, 100

- punctatus......... 98

mbens.......... 88

viridis ......... 99

Rhynehœnus urmeniace......118 avarus........ 127

arurus; var. P. . 128

ecrasi....... 118

pomorum ...... 121

prumi.........119

scmi-cylindricus . . 130

ulmi ....... 127

ulmi...... 127, 128

Richard vert allonge....... 97 
Rothenwerden ........... 122

SAPERDA ............ 112

Saperda bivillata. ......... 144

- candida........6 6i, 144

- migricornis........ 146

- prousta.......... 145

Saperde porte-echelle......... 143

Scarabous coriaceus. . . . . . . . G

- cylindricus....... 68

- eremita........ 64

Scolyle destructew......... 134

— du prunier.......... 135

Scolvtus .............. 131

Scolytus destructor . . . . 6 63, 134

_ pruni........663, 135

- pyri............ 135

- lugulosus. ......63, 136

Silbergraurüssler .......... 110

Silvain................ 74

Silvanus .............. 73

Silvanus unidentalus ......61, 74

- billentatus .......... 74

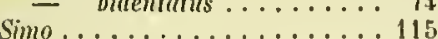

Sinodendre............. 68

SinodendRon ............67

Sinodendron cylindricum. $50,61,68$

Silophilus granarius... . . . . . 130

Stutiorken hofer.......... 134

Synchila evenata.......... 72

Tumnophilus cevasi........ 118 pruni........ 119

The apple tree borer......... 144

Telrops prousta. ......... 145

TImidalus ............. 70

Thymalus limbatus..... 61, 70

- fuscus .......... 76

Tomicus bidens......... 73 , 76

- laricis.......... 73

- fusous.......... 73

Trichie ermile......... 6.1

Trichius eremila ..........6 64

Trichi gymnodi.........6. 61

Tingis pyri. . . . . . . . . 55

Ver blane ............... 66

- Limace ........... 55, 58 58

Vespa vulgaris........... 50

Zweigabsleseher.......... . 89 


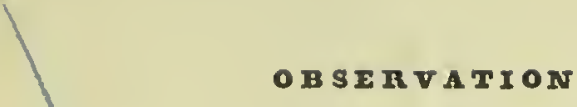 sun UN FOSSILE NOUVEAU, Trouve}

DANS LE DÉPARTEMENT DE LA MDSELLE, Par Mi. Terqueir, anciem Pinarmacien.

La faune palcontologique de notre département est peu riche en ećphalopodes: on y trouve quelques sepia, des belcmnites, dles nautiles et des ammonites; c'est done une bonne fortune que d'y signaler la présence non-seulement d'un nouveau genre, mais encorc d'une espece nouvelle.

Depuis plusicurs années nous avions remarqué dans le grc̀s supraliasique de trois localités (Gong-la-Ville, près de de Longwy, Mont-Saint-Michel, près de Thionville, MontSaint-Quentin, près de Metz.), des corps eylindriques, allongés, légèrement cỏniques, un peu courbes, et ornés de de stries régulic̀res transversales.

Placés presque toujours sur la surface des, blocs, parallèlement ou croisés entre eux, ecs cylindres possèdent une longucur de 40 à $\$ 0$ centimètres, qui ne donne pas leur 


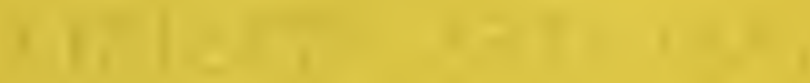


RAPPORT FAIT AU COMICE AGRICOLE DE MET2,

SUR

\title{
QUELQUES INSECTES XYLOPIAGES
}

\author{
L'l' SUR IA
}

\section{DESTRUCTION DES INSECTES NUISIBLES}

$$
\text { EN GÉRLAL, }
$$

Par une Commission composèe de MM. les docteurs Barthelemy, Dreu et Fisot, de M. l'ingenieur Ralland, et GẺms, rapporteur.

\section{Messicurs,}

Le 26 juin dernier, un membre du Comice agricole de Metz, M. Fridrici, vous a lu une uotice sur quelques insectes muisibles qui, depuis plusieurs années, font des ravages considérables autour de Metz. En donnant les indications sommaires propres a faire connaitre la nature des dégals, leur importance ainsi que les principaux moyens d'y porter remede, notre honorable collègue a en méme temps placé sous les yeux du Comice les six especes d'insectes auxquelles il faut, selon lui, attribuer le dépérissement on la mort de la plus grande partic des arbres qui ornent. les promenades et les fortifications de la ville, ou qui bordent les routes de nos environs. Frappé de l'importance des révélations qui lui étaient faites, le Comice a, dans la méme séance, renvoyé la note de M. Fridriei à l'examen l'une Commission, en chargeant celle-ci de faire un rapport al ce sujet et de proposer, s'il y avait lieu, les moyens propres à faire disparaitre, ou tout au moins a diminuer le dommage causé par les insectes dont il était question.

Peu de temps aprés celte communication, l'Académie impériale de Metz fut également touchée des mèmes observations, et, dans sa siance du 28 juillet suivant, cetle savante compagnie décida que la notice de M. Fridrici serait insérée dans ses mémoires, et qu'il serait fait appel aux journaux de la localité pour 
donner la plıs grande publicité possible a des faits quí intéressent, non-seulement l'édilité messine et de nombreux propriétaires, mais encore l'administration du géuic militaire et celle des ponts el chaussées, auxquelles appartienurut une grande partic des arbres attaqués par les Cossus, les Scolytes et les Sćsics.

A la suite de ces communications et de la publicité qui les suivit, une visite générale de tous les arbres signalés fut faite par les délègués des administrations que je viens de ciler. Les Commissions qui procédérent à celte enquête reconmurent l'exactitude des faits observés par M. Fridrici, et décidèrent l'abattage de plus de six cents arbres de la ville el des environs. C'est évidemment à l'exécution de ces mesures et à la publicité donnéc a la notico lue au Conice agricole el à l'Académie impériale, qu'il fuut attribuer le peu d'empressement que mirent á se cons. tiluer les membres de la Comnission nonmée le 26 juin dernicr; lexpérience qui se faisait au grand jour paraissait suffisante, et ce ue fut que pour satisfaire aux exigences du réglement, aimsi qu'aux sollicitations de M. le Président du Comice, que M. Périn vous conmuniqua, dans la séance du $1^{\text {er }}$ décenbre dernier, la notice qu'il avait préparéc sur le mème sujet.

Mais, Messieurs, outre que ce rapport ne faisait connaitre aucun fait nouveau, il passail sous silence plusicurs autres questions tout au moins aussi importantes, qui se rapportent i la propagation des insectes unisibles, a leur destruetion ainsi qu'd la législation qui la concerne. Ce sont ces considérations qui ont déterminci le Comice à unmmer une nouvelle Commission qui aurait à la fuis à examiner la inotice de M. Fridrici et á formuler des conclusious sur les questions incideutes que je viens d'indiquer.

Celte Commission s'est immédiatenent constiluéc, elle a nommé 11. le docteur Dieu pour son président, et elle a bien voulu me confier le soin de vous présenter le résultat de ses délibérations. J'ose espérer, Messieurs, qu'en raison de l'importance du sujel, vous voudrez bien m'excuser pour le temps qui s'est écoulè depuis le $1^{\text {er }}$ décembre, et que le Conice in'accordera en mème temps soa indulgence pour la forme des observations que nous allons soumettre d sou examen.

Lia notice qui rous a été communiquée par M. Fridrici, we 
concerne que les insectes qui vivent sur des arbres de culture, de vígétation el d'usages très-différents, l'Orme et le Peuplier. L'histoire entomologique de ces arbres est encore a faire, mais celle des Scolytes, des Cossus et des Sésies est connue depuis longtemps : la reproduction ici ne serait pas d'une grande utilite el comporterait, d'ailleurs, des descriptions et des développements en dehors du cadre d'un rapport. Je ine bornerai a vous en rappeler les faits indispensables à connaitre, ainsi que les sources auxquelles on devra remonter pour avoir de plus amples renseignements. Je ne réserverai les détails plus précis que pour résoudre la question pratique, el, enfir, les documents les plus importants pour éclairer quelques points de notre législation, en ce qui concerne la destruction des insectes nuisibles. Malgré l'étendue d'un pareil programme, je serai aussi court que possible; et, pour plus de clarté dans mon exposition, j'ai partagé mon rapport en trois partics sur lesquelles j'appello maintenant votre bienveillante attention.

\section{@I.}

De l'Orme et des Insectes qui attaquent cet arbre.

L'Orme, originaire d'Orient, est depuis longtemps acclimalé en Enrope, oú il s'est répandu en l'aison de la dureté de son bois et de la beauté de son feuillage, et deux espéces y sont plus particulièrement cultivées. Dans les environsde Metz, on ne rencontre guẻre que l'Orme champètre (Ülmus campestris, Wildenou); sur nos remparts, on trouve, mélangé a celle-ci, l'Orme a fleurs éparses (Ulmus c/fusa, Wildenou); on distingue en outre plusieurs variétés de chacune de ces especes botaniques. Au point de vue qui doit nous occuper, nous n'avons pas a entrer plus avaut dans ces détails, car tout ce que nous avous à rapporter s'applique indifféremment à l'enscmble des espèces cultivées qui appartiennent au genre Ulmus.

En consultant les auteurs qui, depuis Réaunur et Degèer, se sont occupés des iusectes nuisibles aux plantes utiles, et plus particulierement Ratzeburg et Macquart, on trouve que le nombre total des espéces qui vivent aux dépens de l'Orme ne s'éleve pas 
à moins de cent vingt. II est certain que si toutes vivaicut à la fois sur le méme individı, on si seulement quelques-unes d'entre elles étaient sonvent abondantes, aucun arbre ne résisterait et que mème, si ècétait possible, l'espèce elle-même ne tarderait pas à disparaitre. Heureusement qu'il n'en est pas ainsi et que, suivant le climat, la température, la culturc ou mille autres eirconstances impossibles à prévoir, le plus grand nomb'e de ces parasites ne se multiplieut que dans des limites fort étroites; ce n'rst, an contraire, que dans des cas tout it fait exceptionncls que quelques-uns alleignent des proportions qui les rendent capables de eauser des ravages d'une intensité egrale á celle qui rous a ćlé signalée par M. Fridriei. Si done, en ce qui eoncerne l'Orme, on ne prend en consideration que les especes les plus communciment répandues, on ne tronve plus que quatre Coléoplères (Scolytus destructor; Oliv., Sc. multistriatus, Marsh., Sc. pygmeus, Fabr., et Galleruca calmariensis, Fabr.); trois Lépidoplires ou Papillons (Bombyx ncustria, Linn., B3. dispar, Linn., et Cossus lignijuerda, Limn.); et enfin deux Ilémipléres, Cochenille ou P'uceron (Coccusulmi, Linn., et Aphis ulmi, Linn.); en fout nenf especes, auxquelles il faut encore ajouter deux autres Coléoptéres ou Searabées (IIylesimus varius, Linn., et IIylexcoetus dermestoüdes, Fabr.) que l'on ne reneontre que sur les arbres morts sur pied ou déjả abaltus, mais également nuisilules, puisque, par leurs perforations, ils lialent la décomposition du bois ou le rendent impropre aux usages auxquels on le destine.

Ainsi que je l'ai déjả dil, lous ees insectes sont connus : Ralzeburg, en Allemagne, M. Batlicu, a Naney, et plus récenment encore, M. Eugène Phobert, de Paris, ont publić les détails les plus précis sur leur organisation, leurs mćlamorphoses, la nature des dégâts qu'ils produisent et les moyens de les combaltre. C'est aux ouvrages des autenrs que je viens de citer que j'emprunterai la plupart des renseignements qui peuvent interesser le Comice.

AT. Fridrici ne vous a signalé, comme étant tre̊s-nuisibles à l'Orme, que les Scolytes cicstruclcur el pygmée, il aurait dì y ajouter une troisième espéce intermédiaire, le Scolylzes mullistrialus, Marsh., qui accompagne ordinairement les deux premiers sur les gros arbres, ou qui vil solitaire sur les Ormes de 
grosscur moyenne; quand ees trois especes vivent ensemble sur le mène sujet, elles oecupent chacune une place distinete. Aiusi, lo Seolyte destrueteur, de tous le plus gros, le plus aboudant et lo plus nuisible, ne crense ses galeries que sous l'éeoree du trone, le Seolyte pygmée n'habite que les jeunes branches, et enfin, sur les branches intermédiaires, vit le Seolytus multistriatus. A part ees partieularités, tout ee que nous allons dire peut ètre appliques a tous les Seolytes de l'Orme.

Depuis la fin de naai jusqu'en juillet, on rencontro ees insectes sur le renfement basilaire des jeunes branches de l'aunée, oecupés à y ereuser de petites galeries dirigées de hant en bas vers le eentre de la branche et destinèes à lenr fournir la séve qui se rend anx feuilles terminales. Pendant le restant du inois de juillet et en aoùt, on trouve encore quelques-uns de ees insectes à la surface de l'arbre, mais la plupart ont disparu sous l'éeoree du trone ou des branches, selon les especes, el tans laquelle ils pénètrent ordinairement par le fond des crevasses. Ces nouvelles galeries, dirigées de bas en haut, sont parallèles aux conches de l'aubier, mais n'y pénètrent jamais; leur ouverture reste libre jusqu'à la fin d'aoùt, époque á laquello a lieu la fécondation. Uue fois l'aecouplenent terminé, le male périt misérablement, tandis que la femelle continue à ereuser sa galerie ovifère, dans laquelle clle dépose ses œufs alternativement à droito et ả gauche. lbientot les jennes larves éelosent et, au nois de scptembie, on trouve déjà des vers blanes oeeupés à ereuser des galeries trausversales destinées ả intereepter la séve deseendante qui leur sert de nourriture. On eomprend que tous ees travaux ne peuvent pas s'aceomplir sans la produetion d'une plus ou moins grande, quantité de détritus; aussi, en septembre et en octobre, quelquefois méme beaueoup plus tot, trouve-t-on les erevasses de l'écoree et la base de l'arlore couvertes l'une poussière rougreatre dont l'abondance est en rapport avee la quantité de Seolytes qui ravagent l'intérieur des eouehes eorticales.

Le feuillage des Ormes scolylés ne présente quelquefois aueun signe indiguant une altération ou une interruption de la séve; d'antres fois les feuilles teminales des rameanx avortent en plus ou moins grande quantite; enfiu, dans quelques eas rares, toutes 
les feuilles se flétrissent en quelques jours: alors l'arbre cesse de vígéler et n'est plus bon qü'à ètre abattu. Des perforations pratiquécs par les Scolytes ou par leurs larves, découle une certaine quantité de séve qui produit des phénomènes importants à décrire, car ils fournissent les meilleurs caraclères pour reconnaitre les arbres qui sont allaqués par ees insectes. M. Fridrici vous a déjà signalé la préseuce des Iyménoplères (Guêpes, Frelons, Bourdons, etc.) ou celle des Diplères (Mouches ou Moucherons) qui, altirés par la sève qui mouille l'extérieur de l'écoree, y viennent, souvent en nombre considérable, pour y puiser leur nourriture on pour y déposer leurs æufs. Mais la sẻve qui s'épanche dans les tissus spongieux de l'écorce, s'y altère ou s'y décompose, et produit á la surface des taches brunes ou noiratres, d'autant plus apparentes que l'arbre est plus jeune et que la saison est plus sèche. Ces taches, visibles en toute saison, vont continuellement en grandissant, deviennent confluentes et finissent par' envahir toule l'écorce : on dit alors que l'arbre prond lé charbon, et sa mort peut ètre considérée comme imminente. Pendant l'hiver, et jusqu'an primtemps suivant, aux trous et aux taches que nous venons d'indiguer, on peut encore se servir, pour reconnaître la présence des Scolytes, du son produit avec un petit maillet de bois et qui, en frappant sur l'écorce, fait découvrir les places oủ celle-ci est séparée des couches sousjacentes. En enlevant ces parties décollées, on trouvera les larves des Scolyles logées dans leurs galeries et à divers degrés de développement : les premiers à l'élat adulte, en nymphes ou dejà a l'état parfait, tandis que d'autres n'en sont encore qu'à leur premier ou a leur deuxiẻme àge. Enfin, au printemps, outre les trous d'entrée dont il a été question, on trouve encore des trous de sortie perpendiculairement perforés à la surfaco de l'écoree, et d'autant plus abondants que l'arbre élait plus fortement atlaqué.

Bien que I'on trouve des Scolytes sur des Ormes parfaitement saius, il est incontestable qu'ils sont toujours plus abondants sur ceux qui sont vienx, mal soignés ou à végétation languissante, et s'il est vrai qu'ils ne se portent que Ires-rarement sur des arbres déja aballus, il n'en est pas moins vrai qu'ils continuent a vivre quand ils se trouvent sur un arbre dont leur's attaques 
ont déterminé la mort. Le premier remède qui se présente donc a l'esprit est celui qui consiste d abattre tous les arbres morts sur pied et à les décortiquer complétenent, de manićre a mettre a nu toutes les larves qui $s^{\prime} y$ trouvent et a les faire périr ainsi par leur exposition à la pluie ou au soleil; l'expérience ayant démontré que les larves de Scolytes, une fuis sorties de leurs galeries, n'y rentrent plus et qu'elles ne tardent pas à mourir. Cependant, si l'opération du décorticage est faite au printemps, alors que déjả l'on trouve des Seolytes transformés et qui n'attendent plus que la consolidation de leurs téguments ou la chaleur pour paraitre au jour, il faudra ramasser tous les débris d'écorce pour les brûler', de manière d̀ détruire des insectes qui écloraient quand mème, et iraient ensuite, en nombre plus ou moins considérable, se fixer sur les Ormes du voisinage.

Sur les arbres encore sains, sur ceux qui sont faiblement attaqués ou nouvellement envahis, il faudra, avee un couteau a deux mains, faire sur l'écorce de larges entailles longitudinales allant jusqu'au libel, ou les dessiner de maniẻre a circonscrire presque complétement les parties de l'écorce oủ ces Xyloplrages ont établi leurs galeries. Ces entailles seront d'autant plus nombreuses, d'atutant plus larges et d'autant plus profondes que l'arbre sera plus fortement atteint ou que son écorce ser'a plus épaisse. Par ce moyen, on mel d'abord à nu une grande quantité de galeries de Scolytes, ee qui détermine la mort des larves qui les habitent, et on force ainsi la sére a se porter en plus graude abondance dans les parties conservées de l'écorce, ce qui contribue puissamment a la destruction des insectes qui y sout renfermés.

Sur les jeunes arbres ou sur les brancbes de petiles dimensions, ces incisions peuvent ètre faites au moyen d'une griffe d trois dents (celle du milieu un peu plus courte) que l'on enfonce plus ou moins dans l'écorce, selon l'épaisseur de celle-ei ou sclon l'intensite du mal. Une observation importante il faire encole, e'est quo dans la recherche des arbres scolytés on ne devra pas borner ses investigations a la base du tronc; car, dans les terrains humides, les Scolytes ne descendent guère plus bas que 2 ou 5 mètres aul-dessus du sol. Ce fait, presque général, semble 
indiquer que les Scolytes préfèrent la sécheresse et peut expliquer pourquoi lenr multiplication a élé si considérable pendant Ics années 1858 et $185 \% 9$.

Il serait difficile, si non impossible, d'assigner une date certaine a l'iuvasion des Scolyles sur les Ormes de nos environs. Je dois cependant vous signaler un fait qui, selon moi, parait y avoir contribuc. Ainsi en $18 \%$, en 1833 et en 1856 la plus grande partie des Ormes du Paté, des grlacis de la porte Mazelle et ceux du fort Belle-Croix, les sculs que j’aie observès ì celle époque, a vaient leurs fenilles couvertes des bourses produites par le puceron de l'Orme (Aphis ulmi, Linn.), an point qu'un très-petit nombre d'entre elles avaient conservé les caractères qui sont propres à cet organe dans l'état normal. Or, des feuilles ainsi altérécs dans leur forme el leur structnre, et dont la sève était constamment absorbec par des milliers de pucerons, ne pouvaicnt plus faire qu'incomplétement les fonctions de la respiration; les arbres ont langui et leur force végétative n'a plus été suffisante pour faire périr les premières fernelles de Scolytes qui sont venues creuser leurs galerics ovifères dans l'écorce. Une fois l'invasion opéréc, les années de sécheresse de 1858 d de 1859 , en donnant plus de force aux insectes, ont encore nui à la végétation des Ormes, et les choses n'ont pas tardé à prendre le degré d'intensité que vous a signalé M. Fridrici; car, en ce qui concerne la propagation des insectes, une fois que l'équilibre est rompu, leur multiplication prend des proportions effrayantes en raison de la fécondité énorme qui appartient ả un grand nombre d'entre cux.

Des expériences nombreuses et une pratique de plus de dix années faites șur les Orınes des promenades de Paris, démontrent que les arbres soumis au régine de décortication plus ou moins complète ne paraissent pas en sonffrir et que, dans certains cas mème, leur végétation a paru y gagner notablement. Sans pousser aussi loin que le propose M. Kobert, le nombre ou la dimension des cntailles a faire daus l'écorce des Ormés scolytés, volre Commission, considérant que tous les procédés qui consistent dans l'emploi de liquides acides, alcalius ou iufectes ne sauraient alleindre des parasites protiggés par le tissus mème de l'écorce oủ ils vivent; considerant en ontre que les procédés que nous 
avons indiqués sont recommandés par l'administration des ponts et chaussées, ponr le traitenent des arbres qui bordent les routes, nous avons l'honneur de vous en proposer également l'application aux arbres des environs de Metz, avec la réserve que nous arons signalíe.

Nous ne nous élendrons pas davantage sur les insectes de l'Orme, cens dont il vient d'ètre question comprennent les deux espéces qui sont indiquées par M. Fridrici, et ce n'est que sur cux qu'il y avail urgence d'appeler l'attention du Comice.

\section{§II.}

\section{Du Peuplier et des Insectes qui attaquent oet arbre.}

Le Peuplier est certainement l'un des arbres d'ornements lo plus répandu dans l'Europe tempéréc. Une scule espéce, le Peuplier tremble (Populus tremula, Lin.) est indigène à notre département, oủ l'on rencontre également les autres espèces d'Europe, deux de l'Orient el deux autres d'Amérique, toutes parfaitement acclimaties et indifféremment cultives pour border nos routes et nos promenades. Trous ees arbres ont le bois blane et assez tendre; le nombre des insectes de tous les ordes qui vivent à leurs dépens s'èlève à plus de deux cents. Cette aptitude particulière que possèdent les Peupliers de nourrir un si grand nombre d'insectes, tient a l'analogie qui existe entre ces arbres et les saules, les aunes, les bouleaux, etc., qui, eux aussi, en nourlissent beaucoup, et comme les condilions de tissus, de végétation et de localité sont presque les mènes pour tous ces arbres, il en résulte une sorte de comminauté dans leur faune cntomologique.

Plus souvent que sur l'Orme, quelques-uns de ces parasites acquièrent une multiplication remarquable, mais la force végétatize du Peuplier est telle et sa rusticité esI si grande, que bien rarement il parait souffrir de la présence des insectes et qu'il faut des circonslances tout a fail exceptionnelles pour occasionner des dégals de la nature de ceux qui vous ont été signalés par MI. Fridrici.

Qualre Coléopteres (Saperla carcharias, Lin.; Saperda populnca, Lin.; Linu populi, Fab.; et Lina tremula, Fab.), cinq Lépidoptères (Cossus ligniperder, Lin.; Bomby.x chrysorrhoa, 
Lin.; Bombyx salicis, Lin.; Scsia asiliformis, Lin.; et Sesia apiformis, Lin.), en tout neuf espèces, ont plus particulièrement été signalées, par les entomologistes, comme ayant quelquefois causć de grands préjudices aux Peupliers.

Bien que toutes ces especes se reneontrent indistinetement sur toutes les variétés du Peuplier, il en est eependant qui paraissent choisir de préférence quelques-unes de ses variétés; ainsi, la Saperde chagrinéc se trouve plus ordinairement sur le Tremble, la Sésic asiliforme, sur le Peuplier blanc, etc. Mais comme leurs habiludes n'en sont pas modifiées d'unc manière particulière on peut, dans la pratique, considérer ce que nous allons dire comme également applicable à toutes les espéces de Peuplicr. Pas plus que nous ne l'avons fail pour l'Orme, nous n'entrerons dans les détails longs et techniques que nécessiterait l'histoire, parfaitement connue d'ailleurs, de toutes les espẻces qui, au nombre de quatre, ont été indiquées par M. Fridrici, comme nuisibles aux Peupliers de notre localite. Comme précédenment, nous nous bornerons a signaler les moyens de reconnaitre leur présenee el les procédés à employer pour les 'détruire.

Le Cossus ligniperda, Lin., ou Cossus Gate-lbuis, ou simplement Cossus, Gat, elc., n'est pas, commc on vous l'a dit, la ehenille du saule, mais bien le ver du sanle, la preniére dénomination se rapporte à la chenille du Bombyx salicis, L., espèce qui vit également snr le Peuplier mais qui ne se nourrit que de son feuillage.

Le Cossus est polyphage, on le rencontre sur les arbres Ies plus dissemblables; ainsi on le trouve sur l'Orme, sur Ic Chène, sur le Poirier, etc.; mais e'est surtout dans les arbres à bois blanc (les Conifèrcs exceptés) qu'il se développe le plus ordinairement et qu'il s'y multiplic en plus grande aboudance.

De toutes les espéces do Peuplier, les américaines d'origine (Populus angulata, Pop. canadensis, ete.) paraissent étre plus fréquemment attaquées par cet insecte. En 1857, j’ai déja signalé, a la Socićté d'horticulture de la Mosclle, la grande abondance de ectle ehenille dars les Peupliers des environs de Metz, et, à cette époque, j'annonçais do plus grands dommages ultérieurs, si l'on ne prenait aucune nesure pour en arrêter le développement. 
Le papillon du Cossus, quoique de grande taille, est difficile a reconnaitre et a saisir, car il ne vole que pendant l'obscurité; pendant le jour, il se tient immobile sur le trone des arbres oú sa présence est dissimulée par la couleur grise des écailles qui le recouvrent; ce u'est donc pas sur l'insecte parfait qu'il est possible d'opérer d'une maniére efficace. La ponte a lieu en juillet; chaque femelie dépose ses œufs, au nombre de plus de cinq cents, sur le tronc de l'arbre qu'clle destine à sa progéniture; quelle que soit la hauteur à laquelle celte ponte se fait, les jeunes larves qui en éclosent, pẻnètıent dans l'épiderme de l'arbre par la base de celui-ci. C'est done à cette époque de l'année qu'il couvient de nettoyer complétement le trone des Peupliers, afin de détruire les aufs qui y sont déposés, ou les jeunes chenilles qui s'y proménent pour chercher les endroits favorables à leur introductiou dans l'écorce.

Pendant la prenière année de leur existence, les larves des Cossus restent le plus ordinairement dans les couches superficielles et ne nuisent que fort peu à l'arbre, vu la vigueur de celui-ci. Mais, si leur nombre est considérable, ou si la séve commence à faire défaut par suite de la sécheresse, alor's elles pénètrent plus avant el creusent des galeries longitudinales, dirigées un peu obliquement, de manièrc à se rapprocher du cœur de l'arbre. C'est en ce moment que les ouvertures des galeries, et souvent aussi lo pied de l'arbre, se couvrent des débris de fibres ligneuses rejetés au dehors par les larves. On comprend que des arbres dont l'intéricur est labouré en vingt endroits par des chenilles dont la taille dèpasse souveut six centimetres de longueur, ne puissent continuer à vivre dans de pareilles conditions, surtout si, à de pareilles attaques, la sécheresse vient encore ajouter ses fàcheuses conséquences.

Pour débarrasser les Peuplicrs de parasites aussi dangereux, il faut, du printemps à l'aulonne, tenir le pied des arbics constaminent propre, surveiller arec soin tous les trous par où s'échappent les débris de fibres ligueuses, et une fois les ouvertures des galeries wises ả nu, y introduire des fils de fer recuit terminés en pointe, en crochet ou en hameçon, de maniére à blesser mortellement les larves qui s'y trouvent ou les amener au dehors, 
et, dans ce dernier cas, il faudra les écraser immédiatement, car les larves de Cossus enlevées de leurs galcries, ne tardent pas á en chercher d'autres ou a en creuser de nouvelles, soit sur le mème arbre, soit sur ceux qui sont a proximite. Quand on aura extrail une chenille de sa galeric, il sera bon de recominencer l'exploration de cclle-ci, puisque très-souvent on y rencontre plusienrs individus placés à la suite l'un de l'autre. Enfin, pour complétcr l'opération, il faudra reboucher toutes les ouvertures avec un mastic composé de chaux, de platre et de goudron du gaz, de manière à cmpecher l'introduction des larves venues du dehors, tácher d'asphyxicr celles qui y restent ou arréter la déperdition de la séve qui s'cn écoule.

Ces procédés de destruction pourront paraitre un pcu longs, et votre Commission elle-mème aurait peut-ètre hćsité à yous les proposer il y a quelques mois. Nais, depuis la publication du mémoire do M. Robert, auçuel ils sont empruntés, de nombreuses applications en ont élé faites dans la Moselle et dans la Mcurthe, sous l'habile direction de M.I. les ingénieurs des ponts cl chaussées, Raillard ct Dilschneider; l'expéricnee en a démontré l'efficacité et prouvé que les cantonniers pouvaient en acquérir rapidement la pratiçue el méme une ccrtainc habileté, ce qui a permis d'cn étendre l'cmploi tout en diminuant les frais de maind'œuvie.

Les dcux espéces de Sésies qui, en 1858 et en 1850, ont été si abondantes sur les Peupliers de nos environs, sont cn général beaucoup moins répandues que le Cossus, et ce n'est bien certainement que d'une manic̉re toutc exceptionnelle qu'elles ont pu, à l'aide de circonstances almosphériqnues favorables, sur lesquelles nous reviendrons tout à l'heure, acquérir des proportions aussi considérables. C'est presuje tonjours à la base du tronc, surtout chez les arbres d'une certaine grosseur, que les cherilles des Sisies creuscnt leurs galcries a l'orifice desquelles vicnnent igalemerit s'accumuler des debris de fibres lignenses, mais de dimensions beaucoup plus petilcs que celles qui sont produites par les Cossus. Le plus ordinairement les Sésies se logent dans le trone des jeunes arbres, et comme lenrs galeries sont dirigées presque normalement à l'écorce, elles amẻnent souvent, par lcur multiplication en unt 
méme point, la rupture des jeunes sujets. La chasse aux Sésies devra se faire en avril ou en mai, et an moyen des instruments dont nous avons conscillé l'emploi pour le Cossus. Mais, ce qui sera le plus efficace, et qui se trouve indiqué daus la notice de M. Fridrici, c'est le déchaussement complet des arbres attaqués, de manière à eulever la plus grande partic des chrysalides qui, au inois de mai, se trouvent dans le sol ou a l'orifiec des trous qui débouchent au collet de la racine.

Le dernier des insectes du Peuplier, sur lequel on a appelé l'alleution du Comice, est la Saperde eluagrince (Saperda carcharias, Lin.), coléoptère d'assez grande taille et dont la larve vit également dans le tissus ligneux. C'est, ainsi que je l'ai dejja dit, sur le Tremble que eet insecte se multiplic le plus ordinairement; mais au lieu de crenser ses galeries dans le trone, eomme le Cossus, ou sur le collel de la racine, comme la Sésie, e'est sur les branches de deuxième ou de troisième grandeur qu'elle exeree ses ravages. Iei, Messicurs, votre Conmission est forcée d'arouer l'impuissanee des moyens de destruelion qui préeedent. Rien de pratique ni de rationnel ne saurait vous ćtre proposé pour atteindse facilement et économiquement les larves de co longricorne, dont la présenee ne se déeèle d'ailleurs le plus ordinairenrent que par la mort de la branehe sur laquelle elles se développent. Cependant, comme l'inseete parfait éclot en juin ou en juillet, et que pendant le jour il se tient fixe sur les rameaux de l'arbre, on devra, a ectte époque, seconcr cenx-ei vigoureusement de temps en temps, de maniere à faire tomber les Saperdes qui s'y trouvent, afin de les détruire avant l'accouplement ou tout au moins arant la ponte.

Nous terminerons notre examen, en ee qui eoneerne les insectes du Peuplier, par quelques considérations sur les causes qui, selon nous, ont amené l'état maladif d'un grand nombre des arbres de ectte espece, el dont l'intensité a motivé la communication faite au Comice le 26 juin dernier.

Aux mois de juillet 1835 el 1836 , les Peupliers du Pate el tous ceux qui bordent li route de Magny, étaient presque complítement dẹpouillés de leurs feuilles, par suite de la multiplieation prodigicusc du Bombyx salicis, Hals., dont le papillon 
blane jonchait alors les ehemins de cetto partie des environs de la ville. Ce fait, remarquable en ee qu'il eoïncide avec l'iuvasion du puceron de l'Orme dont il a élé parlé préeédemment, a dù contribuer à affaiblir les arbres que je viens de signaler. En 1857, l'été ayant été chaud et see, la séve a été peu abondante; comme d'autre part la température était favorable au développement des insectes, les Cossus et les Sésies se sont multipliés plus fortement que d'habitude, et ceux qui avaient déjà une année d'existence, ont creusé des galeries plus profondes pour trouver une sève qui leur faisait défaut dans les eonches superficielles. En 1858, les choses se sont passées de la méme manière, avec cette circonstance aggravante que l'arbre était plus malade, tandis que les chenilles étaient au contraire plus nombreuses et plus fortes. Enfin, en 1859 , il faut ajouter, aux causes que je viens d'ènumérer, la déperdition considérable de séve par les ouvertures des galeries des Cossus et des Sèsies, alors cependant qu'une troisième année de sécheresse la rendait plus rare et plus nécessaire à la végétation. 0 v comprend qu'un pareil état de choses ait empéché les fluides nourriciers de flarvenir au sommet de l'arbre en quantité suffisante; de là, le feuillage tardif, maigre ou tout a fait nul que tout le monde a remarquè au sommet d'un grand nombre de Peupliers. La sécheresse a donc contribué à la fois au dépérissement de l'arbre et a la multiplication des insectes; e'est à ce double effet qa'il faut attribuer l'intensité exceptionnelle avec laquelle s'est propagée la maladie dont il est question dans la note de M. Fridrici. Ce qui met celte explication hors de doute, e'est qu'en général ce sont les Peupliers les plus exposés à ressentir les effets de la sécheresse qui ont été les plus fortenent atteints, tandis que eeux qui sont placés dans les terrains restès humides ou dans le voisinage de l'eau ne l'ont élé que faiblement et en petit nombre. A ne voir que la superficic des elioses on à prendre à la lettre les faits qui ont été signalés au Comiee, on serait tenté d'en attribuer l'origine à un seul ordre de phénomẻnes, tandis que ee n'est qu'à leur coincidenee fortuite qu'il faut la rapporter.

Aux considérations qui précèdent, particulièrement applieables aux Ormes et aux Peupliers, votre Commission eroit devoir en 
ajouter d'antres qui ne sont pas moins importantes, mais comme elles sont relatives a tous les insectes nuisibles et qu'elles torchent à des intérêts plus généraux, nous les avous réunics dans un chapitre speccial auquel nous avons donne pour titre:

\section{@III.}

\section{De la destruction des Insectes nuisibles et de la législation qui s'y rapporte.}

Il faudrait, Messieurs, bien peu connaitre la forec d'inertic particuliere a l'homme, pour croire qu'il suffit de lui proposer quelque chose d'utile, pour le lui voir mette en pratique. Ce reproche ne s'adresse pas seulement aux cultivateurs, mais encore aux compagnies savantes qui ont pour but l'avancement de l'agriculture, et dont quclques-unes a peine ont pris des mesures pour vulgariser ou pour expérimenter les procédés indiqués par les entomologistes pour combattre les insectes nuisibles. On connait les moyens de dininuer sensiblentent les pertes que nous font subir les insectes sur les oliviers dans le midi, sur la betleravc dans le nord, sur les colzas ou les céréalcs presque partout, el, cependant, nous ne voyons nulle part ces méthodes entrer dans le domaine de la pratique agricole, mème dans les pays où leur utilité est incontestable. Dans le cas particulier qui a motivé ce rapport, votre Commission prévoit le peu d'application des moyens que nous vous avons proposés, d l'exception, toutefois, de l'administration des ponts ct chaussics et de celle du génie militaire qui, dirigées par des hommes compétents, zélés el actifs, comprendiont l'importance des conseils dictés par la seience ou par l'expérience, et sanront les mettre à profit. Ce sont, d'ailleurs, les résultats obtenus en 1859 , par ces administrations, qui ont conduit votre Commission à examiner si la !igislation ne pouvait pas ètre invoque pour contraindre les indifferents ou les récalcitrants a contribuer à la destruction des insectes nuisibles qui ravagent leurs cultures, et d'oú ils peuvent eusuite se répandic dans le voisinage $\mathrm{cl}$, de proche en proche, envalir toute unc contrée.

Dans les préliminaircs d'un onyrage dont la quatrième párlie 
est à la veille de paraitre, j'il déjá eu occasion de traiter celle question, el j'aurai peu de closes nouvelles i ajouter atux emprunts que je ferai au Mémoje imprimé, en 183\%6, dans le Journal de la Société d'horliculture de la Moselle.

Et d'abord, Messicurs, une loi sur la destruction des insecles nuisibles est-clle utile el, dans ce cas, sonapplication est-clle possible?

La première partic de cette question pent déjá ctre résolue par le simple examen de ce qui précéde. N'est-il pas probable, en effet, que si, en 18506, les Ormes et les Peupliers n'avaient pas ćlc en partic épuisés par les puecrons ou par les cbenilles, leur forec végćtative cût ćté suffisante pour noyer dans la sćve, ou pour expulser, par l'aflux de celle-ci, une grande partic des Scolytes, des Cossus ou des Sésies qui, plus lard, ont trouvé des ¿̇léments si bien préparés el des eirconstanees si favorables à leur développement? N'est-il pas évident que si, en 1857, ou seulement en 18ว̈8, on arait fait ec qui a dé pratiqué en 18539 sur les Peupliers de uos routes, le mal n'aurait pu alleindre des proportions telles, qu'il a fallu procéder a l'aballage d'un grand nombre d'entre eux? N'est-il pas facile de comprendre aussi que toules les préenutions qui ont élé puises, peuvent devenir inutiles, si, dans le voisinage des arbres gui en ont été l'objet, il existe une plantation, ègalemeut envalic par ces insectes, el dans laquelle on ne fera rien pour en arrêter le développement? Il y a done, dans un grand nombre de eas, un moment oú quelques précautions, prises avec ensemble el discernement, pourraient empêcher le développement ultéricur d'une quantité prodigicuse d'insectes nuisibles.

Il y a longlemps que l'on a constate l'instinel merveilleux avec lequel les insecles xylophages savent distinguer les plantes qui conviennent le micux à leur développenent; c'est en général sur celles qui sont déjả malades quüls se jeltent de préfèrence; de sorle que, par leur présenec, ils en bátent encore le dépérissement. Quant aux insectes pbyllophages, on sait quils allaquent indifféremment les arhres dans loutes les conditions de santé possibles; ils paraissent done préparer ceux-ci a recevoir les insectes qui vivent dans le bois : ce qui prouve d'une manicre frappante, selon nous, l'utilité de leur destruetion. L'utilité de l'échenillage une fois reconnue, la nécessifć de le pratiquer en sera la consci- 
quence toutes les fois que le dommage d éviter ou à réparel" dépassera les frais de la mise en ocuvre des procédés destinés i produire ces résultats.

Mais, Messieurs, à quoi bon tant de peines et de sacrifices, si un proprićtaire voisin ne suit pas votre exemple, et s'il laisse iınpunément ses arbres se convrir d'insectes nuisibles qui, en dépit de toutes les précautions que vous aurez prises, ne tarderont pas a envahir les vôtres? A quoi bon abattre des arbres ravagés par les Scolytes ou les Cossus, si on laisse la grume au milieu d'arbres sains, sur lesquels viendront bientot se rendre des milliers de larves ou d'insectes sortant du trone des arbres abattus? Que dans un jardin, enfin, on néglige d'enlever les nombreuses bourses qui protégent, pendant l'hiver, les jeunes larves dn Bomlyy cul brun, et l'on verra si, l'été suivant, les arbres du voisinage ne seront pas envahis par les ehenilles de ce papillon? Unc propriété négligée peut done devenir un véritable foyer d'infection, et l'auteur de cette négligence est responsable du dommage qu'il eause a ses voisins. Il ne fant pas oublier surtout que le mal est à peu prés salls reméde, quand il a atteint nne certaine proportion; c’est donc dès lo début qu’il faut l'arrèter, et ce résultat ne saurait être obtenu sans la contrainte légale.

Votre Commission, désirant vous faire partager ses eonvictions, a pensé que, saus aller au fond de tous les arguments qui peuvent être invoqués en faveur de la néeessité de réglementer la destruction des insectes muisibles, il convient eependant de discuter les principales raisons qu'on oppose a toute intervention, légale ou non, en cette matière; ear, en dehor's des indiflérents, nous avous des adversaires, mème parmi les entomologistes. Les objections dont il s'agit reposent: $1^{\circ}$ sur ce qui se passe dans les autres Litats; $2^{\circ}$ sur l'insuffisance de nos moyens de destruetion; $3^{\circ}$ sur le parasitisme; $4^{\circ}$ enfin sur ee que les plus grandes calamités, causées par les Scolytes, les Bombyx, les Chlorops, etc., finissent par disparaitre d'elles-mèmes sans le seeours d'aucun moyen artificiel. Nous allons, aussi brièventent que possible, examiner chacun de ces motifs d'opposition.

L'Augleterre, les rítats-Unis et l'Allemagne n'ont pas, il est vrai, de législation eoneernant la destruction des insectes nuisibles, 
et cepeudant on ne peut nier l'état prospére de l'agriculture dans ces pays; mais aussi la propriété est loin d'y êlre aussi divisée qu'elle l'est eı France, et, par conséquent, l'iudifférence ou l'incurie d'un cultivateur ne saurait avoir pour ses voisins les inconvénients que nous avons signalés. Les travaux remarquables publies en Amérique et en Angleterve, sous le patronage du gouvernement et sur cette maticre, prouvent suffisamment que, malgré le silence de la loi, on n'en considère pas moins comme tres-utile d encourager la destruction des insectes xylophages; aussi les culfivateurs de ces États, plus éclairès et plus entreprenants que les nôtres, ne négligent-ils aucune occasion de préserver leurs récoltes d'un dommage quelconque. En Allemagne, l'enseignemeut de l'entomologie pratique est fort répandu, et les corps savants y propagent ef y recompeusent tous les travaux qui tendent à vulgariser les procédés qui peuvent faciliter la desIruction de ces insectes nuisibles.

La seconde objection qu'ou nous oppose, et qui est fondéc sur l'insuffisance de nos moyens de destruction est, il faut eu convenir, plus sérieuse que la précédente, ef nous avouons humblement que beaucoup des moyens proposés jusqu'ici sont loin de satisfaire complétement a la solution du probleme. Mais, Messieurs, c'est qu'aussi il n'y a pas bien longtemps que ces questions sont a l'ordre du jour et, en ce moment encore, on peut facilement compter en Europt: les entonologistes qui, abandonnant les abstractions de la science, tachent d'utiliser leur's connaissances au profit de la cause que nous soutenons. Quant aux cultiviteurs, ciest à peine si ceux qui se prélendent les plus éclairés daigneut descendre dans de pareils délails, el cependant, s'ils le voulaieut, de combien d'utilité les observations qu'ils peuvent faire ne seraient-elles pas pour faciliter, luater même, la solution de plusienrs questions d'eutomologie pratique. Ce qui, en France, manque surlout aux ourriels du sol, ce sont les connaissances les plus élénıntaires en histoire naturelle, ef certes, ce n'est pas heaucoup exiger d'eux en leur demandant le savoir au moins disceruer les insectes qui peuvent leur etre nuisibles de ceux qui leur sont de la plus grande utilité. Tous les jours ils détruisent des carabes, des araiguees, des oiseaux 
a bee fin, ete., dont la scule nourriture, cependant, consiste en ehenilles, larves ou autres insertes vivant au dépens de nos plantes eultivées.

Si, dans certains eas, la rapiditi des ravages, le nombre prodigieux des insectes qui en sont eause, le milieu on ils vivent, etc., nons rendent impuissants pour y porter un reméde effieace, il ne faut pas oublier que le mal ne peut alteindre eelte intensité qu'aprés plusieurs génẻrations des insectes envahisseurs, on bien que, loealisés d'abord en quelgues points, ils se sont propagés par le contaet ou par des émigrations en quantité considèrable. Si done, dès le début de leur apparition dans ees centres de propagation, on avait pris quelques prècautions; si, dans le commeneement d'une production anornale des insectes phytophages, le propriétaire de ces arbres couverts de elıenilles ou de pucerons, celui de ce ehamp de blé eceidomyié, avaient employé quelques - uns des moyens employés en pareils cas, n'est-il pas évident que l'on n'aurait pas cu plus tard à subir, dans quelque lieu, les conséquenees facheuses de eette incurie? Dans la question qui nous oceupe en ce moment, tout dépend done du point de départ, et c'est précisément à cause de cela que nous voulons, autant que possible, prévenir ces désastreuses extrémités, on lout au moins les rendre moins fréquentes, en forçant a agir alors qu'il est encore temps.

Le parasitisme est, il est vrai, assez général, et un grand nombre d'inseetes est destiné á cn falire vivro un ou plusicurs autres. Cependant il y a un bon nombre d'inseetes nuisibles pour lesquels le parasitisme n'existe que dans des proportions telles, qu'on ne saurait espérer en tirer quelque parti au point de vue de la diminution des individus. Ainsi, dans le cas particulier des Scolytes de l'orme, sur lesquels vit un petit Iehneumon (Bracon initiator, Wesm.), on a remarqué que celui-ei est en quantité si minime que, sur des arbres eouverts de nilliers de larves de Scolytes, e'est à peine si l'on en a trouvé quelques-uns. Le parasitisme ne peut donc être invoqué que dans quelques cas et, eonme avant son apparition en quantité suffisante pour détruire un grand nombre d'inseetes nuisibles, ceux-ci auront déja pu exercer leurs áavages pendant une ou plusieurs séné- 
rations, ce ne sera souvent que trop tard qu'ils pourront faire rentrer les choses dans l'élat normal. En résuné, si les iusectes deviennent assez nombreux pour endonmager nos cultures, c'est que l'équilibre naturel a été rompu, par conséquent il ne peut se rétablir avec le secours des insectes parasites que quand ceux-ci auront atteint une proportion exagérée. Pendant le temps qui sécoulera jusqu'à ce moment favorable, le mal ne fera que s'ilgrriarer : c'est donc avant ou nonobstant l'apparition des parasites qu'il faut agir, en réservant leur puissant concours pour les cas inalheureusement encore trop fréquents oủ nous ne pouvons plus rien tenter contre les insectes phytophages.

La dernière objection que nous avons signalée, peut dejjà être combattue par les arguments qui précćdent, aussi n'avons-nous que pen de choses a y ajouter. Quaud on étudie la marclıe des épidémies on celle des épizooties, on trouve aussi que ces calaunités, quelles qu'elles soient, finissent toujours par disparaitre cemme elles sont venues, sans canses bien déterminées; cepeudant, Messicurs, peut-il venir à l'esprit de personne aujourd'hui, que nous n'ayons rien i faire pour prévenir ou combattre les ealamités de celte sorte qui viennent frapper l'espèce humaine ou dépenpler uos élables? Relativement aux grandes invasions d'insectes nuisibles, l'assimilation est parfaite et uons ne pouvons ailmettre que, devant les fleaux de ce genre, comme devant les autres, nons u'ayons qu'à nous résigner et à attendre, inaclifs, qu'ils disparaisseut spontanćment. A ces raisons, qui nous paraissent perremptoires, on pent encore ajouter que le moment favorable pour entreprende la destruction d'un insecte rarie pour chaque espece, et que pour chacune d'elles il est souvent limité a un très-petit nombre de jours ou à un temps trés-court de leur existence. Or, il est impossible de prévoir daus quelles conditions météorologiqures la végétation d'une plante doit s'accomplir, au moment oủ clle sera le plus exposée à être la proie des iusectes; faut il donc, avec une pareille inconume; laisser échapper le temps propice pour l'en préserver et rester spectateur indifférent de la multiplication d'une espèce unisible, parce que l'on espère un printemps pluvieux, un été sec on nn hiver rigoureux? 
'Toutes les objections que nous venons d'examiner n'ont donc qu'une valeur relative; el, si on les appliquait a la lettre, il faudrait, pour ête conséquent, non-seulement proscrire toute législation concernant la destruction des insectes nuisibles, mais encore considérer comme oiseux les magnifiques travaux entomologiques publiẻs en France par Réaumur et M. Perris; en Allemagne par Ratzeburg el M. Nordlinger; en Angleterre par M. Westwood, et en Amérique par M. Aza Fitch. Les besoins de la consommatiou, ceux de l'industric ou de la civilisation, forcent l'lomme à rompre constamment quelques-unes des lois qui maintiennent l'harmonic et l'équilibre entre tous les êtres de la création; l'exagération ou le développement de certaine culture, l'amoindrissement ou le cosmopolitisme de quelques autres, sont autant de circonstances qui peuvent amener la multiplication anormale de beaucoup d'iusectes; on a donc eu tort de dire que l'bomme avait trop de présomption en cherchant à lutter contre un état de closes qu'il n'avait pas créé, et qui parait étre la conséquence des lois génćrales qui règlent la proportionualité de chaque espèce dans la nature.

Nous pensons, Messieurs, vous a voir démontré l'utilité et la nécessité, non-seulement de la destruction des insectes nuisibles, mais aussi le besoin de dispositions légales pour la rendre efficace. II uous reste maintenant il examiner quelle est notre législation á ce sujet, de cuelles modifications clle parait susceptible et par quels moyens on peut en faciliter l'application.

Dans le niémoire que j’ai déjả signalé, j'ai démontré que c'était au Parlement de Metz que revenait l'lıomeur d'a voir, le prenier, introduit quelque chose de rationuel dans l'obligation d'écheniller. Fil 1751, toute la France fut ravagée par les chenilles de Bombyx, et pendant I'liver qui suivit, tous les arbres étaient couverts des bourses dans lesquelles hivernent les jeunes chenilles: ce fut pour ancuer la destruction de cette quantité innombrable de nids que furent pris, par tous les Parlements de France, des arrêts dont les gouverineurs provinciaux eurent á réglementer et it surveiller l'exécution. Cette obligation existait bien, il est vrai, avant 1752 , mais les ordonnances rendues a cet effet ne pouvaicut plus ctre invoquées, car toutes sont empreintes de l'igno. 
rance entomologique et des pratiques superstitienses du moyeu àge. La loj du 26 ventose an IV ne ful que la reproduction de ce qui arait élé décrété auparavaut, bien que cependaul á cette èpoque déja, on cùt pu faire beancoup mienx. Depuis la promulgation de cette loi, nous ne trouvons plus que le paragraphe 8 de l'article 471 du Code pénal et la eirculaire ministérielle in 11 avril 1821, qui se rapportent à la législation sur l'èclicnillage.

Sans entrer dans uno discussion approfondie de cette législation, nous vous en ferons seulement remarquer les dispositions qui la rendent insuffisante aujourd'bui. Ainsi, la loi du 26 ventose n'est applicable qu'aux chenilles qui hiveruent dans des toiles filées en commun. Mais en delıors de ces espèces, combien d'autres parıni les Bombyx, les Xylopbages, les Puccrons, les Cícidomyics, elc., qu'il est tout aussi important de faire disparaitre et dont cependant la destruction ne pent être prescrite par celle loi. Outre cette lacune, déjà suffisante pour moliver une réforme, la loi n'est applicable que pendant un espace de temps fort court, de sorte qu'en dehors des limites tracées par elle, les insectes de toules sortcs peuvent inıpunćment, aux yeux du législateur, se développer et envabir tout une contrée. Eufin, la surveillance et, dans certains cas, l'exécution de la loi sont conférées à des agents, zélés saus doulc, mais tout d fait incompétents poul constater efficacement les contraventions. Si, à ces défauts, on ajoute encore la fábcuse execplion créée par la circulaire ministériclle de 1821, en fiveur des forèts ct des lisières d'icelles, qui sont dispensées de l'échenillage inposé aux propriélaires limitrophes, on restera convaincu de la nẻeessité de modifier profondément la législation existante sur l'échenillage.

En 1859, uи noureau projet de loi, élaboré par les soíns de Martin (du Nord), alors ministre de l'agriculture el du commerce, a été présenté a la Clambre des l'airs oú, comme heaucoup d'autres, il u'i pas abouti, malgré les sages dispositions qu'il contenait. En 1848, un nouvel cssai, aussi infructueux, a élé tentes par M. Ricbard (du Cantal). Enfin, on dit que le nourean Cule rnral, en ce moment soumis á la délibération du Sćnat, doil mettre un terne aux réclamations sur la matière; s'il en est 
ainsi, c'est une raison de plus pour désirer la prompte promulgation d'un code réclamé par tous les besoins de l'agriculture.

Volre Commission, Messicurs, pour arriver au but que nous désirons tous atteindre, ne saurait cependant vous proposer de faire peser des charges nouvelles sur l'agriculture, on d'imposer at nos cullivateurs l'application de procédés souvent longs, quelquefois dispendieux et qui, en définitive, nont pas une efficacité incontestable. Nous sommes trop pénélrés des inconvénients d'unc législation qui veut tout préroir on tout réprimer, et nous ne savons que trop bien de combien la pratique du plus grand nombre des agriculteurs est en retard sur les données les moins contestables de la science; aussi saurons-nous limiler uos propositions aux choses possibles et indispensables, sans nous laisser entrainer mème à ce qui serait désirable.

Dans l'intérèt de la santé et de l'hygiéne publique, on impose journellement, et avec raison, à un grand nombre d'industriels des mesures de salubrite dont quelques-unes sout fort gènantes el quclquefois prèjndiciables á leurs inlérèts. Le légrislateur, en rendant le décret de 1810 sur cetle matićre, a voulu garantir les propriélés voisines cu ćtablissant des formalités pour la création et le fonclionnement des industries dangereuses, nuisibles ou incounmodes. Or, nous avons suffisamment démontrẻ qu'un arbre, un champ ou un jardin peuvent, dans certains cas, devenil un véritable foyer d'infection pour le voisinage, et nous pensons que, par les mènes molifs que ceux qui justifient le décret de 1810 , on peut, dans des formes analogues, contraindre un propriélaire à purger ses cultures des insectes qui peuvent devenir dangereux pour les voisins. Nous croyons done que la loi de l'an IV devrait érro élendue á la destruction de tous les insectes, et que son application rationnelle et opportune pourrait ètre réglementéc par des arrclés préfectoraux pris sur l'avis de conseils analogues a ceux de salubrite et d'hygiẻne publiques, et dans lesquels seraient représentées à la fois l'Administration, les sciences naturelles et l'agriculcure.

Dans de pareilles conditions on ne craindra pas de voir imposer aux cultivalcurs des choses impraticables ou hors de proportion avec les résultats a obtenir. La théorie el la pratique, la science 
et la routine, la spéculation et l'observation se trouvant en contact se prêteront un mutuel secours, et l'entomologie appliquée, tout en se vulgarisant, sortira enfin des langes dans lesquels elle est encore enveloppée. Des moyens qui peuvent amener une augmentation d'un cinquième dans la récolte du colza, d'un dixième dans celle dı blè, d'un quart dans celle des olives, etc., valent certainement la peine d'etre recherchés, et les travaux dirigès dans ce but nous paraissent tout aussi dignes d'encouragements que tous les autres. Ce n'est pas sans une certaine amertume que nous lisons souvent les programmes des Académies, des Sociétès d'agriculture, des Comices même, sans y trouver une seule récompense pour les comıunications du genre de celle de M. Fridrici. Votre Commission appelle sérieusement l'attention du Comice agricole de Metz sur les dernicires observations que nous venons de lui soumettre.

Pardon, Messicurs, d'avoir retenu si longlemps votre bienveillante attention, mais le sujet n'est pas sais importance, et nous avons voulu vous éclairer complètement, afin d'obtenir plus sùrement volre assentiment sur les propositions qu'il nous reste encore i vous soumetire.

\section{ERRATA.}

Page 3. Ligue 5. La reproduction ici ne serait pas d'une Lisez. Lal reproduction de ces dèlails ne serait pas ici. 



\section{EN VENTE:}

NO'Tls pour servir a l'histoire des Insectes nuisibles à l'Agriculture, a l'flortieulture et à la sylvienlure dans le dipartement de la Noselle:

No 1. Introduction.

- 2. Insectes qui attaquent les Blés.

- 3. Inscetes du Poiricr. (Premiere partic.)

- 4. Inscetes de l'Orme et du Peuplice.

- b. Insceles du Poirier. (Deuxiène partie.) 


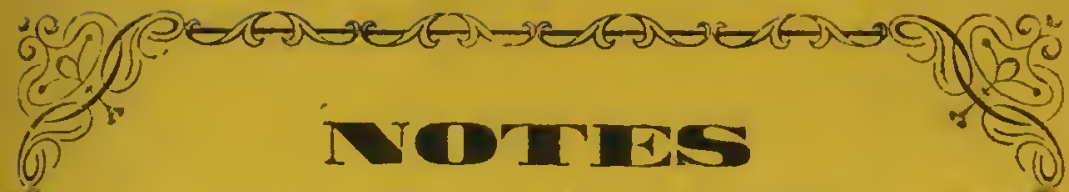

POUR SERVIR A L'HISTOIRF:

DES

\section{INSECTES NUISIBLLS}

\section{A I'AGRICULTURE}

\section{A L'BORTICOLTURE ET A LA SYLVICULTURE}

DANS LR.

DÉPARTEMENT DE IA MOSELLE

P.SN

\section{3.-B. (bélin}

Kembre de plusieurs Socielés sarantes nationales el elirangèpes.

\section{No 5}

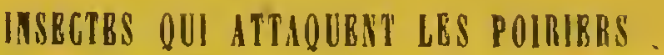

\section{DEUXIEME PARTIP}

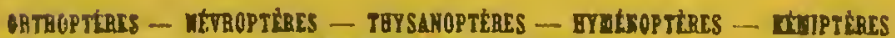
IONOPTERES - DIPTERES.

morvenar

Istrail da ge Bulletin de la Société d'Histoire malorclle th déparitemenil de la lloselle; 1860.

annorno.

METZ

Imprimerie Lithographie de J. YERRONNAIS

(

\section{0}


- 


\section{NOTES}

POUI SERVIR A L'HISTOIRE

\section{DES INSECTES NUISIBLES}

DANS LB

DÉPAR'TEMENT DE IAA MOSELLE

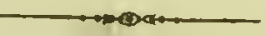

$$
\text { No } 5
$$

INGECTES QUI ATTAQUENT IES POIRIFA

Deuxieme Partie

Orthoptères, Nérroptères, Thysauoptères, llyoénoplères,

Hémipteres, Horuopteres, Miptères. 



\section{NOTIES}

POUR SERVIR A L'HISTOIRE

DES

\section{INSECTES NUISIBLLS}

A L'AGRICULTURE

\section{A L'HORTICULTURE ET A LA SYLVICOLTURE}

DANS LE

DEPPATEMENT DE IA MOSEIIE

PAR

I.-B. Whéhin

Hembre de plusieurs Sociétes sarantes nationales ct étrangeres.

No 5

INSECTRS QUI ATTAQUENT LES POIRIRBS

DEUXIÈME PARTIE

ORTHOPTERES - KLVROPTERES - THYSAYOPTERES - FYMEKOPTERES - MEMIPTEERS HOMOPTERES - DIPTERES.

2urnorian

Exlrait du g* Bulletin do la Socićlé d'Hisloire nalurelle du déparlement de la Hoselle; 1860.

manoun.

\section{METZ}

Imprimerie \& Lithographie de J. VERRONNAIS

1860 


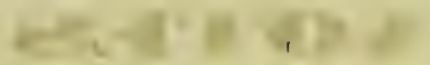

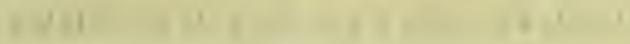

\section{YH.}

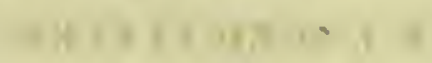

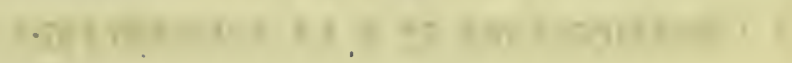

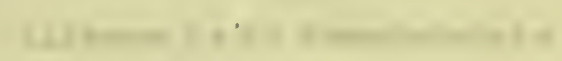

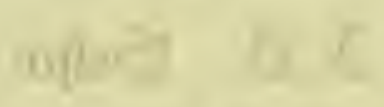

$$
\begin{aligned}
& \text {. }
\end{aligned}
$$

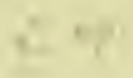

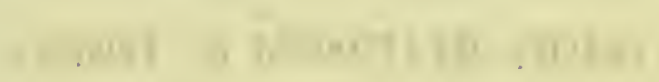

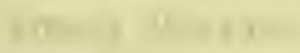

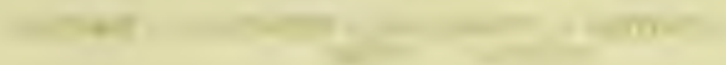

$$
10
$$

\section{In it}
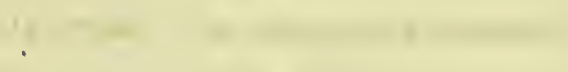

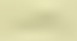




\section{NGTIEAS \\ POUR SERYIR A L'IIISTOIRE \\ DES IMSEGTES NUISIBLES}

DANS LE

DÉPARTEMENT DE TA MOSEITE.

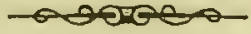

INSECTBS QUI FIVENT SUR LB POIRIER.

$\Rightarrow 0$

\section{DEUXIÈME PARTIE.}

ORTHOPTẺRES - NEVROPTĖRES - BYUÉNOPTÉRES - THYSANOPTÉRES - HEMIPTÉRES BOMOPTERES - DIPTERES.

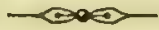

Lorsque, il y a quatre ans, j'entrepris de faire l'hlstoire des insectes qui sont indiqués eomme vivant aux dépens du Poirier, j'étais loin de connaitre l'importanee de ee travail et les difficultés que je devais rencontrer dans son exéeution. Un peu plus de elairvoyanee de ma part, et moins d'inexpérience dans eettc nouvelle voie entomologique, auraient cependant suffi pour paralyser ma bonne volonté, et la monographie que je enntinue aujourd'hui, serait eneore à eommeneer. La bienveillanee avec laquelle la première partie a été aceueillie dans le monde entonologique et les cncouragements préeieux que jai reçus de la part de collègues aussi indulgents qu'instruits, avaient exeité moll ardeur, et, dès 1857, je commençai à mettre en ordre les matériaux qui 
devaient composer la deuxième partie. C'est alors seulement que les difficultés commeneèrent et que le découragement s'empara de moi.

En effet, outre que j’étais resté presque étranger aux détails eoncerriant les inseetes qui n’appartiennent pas à l'ordre des Coléopteres, te manque d'ouvrages spéciaux, les erreur's nombreuses répandues dans les ouvrages d'horticulture, en ee qui eoneerne ees petits animaux, la contradiction manifeste de plusieurs auteurs présentant un égal mérite scientifique, et enfin la diffieulté de recourir à des renseignements plus complets ou plus exacts, dans des ouvrages éerits en allemand ou en anglais, rendaient la táche audessus de mes forees, et bien des fois j’ai été sur le point de l'ahandonner. A ees eauses nombreuses de défaillanec, on peut eneore ajouter la difficulte de l'observation, quand il sagit d'insectes comme les Puecrons ou les Cochenilles; à l'histoire de ees Homoptères, on verra que, malgré les rennarquables travaux de Réaumur, de Degéer, cte., ete., beaneoup de ehoses sont eneore inconnues dans leurs inours et dans leur organisation. Si l'on tient eompte du nombre prodigieux de publications entomologiques, faites depuis une trentaine d'arneces, on eompreudra la diffieulté de réunir en un seul faisecau les travaux de tous mes devaneiers.

L'appel que, en 185̆7, j’ai fait aux observateurs, a été entendu, et j’ai reçu, depuis eelte époque, sur les inseetes dont il est question dans la première partie, de nombreuses eommunications qui me permettront d'apporter d'intéressants développements à mon travail. Pour ne pas changer le eadre que je me suis imposé, je renvoie toutes ees additions au supplément qui devia terminer l'histoire des insectes nuisibles au poirier, supplément déjà prévu en 
1857 , et que je reconnais, dès aujourd'lıui, comme indispensable. Quant aux rectifications à faire, ear il y en a aussi, elles n'ont pas assez d'importance pour nc pas pouvoir ètre ajournées, plusieurs d'entre elles d'ailleurs, ne me semblent pas incontestables et demandent à ètre vérifièes.

Parmi celles qui m'ont été demandées, il en est unc qui parait avoir pour son auteur une importance particulière; je l'ajourncrai cependant, parce qu'clle est formulèe dans des termes peu en rapport avec les lıabitudes scientifiques, et qu'elle n'a été motivée que par une fausse interprétation des 'sentiments manifestés par moi, dans plusieurs de mes écrits. Je n'insisterai pas plus longtemps sur cet iucident et me garderai bien de rien dire qui puisse faire soupẹonner quel est l'autcur de ectte malencontreuse réclamation.

Je dois eependant faire remarquer que plusieurs les observations qui m'ont été faites, touchent plutôt ì la forme qu'au fond de mon travail ; j'en ai tenu compte dans tout ce qui peut, dès aujourd'lui, produirc une amélioration sans elıanger l'ordre que j'ai adopté. J'ajoutcrai encore quil suffira, pour les combattre, de mettre en présence les désirs contradictoires manifestés par plusicurs auteurs, et que quelques-unes de leurs demandes ne se seraient pas produites, s'ils avaient lu avec attention les préliminaires qui précèdent la première partic.

Aux nombreuses difficultés matériclles que je viens de mettre en évidence et qui seront plus particulièrement comprises par ceux qui ont l'habitude de travailler séricusement, il en est d'autres qui, bien que secondaires, pou. vaient amener le découragement à cause de l'incapacité évidente de nos moyens de destruetion, quand il s'agit l'êtres aussi petits que les puecrons, les cochenilles, ete.

Il ne suffit pas en effet, pour résoulle une pareille 
question, d'indiquer un moyen plus ou moins radieal d'extermination, mais il faut aussi, et par dessus tout, proposer quelque chose de pratique, dont l'application soit en rapport avec le dégàt á éviter, et qui n'expose pas la plante à de plus séricux domnages que eeux qui sont causés par les insectes. Il faut convenir que la question, restreinte dans scs véritables limites, est des plus difficile à résoudre, qu'clle réelame le eoneours des cntomologistes et des horticulteurs, et, par conséquent, unc étude complète des mœurs des inseetes, aussi bien que l'examen sérieux des mélhodcs empiriques employces depuis longtemps par les jardinier's, afin d'en dégager l'inutile ou l'absurde et de n'en retenir que le raisonnable.

J'ai déja cu plusieurs fois oceasion de dire que les entomologistes sont loin d'ctre d'aceord entre eux sur les eauses qui déterminent, dans eertaines annćes, ou dans eertains pays, le développement considérable, et souvent subit, de quelques espeeces d'inseetes nuisibles aux végétaux. Je lois ajouter aussi que la mème divergence d'opinion se préscnte, quand il s'agit de détruire ees insectes ou d'cn prévenir le retour. Sans entrer dans le fond de ees difficiles questions, je saisirai toutes les oceasions qui se présenteront de publier les observations qui me paraitront propres à en amener la solution. Sans parti pris d'avanee pour l'une ou pour l'autre des deux opinions, je ferai connaitre eelles de ces obscrvations qui sont favorables aux partisans de la destruction comine aussi eelles qui semblent donner raison aux entomologistes qui, croyant aux eauses finales, admettent que le parasitisme et les variations de l'atnıosplıc̀re sont nos plus utiles, nos plus constants eomme nos plus efficaces auxiliaircs.

Un exemple frappant de ee que peuvent les moycns naturels de destruetion des insectes nuisibles, nous a été 
fouıni dans la Moselle à propos de la cécidomyie du blé, laquclle a causé un défieit énorme dans nos récoltes de 1856. Eı effet, en dépit des avertissements de toute nature et des conseils donnćs par les entomologistes, pour empèeher eet insecte de se multiplier, en 1857, en aussi graude abondance que pendant les années 1855 et 1856 , rien n'a été fait, ni mème tenté, par les eultivateurs, eela va sans dire, mais aussi par les individus qui, se plaçant à la tète du progrìs, préteıdent le diriger. Aussi, qu'est-il arrivé? C'est que, en 1857, bien que la saison ait été peu favorable à la ponte de la ecicidonnic, celle-ci n'en a pas moins été trìs-abondante et, malgrí cette circonsıance, diffieile à prévoir, elle aurait bien eertainement eausé un déficit plus considérable qu'en 1856, sans la présence d'un grand nombre de parasites.

En mime temps que commençaient à disparaitre les éeidomyies, on pouvait observer un nombre de plus en plus considérable de petits ichneumons du genre Platygaster, que nous avons reconnus ètre les parasites de notre Tipulaire*. Ces petits parasites firent si bien, qu'en 1858, e'est à peine sil'on pouvait trouver quelques rares cécidomyies dans les blès en fleurs, et que, en 1859 , l'on ne voyait plus ni céeidomyic ni son parasite; l'équilibre est done rétabli, quant à présent. Ces résultats sont sans doute très-favorables aux partisans du facile, rien à faire, mais leurs adversaires ne sont-ils pas en droit de leur desnander ee qui serait advenu en 1858, si, peudaut l'année priécente, la floraison du blé ne s'était pas faite d'une manière très-rapide et par un soleil ardent? N'est-il pas eertain que nous aurions eu à supporter une récoltc aussi pauvre que celles des années précédentes, et que l'on aurait pu arrèter, lés 1856 , la propagation de la ećcidomyie?

- Voyez notre № 9. Inseeles qui allaquent les blès. Mets, $185 \%$. 
J'arrive maintenant à un argument d'une grande valeur, qui doil ètre opposé aux partisans du rien à fairc, paree qu'il est fondé sur les résultats incontestables que l'on obtient avee les poudres inseetieides.

Dans le Bullelin cles annales de la sociélé entomologique de France publié dans le premier trimestre de 1859, jai donné les prineipaux résultats obtenus par moi au moyen de eette poudre appliquéce à la destruetion des insectes qui attaquent les plantes*. Grảec à l'obligcanee de M. Belhomme, jardinier en elsef du Jàdin botanique de Metz, et a la eomplaisante coopération de M. Thomas, l'habile ehef de eulture de l'établissement de MM. Simon-Louis frères, j’ai pu cette année, continuer et compléter les expériences commenées en 1858. L'importanec desormais acquise ì ce nouvel agent mérite de fixer notre attention, et l'usige que j'en eonseille dans plusicurs eireonstanees, ne eonduit naturellement à en dire quelques mots.

Sous les noms de Poudre inseetieide, Burnichon, Désille, Mismaque, Vicat, ete., on vend en Fıanee, depuis plusicur's aunées, une poudre que l'on obticut avec les eapitules du Pyrethrum caucasicum et qui depuis fort longtemps, sous lc nom de poudre persane, est employée à la destruction de certains inseetes, par les peuples qui labitent l'empire Russe, depuis la mer Noire jusqu’à la mer Caspienne**.

L'efficaeité de eette poudre, pour dètruire les punaises, les puees, etc., est un fait aujourd'lui lors de doute, eependant son action n'est pas eonstante, dans bien des eas, la mort n'est qu'apparente, et l'insecte anesthésié, en quelţue

- Voyez aussi les Annales de la société enlomologique de France, en 1858.

- On a attribué à Paltas d'avoir, le premier, fait connaître celte poudre, mais je ne trouve rien dans la relatiun de ses voyages qui confirme celte opinion. 
sorte, reprend bientòt et peu ì peu ses mouvenents, el ćchappe de nouveau à ceux qui voulaient le détruire. Cet inconvénient a surtout licu avec la poudre grossière des Pyrelhrum Caucasieum et $P$. Roseum, ainsi qu'avec ces poudres fines, mais éventées. J'ai fait, avec toute l'exaclitude désirable, et dans le but de chereher unc succédancic au Purelhrum Caucasicum, des expérienees comparatives sur les poudres de plusieurs plantes, afin d'utiliser, dans le mème but, quelques-unes de nos plantes indigènes que l'on suppose avoir la mème action.

J'ai essayé les Pyrethrum maritimum, Sehmith., Pyrethrum Alpinum, IVilden., Pyrethrum parthenium, Lin., qui sont congénères ou voisines des Pyrethrum roseum, et Caucasicum; Ies Artemisia judaica, Lin. (Semen contra, Barboline, etc.), Arternisia absynthium, Lin. (absinthe), Artemisia maritima, Lin. (absinthe marine), etc., qui sont des plantes voisines des pyrèthres et donées d'une grande amertume; la digitale (Digitatis purpurea, Lin.), la belladone (Atropa belladora, Lin.), et la ciguë (Conium.maculatum, Lin.), qui sont des plantes nareotiques; et enfin la farine ordinaire et la poudre de réglisse, comme étant des poudres inertes et ne pouvant agil que mécaniquement. Les résultats généraux auxquels je suis arrivé, m'autorisent à établir que, a l'exeeption d'une seule, la matricaire, toutes ces plantes sont loin d'avoir une action aussi efficace que le Pyrèthre du Caucase, et qu'il vaut mieux, par conséquent, poursuivre l'acelimatation de eclui-ci, que de eliereher à le remplacer par des plantes indigènes. Gràce aux efforts persévérants de plusicurs membres de la société impériale d'acelimatation, et plus particuliẻrement de M. Guillemot, de Paris, ectte culture facile, commenec ì se répandrc. Avec les moyens de pulvérisation que l'on possède aujourd'bui, 
on peut obtenir cette plante dans un degré de division extrème et à un prix raisonnable. Le enmmerec peut déjà la livrer au prix de $8 \mathrm{fi}$. le kilogr. (bien qu'au détail, on la vende souvent au public à raison le 50 fir. le kilogr.), mais quand la plante sern cultive en grand, on pourra arriver au prix de $\overline{5}$ a $4 \mathrm{fr}$. le kilogr. et mème descendre plus bas, si l'usage en devient général.

Conme il arrive souvent pour une foule de produits, il existe des qualités fort diverses de ectte poudre, et il parait que Jéjà la fraude, pour en augmenter le poids, y a fait introlluire des substances minèrales inertes. Les qualités que l'on doit surtout y recliercher, sont dètre aussi fine que possible, réceminent préparcic et n'ayant pas subi l'action de l'humidite. Le meilleur moyen de l'appliquer consiste à faire usage du soufflet ou de la houppe à souffrer; il faut aussi choisir un temps sec et éviter d'en faire usage, quand les plantes soitt mouillées par le brouillard, la roséc ou la pluic.

Bien que de toutes les plantes indigènes et communes que j'ai citèes plus haut, la maroute ct la digitale soient celles qui, par leur action, se rapprochent le plus de la poudre insecticide, ut que les moyens de pulvérisation convenables (car toutes choses égales d'aillcurs, une poudre agit d'autant plus qu'elle cst plus fine) ne soient pas à la portćc de tous, je n'en conseille pas moins très-vivement la culture du Pyrelhrum Caucasicum à tous les jardiniers pour leur usage et dans le but de répandre cet utile composé ou d'en fairc baisser le prix.

Quant à l'action de la poudre insecticide sur les insectes, voici les conclısions auxquelles je suis arrivé ; elles diffèrent peu de celles qui se trouvent consignées dans les Annales de la sociéle entomologique.

10 Pour la punaise des lits, les résultats sont des plus 
satisfaisants, eependant il est bon de répéter l'opération plusicurs fois, à eause des oufs qui ne sont pas éelos et sur lesquels la pondre est sans aetion ;

$2^{\circ}$ Les puces ne sont pas toujours eomplétement asphyxices, aussi est-il hon de les ramassel et de les jeter au feu ou á l'eau. Celte observation est en tous points applicable anx mouehes;

$5^{\circ}$ Sur le charaneon (Sitophilus granarius, L.) etla teigne du blé (Tinea cereulella, Treist.), lous les deux liès-abondants eette annee, la poudre est sans aetion;

$4^{\circ}$ Sur un grand nombre de chenilles et partieulièrcment sul' celles qui sont velues, la poudre parait d'autant moins agrir sur elles, que es ehenilles sont plus àgées et par eonsćquent plus près de leur transformation en ehrysalicles ;

$5^{\circ}$ C'est eontre les insectes des ordres des llemiptères ou des Diptères que toutes ees poudres ont le plus d'action.

Les autres partieularités que j’aurai à signaler, troureront leur place avec l'histoire des insectes qui y auront donne lieu*.

- Les lignes qui précédent étaient écritcs quand jai rçu de M. Villenıot une broclure intituléc: Dc la destruction des insectes nuisibles oli résumé historique des proprietes du Pyrethrc du Caucase, clc. 32 pages in- $8^{\circ}$, Paris 1859.

Jextrais de ce travail intéressant, au double point de vue de la botaniquo et de l'entomologie appliquće, les passages suivants qui se rapportent plus particulièrement an sujet que je traite en ce montent:

* Il serait trop long d’énunérer iei les plantes pulvériscies dont on s'est scrvi pour la destruction des inscetes et dont on se sert encore actuellenent daus certains pays. Nous nous contentcrons de citcr eclles que nuns avons étudiées.

- La passerage à leuilles ćtroites (Lepidium ruderale, L. - Thlospi ruderale, Desf.), qu'ou trouve quelquefois dans les lieux incultes et les déconbres des 
Enfin, aujourd'hui, pas plus que l'an dernier, je ne suis en mesure de présenter une explication raisonnable sur le mode d'action de ectle poudre insecticide. Mon honorable collegue en entomologie, M. Girard, professeur au college Rollin, a essayć, dans les Annales de la socièté entomologique, d'expliquer l'asphyxie des insectes par ectle poudre, en

environs de Paris s'emploie dans toute la Dalmalie méridionale, sous forme de poudro obtenue de toute la plante, mais presque exclusivement pour la destruction des puces.

"Dans quelques endroits de la Russie méridionale, particulièrcınent en Criméc, - c'est encore une plante très-commune en France, - l'aristoloche clématite (A ristolochia clematilis, L.), qui s'applique à la destruction spéciale des punaises.

- Enfin, certaines sciures de bois aromatiques, particulièrement celle du cédre d'Amérique (Cedrela odorala, L.), circulent dans le commerce comine poutres insecticides, et d'après l'opinion mềne d'un sirsant, la plupart tes produits vendus à Paris en sont composćs. Souvent aussi ces produits sont falsifles à l'aide d'autres sciures de bois. "

"La poudrede Perse, qui paraft êrre la plus répandue, est presquc exclusiventent composée du pyrèthre carné (Pyrcthrum curneum, Biebers). M. le docteur Clı. Koch a rapporté de son voyage en Orient des détails fort curieux sur cotte espéce précieuse, dans les contrées caucasienues. On trourera aussi dans le journal de la Saciété impériale et centrale d'lorticulture de la Seine (tom. 111, 1857, P. 756), l'analyse d'une Note de M. Neumann, de Breslau, sur la culturc et la préparation de la poudre du Pyrcthrum carncum. "

" Mettre lo plus directement possible la poudre de pyrèllıre en contact avec l'insecte nuisible, l'appliquer en quantite suflisante, telles sont les deux raisons principales de succos qu'il ne faut pas perdre de vuc jendant l'opération. C'est mène la partic la plus inportante de tout ce qui a rapport au pyrèthre du Cauease, et qui oflre quelques difficultés dant l'habitude ne tarile pas i se rendre maitresse.

" En effel, si des personnes trop entpressées á nicr l'efficacité du pyrèthre tu Caucase n'out pas obtenu de réussito positive, cela ue nous surprent aucunement, car nous savons que toute innovation, de quelque valeur qu'elle soit, soulive toujours l'antagonisue plus ou moius désintéressé; mais il est 
admetuant locelusion des trachées ou au moins des orifices de ces organes. Sans repousser ccttc théorie d'unc manièrc ahsolue, j'y ferai cependant les objections suivantes :

$1^{\circ}$ Pourquoi les poudres incrtes, de réglissc ou de farine, ou celles qui sont analogues à celles des autres pyrc்thres, n'ont-clles pas la mème action mécanique sur' ecs organes de

évident pour nous, qui savons à quoi nous en tenir, que ces personnes n'avaient pas opéré avec la patience nécessaire dans tout essai, et en Suivant les règles aequises par nous pendant ume longue expérience. „

"De tous les appareils que nous avons cssayés pour projeler la poudre de pyrètlıre sur les̀ végétaux attaqués d’insectes, le souftlet qui s'emploie eneore pour le souffrage de la vigne nous a paru remplir les meilleures conditions.

* Avec le soufflet, on envoie à la fois une faible quantite de poudre, et par conséquent on peut ćviter le plus possible la perte qui serait inévitable de toute autre manière. Il se forme alors un nuage léger; les pareclles de poudre embrassent une ćtenduc assez grande et viennent s'appliyuer directement sur les parties sounises à l'optération. "

* Cette opération relative à la destruction des inscetes nuisibles à l'agriculture et à l'horticulture par la poudre de pyrèthre devra a voir lieu de préférence le matin, par un temps assez sec. Toutefois on ne craindra pas de projeler la poudre sur les planles cọnservant encure un léger restant d'humidité dù à la roséc. De ectte façon, la poudse adhérera facilement aux prarties et conservera encore assez longtemps ses proprićtés pour que les insectes subissent son influenec destructive. Le soleil aura plus tòt absorbé cette humidité que l'air ne se sera cmparé du principe de la pondre.

v On insuflera la poudre à diverses reprises. Ainsi, linsuftlation se fera d'abord sur une branche, de manièro à porter une première alleinte aux insectes. Ensuite, on passera à une deuxième partie, à une troisième et ainsi de suite; puis on reviendra uno seconde fois ou plus, suivant la naluro et la quantité d'insectes à détruire.

"La premièro opération étourdit l'inscete, la seconde l'achéve; il ne tarde pas à perdre la foree qui le faisait se maintenir sur le végétal et il linit jar tomber. Uno fois à terre, il pourra vivre eneore quelqque temıls, mais il sera désormais dans un état qui ne lui permettra plus de nuire. Enfin, il mourra sur le sol. 
la respiratiou? $2^{\circ}$ comment se fait-il que les insectes meurent si promptement dans la poudre de pyrètlıre du Caucase; tandis qu'ils résistent, en généıal, si bien à l'asphyxic, dans le vide ou dans un gaz noll respirable? $5^{\circ}$ pourquoi des chenilles de Bombyx pyri et de Noctua tridens auxquelles j'ai bouché les stigmates avec unc pàte formce de poudre insecticide, ont-clles vécu aussi longtemps que d'autres elrenilles de ces mèmes espèces, auxquelles j'avais bouché les stigmates avec de la gomme? $4^{\circ}$ Comment se fait-il que la poudre insectieide, relativement grossière, agisse mieux que les poudres fines des autres plantes essayées? $5^{\circ}$ Pourquoi le tigre (Tingis pyri, L.) vit-il pendant longtemps dans la poudre fine de pyréthre du Cauease, tandis qu'il meurt presque immédiatement dans celle de digitale? $6^{\circ}$ Enfiı, coinment expliquer la reproduction d'Anobium panieeum, Fab., dans les poudres impalpables d'absintle et de camomille, ou eclle de la calandre du blć (Silophilus granarius, Lin.), dans du blé mélangé de poudre de pyréthre, si l'asplıyxic ou plutỏt l'anesthésic des punaises, des puces, ctc., est produite par une action méeanique de la poudre insecticide sur les organes de la respiration?

A l'appui de l'opinion émise par M. Girard, je ne vois que la différenee d'action, bien constatce, des poudres fines sur les poudres grossières. Sclon moi, il y a licu de recherelıer une autre cxplication, ou tout au moins quelque elose qui la eomplète; ear il est évident que l'on ne pourra arriver à employer judicicusement celte poudre, nu a en rechercher rationnellement une suecédanée, que quand on cennaitra la manière d'agir de ec nouvel agent insecticide.

En faisant paraitre la première partic des Notes pour servir a l'hisloire des insecles qui vivent sur le poirier, j’ai cu soin de réclanıer l'indulgenee pour ce nowvel essai d'ento- 
mologie pratique. Aujourd'lui je la réclame de nouveau, et jc remercie particulièrement MM. Goureau et Ed. Perris, pour les excellents eonscils ct les bienveillants encouragements dont ils ollt constamment soutenu mes efforts. MMI. Amyot, Fridrici, Siclıcl et Thomas, déjà cités précédenment, et MM. V. Signoret et Bigot, de Paris; Louis Brisout de Barneville, de Saint-Germain-en-Laye ; Belhomme et le docteur Ilaro, de Metz, mayant fourni de précicux documents et ayant bien voulu in'aicler dans mes recherchics, ont également droit à ma reconnaissance.

Metz, le $1^{\text {er }}$ décembre 18509.

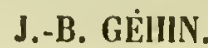




\title{
DEUXIËME PARTIE.
}

\section{Maturar \\ LISTB des espèces d'Orthoptères qui virent sur le Poirier.}

FORFICULIENS. Fonficula auniculania (Lin.). La larve, la nymphe el l'insecle mangent les jeunes bourgeons etentamen! les fruits.

Assez nuisibles.

\begin{abstract}
LOCUSTIENS. Locusta vinidissima (L.). $\quad\left\{\begin{array}{r}\text { Les larves, les nymphes } \\ \text { cl lesinsccles mangent }\end{array}\right.$ Decticus vennucivonus (L.). $\begin{gathered}\text { les feuilles. } \\ \text { Très-peu nuisibles. }\end{gathered}$

GAYLLIENS. GnvLlotalda Vulganis. (Lalr.) Iaa larve, la nymphe et l'insectc coupent Ics racincs.

Très-nuisibles dans les pépinières.
\end{abstract}

XXXIr. FORTICUIA (Linnd)

Andinel-Serville; Orthoplères des suiles à Buffon, page 35.

Corps allongé; deuxième et troisième plaque dorsale ayant un tubereule latéral; antennes composées de 10 ì 1/4 articies distinets; palpes filiformes, les maxillaires de $b$ articles, les labiaux de 5 ; yeux petits, peu saillants; corselet carré; tarses te trois articles, le premier et le troisieme allongès, celui du milieu plus ou moins dilaté; abılomen termine par deux appendiees en forme de pinee. Quand

- Synonymie : Cirfunoura (Latr.). 
l'insecte est développé, les élytres ne se recouvrent pas l'une l'autre, mais sont simplement rapprochées le long de la suture; les ailes sont longues, au moins de la longueur du corps, pliées en éventails dans le sens longitudinal et deux fois sur elles-mèmes cu forme d' $\mathrm{N}$, de manière à pouvoir se loger plus ou moins complétement sous les élytres; celles-ci laissent toujours à découvert la première partic de l'aile qui a une consistance coriace et qui, par sa position, scmble former le prolongement des véritables ćlytres; chez ees insectes la partic membraneuse de l'aile se trouve ainsi protégéc par deux couvertures.

Les Forfieules, vulgairemeut nommées Perce-Oreilles, sont des inscetes bien connus de tout le monde. Leur forme rappelle celle des Brachélyıres de l'ordre des Coléoptères; et, comme eux, ils relèvent l'extrémité de l'abdomen, quand on les inquiète. Leur métamorphose est ineomplète, e'est-à-dire que, à l'execption des ailes et des élytres, les jeunes sont, au sortir de l'ouf, presque semblables à leurs parents. Ce n'est qu'après aroir changé de peau trois ou quatre fois que ces larves passent à l'élat de nymphe, e'est-à-dire, que les ailes commeneent à se montrer; alors les anneaux du thorax prenuent plus de développement; les antennes aequièrenı quelques artieles de plus, enfin, après une dernière mue, parait sculement l'iuseete parfait, apte dès-lors à reproduire son espèce. Les deux sexes sunt assez semblabes, eependant, chez les màles, les pinces sont plus fortes, plus arquées et munies d'épines plus saillantes. Les Forfieules sont polyphages, clles préfèrent les matières suerées, les fruits múrs, ete. Elles sont nocturnes, et pendant le jour, elles se tiennent eachées sous les pierres, sous les ćcorees, dans les fentes des murailles, ete. On en connait une vingtaine d'espèces d'Europe, d'Orient, du Sénégal et d'Amérique, dans le département de la Moselle on en tronve deux espèces dont une seule doit fixer notre attention, c'est la : 
52. TORFICUIA AURICULARIA (linné).

Audinet-Serville; Orthoplères; suiles à Buffon, page 36.

Synonymie: Forficule; - Perce-oreille; - Fourchelle; Ohrwurm des allemands; - Le grand Perce-oreille, Geoffroy*.

De 12 à 15 millimètres de longueur, non eompris la pince qui varie de einq à sept millimètres. Corps d'un brun de poix plus ou moins foneé selon le temps qui s'est écoulé depuis la dernière mue; eorselet plus clair sur les bords, antennes et pattes testaeées plus ou moins elair; èlytres bordées de testacé pàle; extrémité visible des ailes de eouleur testaeẻe et formant une sorte de taelıe à l'extrémité des غ̀lytres; antennes de 15 à 14 artieles granuleux; pinees brunes à l'extrémité, moins foneées à la base, à branelies presque droites et mutiques ehez les femelles, dilatées à l'origine et fortement arquées ehez les màles. L'un et l'autre sexe dégagent une odeur partieulière désagréable et trèsforte, quand ils sont aglomérés en eolonies.

La Forficule est rẹ́pandue dans toute l'Europe, elle est trèscommune dans le département de la Moselle. Cet insecte vit en société, vole très-bien et fuit la lumière. Pendant le jour il se tient cachć sous les pierres, sous les écorees, dans l'aisselle des fcuilles, sous la mousse, dans le ealice des fleurs, dans la tige de quelques plantes fistuleuses, entre les tuteurs et les arbres, souvent aussi, en plein solcil, sur les fleurs d'ombellifères. les fruits suerés sont plus particulièrement de leur goủt, aussi la trouve-t-on le plus souvent sur les poires müres, les abricots, les pêches et même les pruncs, dans les eavités de ees fruits entamés par les guêpes. Ces orthoptères ne bornent pas lả le

- M. Fischer de Fribourg (Orthoptera Europearum) a figuré cet insecte pl, VI, fig. 11 et $1 \mathrm{l}$ a i 11 , ; la larve et la nymphe, pl. VI, fig, 11 r -11 s. 
dommage qu'ils nous causent, car avant la maturité des fruits, on trouve souvent ces insectes, lcurs larres ou leurs nymphes occupés à mangcr les jeunes boutons à fruits dont la sève sucrée remplace la pulpe du drupe qui doit plus tard former la nourriture presqu'exclusive de l'insecte parfait. Ce sont plus particulièrement les boutons des pêchers et les jeunes pousses des cillets qui ont à souffrir de leur voracité, et cela au point de compromettre sonvent la fructification des uns et la floraison des autres.

Quand on touche ou que l'on dérange les Forficules, elles relèvent la partie postérieure de l'abdomen en écartant les branches de la pince tcrminale et prennent ainsi un air redoutable qui en impose aux enfants, mais qui n'a rien de sérieux, pas plus du reste que la crainte généralement répandue dc les voir s'introduire dals la conque de l'oreille, où, comme dans toute antre cavité à l'abri de la lumière, elles peuvent chercher à se réfugier mais oì elles ne sauraient vivrc à cause de la partic grasse dı cérumen qui ne tarderait pas ả les asphyxier; onsait d'ailleurs que la membrane du tympan s'oppose à leur pénétration plus avant.

Quand l'été a été chaud et favorable, lcs Forficules atteigncnt vite leur enticr développement et, en septembre ou eu octobre, on peut en observer l'accouplement; celui-ci a lieu, comme chez les papillons, bout à bout, les pinces de la fcmclle entre celles du mâle et les branolics des pinces de l'un des sexes appliquées contre l'abdomen de l'autre. La ponte sc fait toujours au printemps suivant, dès le commellccmeut d'avril. Les œufs sont blancs, lisses et allongés (de un à deux millimètres de longueur); on les trouve sous les pierres, sous les écorces, etc., aglomérés en paquets de dix, vingt ou trentc. La femelle nc quitte pas ses cenfs, ce qui a fait dire qu'elle les couvait, opération qui ne saurait avoir lieu que par un développement de température bicn difficile à comprendrc chez les animaux respirant par des trachées! Je crois tout simplement que la femelle garde ses aufs contre la voracité des autres insectes ou même celle de ses 
eongénères, ainsi qu'elle le fait encore pour les petits qui, dans les premiers jours qui suivent leur naissance, se réfugient autour d'elle au moindre danger. On a dit aussi que la femelle transportait ses oufs quand elle était inquétéc, je n'ai pu vérifier ce fait, qui, füt-il vrai, ne prouverait rien en faveur de la prétenduc inculuation.

Dans le courant du mois de mai, einq ou six semaines après la ponte, les jeunes sortent de l'œuf, mais tellement gros $\mathrm{ct}$ allongés que l'on a de la peine à coneevoir comment ils ont pu être contenus dans une si petite enveloppe. Ces jeunes sont presque blancs, et on n'aperçoit que la place des yeux qui soit un peu plus colorée; au bout de quinze jours, au eommencenent de juin, ils ont déja quatre millim. de longueur (non compris les pinces); ils sont d'un lestacé pále aree les yeux et une grande partic des mandibules noiratres. $\boldsymbol{\Lambda}$ cet áge les jeunes Forfieules ue se sont pas eneore beaucoup éloignées de leur mère; ce n'est que vers la fill de juin (probablement après aroir subi une première muc), que l'on commence á les reucontrer voyageant isolément à la recherche de leur nourriture et que l'on ne trouve plus de vicilles mères dans les colonies de cet insecte. Leur taille est d'environ cinq millimètres, non compris la pince, celle-ci est droite; les autennes n'ont que huit articles et la couleur générale de tout le corps est d'un brun clair uniforme. Les nouvements des jeunes Forficules sont assez lents et leurs téguments ont peu de consistance.

Bien que les Forfieules soient très-communes, on ne sait pas encore eombien elles subissent de inues avant d'arriver à l'état de nymplıe. Celle-ci diflère de la larve par la présence des rudiments des ailes et des élytres, le nombre des articles des antennes qui est déjả de onze ou de douze el enfin par sa conleur plus foneée et ses pattes páles aunulées de couleur obscure ehez le plus grand nombre des individus.

L'instinet soeial que nous venons de remarquer chez les jeuncs Forficules, persiste malgré la disparution de la mère; peudant tout le reste de lamnée on les trouve vivant en eolonies plus ou 
moins nonbreuses et dans lesquelles on reneontre sourent des débris d'inseetes (ehenilles et eocous du Bombyx dispar; élytres de hannetons, ete.), qui attestent que ees insectes sont polyphages et que ee n'est pas toujours aux dépeus de nos fruits qu'ils parviennent à se nourrir. Selon Degéer, les Forfieules mangeraient même les individus morts de leur propre espèce.

Quoique les Forfieules préfèrent les matières sucrées, ee n'est pas en attaquant nos fruits múrs qu'elles nous eatisent le plus de mal, mais bien en mangeant ou en entamant les bourgeons alors que gonflés de sève, ils préparent la récolte de l'année suivante. Il est done indispensable, pour les hortieulteurs, de leur faire une guerre ineessante, surtout pendant l'été. Mallıeureusement nous ne eonnaissons eneore aucun moyen pratique pour empêcher leur multiplication, ee n'est qu'isolément qu’on peut en détruire les individus. Pour atteindre ce but on a proposé une foule de procédlés plus ou moins ingénieux, parni lesqnels je recommanderai les suivants :

On fait, avee des brindilles, des tiges rides de rnseau, de sureau ou de topinambour, de petites boltes que l'on suspend a proximité des arbres que l'on veut protéger, les Forfieules s'y réfugient pendant le jour, on peut alors les atteindre et en détruire uin grand nombre en secouant ees piéges de refuge audessus du feu on d'un baquet d'eau*. Lor's de la maturité des fruits on leur tend des piéges aree de petits cornets de papier, dans l'intérieur desquels elles se réfugient et dont on peut les extraire pour les déltuire. Dans les environs de Longwy, où ees inseetes sont extrêmement abondants, on rceouvre l'extrémité des tuteurs arec un moreeau de vase de verre ou de terre eassé, sous lequel les Forficules vout s'abriter et d'où il est facile de les falire tomber pour les écraser.

- Plusieurs auteurs conseillent d'employer les brindilles que procure la taille des arbres pour former ces piéges; mais je crois que dans aueun eas, on ne doit laisser ees brindilles dans le voisinäge des arbres, et qu'il vaut toujours mieux les détruire et, avec elles, les cufs et larves qu'elles recèlent souvent. 
Au mois d'oetobre 1859, j'ai trouvé une larve de Forfieule d'entiron dix millimètres de longueur, de couleur assez foncéc et ayant la tête presque noire; les anneaux des pattes avaient disparus; les anıennes avaient 11 artieles. Cette larve provenaitelle d'une ponte faite en juillet, ou d'œufs pondus plus tót, mais qui auraient été retardés dans leur éelosion? on la larve elle-même a vait-elle subi un retard exeeptionnel? e'est ee que je ne saurais dire, mais e'est ee qui peut faire penser que dans eertaines années il $y$ a plusicurs générations.

\section{IOCUSTA (Fabricius)"}

Audinel-Serville; Orthoptères; suites à Buffon, page 527.

Tète vertieale avee un tubercule sur le front; antennes sétacées, très-longues et très-minees, le premier artiele assez gros; prothorax rétréei en avant, earéné latéralement; pattes longues et fortes, les jambes des deux premières paires fortement épineuses; les jambes postérieures propres au saut, garnies au-dessus de dents fines et serrées; tarses de quatre artieles; mandibules fortes; palpes maxillaires plus longues que les labiaux; élytres plus longues que l'abdomen; ailes de la mème longueur; abdomen terminè dans les deux sexes par dettx appendiees gros et eourts; la femelle ayant une tarière ou oviseapte, long, presque droit, élargi à la base et pointu au bout.

Les sauterelles sont aussi des insectes très-connus de tout le monde; chez ees orthoptères, les quatre pattes antéricures sont proportionnellement assez eourtes, tandis que les deux postéricures sont fort allongées, eette disposition, très-favorable pour le saut, rend la marehe diffieile ehez ees inseetes. Les ailes sont disposées en toit pendant le repos; le vol est lourd et peu

-Synonymie: Gayluds (Linné); - Conocephalus (Thunberg); - HexAcentrus (Aud. Serv.); - Sauterelle. 
soutenu. L'espèee de sabre qui terminc l'abdomen des femelles est composé de deux lanes allant en sc rétrécissant et entre lesquclles s'éeoulent les œufs au moment de la ponte. Lorsque celle-ci doit avoir lieu, la femelle choisit une terre légère et meuble, y introduit son oviscapte, at, cn éeartant les valves de celui-ei, laissc tomber les oufs dans le fond du trou.

Les élytres des màles sout pourvues ȧ leur basc d'une membrane transparente, semblable à un petit moreeau de talc, entouré d'une nervure forte et saillante, de sorte que l'insecte mcttant eette membranc en vibration, en frottant les élytres l'une eontre l'autre, fait entendrc ce chant monotone, hien eonnu dans nos contrées, oú on la prend pour celui des eigales du midi de la Franee. Les sons ainsi produits varient en intensité selon les espèces; ils sont en général très-aigus, sc font entendre de loin, mais ccssent aussitôt que l'aniral cst inquiétć par le moindre bruit.

Selon M. Brullć, les sauterelles ehangent quatre fois de peau avant d'arriver à l'état parfait, d'autres naturalistes disent cinq fois; selon le même auteur, depuis la sortie de l'ouf jusqu'ả la secoude mue, les deux sexes sont entièrcment semblables, l'oriscapte des femelles ne se montrant qu'après le deuxième eliangement de peau. Les sauterelles sont phytophages, mais les dégàls qu'elles peuvent oeeasionner sont toujours asscz limilés à cause du nombre restreint des individus.

Le genre Locusta, circonscrit comme il l'est aujourd'hui, ne renfermc plus que quatre espèces dont deux habitent la Franec, une la Nouvelte-Hlollande et l'autrc les Iudes oricntales.

53. LOGUSTA VIRIDISSIMA (Fabr.).

Audinet-Serville; Orthoptères; suites à Buffon, page 529.

Synonymic : Gryllus viridissimus (Linné); - Conoccphalus viridissimus (Thumb.); - la Sauterelle à coutelas, de Gcoffroy; - la Grande Sruterelle; - Sauterclle verte. Longucur du corps de 25 ì 55 millinc̀tres, cntic̀rement 
vert plus ou moins fonec; ; le corselet a souvent une tache brunàtre plus on moins apparente at milieu, les élytres ont aussi quelquefois une étroite bordure de celte couleur at cóté interne; antennes vertes à la base, jaunes à l'extrémité; ailes presqu'incolores, transparentes, de la longueur des ćlytres; celles-ci longues, dépassant de moitić l'extrémitć du (corps; abdomen vert avee le dos et les còtés souvent teints de ferrugineux; organes stridulants tlu mále, grands, celui de gauche inégal, un peu ferruginesx, opaqute, celui de droite arrondi, transparent et irisé; tarière de la femelle, droite et presque aussi longue que le eorps de l'animal.

La saulerclle verle est assez commune daus le département de la Moselle, uủ on la désigue improprement sous le nom de cigale, erreur fort ancienne du reste, car e'est de cet insecte quc le pocte Lafontaine a parlé dans sa fable, de la Cigale et de la Fourni.

C'est dans les prairies qu'on la rencontre le plus souvent; et, bien 'qu'elle ne dédaigne pas le feuillage des arbres, et surtuut celui des jeunes arbustes, on the saurait la considerer comme trés-nuisible ȧ l'arbre dont j'ai cntrepris de faire l'listoirc entumologique. Mais la sauterclle verte étant indiquéc par plusieurs auteurs comme nuisible au poiricr et aux arbics fruitiers en gćnéral, je dois la faire figurer dans mon Iravail.

C'est en aoùt, ou en septembre, que les sauterelles sont parvenues à l'ćtat adulte et que, pendant les belles journées, on cntend striduler le málc pour attirer la femellc. Pendant le jour, il se tient de préférence sur les arbres et les haies, le malin et le soir, dans l'herbe ou dans les champs d'avoine. L'accouplement terıniné, le mảle disparait. La ponte a ordinairencut lieu en aoút ou en septembre, selon que l'annéc a étć plus ou moins farorable, et que, par consėquent les jeunes se sont développés plus ou moins rapidement. Quoiqu'il en soil, cette opération se fait comme je l'ai dit précédemment, et la femelle, après avoir remué la surface du sul pour reboucher le trou formé par 
l'oviseapte, abandonne ses $œ u f s$ qui ne devront éclore qu'au printemps suivant. Dans les premiers beaux jours d'avril et de mai, les petits sortent de l'ocuf; ils sont alors blanchàtres et acquièrent rapidement assez de volume, subissent deux ou trois mues en mai et en juin, puis, à la fin de ec dernier mois, ils présentent le eommeneement des ailes et des élytres, et passent ainsi à l'élat de nymphe.

Un insecte comme la sauterelle, dont les ocufs sont cachès daus la terre et dont les larves ou les nymplecs ne se nonirent qu'isolément, est assez diffeile à atteindre; aussi suis-je foreć d'avouer que je u’ai aucun moyen pratique à indiquer pour le détruire, fort heureusement, ainsi que je l'ai déjà dit, le nombre des individus de cette espèce est assez restreint.

J'ajouterai en terminant, qu'il ne faut pas confondre, comme on le fait ordinairenent, les sauterelles du genre Locuste avee les Criquets on sautcrelles royageuses dont l'invasion, dans nne eontréc, est un véritable fléan. Les premières ont les antennes trèslongues, composées d'un grand nombre d'articles, très-fragiles, tandis que les Criquets en diffèrent par des antennes très-courtes, par l'absenee de tarière elıez les femelles et de miroirs stridulants ehcz les mảles.

\section{DECTICUS (Audinet-Serville)*}

Aud.-Serville; Orthoplères; suites ì Buffon, page 482.

Tète grosse, large, mutique; palpes grèles, les maxillaires dı double plus longs que les labiaux; mandibules tris-fortes; antennes très-longues, écartécs l'une de l'autre à lcur inscrtion laquelle a licu dans unte petitc cavité; ycux grands, peut saillants; corselct caréné latéralement; élytres étroites, dépassant à peine les ailes; abdomen gros et coutt; oviseapte de la femelle recourbé en dessus; pattes longues, les postéricures surtoutt; tarses allongís, de quatre articles.

- Synunymie : Gnvllus (Linnée); Locusta (Fabr.); SAutenelte. 
Les Decliques different peu des sauterelles auxquelles nous aurions pu fort bien les réunir, si nous n'étions fermement résolu à nous conformer, comme précédemment, à la nomenclature de l'autcur le plus compéten I, sur la famille à laquelle apparticunent lcs insectes dont nous aurons à écrirc l'histoire. On peut appliquer à ces insectes ee que nous avons dit des nours et des habitudes des espèces du genre Locuste. J'ajouterai seulement que les Dcctiques onl, en général, des couleurs grises, et que presque toutes les espèces connucs appartiennent à la faunc française; deux ou trois se trouvent dans notre département mais une seule doit nous occuper, c'est le :

54. DECTICUS VERRUGIVORUS (Linné).

Audinet-Scrville; Orthoptères; suiles à Buffon, page 484.

Synonymic: Gryllus verrucivorus (Linné); - Locusla verrucivora (F.); - la Sauterclle à sabre (Geoffroy).

Long de 3 à 4 centimètres; varie pour la couleur, du vert at brun clair; tête lisse, luisante; antennes longues, le premier artiele rosé, une douzaine des suivants verdâtres, le reste brunàtre (après la mort de l'insecte les antennes (leviennent entièrement de celle dernic̀re couleur); corselet très-fortement caréné, rose sur les còtés et vert en dessus; élytres opaques, vertes ou brunâtres, avee le bord interne ferrugincux et deux ou trois rangées de taclıes carrées et noiràtres, les élytres sont étroites, dépassent plus ou moins le corps et sont arrondies a l'extrémité; ailes ineolores, transparentes, ne dépassant pas les élytres et ayant souvent a la base une tache sulfurense; abdomen brun avec des taches plus fonećes et disposées sans ordre; tarière de la femelle, souvent aussi longue que le corps, fortement recourbéc ê dessus à l'extrémité, rosée à la base, brune au bout; pattes de la couleur du corps. 
Celte espèce est aussi commune que la précédente; les femclles sont plus grandes que les màles; on les troure en septentbre ayant, atteint tout lcur dévcloppement; les organes sIridulateurs du màle sont très-développés.

Les obscrvatious faites à propos des dégàls cansés par la sautcrelle verte, sont également applicables á ceux qui sont produils par la sauterclle à sabrc. Jc compléterai ce que j’ai a en dirc par la citation suivaute que j'cmprunte a l'ouvrage l'Audinet-Serville:

* Les mandibules de celte espèce ont une telle force qu'elles entament la peau jusqu'au sang. Dans le nord de l'Europe, les paysans saisissent exprès cet insecte pour lui faire inordre les verrues qu'ils ont souvent sur les mains; ils pensent que la liqueur aere et brune que ce dectique répand cn même temps dans la plaie, fait sćeher et disparaitre les verrucs. C'cst pourquoi ils lui ont donné le nom de $\mathbb{V}$ art-bit, qui veut dire : Ronge+ verrue. $y$

\section{GRYIIOTAIPA (Tatreille)".}

Audinet-Serville; Orthopteres; suites $\dot{a}$ Buffon, page 300 .

Corps allongè; tète petitc, avancée; yctıx saillants, ayant en outre deux ocelles ou yeux lisses places obliquement sur le front; mandibulcs fortes; palpcs maxillaires très-allongées, les labiaux fort courts; antennes assez longues, sćtacées, pcu écartćcs à la base; prothorax grand et ressemblant ì la carapace des écrevisses; élytres courtes ; ailes longues, pliécs en éventail en forme de filet d, dans le repos, dépassaut de benucoup les élytres; abdomen grand, ayant les appendices terminaux non articulés; pattes antćricures propres à fouir; lranclics très-larges, cuisses courtes et fortes, jambes larges, courles, imitant unc sorte de main, terminćes par des dents

\footnotetext{
- Synonymie: Gnvllus (Linné); -- Acheta (Fabr.); - Countilięre.
} 
fortes, fixes et acérées; pattes interınédiaires rapproelı́ces à leur insertion eomme les précédentes, cuisses forles; pattes pustérieures plus longues, fortes, mais inmpropres au saut; tarses de trois articles, ecux dles pattes antéricures se logeant dans une rainure de la jambe; tarière de la femelle rétractile et non apparente; les filets abdominaux épais et courts.

Les Courtilières sont assez peu nombreuses en espèees, mais les indiviclus sont très-abondants. On trouve des représentants de ce genre dans toutes les parties du monde, mais on ne sait rien des mours de celles qui sont étrangères à l'Lurope. Ce qu'il $y$ a de remarquable, c'est que presque toutes ont des couleurs analogues et que les espèces exoliques sont plus petites que celles de notre pays, tandis que, dans la elasse des inseetes, e'est ordinairement Ic contraire qui a lieu.

55. gRTLLOTALPA VUzGaRts (Latreille).

Audinet-Serville; Orthoptères; suites ì Buffon, page 306.

Synonymis: Gryllas gryllo talpa (Linnć); - Acheta gryllo lalpa (Fubr.); - Courtiliere (Geoffroy); - TaupeGrillon; - Werre; - Werle; - Erd Krebs; - Erd Wolf; - Riehmaus; - Reil Krote, ete., des allemands.

Longueur du eorps de quatre a cinq eentimères; d'un brun roussìtre plus ou moins foncé et velouté en dessus, plus clair et plus pubeseent en dessous. Elytres tecouvrant a peine la moitié de l'ablomen, à ncrvures fortement aecentuées et d'un brun foncé ; ailes plićes col lanières et dípassant l'abdomen de einq à dix millimètres. Pattes d'un fauve ferrugineux, pubescentes, les dents des jambes el des tarses, grandes, aiguës et noires à l'extrémité.

La Courtilière est connue depuis la plus haute antiçuité; elle a fixé l'altention des agriculteurs, dans toute les eontrées de l'Lurope, a cause des dommages qu'clle cause souvent daus 
les cultures, et celle des naturalistes, en raison de sa singulière organisation. C'est égalennent en considération de celle-ci, et de celle de ses moeurs particulières, qu'elle a reçu des noms si nombreux dans les différentes parties de l'Mllemagne. Ces insectes sout nocturnes, vivent presque constamment sous la terre, dans laquelle ils creusent une galerie verlicalc de 20 à 40 centimètres de profondeur, de dix à quinze millim. de dianètre, autour de eelle-ci s'élendent des galeries horizontales secondaires. Bien que les ailes membraneuses soient assez développées, l'insecte en fait rarement usage. C'est ordinairement le soir, et au moment de l'accouplement, qu'on les roit voler, mais leur vol est lourd, peu élevé et de peu d'étendue; quand ils se posent, ils se inettent aussitó a fouir la terre, el, en quelques minutes, on ne trouve de leur trace qu'une petite motte de terre semblable a celle que forment les taupes mais qui n'a que un à deux centiuctres d’élévation.

C'est pendant les soirées ou les nuits chaudes de juin ou de juillet que se fait l'aceouplement. Les élytres du màle n'ont pas de miroirs comme celles des sauterelles, mais clles produisent cependant une stridulation qui sert a appeler la femelle. Celle-ci unc fois fécondée se retire et va trarailler a la construction d'une galerie spéciale dans laquelle elle doit operer sa ponte. Cette nouvelle galerie, qui portc également le nom de nid, prend naissanec à la galerie verticale qui sert d'introduction. Elle se dirige latéralement, s’élargit considérablement à la distanec de 6 à 8 centimètres, puis se continuc circulairement pour rejoindre la galerie principale ou l'une des galeries horizontales scconclaires.

Pendant ce travail, le ventre de la femelle augmente de rolumo ct bientòt, en juillet le plus ordinairement, elle dépose dans la chambre qu'elle a eonstruite, et donl le diamc̀tre varic de $\mathbf{s}$ à 15 cent., des aufs verdàtres, gros comme des grains de millet et dont le nombre, toujours assez grand, s'élève parfois a 230 on 500 . Quelquefois cependant on trouve des nids dans lesquels il n'y a que huit ou dix oufs. Ces ceufs sont elliptiques, d'un vert sale, lisses, diffieiles à écraser, remplis d'abord d'un liquide lıuileux 
et jaunảtre. Si la température du sol est favorable, ils ue tardent pas à éelore, les jeunes paraissent au bout de dix ou douze jours; quelquefois eependant ee n'est qu'après vingt-einq ou trente jours; le terme moyen ordinaire est de deux semaines. Selon Rosel, la ponte dure de douze à vingt-quatre heures; mais on eonsprend que la longueur de eette opératiou soit subordonnce à une foule de eireonstanees qui doivent la faire varier cousidérablenent, et dont la prineipale est eelle qui eoncerne le nombre des œufs. Cette observation est d'ailleurs de peu d'importanee, mais ee qu'il est bon de signaler, 'e'est que la femelle ne quitte pas ses $\alpha u f s$ une fois qu'ils sont pondus, ou tout au moins ue s'en éloigue guère. Ces aufs sont disséminés sans orlre dans toute l'étendue du uid, et s'ils étaient réunis, on n'aurait pas manqué bien eertainement de dire, ainsi qu'on l'a fait pour la Forfieule, que la Courtilière couvait ses œufs. Il est évideut iei que la femelle garde le produit de sa ponte, non-seulement eontre la voracité des autres inseetes, nais aussi contre celle des individus de sa propre espèee.

Les petits, nouvellement ćelos, ont de 4 à $\mathbf{5}$ millimètres de longueur, ils sont d'un brun elair avee le dessus du eorps tacheté de couleur un peu plus foneée. La tête est proportionnellement plus grosse. Ces jeunes larves eommeneent à se nourrir des radieelles qui sont à leur portée, ou, comme les jeunes larves de lianneton, elles absorbent l'humus; et, toujours sous la proteetion de leur mère, elles ne tardent pas à ereuser des galeries dans tous les sens. Ces faibles ressourees mises a la disposition de deux ou trois eents jeunes larves, expliquent eomment il en meurt un si grand nombre. Leurs dépouilles, après la nuort, servent à la nourriture de leur mère, et peut-être aussi à celle de leur frère, c'est ee qui a fait eroire à Bouehé et à d'autres, que les fenelles mangeaient quelquefois leurs petits. Toujours est-il que j'ai entendu répéter sourent par des jardiniers, counaissant très-bien les habitudes de la Courtilière, que sur un nombre eonsidérable d'oufs ou de jeunes larves, quelques-uns seulement arrivaient à l'état d'insecte parfait. Cette observation 
est d'ailleurs parfaitement conforme à la loi d'harmonic générale, laquelle loi veut que la fécondité d'une cspèce soit cn rapport arec lcs causes de destruetion auxquelles cette même espèce se trouve exposée dans les diverses phases dc son existence.

$\Lambda u$ bout de quinze jours, les galeries crcusées par les jeunes larves ont déjả de 10 à 15 cent. de longucur, et, au bout d'un nois, de 20 à 25 centimètres; alors lcs larves ont cnviron un centimètre de longueur, changent de peau pour la premièrc fois et aequièrent une eouleur un peu plus foncée en tnême temps quc les taches disparaissent. C'est à cette époque (fin d'aout ou commencement de septembre) que l'on commenec à s'apercevoir, à la surface du sol, de la présence de ces insectes, dans les prairies ou dans les luzernières, par la teinte jaunatrc des plantes dont les racines ontété attaquées. Ces plaques jaunåtres ont souvent de trois à quatre décimètrcs carrés. Dans les pépinières on ne s'aperçoit du dégàt que beaucoup plus tard, et alors souvent qu'il n'cst plus temps d'y porter remède.

Dans le courant de septembre, les jeunes ont environ 15 mill. de longueur, muent pour la dcuxiènc fois, croissent rapidement jusqu'en octobre ou le commenceinent de novembrc époque a laquelle ils ehangent de peau pour la troisième fois; alors ils ont de vingt-cinq à trente millimètres et sont de couleur brun elair.

Aux premiers froids, les Courtilières s'enfonecnt plus ou moins profondément dans le sol, sclon la nature de eelui-ci, son état lyggrométrique, l'état extérieur de la températurc, ete. Pendant tout l'hiver, ecs larves prennent peu ou point de nourriture, aussi, all printemps, les retrouve-t-on à peu près dans lc méme ćtat qu’a l'automnc, les plus grands n'ayant guèrc plus de 5 cent. de longueur. Lans le courant d'arril ou au commenccment de mai, ont lieu la quatriène mue ct le passage à l'état de nymplıe; cnfin dans lc courant de juin, a licu, après une dernière mue, le dévcloppement complet de l'inscetc. Après eliaque eliangement de peau le uouvel insecte est de coulcur testacéc plus ou moins claire, mais cette teinte ne tarde pas à se foncer; et, dans les 12 ou 24 heures qui suivent, elle a aequis les couleurs que nous arons indi- 
quées; en même temps, les tíguulents du corps se raffermissent et prennent la consistance nécessaire pour creuser des galeries dans un sol souvent très-compacte ou endurci par la sécheresse.

Lin donnant les caractères du genre Courtilière, j'ai dit que la jambe des pattes ant iricures est courte, large, aplatie en forme de main et très-fortement dentéc. Si maintenant j'ajoute que le tarse vient se loger dans une petite rainure placée à la partie externe de cette jambe, et que les deux premicrs articles de ce tarse sont en outre armés chacun d'une forte dent, on comprendra facilement qu'il résulte de cette disposition que le tarse et la jambe se meuvent l'un sur l'autre comme le font deux branches de ciseaux dont les deux lames seraient dentées en scie. Les jambes antérienres de la Courtilière lui servent done non-sculement à creuser ses galeries, mais encore à couper les racines qui s'opposent à son passagc. Avant d'entrer dans quel $c_{\text {jues }}$ détails sur les habitudes de ces animaux, il était indispensable de revenir sur ee mécanisıne qui permet de comprendre facilement la nature des dégáts causés par Ics Courtilières.

La Courtilière se rencontre dans les bois, daus les prés, dans les champs et dans los jardins. Tous les terrains semblent lui convenir, même les terres sablonneuscs, et les tourbières pendant les grandes sécheresses. Cependant elles préfèrent les terrains secs, meubles et exposés au midi. On la trouvc dans toutes les contrées méridionales et tempérćes de l'Europe. Dans la itloselle, elle parait assez rare dins les envirous de Bitche et dans le pays haıt, tandis qu'au Sablon, près de Metz, elle y est toujours trèsabondante, surtout dans les jardius les plus rapproclićs de la Seille. Dans les vignes de Queulen (rirc droite de la Seille), elle $y$ est aussi très-commune, mais comme la vigne a des racincs très-longues el très-fortes, la Courtilière ne saurait lui nuire bcaucoup, tandis qu'au contrairc ellc muit aux autres plantes parasites qui croissenl dans les vignob!cs.

Ia Courtilière est un animal nocturne, très-craintif et qui ne peut en aucune façon légitimer le nom impropre de scorpion qu'on lni donne clans notre département. J'ai dlans ma collection 
un insecte de ce genre pris en 1854, dans la rue du Pontiffroy, oú il avaiı donné lieu à un véritable rassemblement, dans lequel on discutait séricusement de quels mallicurs la présence d'un tel monstre était le précurseur! Cet insecte court assez vite, nage parfaitement, et, quand on le touche, il jctte par l'anus un liquide noirâtre; e'est là sa seule arme défensive. Ainsi que je l'ai déjả dit, le mále est pourvu d'organcs de stridulation, c'est surtout le matin et le soir au eouelier du soleil, qu'il fait cntendre un bruit analogue à celui du grillon unais moins aigu et plus souvelt interrompu..

Comme beaucoup d'Orthoptères, eet insecte est à la fois earnassier et phytophagc, et ce u'est pas par son alimentation qu'il est le plus nuisible. Les nombreuses galeries qu'il ereuse et pour l'établissement desquelles il cst obligé de couper tous les obstacles que lui présentent les racincs et les radicelles des végétaux, sont la cause prineipale de tous les dommages qu'il nous fail subir. En ce qui concerne le poirier, on comprend que pour des arbres d'une ecrlaine importance, on puisse le considérer comme presque complétement inoffensif, mais il est loin d'en être ainsi dans les pépinières où l'on éléve ecs arbres, el c'est à ce point de vue que je me suis placé en entrant dans d'aussi longs détails au sujet de cet insecte.

Une terre trop séche lui est nuisible ainsi qu'un sol trop lıumide dans lequel ses aufs s'y altèrent en quelques jours. Les porcs sont très-friands de cet insecte et on a tort de dire que ce genre d'alinent les fasse périr.

Les taupes, la salamandre terrestre et les carabes en détruisent un bon nombre à l'ćlat de larves ou de nymphes. Il parait que la fumure des terres leur est favorable, probablement cn risison de l'aliment azoté et liquide qu'elle fournit aux jeunes larves. Uu sol trop enclrevetré de racines les fatigue et les force à émigrer, aussi conseille-t-on de semer dru dans le tcrain oủ elles sont abondantes.

Comme pour le lanneton, on a conseillé pour la détruire, un grand nombre de proeédés, la lessive, l'eau de savon on l'luile 
versées dans ses galeries, sont de bons moyens, mais un peu longs et impossibles à pratiquer en grand. Les arrosages à l'eau de eliaux mélangée de fleur de soufre et de eendre, ou ceux qui sont faits avec de l'eau acide ou mélangée d'essence de térébenthine, ou d'huile de pétrole, ete., ne sont efficaces qu'à des doses qui deviennent préjudiciables aux plantes que l'on veut protéger. L'usage du plısplıore, du fumier de pore, du poisson mort, etc., ne semble pas non plus étre très-efficace. L'emploi des pots de terre placés à fleur du sol, et dans lesquels on met de l'eau, rceouverte d'une couche d'huile, réussit parfaitement pendant les moins de juin et de juillet, époque à laquelle les Courtilières adultes sortent de leur galerie pendant la nuit; en cherchant à s'aceoupler, ou en eliassant les pelits insectes dont elles se nourrissent, elles tombent dans ees piéges et y sont rapidement asplyyxiées. Mais de tous les ınoyens proposés, le plus rationnel est celui qui eonsiste a recliercher leurs nids au moment de la ponte ou pendant le jeune áge des taupes-grillons, et a en détruirc le contenu, en écrasant les jeunes ou en exposant les $\propto u f s$ au soleil qui les dessèche. La couleur jaunatre que preunent les plantes dont eet insecte a eoupé les racines, l'existence des trous vertieaux par lesquels il se rend de la surface du sol dans l'intérieur des galeries, la présence des petites mottes de terre, dont nous avons parlé, sont autant d'indiees qui suffisent aux jardiniers expérimentés, pour leur faire découvrir ces nids, qui, placés à 10 ou 20 centimètres de profondeur seulement, sont faciles à enlever avec un coup de béelıe.

Avant de terminer, je ne saurais trop inviter les horticulteurs à se mettre en garde contre certains industriels qui vont colporter de prétendues recettes, pour la destruction complète de ces insectes. Ce sont autant de clarlatins, dont tout le secret eonsiste ordiuairement á introduire, dans les galeries, de l'eau dans laquelle ils ont fait dissoudre une substanee dont le nom tenu seeret forme tout le mérite. C'est le plus ordinairement du sayon, du sulfate de zinc, on du sulfure de potassium. 
Liste des Espèces de Nérroptères, qui vivent sur le Poirier.

MYRMELEONIENS. Hemenoutus PEnLA (Linné). $\left\{\begin{array}{l}\text { Les tarves de ces in- } \\ \text { sectes sont très- } \\ \text { utılesparlagrande } \\ \text { quantité de puce- } \\ \text { rons qu'elles dévo- } \\ \text { rent. }\end{array}\right.$

XXXVI. HEMEROBIUS (IiEné)

Rambur; Nérroplères; suiles ì Buffon (Ed. Rorel), page 423.

Corps ınou, tèle non prolongiee en avant, mandibules forles, dernier artiele des palpes maxillaires plus long que le précédent; antennes longucs, sćlaećes, insérèes entre lẹs yeux, composćes d'un très-grand nombre d'articles evurts; ailes larges, transparentes, à réseau rétieulẻ très-ạpparent, disposées en toit pendaut le repos; aludomen peu allongé; palles grêles, ayant aux tarses einq artieles, dont le dernier plus large; les autres portent deux erochcts écarlés, ayant entre eux une petite pelonte.

Les liéméroles sonı des iusectes qui ressemblenı assez aux Libellules ou demoiselles, mais qui ont l'abdomen beaucoup plus eourt que ees deruières; les ailes, fortensent rétieulées, sont transparentes ou simplement translucides, quelquefois obseures ou hyalines; les yeux sout saillauts et souven d'une belle eouleur métallique (pendan ta vie de l'inseete au moins). Leur eorps exhale ordinairement une odeur désagréable, que l'ou a comparée avee raison, au moius pour les espèces européeunes, à celle du sulfhydrate d'ammoniaque. Les larves des Ilémérohes sont

- Synonymic: lléménoue; - Lan nes Pucenoxs; - Flon-Flegex. - Cunrsopd. (Lepel.) 
connues depuis longtemps, et leurs labitudes leur ont valu de la part des Entomologistes du siècle dernier, le nom de Lion des Pucerons, a eause de la guerrc acharnée qu'elles font a ces Homoptères; ccux-ci forment en effet la nourriture presque exelusive des larves d'Hémérobes.

Les fcmelles d'Hémérobes, après avoir passé l'hiver sous divers abris, se rendent dès lcs premiers beaux jours, pour $y$ pondre leurs oufs, sur les feuilles des groseillers, des poiriers, des pommiers, du sureau, etc., vù d'ordiuaire, pendant l'été, on reneontre des eolonies de puecrons. Ces $\propto u f s$, ordinairement en assez petit nombre, sont posés sur les jeunes ḅranches, sur les feuilles, quelquefois même sur les fruits; ils sont blanelıàtres, de forme ovoïdc et présentent à leur base un pédieule plus ou moins long, de sorte que ces œufs, ainsi pédicellés, ressemblent a dc petites plantes eryptoganiques, mousses ou champignons, en fructifieation el sortant de la plante sur laquelle la femelle a pondu.

Vers la fin de mai ou daus le commencement de juin, les jeunes larves écloseut, et se rendent au milieu des colonies de Pueerons. En saisissnut ceux-ci au moyen de leurs longues mandibules aiguës $\bullet$ arquées, clles en suecut les parties molles intérieures, et en font unc très-grande consommation. Quelquefois, à défaut de Puecrons, clles attaquent les jeunes Chenilles, qu'elles détruisent arec la mème avidité et de la même manière. Ces larves, dont le corps est aminci aux deux cxtrémités, sont d'unc graudc agilité; parvenues à leur ćtat adulte, elles ront se meltre à l'abri sous unc pierre ou dans les feutcs dc l'écorce ce certains arbres; la elles se filent une coque sphé. rique et soyeuse au moyen d'une filière plaeće à l'extrćmité de l'ablomen, et non sous lc menton, eomme eela a lieu ehez les Clienilles et les fausses Chenilles.

Jes larves d'Ilémérobes sont tellement voraces qu'clles s'entuedévoreat; elles nc meltcnt guère que 15 à 20 jours prour alteindre tout leur développennent. Ia coque filée par clles a environ cinc millim. de diamètre; quelques jours après qu'elle 
est terminée, on n'y trouve plus que la uyinplie, laquelle differe de la larve surtout par l'absenee des mandibules; environ quinze jours ou trois semaines plus tard, l'iuseete parfait sort de son enveloppe de soie, dans laquelle il était replié et où ses ailes étaient ehiffonuées, pour pouvoir se loger dans un si petit espace. Souvent (je erois mème que puur les deux espèces de nos environs, e'est le cas le plus ordinaire), il y a deux générations par année; alors la seconde ponte se fait fin-juillet, et les œufs éclosent au mument où les Puecrons sont le plus abondants. Les larves parvienuent vite à leur élat parfait et les nymples passent l'hiver dans leurs eoques pour éelore au printemps suivant; e'est ce qui explique les accouplements nombreux que l'on peut observer en avril, et le nombre prodigieux de ees insectes, que l'on reneontre sur les premières feuilles des jeunes arbusles de nos jardins. Le vol de l'Hémérobe a peu de portée. Le nombre total des espèces du genre s'élève à une vingtaine dont la moitié appartient à l'Europe.

D'après ee qui préeède, il est facile de voir que les Hémérobes sont des insectes éminemment utiles, qu'il eonvient de les ménager, plutòt que de les détruire, et que les œufs qu'ils déposent sur des filamenls semblables à ceux de eertains Cryptoganes doivent ĉtre eonservés. C'est dans le but d'empèelser la destruetion de ees insectes utiles, que je donne iei la deseription des deux espèces qui habitent notre pays.

56. HEMEROBIUS TERTA (LinnÉ).

Rambur; Névroptères; Suiles ì Buffon, page 424.

Synonymic: II imérobe-perle; - Florhflieg; - Stinckflieg (de Ratzeburg). - Lion des Pucerons (Geoffroy).

Longueur huit à neuf millim.; envergure vingt-cinq millin.; variant du jaure verdàtre au vert, quand il est vivant ott nouvellentent éclos; roussàtre après la mort; yeux d'ıtne belle eouleur d'or pendant la vie; vertex bossu; antennes très-rapproelıćes à la base, un pett obseures vers l'extrémité, 
et presque aussi longtues que le corps ; eorselet presque earré, ayant une eòte éleviée au milieu; abdomen ayant une bande verte sur les eótés et quclqques taelıes plus ou ınoins apparentes de cette couleur en dessus; ailes transparentes, ltuisantes, ayant les nervures et les nervules entièrement verdatres ou au moins jaunatres, eiliẻes sur les bords, avee une trés-faible taelse sigmatale obseure, pattes verdảtres, tarses obseurs.

Cette espèce est la plus commune, e'est celle que les auteurs ont déerile en domnant l'histoire de la larve; celle-ei est de couleur jaunàtre arec des taehes brunátres formant deux lignes longiludinales irrégulières sur la partic antérieure du eorps; ces taches sont plus petites sur la partie dorsale des anneaux; ceux-ei sont latéralement bordés de huppes soyeuses. Les mandibules sont jaunáıres, longuẻs, aiguës, arqquées et paraissent plutót des eornes, que des organes de la bouehe. Cette larve saisit les jeunes Pucerons avec ses longues pinces, les suce, et, après quelques instauts, n'en laisse plus que la peau vide. Les premiers individus paraissent en avril, et eeux de la seconde génération en aout.

57. HEMEROBIUS GRRYSOPS (Linné).

Rambur; Néuroplères; Suiles à Bu/lon, pages 427.

Synonymie: Chrysopa Reticulata; Burmeister.

Longueur: dix à onze millim.; eorps varié de noir et de vert ou de jaunatre, le noir donine dans eertains individus, dans d'autres, e'est le vert; bouehe et base des antennes entourées de noir ; antennes d'un jaune verdatre ou brunâtre; ailes fortcment eilices, pltts larges proportionnellenent que dans l'espéee préeédente, transparentes avec les nervures et les nervules prineipales vertes, le reste noir on noir varié de: verdatre; yeux dorés et très-brillants; pattes verdatres avec des taehes noires. 
Celte espèee, facile à distinguer, est moins communc que la prćeédente dans notre dépaytement; au moins, n’en ai-je jamais trouvé que des individus isolés; peut-être cette eirconstanee dépend-elle de ce que paraissant plus tard, elle trouve un plus grand nombre de plantes oủ elle peut faire sa ponte et par conséquent sur lesquelles elle se disperse plus facilement. On la trouve plutót sur les péchers et sur les rosiers, où d'urdinaire les pucerons se montrent en grande abondance. La ponte se fait comme celle de l'Hémérobe perle; la larve est de couleur rosée avec de nombreuses taches irrégulières et jaunes, elle est aussi plus eourte et proportionnellement plus arrondie que la précédente; sa peau est, suivant Gcoffroy, car je ue l'ai jamais rcmarqué, souvent reconverte des débris de la peau des pucerons dont elle a sucé les parties internes. Selon le même anteur, sa larve file une coque semblable à celle du lion des Puccrons.

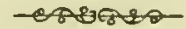

liste des Thysanoptères qui virent sur le Poirier.

TIIRIPSIENS. Tunips Vulgatissima (Ilaliday). Linsecte et la larve dans les fleurs; peu ou pas nuisibles.

Tumps newommorbalıs (Bouchè). Sous les feuilles et dans les serres où il est trèsnuisible.

XXXVII, THRIPS (Iinne)"

Blanchard; Ilistoire des animaux articules, tome 2, page 272.

Corps linéaire, allongé, plat, lisse ou rélieulé ; mandibules longues, machoires aplaties; palpes maxillaires de trois

- Synonymie: La synonymie du genre Tlırips est très-diffieile à établir, M. Ilaliday, auteur anglais, ayant forné une quarantaine de coupes génériques avee Ieg insectes désignés par les auteurs sous le nom te Thrips: 
artieles; antennes de huit arlieles, assez longues, filiformes, les derniel's artieles presque soudés; yeux grands, lateraux, trois oeelles au somınet de la thic; ailes longues, fort étroites, posées horizonlalement le long du dos pendant le repos. Les ailes supérieures sont les plus grandes, elles ont deux nervures longitudinales parallèles, sans nervures transversales. Les ailes inférieures sont membraneuses, velucs et frangécs. Abdomen velu, glabre ou ćcnilleux; pattes eourtes; tarses vésieuleux, de deux artieles. Femelle ayant une tarière ou aiguillon en forme de valve.

Les Thrips sont des inseetes de taille excessivement petite, (de deux à trois millim.) et très-répandus dans toutes les contrées du globe. C'est le plus ordinairement dans la corolle des fleurs qu'on les reneontre; cependant, on en trouve aussi sous les écorees et sur les feuilles.

Ces insectes ont des métamorphoses incomplètcs, e'est-à-dire, que la larve ne diffère de l'insecte parfail, que parce qu'elle est privée d'ailes; celles-ci ne paraissent qu'après le deuxième ou le troisième changement de peau. Ces larves sont allongées, très-agiles; et, quand on les inquiète, elles relèvent leur abdomen comme le font les Staphylins et les Forficules. De couleur ordinairement blanchàtre ou jaunàtre, ces larves sont quelquefois d'un beau rouge vermillon, conme celles du Thrips Cerealium qui vit dans les épis du froment; rarement elles sont de couleur foneéc, tandis que les insectes sont au contraire le plus souvent de couleur noire, avec des ornements blanchàtres sur les élytres ou sur les pattes.

Les mœurs des Thrips sont encore très-peu connues; l'organisation de leur bouelıe, qui est un mélange très-remarquable de

les earactères dont il s'est servi, étant en général très-peu apparents ou difficiles à vérifier pour les persornnes peu versées dans l'étude entomologique, je crois que, sans grand inconvénient, on peut eneore laisser intact le genre Linnéen, devenu le type de la petite famille des Thripsiens. 
eelle des Hémiptères et de celle des Névroptéres, semble cependant indiquer qu'ils sont broyeurs, tandis qu'il parait que leurs mandibules ne leur servent qu'à percer l'épiderme pour pouvoir ensuite sucer, à la manière des Pucerons, le suc ou la sève du végétal sur lequel ils vivent. Ordinairement on en rencontre un certain nombre dans la même flcur, ec qui semble indiquer qu'ils vivent en sociéti. L'insecte, comme la larve, est très-agile et s'envole facilement.

Il est Irès-peu de fleurs, dans le fond desquelles on ne rencontre de ces inseetes; la même espèce se trouve quelquefois sur plusieurs plantes différentes. Ordinaircment ehaque espéce de plante nourrit la sienne propre; mais les entomologistes n'ont pas encore démontré que leur présence fut réellement préjudiciablc aux végétaux.

58. TRRIFS VULGATISBIMA (Ilaliday).

Blanchard; Hisloire des animaux articules; Suiles i Buffon, page 217.

Synonymic: Thrips Physapus? (Degécr); — Physapus Vulgatissima (Amyot).

Longueur: trois millim. Cor'ps allongé, noir, lisse, dépourvu de poil ; ailes supéricures d'un noir brillant; pattes blanches, ainsi que les ailes inféricures.

Cet insecte se trouve au printemps, dans les fleurs de plusieurs arbrcs fruitiers, poiriers, cerisiers, ponmiers, etc. Mais, je nc crois pas que sa présence soit bien uuisible à la fructification de ccs arbres. Nalgré mes rccherches, je n'ai jamais pu rencontrer que des larves d'une certaine grandeur, et la plupart sur le point de passer à l'état de nyunplic. Il est donc permis de supposer, ou que la ponte n'a pas licu dans la fleur, ou, ee qui cst moins probable, que l'évolution des jeunes larves est très-rapide.

L'obscrvation est d'ailleurs extrêmement difficile sur des insectes aussi pctits et aussi délicats; aussi, ne faut-il pas s'étonner si leurs mocurs sont encore presque complétement incounues. Tout ce que jc puis ajouter au peu que l'on cu connait, c'est que 
l'accouplement se fail en mai, au plus beau noment de la floraison des arbrcs, que les mảles survivent plusieurs jonrs à l'acconplement, et que les femelles pondent de 10 a 15 acufs; ceei du reste, résulte du nombre d'œufs que j’ai rencontrés dans lcur ovaire; car, malgré toutes mes tentatives, je n'ai pu les voir dépuser leurs oufs, soit parce qu'elles périssaient avant la Inaturité de crux-ci, soit parce qu'elles ne trouvaient pas un lieu à leur convenance, daus les flacons où je les tenais renfermées.

59. THRIPS hOEMORRHOIDALIS (Bouché).

Bouché; Naturgesichle der Schudlichen, etc., page 12.

Synonynie: Thrips des serres, de nos Jardiniers.

Longueur: deux millim. Corps allongé, étroit, d'un noir mat; tète arrondie; yeux saillants; antennes de sept artieles, jaunàtres, avec la base et l'extrémité d'un brun noir; eorselet oval, aplati; jambes eourtes, d'un jaune clair, avee les euisses blanchâtres; élytres d'un brun jaunàtre, avec la base blanche; abdomen allongé, pointu à l'extrimité, les deux ou trois derniers anneaux rouges.

Cet insecte est excessivement commun dans les serres chaudes et tempérées ou il eause de grands dommages par sa prodigieuse multiplication. J'en ai trouvé un grand nombre sous les feuilles des poiriers élevés en espaliers et exposés au midi. La larve est d'un jaune clair avec l'abdoınen transparent. Selun Bouclıé, qui n'a observé cet iusectes quc dans les serres et dans les orangeries, les $\propto u f$ sont rouds et blanehàtres.

Pour détruire les Tlurips, on peut avec suecés faire usage de la pondre insecticide. Dans les serres, on pent éeraser un grand nombre de leurs larves en brossant les feuilles sur lesquelles elles sont fixées. Ainsi que je l'ai déjả dit, ccs insectes me paraissent peu nuisibles au Poirier, aussi u'cu anrais-je pas fail mention si je n'avais trouvé fréquemment des Thrips, à divers étals, dans les fleurs et sous les feuilles de cet arbie. 
Liste des espéces d'Hyménopteires qui virent sur le Poirier.

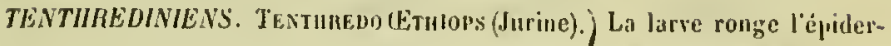
- Apumanata (Klug). $\left\{\begin{array}{l}\text { me supérieure des } \\ \text { feuilles. }\end{array}\right.$ Loma Silvarica (Linné). ） La larve mange les - Proi (Schranck). $\quad$ Pen nnisibles.

Cepuns Comphessus (Fabr.). La larve vit dans les jeunes pousses.

Assez nuisibles.

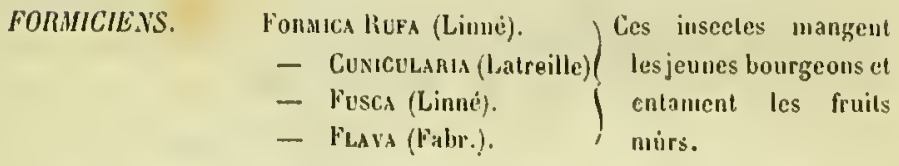
GUEPPIENS

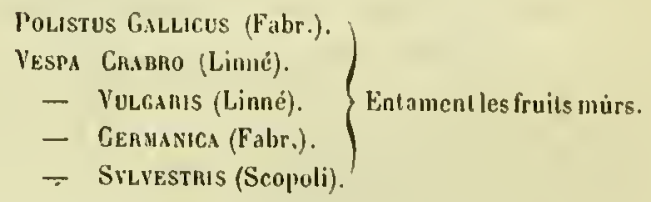

- Vulgams (Linné). Entament les fruils mürs.

- Geranica (Fabr.).

- Silvestais (Scopoli).

Megachile Praina (Lepall). Coupe quelques feuilles. Pas nuisible.

XXXVIII. TENTHREDO (Linné)".

Lepelletier de Saint-Fargeau. Monographie des Tenthredides, page il.

Téte large et eourte; mandibules longues, aplities, bidentées au còlé interne; palpes maxillaires de six artieles, palpes labiaux de quatre. Antennes de neuf articles; abdomen. sessile, et tellement uni au corselet qu'il semble en faire partie; ailes ayant deux cellules radiales igales el quatre cellules cubitales inégales, la premiere arrondic, la seconde

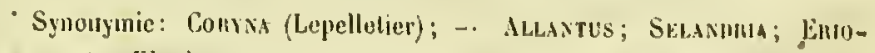
САнР, Cls. (Klug). 
très-allongée, la quatrième alleignant l'extrémité; paltes courtes, fortes, les quatre tibias postéricurs sans épines.

Les insectes qui appartiennent au genre Tenthrède sont, ell général, de petitc taillc (dc la grandeur d'une mouche ordinairc) et n'ofirent, quant à leur coloration, ricn qui puisse attirer l'attention des personnes ćtraugères à l'entomologic. Leurs labitudes à l'état'parfait sont aussi très-peu connucs, à l'cxception toutefois de la ponte des femclles. Cellcs-ei, en effet, sont pourvues d'une tarière d'une forıne toute spéeiale, laquelle se rapproche de eelle d'une scic appclée queuc de rat et au moyen de laquelle elles pratiquent, dans les plantes, des ouvertures ou elles déposent leurs ocufs. Ou sait aussi que plusieurs insectrs de ee genre-fréquentent les fleurs et plus particulièrement eclles des Ombellifères, et que si, en génćral, les Tenthrèdes vivent du suc de ces plantes, il en est aussi quelques espèces qui sont carnassières et qui attaquent même des inseetes appartenant à l'ordre des Colćoptères.

La taric̀re des femelles qui est double, mobile, écailleuse, est logée cntre deux lames qui lui servent de gaine; elle leur sert à perforer les feuilles ou les jennes pousses des plantes. C'est à la strueture de cet organe, aussi bien qu'à la manière dont il fonctionne, que les Tenthrèdes doivent leur nom vulgaire de Porte-seic ou de Mouches à seie. La prćsence d'un reuf de Tentlırède dans unc partic de la plante, y détermine quelquefois la formation d'une galle ou d'un boursouflement dans lequel la larve se développe; d'autres fois, et c'est le cas le plus ordinaire, la larve vit à découvert sur' les parties vertes de la plantc dont elle se wourrit. Ces larves sont beaucoup micux eonnues que les inseetes qu'elles - produisent, et plusicur's d'entre elles se multiplient tcllement, qu'elles causent souvent de trés-grands don mages à la vćgétation. La forme de leur corps et leur eouleur sont assez variables, mais In graud nombre d'entre elles oflient une ressemblanee rcuarquable avec les Chenilles ou larres des papillons. C'est ectic circonštance qui leur a fait donner le nom de fausses chenilles, sous 
lequel on désigne presque toujours ces larves. Mais on distinguera toujours facilement les chenilles des fausses chenilles, en ce que les premic̀res n'ont jamais plus de rinq paires de fausses pattes, tandis que les secondes en ont toujours sep $\ell$ au moins; les unes et les autres ayant en outre six pattes éeailleuses, il en résulte que, dans les chenilles, le nombre total des pattes ne dépasse janais seize, landis qu'il est toujours au moins de vingt ehez les larves de Tenthrèdes.

Quelqques larves de Mouches à scie sont recouvertes d'un enduit visqueux qui les fait ressembler à de petits mollusques dépourvus de eoquille; d'autres sont lissés ou plus ou moins vclues. Cellcs-ci rampeut sur les feuilles, celles-lá se tiennent sur la tranclie ayant le corps rccourbé en forme d'S, tandis que d'autres se roulent en spirale et ressembient, dans cette position, à la coquille des Planorbes. Tous ces caractères ont servi de base à quelques auteurs pour diviscr le genre Tenthredo $\mathrm{cll}$ plusicurs sous - genres.

Après plusieurs clangcınents de peau, la larve de Tenthrède se retire dans la terre où elle se file une coque, ou bien elle s'en construit une en aglutinant les grains de sable, au moyen d'une sécrétion visqueuse, de nature gommo-résineuse, imperméable à l'eau. Là, clle reste souvent six, sept, huit et même neuf mois, sans subir de transformation cl sans éprouver d'autre changement qqu'un amaigrissement général et une contraction plus ou moins prononcée de son corps. Ce long jeùne écoulé, elle se transforme en nymphe et bientòt après en insecte parfait.

En général, les máles de Tenthrèdes sont exeessivement rares et beaucoup d'espèees ne sont encore eonnues que par la description de la femelle.

Ainsi que je l'ai déjà dil, les labitudes des larves de Tentlırèdes sont très-variées: les unes vivent sur les feuilles des arbres on des arbrisseaux, d'autres sc crcusent des galeries daus l'intérieur du eanal médullaire des jeunes pousses, d'autres enfin vivent dans le bois en décomposition ou sous les éeorces des rieux 
arbres. Celles-ei ne vivent que d'une seule espèce de plante, relles-lá, au eontraire, sont polyphages et se uourrissent indifféremment des plautes de la même famille ou de celles qui, au point de viue botanique, sont les plus diffèrentes.

Le nombre des cspéces connues est assez considérable; on en a ınème décrit quelques-unes qui sont étrangères a l'Lurope. En Amérique, une larve de ce genre, dont le corps est visqueux, et que l'on y désigne sons le noun de Slecrn- $W$ urm, eause des dégáts souvent considérables sur les cerisiers, les pruniers, les poiriers et les coignassiers. Notre clépartement en nourrit un bon nombre d'espèces, dont deux doivent plus particulièrcment fixer notre attention. Comme il règne beaueoup d'incertitude sur la détermination exacte de l'insecte qui, dans notre pass, provient de la larve que nous allons décrire, que d'ailleurs aussi, il parait que plusieurs espèces roisines ont des larves qui diffèrent très-peu entre elles, je vais d'abord donner l'histoire de cette larve; et, en décrivant ensuite les deux insectes qui en proviennent, selon que l'on s'en rapporte à tel ou tel auteur, je ferai connaitre les opinions direrses qui ont été émises à ce sujet, ainsi que les raisons qui ont pu amener la confusion entre les larves et les insectes des espèces roisines.

Sur la fin du mois de juillet, etplus ordinairement dans le commencement du mois d'aoút, on rencontre sur les feuilles des poirier's élevés en espaliers, très-rarement sur les quenouilles, et pas que je saelıe, ici du moins, sur les hauts-vents, de petites larves noirảtres très-luisantes el courertes d'un encluit visqueux. Si l'on examine attentivement cette larve, on voit qu'elle a six pattes ordinaires et sept paires de fausses pattes; c'est donc unc fausse eltenille, ou plutỏ une larre de Tenthrède du sous-genre Blennocampa de Klug, lequel a précisément pour caractère cette yiscosité de lat larve. Ceelle-ci a le corps renflé en avant et presque eylindrique dins les deux tiers de la longueur postérieure, ce qui lui donne une resseublance assez marquée avec le 'Tétard; sa conleur est d'un rert plus ou moins foncé, et, comure le dit Réaunur, de la coulcur de Nostoc. Si, ayant les 
doigts secs on touelue cette larve, la ınucosité est facile à cnlever, et alors la couleur du corps est d'un vert jaunatre sale, avec úne raie sinucuse sur le dos, laquelle est produite par l'intestin que l'on voit alors par transparence. Si, aprés cctte opération, on rend la larve a la liberté, il faut deux ou trois jours (quelquefois, en caplivité, un jour suffit) pour quc la nıncosité reparaisse entièreinent.

Dans les premières phases de son déreloppement, celle larve élant tris-petite, on ne s'apergoit pas de son existence; ce n'est le plus souvent qu'au unois de septembre, lorsqu'elle a déjà alteint la longueur de cinq à six millimètres, ou que par les taches qu'elle détermine à la surface des feuilles en cn enlevant le parenclyyme supérieur, que l'on commence i soupconner sa présence. Je pcnse qu’à celte époque la larve a déjà subi une prenière mue; dans tous les cas, clle en subit une vers le milien du mois. Alors, la tète est couvexe, la bouche de couleur plus claire que le dessous du corps; les mandibules sont quadrangulaires et tridentées, les palpes maxillares de trois articles et les palpes labianx à peine visib!cs. Les antennes sont coniques et très-courtes; les ycux latéraux, très-visibles. Ces larves mangent constamment et reudent par l'auns de petites crolles dont plusicurs restent souvent attacliées à la peau visqueuse qui les recouvre. Quoiqu'elles soient peu agiles, leur têtc cst très-mobilc, cc qui leur permel de brouter une assez large sutface (comparativement a leur tiille, bien entendu) sins se déplacer; et, quand clles le font pour ronger une nouvelle portion d'épiderme, c'est toujours à reculons.

Le dessous dn corps et tontes les pattes sout, à l'état nornal, de la mèmc couleur que le corps, mais dépourvus de la matière gluante qui recourre celui-ci. Quoique ces larves soient souvent très-aboudantes sur les feuilles du poirier, on n'en voit eependant qu'une ou deux sur la même fenille. Presque constimment elles restent ou semblent rester inmobiles; car, en y regardant de près, on les voit presque tonjours occupées à brouter, marelıant à reculous à unesure que la surface rongée augmentc. L'épiderme 
supérieur de la feuille une fois enlevé, il ne reste plus qu'un réseau blanchàtre d'abord, náis qui ue tarde pas à se colorer, a brunir et ả ressembler aux brúlures produites par le soleil. Degéer a eu tort d'affirmer qu'elles ne eliangent de place que pendant la nuit; car si l'on en réunit dix ou douze sur une urême feuille, elles ne tardent pas, mème en plein soleil, à se disperser, conure si elles ne pouvaient vivre en compagnic. Quelques auteurs disent aussi que, pour clianger de peau, elles se retirent sous la page intérieure des feuilles; ce fait est une exception; pour s'eu convainere, il n'y a qu'à examiner la page supérieure, on y rencontre einq fois sur six, les débris de la peau que vient dé quilter la larve; celte vicille peau ressemble à un petit filct noir ayant un renflement considérable en avant, le tout visqueux et brillant quand il est frais. M. Delacour a très-probablemeut eommis un lapsus calami, quand il a écrit que la larve, avant de changer de peau, est quelques jours sans manger; c'est quelques heures qu'il fallait dire, pour se conformer à ce qui se passe réellement dans celle circonstance, ainsi que je viens de le vérifier sur des rers linnaces que j'élève en ce moment en captivité.

Selon M. Gorski, c'est dans le milieu du jour, qu'à l'air libre, les larves de Tenthredo adumbrala changent de pealt. Il pense avec raison, que la températurc plus ćlevée est la cause de ccltc particularité; il se fonde, pour établir son opiuion, sur le fait qu'en captivité, e'est-à-dire dans un lieu où la température est a peu près constante aux inois de septembre et d'octobre, les mues se font tout aussi bien la nuit que le jour. J'ai eu lieu de vérificr ce fait sur les larves que j'ai nourries dans mon calsinet, et j'ai les mèmes raisons que l'cntomologiste russe pour partager son opinion.

Dans le plus grand nombre des cas, c'est sur la face supérieure des feuilles de poiriers en espalicrs que l'on rencontre ces fausses chenilles que l'on désigne habituellcment, depuis Degéer, sous le nou de ver linace. Que le soleil soit ardent ou que la pluie soit des plus battante, rien ne leur fait, et presque toutes 
semblent complétement indifférentes à ces manifestations atmosphériques. En général, quand elles ue sont pas trop abondantes, les larves paraissent également réparties sur toutes les branches de l'arbre; mais dans quelques cas, notamment daus ceux où elles sont très-nombreuses, ce sont les parties supéricures de l'arbre qui sont les premières en rahies; je crois également avoir remarqué, au moins sur celles que j’ai élevées en eaptivité, qu'au ınoment où elles vont changer de peau, elles se rendent de préférence sur les feuilles des rameaux inférieurs.

Dans le courant d'oetobre, ordinairement dans la première quinzaine, la larve change une dernière fois de peau, mais alors elle semble aroil perdu la faculté de sécréter la matière visqueuse dont nous avons parlć. La larve est d'un vert clair, sale et presque jaune, ses anneaux sont plus saillants; le renfleınent antérieur du corps, produit en grande partie par la peau du thorax et le devant de la tête qui débordent pour cacher les pattes, n'est plus aussi marqué; et, comme ses pattes sont trèscourtes, elle ne tient plus sur la feuille. Alors elle tombe sur le sol et s'enfouce dans la terre ou elle forme une coque non soyeuse, composée de grains de terre aglutinés. Le cocon est elliptique, lisse intérieurement, long de 5 will. et large de 5. C'est dans eelte enveloppe que les larves d'allantus séjournent pendant un temps que je ne puis limiter, n'ayant pu parvenir jusqu'iei à mener à bonne fin les édueations que j'ai commené pour obtenir l'éelosion de l'insecte parfait. Cependant en jugeant par analogie au moins, sur ce qui s'est passé ailleurs, on peut admette que le changement de la larre en nymplie ne se fait qu'au mois de juin suivant, et par conséquent que c'est seulement yers la fin de ce mois, ou au commencement de juillet, que l'insecte parfait sort de sa dernière euveloppe.

Voila, aussi succinctement quc possible, l'histoire du ver limace que j'ai pu observer à Metz en 1857, en 1858 et en 1859. Mais, je le répète, je n'ai pu obtenir l'insecte d'éclosion ni une procurer d'Allantes an vol, lors de la saison farorable à la ponte que je suppose devoir se faire eu juillet. 
Une chose qui me parait devoir être signalie, éest que dans les trois invasions de 1857, de 1858 et de 1859, celie fansse elienille a varié chaque année son lieu d'ćlection; il n' $y$ a eu de constant que l'époque de l'apparition et la grande quantité d'individus. Ainsi, en 1857, les espaliers de Grimont, de SaintJulien at de Vallières ell étaient couverts; en 18\%8, c'étaient d'abord ceux qui sont limitroplies de Plantières el de Borny et ensnite ceux qui avoisinent le Sablon el Montigny; en 1859, c’était à Ars-sur-Moselle el à Vaux qu'elles étaient le plus ahondantes, et à Metz où un espalier du jardin le l'école d'applieation d'artillerie et du gánic en était littéralement couvert. La propagation s'est done faite, pendant ces trois dernières années, en snivant une direction du nord-est au sud-ouest.

Bien que, en génceral, on considère coune insignifiants l'enlìveinent de l'èpiderme supérieur des feuilles, opéré dans les conditions que je viens d'indiquer, il parait cependant loors de doute que si eette inıtilation n'est pas préjudiciable à la récolte pendante des fruits, elie a une grande influence sur la réeolte de l'annce suivantc, et on recomnait, quand on veut y faire attention, que les boutons a fruits sont plus rares, plus unaigres et que leurs produits sont moins beaux que l'année précédente. II est donc utile de se délarasser autant que possible de ces hótes malfaisants.

Sur l'insecte lui-même, ii n'y a rien à tenter, ni à proposer d'efficace; mais sur les larves, on derra des leur apparition it au moyen de ia houppe i soufrer, saupoudrer les feuilles avec des cendres de bois très-fincs et très-alcalines. En faisant, par un temps sec, cette opération plusienrs jours de snite, on détruira le plus grand nombre des vers limaees, et eeux qui échapperont, seront en trop petit nounbre pour eauscr, a lat récolte future, un dommage appréciable. La cliaux en poudre est d'une application plus difficile ct elle ne semble pas plus efficace; j'ai essayé sans ı’ésultats plus satisfaisants, la poudre insecticide. Les arrosages sonı également sans succès, la matière visquensc qui recourre les larres servant a les protéger contre les sulss- 
tanees liquides, acides, icres ou alealines, tandis qu'elle retient les Duatières pulvérulentes.

En terminant, je dois dire que pendant les trois années que je viens de signaler, je n'ai tronvé le ver limaee que sur le poirier, que, malgré toutes mes icclierclıcs, je ne l'ai rencontré ni sur le ponmier, ni sur le prunier, ni sur le cerisicr, mêtne en espalier. Deux ou trois de ces larves se sont eependant rencontréessur un abricotier, mais celui-ei était élevé en espalier et il était voisin de poiriers qui en étaient eouverts. Enfin, en 1859, j'ai nourri chez moi en captivité, un bon nombre de ces larves; j’ai constaté que, indistinctement clles changeaient de place sur un bouquet composé de branches de poirier, de cerisier, de prunicr et d'abricotier; le duret soyeux qui courre les feuilles du pommier ne paraissant pas leur eonvenir, j'avais cessé de leur donner de ces feuilles dès les premiers jours. On peut done présumer que ecs larves sont polyphages ct que, dans certains cas du moins, elles peuvent fort bien prospérer sur des arbres de la même famillẹ que le poirier.

Examinous maintenant à quclle espèce du genre Tenthredo il eonviendrait de rapporter lc ver limace dont je viens de parler.

Ein consultant les travaux de Réaumur, de Degéer, de Bouché, de Ifartig, de Ratzehurg, de Nordlinger, de Westwood, de Gorski et de Delacour, le doute ne saurait avoir lieu que pour l'unc des deux espéces que les auteurs ont fait connaitre sous Ics noms spécifiques de Adtumbrata "t OEthiops, que nous allons maintenant examiner.

58. TENTHREDO ADUMBRATa (Ḱlug).

Klug; Monograph. des Tenthrédides, Jarb. vin, no 36.

Synonymic: Allanlus adumbrala (Klug); - Selandria (s. y. Eriocampa) adumbrata (Ilartig); - Tenthredo (s. g. Blennocampa) adumbrata (Klug); - Selandria atra (Stephens).

Longucur de la femelle quarante-cing mil., envergure 
Jouze à quinze mil.; noire el luisante, éeusson lisse; tibias antérieurs d'un lırun pàle ; ailes supiricures claires, ayant aı milieu une bande transversale brunàtre, veines et sigmas presque noirs; ailes infërieures un peu plus obscures vers le bout.

Cette description est celle de la femelle (le male est inconnu), je la dois à l'obligeanee de M. le docteur Sielıel*"

Réaumur est le poremier qui ait parlé des larves gluantes qui détruisent l'épiderne supérieur des feuilles de plusieurs arbres fruitiers. Je erois que, dans l'intérèt de la diseussion synonymique

- Conme il importe de pouvoir au besoin distinguer eomplétement l'une de l'autre les Tenthredo Ethiops et T'. Adumbrata, je vais compléter la description faite par M. Sichel, des renseignements fournis par M. Gorski dans l'excellente brochure qu'il a publiée à ce sujet, en 1856.

- Les antennes de la T. Allumbratr, sont composées de neuf articles. dont le troisiome est le plus long; elles sont un peu plus minces aux deux extrénités qu'au milieu; r'cdressées quand l'insecte est vivant, elles se recourbent en arc quand il est conservé dans l'esprit de vin ou s'arrondissent autour de son corps quand celui-ci est desséchlé et fiché. Le corps de l'insecte vivant et au repos est tout à fait eylindrique, luisant et glabre. On peut voir sur la têtc et le tlıorax, à travers une bunne loupe grossissant beaucoup les objets, quelques poils de longueur modique et resserrés. La couleur de l'inseete est nuire, mais, sur' l'abdumen elle se rapproehe quelquefois de celle de ls poix.

"L'insectc desséclié est plat et par cela même plus ovale et dilaté ; son eorselet est de la même eouleur noire et luisantc; les quatre parties dorsales en forme de bourrelet, sont très-distinctes; les Cenchri ont la eouleur dc la poix et ne se laissent apercevoir qu'avec difficulté. Les ailes sont d'un gris sảle, iridcscentes, avec des nervures très-prononcées. Les veines, Ic punctum stigmaticum et le côté intcrne sont bruns. Les deux aréoles radiales sont inégales, l'extérieure sans appendice; les aréoles cubitales sont au nombre de quatre, la première est ronde, les autres soni courbées trapézoidales et grandissant à l'extrémité, la seeonde jusqu'au ınilieu, la troisième un peu en avant, sont claeune interceptées par unc veine réeur. rente transversale; les aréolcs disevïdales sont au nonbre de trois. La première aréole radiale, les deux cubitales et la diseoĩdale extérieure sunt 
que je vais examiner, il n'est pas inutile de eiter les passages les plus importants, dans lesquels cet auteur parle de ees insectes.

Tome V, mémoire III, page 97 . « Un autre genre de fausse chenille qui s'éloigne extrêmement de la figure la plus ordinaire aux fausses elıenilles, est un genre dont il n'est pas aisé de caractériser les espèces. On trouve de ces fausses chenilles sur diverses sortes d'arbres fruitiers, sur les pruniers, sur les eerisiers, mais surtout sur les poiriers. Les arbres fruitiers, ne sont pourtant pas les seuls sur lesquels on puissc les voir, car j'en ai vu sur des chênes. I.es unes et lcs autres se tiennent sur le dessus

teintées d'une couleur enfumée, mais si peu distincte, si peu visible, que la bande transversale enfuméc ne s'y laisse pas assez distinguer. L'abdomen, cylindrique, est composé de neuf anneaux dont le dernier est aminci à son extrémité. La tarrière, insérée dans les coulisses de deux segments annelés, est jaunâtre. Ses pattes sont courtes, leurs attaclies et les deux pattes postérieures sont d'un brun noir uniforme; la couleur des cuisses intermédiaires est, à l'extrémité, noins prononcée que celle de la partie correspondante des cuisses antérieures, laquelle est d'un brun testacé; les jambes et les tarses des quatre pattes antérieures sont de la même couleur brun testacé, mais plus clair dans les deux patles du devant. Toutes les jambes sont armées de deux petils éperons (calcaria) de couleur blême. Les tarses sont de cinq articles, dont le premier (métatarse) est le plus long et le quatrième le plus court; leur dernier article est muni d'une pelotte et de deux crochets simples.

- La Tenthredo adumbrata, daus son état parfait, atteint la granileur d'une mouche ordinaire (M/usca domestica, Linné). Lorsqu'elle est en mouvement, elle fait vibrer ses antennes du haut en bas avec une grande vitesse, i la manière de quelques Entodons de la famille des Pteromalins. L'insecte étant pris en main, coınme la plupart des petitcs espèces de ce gente, il replie ses antennes sous son corps, retire ses pattes et fait semblant d'ćtre Inort; mais aussitòt qu'il voit que le danger est passé, il se relève avec vitesse el s'envole brusquement."

A cette description minutieuse je dois cependant ajouter que la figure 2 de la planche 3 de la brocliure de M. Gorski, ne correspond pas tout à fait aux earactères qu'il donne de cet insecte, car la bande transversale inférieure y est bien distincte et, en cela, la figure 2 est parfaitement d'accord avec la description de Klug , citće plus laaut par M. Sichel. 
des feuilles, et n'en mangent quc lc parenclıyme supérieur. Elles ont une peau toujours gluante.... je n'ai trouvé que vingt jambcs à cclles de ces fausses chenilles qui se tiennent sur les feuilles du poirier.

A la page 134, à l'explieation dc la planclic XIII, nous lisons les passages suivants non moins importants à eiter : «La figure $\leftrightarrows$ représente la mouche dans laquelle se transforme la ehenille Tétard de la figure 1 (e'cst-à-dire, celle qui esı représentée sur une feuille de poirier)..... La figure 6 , est celle d'une mouehe venue d'une fausse chenille Télard qui avait véeu sur le cerisier, ectte mouclıe est assez semblable à eelle de la figure préeédentc, et je ne suis pas sủr qu'elle en diffẻre spéeifiquement, elles sont l'une et l'autre de la classe des mouelies qui ont une bouehe et des dents. 》

Il est impossible de reeounaitre les espèees représentées sur les planehes de l'ourrage de Réaumur, par les figures 5 et 6 , mais il n'en résulte pas moins que, pour lui déjá, il y avait probabilité que les vers limaees du eerisier ct du poirier appartenaient à deux espèces distinetes, ear il ne les eủt pas fait représentcr si elles lui eussent paru identiques, ct si mème elles nc lui eussent offert des différenecs assez seusibles, quoiqu'il ajoute: «e ne suis pas sủr, etc.; 》 mais il ne faut pas oublier, qu'à ccttc époque, on n'était pas si rigoureux qu'aujourd'luui dans la détermination des inscetes, ni surtout dans la séparation des espèees voisines.

Linné a donc eu raison de eiter Réaumur, pour la figure de la Tenthredo eerasi, malheureusement la phrase deseriptive qu'il en donne est trop courte et peut s'appliqquer à la T'enthréde ducerisier tout aussi bien qu'à la Tenthredo adumbrata décrite plus tard par Klug. Les diagnoses données par Geoffroy, Fabricius, ete., sont tout aussi insuffisantes que celles de Linné et Dcgéer est le premier qui ait donné une earaetéristique suffisante de la Tenthredo cerasi. J'ajouterai enfin que Fabricins, en décrivant eet inseete. (Systema, page 520), eile lcs figurcs 1 ì 5 de Réaumur, au lieu de la figure 6 qui représente la mouche provenant d'une faussc chenille du eerisier. 
C'cst cn 1847 seulemcut, que M. Gorski, dc Wilna, obtint d'cclosion un grand nombre dc Tcuthrc̀des au moyen de larves élevées par lui et nourrics en captivité, de fcuilles dc plusieurs arbres fruitiers. Par la comparaison dc ees inscctcs avec lcs types de Klug couservćs dans la collection du musée de Berliu, il reconnut quc Ic ver limaec qu'il avait élcvé et qu'il rencontrait en abondancc dans son pays, sur les cerisiers, les pruniers, les poiriers ct mêmc sur' les framboisiers et l'anygdalus nana, était la larve dc l'insectc décrit par Klugi, sous lc nom de Tenthredo adumbrata.

En lisant Ic mémoire de M. Gorski, on cst frappé du grand nombrc de points de rcssemblanec qui cxistent entre le ver limaee de nos cuvirons et la larve de la Tenthredo adumbrata. Cepeudant, cn raison des différences suivantes qui existent dans les habitudes de ccs larves, j’hésite eneore à prononeer la eomplète identitć des deux inscctes qua produisent ecs fausscs clienillcs.

1 "Les larves que j’ai pu observer, ont toujours ćté reneontrées sul' des poiriers; et tous lcs renseignements quc j'ai pu recueillir dc nos jardinicrs, confirmcut mes obscrvations. Bien qu'ellcs soieut polyphages, aiusi que me l'unt démontrć lcs édueations faites chez moi, ct que les cerisiers soicnt très-comununs daus plusieurs contrées de nos environs, notamment à Famee, à Koenigsmaeker, à Mćtrich, ctc., lcs larves dc cctte Tentlićdide y sunt complétconent inconnues; $2^{\prime 2}$ jc n'ai jamais observé l'odeur partieulic̀rc de malatc de fer, indiquéc par MM. Gorski et Delacour, conme appartcnaut aux larves qu’ils ont étudiées; $5^{\circ} \mathrm{je}$ n'ai jamais remarqué quc la larve fit, quand on l'inquiète, un moumveunent particulier arce la partic postérieure du corps, et cela, malgré un graud uombre de Icntatives faitcs sur des individus fix és sur les espaliers et sur ceux qui ćtaicnt élevés en eaptivité; $4^{\circ}$ enfin, lor'sque la larve a subi sa dernière mue, et qu'clle est sur lc point de s'cnfermer dans une eoque, sa cou. leur est, comme l'indiquc Degécr, d'un brun clair et jaunátre, mais nou d'un june dor é comme lc dit M. Gorski. D'aillcurs, la forme du cocou, ses dimcnsions, eelle de la larve, quand 
elle y est renfermée, sont parfaitement eonformes à la description de l'entomologiste russe el permetlent de faire eroire à l'identilé des espèees de Metz et de la Lithuanic.

En terminant, j'ajouterai que M. Gorski, sur plusienrs eentaines d'éclosions qu'il a obtenues, n'a pas eu un scul mále, el que deux espèces d'Iehneumouides du gente Tryphon (Tr. Gorskii, Ratzeburg, et Tr. Ralzeburgii, Gorski) vivent, en.Lithuanie, aux dépens des larves de la Tenthredo adumbrala de Klug.

59. TENTHREDO OETHIOPS (Fabr.).

Lepelletier de Saint-Fargeau; Monoyruph. des Tenthrédides, page 112.

Synonynie: Allantus athiops (Jurine); - Nemalus othiops (Spinola); - Selandria athiops (Hartig); - Tenthredo cerasi(Linné); - Celandria cerasi (Blanchard in litteris). - Tenthredo (s. g. Blennocampa) athiops (Klug); — La larve: Ver limace.

Lorgueur de la femelle quatre mill. ; envergure treize mill.; noirc, lisse et luisante; abdomen épais, presque ovale, un peu eomprimé; antennes un peu plus courtes que le eorselet; jambes antéricures avee les genoux d'un brun rougentre; tibias at eòtés interues des tarses de la mème eouleur; ailes obscures, entières, presque de la longueur de l'abdomen. A ectte deseription que je dois, comme la préeédente, à l'obligeanee de M. le doctcur Sichel, j'ajouterai eneore que la T. adumbrata a deux cellules diseoïdales aux ailes postérieures tandis que la $T$. oethiops n'en a jamais qu'une*.

- Pour ne laisser aucun doute dans l'esprit' de ceux qui voudraient délerminer celte espèce, je traduis iei la description donnée par M. Gorski pour la femelle de la $T$. cethiops.

" Corps de forme intermédiairc entre la cylindrique et l'ovale, ne présentant du reste rien de particulier dans la pubescence ou la ponctuation; 
Sclon Bouché, la fcmelle de la Tenthredo othiops pond ses $œ u f s$ à la partic inférieure des feuillcs; ces œufs sont petits, ovales, un peu aplatis en dessus et d'un jaune pále; pondus en aoủt, ils éclosent en septembre.

En nous rapportant au texte et aux planches de Réaumur, nous avons établi plus haut que, selon toute propabilité, cet auteur croyait différentes les Tenthrèdes du poirier de celles du cerisier. Malgré les pctites différences que j’ai signalées entre les larvcs observées á Metz ct cel!cs dont MM. Gorski et Dclacour out écrit l'histoire, on peut admcttre, sans grand cffort, que le vcr limace du poirier est bien Ja Jarve de la Tenthredo adurnbrata. Nous nc devrions donc pas nous occuper plus longtemps de la Tenthredo oxthiops, 'si, en consultant les ouvrages de Ratzeburg et de M. Nordlinger, on n'y voyait le ver limace, qui vit sur les arbrcs fruitiers en général, indiqué comme étant la larve de la Tenthredo axthiops. C'est en effet sous ce uom que, sans aucune

antennés noires, opaques, de la longueur de l'abdomen, sur un individu j'a su un dixième artielo extrèmement pelit. Clypeus légèrement émarginé, bulche noire intćrieurement, extrémités des mandibules brunes extérieurement. Cenchri sans couleur partienlière; pattes antérieures et intermédiaires avec les tibias et l'extrémité des euisses d'un jaune pâle, les tarses de ecs mêmes pattes sont d'une couleur pâle ou d'un jaune rougeảtre vers Ics genoux. Ailes visiblement marquuces de points un peu argentés; le bord et les nervures d'un noir un peu obseur. Les cellules externes sont incolores et la deuxième arcole eubitale est marquée d'un petit point noir."

M. Nordlinger donne la description suivante:

" Allongée, abdomen épais, presque ovale et un peu comprimé; antennes un peu plus courles que l'abdomen. Corselet noir, brillant et légèrement velu; une partie de la bonelıe, le corselet, la plus grande partie des tarses antérieurs d'un jaıne-brun ainsi que les genoux. Ailes enfumẻes avec lo bord d'un brun noir."

Le mảle de cette espèce est également décrit dans la broclure à laquello je fais ces emprunts, mais, pour ne pas trop allonger ces eitations, je reuvois à cel ouvrage los personnes qui voudront approfondir l'étude de ees inseetes, elles y trouvcront d'ailleurs d'autres détails très-intéressants sur leurs mœurs, lours habiludes, etc. 
espèce d'hésitation de lenr part, ces auteurs décrivent la fausse ehe. nille qui ronge l'épiderme des feuilles de poirier. Sans nous occuper des doutes émis par quelques lyyménoptérologistes sur l'identité spéeifique des Tenthredo cerasi de Linné et T. œethiops de Fabricius, je crois qu'il faut nous arrêter, pour la diseuter, a l'opinion des deux entomologistes allemands en contradiction avee M. Gorski. Nous aurons d'autant plus de raison d'en agir ainsi, que la plupart des ouvrages d'lorticulture oit il est question du ver limaec, le rapportent tous au 'Tenthredo cerasi de Linué.

Je coumence par faire observer que, ni liatzeburg, ni 11. Nordlinger, ne disent avoir obtenu la Tcnthredo athiops d'éclosiou et que par conséquent on peut laisser à l'état conjeclural la détermination exacte de l'insecte dont, sous le nom de Tenthredo cerasi, ils out voulu faire l'histoire. Malgiré l'autorité scientifique de ces deux auteurs, on ne siurait se fonder sur leur opiuion pour mettre eu doute les résultats anuoncés par M. Gorski. Ils ont adopté a ce sujet la manière de voir généralement aduise, et voilà tout. Quant à M. Delacour, dans la brocluure qu'il a publiéc, en 1856, au sujct de l'insecte qui nous occupe en ce moncnt, non-seulement il rapporte le ver limace de Degéer à la T'enthredo adumbrata de Klug, mais il ajoute eucore que la Tenthredo oethiops ne vit que sur les rosiers. Si ce dernier fait est exact, la question relative au nom spécifique qui appartient définitivement à la fausse chenille du poirier serait compléteinent résolus; mais je crois bien que lcs choses ne sout pas aussi ayancées que l'aunonce Mr. Delacour, et j'ai tout lieu de croire que la larve observée sur les rosier's par cet auteur, n'est pas celle du Tenthredo athiops. En effet, qu'une larve polyphage, comme l'est le ver limace, quelle que soit d'ailleurs l'espèce à laquelle on la rapporte, vive sur les rosiers, il n'y a lá rien de bien extraordinaire; mais il est bon de remarquer que si les auteurs que j'ai cités ne sont pas d'accord entre cux sur les noms spécifiques des insectes patfaits, il n'en est aucun qui diffère d'une unanière notable, sous le rapport de la descrip- 
tion de la larve. Les différences signalées sont très-légères, et c'est précisément à cette similitude de description que l'on doit attribuer la confusion qui a été comnise par les auteurs que j’ai cilćs plus haut. Or, voici textueliement ce que dit M. Delacour, de la larve qu'il attribue à la 'l'enthredo athiops: «C'est lorsque l'arbre, prêt à ouvrir ses fleurs brillantes, a besoin de toute sa vigueur, qu'on voil les feuilles prendre tout à coup une couleur brun pảle comme si elles avaient élé brúlées par quelques rayous du soleil; en les examinant avec attention, on reconnait que leur face supérieure a élé rongée en tout ou en partie, comme si ellc avait été écorchée, tandis que la face inféricure est tonjours eutière. Il faut beacoup d'altention pour découvrir l'atuteur du dommage, car sa conleur se confond arec celle de la feuille, ce qui l'avait fait échapper à I'attcntion.... C'est une Jarve cylindrique, ayant cnviron treize mil. de longueur, d'un vert jaunatte assez pàle, avee unc ligne plus foncée sur le milieu du dos; la icte cst couleur orange, arec deux pelites taches noircs de chargue cỏté, le segment, l’anus portent aussi une derniérc paire de pattes, eir sorte que leur nombre total s'clère à vingt-deux ${ }^{\star}$.

De ce qui précéde, il résulte incoutestableurent : que l'insecte dont M. Delacour décrit la larve et qu'il ne parait pas d'ailleur's a roir obtenu d'éclosion, n'est pas la Tenthredo aethiops de Fabr.; ou bien, que tous les auteurs qui ont rapporté la Tenthredo aethiops de Fabricius au 'T. Cerasi de Limné, se sont trompés.

Lcs vers limaces décrits par Réaumul, Gorski, etc., ne donnant pas tous des Tenthredo adumbrata, il faut rechercher quelles pcurent être Ics espèces (ju'clles produisent. Car, dit M. Gorski en terminant, « tous ces doutes et toutes ces incertitudes ne peurent étre levées autrement, qu'en élerant en différentes contrées les larres gélatineuses qu'our rencontre sur les cerisiers et les autres drupacées, afiu d'obtenir l'iusecte parfait, et en observant scrupuleusement le nombre des cellules des ailes

- C'est ici le cas de rappeler que Réaumur u’a trouvé que vingl jaubes aux larves dont il parle dans le $3^{e}$ mémoirc du tome $\mathrm{V}$. 
postćrieures, ainsi que la conformatiou des antennes. Ce n'cst que de cette manière qu'on pourra fixer avec ccrtitude le nombre des espèces analogues aux ' $T$. . othiops et $T$. adumbrata, qui ne diffèrent entre elles, d'après mes observations, que par le nounbre différent de ces cellules, nonobstant qu'ils proviennent de larres presque tout à fait les mêmes. »

Pour en finir avec la Tenthredo athiops, je dois encore signaler deux contradictions importantes à rćsoudre : ainsi, Zenker, selon M. Nordlinger, dit que le ver limace du cerisier, avant de sc filer une coque, s'cnroule dans une fcuille; cette assertion cst en contradiction arce tout ce que l'on a dit et obscrvć des vers limaces, el j'ajoute, qu'elle est même peu admissible, pour une espèce roisine, ear on ne connait aucune Tenthrédide s'enroulant dans une fcuille pour se transformer. D'autre part M. Nordlinger dit qu'après aroir subi sa dernière muc, elle s'enfonce dans la terre et que là clle se file une coque en forme de tonneau. 01, MI. Gorski dit positivement qu'il n'a pu découvirir la filièrc de la larve de la $T$. adumbrata, et nous avons vu plus haut quelle est la forme ct la contexture des cocons formés par les vers limaces ćlevés par moi.

De tout ce qui précèdc, il résulte quc très-probablcment la Tenthredo cerasi, de Linné, est unc cspèce collective et qu'il arrive dans le genre Tcuthredo, commc dans beaucoup d'autres genres d'insectes, que plusicurs espèces roisines oni des larves presqu'identiques, ou qui ne diffèrent que par des caracteres difficilcs à saisir. C'est dans le but d'arriver à la solution de toutes ces questions, que je me propose de continuer mes tentatives sur des larves élevées par moi, afin d'en obtenir les insectes parfaits, et que j'invite tous les entomologistes qui s'occupent des inseetcs nuisibles, à tn'aider de lcur's conseils et de Icur expéricnee pour attcindre ce résultat. 


\section{CEPHUS (Fabr.)}

Lepellelier de Saint-Fargeau; Monogr. des Tenthr, prge 18.

Corps court, groilc et comprime; mandibules longues, aplaties, tridentées, la dent du milicu assez petite; palpes maxillaires de six articles; antennes de vingt et un articles, un peu plus grosses à l'extrémité quäa la base, le premier article distinct, les trois suivants un peu plus longs, tous les autres tris-petits; ailes avec deux cellules radiales, la première presque quadrangulaire, la scconde grande; quatre cellules cubitales, la deuxieme avec une nervure recurrente, la quatrième atteignant l'extrénité de l'ailc; abdomen sessile, intimement uni au thorax, comprimé; tarière courte, à peine saillante. Pattes courtes ave les quatre tibias postéricurs bi-épineux au milicu.

Les Cèphes sont de petits insectes lyyménoptères rcmarquables par la longuenr proportionnelle de leurs antennes. Les fenelles sont pourvues d'une tarière en forme de scie, avec laquello clles introduisent leurs $x u f s$ dans les tiges des végétaux.

Les larves qui en proviennent sont molles, génèralement de couleur de chair, elles ont six pattes éeailleuses ct seulement deux unamelous sur le dernier anıeau; ee qui leur domne une physionomie tout à fait différente de celle des genres voisins. Leur corps est aminci postérieurement; elles vivent dans l'intéricur des tiges de divcrses plantes, dont elles mangenı lc tissus cellulaire qui forme le canal mćdullaire.

On comuait une quinzaine d'espéccs du genre Cèphes, unc est du nord de l'Afrique, les autres sont d'Europe ct beaucoup font partie de la Faune franģaise. Une d'elle, le Cephus pygmous

* Synonymie: Sinex (Linnè); - Bakchus (Panzer); - Tenthredo (Kourcroy); - Astatus (Klug); - TaAchelus (Jurine) : - CÈphe; -- Нal\$WESIE. 
(Fab.) vit à l'élat de larve dans l'intérieur des liges du blé, einpéche leur développement et produil très-sourent un déficit considérable dans la récolıe. Unc autre vil sur le poirier, e'esı le:

60. CEPKUS compRESSUS (Fabr.).

Lepolletier de Sainl-Fargeau; Mongr. Tenthred., lage 18.

Synonymic: Trachelus Compressus (Fab.);-Sirex Compressus (Coqueb); - Tenthreclo prolongala (Fourer);-Cephus abdominalis (Latr.)??

Màle: Longueur lınit millim. T'ète et antennes noires; mandibules el palpes testacées; corselet noil avee une petite ligne transversate en avaut et quelques taches en arricie d'un jaunc clair; ailes diaphanes, légèrement irisécs el enfuncés; tontes les pattes d'un beau jaune testacé dail avec les tarses antéricurs et internédiaires plus fonećs et les postéricurs roussitres; abdomen ferrugineux ave; le premier anneau plus ou moins noil en dessus.

Femelle : Longrueur huit millim. 'T'ite et antennes noires; mandibules et palpes d'un lestacé ferrugineux; corselet noir" avec une tache triangulaire, dont la pointe est dirigice el avant, jaune sur le derricre; ailes lortement irisées, diaphanes el enfumées; pattes noires, les antélieures ayant les jambes et les tarses d'un testacé ferrugineux elair; les palles postéricures ont les cuisses noires, la moilié des tibias testacec et le reste: de la jambe, y compris le tarse, d'une couleur obseure; toutes les euisses ont en ontre une tache blanchitte a la base; abdomen ferrugineux, avec la base ct l'extrémitć noires.

Celle description esı faite sur deux insecles que je dois al'obligeance de M. Sichel, de l'aris; comme on le roil, les deux sexes sont très-différents l'un de l'autre. Lepellelier de Saint-Jíngeau (Loc, cit.) a dil, d'apris Jurine, que le mále esı semblable à 
la femelle; le naturaliste de Genève arait donc commis une erreur qu'il importait de rectifier, puisqu'elle a ćté bien sourent reproduite depuis.

Dans la première partie de cet ouvrage (pagc 65), en parlant du Rhynchiles Cuprœus (Gyll), je disais que. les œufs pondus sur les jeunes pousses de poirier et qui étaient disposés en spirale, ne me paraissaien! pas appartenir, ainsi que Ml. Nordlinger le croyait, à eet insecte Dès la fin de 1857, en effel, M. le colonel Goureau m'annonçait qu'il avait découvert le véritable auteur de ees trous disposés en spirale, cl que c'était un insecte hyménoptère de la famille des Tenthrédides, dont il avait suivi les métainorphoses et dont il me donnerait le nónt aussitót que l'insecte parfait sortirait des larves qu'il avait élcvées.

Depuis lors, dans le Bullctin de la Socićtć entomologique de France, page ccxxxı. M. Goureau a publié le nom de cet insecte, qui est le Cephus Prgmous (F.) et sur lequcl il donne déjà quelques délails. En 1859, mon honorable correspondant a eomplété ses observations, et aujourd'hui, grảce à son extrême obligeance, je puis compléter l'histoire du Cepluts Pygmous.

«Il n'est pas rare, m'écrit MI. Gourcau, de remarquer dans un jardin, pendant le printemps et l'été, des jeunes pousses de poirier qui se flétrissent gradnellement, qui se desséelıent, noir. eissent el meurent. Si on les examine avec altention, on $y$ observe de petites piqủrcs noires, également cspacćes, disposées cn ligne spirale autour de la branche dout elles font une ou deux fois le tour. La petite branche cst un peu renflée dans la partie blessée, et se casse assez facilentent en ee point. lin fendant par Ic inilieu, daus le mois de juillet ou d'aouit, eette pousse noircíe, on tronve à son centre une larve blanche qui cna rongé le coeur, laquelie s'avance en galeries, vers le point d'où part la pousse, el qui laisse derrice elle une masse de ponssiere noirâtre, formée de scs exeréments et des débris du bois qu'elle a rongés. Parvenue à toute sa taille, en septembre, elle s'cufermc dans un léger coeon de soie blanche, à l'extrémité de sa galerie. pour y passer l'hiver et altendre le moment de sa métamor- 
phose en chrysalide et en insecte parfait, ce qui ne doit avoir lieu qu'au printemps suivant. L'insecte parfait ouvre un trou rond dans la paroi de sa prison et s'échappe à la fin de unai. Cet insecte nuisible au poirier, est une Tenthrédide du genre Cephus, qui se rapporte au Cephus Compressus de St-Fargeau.

„La femelle du Cephus pond ses oufs au mois de mai, sur les bourgeons du poirier, un seul ocuf sur chaque hourgeon; à cet effet, elle percc avee sa tarière l'écorce de la pousse très-tendre, et y fait uue série de pirquires également espacées, disposées en ligne spirale faisant une ou deux fois le tour du bourgcon. Fille ne ponıl qu'un seul $\propto u f$ qu'elle laissc dans la dernière piqûre, c'est du moins ee que je suppose; après quoi elle passe à unc autrc branche où ellc exécute la méme opération, puis à une troisième el ainsi de suite tant qu'elle a des œufs à pondre. La sève ne circule plus librement au-dessus de la partie blessée qui se fanne, sc dessèche et noircit; elle s'accumule dans la région des plaies qui se gonflent et. se tuméfient un pcu. L'œuf éclot dans les premiers jours de juin, la petite larve ronge la moelle du bourgeon et s'avance cu marchant vcrs sa base, sa galerie s'élargit à mesure qu'elle croit, parvenuc à la base du bourgeon son accroissement est aecoinpli.

* Cctte larve n'a aucune ressemblance avcc cclle de T'enthrédides, inais clle se rapproche de celle de Urocérides, ce ipui indique quc Linné avait placé, avec un tact exquis, dans le genre Sirex, devenu la famille des Urocérides, le Cephus pygmœus, la seule espèce qu'il connút. 》... * Arrivée à l'état adulte, la larve s'enveloppe dans un cocon où elle passe l'hiver et se transformc cn chrysalide vers le 18 mai; l'insccte parfait s'envole, vers la fin de mai pour reproduire son espèce, il sort par un trou qu'il a pratiqué avec ses mandibules à la paroi de sa cellulc. »...« Lorsque la larve du Ceph. compressus a alleint toute sa taille, clle a six mill. de long, sa tête est roude, blanclıc, luisante; ellc est arncée dc deux fortes mandibules brunes; d'un labrc de la mênuc coulcur; de deux màchoires et d'une lèvre qui se manifestcnt par les pointes qui les tcrminent; on $y$ distinguc deux très-petites 
antennes et deux points oculaires bruns. 'Tout le corps est blanc, arrondi, courbé en $\mathbf{S}$, les trois segments thoraciques sont plus gros que les autres et s'élèvent au-dessus de la tềte, ce qui donne a la larve une apparence bossue; ils portent au-dessous trois paires de très-petites pattes. Les aulres segments sont à peu près de même grosseur et privés de pattes; le dernier uu peu plus grand que les précédents, relevé en dessus et terniné par un petit appendice eaudal brun, corné, granuleux, eouvert de poils sortant des granules. On distingue nne ligne dorsale qui aboutit près de l'appendiee. \... \& Celte larve, pendant sa vie, est exposie à l'atteinte d'un parasite qui la blesse à travers les parois de son logement et laisse un ouf dans son corps. Lorsque la larve du parasite a dévoré celle du Cephus, elle se ehange en ehrysalide dans la cellule de cette dernière, puis en un insecte parfait qui peree un trou daus le bourgeon pour s'échapper dans la eampagne. Ce parasite a paru chez moi (à Sautigny, Yonne), le 14 aoùt 1857, et sortait de petiles branches de poirier qui u'ont donné leur Ceplıus que le 51 mai 1858. C'esı un Jehueumonien du genre Pimpla qui m'a paru se rapporter au $\boldsymbol{P}$. stercorator de Grav.

¿ Le moyen de combattre le Cephus compressus est simple et évident. Il faut eulever toutes les jeunes pousses, flétries ou noireies en les coupant eontre la tige ou la branche qui leur sert de base et les brüler.

Je n'ai pas encore pu rencontrer ect insecte dans le département de la Moselle; mais, comme mes reeherehes ne datent que de cette année, je conserve l'espoir" de l'y trouver plus tard et d'y suivre l'histoire de ses métamorphoses.

XXXIX. IYDA (Fabr.)*.

Lepelletier de Saint-Fargeau; Monog. Tenthrédides, page 4.

Corps épais, court; mandibules grandes, bidentées et aplaties; palpes maxillaires de six artieles; antennes sélarées

- Synonymie : Pasphilius (Latr.); - Ce.phaleia (Jur.); - 'Jenthrado (lin.). 
de 18 a 36 articles petits, le premier et le troisiéme phas longs que les autres; ailes ayant deux ecllules radiales, la premiere eireulaire, la seconde allongce; quatre cellules cubitales presque égales, la deuxieme recerant la premiére nervure récurrente, la troisième cellule recevant la sceonde, et la quatrieme ecllule n'atteignant pas le bord de l'aile; pattes cotrres avec les quatre tibias postérieurs tridentés au milieu; abdomen sessile complétement uni au thorax.

Les insectes qui appartiennent au genre Lyda, se reneontrent dans toules les parties du monde, plusieurs se irouvent dans uolre pays. Leurs larves, assez. allongées, snnt dépourrues de patles membraneuses, ce qui fail qu'elles ne marchent que trèsdifficilement et par une sorte de reptation. Pourvues d'une filière eomme les chenilles, elles se suspendent sourent par un fil pour changer de place; quelques-unes se filent des snrtes de luyaux d'nn tissu très-láche el dans lequel elles progresseut en contractant les anneaux du corps.

Les femelles sont pourvues d'une tarière, ordinairement sail lante, ct arec laquelle elles introduisem leurs œufs dans le tissu des végétaux dont les larves se nourrissent. Celles-ci vivent sonvem cn socićtés très-nombreuscs; $\mathrm{ct}$, comme elles sont très-roraces, elles occasionnent quelquefois des dégàts aussi considérables que les chenilles de certaines espèers de papillons des genres Orgya, Liparis, etc. Ces' lar'es rémuissenl souvent un grand nombre de feuilles en paquet et vivent au milieu de ce nid commun; d'autres fois, elles enveloppent de lenrs fils l'extrémilé d'un rameau et vivent a milieu de ecte toile snit en commun, soit d'une manière isolće en se filant ehacune un tuyau soyeux propre.

C'est le plus ordinairement sur les coniféres que l'on rencontre les diverses espèces de Lyda; cependant plusicurs autres arbres en nourrissen aussi qui leur sont propres, les autcurs en signalent deux espèces sur le poiricr. 
63. ITDA PIRI (Schranck)

Synonymie : Lyda Clypeata (Klug); - Lydda flaviventris, (Fallen non Relz). Birnblallwespe, des allemands. Tenthredo IInemorrhoidalis? (Sclımidtberger).

Longueur de onze ì douze millimètres; envergure de vingt a vingt-quatre millimètres, pour Ies deux sexes. Anternes d'un gris noiràtre et sale avee la base jaune; une taehe jaune eordiforme sur le front de la fenelle; parties de la bonelie également jaunes; corselet profoudément et grossièrement ponetuć; jambes de devant et eelles du milicu ainsi que les hanehes et un anneau bien marqués en noir; abdonen d'un noir blettitre avee le bord visiblement denté en dessous.

Dans le mâle, tout le devant de la tète est noir comme le reste du eorps ; l'abdomen est d'un jaune brun avee une taelre obseure.

Cette espèee, dont j'emprunte l'histoire à M. Nordlinger, se trouve daus toute l'Europe, depuis la Suéde jusqu'en Autrielie. Elle est polyplage et se reneontre sur un très-grand nombre d'arbres très-différents. Dans les jardins eependaut, e'est ordinairement sur les poiriers, espaliers, quenouilles ou haut-vents, qu'on reneontre sa larve. C'est le plus souvent en mai qu'apparait l'inseete parfait; mais dans les années tardires, on en troure eneore dans le milieu d: mois de juin. La femelle pond de 40 à 60 aufs, allongés, jaunes et 'assez gros. Ces oufs sont rangés en lignes sur la faee inféricure des feuilles et reeouverıs d'un enduit glutineux. Quelques jours après la ponte, on

- Dans la Monogruphic des T'enthredides de Lepelletier de Saint-Fargeau, un ne trouve pas de Lyda Pyri. A la table, on trouve Lyda Clypeala Klıg, avec le no 21. Mais dans le texte, le no 21 donne Lyda Circumcincte de Klug, espèce américaine, dont la description n'est pas du tout applicable à la Lyda Clypeata dont il est ici question, et qui ne figure pas dans la partic descriptive de l'ouvrage. 
aperçoit de petites larves d'un blane jaunâtre. Elles ne tardent pas à changer de peau, quoiqu'en dise Schmidtberger. Bientót après leur sortic de l'œuf, es petites larves commencent à filer une toile qui embrasse une eertaine longueur de la branche et qui ressenbble assez à celle que filent, aussi en comınun, les chenilles de la Tinea cognatella. Dans ces toilcs, on reneontre un nombre variable de fausses elienilles, souvent huit ou dix, quelquefois plus de vingt.

A mesure que les feuilles comprises dans leur toile se consomment, elles allongent leur réseau pour en prendre de nouvelles. Bien qu'elles soient très-voraces, il ne faut cependant pos prendre à la lettre ce que dit Locw, que 30 ou 40 de ces larves suffisent pour dépouiller, en quelques jours, un arbre de toutes ses feuilles. Pendant les instants de repos, elles se séparent et se suspendent, au inoyen de leurs pattos, aux fils qui eomposent la toile eomnune; ou, si on les inqquiète, elles sortent du nid et restent au dehor's suspendues à un fil jusqu'à ce que le danger soit passé uu que le besoin de nourriture se fasse sentir. $\Lambda$ près quatre à cinq semaines (vers le commencement d'août, à Metz, en 1859), les larves arrivent à l'état adulte; elles ont alors de 20 à 24 mill. de longucur, sont d'une couleur jaune d'œuf (celles que j'ai observées étaient de couleur mains foncée), arce la tête noire, luisante et une plaque cornée sur le premicr anneau. Les antennes sont noires et assez longues pour une larve, le dernier anneau a en outre deux petits cornicules très-visibles. Les trois premiers segments du corps portent chacun deux pattes eoniques et ćeailleuses analogues à celles des chenilles mais qui paraissent presque inutiles au mouvement. Les autres fausses pattes qui se reneontrent sur un grand nombre de larves de la famille des Tenthrélides, font également défaut dans le gemre Lyda.

Quand les fausses chenilles de Lyda ont vécu sur des arbres de petite taille, leur nid deseend souvent jusqu'à une faible distanee du sol; alors elles filent un tuyau dans lequel elles rampent pour gagner ainsi la terre dans laquelle elles s'enfoneent de 8 a 10 cen. timètres et où elles se construisent une coque soyeuse pour y passer 
I'hiver. Elles ne se transforment en chrysalide que peu de temps avant d'apparaitre à l'état d'insecte parfait, ee qui a licu au mois de mai suivant. Quand, au eontraire, l'arbre est élevé, la fausse ehenille gagne le sol à la manière de beaucoup de elıenilles fileuses, e'est-à-dire en se suspendant à un brin qu'elle laisse filer jusqu'i ee qu'elle ait atteint le lieu dans lequel elle doit subir sa dernière métamorplıose. C'est, je erois, le eas- le plus ordinaire et du reste le seul que j'aie pu cunstater.

Souvent, quand les colonies sont nombreuses, elles étendent: leur toile sur de nouvelles parties de l'arbre; et ee n'est pas le besoin de nouvelles feuilles qui les fait proeéder à ectte extension, tnais le but d'éviter l'encombrement et les erottes qui restent attaeliées anx nombreux fils forinant la trame du réseau génćral. Quelquefois aussi le déplaecnent est déterminé par les dérangements qu'on leur fait supporter et qui paraissent leur être plus importuns qu'aux autres espèces de ehenilles qui vivent en so eiété. A ussi ees larvesproduisent-elles des toiles d'une très-grande dimension et peu en rapport aree leur nombre ou leur besoin, ce qui fait paraitre leur présenee plus nuisible qu'elle nę l'est en réalitć. C'est trìs-probablenent eela qui a fait dire à Loew que 30 ou 40 de ees fausses-ehrenilles peuvent dépouiller un. arbre en quelques jours.

D'après Sehnidıberger plusieurs parasites vivent aux dépens de la larve de la Lyda pyri, notamment la larve de l'Oplaion mercator, Grav. D'autre parl, le temps eonsidérable que ees larves passent dans la terre sans s'y transformer en nymphes, les. expose aussi à des alternatives d'lumidité et de sćeheresse, aux gelées, ete., ee qui doit en faire périr un grand nombre. C'est, sans aueun doute, à ees diverses eireonstances que l'on doit attribuer ee fail, heureux du reste, que très-abondantes une année, elles sont souvent en si petit nombre l'anuće suivante qu'on . a de la peine à ell reneontrer quelques-unes.

Je n'ai pas eneore reneontré la Lyda pyri dans les envirous de Metz, bien que eelte anuée j’aie trouvé sur un jeune eerisier, plusienrs larves vivant ensemble dans une toile eommune. Je 
n'ai pu conduire ces fausses-chenilles au delà du commencement de leur cocon, et cepcndant leur description se rapporte tellement (sauf la couleur) à cellcs qui sont décrites par Ratzeburg, Nordlinger et Macquart, que je n'hísitc pas à rapporter ces larves à l'espècc indiquée par ces auteurs sous le nom de $L y d a$ pyri.

\section{IXDA BXIVATXCA (Fabr.).}

Lepelletier de Saint-Fargeau; Monogr. Tenthr., page 9.

Synonymie: Tenthredo Sylvatica (Lin.);-Pamplitius sylvalicus (Latr.); - Cephaleia nemorum (Panzer); - Cephaleia Sylvalica (Lat1.); - Tenthredo fulvipes (Rets);-Lyda nemorum (Fabr.); - Wald Blaltwespe, en allentand.

Femelle : Antennes jaunes avee le premier artiele noir en dessous; tète noire; mandibules testacées ainsi que les palpes; une taehe jaunátre sur le derricire de la tćte; eorselet noir avee l'ćeusson et un point jaune à l'insertion des ailes; abdomen noir; pattes jaunes avee les hanches et la bose des euisses noires ; ailes diapltanes.

Måle : Se distingue de la femelle par le bord du eltaperon, qui est jaune, ainsi qu'une ligne transversale interrompuc en avant des yeux.

Longucur des deux sexes de dix à douze mill.

La Lyda sylvatica se rencontre à l'état parfait dans le département dc la Moselle oú ellc parait mème (dans nos cnvirons) êtrc la plus commune du genrc. Selon Ratzeburg, sa larve vit sur le chêne et n'est pas de la même coulcur que celle de la Iyda pyri, dont nous venons de lirc l'histoirc. M. Nordlinger n'cn parle pas non plus, ce qui mc fait supposcr qu'il ne l'a jamais observíe sur les arbres fruitiors. Macquart gardc le même silence. M. Goureau n'a jamais trouvé sur les poiriers qu'une larve dont il me donne Ia description et qui est bien évidemment cclle de la $L y d a$ pyri.

M. Blanchard est à ma connaissancc lc seul auteur qui indique 
la larve de la $L y d a$ sylvatica eoınme vivant sur le poirier; voiei du reste ee qu'il en dit : “ "On a déerit plusieurs larves de Lydas; quelques-unes d'entre elles vivent sur le poirier; telle est la Lyda des forêts (Lyda sylvalica), l'une des espèees les plus eommuues en Europe; elle est noire avee les antennes et les pattes jaunàires, exeepté leur base; sa larve est jaune avec la têtc noir. \Cette diagnose eonvient bien a l'insecle parfait, mais ee qui est relatif à la larve, semble plulót se rapporier á eelle de la Lyila pyri. Selon M. le docteur. Sielıel, dont on ne saurait mettre en doute l'autorité en cette matière, la $L y d a$ hortorum est la seule espéce du genre Lyda qui se reneontre dans les jardins des environs de Paris. Il est done permis de supposer que c'est de eette dernière espèet, dont la larve est également jauue, que M. Blaneliard a voulu parler.

In terminant ee qui est relatif aux espèees de $L y d a$ qui vivent sur les poiriers, je dois dire que l'éelıenillage, e'est-à-dire l'eulèvement et la destruelion des nids, est le seul moyen efficace pour se débarrasser de ees inseetes. ?

\section{FORMrioA (Iinné).}

Lepellctier de Saint-Fageau; Suites à Buffon, tome 1, page 199.

Tc̉te assez grande proportionnellement; mandibulcs triangulaires, très-fortement dentècş; antennes insérécs sur le front, très-mobiles, coudées après le premier article, eclui-ci presqu'aussi long que la moitié de tous les attres réunis. Corps ètroit; abdomen pédiculé, de forme ovalaire, ayant son premier segment en forme de noend; pattes de moyenne grandeur.

Les måles sont toujours ailés; les ailes ont une cellule radiale et deux eellules eubitales dont la scconde n'atteint pas l'extrẻmité; l'abdomen est armé d'un aiguillon à sou extrénitic.

- Histoire des Insectes. E. Blanchard. Paris, 1845, tome 1, page 190. 
Les femelles sont aussi ailées, mais elles perdent souvent leurs ailes apres l'aceouplement; leurs antennes vont visiblement en grossissant, ee qui permet de les distinguer des måles, quand elles ont des ailes, et des neutres, quand elles les ont perdues.

Les Neutres ont le labre grand et corné, et sont toujours aptères.

Les fourmis sont des insectes bien connus de tout le inonde; ce sont les individus privés d'ailes que l'on rencontre le plus ordinairement; on les désigne sous les noms d'ouvrières et de neutres, bien qu'il soil cependant parfaitement démontré que ec sont des femelles ehez lesquelles les ailes et les organes de la génération ont avorté. L'histoire des fourmis a été faite par Iluber au sièele dernier, et depuis elle a été complétée par Lepelletier de Saint-Fargeau. Bicn que ees obserrateurs aient fait connaitre une foule de particularitćs fort intéressantes et généralement peu connues, je ne n'y artèterai pas, en raison du peu d'importance qu'out ees petits inseetes relativement à l'arbre dont je déeris la faune entomologique; je me bornerai done, non a les rélıbiliter complétement, mais à détruire à leur égrard plusir.ırs préjugés très-r'épandus*.

- Voiei quelques passages do l'ouvrage de Lepelletier de Saint-Fargeau, eité plus liaut et dans lesquels l'auteur entreprend la défense des fourmis:

Page 164. "Il est eertain que les fourmis détruisent partout beaucoup d'insectes et d'autres animaux nuisibles. Mais la petilesse des espèces de notre pays nous empêche de remarquer do quelle utilité elles sont sous ee rapport. En ramassant la liqueur suerée que rejettont les pueerons el les gallinsectes, elles rendent un éıninent service aux végétaux; ear lorsque la miellée, nom qu'on donne assez généralement à celle liquour, tombo sur les feuilles, elle en bouehe les pores, et alors, à moins qu'une pluie bienfoisante ne vienne les laver, ees feuilles dépérissent et tombent bientôt. Il arrive eneore que, moyennant eet enduit collant, la poussière se fixe sur les feuilles, voile leur verdure et bonche leurs pores, ee qui produit un effet désagréable à nos yeux et empéelıc les feuilles de reevoir de l'atmo- 
Les fourmis vivent cn société fort nombreuses et composées de trois sortcs d'individus: $1^{\circ}$ de màles qui sont ailés et qui ne paraissent avoir d'autres fonctions que celle dc féeonder les femelles; ils sont retenus en captivité jusqu'à la sortie de celles-ci et meurent pendant ou immédiatemcnt après l'accouplement.

sphère les principes qui constituent l'espèce de sève qui sert particulièrement a la nourriture des fruits. "

Pages 166 et 167 . a Les acides ayant la propriẻtẻ de crisper, et les parties des végétaux oủ se rendeut, pour leur réeolte, nos fournis, étant souvent erispées, on a accusé de ces déformations l'acide formique et par conséquent nos inseetes. De la nos jardiniers, qui voient les feuilles el les jeunes branehes de lcurs arbres fruitiers, ou même d'agrément, rabougries et contournèes, eherehent à détruire leurs retraites. Nous pouvons cependant assurer que ees aecidents ne sont causés que par les piqquires réitérées des gallinseetes et des puccrons. Ce fait paraîtra elair à tous ceux qui, n'examinant pas superfieiellement, trouveront beaueoup de branches contournées, de feuilles rabougries, plissées ou eloquetées, sans que les fourmis y soient parvenues. La seulc inspection des branches oi se tiennent les pucerons et les gallinseetes prouve suffisanment, dinsi que nous l'avons observé souvent nous-même, ee que dit Réaumur à propos des figures 2 et 3 de la 230 planche, 90 mémoire, pages 29.1 et 295 , tome 3.

"Comme la tige en eroissant, tend à s'èlever, et que les pucerons qui la " suivent jusque dans sa plus tendre extrèmitè font perdre au côtè contre le"quel ils sont appliqués, beaucoup de suc nourricier, les courbures que prend " successivement eettc tige doivent faire, par la suito, différents tours arrangés " i peu près comme ceux d'un tirebourre. "On voit que cet observateur n'attribue la déformation des végétaux dont il s'agit qu'aux inseetes qui en sucent la sève. Si l'acidc formique, ct par conséquent la prèsenee des fourmis qui l'exhalent continuellement, pouvaierıt la causer, combien de parties de mème nature des mêmes végélaux sont elles parcourues par un nombre considérable de fourmis, sans çtre déformées, Iorsqu'elles vont visiter les pucerons! On voil même, dans le sol des fourmilières, des végétaux qui sout eertainement là dans une atınosphère saturée d'acide formique, et qui cependant n'ẻprouvent aueune déformation. C'est done à tort que les fourmis sont accusées de nuire par leur acide aux végétaux. „.... " Je serais aussi satisfait s'il m'étail possible de disculper de tout reproehe, ees insectes si renırquables par leur industric, par leur union sociale la plus perfectionnce qui se trouve dans les insectes, et par leur esprit de comparaison, ctc." 
$2^{3}$ de femelles, dont tout le soin eonsiste à pondre des oufs en trc̀s-grande abondance, de inanière à augmenter continuelle. ment la population de la petite république; ees femelles sont également retenues en captivité après leur fécondation; $\mathrm{et}$, souvent pour assurer plus complétement leur dépendanee, les neutres leur arrachent les ailes. C'est d'ailleurs toujours dans les parties les moins aecessibles de la fourmilière que ees femelles sont obligées de pondre. Leur taille est toujours plus forte que eelle des neutres, et souvent aussi que celle des måles. La troisièue sorte de fourmis qui, ainsi que je l'ai déjả dit, u'est conposéc que de femelles aptères et infécondes, est toujours fort nombreuse. Ce sont ees neulres on esclaves qui procurent la nourriture aux femelles fécondécs, qui coustruisent la foumilière, qui preunent soin des œufs, des larres, des nymphes ct mẻme des fourmis nouvellement ćeloses; ee sont eneore les ueuttes qui retiennent les mâles en raptivité, qui nourrissent les autres ouvrières restées à la unaison, et cnfin qui amassent les provisions d'lliver.

Les fourmis établissent leurs nids dans la terre, dans le ercux des arbres, sous les ćcorees, au pied des vieux murs, entre les racines d'un grand nombre de plantes, ete. Sourent ees nids sont surmontés d'un anas plus ou moins considérable de débris de toutes sortes où le bois et la terre forment eependant la majeure partie. Ces débris de bois provienneut le plus ordinaireınent des arbres qui, déjả endommagés par d'autres iuseetes, ou par des aeeidents, ont les bords de la platic ramollis par l'effet de l'air el de l'humidité. Le bois ainsi plus ou moins décomposé, se laissant faeilement eutamer, est déehiré par les fourmis et transporté en forme de grains de seiure dans l'labitation coumune. Ce genre de dommage, causé par les fourmis, est souvent très-considérable et amène en quelques années, la perte d'un arbre déjà perforé par quelques grosses larves, ou aceidentellcment blessé. On devra donc boucher avee soin, au moyen de mastic à gleffer, de poix, de platre ou de mortier, selon leur importanec et leur étendue, tons les trous que l'on pourra reuar. quer sur les arbres fruitiers ou autres. 
Dans un grand nombre de eas, les fourmis qui labitent les jardins, viennent établir leurs nids aux pieds des murs, sous les racines des espaliers, qu'elles inellent ainsi à décourert, en déeoupent les radieclles, ete., et leur eausent en définitive un très-grand préjudice; on doit alors se hàter de détruire la fourmilière, au moyen de ehaux vive, d'eau bouillante ou d'eau benzinée, et non se eontenter, comme on le fait trop souvent, de la bouleverser; ear, dlans ee cas, les industrieuses fourmis ont bientoot réparé le désastre et remis les elıoses sur l'aneieu pied.

Ainsi que je l'ai déja dit, les larvés des fourmis sont apodes et iueapables de pourvoir ì leur nourriture; ce sont les individus neutres qui sont elıargés de ee soin et obligés d'aller au dehors elıercher le liquide sueré qu'ils viennent ensuite leur offrir en le ramenant de l'estomae dans la bouehe où la larve va le puiser, reeerant ainsi une sorle de becquic. Ce liqquide sueré dont se compose exclusivement, il parait, la nourriture des jeunes fourmis et de leurs larves, est emprunté aux plantes et plus partieulièrement à la sève qui déeoule de quelques plaies, a celle qui tuméfie ou liumeete les jeunes bourgeons, au suere que eertaines fleurs renferment dans leur ealice et enfin dans le parenelyme des fruits sueeulents comme les prunes, les poires arrivées à leur maturité, ete. Il est done naturel, d'après ee qui préec̀de, de supposer que les four'nis ne parcourent constamment les diverses parties des arbres ou des plantes que pour y rechereher l'aliment sucré dont il vient d'ètre question. Cependant, d'après les observations de Dalbret, Lepère, Hardy, ete., il est hors de doute que ees animaux eutament souvent les jeunes bourgeons pour en faire conler la sève. C'est

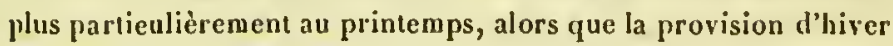
est épuisće et qu'elles n'ont pas encore d'autres endroits oủ elles peuvent aller ehereher la páture des habitants de la fourmilière, que les fourmis allaquent les boutons à fleurs ou i bois. A l'autoune, au eontraire, elles altaqueront les fruits murs, parce que, à eette saison, la sc̀ve leur fait défaut et qu'il n'y a plus pour elles d'autres ressources que eelle-là. Voilà les seuls et mais 
dommages dircets que peurent nous occasionner ees insectes, et si, en récoltant les fruits à temps, on peut en quelque sorte les sauver de la dent des fourmis, il est loin d'en être ainsi, en cc qui concerne les jeunes bourgeons dont un très-grand nombre sont souvent détruits ou déformés pat les mandibules de ccs insectes. On devra done, dès les premiers beaux jours, placer sur les arbres à préserver, de petites fioles à moitić remplies d'eau iniellic ou sueríe et dans lesquelles un grand nombre de fourmis iront se noyer. Ce unoyen, très-commode et peu dispendieux, est génćralement employé, mais il l'est tonjours un peu tard, alors qu'il u'y a plus que les fruits á préserver. En le mettanten pratique dès le printemps, on garantira lcs bourgeons ct on empêchera les fourmilières de prendie de trop grands développcments.

Bien que la plupart des anteurs aient démontré depuis longtemps, quc ce ne sont pas les fourmis qui amènent les pucerons sur les végétaux, il y a cncorc un très-grand nombre de jardiniers qui les accusent de ce fait.

On sait, en effet, que les pucerons et les cochenilles vivent de Ia sève des plantes sur lesquelles elles se fixent en cnfonçant leur trompe dans les couclies corticales, soit d'une manière permanente comme le font les eochenilles à certain àge, soit d'une manic̀re intermittente comme le font les pueerons; la sève, attirće par la suecion, s'épanche souvent en delıors par les bords de la plaie, par celle-ci, quand l'insecte retire sa trompe, ou par les cornicules des pucerons, et c'est là que les fourmis vont ehereher leur principale provision. C'est aussi pour se la procurer plus facilement ou plus abondanment, qu'on les voit constamment róder autour des parasites que nous avons indiqués, les toncher de Icurs autemnes pour les exciter à lảcher le liquide sucré qu'ils tiennent en réscive, ou pour lenr faire abandomer Icur place afin de pomper la sève à l'orilice du tron formé par la trompe. Tous ces faits sont depuis longtemps liors de doute, et on a lien de s'étonner de voir eneore un aussi grand nombre de jardiniers accuser Ies fourmis de l'invasion des pucerons ou des coclic- 
nilles, tandis que ec sont au contraire ees parasites qui les attirent.

En résumé les fourmis ne sont pas aussi coupables qu'on le croit en général, car, dans une foule de circonstances, elles nous sont, au contraire, trèsutiles; quoique très-avides de choses sucríes, elles n'en font pas moins la guerie à beaucoup d'insectes et particulièrement aux jeunes chenilles qu'elles transportent dans leurs magasins poui subsister quand la bise sera venue. 0 n a dit aussi (mais je n'ai pu vérifier ce fait indiqué, pour la première fois, par ILuber) que souvent, afin d'avoir constamment i leur portée l'élément liquide et sucré qui transcude des cornicules des pucerons, les fourmis transportent vivants ees homoplères dans leurs fourmilières; si ec fait est vrai, ec dont je doute fort, les fournis diminuant ainsi le nombre des pucerons, nous rendent alors de véritables services et c'est alors que, suivant la pittoresque expression de Linnéc, on peut dire que ceux-ci sont les vaches laitičres des fourmis.

Le noinbrc des espèces du genrc Fourmi est assez considérable et on en trouve des représentants dans loutes les parties du monde. Comme presque toujours les espèces méridionales et exoliques soul plus grandes que les nótres. On peut estimer à une dizaine d'espèces, celles qui font partic de la Faune de la Mosclle, et par conséquent eelles auxquelles on peui appliquer les généralités qui préec̀dent, et aussi les moyens de destruction dont nous allons maintenant uous occuper.

Quand il s'agira d'un uid de fournis, les moyens précédemment indiqués pourront être mis en nsage pour les fourmilières ćlablies dans les jarlins, dans les conclıes ou dans les serres; dans les bois, les fourmis nous paraissant plus utiles que nuisibles, on fera bien de ne pas toucler a leurs nids. Quant aux individus isolés il fiut, pour les éloigner, bouelıer les trous des arbres avec du platre ou du morticr daus lequel on aura mélaugó une substance amère comme li suie, la eoloquinte ou l'aloès. On peut aussi disperser les matériaux qui eomposent le uid, mais il ne faui employer ee moyen que par un temps de pluie. Lors- 
qu'on dérange souvent les travaux d'unc colonic de fourmis, ou a aussi remarqué qu'elles finissent par émigrer. Enfin, un procédé qui réussit bien, disent les auteurs, consiste à verser dans les fourmilières, de la saumure, de l'cau de savon, de l'acide sulfurique très-ćtendu, des solutions de sulfate de fer ou de zinc, etc., etc. Je crois que l'on fera bien de ue pas trop se fier à ces procédés, infaillibles e'cst possible, mais qui me paraisseut aussi avoir un autre résultat ćgalemeut infaillible, celui de faire périr les plantes du voisinage. En plaçant des pots à fleurs renversés sur la terre de manière à ce que les fourmis puissent aller y butiner quelques mielles de suere qu'on aura $\mathrm{cu}$ le soin d'y répandre, on en attirera un bon nombre et oll pourra les écraser de tomps en temps en changeant le pot de place*.

Enfun, on a conseillé, pour se débarrasser compléteuneut et facilcment des petites espéces de fourmis que l'on reneontre le plus souvent dans les fruitiers, dans les serres, dans les couclies ou dans les jardins, de les fairc chasser par la grande fourıni des bois. On a en effet remarqué que celle-ci ue peut vivre en compagnie des espèces précédeutes, qu'elle leur fait une guerre acharnéc et tellement persévérante, que celles qui échappent

- Lepelleticr de Saint-Fargeau (Loe. eit., page 168) termine ainsi ce qu'il dit des Fourmis : "Ces dégáts, quoiqu'ils se bornent à bien peu de choses, ont fait employer contre les fourmis des moyens de répression et de destruetion. Les moyens de répression eonsistent à placer sur leur passage des corps sur lesquels elles répugnent à marcher ou qqui les font tomber. Ainsi, lorsqu'elles ont à monter une ligne tracée avec de la eraie, celle-ei cmpêclıe pendant quelque temps leur passage... Un cordon de lainc oppose également à leur marche aseendente l'entrelacement des fils qui le composent et leur mobilite. Une ligne d'huile tracée par lo pineeau est aussi un obstacle qu'elles redoutent de franchir. Mais est-il nécessaire pour les fournis d'aller oủ elles vont: les atomes mobiles de la eraie tombent avec les premières fourmis qui veulent franehir la ligne; les autres sont solides et n'empêcheront plus la Inarche. Les fils de laine se cumpriment sous les efforts de la multitude; le chemin devient praticable. L'luile se sèche et u'oppose plus d'obstaeles, ete. 
au désastre ne tardent pas à fuir et à vider les lieux. Ce moyen n'est pas cependant d'une efficaeile absolue, car il arrive, ou que les grandes fourmis trop peu nombreuses ne tardent pas à disparailre, ce qui permel aux autres de revenir impunémenl, ou bien que ces grandes fourmis se propagent et se multiplient à tel point qu'elles deviennent plus incommodes que celles qu'elles ont remplacées. Il y a donc là, comme en toutes elıoses, un juste milieu à atteindre et à conscrver et que l'observation seule peut conduire à trouver. Depuis quelques années il existe, à l'entréc de la ville de Boulay, une faisanderie qui, bien dirigée, promet de devenir considérable. Pour nourrir les jeunes faisans qu'on y élève, on fait une consommalion prodigieuse d'œufs, de larves et de uymplies de celtc fourmi que l'on va chereher dans les bois des environs; on pourra done s'adresser à ces nouveaux industriels pour se procurer eet insecte qu'ils désignent sous le noin de Grosse fourmi.

Il me resterait maintenant à donner la description des espèees de Fourmis qui peuvent êlre rencontrées sur le poirier, ce qui probablement me conduirait à faire l'histoire de presque toutes celles qui habitent le département de la Moselle. Ce travail serait à peu près inutile, bien qu'au point de vue entomologique une pareille monograplice ne serait pas sans intérêt, mais fidèle au cadre que je me suis imposé, je ue ferai la description que des espèces qni sont désiguées, par les auteurs que j’ai consultés, comme vivant sur le poirier.

65. ronmra nora (Linné, $\left.n^{n} 1\right)$.

Lepelletier de Saint-Fargeau; Suites a Buffon; tome 1, prage 201.

Synonymic : Fourmi fauve; - Grande fourmi des bois; -

Rothe ameise (Ratzeburg); - La fourmi brune à corselet fauve (Geoffroy).

Ouvrière: Longueur sept mill. Corps presque glabre; tète plus large que le corselet, d'un rouge assez, vif, front noir avee unc petite ligne enfoncẻc au milicu, trois ocelles 
ou yeux lisses, visibles is la loupe; corselet enfoneé vers le milieu du clos, d'un fauve vif avec le dessus' du dos plus ou moins foncé et variant jusqu'au noir ; premicr segment de l'abdomen fauve avec le hord supéricur plus ou moins foncé; les autres segnients de l'abdonien formant une inasse presque globulcuse, d'un noir brun ou un peu cendré, avec les poils trés-courts; pattes d'un brun noiritre avee la base des cuisses et les genoux rougentres.

Màle: Longueur de neuf à dix mill. Corps noir ainsi que les antennes; tète plus petite que chez les neutres; mandibules faibles, peu dentées; corselet grand, pubescent et comprime; les segments de l'abdomen qui suivent le noeul, en masse presque conique, d'un noir luisant avec l'anus roussattre et allongé; paltes d'un rouge livide, euisses noiratres en dessous; ailes obseures avec les nervures d'un brun clair.

Femclle : Longueul de dix à onze millim.; assez semblable à l'ouvrière dont elle diffère eependant par les ailes et la taille, qui est plus grande; les yeux lisses très-distinets; la tête n'a qu'une très-petite tache de noir au-dessus de la bouche; l'abdomen est court, presque globuleux, d'un noir un peu bronzé, tris-luisaut, obtus et fauve en avant; pattes plus ou moins foncées, cuisses rouges; ailes enfumécs, nervure et le point épais, noirátres.

Cette espèce est indiquéc par M. Dubreuil comme allaquant les poires á leur naturité. C'est l'une des plus grandes de uotre pays et eelle dont il eonvient de se servir pour se débarrasser des autres fourmis. Elle fait ordinairement son uid dans les bois ou les taillis. Elles n'ont pas d'aiguillon mais elles éjaculent une graude quantité d'acide formique dont les vapeurs pénétrantes se font douloureusement sentir aux mains et aux yeux de eeus qui remuen leurs fourmilières. 
L'aceouplement de eetle espèee a lieu fin de mai ou eommencement de juin.

66. Formica rusca (Latreille, no 3).

Lepelletier de Saint-Fargeau; Suiles ì Buffon; tome 1, page 205. Synonyınic : Fourmi noire cendrce (lluber).

Ouvrière: Longueur einq mill. Corps d'un noil un peu ecndré, luisant; la base des antennes rougctitre; une carène. sur le devant de la téte et trois yeux lisses distinets; les derniers segments de l'abdomen (après le noud) en masse presque globulcuse, un peu velus à l'extrémité; paltes d'un brun rougeàtre avee le bas des cuisses de couleur plus foncece.

Màle : longueur de six à sept mill. Corps noil', très-luisant et presque glabre; antennes généralement noires, mais quelquefois brunes à la base; anus et pattes d'un rouge pàle aver: les hanches noires; ailes obseures it nervures d'un jaune foneć.

Femelle: Longueur de six à sept millim. Corps d'un noir très-brillant avee un reflet bronzć; antennes noires avee le premier article seulement brun; ailes enfumées, nervure et point épais noirâtres; pattes d'un brun rougeatte avec le bas des euisses de couleur plus foncéc.

Cette espèec niehe ordinairement sous les pierres et sous l'écoree des vieux arbres, elle n'est pas rare dans les eouches dc nos jardins.

67. römica reava (Fabr., no 4).

Lepelletier de Saint-Fargeau; Suites à Buffon; tome 1, page 208.

Synonymic : Fourmi jaune.

Ouvricre: Longueur trois à quatre millim. Corps d'un jaune fauve plus ou moins fonec, luisant, un peu pubeseent; 
l'abdomen ordinairement de coulcur un peu plus foneée que le reste du corps.

Mâle : Longucur de trois à quatre mill. Corps d'un brun clair; les antennes è les pattes de couleur plus pille; ailes blanclies, diaphanes, avee des nervures jaunatres, quelquefois les ailes sont très-légèrement enfumées.

Fenılle : Longueur einq à six millim. Corps d'un lrun roussåtre foneé ; antennes et paltes d'un roux jaunatre elair; devant de la téte, partic inférieure et eótés du eorselet brunàtres; ailes l'un jaune obseur, surtont à la base, avec les nervures et le point épais jaunàtres.

Cette espèce, l'une des plus petites du genre, n'est pas Irèseommune dans le déparlement de la Moselle, clle est égalcment indiquée par M. Dubreuil. Elle établit ordinairement son uid à la raeine des plantes et plus parlieulièrement dans les pots de fleurs. J'en ai aussi trouvé un nid assez abondant à la raeine d'un jeune poirier dans les pépinières de MM. Simon frères.

68. Tonmian comiodiania (Latreille, no 2).

Lepelletier de Saint-Fargeau; Suites à Buffon;_tome 1, page 203.

Synonymic : Fourmi mineuse.

Ouvrière : Longueur einq millim. Corps presque glabre; antennes d'un rouge noiràtre ou noires, avee le premier' article jaunàtre; téte noire, environs de la bouche rongeảtres, une ligne légèrenient eufonece sur le front; trois oetelles apparents; corselel d'un jaune plus uu moins foncé, avec une taehe noire sur le dos; les segments qui suivent le nœud réunis en masse d'un noir cendrí et pubeseents.

Male : Longneur six à sept millim. Corps noir et trèsluisant; abdomen soyeux; patles noires ou d'un brun trisfonce; ailes obseures, enfumées, à reflet irisé, nervures d'un 
brun jaunàtre et point épais, noir; antennes noiràtres, soyeuses, surtout ì l'extrémite.

Femelle: Longueur huit à dix millim. Corps grand; antennes et tête semblables à eelles de louvrière; corsclet fauve avee trois taclies noires sur le eorselet; abdomen noir ; pattes fauves; ailes transparentes avee les nervurcs jaunatres ct le point obscur brun.

Celle cspèce est des plus communes, elle fait son nid au pied des espaliers, dans les couches, près des scrres, etc. C'est elle que l'on rencontre le plus ordinairement sur les arbres à la reclicrchc des puccrons ou des cochenilles, el aussi, d'après M. Goureau, près de la $P$ sylla rubra.

XII. VESPA (Linné)"

Lepclletier de Saint-Fargeau; suiles i Buffon; tome 1, pagc 504.

Corps ipais; màchoires longues mais non en forme de trompe; labre bifide; mandibules presque aussi larges que longues, dentées, la pronière dent très-courte, obtuse et éloigince des autres, la seconde dent plus large que les deux inféricures, celles-ci portées sur une mènı base; yeux ćehancrés; antẹnnes vibratiles, légèrement renflécs à leur extrémitć, le premier artielc très-long, le sccond très-court. Prolongement du milieu du ehaperon obtus avec une dent de ehaque eôté; thorax ovalaire; abdomen presque sessile, le premier segment eoupé droit, sans tubereules latéraux; ailes supćricures plissíes longitudinalement dans le repos, ayant une cellule radiale, ne s'avançant pas beateoup plus près du Lout de l'aile que la troisième cubitale, qui est en earrélong; pattes fortes, jambes postérieures bi-ćpincuses à leur cxtrémité; articles des tarses non dilatés en forme de palettes pour la rícolte du pollen.

"Synonymie : Guere; - Wespe (en allemand). 
Trois sortes d'individus, tous ailés, les femelles les plus grandes, les màles dípourvus d’aiguillon.

Sans être aussi abondantes que les Fourmis, lcs Guêpes sont assez communes pour être connues de tout le mondé, soit à causc de la piqúre douloureuse quc eause leur aiguillon, soit en raison de la perte qu'elles oeeasionnent en entamant les prunes, les poires, le raisin, clc., à leur inaturitćt.

Les Guìpes formen! des sociétés don! la durée est annuclle. Lues fcmelles fécondées à l'autounne se eaclient pendant l'hiver dans les trous des arbres, dans les crcvasses, sous les ćcorecs, dans la terre, etc., et y restent engourdies jusqu'au printemps. Dis les premiers beaux jours, clles quittent leur retraite, et c'est alors qu'on les voit butiner dans les premières fleurs de l'aubépine, des cerisiers, des poiriers, ctc., pour y puiser quelques sucs destinés à réparer les forces perdues pendant l'hiver el à les disposer a remplir les nombreux travaux qn'clles vont entreprendie.

Bientiot, en effet, ces femelles chcrchent une place conventable pour y construire leur nid, les uncs dans la terre, les autres dans lc creux d'un arbre, une troisième espèce sur les branches d'uu buisson ou sous la toiture d'un bàtiment. Ces nids sont formés d'une patte papyracée que la Guêpe prépare ellc-même arec des débris qu'elle arrache soit á du bois mort, soit à l'écoree ou au liber des arbres vivants. La pate dont elle compose son nid est

- La douleur eausée par la piquare de la Guêpe est déterminée par le liquide que ect insecte verse dans la plaie au moment où il enfonec son aiguillon. Un préjugé vulgaire, assez généralement répandu, veut qu'en écrasant l'aninal sur la plaie on fasse disparaître la douleur. Cetle pratique, au contraire, est des plus mauvaises, en raison do la eumpression, propre à laire pénétrer l'aiguillon plus avant, s'il est resté dans la plaie, ou le venin si l'aiguillon a été retiré, ou enfin l'un et l'aulre, si la Guêpe n'est pas eneore envolée au moment où on lui applique le eoup. En définitive ce moyen ne saurait produire d'autre soulagement quc la salisfaetion qui résulte d'un cnnemi vaincu all momeut de la surexcitation causéc par la doulcur. 
pétric entre ses mandibules, adınirablenıent disposćes pour cela, et liéc par une sorte de salive gommense dégorgée pendant le pétrissage.

Ce n'est pas ici le eas d'cutrer dans de longs détails sur les différentes formes données à ces nids, qui rarient sclon les espèces, ni de décrire les procédés que ces ingénieuses femelles mettent en usage pour construire les premières eellules liexagonales qui forment la base de la maison commune. Je me bornerai à indiquer sommairement les diverses phases de la vie de nos guêpes.

A peine les premiercs eellules sont-clles construitcs, que la femelle dépose un cuf dans chacune, ect ouf ne tarde pas à éelore et à donner naissanee à une larve apode, mais ayant des mandibules susecptibles de broyer certains aliments. La femellc eesse la construction des cellules et sn ponte pour ne s'oecuper que de procurer la nourrilure à ccs larves. Celles-ci ne tardent pas à prendre de l'accroissement et bientót elles bouehent leurs ecllules et se transforment en "ymplies. Au bout de quelques jours, chaque nymplie produit une guêpe qui sort de sa prison en rongeant la cloisun qui cll formait l'cutréc. Tous les individus de celtc première ponte, ainsi que relles des suivantes, sont des ouvrières, e'est-i-dire des femclles dont les ovaires ont avorté.

A partir de ec moment, la mère nc s'occupe plus ni de la construetion de nouvelles cellules, ni de la nourriture des nouvelles larves, ce soin est désorınais l'unique partage des ouvrières. La ınère commune, en effet, n'a plus qu'à pondre des œufs dans les nouvelles loges qui vont être construites, ou dans les anciennes quc les ouvrières ont débarrassé des débris de nymple ou des cxcréments laissés par l'luabitant précédent. Les choses continuant ainsi pendant une grande partic de l'été, la république augmente considérablemeut d'labitants; quelquefois le nombce de ccuxci atteint deux ou trois mille.

Vers le mois d'aoùt, on commence à voir des cellules plus grandes desquelles sortiront des jeunes mảles et d'autres ecllules plus grandes encore, d'où sortiront des femelles. Les individus 
de ces deux sexes, arrivćs à l'état adulte, sortent du guêpier pour ne plus y rentrer; c'est alors que l'accouplement a lieu; les femellcs hivernent, comme je l'ai dit en commençant, tandis que les mâles, subissant la loi naturelle parmi les insectes, ne tardent pas à mourir. Quant aux ouvrières, elles continuent à donner leurs soins aux larves restées dans le guêpier jusqu'au moment oủ les ressources coinmencent à faire défaut, ou bien jusqu'aux prcmiers froids un peu vifs, « alors, dit Rćaunur, il se fait dans les guêpiers un singulier et eruel changement de scène. Les guêpes alors cessent de songer à nourrir leur petites larves: elles font pire, de mères ou nourrices si tendres, elles devicnnent des maratres impitoyables; elles arrachent des cellules les larves qui ne les ont pas encore fermćes; elles les portent hors du guêpier; c'est alor's la grande occupation des ouvrières. Le massacrc est général. \ Car, ajoute Lepelletier de Saint-Fargeau, \& le froid les privant subitement de nourriture, elles savent bien ne pouvoir les élever. 》Bientót ces ouvrières périssent elles-mêmes et le nid est eomplétenicnt abandonnć.

Pendant les premiers moments, les jeuncs lạres semblent n'être nourries que par des substances sucrées récoltées dans le calice des fleurs, de la sive des arbres ou aussi du miel volé aux abeilles. Plus tard, c'est avec des morceaux dc fruits plus ou moins triturés, qu'ellcs achèveront de prendre tout leur développement. Cependant, quand ces aliments font défaut, les guêpes s'attaquent aux autres inseetes, les engourdissent avec une piqủre de leur aiguillon, ou les décapitent avec leurs mandibulcs, en liument les parties molles ou les pétrissent avec leur bouche, en forment une sorte de boulettc qu'elles vont porter à leurs jeunes élèves.

Le genre guêpe est nombreux en espèees; on en trouve des représcntants dans toutes les parties du monde, leur eoloration est assez uniforme el composce de noir, de jaune et de fauvc. Quatrc espèces vivent dans le département, et on peut leur appliquer les généralités qui précèdent, ainsi que les moyens de destruction dont il va être question. 
Ainsi que nous l'avons vu précédemment, e'est plus partieulièrement aux fruits mùrs que les guèpes vout demander la subsistanee de leurs larves; un relard de quelques jours apporlé à la réeolte des poires lors de leur malurité, peut souvent amener la perte de tous les fruils d'une quenouille ou d'un espalier. Un fruit entamé par une guêpe ne tarde pas à ètre envahi par les fourmis et les forficules, qui ne font qu'augmenter la blessure, et la pourriture vient bienıôt ell acherer la destruetion complète.

Pour détruire ees inseetes, je ne puis conseiller que l'usage des petites fioles à noitié remplies d'eau sucrée comme il a été dit pour les fourmis; quant aux nids, leur dest'uetion ne doit être entreprise qu'avec précaution, et le soufrage, au moyen d'une unêche introduite daus l'une des issues (les autres étant préala. blement bouchées) est, de tous les inoyens indiqués, eelui qui réussit le mieux, comme aussi celui qu'il est le plus facile à $\mathrm{em}$ ployer. Cette opération devra toujours ètre faite le matin avant le lever du soleil, ou le soir après son coucher, ou mieux encore par une forte pluie.

\section{VEBPA CRABRo (Fabr.).}

Lepellelier de Saint-Fargeau ; suites a Buffon, tome 1, page 309.

Synonyınie : Guépe frelon; - Hornisse en allemand.

Longueur du màle et des ouvrières : vingt-quatre à vingtsix millim.; femelle: trente-deux à trente-six millim. Túte ferrugineuse; chaperon, echanerure des yeux, tache triangulaire sur le front, base des mandibules de eouleur jaunc; antennes bruncs, les trois premiers artieles d'un roux elair'; eorselet ferrugineux plus ou moins foneé; éeusson et deux lignes sur la partic antéricure du dos d'un roux elair ; dessus de l'alidomen et la base du premier segment roux, le milieu brun et ture ligne étroite jaune sur le bord postérieur, leuxième segment, brun à la base, jaune postéricurement, ces couletrs séparées par tune lignc très-sinuce; le troisième 
segment jamne avec la base brune, les deux segnients suivants et l'anus jauncs avec un point brun de claque cóté; en dessous, le premier segment est brun ct les quatre suivanls ont leur basc de celte dernièrc couleur ; pattes ferrugincuses; ailes rousses; corps couvert de nombreux poils roux.

Les femelles se distinguent par leur taille plus grande, et les màles par des coulcurs plus claires ainsi que par l'absence d'aiguillon.

Ja Guĉpe frelun est répandue dans toute l'Europe, e'est la plus grande des espéces de ce genre qui se reneontrent daus eette èntrée. Elle construit son nid dans les cavités des vieux arbres, re nid, sourent fort considérable, est furmé d'une substanee papyracće jannàıre plus ou moins foncée et très-friable. Les frelons, dit Marquarı, doivent être considérés comme les plus grands destructeurs de la Reine-Claude. Ils attaquent aussi les poires, et comme ils sont plus grands que les autres espèces de guêpes, ils font aussi plus de mal à ees fruits.

La piquire du Frelon est Irès-dangercuse, surtout quand il est irrité, et on sait que les Guêpes, en génćral, sout tris-irritables, aussi devrat-on prendre les plus grandes précautions, quand il s'agira de la destruetion cl'un nid de ees insectes.

70. VEsfa GERMagica (Fabr.j.

Lepellelier de Saint-Fargeau; suites à Buffon, tome 1, page 515. Synonymic : Guipe germanique; - Vespa vulgaris (linuc).

Longucur du malle: dix-lıuit mill.; de la femelle: vingteinq millim.; des ouvrières : quiuze millim. Antcunes noires; tète jaune avec uuc tache et une ligne Iransversale noires plus ou moins interrompues; corselet noir, avec unc bordure, deux lignes de chaque cóté et unc lache jaunes; segments de l'abdomen ayant leur hase noirc et la partic postéricure jaune, celle-ci prolongéc au milicu avec un point 
noir de chaque eòté, ecs points souvent réunis entre cux ou avec la base; anus jaune des deux còtés; palles jaunes avec le dessus des cuisses noir; ailes transparentes, un peu enfunces, nervures ferrngineuses; corps couvert de poils assez longs, et de la couleur de la partic sur laquelle ils sc trouvent implantés.

Outre la taille qui est différente, on leconnail'a les ouvrieres à l'anus qui est presqu'entièrement jaune; les mâles se distinguent par l'absence d'aiguillon, par le sixiène segment et l'anıs qui sont presque complétenıent jaunes, el enfin par la forme allongée de leur abilomen.

Cetue espèce fail son nid en lerre; ec nid est composé d'une sorte de papier gris eendré, trìs-ıninee, luisant el non cassant.

La Guêpe allemande est rare aux environs de Metz, mais j'en ai reçu de nombreux individus pris sur les poires, dans les jardins de Boulay, de Saint-Avold, de Bitelse et de Briey. Probablemenı que, dans ces loealités, elle remplace la Guêpe ordinaire.

71. VEBPA VULGARLB (Fabr.).

Lepelletier de Saint-Fargeau; suiles a Buffon, tome 1, page 516.

Synonymie: Guepe vulgaire; - Guepe; - Wespe en allemand.

Longueur du mále et des ouvrières : quinze à scize nill.; de la femelle: vingt a vingt-et-un mill. Antennes noires; téle jaune, lout le vertex et le derrière de la lète, le bord des mandibules, une tache entre les antennes noire ainsi que le bord antéricur du claperon; eorselet noir, bordé de jaune en avant et le long des épatıletles; une taclıe sous les ailes de coulcur noire ; écusson et porte-ćcnsson ayant de chaque còte une petite ligne jaune; segments de l'abdomen noir's ì la base et jaunes postéricurement, celle partie postérieure 
prolongée au milieu avec un point noir de chaque eóté, ees points jlus ou moins eonfluents entre eux ou avee la bande; anus presqu'entierement jaune; pattes jaunes avec les cuisses en grande partic noires; ailes assez transparentes, enfuniées et à uervures rousses; corps parsemé de poils assez longs, noirs en dessus, blanchritres en dessous et sur les còtés.

Les ouvrières se distiuguent par une tache jaune de elaqque cóté du métathorax et les inàles par le dessous du premier article des antennes, qui est jaune, ainsi que par l'absence d'aiguillon et par leur forme allongée.

Cette espèce est la plus comusune du genre; comme la préeédente, elle niehe en terre, et son guêpier a la mêtne forme et la mêne composilion que eelui de la guêpe germanique.

\section{VESPA BYLVESTRIS (Scopoli).}

De Saussure; Monograph, des Guêpes sociales, pagc 123.

Synonynic : Guepe Sylvestre.

Longucur : Mảle et ouvrière seize mill.; femelle dix-lıuit mill. Antennes noires avee le premier article jaune en dessous; téte jaune avee le vertex et le derriére des mandibules noirs; bord intéricur des mandibules noiràtre; corselet noir, bordé de jaune en avant et le long des épaulettes, une très-petite tache jaune sous les ailes; écusson ayant de cliaque eỏté une tache jaune assez grande; porte-ćcusson entic̀rement noir ou ayant seulement une trés-petite tache jaune de elıque eótć; segments de l'abdomen avece la base noirs et le bord postéricur jaune, la ligne de démareation asser régulière; antus presqu'entièrement jaunc; pattes jaunes, euisses noires dans les trois quarts de leur longueur; ailes transparentes, très-faiblement enfumées avee les nervures d'un ferrugineux elair. Tout le corps est convert de poils assez longs de couleur 
blanchàtre, exeepté sur le vertex où ils ont tune coulcur obseure ou noiratre, selon les individus.

On distinguera toujours facilement la Vespa sylvestris des deux précédentes, parce quc celles-ci ont les yeux prolungés jusqu'à la base des mandibules, tandis qu'il y a un espace libre distinct entre ces deux organes dans la Guêpe sylvestre; clle parait d'ailleurs assez rare dans notre département.

L.es femelles se distinguent par leur taille plus grande; les mảles par l'absence d'aiguillon et la longucur proportionuelle de leur abdomen.

J'ai reçu, de Bitche, un individu de cetıe espicec, qui avait été pris sur des poires múres, en mêne temps que d'autres individus neutres de la Vespa germanica.

\section{POIISTES (Latreille)*}

Lepelletier de Saint-Fargeau; suiles à Buffon, tome 1, page 518.

Ce geure est très-voisin du préeédent. Les Polistes différent des guépes par la première dent des mandibules qui est rapprochéc des suivantes, par la forme en eloche du premicr anneau de l'abdomen qui est presque pédicellé, par le corps plus allongé el enfin par le hord antéricur du chaperon, qui est anguleux chez les premicrs et tronqué chez les secondes.

Lc genre Polistes cst très-nombreux cn cspèccs, trois sculcment sont européeunes. L'uue d'entre elles est très-communc dans le département de la Mosclle où on la confond vulgairement avec les guêpes. Les mœurs de ces insectes ont beaucoup d'aralogic avec eclles des espèces qui précèdent, coume clles, ils vivent en sociétés annuclles, peu nombreuses il est vrai, mais daus lesquelles les ehoses se passent à peu près de la mèmo manièrc.

\footnotetext{
- Synonymic : Vespa (Lin.); — Guêre (Rẻaumur).
} 
Les uids de Polistes ne sont pas cacliés eomme ceux des Guẹpes, ee sont eux que l'on renennlre si souvent fixés aux plantes, aux lutenrs, aux murs des jardins et méme à la tige des graminées.

\section{POLISTES daLIICUS (Fabr.).}

Lepelletier de Saint-Fargeau; suiles a Bufon, tome 1, page 597.

Synonymie: Vespa Gallica (Fabr.); - Guêpe; - Poliste francuise.

Longucur seize à vingt millim. Corps allongé, glabre et noir; clıaperon jaune, ainsi qu'une tache devani les yeux et une autre à eóté, une partic de l'orbite postéricur et une ligne sinueuse sur le front; antennes jaunes avec les trois articles de la base noirs en lessus; corselet avee des taches arrondies et des lignes jaunes ainsi que le bord inférieur de lous les segments de l'abdomen.

Les ouvrières sont plus petites que les femelles, et les mäles n’ont que le dessus des cuisses noir.

Cetle espèec est excessirement eommune, surtout pendaut les inois de juillet et d'aoúl. Elle est beaueoup moins irritable que les Guèpes ordinaires ou les Frelons; elle altaque plus volontiers les baies de raisin, les prunes ou les mirabelles que les poires.

Son nid est petit, presque loujours en évidenee el par conséquent très-facile à détruire. (On trouve souvent du miel au fond des cellules de ees nids et les enfants les reeherehent pour en sueer la matière sucrée.

\section{XIIV. MEGACHILE (Latreilte)}

Lepelletier le Saint-Fargeau; suites à Buffon, lome 2, page 330.

Màchoires et lèvres allongées en forne de trompe, lévre inféricure allongée; palpes maxillaires de deux arlicles;

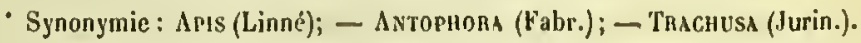


mandibules quadridentées; abdomen aplati en dessus et garni en dessous de plusieurs rangs de faiseeaux de poils pour la réeolte: du pollen; premier artiele des tarses avee une scule brosse; ailes étendues pendant le repos; deux sortes d'individus seulement: des màles et des femelles, pas te neutres.

Les Mégachiles ressemblent assez à des abeilles dont elles diffèrent beaucoup par la manière dont clles ramassent le pollen. Les fenclles construisent des nids composés de cellules diverscment grompées, ayant l'apparence d'un dé à coudre, et formés de morceaux de feuilles découpés fort ingénieusement par ces femelles. Celles-ci pondent un ocuf dans chacune de ces celluics et y accumulent une certaine quantitć d'un micl plus ou moins liquide et qui doit scrvir à la nourriture de la larve.

I)'après ce qui précc̀de, on peut déja soup̧̧onucr que les Mégachilcs ne sauraient fairc grand tort aux plantes et plus particulièrement aux poiriers. Je n'en aurais pas parlé non plus, si, en 1859, un jardinier de Jouy-aux-Arches ne m'avilit apporté un grand nombre de feuilles de poiricr couvertes des conceptacles de l'OEcidium canccllalum, et qui, outre cette plante cryplogamique assez commune, présentaient des échancrures plus ou moins profondes, de forme quadrangulaire ou arrondie, et qu'il supposait être l'ouvrage de quelque chenille ou de quelque larve habitant l'intéricur du petit champignon parasite. Les disques enlevés pouvaient avoir un centimètre de dianètre, et les quadrilatères cnviron deux centimètres dans le scns le plus allongé; beaucoup de feuilles portaicnt aussi des traces de coupures commencécs et interroinpucs à la rencontre des partics jaunes qui cntourent toujours la hase des conceptacles de l'OEcidium cancellatum. Ce fait met pour moi hors de doute l'origine de tontes ces entiilles régulières, semblables à celles que l'on rencontre sur beaucoup d'autres feuilles, notamment sur celles des rosiers, et qui sont faites par une espèce du genre Meigachile lors de la construction de son nid. 
74. MEonchire Ptarka (Lepellelier de S.-F.).

Lepellelier de Saint-Fargeau; suiles a Buffon; tome 2, page 334.

Synonymie: Apis marilima (Kirby); - Apis Lagopoda (Kirby); - Megachile du poirier.

Longueur quinze inill.; noire, couvertes de poils assez longs et d'un roux cendré; les deux ou trois premiers anneaux de l'abdonien plus velus que les suivants; tous les segınents bordés de poils eendrés assez serrẻs; ailes transparcultes avec les nervures noires; pattes noires, les jambes blanchàtres vers le bout, les quatre premicrs arlicles des tarses dilatés, blancs en dessus ct ciliès de roux foncé.

Le màle est plus petit que la femelle et a le dernier article des antennes comprimé, plus large et plus long.

Après la description de celle espèce, Lepelleticr de SaintFargeau, dit : a Environs de Paris; asscz commune. Celle espèce fait assez souvent son nid daus lc terrean des arbres pourris el creux. Elle sc scrı pour l'enveloppe, de morecaux de feuilles, tcls que le poiricr ou le marronnier d'Inde. 》

Bien que je n'aie pas encore rencontré la Megachile pyrina dans uolre départemeul, conme celte espèce est commune dans plusieurs partics de la France, je n'hésiłe pas à lui atıribuer les coupures faites aux fenilles dout il a élé question, et je pense que personuc ne sungera à regarder eet insecte comme véritablement nuisible. 


\section{Liste des Hémiptères qui vivent sur le Poirier.}

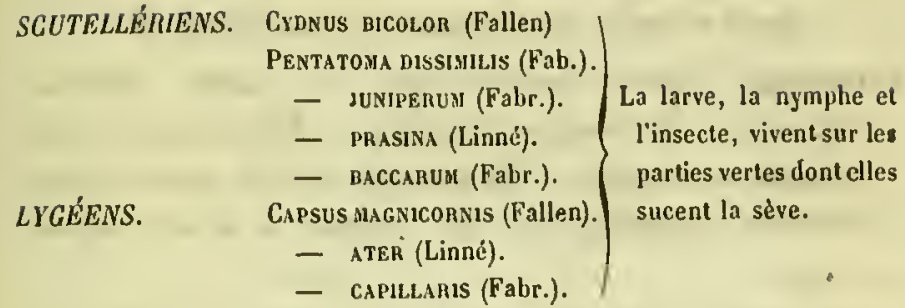

RÉDUVIENS. TINGIS PYHI (Linné).
La larve, la nymphe et l'insecte, sous les feuilles.

Très-nuisible.

\section{CTDNUS (Fabricius)*}

Blanchard; IIisloire des insectes; tome 2, page 446.

Corps ovalaire, assez large; tète assez petite; antennes assez greiles de einq artieles, allant un peu en grossissant vers l'extrémité; écusson grand, presque triangulaire, mais ne couvrant pas tout le rorps; pattes courtes, jambes grêles, garnies de fortes épines dans toute leur longueur, tarses robustes; les ailes supcrieures ont la partic coriace plus grande que la partie membraneuse.

Ce genre est nombreux en espèces; quelques-unes d'entre elles sont assez communes dans le département de la Moselle. Leur couleur est ordinairement uoire, variée de blanc ou de jaune. Ces insectes virent sur les parties vertes d'un grand nombre de plantes dont ils sueent la sève. Les fenelles pondent leurs oufs sur les feuilles et les larves qui en éclosent, vivent squvent en sociétés

- Synonymie : Cimex (Fabr.); - Pentatoma (Lepel. et Serv.); - Punaises. 
assez nombreuses. C'est dans ee eas senlement qu'on peut les eonsidérer eomme nuisil)es. Une scule espècc est indiquée eomme vivant aux dépens du poirier, e'est le :

75. CYDTU Brcozon (Fabr.).

Amyot et Serville; suites à Buffon; llémiptères, page 98.

Syıonymı : Cimex bicolor (Lin.); - Cimex nubilosa (Harris); - Trilomegas (Amyol); - Trilomegas bieolor. (Amyot et Serville); - La punnise noire à quatre taehes blanehes (Gcoffroy); - La punaise à deux eouleurs (Stoll).

Longueur : sept millim. Corps d'un noir luisant, finement ponetui; une taclic longitudinale assez grande el sinuce irrégulièrement en dedans sur le bord antérieur du protlıorax, uıc autre ıaclıe assez large, en croissant irrégulicr, a la base des élytres, et une tache moins grande à l'angle extéricure de l'extrémité de la partie coriace, blanche; partie membrancuse de l'élytre transparente et blanchatıe; còtés de l'aidlomen tachés de blane.

Celte espèee est très-commune. Elle se reneontre pendant les mois de juin, de juillet et d'aoút sur un grand nombre de plantes potagères, ainsi que sur les pruniers, les pommiers et plus ordinairement eneore sur les poiriers. Comme M. Nordlinger, je l'ai plus souvent rencontrée sur les espaliers que sur les quenouilles ou les lıauts-vents; mais si, à Stuttgard, le poirier bon-elırétien est plus souvent que les autres visité par la punaise noire à quatre taches blanehes, il n'en est pas aiusi à Metz où je ne l'ai trouvée ell abondance que sur le doyenné d'hiver.

Ces inseetes plantent leur trompe indifféremment sur les jeunes pousses, sur les feuilles et sur les fruits, ils en sueent la sève et, quand ils sont nombreux, ils épuisent la plante, en font jaunir les parties vertes et empéelıent ainsi la maturité dı fruit et le dé. veloppement des boutons qui doirent produire l'année suivante. 
On peut, pour les délruire, employer avec succès contre cux les insufflations de poudre inseclicide; mais, comme le plus ordinairement eelle-ci ne fait que les cngourdir, il faudra pour obtenir un résultat satisfaisant, ramasser les insectes tombés et les jeter au feu, ou bien arroser avee de l'ean houillante le sol sur lequel ils sont tombés.

\section{XeVI. PENTATOMA (Olivier)"}

Anyot et Serville; llémiptères, suites à Buffon, page 128.

Téte peu avancéc, yeux latéraux, ocelles placés en arrière; anteunes de sinq artieles, le premier court; bee atteignant la base de l'abdomen; prothorax lexagonal, ćlargi postérieurement; écusson triangrulaire grant, allongé, dépassant un peu le nilieu de l'abdomen; élytres grandes et larges, la partie coriace plus grande que la parlic membraneuse, dépassant un peu la longueur du corps; ailes inférieures blanches et transparentes; abdomen large, ramassé, ınutique, a bords trancliants, pet convexe en dessus et en dessous, sans sillon ventral ; pattes courtes, grẻles et mutiques, à peu près d'çgale longueur.

Ces inseetes sont bien eonnus; on les désigne ordinaircment sous les noms vulgaires de Punaises des bois, Punaises des jardins, ete. Quand on les touche, ils répandent une odcur fortc, pénétrante, désagréable, ct qui rappclle celle de la punaise des lits. Il suffit même sourent du passage d'un seul pentatoma sur unc fleur ou sur un fruit pour lenr eommunicuer une odeur ou un goùt repoussant.

C'est plus particulièrement sur les plantes potagères de la famille des crucifères qu'on les rencontre le plus souremt dans les jardins. Cependant on en troure plusicurs espèces sur les arbres fruitiers. Les deux sexes sont assez semblables entr'eux;

- Synonymie: Cimex (Linné) ; - Eoessa (Fabr.), 
lors de l'aecouplement ils se tiennent bout à bout; la femelle pond des oufs de forme ovoïde et en général de couleur verdàtre, clle les fixe sur les feuilles an moyen de l'enduit glutineux dout ils sont revêtus eu sortant de l'oviducte. Au bout de quelques jours, ces oufs éelosent; il cu sort de petites pumaises qui diffèrent des individus adultes par des couleurs plus tencires, l'absence des ailes et une forme proportionnellenent plus allongéc. Ce n'est qu'après un certain nombre de changements de peau que ces larves aequièrent tous leurs organes et sont propres a reproduire leur espèce.

Le nombre des espèces de Pentatome connu est très-considérable, toutes les parties di monde en possèdent, et nos jardins eı nourrissent une dizaine d'espèces dont quelques-unes sont des plus eommunes. Le poirier n'en nourrit pas d'espèees qui lui soient propres; mais, comme presque toutes sont polyplages et sucent indifféremment la sève de plantes très-disparates, on rencontre souvent, sur les parties vertes le cet arbre, des individus isolés. On a plus particulièrement signalé les espèces suivantes :

76. PENTATOMA DIssImIrs (Fabricius).

Amyot el Serville; Ilémiptères; suites à Buffon, page 131.

Synonymie : Cimex dissimilis (Fabr.); - Cimex prasina (Degéer); - Pentatoma juniperina (Lepelletier et Serv.); - Pentatome dissemblable (Amyot et Serville); - La munaise verte (Gcoffroy).

Longucur : dix ì dotze mill. Corps vert en dessus, jaune, vert ou rougeàtre en dessous; lobes latéraux de la tête dépassant le milicu de celle-ci, yeux noirs; antennes fauves avec l'extrémité des dernier's articles plus fonecie; tète, corselet, écusson el partic coriace des élytres couvertes de points enfoncés, nombreux el serrés; partic postérieure de la léte, cotés latéraux du corselet el angles huméraux des élytres étroitement bordés de jaune; pattes fauves, tarses roux; un 
point noir sur la partic antérieure de chaqque euisse et un autre de mème eonleur, à la plaee des stigmates, sul' les bords latéraux de l'abdomen; partie membraneuse des élytres brune.

Cette punaise est commune dans les jardins, mais le peu d'individus que l'on en reneontre sur les poiriers doit rendre son dommage insignifianı. Je n'en ai pas observé les mélamorphoses; selon M. Signoret, elle pond en juin. Selon M. L. Dufour, le dernier segment abdominal est largemeu échancré daus le mảle.

\section{PENTATOMA JUNIPEROM (Fabr.).}

Amyot et Serville; Iremiptëres; suites à Bufion; page 132.

Synonymie: Cimex juniperinum (Lin.); - Pelidia (Amyo ).

Longueur de onze à treize millim., largeur de six à sept mill. Corps entièrenent d'un beau vert elair en dessus eomme en dessous; tìte et eorselet chagrinés; antennes vertes ì la base, noirâtı'es à l'extrémité ; une légère bordure latérale delrière les yeux et l'angle lıtıméral externe des élytres jaunâtre; extrémité de l'éeusson blane ou blanchitle; partie membraneuse des ćlytres blanche mais paraissant grise, quand elle repose sur le fond noir du dos de l'abdomen, bord externe de eelui-ei jaunàtre.

Cette espèee, qui, à la première vue, peut aisément se confondre avee la précédente, s'en distinguera facilement par la couleur différente de la membrane des élytres. Plusieurs auteurs l'ont indiquée eomme vivant sur les arbres fruitiers, mais, dans les euvirons de Metz, je ne l’ai jamais reneontrée dans les jardins. Daus le départenent de la Moselle, elle parait même assez rare, et elle ne s'y trouce que sur le genêvrier. Dans la Champagne, elle seinble y être très-commune; ear, en 1859 , j'en ai trouvé une graude quantité dans des baies de genièvre récoltées au mois de septembre dans eette partie de la France. Ce n'est donc, selon 
moi, que très-accidentellcment qu'elle a pu se trouver sur le poirier, et il ẻst mêtuc permis de supposer que sa grande ressemblance avec l'espèce précédente l'a fait confundre arcc ellc et a fait croire à son existence dans nos vergers.

78. PExtatoma pRasixa (Linné).

Amyot et Serville; llémiptères; suites à Buffon, page 131.

Synonymic : Cimex prasinus (Linné); - Cimex dissimilis (Wolf); - La Punaise verte des choux.

Longueur dix millimètres. Corps d'un vert pré, finement ponetué de brunattre en-dessous; milieu du front aussi avaneé que les lobes latéraux; membranes des élytres d'un vert pàle ; extrémité des quatre premiers articles et tout le cinquième article des antennes ferrugincux; pattes vertes.

Celle pentatome est des plus communes. Elle vit dans les chanps, d'ordinaire sur les gramiućcs ct sur les choux; dans les rergers, on la rencontre en effet sur le poirier, mais accidentellement, el elle ue doit y causer aueun dommage bicn scnsible.

79. PExTATOMA BAGCAROM (Linné).

Amyot et Serville; Ilémiptères; suites a Buffon, page 132.

Synonymic: Cimex vorbasci (Degíer); - Pentatoma confusa (Westw); - Penlatoma depressa (Linné); - Cimex baccarum (Linnć); - Pentaloma nigricomis (Fabr.); - Pentatoma eryngii (Germ.); - Pentatoma Willinsonii (Hope); - Pentaloma bihamala (Kolenati); - Punaise brune à antennes el bords panachés (Geoffroy); - Pentaloma (Amyol).

Longucur de huit à neuf millim. Corps d'un roux plus ou moins verdittre en dessus, le dessous est d'un jaune testacé avec de nombreux points noirs; lobes latéraux de la tète notablement plus larges que le lobe médian; antennes 
jaunes, le deuxième el le troisieme article et quelquefois te quatrième de couleur très-foncic ou noir à l'autre cxtrémité, ledernier plus ou moins eomplétement noir ; cótés de l'abdomen tachís de noir et de jaunc ou de rouge en dessus ; pointe de l'ćcusson jaune; palles fauves.

Celte cspèce est, comme la précédente, extrêmement cominune; on la reneontre partout et sur un grand nombre de plantes d'espèees différentes; souvent elle vit en société et alors elle devient nuisible tant à cause du tort qu'clle fait à la plante qu’à cause de l'odeur pénétraute et fort désagréabie qu'elle coinmunique aux fruits sur lesquels elle a séjourné ou dans la pulpe desquels elle a puisé sa nourriture. Les framboises ct les inùres sont plus particulièrement exposées à eet inconvénient; quant au poirier, sur lequel on la reneontre souvent, il ne parait pas beaucoup souffrir de sa présence. Les deux sexes sont semblables. Sclon M. L. Dufour, les ocufs sont éehancrés sur un còté. Je n'en ai pas suivi les métamorphoses; et, bien qu'elle soit trèscommune, je n'en connais ni les acufs ni les larves. En terninaut, j'ajouterai eneore que M. Nordlinger dit qu'elle se trouve souvent sur le bouillon blane. Iei je n'ai pu, au mois de juillet et d'acut de 1858 , en trouver un seul individu sur cetle plante, saurage ou cultivéc, bien que l'espèce elle-mème ne füt pas rare ì cette époque.

\section{Xevir. capsus (Fabricius)*}

Amyot et Serville; llémiptères; sutites i Buffon; page 280.

Corps en général elliptique; tète arrondic, non prolongéc en pointe; antennes grèles, insérées au-dessous des yeux, le deuxième artiele notablement élargi ou épaissi en massue à l'extrémité, le troisième et le quatrième d'égale longueur entre cux; bec court, atteignant ecpendant l'insertion des

\footnotetext{
- Synonymie : Miris (Fabr.); - Lrgeus (Fabr,); - Gloniceps (Encyclop.); P'ecilosoma (Sléplens); - Pimtoconis (Macq.); - Cisra (Fabr.).
} 
pattes intermédiaires; partic coriace des ílytres peu eonsistante; pattes grèles, assez longues, les postérieures les plus longues.

Le genre Capsus se compose de plusicurs espèces, la plupart de petite taille, une grande partie se trouve dans nos contrćes et $y$ ont produit un très-grand nombre de variétés.

Selon M. Léon Dufour, l'abdomen du màle est formé, dans une grande étendue, taut cn dessus qu'cn dessous, par une seulc pièce cỏnoïde très-obtuse, appartenant à l'armure copulatricc; selon le même auteur, les cufs de Capsus qu'il déclare n'avoir jamais vus pondus, sont, dans l'ovaire, allongés, eyliudroïdes, tronqućs à un bout et légèrement arqués. Ces inscctes vivent en soeiété sur beaucoup dc plantcs herbacées ou ligncuscs; et, quoique lcs individus en soient très-souvent nombreux, les métamorphoses en sont encore inconnues.

80. CAPSUS manxcoRnis (Fallen).

Fallen; Hémiptères de la Suẻde; page 119.

Synonymie : Capsus mali (Meyer); - Phylocoris magnicornis (Maeq.).

Corps noir, brun en dessus ; antennes noires ou brunes à la base avee le second artiele fusiforme et l'extrémité composée d'artieles plus minces et blanes; téte et corselet obseurs ; élytres brunes ainsi que l'écusson; euisses postéricures renflies, jambes plus elaires garuies de petites ipines.

Cet insecte m'cst complćtement inconnu. Il est indiqué par Macquart comme vivant sur les feuilles du poirier et du pommier. Kirclibaum, dit que l'accouplcment a lieu en juillet et $\mathrm{cn}$ août et que cet insecle vit sur le poirier. Il ajoute encore que la Phytocoor is mali, do Meycr, qui vit sur le ponmier, est identiquc avec cct insecte. M. Signoret, auquel je dois la description de cctte cimicide, faitc par Falleu, m'écrit encore à ce sujct: a Pour moi conme pour Kirchbaum, Phyt. magnicornis me 
semble synonyue de $P h$. mali de Meyer, que ec dernier indique toujours plus grand que magnicornis. Je le possède, pris sur le pommier par moi-même, et il est évidemment plus graud que ceux qui m’ont été envoyés de Suède par M. Bolıéman. De même j'ai reçu de Meyer, $C$. Hali et C. magnicornis (Fall.), sans que je puisse assigner de caractères distinetifs à ces deux insectes. \Enfin pour en finir avec les incertitudes qui semblent se rapporter a Capsus magnicornis (Fall.), j’ajouterai quc sous le nom de Punaise ligre, Gcoffroy a déerit une espèce voisine connue des entomologistes actuels, sous le nom de Phylocoris clavicornis (Linné), elle vit exclusivenent suı le T'eucrium chamadrys; il ne faut pas la confondre avee une autre einicide de la mème tribu, que les jardiniers comnaissent aussi sous le nom de Punaise tigre el qui sera décrite plus loin (voyez Tingis pyri).

81. OATSOS ATER (Linné).

Amỹot et Serville; Hémiptères; suiles ì Buffon; page 281.

Synonymic : Cimex aler (Linné); - Cimex semiflavus (Lin.);

- Capsus tyrannus (Fab.); - Lygaus tyrannus (Volf); - Capsus flavicollis (Fabr.); - Lygaus flavicollis (Woll').

Longueur cinq millim. Corps plus ou moins bruı dans toutes les parties ou dans quelques-unes seulement; palles rousses, avec ou sans anneaux, de coulcur plus foncéc.

On trouve cet insecte sur toute sorte de plantes, dans les prés, dans les bois ou dans les jardins, souvent isolé; il n'est eependant pas rare d'en reneontrer des eolonies de 25 on 30 individus, vivant en parasites sur le mème régétal. C'est dans ee eas seulement 'qu'on peut le regarder eomme nuisible et qu'il peut ĉtre utile de clierclier à le détruire au moyen des insufflations de poudre insecticide. 
82. capsus gapiziaris (Fabricius).

Amyot et Serville; Ilémiplères; suites à Buffon; page 281.

Synonyunic: Phylocoris capillaris (Blanch.); - Capsus tricolor (Fabr.); - Capsus danicus (Fabr.); - Cimex tricolor (Fabr.); - Cimex flavomaculalus (HlerichSchœlf); - Piggudus (Amyol); - Capsus pyri (Maequarl).

Longueur six mill. Corps jaunatre ou rougeatre; élytres unicolores ou ayant une tache ronge el un point noir a l'extrémité; pattes de la couleur du corps, cuisses noircs à la base.

Cette espèce raric encore plus que la précédente et présente, sur le corselet et sur les ćlytres, comme les coecinelles, des variations, par excès ou par défaut de couleur. Elle est également polyphage et, comme la $C$. ater, elle vit en socićté on solitaire sur un grand nembre de plautes culirécs ou sauvages, lierbacécs ou ligncuses. Dans Ies jardins ecpendant on la trouve le plus ordinairement sur les rosiers et sur les groseillers, aceidentellenent on peut en rencontrer quelques individus sur les arbres fruitiers, mais elle ne peut guère être considéréc comme étant nuisible à ces arbjes.

Beaucoup de variétés, les plus communes et les plus constintes surtout, de cette espèce, ont ćtć considérécs et décrites, ou au moins indiquécs comme espèces distinctes, par quelques auleurs. C'cst ainsi que Macquarl cite comme ćtant nuisible au poirier, lc Capsus pyri. 0r, aucun des auteurs que j’ai consultés ue contient de Capsus pyri, et comme le sarant diptérologiste français ne donne aucune description de l'insecte qu'il désigne sous ce nom, je suppose qu'il a voulu parler d'une variété bien tranclaće des Capsus ater ou capillaris et que, conuaissant déja de nom la Capsus mali, sans en donner la description dans un calalogue où il n'étail pas nécessairc de pousser l'exactitude d'une manière bien rigourcuse, il aurit voulu earactériser l'espèce qu'il observait par le nom de Pyri. 
XIVIII. TINGIS (Fabricius)"

Amyot et Serville; Ilemiptères; suites ḋ Buffon ; page 296.

Corps très-aplati; tête rètrécie à son insertion; antcuntes de quatre articles, le prenier eylindrique, le second plus court, le troisièmo: gràle et plus long que les deux préećdents réunis, le quatricme en massue globıleuse; bee pouvant se $\operatorname{loger}$ dans un sillon assez marqué et qui s’étend jusqu’a l'extréulité du sternum ; prothorax prolougé postérieurement en pointe de manière à couvrir l'ècusson, celui-ei très-petit ; Ic corselet présente en outre trois lignes longitudinales élevées dans son milicu, les cỏtés sont fortement dilatés et forment unc expansion membrancuse, tandis que le disque est fortement relevè et comme vésieulcux; ćlytres ovalaires, plus longues et plus larges que l'abdonen, dilatées latéralement et renflées sur le disque; toutes ees parties vésiculeuses et foliacies, d'une transparence membrancuse et présentant un rèscau à petites ecllules formées par de fines nervures; pattes courtes, grc̈les et d'égale longueur; tarses de trois articles.

Les insectes de ce genre sont très.remarquables par les expansions foliacées du corselet et des ćlytres et le renflement vèsiculcux du corselet qui, dans quelques espèces, forme une sorte de capuchon au-dessus de la tête de l'insecte. Ils sont phytophages et se multiplient quaclquefois en telle quantité qu'ils causent un préjudice considérable aux plantes sur lesquelles ils vivent. Quelques espèces déterminent la formation de sortes de galles par les piquures réitérées qu'clles font avec lcur trompe sur les parlies lierbacées des végétaux. Parmi les espéces de ec genre qui habitent la France, une seule mérite de fixer notrc altention d'une manière toute particulière.

* Synonymie: Acantila (Wolf); - Mounatha (Schoffi); - PIesma (Burnteistcr). 
83. Trwars PXRI (Fabrieius).

Amyot et Scrville; llémipléres; stiles à Buffon; page 297.

Synonymie: Acanthir pyri (Fabr.); - Tingis appendicens (Fill.); - Cimex pyri (Fallen); - Punaise à fruise antique (Gcoffroy); - Tingis (Amyot); - Dyctionata pyri (Stçphens); - Tigre ${ }^{*}$; -- Punaise du poirier (de Bose).

Longucur deux mill. Corps brunatrc ou noiràtre, dilatition des élytres et du corselet, blanches; celui-ci avec un

- Sous ce noun de Tigre, les jardiniers, ainsi que plusieurs auteurs d'ouvrages sur l'arboriculture, désignent des inseetes et des choses bien différentes, et pour lesquelles il est néeessaire de bicn nous entendre pour iviter la confusion :

On distingue d'abord deux sortes de Tigrcs: le Tigre sur bois et le T'igrc sur feuille. En ee qui regarde les arlıres fruitiers, en général, le Tigre sur bois n'est autre chose que la coehenille, soit qu'elle affecte la forme hémisphérique eomme la eochenille du jêeher ou celle du pommier, soit qu'elle ait la forme conehylienne comme l'Aspidiolus, dont il sera question plus loin.

Le Tigre sur fcuilles se dit des inseetes qui, eomme les eochenilles des Camélias, du Isauricr rose, de l'Oranger, etc., forment avec leurs corps des taches blanchâtres ou jaunatres plus ou moins nombreuses sur les partics herbacécs d'un grand nombre de plantes eultivées dans nos serres tempérées; ou bien encore des inseetes qui, par leurs piquures ou leurs déjections, maeulent plus ou moius les feuilles de eertains arbres, comne e'est le eas pour le Tingis Pyri dont nous nous oecupons.

Mais en dehors de eette dénomination de Tiqre, appliquée it des inseetes ou a leurs jroduits, les jardiniers désignent eneore sous ce nom des taches ou des ntaculatures produites sur les feuilles sans la coopération des insectes, soit d'ailleurs qu'ils eonnaissent cctte indépendance, soil, au contraire, qu'ils attribuent a tort à des animaux la production de ees taches.

De ce nombre, sont les taches jaunes ou orangées qui se reneontrent sur les feuilles du poirier en juillet, en aout on en septembre, et qui sont produites par le Iycelium de l'Gicidium canccllatum: Les taelıes noires furoduiles sur les leuilles de bcaueoup de plantes par le Mycclium de 
renflement vésieuleux grand et en forme de capuchon, ou de mitre avancé sur la tète, les cótés et la earène médiane

petits champignons appartenant aux genres Puceinia, Ecidium, Uredo, cte. : les taches rouges, brunes ou noires produtes par la brülurc ou par les larves mineuses des Cècydomyics, des Tinéides, ete.

Pour nous, nous conserverons le nom de Tigre au Tingis Pyri, cn faisant observer encore que la synonymie qui précède a, sans doute, été cause de la confinsion commise par plusieurs auteurs, et particulièrement par Dalbrct, qui attrihue à un seul insecte (le véritable Tigre), plusicurs altírations produites par des insectes tout à fail diffẻrents. M. Dulırcuil pousse eneorc la confusion plus loin; ainsi, il commence par décrire une espece de coclıenille qui resscmble $\dot{a}$ du son et qui se fixe sur l'écoree an mois d'oetobre, ou elle reste jusqu'au mois de juin de l'année suivante; it cette époque, M. Dubreuil la fait ehanger de peau et produire le Tingis Pyri!!!!

Lejère va plus loin: ainsi, il nomme Grise le tigre sur fcullle, ct d'après ec qu'il en dit, il est impossible de ne pas reconnaitre le Tingis pyri dont il cst ici question; cependant la Grise, est un insecte qui détcrmine sur les plantes oú il sc trouve une affection bien différente de celle dont il a étó question jusqu'ici, et qu'il n'est pas inutile de faire connaître.

Si, pendant l'été, on examine la page inférieure des feuilles dı Pècher, du Poirier, du Nelon, elc., on remarque que celle face est couvcrle d'un réseau de fil très-fin, de couleur blanchâtre, assez serré, cl qui donne à la feuille la couleur particulière qui a valu le noun de grise a cette affection. Au milicu de ce réscau, dont la présence doit nécessairement nuire aux fonctions respiratrices de la fcuille, on trouve ordinairement un petit aearicn ayant le corps globuleux, luisant, de couleur grise et se mouvant arce agilite. D'autres fois, seul ou cn compagnie dujpréećdent, un sccond araclınide de taillc un pou plus grande, de couleur verdatre avec deux taclıes brunes sur les angles huméraux de l'abdomen, lc ceplalothorax gros, les palpes longs et très-mobiles. Ces deux petits animaux aticulés sont excessivement abondants, l'arachnidien encore plus que l'acarien, et souvent ou les trouve, sur la mème plante en compragnie de larves ou d'insectes parfaits. Sur lo Poirier particulièrenent, la Grise se rencontre avec les Tingis pyri, et de pelits oufs blanes et sphériques qui pourraient bieı être ceux de notre Tigre, et enfin avec des larves apudes et blanehattres qui paraissent servir a la nourriture de la petite araignée, car celle-ci les toumente cunstamment. 
dilatce en foliotes visib!ement réticulées. Elytres présentant de elıaque còté et vers la base une tache brune, et une autre scmblable placéce aussi de elaque eótć, vers l'extrémitć; ees taehes sont plus ou moins grandes et se réunisscut quelquefois de manière á former une taelıe cruciforme. Le dessous du corpsest d'un ver' olivàtre plus ou moins fonce avec le bord des anneaux noiràtre; aprés la mort de l"inseete, toute eette partie du corps se fonee et devicut souvent noire; les pattes sont páles. Les deux sexes ne paraissent pas différer, et la deseription qui précède leur est egalement applieable.

Le viritable Tigre, le T'ingis pyri, le seul dont il scra désurmais question dans ee travail, a été signnalé, il y a déja bicu longtcinps, par bcaucoup d'cutounologistes et d'arborieultcurs, :omme étant nuisible au poiricr.

Il est ecpendant très-étonnant de ne pas en trouver la deseription, ni mème la eitation, dans le jeuarquable ouvrage de M. Nordlinger; mais ee qui doit paraitre eneore plus extraordinaire, e'est que ee n'est qu'en 1859 qu'on parait l'avoir observé pour la premic̀re fois dans les jardins des cuvirous de Melz. Tous les jardiniers que j'ai consulıćs à cet effet, m’ont donné des lenseigucments identiques et qui eonstatent que, depuis bien Jongtemps, on n'arait ru eet insecte dans nos jardins. C'est en faisant cette sorte d'enquètc entomologique, 'que j'ai rceueilli les éléments nćeessaires pour élallir la synonymie indiquée plus laaut pour le Tigre. Malgré l'absenee d'observations affirmatives et ru le nombre vraiment prodigicux de 'T'ingis pyri reucontrés sur quelques poiriers, il me semble plus rationnel de penser yue cet inscele existait déjà dans notre département et qu'il y a pris tout a coup un développement considérable, gràec à un coneours de cireonstanees favorables à sa multiplieatiou et 'jue jusqu'iei il ne m’a pas été permis de préeiser.

Lc Tingis se reneontre le plus ordinairenent à la page inférieure des feuilles du poirier et de préférence sur ecux qui sunt 
ćlcvés en espaliers. Quelquefois on le trouve sur les pêchers et plus rarement sur les pommiers, le duvet cotonncux qui recourre les feuilles de ect arbre, est très-probablennent la causc de l'iumunité dont il jouit. Maequart cite aussi unc cspèce de Tigre comme vivant sur les feuilles du laurier (Laurus nobilts), mais je erois que daus ec cas, cet autcur s'est trompé, comme M. Dubreuil, el qu'il a fait comme les jardiniers qui nomment tigre la cochenille de l'oranger, si comununc sur la plupart des arbres de la fanille des Laurinées quc nous cultivons.

C'est dans le commencement du mois de juillet 1859, que l'on a commencé a remarquer la préscnce de cel insccte sur lcs feuilles du poirier; mais alors out ne trouvait que des larves ou des nymplies arec un nombre relativement tres-minime d'inscetes adultes et presque toujours accouplés. Dans lc courant du mois d'aoùt, le nombie de ces inscetes est deveru si considérable que de tous côtés il a altiré l'attcntion des horticulteurs; $\mathrm{cl}$, cliose remarquable, e'est quc, dans la même scmainc, j'cn ai reçu en communication de plusicurs points du département de la Moselle ct méme des départcments de la Mcurthe et des Yosges. Partout ils apparaissaicnt pour la première fois, ct, partout aussi, on mc signalait la rapidité arce laquellc ils se propageaicnt, et l'intensité du dommage qu'ils causilient aux poiricrs.

Dans le courant du mois d'aoùt ou de scptcmbre, on trouvc, sous ehaque feuille des arbres envahis, de vèritables colonics de T'ingis, composées de larves, de nymphes cl d'insectes parfaits; ccux-ci sont alors en grande majorite et cependant les accouplements cu sont très-rarcs. Malgré mes rccherclıcs, jc n'ai pu obscrver ni la ponte ni l'éclosion des oufs. Les plusjeuncs larres que j’ai puétudier avaicnt cnviron un millinètre de longueur. En ce moncint clles sont blanches, à l'cxecption du premicr ct du quatrième article des antennes, ainsi que les tarses, qui sout d'un brun plus on moins foncé, quclquefois noir. La tétc porto trois pointes aiguës, allongées et dirigécs horizontalcment en avant. Les cxpansions latéra!cs du corselet sout blanclics, opaques et non encore réticulécs. L'abdomen cst cordiforme, deux fois 
aussi long que la tẻte et le corselet réunis, avec deux taches noiràtres plus ou moins grandes aux angles liuméraux; sur la moitié postérieure de l'abdomen se trouve cu outre une taclıc transversale plus ou moins grande, plus ou moins dilatée dans son milieu et de couleur brune ou noiratrc. Le dessous du corps cst blanc, avec des taches brunes sur les còtés de l'abdomen et de la poitrine. Antennes de la longucur des deux tiers du corps; pattes longues et grêles. Les bords postéricurs et latćraux de l'abdomen sont hérissés de longues épincs, dirigécs horizontalement comme celles de la tête; tout le dessous du corps porte également de ces épines placécs normalement à la surface ct de couleur noire ou blanche, selon la teinte de la partic du corps sur laquelle elles sont implantées.

On troure aussi souvent des larves de taille beaucoup plus grande et presque entièrement blanches, c'est qu'en ce moment elles vont subir une mue, probablement la deuxiène; quand ce changement de peau est terminé, le disque du corselet commence à se boursouffer, les cótés latéraux se dilatent, et l'ou aperçoit distinctement les moiguons d'oủ sortiront les ailes de l'insecte. Ces nymplies diflèrent cncore des larves par l'absence des épincs que nous avons signalćes sur le corps de la larre, par le dessous du corps qui n'cst plus blanc, mais brunatre, ct enfin par le dessus de l'abdomen qui est blanc avec une taclie discoïdale assez grande de coulcur foncéc.

Ainsi que je l'ai dit plus haut, les Tingis virent en sociétés fort nombreuses sous les fcuilles des arbres et, à pcu d'exception près, sous les feuilles des poiricrs en cspaliers, sans qu'il soit possible de trouver une variété qui en soit moins affectée que toute autre. Les larres, les nymplics et les inscetes parfaits marchent lentement et avec hne sorte de gravité.

Jien que ces Ifémiptères paraissent peu agiles, ils s'envolent facilement au moindre danger, et quand on vicnt a secoucr un arbre habité par cux, ils forment, par lcur nombrect lcur coulcur, une sorte de nuage peu étendu et qui ne tarde pas à sc dissiper, parce que tous ces inscctes relournent rapidement sous leur abri. 
Dans leurs colonies, les Tingis paraissent continuellement en mouvement, et quand ils enfoncent la trompe dans la parenchyme de la feuille, c'est pour un instant si court qu'on ne peut supposer qu'il suffit pour y ponper quelque nourriture, peut-êtré cependant que pendant la nuit, ou quand ils ne sont pas inquiétés, restent-ils fixés plus longtemps ct prennent-ils le temps d'en puiser la sève dont bien certainement se compose leur alimentation?

On comprend toutefois que ces nombreuses piqüres doivent Atrc très-préjudiciables à l'arbre, mais ce qui l'est davantage encore, selon moi, c'est la déperdition de sève qui s'opère par les millicrs de piquúres dont se trouve labourée la face iufẻrieure de la feuille. Cette sève cxtravasée s'agglomère en goutlelettes, s'altère, se dessèche et forme une grande quantité de petites taches visqueuses, luisantes, bruncs ou noiritres sur lesquelles l'inseete a de la peine a mareher et qui font par leur ensemble paraitre la feuille comme tigrée, de là peut-être l'origine du nom donné à l'insecte qui en est l'auteur. Cette matière gluante continuant à se dessécher et à augmenter, les pores de la feuille s'cn trouvent obstrués; alors celle-ci ne respirant plus, jaunit à la face supérieure, se dessìche et finit par tombèr, ou, si elle r'este attachẻe à l'arbre, elle lui donne l'aspect d'un arbre mort. C'est seulement alors que l'on commence à s'apcrcevoir de la présence du Tigre , car, jusque-lá, la coulcur et la taille de l'insecte ne permcttaient guère de les distinguer an milieu des maculatures de la feuille.

On comprend dès-lors qu'un arbre placé dans de telles conditions, et cela pendant les mois d'aouit et de septembre, ne tarde pas à languir; les fruits restant petits et chétifs, et les boutons a fruits ne se devcloppant pas du tout, la récoltc future est également compromisc. Une ehose singulière, e'est que les 'Tigr'es, même cenx qui sont arrivés à l'état parfait, ne quittent pas des feuilles qui ne fonctionnent presque plus ou qui neême sont eomplétement desséchées. Ce n'est que quand ces feuilles sc détachent de l'arbre, ou seulement quand le nombre des individus de lat colonic devient trop considérable, qu'on voit émigrer lcs larves, les 
nymphes ou les iusectes, pour se porter sur les feuilles les plus roisines, de sorte que de proche en proche l'arbre tout entier finit par êre complélement envalii. C'est dans ees envalissements progressifs que les pêchers, les abricotiers, les pommiers et mime les pruniers roisins se couvrent également de ces insectes sans que toutefois.ils s'y unuliplient en aussi grande abondance, ct aussi sans que leur présence paraisse être aussi nuisible à ces arbres qu'ils le sout aux poiricrs.

D'après ee qui précède, il est évident que le dommage causé aux poiriers par le 'Tigre est déterminé d'une part, par l'absorption et l'excudalion de la sére, el d'autre part, par l'obstruction des pores de la feuille oceasionnée par l'accumulation simultanćc des déjections de l'insecte et du liquide visqueux et noirâtre dont j’ai expliqué plus laut l'origiue.

Par conséquent, tous les auteurs, au nomotc desquels je suis fort étonné de trouver Macquart, qui ont dit que cet insecte délruic le parenelıyme des feuilles, lui out allribué des dégàts qui étaient eausés par d'autres insectes, avint, pendant ou après le passage de celui-ci. Enfin, le fail assez rare, d'insectes persistant à habiter une feuille dans laquelle la sève ne saurait plus eirenler, un'a fait supposer que les nombreuses piquiues faites par les Tingis pour faire écouler le liquide sueré an dehors, avait surtout pour but d'amener une accunulation de celui-ci el de créer une réserve de nourriture pour l'insecte, quand la feuille est ineapable d'en produire directement par la suecion. Je livre cette supposition pour ee qu'elle vaut, me r'éservant de faire des observations qui permettent de la confirmer ou de lui en subslituer une plus conforme í la réalilé. Qnelle que soit d'ailleurs la solution de cette question, le résultat est le même, au point de vue horticole; il importe maintenant d'examiner par quels moyens il est possible d'arrêter les progrès du mal aussitôt qu'on aura reconuu la présence du Tigre, et sauver ainsi la récolte pendante et l'arbre lui-même.

Ia poudre insectieide, appliquée au soufflet on à la louppe, semble d'albord réussir parfaitenient; dès les premières insuffla- 
tions, on voit tomber des quantités considérables de larves, de nymplies et d'inscetes plus ou moins engourdis. Si l'on se borue à ce premier résultat, on n'a qu'à rechereher le lendemain les tigres tombés sur le sol, et c est à peine si l'on en retrouvera quelques-nns. C'est qu'en effet bien peu meurent aussi vite, le plus grand nombre n'est qu'engourdi, et en quelques licures ils reprennent assez de vigueur pour remonter sur les feuilles et y continuer leurs dégâts. Pour obtenir un suceès plus eomplet, il faut aussitót après l'aspersion de la poudre, avoir le soint d'arroser le sol couvert des insectes endormis, aree de l'ean clatude ou une lessive légère et l'on en achève ainsi la destruction.

Ces opérations, surtout celle du souflage de la poudre, derront se faire le matin s'il n'y a pas de rosée, ou le soir clans les temps sees, de manière a déranger le moins ees insectes et empécher leur dispersiou sur les arbres roisins. En outre, comme la ponte est continue, puisque pendant deux mois au moins on trouve a la fois des jeunes litres, des nymphes el des insectes adultes, il sera bou de renouveler plusieur's fois et à quelques jours d'intervalle, l'application de la poudre inseeticide. Quoique souvent effieaces, les moyons qui précèdent ne produisent quelquefois d'autre effet que lit dispersion de quelques colonies, mais, dans lous les eas, il sera bon d'employer suecessivement quelques-unes des méthodes anciennement eonuues.

Les jardiniers eonseillent les aspersions avee de l'eau de saron, de la lessive, une décoction de tabac, ete., mais comme le tigre se tient presque toujours à la faee inféricure des feuilles, il en résultc que le plus grand nombre n'est pas atteint par ees liquides dont l'effet me parait d'ailleurs assez problématique, tant qu'on n'emploie pas des solutés d'une certaine force, et par eonsécruent dans des conditions où ils deviennent eux-mêmes nuisibles aux végétaux.

Les fumigations de tabae sont de tous les moyens à employer, celui qui réussirait le micux, s’il étuit facile à metre en pratique. Bien qqu'en effet ce moyen soit presqu'impratieable en grand, je dois le conseiller, paree qu'il y a souvent urgence à employer un 
remède radieal, si l'on veut saurer une rćeolte de fruits et souvent l'arbre lui-ınemc. Comme d'ailleurs il est à peu prìs le seul qui, dans eertains eas, puisse être employé eontre les pucel'ons, les détails dans lesquels je vais entrer trouveront leur application plus tard, et je n'aurai plus à y revenir en faisant l'lıistoire de ees Ilomoptères.

On eommenee par eouvrir l'arbre d'une toile fermée par le lıaut et sur les eùtés, de manière à retenir autant que possible les vapeurs emprisonnées; puis, avec le souflet que je déerirai tout à l'heure, on dirige la fumée du tabac sous la toile et autant que possible en faisant arriver le plus fort du jet sous les feuilles les plus chargées de Tingis ou de Pueerous. On coutinue la fumigation jusqu'à ce que l'on ait atteint toutes les parties infestées et que l'on ait brủlé, pour un espalier de moyenne grandeur, de 20 à $50 \mathrm{gr}$. de tabae.

Deux ou trois heures après eette opération, on enlève la toile et on proec̀de, aree de l'eau ordinaire, à un bon arrosage de tout l'espalier, on terminera enfin en piétinant fortement le sol mouillé sur lequel sont tombés les inseetes. $\mathbf{A}$ part la difficulté d'exécution, ee moyen est exeellent, unais il ne fait pas périr tous les inseetes, et ceux qui éelrappent à celte asphyxie eontinuant à se reproduire, on est dans la néeessité de leur faire une nouvelle fumigation, quinze ou viugt jours après la première, si le temps est elıaud; vingt-cinq ou trente jours seulement après, si l'on est en août ou en septembre.

La plupart des jardiniers qui font des déeoctions ou des fumigations de tabae, ont l'labitude d'enployer à cet usage des tabacs de eontrebande ou des tabacs de qualités inférieures qui sout livrés à bas prix par l'administration. L'économie, dans ee cas, est plus apparente que réel!c, et il vaut mieux employer, eu le découpant menu eonme pour le fumer, le tabae à ehiquer en rolles. Ce talsae produit, à poids égal, une déeoction beaucoup plus elıargée et une fumée au moins quatre fois plus forte que eelle que l'on obtient des autres qualités.

Le souffet à fumigations se compose d'un soufflet ordinaire 
a la bouclse duquel out adapte un petit cylindre en tóle de quatre à six centimètres de diamètre. On place dans le fond de ce cylindre un morceau d'amadou allumé; on y ajoute, cn le tassant légèrement, le tabac découpé, et on fait agir le soufflet. On peut, si l'on veut, surmonter le cylindre-foyer d'un couvercle portant un tuyau souple ou rigide, et au moyen duquel on dirigera la funce sous les cloches, dans les bâches, dans les serres, etc.

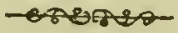

Lisle des Homoptères qui virent sur le Poirier.

PSYLLIENS.

APIIIDIENS.

PSYLLA ALNI (Linné).

- Pya (Linné).

- Prricola (Fœrster).

La larve, la nymphe et l'insecte, vivent sur les

- Apiophyla (Fœrster). feuilles dont elles su-

- Pynisuga (Forster).

- Rubha (Fœrster). cent la sève.

- Aubantiaca (Gour.). sont nonibreux.

Apilis mali (Degéer).

- Pyni (Koch).

- Pruni (Fabricius).

Inscetes vivant sur les

jeunes pousses.

- SonBs (Kaltembach). $\begin{gathered}\text { Très-nuisihles quand ils } \\ \text { sont abondants. }\end{gathered}$

Schizoneura lanigema (lllig.) Vit sur le tronc.

Penilugus pyn (Aza-lítelı). Espèce américaine qui vit sur la racine.

Coccus aram (Schranck).

LECaniua PYM (Schranck).

Aspidiotus concinformis (Gmélin). $\{$ Très-nuisible. 


\section{Xexx. PExiza (Geofroy)'}

Amyot et Serville. Hiemiptères, suiles ì Buffon, page 591.

Corps allongè, tète inelinéc et aplatie cn dessus, ayant leux yeux globulcux, saillants, trois óeelles disposés en triangle, un dc ehaque eòtc derrière les yeux, le troisième sur le front; antennes insérécs devant les yeux, filiformes, de huit à dix articles, de la longueur du corps, le dernier article terminé par deux soies fines et raides; bee très-eourt paraissant maitre de la poitrinc, en arric̀re de l'insertion des pattes antírieures, formé de trois articles, restant perpenlieulaire à l'axe de l'inseete, eorselet très-eonvexe en dessus et portant deux petites pointes élevées; cieusson grand; ailes supérieures liyalines, plus longues el de eonsistanee plus ferme que les ailes inféricures, les ailes supéricures ont trois nervures prineipales, longitudinales, dont l'intermédiaire fourchuc forme à l'extrémité une espèce de cellule triangulaire; ailes inférieures avee quelques nervures longitudinales à peine sensibles; abdomen eonique, intimement uni at eorselet, termine par une sorte de pointe; pendant le repos, les ailes sont disṕosies en toit aigu et dépassent l'abdomen; pattes postérietures, propres au saut; tarses de deux artieles, le dernier plus long, muni de deux eroehtets ayant entre eux unc pelote membraneuse.

Les Psylles sont de très-petits insectes qui vivent sur les plantes dont ils poinpent la sève. Ies espèces en sont nombreuses et très-diffieiles à earaetériser. Selon M. Foerster, la plupart des auteurs. qui s'en sont oceupés, ont comınis de nombreuses erreurs, surtout en ee qui coneerne les veelles, la trompe, les ailes, les organes génitaux, ete. On connait l'histoire complète

\footnotetext{
- Synonymie : Psylue; - Chermes (Linné); - Faux Pucerono (Réaumur).
} 
de plusicurs espéces de Psylles. M. Léon Dufour a écrit eelle de la Psylla ficus, Lin.; Réaumur, celle de la Psylle du buis ( $P$ sylla buxi, Mraeq.); Schmidberger, celle de la Psylla pyrisuga, Farst., etc.; les différenees qui existent dans la manière de virre de chacune de ees trois espèces, sont assez notables pour justifier la division du genre Psylla des auteurs en plusieurs sous-genres, ainsi que l'a fait M. Forster

- Le remarquable travail de $M$. Forster est peu connu el eomme il a été publié en allemand, je erois rendre strvice aux entomologistes français, qui ne eonnaissent pas eette langue, en donnant ici la traduetion du tableau synoptique de la division des genres qui eomposent aujourd'hui la famille des Psylliens.

1

2

$\int$ Tête avee deux tulereules frontaux 3 .

- sans tubereules frontaux 6 .

Nervure prineipale des ailes supé-

rieures bifurquée ..........

3

- trifurquée...........

4

Ailes supér. ayant un sigma obseur

Iémiélytres coriaees, très - visi-

blement ridées .............

5

Hémiélytres visiblement membra-

neuses............... Genre Anvtana, Ferster; le type

Hémiélytres sans sigma ...... ๆ.

6

Un sigma bien caraetérisé aux hémiêlytres.............
Genre: Tnroza, Fœrster; le type est la Trioza urlice; Chermes urlicce, Lin.

Genre: Issula, Geoffroy; le type est la Psylla alni, Lin. 5.

Genre Livilla, Curtis; le type est la Livilla ulicis, Curtis. est la Arylaine Spartii, llartig ; Psylla Spartii, Hartig. - Chermes quercus, Linuc.

Ia Livia Jincorum, Latr. .

Genre Rhinocola, Focrster, le type est la Rhinocola erica, Curtis; Psylla erice, Curtis. 
Le genre Psylle, restrcint commc l'indique M. Focrster, renferme encore une quarantainc d'espèces, presque toutes sont proprcs à l'Europe et sept au moins vivent aux dépens du poirier. Les Psylles ont les paltes courtes, les cuisses renflées, fusiformcs, celles des pattes postérieure; sont proprcs au saut; comme ees insectcs sont très-agiles et qu'ils volent facilement, il cn résulte que ce double moycn de locomotion les rend très-difficiles à saisir à l'état parfait, tandis que leurs larves et leurs nymphes sont très-lourdes et inarchent lentement. Les femelles ont unc tariè̀e avec laquclle elles percent l'épiderme des plantes pour y déposer leurs œufs; celle opération détermine, dans plusieurs circonstances, la formation d'excroissances ou de fausses galles.

Plusicurs larves sćcrètent une matière cotonneuse blanchàtre dont elles sc recourrent, d'autres au contraire, rejcttcnt par l'anus unc matière sucréc souvent assez abondante pour salir les fenilles ou les jeunes pousses des végétaux et y attirer Ics fourmis. Par l'enscublc de leurs caractires el de lcurs formes, Ics Psylles ressemblent assez aux petites Cicadelles. Lcs espèces sont en gćnéral assez difficiles à distinguer parce quc leurs couleurs varient beaucoup selou qne l'insectc est virant ou mort, qu'il y a plus ou moins de temps qu'il cst transformé, ctc. Les

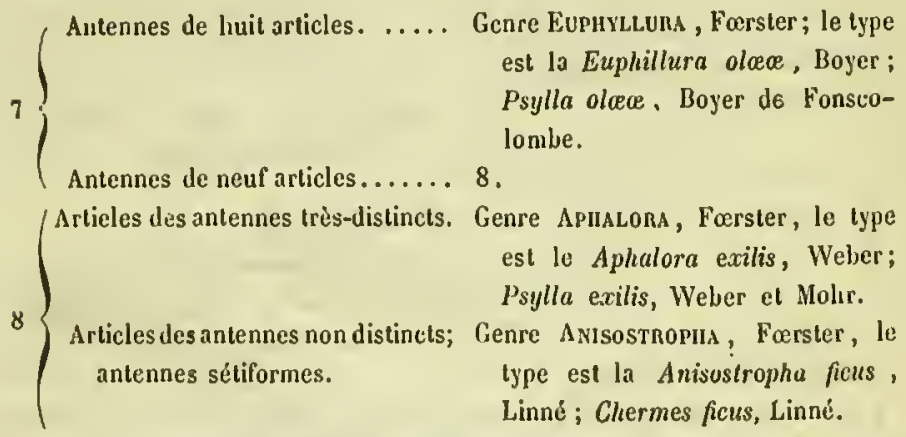

Les divisions systématiquement établies par M. Fœrster, sont cependant si nalurelles que ehacune d'elles ne renferme plus qne des espèces ayant des mœurs semulables à l'état de larves, de aymples ou d'insectes parfaits. 
pointes frontales offrent, par leur forme ct par leur position, les caractèrcs spécifiques, les plus visibles ct les plus consıants.

\section{PBIZIA AIXI (Linné).}

Forster; Rèvision du genre et des espèces de Psylles, no 1, page 70. Synonymic: Psylle de l'Aune.

Longueur de deux ou trois millimètres. Corps d'un vert un peu jaunảtre en avant; extrémitć de la trompe et tarses brunâtres; pointes frontales courtes, larges et tronquées; articles des antennes jaunàtres au sommet à partir du quatricme, les trois avant-derniers plus de la moitic jaunatres, dernier artiele entièrement brun; ailes jaunátres avee les nervures jaunes.

Cette espèce est très-communc dans le départcmcut de la Moselle où on la rencontre sur bcaucoup d'arbics d'espéces différentcs. Jc ne l'ai cependant janais trouvéc sur le poirier; mais Macquart, sans donner aucun détail sur scs habitudes, dit qu'clle habite également le poirier et d'auttes arbrcs fruitiers.

\section{RBTIIA RXRI (Linné).}

Forster; Révision des genres et des espéces de Psylles, no 21, page 77.

Synonymic: Chermes Pyri (Linné); - Aprophylla (Amyol);

- Psylle du poirier.

Longueur : leux millim. à deux mill. et demi. Corps d'un jaune rougedtre sale; téte et thorax avee des taelies et des raies brunes; abdomen avee de larges bandes lirunes et le bord postericur des segments rouge; pointes frontales médiocrement longues, larges à la base, obtuses au sommet. Antemnes presquientièrement brunes à partir du quatrième article; euisses et tarses bruns. Les nervures des ailes sont d'un brun fonec a l'exeeption de la nervure eostale, qui est jaunàtre jusqu”i sa bifureation; cntre les nervures, se trouvent 
des taches brunes plus ou moins allongies, plus ou moins apparentes, enfin sur le bord inférieur, près de la première cellule, se trouve aussi une tache plus foncée.

A celte description, que je traduis d'après l'ouvrage de M. Forster, eet auteur ajoute eneore : « Cette espèce, de laquelle je n'ai qu'un màle provenaut de la collectiou de M. de Ileyden, vient de Bingen et doit être uuisible au poirier; il est probable que c'est le Chermes pyri communis, de Linné et de Degéer, au moins la deseription, ou plutót la diagnose (Alis fusco macuLatis) convient-elle micux à cettc espèce qu'aux Psylla pyricola et Psylla apiophila. »

Celte opinion de M. Forster n'est pas celle de Selımidberger, qui pense que la Chermes pyri, de Limné, est la Psylla pyrisugra. D'autre part, M. Nordlinger, en donnant de eet insecte une description qui n'est pas du tout applicable a eelle de Focrster, dit que la difrérence vient peut-ĉtre de ce que les auteurs, pour fairc leur description, ont pris des individus plus ou moins àgés, vivants ou morts.

Du reste aueun des auteurs qui se sont occupés de cet insecte, n'a parlé de ses liabiludes. Tous se contentent de dire qu'il vit ou qu'il a élé trouvé sur le poirier. J'ajouterai enfin que pour M. Signoret, si compéteut daus l'étude des Hémyptères, la Psylla pyri (Burmeister') est bien la Chermes pyri de Linné et qu'il l'a également trouvée, aux environs de Paris, sous les feuilles du poirier.

La Psylle du poirier, dont il est ici question, n'a pas eneore, que je sache du moins, été observéc dans notrc département, unais comme elle se trouve sur les bords du Rhin, cu Belgique, à l'aris et en Bourgogne, c'est-à-dire dans des localités qui forment une ceinture autour de la Moselle, je n'llésite pas à la conprendre parmi les insectes nuisibles aux poiriers que nous cultivons. 
86. PEIIIA PTRICOIA (Forster).

Foerster; Révision des geures el des espèces de Psylles, no 25, page 77.

Synonymie: Psylla similis (de Heyden).

Longueur: deux à trois millim. Corps d'un jaune rougeàtre avee des tachies ou des raies brunes sur la tète et le dessus da eorselet; abdonien avee des bandes, le bord des segments pàle, la poitrine de eouleur plus pàle en arrière. Antennes jaunes avec les artieles, a partir du quatrième, annelís de brun à l'extrémité', les deux derniers entièrement bruns. Les pointes frontales pàles, un peu eourtes, larges à la base et obtuses au sommel; base des euisses brune; ailes jaunàtres avee les nervures jaunes et une taehe brune au bord inféricur, avant la première eellule.

J'ai pris, dit M. Forster, une femelle de eette espèce près d'Aix-la-Cliapelle; deux autres femelles, sous le nom de $\boldsymbol{P}_{\text {sy }}$ lla similis, m'ont été envoyées par MI. de Heyden; elles avaient été prises par lui à Soden, près Francfort-sur-lc-Mein, sur le Pyrus communis. L'auteur, auquel j'emprunte eette citation, ne dit pas si e'est sur le poirier sauvage ou sur le poirier eulivé, et ne donne aucun détail sur les habitudes de eet insecte ni sur la nature du dommage qu'il peut veeasionuer.

87. pgtiza aprophiza (Forster).

Forster; Révision des genres et des espèces de Psylles, no 26, page 78.

Un peu plus petite que la préédente, du reste assez semblable pour la couleur; tète et thorax comme dans la Pyricolta; abdomen ayant également des bandes de eouleur rouge, mais les bords des segments sont d'un rouge vermillon; les tubereules frontaux sont beaueoup plus eourts encore que dans l'espèee ì laquelle je la eompare, ec qui l'en fera très-facilement disthnguer, ils sont pàles au sommet et tronqués de la mème manière. Les ailes sont plus trans- 
parentes ct la tache brune du bord inférieur plus foneece ct plus apparente.

M. Forster ajoute qu'il a trouvé, sur des poiriers en espaliers, les deux sexes de cette espèce à Aix-la-Chapelle et à Boppart, que M. de Ileyden lui en a enroyé plusieurs de Soden, qui se trouvaient aussi sur des poiriers en espaliers et qu'enfin M. Walker lui en a envoyé d'Angleterre, mais sans indiquer ia plante sur laquelle il les avait trouvés.

\section{TBYLLA PYRIsUGa (Fœrster).}

Fœrster ; Révision des genres el des espéces de Psylles, no 27, page 78.

Syronymic : Psylla pyri (Schmidberger); - le grand Suceur de poires (des allemands).

Longueur: trois millimètres. Corps d'un rouge foneé teinté de brun, eette dernic̀re couleur donine ordinairement; les pattes ont les genoux, l'extrémité des tibias et les tarses jaunes; antennes jaunes, les articles, à partir du troisièıne, sont annelés de brun à l'extrémité, les deux derniers articles entièrement de cette eouleur; les tubercules frontatx courts, fortement tronqués etordinairement de la eouleur de la tète, quelquefois eependant l'extrémité est plus foncée; ailes passablement transparentes avee les nervures et ttit sigma de coulcur rougeatre.

Cett: espèee, ajoute M. Fœister, est, parmi toutes eelles qui vivent sur le poirier, la plus grande et la plus remarquable; j'en ai Irouvé onze femelles dont l'abdomen arait des bandes brunes et un bord très-étroit de couleur vermillon, et un mále qui ne présentait pas ee earaelère. On la trouve sur les poiriers en espaliers dans les jardins d'Aix-la-Chapelle et de Boppart; eette espèec ne parait pas se reneontrer en Angleterre. A Melz, en 1859, j’ai trouré, sur un jeune poirier en espalier, deux Psylles qui se rapportent assez bien à la deseription que je viens de donner. 
J'ai déjà dit plus haut que, selon Sehmidberger, la Psylla pyrisuga est le Chermes pyri de Limné et de legéer, tout en reeonnaissant eependant que la deseription donnée par ce dernier auteur ne soit pas eomplétement applieable au grand suceur de poires.

Sans entrer dans l'examen des raisons qu'il donne pour justifier eette opinion, et partageant à ee sujet la inanière de voir de M. Focrster, le savant monographe de la famille cles Psylliens, je vais rapporter l'histoire de la $\mathbf{P}$ sylla pyrisuga de Sehmidberger, d'après ee qu'en a publié eet habile observateur.

Le Sueeur de poires parait en abondanee tous les ans, depuis le milieu du mois d'arril jusquau milieu de mai, sur les poiriers, quelquefois, mais rarement et d'une manière isolée, sur les pommiers. Au moment de la pousse des feuilles et de l'épauouissement de la fleur ees inseetes se posent sur les pétioles, sur les pédoneules et quelquefois sur la fleur elle-même. Souvent on reneontre les deux sexes réunis; ils sautent ou s'envolent au moindre attouehement et sont très-diffieiles à saisir. Pendant l'aceouplement, le màle est plaeé auprìs de la femelle, eelle-ei a les ailes fermées en toit, tandis que le måle maintient relevé l'aile supérieure qui se trouve du eóté de la femelle. Celle-ei étant féeondée, on la voit bientôt pondre ses cufs. Pour eette opération, elle introduit sa tarière dans le pétiole, dans la jeune feuille, sur la fleur ou sur le fruit nouvellement noué, mais toujours sur les parties légèrenent velues, et y introduit un acuf. Cette opération dure environ une minute pour ehaque xeuf; les ovaires étant très-gros, ees inseetes sont très-féeonds. C'est surtout en inai que l'on trouve ees oufs; ils sont jaunes, plaeés les uns près des autres, sans eependant se toueher; peu de jours après qu'ils ont été pondus, la partie de la pousse sur laquelle ils sont fixis se eontraete, prend une eouleur différente et devient faeile à reeonnaitre. En quelques jours l'éclosion a lieu et produit des petits très-différents de la mère.

Les larves de Psylles ont le eorps allongé et eylindrique; les antennes sont blanelátres ainsi que les pattes qui paraissent 
diformes. La trompe sétiforme est fort longue, les yeux sont ronges et le reste du eorps d'un jaune foneć, au moins pendant les preniers jours de leur existenec. Ces petites larves ne tardent pas a subir unc première mue, alors elles clangent de couleur et deviennent brunes avec des raies transversales blanchàtres sur le dos; le corselet est rougcatue avec des points et des bandes noires; enfin, la poitrine, le rentre, les pattes et les antennes sont d'un vert pảle avee quelques points noirs. Peu de temps après ce premier cliangement de peau, les larves de Psylles quittent les fleurs ou les feuilles ou elles étaient, et vout se placer les unes à cóté des autres, à la base des rameaux de un á deux ans.

Schmidberger pense, et je erois avee raison, que les larves de Psylles une fois fixées changent encore une fois de pean et que e'est alor's seulement que l'on voit apparaitre les moignons des ailes; le corps devient obtus à l'extrémité, se termine par dcux petites soies et prend l'aspect général d'une punaise aplatic.

C'est en ce moment que, par les dejections qui sortent goutte a gontte de l'anus avec la eonsistanee d'un liquide ćpais et visqueux, les nymphes de Psylles commencent à salir la branche ou elles se trouvent fixées. Les fourmis, attirées par ee liquide, les visitent souvent et serveut à les fairc déeouvrir, car une fois fixées, les nymphes ne changent plus de place jusqu'à leur complètc transformation, à moins quon ne les inquiète.

Pour subir leur dernière métamorplıose, les nymphes sc séparent ordinairement et vout se fixer isolément sous une feuille à proximité; alors la peau se fend sur le front, ct ordinaireıneut une heure après, l'insecte parfait se trouve complétement dégagé de sa dernière enveloppe. Dans le premier moment, il est d'un beau vert, les yeux sont roses, les ailes délicates et transparentes. Les couleurs ne se foneent et ue prennent les earaetères que nous avons indiqués en commeneant qu'au bout d'un temps assez long. Comme pendant l'été et pendant l'automne on ue voit ni accouplements ni nouveaux ocufs, Sehmidberger en conclut que les deux sexes hiveruent et que les femelles ne sont féeondées qu'au printemps suivant. 
Tous les auteurs sont d'aceurd pour considérer cet inseete comme très-nuisible, au moins à l'élat de nymplee, qu'il passe, aiısi que nous l'avous vu, fixé à la base des pousses qui se fanent, se flétrissent, se contractent en se desséeliant et finissent par mourir si l'on ne vient les délivrer des parasites qui leur enlèvent le sue nourrieier. C'est au moyen de frietions faites sur les parties de l'arbre envahi par les Psylles, que Schmidberger propose de dérruire ces insectes; il faut, dit-il, employer, pour celte opération, une brosse ou un pineeau de soies raides. L'autenr auquel j'emprunte tous ces délails termine en disant qu'il a vu souvent cette psylle sucée par un liémiptère du genre Lygaus.

89. FEYLA RUBRA (Fourcroy).

Synonymic: P'sylla Pyri?? (Linné). - Psylle rouge de Gicolfroy.

Longueur : deux millim. et demi. Corps brun fertugineux; tẻte d'un brun ferrugineux marqué de taches rouges; antennes brunes; thorax brun ferrugineux avee quatre lignes longitudinales sanguines sur la mésothorax; écusson brun ; abdomen brun, ayant le bord des segments sanguins; pattes d'un brun noiratre avee les articulations des tarses ferrugineux; poitrine tachée de rouge ferrugineux; ailes hyalines à eòtes et nervures ferrugincuses.

Cette description, que je dois à l'obligeance de M. Goureau, est eclle d'une espèce très-voisine de eelle que Focrster a décrile sous le nom de Ps. pyri, et il ponrrait bien se faire que les différenees fussent dues à l'àge des inseetes déerits ou à des. variétćs locales. Quoiqu'il en soit, M. Goureau complète eette deseription par les détails suivants, que je transeris littéralement: "Isa Psylla rubra, Foureroy, Psylle rouge de Geoffroy, est, je erois, la mème que la $P_{\text {sylla }}$ prori de Lin. La femelle poud ses aufs sur les bourgeons des poiriers vers le 15 inai, ee sont des atỏmes jaunatres, oblongs, ayant une très-courte quene. Les 
larves qui en sortent, observées lc 28 mai, ont environ un tiers de millimètre de longueur; elles sont ovales et jaunàtres, un peu plus grosses du cóté de la tête, qui n'est pas détachée du thorax; on y voit deux petites antennes de quatre articles, dont le dernier est terminé par deux poils. On remarque sur le dos du thorax deux lignes longitudinales parallèles de quatre points plus colorés que le reste du corps; l'abdomen n'est séparé du thorax que par une ligne transversale, les segments sont a peine indicués; le dernier est bordé de poils eourts. Les paltes sont au nombre de six, arliculées, terminées par deux eroehets; le suçoir ou bec se voit entre les deur premières pattes eomme un point noir.

»Ces larves sont serrécs l'uue contre l'autre atutour du bourgeon; elles eroissent rapidement sans ehanger de place. Le 14 juin, elles étaient toutes elıangées en nymphes, et déjả plusieurs inseetes s'étaient envolés. Cette nymphe est presque eirculaire, très-déprimée, longue d'un denn-millimètre et de eouleur brunàtre; on distingue très-bien sa tête, son thorax, son abdomen et les fourreaux des ailes; les antemnes sont blanehes arcc l'extrémitć noìe, le eorselet est rougeàtre, orné de deux lignes longitudinales dc points noirs, ehacune ayant entre elles une ligne médiane blanehătre qui se prolonge sur la tête et sur l'abdomen; les fourrcaux des ailes portent uue taehe blanehàtre dans leur milieu, les pattes sout de la mème couleur; le dessous du thorax et de la base de l'abdomen sont d'un vert-pré luisiut. Les nymphes sont serrćes l'une contre l'autre et immobiles comme les larves. Elles se transforment sur place.

Ces détails se rapportent d'une manière remarquable à ecux que j'ai fait connaitre plus haut, d'après Sehmidberger, pour la $P_{s}$. pyrisuga, et montrent avee quelle exactitude ees deux observateurs ont suivi les diverses phases de la vie de ees petits inscetes.

90. PStrla adrantica (Goureau).

Longuetr: trois millim. Corps de couleur otange; antemnes jaunattes, ayant l'extrémité de la tige noiràtre et le 
dernier noir ; tète et corselet d'un jaunc-orangé foncé avec la partic antérieurc de la première bifide, blanchàtre, les ycux noiratres et les stcmmates (ocelles) rougcàtres; abdomen vert, l'extrémité d'un jaune orange; pattes testacées; ailes hyalines ainsi que les hémiélytres; ees dernières à nervures testacées.

Cette deseription, que je dois égaleınent à l'obligeanee de M. Goureau, ne saurait s'appliquer à aucune des espèces déerites par Focrster. Elle parait nouvelle et provient de Sautigny, département de l'Yonne, oủ M. Groureau l'a trouvée sur des poiriers. M. Signoret la eroit également nouvelle, il l'a aussi trouvée sur le poirier aux environs de l'aris.

Voici ee que M. Goureau ajoute sur eet insecte: « Je ne sais pas si la Psylle que j'ai appelée aurantiaca a déjà été nomméc. M. Signoret pourra vous le dire, ear je lui en ai donné des cxemplaires. Son histoire est semblable a celle de la Psylla rubra, mais ee n'est qu'au mois de juillet que je une suis aperegu de sa présence. Jc n'ai pas vu l'œuf ni la larve, eette espèee était à l'état de nymphe lorsque je l'ai treneontrée.

La nymplıe a deux millimètres de long sur un et demi de large; elle est ovalaire, plate, brunàtre; ses antennes sont filiformes, de la moitie de la longueur du eorps; la tête est arrondie en devant, aussi large que le thorax dont elle n'est séparée que par un siuple trail; celui-ci est ridé transveisalement et porte de ehaque eôté un disque presque rond dans lequel les ailes sont renfermées; l'abdomen est aussi de la largueur du thorax à sa base, de la longueur de ee dernier, arrondi à l'extrémité qui est un peu atténuée. Les six pattes sont courtes; le dessous de l'abdomen est d'un vert-pré au milieu et brun sur les eótés.

L'inseete parfait eommenee à s'envoler dès le 5 juillel, il se transforme sur place; enfin, les antenues ont neuf articles et outre les deux yeux latéraux il n'y a que deux ocelles, les autres earaetères sont ceux des Psylles. 


\section{APHIS (Iinne) ${ }^{\circ}$.}

Amyot et Serville; Hémiptères, suites à Buffon, page 597.

Téte petite, ycux globuleux ordinairement saillants; antennes de cinq, six ou sept articles, quelquefois micme de trois seulement; trois ocelles chez les individus ailés, un entre les antennes, les deux autres de chaque còté de la tète, derrière les ycux ordinaires; aplères sans ocelles; les deux premiers articles des antennes conrts, les autres plus ou moins longs, le dernier arrondi ou pointu, ordinairement petit et maigre. Bec en forme de trompe, composé de trois articles, paraissant sortir de la poitrine sur liqquelle il cst implanté, ordinairement long jusquà atteindre la première ou la scconde paire de pattes, quelquefois atteignant on dépassant mème l'inscrtion de la troisième pairc. Prothorax court et transversal. Ailes uulles ou au nombre de quatre, posées horizontalement ou en toit pendant le repos. Les deux supéricurcs hyalines et offrant, ainsi que les infëricures, des nervures dont les dispositions présentent de très-bons caractères pour l'arrangement des espèces. La prenièrc nervure (nervure costale) commence à l'origine de l'ailc supéricure, clle s'étend presque parailèlement au bord externe jusquà son cxtrémité où elle se termine par une tache plus out moins grande et obscure (sigma) de laquelle sort presque toujours une petite nervure courbe qui attcint l'extrémité dc l'ailc.

De la nervure costale naissent d'abord deux nervures transversalcs qui vont rejoindre Ic bord interne de l'aile; puis une troisic̀ne nervure transversale aussi (cubitus) qui

\footnotetext{
- Synonymie : Puceron; -- Blattlaus, en allemand.
} 
rejoint également le bord externe après avoir émis en avant ct vers l'extrémité de l'aile une nervure simple ou fourchue touchant aussi le bord externc. Les ailes inféricures sont transparentes, clles ont unc nervure costale mais pas de sigma, et deux nervures transversales partant de la précédente et rejoignant le bord internc de l'aile; ablomen plus ou moins soudë avec le prothorax, peu distinet de celui-ci chez les individus aptères, ordinairement gुros, renflé et couvert dans beaucoup d'espèces, ainsi que le reste du corps dans quelques-unes, de poudre blanchåtre ou de filaments laincux plus ou moins longs et de couleur blanche. Pattes assez. longues, grèles; les postérieures quelquefois plus longucs sans itre cependant propres au saut; les tarses sont nuls ou composés de un ou de deux articles.

Sur le dos de l'abdomen et ver's l'extrémité existent cliez. un trìs-grand nombre de pucerons, deux petils appendices ordinairement dirigés d'une manière normale ì la surface sur laquelle ils sont implantes, et plus ou moins longs suivant les espèces. Enfin l'abdomen est terminé par une petite queuc plus ou moins apparcntc.

Les caraclères qui précèdent et que j’ai à dessein exposés un peu longuement, sont ceux qui conviennent it la grande majorité des inseetes qui sont connus de tont le monde et deshortieulteurs surtout, sous le nom de Puecrons. De tous les insectes, aucun ne mérite plus que ecux-ci de fixer l'attention des personnès qui s'intéressent à la eulture des plantes ou à l'étude de l'histoire naturelle. Leur organisation particulic̀re, leurs habitudes, leur mode de génération surtout, sont en effet des plus intércssants à eounaitre, tandis que lenr prodigieuse fécondité, le tort que dans eertain eas ils eausent aux plantes cultivées, expliquent suffisamment les détails dans lesquels je vais entrer et qui résument l'ensemble des connaissances aequises sur ces inscetes jusqu'à nos jours. 
Il $n^{\prime} y$ a pas encore deux cents ans que Godart ${ }^{\star}$, peintre naturaliste lıollandais, disait quc les pucerons naissaient d'un liquide que les fourmis déposaieut sur les plantes. Environ einquante ans plus tard, Lœvenhock détruisait cette grossière errcur en démontrant la présenec des jcunes pucerons dans le ventre de la mèrc. Ce n'est guère que vers 1730 , que Réaumur publia ses ınagnifiqucs travaux et qu'il fit eonnaitre eeux de Ccstoni, de Lahirc, etc., lesquels mirent hors de doute, non-seulement que les pucerons mettaient au monde des petits vivants sans féeondation préalable, mais encore qu'une seule féeondation suffisait pour toute une génćration de pucerons, de laquelle pourraient encore sortir jusqu'a dix générations nouvelles, sans l'intervention d'aucun accouplement. Bonnet et Degéer, vinrent ensuitc confirmer ces découvertes et les compléter par des observations faites sur un grand nombre d'espèces différentes.

On comprend sans peine que des résultats aussi extraordinaires et si contraires aux idées admises alors sur la génćration dcs inscctes, excitèrent l'émulation des naturalistes; et, chosc remarquablc, e'cst que plus on faisait de déeouvertes dans celle voic, plus les pucerous semblaient être en dehors de la loi communc; l'hcrmaphoditisme fut alors la seule supposition faite pour expliquer ce nouveau mode de reproduction.

Linné, Fabricius et Schranck ne paraissent pas avoir fait d'observations anatomiques nouvelles sur ces insectes, ni d'études sur leurs mocûrs. Ils se sont contentés d'en décrire un eertain nombre d'espèces, inais a vec des phrascs diagnostiques si courtcs quiil est maintcnant tris-difficilc, sinon iupossiblc, de reconnaitre les inscetes dont ils ont voulu parler; cctte difficulté est encore augmentéc par l'babitude qu'araient ces autcurs de prendre pour noms spécifiques des pucerons, les noms des plantes sur lesquelles ils se trouvaient, ignorant sans doute que la même espèec de pucerons vit souvent sur plusieurs plantes

- Metamorphosis et llistoria naturalis insectorum, 3 vol. in-80, 1658. 
différentes et que la mêne plante est souvent habitée par plusirurs espèces de ces insectes. Cetle deuxième période de l'histoire scientifique des pucerons correspond à la dernière moitié du sièele dernier; elle a été suivie par une troisième période qui s'étend jusqu'à c'es dernières années et qui est surtout signalcée par les travaux remarquables et ies belies recherehes anatomiques de Dutrochet, Léon Dufour, Siebold, Kyber, Morren, etc., et les curicuses observations de Schmidberger, lesquelles sans expliquer encore la manière dont s'opère en une fois la fécondation de plusieurs générations, mirent cependant hors de doute l'existence des deux sexes, la nécessité de leur réunion dans certains cas déterminés et détruisirent ainsi la supposition d'hermaphrodisme, faite par leurs devanciers. $\Lambda$ ces découvertes déjà trèsimportantes, il faut encore ajouter l'observation que certains pucerons produisent tantót des acufs et tantót des petits vivanls, d'autres toujours des petits vivants, tandis que quelques espèces, rentrant dans la loi générale, paraissent toujours pondre des cufs.

Dans une dernière période, qui conıprend l'époque actuelle, les traratux sout plus particulièrement technologiques comme le sont eeux de Hartig, de M. Walker, de M. Koeh, de Burmeister et de Kaltembach. Ce dernier auteur est celui qui m'a servi de guide dans la description des espèces de pucerons qui vivent sur le poirier; bien que son ouvrage ait été publié en 1843, pour des raisons que j'aurai plus loin l'oceasion de dèvelopper suffisamment, je l'ai préfërè à celui de M. Koch, qui a été publié en 1857.

En général, les auteurs qui, en France du moins, se son, occupés des pueerons, ont pris pour types les espèces les plus répandues (celles du rosier, du pêelıer, du poinmier) et semblent aduettre que toutes les autres ont des liabitudes analogues. Kaltembach, bien qu'il n'ait pas le premier signalé les différences qui existent sous ce rapport, a établi trois divisious, selon que les pucerons se reproduisent par des aufs et par des petits vivants, conme c'est le cas le plus général, ou qu'ils pondent toujours des oufs, ou enfin qu'ils ne font que des petits vivants. 
Il désigne ces trois eatégories par les noms de vivi-ovipares, ovipares et vivipares, qualifieations qui se comprennent d'ellesmêmes. Je suivrai les mềnes divisions en faisant remarquer, dès à présent, que les deux dernières renferntent des insectes eaeore peu eunnus et sur lesquels il y a encore une ample noisson de faits nouveaux à observer.

Si, pendant l'lriver, on examine avec attention les pousses de certains arbies, un trouve sur quelques-unes, quelquefois en grand nombre, des grains noirs, brillants, ovoïdes et qui sont fixés sur l'écorec par un enduit glutineux. Quelle que soil la rigueur de l'hiver, si l'on ouvre ees corps ovoïdes au printemps, on trouve un jeune puceron dans leur intérieur. C'est qu'en effet ce sout les œufs des pucerons dont nous allons suirre les évolutiuns.

Dès que la température conmence à ètre plus douce et moins variable, mais surtout quand la sève commence à circuler dans les plantes, les jeunes pueerons sortent de l'euf en poussant devant eux une sorte de eutrerele qui le termine à un bout; presque tous les œufs éclosent a la mểne époque; et, en quelques jours, deux ou trois tout au plus, toute la nichée est sortic de la eoquille. Au moment oú le jeune puceron sort de l'œuf, il est tout à fait semblable à la mère aptère dont il provient, sauf eependant la conleur qui d'ordinaire est plus pàle, au moins dans quelques parties du corps. Les anatomistes ont constaté la présence de jeunes embryons dans le corps de ces pueerons qui viennent de naitre.

La jeune larve eroit assez rapidement de volume, probablement par l'introduction de l'ait dans ses organes, et au bout de quelques heures, elle va se fixer sur la partie du végétal où elle doit trouver sa nourriture. C'est le plus ordinairement sur les parties vertes et tendres, les bourgeons, les jeunes feuilles, les pousses en train de se développer, ete., qu'ils se rendent et où ils se fixent les uns près des autres, la tête tournée dans le même sens ou vers un centre commun; là ils enfoneent leur trompe dans le parenchyme de l'épiderme et pompent le suc ou la séve 
qui doit leur servir de nourriture. On comprend dè-lors que ces nombreuses piquires et la déperdition de sève qui en résulte, sur des pousses en voie de développement, amènent dans leur organisation une perturbation qui les déforme plus ou moins et produit ici la formation de galles ou d'exostoses, là une courbure des feuiles ou des pétioles, leur décoloration, etc.

La déformation particulière des feuilles du pêcher, du poirier, des pruniers, etc., que les jardiniers appellent la cloque, n'est cependant pas toujours produite par les pucerons. On comprend en effet, que lors du développement des feuilles, s'il survient uu changement brusque de température, la sève s’arrète dans les vaisscaux de la feuille, celle-ci tourmentée par l'affluence du liquide nourrieier et ne pouvant plus l'élaborer, se déforme, se contourne, se crispe, en un mot commence à se cloquer. Si alors quelques puccrons paraissent, ils trouvent un abri convenable et une nourriture toute prête pour leur subsistance. Ils ne feront ensuite, par leurs nombreuses piquires, que favoriser le développement du mal qui les a précédés, et à la permanence duquel ils ne font souvent que contriluer pour une faible partic. Il me parait bien démoutré que la cloque peut exister sans les pucerons; outre la preuve qui précède, on peut encore dire que eet aceident se montre tres-souvent en avril et en mai, époque de l'année où les pucerous n'out pas cneore paru, ou ne sont pas très-abondants. Une teinpérature chaude et soutenue pendant quelques jours la faitsouvent disparaitre, bieu que celte circonstance soit, ainsi que nous le verrons plus loin, très-favorable à la multiplication des pucerons.

Un arbre sain et vigoureux peut quelquefois être abondamment pourvu de pucerons sans apparence de cloque, tandis que celleci se montrera tout à coup s'il survient un abaissement brusque de tempéralure ou si les racines de l'arbre viennent à péuétrer dans un sol moins favorable à sa végétation. Enfin, un dernier argument pour détruire une erreur assez généralement répandue, e'est que les arbres, les pêchers exceptés, abrités ou bien exposés, sont moins sujets à ln cloque que les autrcs, et que cependant il 
est bien reconnu que ces circonstances sont plus particulièrement favorables à la multiplication des pucerons. Quant à la formation des bourscs ou des ressies que l'on rencontre sur les feuilles de certains arbres et dans lesquelles virent des pucerons, Réau unur en a donné une explication si claire et sinette, que je crois inutile d'insister davantage sur ce point.

Les pucerons nouvellement nés ou fixés sur les feuilles ne tardent pas à subir un premier cliangement de peau, c'est ordinairement le troisième ou le quatrième jour après leur naissance que cette mue a lieu; elle cst suivic de trois autres mucs à des intervalles de quatre ou cinq jours au plus. Les pucerons ćtant des insectes à métanorphoscs incomplètes, on est très-étonné de voir ces clangements de peau se succéder sans que la larve présente, après la secondc ou la troisième mue, les moignons caractéristiques de la présence des ailes. C'cst qu'en effet ces larves the doivent pas en acquérir, mais rester aptères pendant toute leur vie, comine les femelles de plusieurs insectes des autres ordres. Souvent, le jour même de celte dernière inue, le plus ordinairement le leudemain, on voit les femelles de pucerons pondre des petits vivants, sans qu'il y ait cu de fécondation, puisque tous les ocufs ne donnent naissance qu'à des fenielles. C'cst au moment de cctte dernière transformation que les pucerons acquièreut la petite queue qui termine leur abdomen, ou tout au moins que cet appendice prend le plus de développement.

Ce premier accouchement, qui a urdinairement lieu du neusième au douzième jour qui suit la sortie de l'œuf, ne tarde pas à être suivi de plusieurs autres qui se succèdent à des intervalles plus ou moins rapprochés: suivant les especces et suivant la température. Il est incontestable pour moi, que celle-ei exerce une influence extraordinaire sur la plupart des phases de la vie et du déreloppemcut des pucerons, ct particulièrement sur'la ponte, la durée de celle-ci, sa fréquence, le temps pendant lequel elle peut ayoir lieu, etc.

On peut facilement observer l'accouchemeut des pucerons; car 
dans les colonies nombreuses, et par un tenups favorable, il y. a presque tuujuurs quelques femelles occupées de ec soin. Le petit puceron sort du ventre de la suère le derrière le premier; presque toujours on lui voit remuer les pattés avant d'cttre complétement dégagé. En ce moment les pucerons sont un peu allongés et trèspeu colorés dans la plupart des especes; les yeux sont souvent les seuls organes qui aient une teinte plus foncée et qui soit en rapport avec celle qu'ils doivent avoir plus tard. La ponte d'un petit dure plus ou moins de temps, selon la vigueur de la mè̀e el l'état de l'atnosphère. J'ai vu quelquefois cette opération ne durer qu'un quart d'heure, tandis que d'autres fois, je l'ai vue ne se terminer qu'au bout d'une heure.

Les accouchements se succèdent a des intervalles de denx ou trois heures, et les mères n'ont pas l'air de s'occuper de ce que devient leur progéniture. Lc jeune pucerun, une fois dégagé tout a fait, ce qui a ordinairement lieu à la suite d'un mouvement de haut en bas que la mère fait avec son abdomen, se cramponne à l'aide de ses pattes aux poils de la feuille ou de la pousse, remue ses antcunes, dresse ses cornicules et marche enfin pour aller se placer à cóté de ses aỉnés, frères on cousins on mème sur eux; une fois eu place, il enfonce sa troupe dans l'épiderme de la plante et y pompe le liquide qui lui sert de nourriture.

Schuidberger, qui a vu des fenclles accoucher encure après le coucher du soleil, dit qu'elles ue pondent pas pendant la nuit. Chez moi, jai vu souvent accoucher des pucerons du rosiev et des pucerons dı pommier à la lumière artificielle; el, pendant le jour, mais daus l'obscuritć, j'ai également constaté la naissance de plusieurs petits puccrons; je crois donc que, si, la nuit, la ponte est interrompue, cela tient uniquement à l'abaissemeut de la température et que les choses se passent alors comme il arrive pendaut les mauvais temps durant lesquels on ne voit plus que de rares accouchencuts, lesquels sont toujours plus longs à se terminer que dans les tcmps ordinaires. Après avoir pondu des petits pendaut huil, dix ou douze jours, la incre puceron se retire à l'écart, maigrit et linit par périr, après aroir pris souveut 
une couleur et une forne si différentes que l'on croirait facilement à l'existence d'un nouvelle espèce, si l'on n'avait pas suivi tous les ehangements suceessifs qu'elle vient de subir.

D'après ce qui précède, on voit qu'à moins de cireonstances tout à fait exceplionnelles, une femelle de pucerons ne saurait produire plus de quatre, six ou huit jeunes au plus par jour, et comme aussi, à mesure que la mère vieillit, le nombre des accou. chements faits en un seul jour diminue, il en résulte que le nombre total des jeunes produils par une seule femelle est d'environ .40 à 50 , très-larement plus, et plus rarement encore de 80 à 90 , comıne le dit Réaumur.

Les pucerous de la deuxième génération vont chacun suive la mêne évolution que leur mère, c'est-a-dire qu'ils subiront trois ou quatre mues sans acquérir d'ailes. Tous sont des femelles, lesquelles vont à l'àge de huit, neuf ou dix jours, toujours selon la température, pondre de nouveaux pucerons sans cependant avoir été fécondées.

Si le tenups est peu favorable, tous les jeunes pondus seront eneore des femelles qui ne preudront pas d'ailes et qui pondront aussi des petits sans avoir reçu l'approehe du mále. Dansla najorité des cas, si le temps est convenable, les premiers jeunes pondus seront aptères, mais ceux qui les suivront, après avoir subi deux elıangements de peau, présenteront des moignons d'ailes; res uymphes subissent une nouvelle nue, après laquelle les ailes deviennent encore plus apparentes, et enfin à l'ảge de lıvit ou dix jours, elles se retirent à l'écart, changent une quatrième fois de peau, et acquièrent des ailes transparentes présentant les caractères que j'ai indiqués plus haut. Ces individus ailés, qui sont toujours des femelles, restent deux ou trois jours tranquilles, elles ne sont pas fécondées, et cependant quelquefois, après avoir pondu un ou deux aufs à còté de leur sœur, elles s'envolent pour aller sur d'autres plantes fonder de nouvelles colonies. La troisième génération n'est donc composée que defemelles aptères ou ailées, lesquelles, sans ètre fécondées, pondrout des petits qui, selon les circonstanees, ne seront non.plus que des femelles ailées ou aptères. 
La présence des individus ailés correspond ordinairement ȧ la troisième, à la quatrième ou à la cinquième génération, quelquefois à une seule ou à deux de ces générations, naais au delà on ne trouve plus, encore une fois, que des générations counposées uniquement de femelles aptères et, clıose remarquable, e'est que l'on n'olsserve pas de différence eutre les pucerons qui proviennent des femelles ailées et ceux qui sont produits par les femelles aptères.

Les choses se passent ainsi pendant un certain temps, variable saus doute selon los espècès et selon Ics influences extérieures; Bonnet a compté onze générations successives, Schmidberger en a observé jusqu'à dix-sept, et cela sans aucune espèce de fécondation!

Au moment oủ la végétation commence à perdre de sa vigueur, vers le mois de septembre, pour les pucerons du pommicr et du poirier par exemple, on trouve cncore une fois dans les colonies des larves qui sont pourvues de moignons renfermant les rudiments d'ailes, e'est qu'en effet ces nymphes doivent donner des pucerons ailés, mais qui presquc tous seront des mảles. Si, dans ce moment, on ouvre une femelle aptère on ailée, mais de la mèute génération que ces máles, on ne trouve plus que des oufs daus le ventrc et pas d'embryons de pucerous prêts à naitre. C'est à cettc époque qu'a lien l'accouplement. Les màles sont très-ardents, ce qui est nécessaire ru leur petit nombre relativenent à celui des femelles; un seul suffit ordinairenent pour convrir quatre ou cinq femelles aptçres ou ailćes. Après avoir assuré la fécondité extraordinaire de celles-ci, par un procédé dont on n'a pas encore pu pénétrer le mystẻre, les màles subissent le sort commun et périssent misérablenteut. Les femelles atilées et qui n'ont pas été fécondées, aiusi que les fenıelles aptères qui ne l’ont pas été uon plus we pondent pas leurs œufs et meurent avec ces aufs stériles dans le ventre*. Quant aux femelles aptères qui out été fécon-

- Kaltembarlı dit que les femelles ailées, fét:ondées, périssent ėgalenent sans pondre ni petits, ni oeufs, ce qui me parait une nuuvalle anomalie. 
dèes, les unes, et c'est le très-pelit nombre, meurent avant de pondre, les autres se mettent à pondre des oufs, ou plutỏt à accoucher d'ceufs; ear chez les pueerons que j'ai rus pondre, les cufs sortent presque aussi lentement quc les petits; le nombre des ceufs, la rapidité de la poutc... ete., paraissent dépendre des mêınes influences que celles qui ont été rapportées plus haut; ainsi à l'air libre, et pendant la uuit, il n'y a que peu ou point d'oufs de pondus; ell eaptivité, et par une chalcur convenable, la ponte a lieu uuit et jour sans interruption. Les $\alpha u f$ des pucerons sout gros, ovoïdes, enduits d'un liquide visqueux au moyen duyuel ils restent fixés sur la plante; ilssont d'une couleur claire, qui se fonce ou se noircit après deux ou trois jours.

$\Delta$ la premièrevue, les $\alpha u f s$ des pucerons semblent disposés sans ordre; cependaut, on les trowve cu plus graude abondance près des boutons, ou du cóté de la branche la moins exposéc au mauvais temps. Ces ceufs passent l'hiver et supportent souvent les froids les plus rigoureux sans perdre la propriété qu'ils possèdent de renfermer la fécondation de dix-sept générations. Cette propriété, d'après les abservations de $\mathrm{K}$ yber, se conserve pendant plusicurs années sans perdre de sa foree. Quelquefois les cufs éclosent déjà à l'automne, alors les femelles aptères qui en résulteut, se réfugient, aux premiers froids, dans les crevasses, sous les feuilles tombées a terre, sous les ćcorees, etc., passent l'hiver engourdies, et, au printemps suivant, elles vont continuer leur évolution comme leurs sours qui n'écloront qu'à cetle époque.

Les nymphes de pueerons se font reconnaitrc par les moignons cylindriques et la coloration blanchatre de ees appendices. Mais les pucerons ailés, màles ou feınelles, sont quelquefois si différents des femelles aptères que l'on a peinc i croire qu'ils appartienneut à la même espèce; lcur corps plus étroit est toujours coloré d'une manière plus variée que celui des individus privés d'ailes. Ces inscetes, màles ou femelles, ne peurent sc conserver sans altération pendant quelques jours seulement : Icur forme varic, les coulcurs s'altìrent, les caractères les plus essenticls 
s'oblitèrent ou changent au point qu'il n'est plus possible d'en fairc la description; e'est celte difficulté qui empêche de les conserver dans les collections; c'cst aussi ce qui rend leur élude si compliquéc et si peu répandue.

En général, les pucerous aiment unc température élcvée acconpagnée d'une atmosphì̀re lıumide; c'est pour celtc raison que leur nombre aummente à mesure que l'on s'approche du midi, et que, dans les serres, oủ ils trouvent toutes ces conditions réunies, ils se propagent avec tant de facilité et tant de persistancc. Les variations brusques de température leur sont trèspréjudiciables, ainsi que les coups de vcut, les giboulées, les orages ou l'cxposition au nord, sur les terrains arides élevés ou décourerts. Les pays de montagnes ou les bois couveris dans lesquels le soleil ne pénètre jamais, leur sontégalement peu favorables; le fond des vallées, les jeunes taillis, les enciroits abrités, les jardins clólurés, ceux qui sont cxposés au midi ou à l'est, leur conviennent au contraire parfaitement. Mlais ce qui par dessus tout leur est plus particulièrement favorable, c'est une végétation vigoureuse et une sèvc abondante, circonstances qui dépendeut páticulièrement des soíns donnés à la terre, des produits qu'on $y$ cultive, et enfin de la taille qu'on pratique sur les arbres, laquelle détermine la formation de pousses tendres qui rćunisscut toutes ces conditions. Or, comme on ue trouve ees conditions réunies que dans les jardinş ou dans les licux cultivés, il cn résulte que c'est à lcur présence qu'il faut attribuer ce fait, observé depuis longtemps, que les puccrons sont surtout abondants près des villes, près des villages ou des cndroits habités, tandis qu'ils diminucut sensiblenent eu nombre et en espèces dans les'forèts, dans les claanps ou dans les terrains incultes.

En général, les plantes herbacées en wourrisscnt proportionncllement moins d'espèces que lcs plantes vivaces, et cellcs-ci encore moins que les arbres ou les arbrisseaux. Ainsi les Conifères cn nourrissent ueuf ou dix espèces; le treruble, lsuit; lc poirier, sept; le clıéne, six au moins; le peuplier, cinq; l'orme, quatre; Ic groscillicr, trois seulenent; le rosier, deux; sur le fran- 
boisier autant, etc. La plupart des plantes herbacées fabsinthe, avoine, betterare, chardon, cerfeuil, ciguë, ćpilobe, fève, ortie, la plupart des Composées, eté), n'en nourrissent que deux especes; on n'en troure qu'unc seule espèee sur un plus grand nombre encore. Il y a eependant quelques exceptions : ainsi on conuait trois espèces de pucerons qui vivent sur la Tanaisic, autant sur la Millefeuille et cinq sur la Capsella bursa pastoris. Certaines espèces vivent sur plusieurs plantes à la fois, mais en général, ees plantes apparticnneut au même genre ou tout au moins á la même famille. Celles qui vivent sur les plantes vivaces sont moins polyphages; et, à part quelques espéces très-comnunes, on n'en connait gnére qui vivent a la fois sur des arbres appartenant à des familles 0.1 à des genres éloigués daus la elassifieation. Enfin, quand un arbre nourrit plusieurs espèces de pueerons, il arrive souvent que elıacune d'elles affecte un lien particulier d'éleetion; ainsi, le cliène en présente une qui vit sur le trone, une autre sur les rieilles branclıes, une troisième sur les jeunes rameaux et les autres sur les feuilles; sur le poirier nous en trouvons sur les racines, sur le tronc et sur les feuilles ou sur les pousses vertes.

De même qu'il y a des pucerons qui peuvent sc nourrir indifféremment du sue de plısieurs plantes, de mème, il y a des espèces qui vivent sur une espèce botanique dans une loealité et sur une antre dans un antre lieu. Enfin on trouve des plantes sur lesquelles on ne reneontre jamais de pueerous et dont la sève parait ĉtre un véritable poison pour ees petits animanx; les Cryptoganies sont plus particulièrement dans ce eas et jusqu'ici ón n'en a encore signalé ancıue espècec sur les plantes de eette famille. Les Labićes u'en nourrissent que denx, les Graminées et les Cypéracécs trois seulement; cn général, les Monoeotylédones sembleut très-pen conrenir à ces insectes.

Il existe, au contraire, des familles végrétales qui scmblent plus partienlièrement destinées à servir de nourriture aux puecrous, daus ec cas se trourent les Composées et les Crucifères parmi les plantes berhacées; les Conifères, les Amentacées et les Rosa- 
eées parni les arbres ou les arbrisseaux. On comprend d'ailleurs que la longévité d'une plante influe autant sur le développement des pucerons que les autrcs circonstances de la végétation de cette. plante ou la tempćrature, ete.

Les pucerons sont en général de très-petite taille, de un à quatre millimètres au plus, et de couleurs variées; cependant les noirs et les verts sont les plus répandus; lcur corps est mou et leurs mouvements sont très-lents. Lcs cornicules qu'ils portent sur l'abdomen sont de forme et de longueur différentes et fournissent de très-bons caractères spécifiques. It parait ccrtain que ccs organes, exclusivement propres à ces insectes, servent à la respiration; cependant à leur base interne, il existe des glandes qui sécrètent constamment un liquide sucré, diversement coloré, suivant lcs cspèces, et qui s'écoule cn petites gouttelettes par l'extrémité de ces cornieules. En faisant l'histoire des fournis, nous arons,vu que c'est à la recherche de ce liquide sucré qu'il faut attribuer leur présence parmi les pucerons, et je répétcrai encore une fois ici que ce sont bien les pucerons qui altirent les fourmis et non cellcs-ci qui amènent ces Homoptères sur lcs plantcs.

Les pucerons vivent en sociétés plus ou noins nombreuses; dans ecs colonies on rencontre, suivant l'époque de l'annce, des femelles aptères et des jeunes sculement, ou un mélange d'insectes aptères, de jeunes larves, de nymphes, d'insectes ailés, de femelles qui commencent à pondre, d'autres qui meurent de vieillesse, etc. Cependant, qucl que soit le nombre d'habitants de ces colonies, le muavement et l'agitation ne s'y rencuntrent jamais ; c'est qu'en effet les pucerons sont lents, cmbarrassés dans leur démarche, et c'est à pcine s'ils cherchent à échapper anx nombreux ennemis qui leur ont été donnés par la nature et qui vivent sans obstacle au milicu d'cux. Quelques individus aptères, quand on les inquiète, contraetent quelquefois leurs membres, contrefont le mort et se laissent tomber sur le sol; les insectes ailés sont plus agiles et s'enrolent facilement au moindre danger. Les Pucerons qui vivent dans les vessies ou les galles qui 
se développent sur les plantes à la suite de leurs piqúres, som encore plus lents it se mouvoir et ils sont quelquefois si pressés dans leur habitatiun, qu'on a peine it comprendre comment ils peuvent tous approcher de la surface pour y puiser leur nourriture; leur trompc n'étant ordinairement pas aussi allongée que celle des espèces de pucerons qui vivent sur le bois on sur les écorces.

D’après ce qui a étć dit plus liaut de la fécondité des pucerons, on roit que Ic nombre des individus produits par une seule femelle sc compose de la somme des ternics d'une progression géométrique dont la raison est égale au nombre des individus prurluits par elıaque génération, et le nombre des tormes égal à celui des générations produites par la unème femcllc. Or, si l'on prend pour moyeunc le chiffre de 20 individus, qui cst bien certainemcut uu mininum, pour lc nombre des petits pandus par unc femellc, et 8 seulement pour le numbre des gćnérations, on trourera que celle famille se compusera de près de trente billions d'individus. C'est-à-dire que la punte de quelques femelles suffirait pour couvrir de pucerons tuutes les plantes d'uue eontrée sur lesquelles ils peuvcut vivre, ct que par l'cnvahissement successif de ces insectes, In végétation aurait bicntót disparu. lleureusement que celui qui a donué à ces petits animaux une si prodigiense féeundité, leur al dlonué en mème tcmps un nombre d'ennemis tcl, qu'il est souvent cxtraordinairc qa'il cn échappe quelques-uns, ct que si toutes les générations de pucerons étaicnt compusécs d'individus aptères, leur race finirait par dispraraitre. En effet, les pucerons non ailẹ́s sont si peu aptes aux émigrations que, si l'un met me branche de poirier couverte de pucerons dans un vase sans eau, ces insectes périssent en grandc partic à mesure que les fenilles se dessèeliént on se fanent, bicn qu'ou ait pris la précaution de incttée à leur portée des branches toujours fraiches et sur lesquelles ils trouveraicut unc nourriture abondante et semblable à cclle quc l'arbre vivant lcur fuuruit. Cependant, si par accident ou autrement, ils sunt enlevés de la plante ou ils vircut, ils uc tardent pas à y revenir, si tuntcfois 
ils ne rencontreut pas en chemin un de leurs ennemis au rang desquels on est étonné de trouver la fourmi qui, oubliant les services rendus, s'empare de l'animal et l'einporte comme proie dans la fourmilière *.

Si donc la nature n'avait pas pourvu a celle apathie des pucerons, ou, si l'on veut, ál'amour du lien de la naissance, par la création de femelles ailées chargées de la dissémination de l'espècc, il serait arrivé infailliblement que la inultiplication des pucerons sur la même plante aurait amené la mort de celle-ci, et que, par suite, toute la colonie aurait péri á soll tour; cette double conséquencc est trop en delıors des admirables lois qui régissent l'équilibre dans toute la série des êtres organisés, pour que le Créateur n'ait pas donné des ailes aux femelles chargécs de porter au loin les produits de leur race, et assurer ainsi la conservation de l'espèce, par de nouvelles colonies placées en deliors des conditions oi sont les générations qui doivent continuer à vivre au berceau de la famille.

Sans parler des oiseaux ou d'autres animans qui font une ample consommation de pucerons, uous trouvons parmi les insectes, et presque dans tous lcs ordres, les ennemis lcs plus noinbreux et les plus roraces. Dans la première partie de ces notes, j’ai déjà eu occasion de citer les Coccinelles à l'étal de larres et d'insectes parfaits, conme faisant une guerre à ınort à ces petits inseetes. Plus haut, j’ai indiqué les larres d'llćmćrobes. Un bon nombre d'llyménoptères de la famille des Ichmeumoniens appartenaut aux genres Ephedrus, Pempliredon, eie., déposent leurs aufs sur les femelles aptìres, et les larves qui en éclosent, virent aux dépens des pucerons; quelques espèces du genre Crabı approvisionnent leurs larves avec des quantités notables de pucerons. Une ehenille de papillon du genre $L$ y'macoles, mange les pucerons

- C'est probablement ee fait, que j'ai souvent observé, qui a fait dire d lluber et répéter depuis par un grand nombre d'auteurs, que les fourmis emportent des pucerons dans leurs fourmilières pour se procurer eonstamment le liquide sueré qui sort der eornieules de ces insectes. 
du pêclıer ; parmi les Hémiptères, on cite aussi le genre Lygœus. Mais tous ces ennetnis réunis sont peut-être encore loin de faire cnsemble autant de mal aux pucerous que quelques espèces de Diptères appartenant au genre Syrphus; voici comment Macquart parle de ces larves ct du mal qu'elles font aux pucerons. a Les larves de Syrphius, ou au moins celles d'une partic du genre, éclosent sur les tiges ou les feuilles couvertes de pucerons dont elles sout des ennemis aussi redoutables que les larves des Hémérobes, quoiqu'clles soient conformées d'une manière bien moins hostile en apparence. Semblables à celles des autres diptères, elles sont sans pieds et sans yeux; mais, nées au milieu des groupes d'une race stupide, il leur suffit d'allonger le corps et de porter la tête de cóté et d'autre pour trouver leur proic; quand elles out dévoré tout ce qu'elles ont á leur portée, elles avancent ell rampant, et en rencontrent de nouvelies. Leur bouclie est armée d'un organe de succion qui a été décrit avec beaucoup de dévcloppement par Réaumur... Lorsque le développement des larves cst arrirć ì son terme, elles se fixent sur les tiges ou sur les feuilles, en s'y collant au moyen d'one liqueur visqueuse qu'elles font sortir dc la boviche. Le corps se raccourcit, la peau se durcit et elles passent ainsi à l'état de nymplic. 》

A toutes ces causes de destruction, il faut encore ajouter les orages, les coups de vent, les gelées tardives du printemps ou les gelées précoces de l'autonne, et l’on sera convaincu que la fécondilé des puccrons n'est pas trop considérable pour contrehalancer leurs pertes. Aussi, gràce à ces admirables conditions d'équilibre, les pucerons restent-ils, en général, dans les limites ordinaires de tous les insectes nuisibles que nous avons eu occasion d'étudier jusqu'ici, c'est-à.dire que, sauf de rares exccptions, ils ne causent qne des dommages partiels et plus ou moins limités à une petite étendue de terrain. C'est cependant á l'absence de l'un ou de plusieurs des éléments de destruction que je vicns de signaler, et probablement aussi à un concours particulier de circonstances favorables, que l'on doit attribuer les invasions 
extraordinaires de pueerons qui ont été sigualées et qui ne peuvent se comparer qu'ả eelles dont les sauterelles douneut de si fréquents exemples dans le nord de l'Afrique et en Orient. La plus remarquable de ees invasions est eelle que M. Morren rapporte de la manière suivante : « L'lliver de 1833 à 1834 fut extrêmement doux, el l'été de 1834 extrêmement elıaud et see; il se passa des mois entiers sans pleuroir. Un hortieulteur, Van Mons, prédit dès le mois de mai que tous les légumes seraient dévorés par les puecrons. Le 28 septembre suivant, alors que l'épidémie du elıoléra venait d'étendre ses ravages en Belgique, tout à eoup, une unée de pueerons ( $A$ phis persica, Morren) parut entre Bruges et Gand. Le lendemain on les vit à Gand voltiger par troupes en telle quantité, que la lumière du jour en était obseurcie. Sur les remparts on ne pouvait plus distinguer les murs des habitations, tant ils en étaient eouverts. On se plaignit du mal qu'ils faisaient aux yeux. Toute la route d'Anvers à Gaud était eouverte de leurs innombrables légions; partout on disait les avoir vues subitement; il fallait se couvrir les yeux de lunettes et le visage de mouehoirs, pour se préserver du elıâtouillement qu'oceasionnaieut leurs six pattes. Il parait que ees inseetes étaient interrompus dans leur marehe par des montagnes, des eollines, des ondulations de terrain, mème peu élevées, mais suffisantcs pour influer sur le vent. Les différentes directions que l'on a eonstatées, doivent faire supposer que l'émigration a eu un centre, et que ee foyer était un point d'irradiation, puisque des troupes ont émigré vers le nord, vers l'est et vers le sud, l'ouest étant la eờte maritime elle-même.»

Si les émigrations en masses sont rares, l'invasion de eertaines espèces de pueerons dans des eontrées où elles n'étaient pas conıues auparavant, n'est malheureusement plus un fait à rérifier. Les éehanges qui se font entre des eontrées très-éloignées, de plantes ou d'arbres eultivés, favorisent ees introductions et ees aeelima. tatious peu désirables. Ainsi, le pueeroulaniger était inconnu en Angleterre, a la fin du sièele dernier; de lá il s'est répandu sueeessivement en Bretagne (1812), en Normandie, dans la 
Flandre et en Belgique, où l'on ue remarqua sa présence qu'en 1820. Cet insecte parait nous venir d'Amérique où nous avons envoyé notre puceron du pommier, lçuel s'y est si bien acclinaté que, malgré les nombreuses espèces d'Hémérobes qui vivent dans l'Amérique du nord, M. Aza-fitclı en a déjà décrit neuf variétés bien caractérisées.

D'après tout ee qui précède, il ne parait pas doutenx que les pucerons ne soient des insectes émineurment préjudieiables aux arbres ou aux plantes sur lesquels ils vivent, et que dans bien des cas on les ait considérés comme de véritables fléaux. Cependant, tous les auteurs sont loin de partager cette opinion; et, après avoir défendu ces insectes contre ceux qui les accusaient de la cloque, il nous faut maintenant prouver qu'ils sont bien réelleuent nuisibles à l'agricultıre.

S'il est vrai en effet que, dans certaines limites, une plante forte, saine et vigoureuse peut facilement supporter la déperdition de sève que lui canse des pucerons, on peut bien eoncevoir cependant qu'il ne saurait en être toujours ainsi. Une plante cultivée, c'est-îdire en état de dégénérescence parl'hybridation ou une culture forcée, ou un arbre mutilé par les tailles suecessives, l'ćbourgeonnement ou toute antre pratique destinée à le eontraindre à donner des frints ou des fleurs, ou enfin, une plate de serre forccic de sc développer rapidement et dans des conditions qui s'éloigncnt plus ou moins de son état de nature, ne peuvent, sans grands ineonvénients, perdre eneore une partie, quelque faible qu'elle soit, des sues nutritifs dont elle a besoin et qui, dans bien des cas, sont déjả trop pauvres ou sont dans un état maladif. Schranck, Kaltenıbaelı, et d'antres encore, auront bean dire que des groseilliers ayant souvent leurs fenilles couvertes de pucerons leur frnelification est cependant aussi abondante; que des rosiers infestés de pucerons n'eu donnent pas moins leurs brillantes fleurs au nois de juin, ou que les tiges du sureau, du nerprun, du fusain, sont quelquefois convertes de ces insectes, sans qu'aneun fruit de ces arbrisseaux paraisse avoir avorté ctc.; je répondrai que des plantes cultivées ne somt pas dans un état de vigueur comparable à eelles 
que l'on eite, que les plantes de serres ont une eonstitution qui les rend propres à se courrir de pucerous et qu'clles sont, par dessus tout, bien moins en élat de supporter ees insectes en aussi graud nombre ou aussi longtemps sans sonffrir de leur présence.

D'ailleurs, et en dernière analyse, le mal causé par les pucerous est relatif et proportionné à l'importance que l'on attaclı aux plantes qui en sont envalıies. Cequi est insignifiant dans une forêt ou daus un elıamp de luzerne, ne l'est déjá plus, quand il s'agit des poiriers, des pommiers ou des pruniers, et derient un dommage réel, quand il s'agit de la eloque du pêcher déreloppée ou aggravée par les pucerons. Enfin, ecux-ei causent de véritables désastres, quand il s'agit des plantes eultivées dans une serre, quii réunissent, comme on le sait, toutes les conditions pour amener un rapide aecroissentent dans le nombre de ces inseetes

Si l'on voulait passer en revue tuutes les publications faites en vue de la destruetion des pueerons et diseuter la valeur de tous les procédés qui ont été proposés, il faudrait faire un travail considérable et dnquel il ne sortirait rien de bien important ni de bien instruetif. Les procédés les plus bizarres, les pratiques les plus contradietoires et les plus en désaceord avee les habitudes de ees insectes ont été préconisćes avee plus ou moins d'enthousiasne et toujours présentées, par leurs auteurs, comme infaillibles. Sans cntrer dans ces détails, je vais seulement indiquer bric̉vement les moyens qui me paraissent les plus recommandables, en y ajoutant les olsservations faites depuis plusieurs anućes par les jardiniers les plus eompétents de nos environs.

L'éerasement direet des insectes au moyen de la main; d'une brosse, d'unc épouge, est tris-efficaee, mais il est tellement long, qu'il n'est guère applicable qu'aux plantes de serres auxquelles on attaelie un soin tout particulicr. Les arrosages avee l'eau, la lessive, les solutions alealines, l'urine, l'eau salće, les décoetions de suie, d'aloës, d'absinthe, de noyer, de tabae, ete., réussisseut rarenent, paree que les pueerons, qui eherehent toujours à sc mettre à l'abri cle la pluie, se trourent par eela même à l'abri de ees irrigatious. Ia plupart de ees solutions ne peurent d'ailleurs 
devenir effieaces que si elles présentent un ecrtain degré de eoncentration; ce qui, dans ee eas, est plus nuisible anx plantes qu'aux pucerons.

Le barbouillage des plantes avee le goudron, l'huile, ele., présente à la fois l'ineonvénient de l'éerasement par sal longueur et eclui de l'arrosage par le tort que ces substanees font aux plantes. L'insufflation des eendres fines, du plâtre ou de la elaux ne parair produire aueun effet; la poudre iusectieide agit beaucoup m!eux, quand elle est appliqquée comme il a été dit plus haut a propos du Tigre. Seulement il faut de toute nécessité recom. mencer l'opération tous les luit ou dix jours, ee qui devient dispendieux.

Les funigations faites dans les serres ou sur les espaliers, aree le tabac a chiquer, réussissent on ne peut mieux, mais, comune pour les insufflations de poudre, il faut les l'ecommencer souvent, dans les serres surlout, parec que les puecrons qui y ćehappent, ne manquent pas de eontinuer à pondre el a produire de nouveaux individus. La taille faile an printemps et de bonne lieure, supprime une quantité eonsidérable d'oufs déposés sur les pousses des arbres fruitiers. Pour préserver les greffes ou les pousses qui doivent être eonservées, il faut les barbouiller de terre délayée avee du sang de bouf qui, en se coagulant, devient insoluble et empèche la pluie d'entrainer ec banigeon préservatif. Enfin, dans les serres, on pourrait, avec de grandes ehances de réussise, tenter l'édueation des Coeeinelles on des Iléncirules, qu'il est faeile de se procurer et dont les larves, trnuvant une patture abondante, ne manqueraient pas de prospérer.

La deuxième catégorie de pucerons, que Kaltembael désigne sous le nom de pucerons oripares, ne eomprend, jusqu'ici, qu'un très-petit nombre d'espèces qui toutes vivent sur les Conifères, les unes renfermées dans des galles, dont elles détermineut la formation par leurs piqûres, les autres à déenuvert sur les aiguilles de ees arbres. Ces derniers pucerons pondent des oufs, de ees ceufs naissent au printemps des petits aptères ct d'autres pucerons ailés 
- qui, sous l'une comme sous l'aulte de ces furmes, doivent encore pondie des $\alpha u f s$; celle fois il ne sort de ces aufs que des femelles aplères qui se fixent sur l'arbre. Ceux des puecrons ovipares qui vivent dans les galles, pondent des oufs à l'aulonne, lesquels produisent des pucerous aptères qui passent l'hiver dans ect élat; au printemps suivant, el saus qu'il y ait eu de fécondation, ces femelles sans ailes produisent une deuxiènc génération eoruposée d'individus remarquables par la séerélion laiucusc qui recouve leur corps. Les observations failes sur ecs insecles sont cneore peu nombreuses, et il reste bieu des particularités de leur listoire a fairc commaitre, mais malleareusenent leur élude est encore plus difficile que celle des pucerons ordinaires.

Quant aux puccrons qui font parlie de la elasse que Kalıcmbach noume viripares, ils sont aussi peu connus que les précédents, el commta deux especes appartenant it ee groupe vivent sur le poiricr, nous reviendrons plus loiu sur les faits connus de l'histoire de ces inscetes:

I.e nombre des espèces de pucerous est certaincment tris considérable; M. Koch en décrit plus de denx cents espéees dont uu très-grand nombre doil se trouver dans le déparlement de la Moselle, à en juger par le nom des pliutes saurages ou cultirées sur lesquelles ecl auteur les a lencontrées; mais quand on songe a la richesse de la flore des contrées méridionales et aux condilions, parlieulièrement farorables à leur déreloppement, que l'on rencontre dans ees pays, on sera élonné du nombre d'espèces a faire connaitre, el l'on eomprendra la leutative faite par plusicurs entomologistes pour diviserl 'ancien gente Aphis, de Limé, derenn maintenant le type d'une petite famille parfatitement limiléc *.

- L'ouvrage de Kaltembach est peu répandu en France, et comme il est publić en langue allemande, je crois, avant de passer à la description des pucerons qui ont été signalés sue le Poirier, qu'il ne sera pas sans intérit de donner un tableau des groupes ou des genres nouveaux almis par Kaltembach dans l'ancien genre Aplis, de Linné.

(Ioin le Tablean d'mulre parl.) 
91. Агнтв mazi (Fabr.).

Kaltembach, Monographie des Pucerons, n० 52, page 72.

Synonymic: Aphis mali (Fabr.); - Aphis pomi (Degécr);

- Aphis oxyacantho (Schranck); - Puceron du pommier (Golze); - Aphis pyri mali (Sclımidberger).

Longueur: un à deux millimètres.

Aptènes: Antennes plus courtes que l'abdomen, d'un jaune

Tahleau asialytįue des gentes de la famille des aphidiens.

1

Des ailcs. 2............. APHIDINA.

Pas d'ailes....................... Hyponomeuta.

( Cubilus des ailes émeltant un rameau bifurqué. 3.

2 - - - - - simple... 4.

Antennes de 7 articles, ordinairement

3

aussi longues que le corps ...... Genre ApHis, Linné. Type A. Rosce, L.

Antennes de 6 articles, pas plus longues quela téte et le thorax réunis Genre Lachnus, Illiger. Type $A$. Fagi, Linné.

Antennes de 6 articles; ailes en toit dans lc repos, les inférieures avec 2 nervures transversales .........

Antennes de 5 articles: ailes horizon-

Genre Schuzoneura, Hartig. Type. A. Luniger, llausm.

tales, les inférieures avec une nervurc transversalc.............

Antennes de 6 articles; ailes antéGenre Vacuna, Hayden. Type A. Dryophila, Schranck. rieures avce quatre ncrvures transversales 6.

Antennes de 5 articles; ailes en toit, les antérieurss avec trois nervures transversales ...............

Antennes de trois arlieles; ailes hoGenre Caienases, Linné. Type $\mathrm{Ch}$. Laricis, Linne.

rizontales, lcs antérieures avec trois nervures transversalcs

Gente Puylloxfuna, Boyer de 5 . Type Vacuna Coccinen, Heyd. 
blanchátre avee les trois derniers articles d'un brun noir; yeux d'un brun obseur; trompe d'un jaune blanchátre; le bourrelet et les deux derniers artieles de la trompe brunátres; la léte est rougeátre; le dessus de la poitrine jaunátre; le tour du col d'un jaune verdàtre est épineux. Les cornicules sont noirs, assez longs, amineis vers le bout; la petite queue est noire chez le plus grand nombre, jaunátre

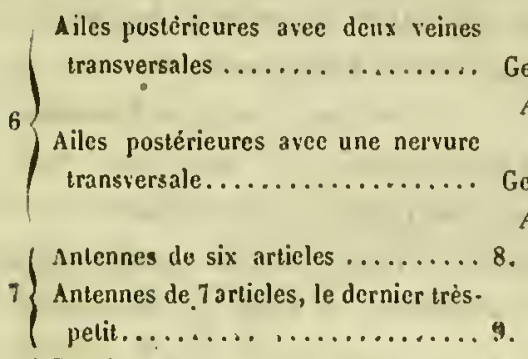

Dernier artiele des antennes arrondi et plus grand quo le précédent... Genre Rnızoвıs, Bu'm. Tỵpe 8

Dernier article des antennes puintu, bezucoup plus petilque le précédent. Genre Fonds, lleyden. Type F. Formicaria, lleyd.

Jambes postérieures longues et sans

Genre Trama, Heyd. Type $T$. Troglodyles, lleyd.

Jambes pustérieures de longueur ordinaire; tarses de deux arlicles ... Genre Panacletus, Heyden. Type P. Cimiciformis. Heyd.

Comme on le voit, le genre Aphis ne comprend plus, d'après Kaltembach, que les puecrons qui ont des autennes de sept arlicles, aussi longues ou presque aussi longues que le corps, et dout les ailes supéricures ont un Cubitus qui émet une nervure divisèc en deux branches à sa jonction au borl extirieur de l'aile.

N. B. Les genres Apus et Lacuxus sunt vivi-uvipares; les genres Chermes, Pnylloxeuna et Vacuna?? sunt ovipares, et enfit, les genres Tetnaneura, Pempuigus, Scinzonelia, et peut-être les autres sunt vivipares. 
chez quelques-uns. Cene queue a les deux einquiemes de la longueur des cornicules; l'anneau anal est d'un jaune brun; les pattes sont jatuatres arec les genoux, l'extrémitć des jambes et les larses noirs.

Altes: Antennes noires, un peiu plus courles que l'abdomen, le troisiène article dentelé en dessous; les yeux d'un brun noiratle, les ocelles d'un jaune blanchàtre; la trompe va presque jusquà la naissance de la deuxieme paire de pattes: elle est d’un jaume pàle avec l'extrémité brunàtle; la téte est noire, l’anneau du col est brun bordé de vert et d'épines obliques; l'anncau pectoral est d'un noir brillant; l'aldomen entièrement d'un vert-pré, les cornicules sont noirs, minces el de longucur moyenne; la pelite queue et le segrment anal varicut du brun au noir; les pattes antérieures sont d'un jaunc sale, avec les genoux, le bout des jambes et les tarses bruns; les quatre pattes postéricures sont d'un brun olsecur avec les jambes el la base des euisses jaumitres. Ailcs unusjarentes avee la nervure costale el le sigma jaunc: grisitte, les autres nervures sont tres-visibles, brunes et termiminćrs par une petite fourche.

Ciclte description est traduite exactement de l'ourrage de Kaltembaeh; elle convient à la majurité des pucerous que l'on ureure en si grande abondauec, daus nos curirous, sur le poumier et sur Ic poiricr. Mais si on lui compare la description que dome M. Koeh (page 107, n"4, fig. 143 el 144), on y lrouve des diflérenees remarquables; lesquelles proureul yue les deux entomologistes ont décrit des iudividus d’àges différents, ou que l'espéce varie aree les localités. Ainsi, M. Koels dit que la tète et le corselet sout noirs mat; qu'il y al sur les auneaux de l'abelumen des ailés, des taehes Iranswersales noires, ete. M. Nordlinger donne du mène insecte une deseription qui se rapporle mieux aux individus de nos envirous qu’à ceux qui sont décrits par Kaltembacł et surtout par MI. Kocls. Ces sortes de coutradietions se reprodui- 
ront eneore plus loin, a propos des autres puecrons qui vivent sur le poirier, elles prouvent eombien la unême espèce varie, el, par conséquent, qu'il ne fant pas aecepter sans contróle toutes les espèces qui se trouvent déerites comme nouvelles dans les auteurs mudernes.

Quand le puecron, dont il est ici question, vit sur le poirier, il présente les caractéres suivants : les individus jeunes et aptères sont d'un vert clair, et leur petile queue est i peine indiquée. Quand ils sont plus grands e qu'ils viemue:ıl de clanger de peau pour la troisième ou la quatriène fois, ils sont d'un jaune verdàtre. Enfin, quand ils sont arrivés à l'état adulte et qu'ils commeneent a pondre, ils sont d'un vert plus ou moins foncé, et leur quene est très-apparente. La couleuc noire de l'extrónité des antennes s'étencl sur un plus on moius grand nombre d'artieles, quelquefois mème, il ı'y a que le aleruier qui soit de celle couleur. Quand ee puceron est jeune ou qu'il vient de changer de peau, les antennes, la trompe et les patles sout entièremenı jaunàlues. La téte et le corselet présentent souvent une teinte roséc ou rongeitre, surtout cluez les vieilles meres; eelles-ei ont l'abdomen plus on moius reulle, il se déprime et se fronce sur les cótés a mesure qu'aurmente le nombre des pucerons ou eelui des ocufs qui sont pondus.

Cette espéce, qu’à la deseription 'qui préeède, ou pourra recon. nailre à tous les àges, se reneontre sur le poirier, sur le poumier, sur l'aubépine el mème sur le sorbier, le coignassier et le uêtlier. C'est pendant les mois de juillet et d'itout qu'elle est le plus abondaule sous les feuilles qu'elle fait crisper; quelquefois aussi on la trouve sur les jeunes pousses et sur les drageons des racines. L'aeconplement se fait en septembre on en octobre; les acufs, d'abord verdatres, deviennent bruns à la fin du prentier jour et noir brillant trente-six ou yjarante heures aprés leut ponte; celle-ci cesse dès les premiers froids. Les cufs, qui paraissent d'abord disposés sans ordre, sont cependant, sur les e's. paliers, plus abondauts autour des bourgeons, rt placés plutót rlu cóté du urur qu'en avant; rarement on en trouve sur les pétioles, presque jamais sur le limbe de la leuille. 
Réaumur a décrit ct figuré lc puceron du pommier; Degéer a fail ses observations sur cetle espèce, en a décrit l'accouplement et a sigualé, sur l'abdomen des ináles, les taches noires transversales dont parle M. Kocls, que Kaltembach passe sous silence et que je n'ai pu observer moi-même. C'est également sur ec pueeron qqué, en 1828 et cu 1829 , Schmidherger a fait ses belles recherches sur la génération de ces inscetes*.

* Voici comment cet auteur rend comple des phénounènes qu'il a observès sur le puceron du pommier (Aphis mali, Fabr.): L'accouplement se fait en septembre ou en octobre, la ponte commence aussitot. Au printemps, les ceufs éclosent aux premiers nouvements de la végétation; les pctits sont aptères, de couleur verdatre, avec les ycux et les articulations rougeâtres; quelques instants aprés leur ćclosion, ils se rendent à la pointe des bourgeons ou sous les feuilles, oil ils se fixent le long de la nervure médiane, se serraut les uns près des autres, quelquefois en si grand nombre, que toute la feuille en est couverte. Une première mue a licu le deuxième ou le troisiène jour après la naissance. Si les pucerons ne sont pas dérangés, ils restent corıstamment sous la même feuille et y subissent a des intervalles de deux, trois ou quatre jours, trois nouveaux chnngements de peau, et, comme je l'ai déjà dit, ils n’acquièrent pas d'ailes dans ce's diverses mutations dont la dernière a ordinairement lieu vers le dixiène ou le douzième jour après la naissance. La ponte des jeunes counnence le lendemain de la dernière inue, souvent le mème jour; les accouchements, qui durent d'ordinaire une demi-lıeure ¿ une heure. se succèdent à des intervalles de deux ou trois heures, et comme ils sont interrompus pendant la nuit, ou comprend que la femelle ne saurait pondre plus de cinq, six ou sejt petits par vingt-ıuatre heures. Après dix ou douze jours, la mère cesse de pondre et ne tarde pas a mourir. Schunidberger a cependant vu des feuselles vivre quinze ou vingt jour's après le commencement de la ponte apris avoir donné le jour a trente ou quarante petits; une seule en produisit quarante-deux. Les jeunes jucerons nés le vingt-six avril commencèrent déjà à pondre le deux uai, c'est-à-dire à l'âge de sẹt jours seulement. Cette truisième gèuération de pucerons était composée d'individus aptères, qui restèrent tels jusqu'i leur mort, et d'autres qui, après la deuxième inue, prèsentaient déjà les moignons cylindriques et blanclrâtres, oủ sont renfermécs les ailes ì l'état rudimentaire, lesquelles parurent en eflet complétemont développées après le quatrième changement de peau. Ces founellos ailées restèrent oncorce 
92. АPHIS воRBI (Kaltembach).

Kaltemb. Jonographie des Pucerons, no 51 , pago 70.

Synonymie: Aphis mali (Schmidberger); - Le Puceron brun café (de Réaumur); - Puceron du Sorbier.

Longueur: uII millisnètre et denii à deux millinètres. Aprèrrs: Antennes plus courtes que lé eorps, brunàtres, avec le troisième article et la moitié inférieure du quatrième jaunàtres; la trompe est jaunattre et son extrémitćbrune, elle atteint ia naissanee de la sceonde paire de pattes ; yeux noirs ;

en place deux on trois jours avant de s'envoler pour aller pondre leur's ouls sur d'auties arbres.

Sans suive l'auteur alleunand dans tous les détails qu'il doume sur les dix-sept générations qu'il a oblenues d'une scule femelle, je crois qu'il sera très-inléressant d'en consigner ici les principaux résultats, lesquels compléteront d'ailleurs co qui manque à l'histoire générale oes pucerons, que j’ai tonnée en exposant les génćralités dı genre. les gínéralions qui suivirent et qui provinrent les unes de femelles aptìres et les autres de femelles ailées, domrèrent des jeunes qui tous étaient aptères, on lont les premiers nés étaient d'abord aptères, tandis que les derniers pondus par la mème mère. aequéraieut des ailes; ure seule fois des femelles ailées naquirent dés le commencement de la pronte. De la troisième génération à la dixième inclusivement, il obtint des jeunes aptives ou ailés, mais à partir de la onzième gćnération, jusqu'a la dix-septième, il ue vit plus que tles femelles aptìes. Schunidberger n'hésito pas à attribuer ce phénomène à l'abaissement de la terapériature el à la diminution de la sćve dans les plantes sur lesquelles vécurent ces pucerons eaptifs (tu 10 aout au 23 septcmbre pour les dernières générations). I l'apjui de celle supposition, il cite ce fait remarquable, qu'en 1828 et en 1829 , par' un temps froid et pluvicux, les quatric̀mes et les einquiènes gèućrations ne renfermaient que des indivilus aptires, tandis que tans les circonstances ordinaires, ce sont ces générations qui jroduiseut les premières femelles ailćes.

l.es femelles de pucerons qui sout aptéres, conmencent luit ou tix jonrs après leur naissance à accoucher de petits vivants, tandis que les fenrelles ailées n'aceuuclıent, en général, qu'aprés ılouze ou y̨uatorze jours, c'est-ì- 
sur le sommet de la tíle se trouvent deux petils lubereules demi-splıcriques el peu brillants, ressemblant à des yeux lisses; la tetc est perite, un peu rougeatre ainsi que le ventre, et les environs de l'anus; sur le hord et les cótes du col se trouvent deux pelits tubereules arrondis et verdatres, de la grosseur de la moitié des yeux réticulés. Sur les bords de l'aldomen, ainsi que sur ses deux derniers segments, se trouvent, en dessus, iles petits tubereules sensiblement plus forls que les granulations aualogues des autres especes du prenier groupe. La poitrine est saupoudrée de blane, et le gonflement extraordinaire du corps lait que lous les anneanx sont i peine distinets. Les cornicules sont minees, d'un jaune pále et brunatre a l'extrémite, de longueur moyenne (trois quarts de longueur normale); la pelite queue est noire el tris-courle; le segment anal est brun aree le bord plus clair; les palles d'un jaune pále arec l'extrénite des jambes et les tarses hr'Ins.

Antés : Antennes un peu plas conrles que le corps, noires,

dire truis on ruatre junrs appres lit dlernière nne. La vie moyenne lles pucerons tu pounnier est d'environ vingt jours, unais à mesnre que la saison avance, celte durée diminue, ainsi que li vigueur des individus; il arrive souvent alors que des lemelles ne produisent que huit ou dix jeunes en tout, une lemelle de la quinziène générution ne produisit même qu'un seul petit.

Si lin température a une certaine intluence sur la production des femelles ailées, il ne l'ant pas s'étonner si les mîles, qui sont tunjours ailés, sont si peu abondants, fuisqu’ils n’apparaissent qu'ä l'arrière saison, alors que les femelles sont moins fécondes. Comme, à celle époque de l'annéc, on trouve aussi quelquefois des femelles ailées et que les lemelles qui sont lécondées poudent des neufs, tindis que celles qui ne l'ont pas été, aćcouehent encore de petits vivants, il en r'esulte, qu'an mois d'octobre, on trouve sourent dans les colonies a la lois des máles et des femellos ailées, les fenelles aptères pondant des veuls ou des petits, et enfin des nymphes, des lapres ou tes auls. 
avee le troisième et le quatrième artiele, granuleux; la trompe est brune avee trois' anneaux jaunes; elle atteint la base: de la deuxiènc paire de pattes; les ycux bruns noiratres; il y a deux tubercules pointus entre les yeux lisses et les petites fosseltes frontales. La poitrine est d'un brun noir et luisant; sur elaque cóté du col se trouve une épine allongée; l'ahelo. men est brun avee la base et le bord plus elair ou d'un jaune rougedu'c; sur le demier segment deux, et quatre șur l'avantdernier petit tubereule; les cornieules de longueur moyenne eylindriques et bruns, avee le milieu plus clair ; la queue trèscourte ex brunc eomme le segment anal, le denxicme ann'au est d'un rouge-brun comme le ventre. Les jambes diun jaune sale avec les tarses bruns aninsi que les jambes et les enisses. Ailes trausparentes avee les ncrvures d'un brun jaunatue; la nervure costale et le sigma sont blanehitres.

Cetle deseription est celle de Kaltenubacls; alle differe anssi de celle de M. Koch; cependant, on reeonnait que les deux auteurs ont vachln déerire le même insecte; celni de M. Koch avait seulement des couleurs plus foncées. Bien que, d'après la figure que cel inteur donne de la femelle aptère, les eouleurs de eelles-ei soient beaucoup plas pàles que ne le dit Kallembach; ce que j’ai observé moi-méme, je pense, arec plusieurs auteurs, que éest à cet insecte que l'on doit rapporter le puceron d'un brun cafí, yac Réaumar a observé sur le poirier. Pour expliquer ces diflérences de couleur, il suffit en effel, d'admeltre, que cela lient à l'àge des individus observés, ou, ce qui est très-probable, a ce que l'éporjue de l'année élait diff'rente.

Le puceron da sorbier parait en juin. On le rencontre sur le sorbier, sur le pommicr, mais jamais on aussi grande abondance que l'especece précédente.

Dars le département de la Moselle, on tee l'a pis encore tronvé sur te poirier, il parait cependimt qu'il peut égrament vive sur cet arbre, el comne il est assez commun sur le sorbier 
des oiseaux, il ne peltt paraitre extraordinaire qu'on l'ait rencontrć sur le poirier sauvage ou même sur les poiriers cultivés. C'est principatentent sur les pètioles el la nervure inédiane que se fixent les pucerons du sorbier; la feuille commence par se plier vers la pointe et fiuil par se rouler complétement sur ellc-nème, de manière à couvrir loute la génération des pueerons; ees feuiltes perdenı leur couleur verte en juin, se noircissent bientò el finissent par tomber. Les jeunes pousses se courbent également, ne croissent plus et les fruits restenı pelils et saus saveur. Schmidberger dit qu'il a vu des espaliers mourir par suite de la trop grande aboadance de ces pucerons.

\section{APHIs QRATOzGI (Kallemb.).}

Kallembach, Monographie des Pucerons, page $65, \mathrm{n}^{\circ} 45$.

Synonymic: Aphis discrepans (Koch in lilleris).

Longneur : deux millimètres.

Aprénes: Corps ovoïde, luisant, fortement boulie, d'un vert gris, saupoudré d'une poussière bleuàtre; antennes courles, du liers de la longueur du corps seulement, brunes, le troisic̀me article plus pále à la base. Yeux bruns; trompe verdàtue, atteignant la deuxiène paire de paltes et ayant le deuxiẻme ct le troisième article brunàtres; tète el premicr anneat de la poitrine tirant sur le brun, les trois anteaux du bout de l'abdonen ayant des bandes noires, itroites en dessus el llatteignant pas le bord externe; dessous du corps vert avec le segrnent anal brun. Connieules courts (denx tiers de la longuenr normale), noirs, anineis à l'extrénite; le tour des cornicules esl jaune rougeatle; la queuc très-courte, feu apparente et brune; palles noires.

Auls: Antennes plus courtes que le eorps, lloires, grossicrement granuleuses; yeux bruns ; la tronupe atteint l'insertion de la troisieme paire de pattes; elle est jaunàtre avee les deux 
artieles dı bout noiràtres; tète et thorax d'un noir brillant; l'abdomen noir en dessus, le ventre blanehátre ainsi que la base du premier et du seeond segment, et sotuvent aussi le bord du troisième et du quatriẻme segment. Les cornicules minces, variant du noir au brun, de longueur moyenne (denx tiers de longueur normalc); la pclite queue a peine visible, noirc, ainsi que le segment anal. Les pattes noires avee In base des cuisses jaunes; Irs ailes transparentes avec les uervures brunes, la nervure costalc el le sigma blanchatres.

A celle descriplion, emprunlée à Kaltembach, cet auteur ajoule que les individus aptères de celte espéee sont très-rares; que presque tous ceux qu'on rencontre, sont ailés ou portent les moignons des ailes qu'ils doivent acquérir. Les premiers sont d'un jaune verdảtre ou rougeảıre, et recouverts d'une poussière gris bleuátre; les antennes sont pàles, brunes à l'extrémité; les pattes et les cornicules páles, l'extrémilé de ces derniers et seulement les tarses bruns.

Cette espèce est décrite et figurée par M. Koch $\left(\mathrm{n}^{\circ} \mathrm{4} / 4\right.$, page 108; fig. 145, ailé; fig. 146, aptère), sous le nom de Aphis pyri, de Boyer de Fonscolombe; mais c'est une erreur. Le fuccron décrit dans les Anuales de la société entomologique de France (page 109 , tome $\mathrm{X}, 1^{\text {te }}$ série) sous le nom d'Aphis pyri ne saurail, en ancune façon, se rapporter à la descriplion qui précede; d'ailleurs, M. Koch compare l'insecte qu'il décrit à l'Aphis grossularie, et.celui-ci ressenble en effet beauccup plus à l'Aphis cratogi de Kaltembach, qu'à toutc autre espèce; ces deux dernières espèees sont trìs-roisines, et le rapprochement qu'en fait M. Koch, ne laisse pour moi aucun doute sur l'erreur synonymique qu'il a commise. En terminant ces observations, je ferai encore remarquer que lc puceron décrit par M. Koelı, sous le nom d'Aphis cratogi $\left(\mathrm{n}^{\circ} \mid 1\right.$, page 64), est une autre espèce qui n'a de rapport avec celui doni il est question dans cet articlc, que parce que, counme lui, on le trouve aussi sur le Cratagus oxyacantha. 
L'Aphis cratagi est exeessivenent commun dans nos environs; on le réneontre déjá au muis de mai, mais plus abondanment en juin, par colonies très-nombreuses, sous les feuilles de l'aubépine, dn ponmier, du poirier, du prunellier même quelq̣nefois. Kaltenbach dit que les individus aptères sont plus rares que les ailés, on que cenx qui doivent le devenir; M. Koch, dit le eontraire; et, ici du moins, les faits sembleut lui donner raison. La différence observéc à ce sujet par les deux anteurs tient, sans aucun doute, à la différence de température qui existait au moment oủ ils firent leurs reclierches.

I.es feuilles habilées par ces pucerons se roulent sur les bords, se contournent el se déforment complétement, puis elles se colorent en rouge d'abord, en jaune ensuite, el finissent par se desséeher entiçrement. C'est partieulièrement dans les lıaies d'aubépine que l'on tronve ee puceron; et, cliose qui est toute naturelle du reste, j’ai remarqué que dans les jardins eutonrés dé haies d'aubépine ou de pruncllier, il était, en 1858, sur les poiriers en espaliers, plus abondants que partoui ailleurs. Kaltembach pense enfin que èest à celle espèce qu'il faut rapporter III puceron qui se trouve souvent sur le pommier en compagnic de l'Aphis mali, Lin., avee lequel on l'a confondu et dout il se distingue eependant par sa taille plus grande el sa couleur rougreàtı.

\section{APHIS RRUNI (Fahr.).}

Kaltembach, Monographie des Pucerons, page 52, $\mathrm{n}^{\circ} 37$.

Symonynic: Aphis pyri (Boyer le Fonseolombe); - Iyaloplerus muni(Koclı); - Prunifex (Amyot); - Puceron du prunier (Geoffroy).

Longueur : un millinı̀tre et deni.

Aptinis: Anlennes un peu plus longues que le corps, verdailres, avee le septième article et la pointe du sixiènı frrunties; les yeux d'un brun rougeatre; la lrompe verte avec la pointe brurie et s'ćtendant jusquäi la seconde pairc 
de pantes; le dessus du corps d'un vert elair avec urois lignes dorsales d'un vert-pré, les cornicules très-conrts (moitic sculement de la longueur normale), mines, bruns, avec la base vertc. La queuc est verte, plus longue que les cornicules. Le dessous du corps, les palles et le segment anal ver's; les tarses hruns.

Autés: Antennes plus courtes que le corps, jaunaties, poudreuses, la base et la pointe un pen brunàtres. Yeux d'un rouge brun; la trompe verte, brunàtre à l'extrémité et n’attcignant pas la deuxième paire de paltes. Téte el poitrine bruncs, fortement saupoudrécs de blane, particuliẻrement en dessus, dans la partic la plus visible. Chez les jernes individus, le sommet de la tête est vert ainsi que lànneau du cól. L'abulomen est d'un vert jaumitre avec trois lignes longitudinales d'un vert-pré; les cornicules, la petite queuc et le ventre, comme clicz les aptères. Le segment anal à peine marqué d'unc efllorescence olscure. Palles verdilles avec l'extrimité des jambes et les larses brums, genoux ex extrémité des cuisses postérieures brunattres. Ailes Iransparentes, souvent poudreuses, avec les nervures bruncs; la nervure costale et le sigma pàles.

Celte deseription, traduite de l'ourrage de Kaltembach, est incontestablement applicaljle all puceron décrit par Boyer de Fonscolombe, sous le nom de Aphis prri. Ia description dounce par M. Koch (page 22, $1^{\circ}$.5), pour soa IJ alopterus pruni, est égalenent applicable à notre insecte, et je ne compreuts pas comment eet auteur a pu confondre l'. Aphis pyri de Boyer de Fonseolombe, avec l'. Iphis cralogi, de Kaltembach, "t qu'il n’ait pas reconnn l'identité des deux espèees en présence des doutes exprimés par Boyer lui-même, qui met, arec un point de doute, toute la synonymie de Fabricius a la suite du nom de son Aphis pyri.

Une autre erreur de M. Koch, qui est non-seulement incom- 
prçhensible mais cncore inexcusable, c'est qu'après avoir déerit le puceron de Boyer, sous le nom d'Aphis pyri que lui donnait cet auteur, il ait décrit une autre cspèce du méme genre sous le nom d'Aplis pyri, Koclı; s'il élait permis de donner ainsi un unème nom spécifique à deux espèces du mème genre, ou même de genres très-voisins et susccptibles d'être réunis, la nomenclature entomologique, déjả si embronillée, ne serait bientôt plus qu'un véritable chaos. N'ayant pas à fairc ici la critique de l'ourrage de M. Koclı, sans in'étendre plus longuement sur les contradictions qui s'y trouvent, je crois qu'on comprendra ma détermination à ne prendre mes descriptions et mes renseignements que dans l'ouvrage de Kaltembach, bien qu'il soit antérieur à celui de M. Koch, et qu'il ne soit pas, eomme l'cst celui de ce dernier auteur, aecompagné de planclies colorićes.

Ce pnceron, qui parait en mai, est le plus abondant en juillet; il vit sons les feuilles du prunier, du covetschier, du prunellier, du poirier, sclon Boyer de Fonscolombe, et de l'abricotier, selon Degéer. Ces insectes sont complétement verts, mais la forme oroïde de leur abdomen les fait déjá distinguer. Plus tard, quand ils vieñueut de clanger de peatu, leur coloration est également plus verte et plus uniforme. Chez les vieilles mères, le corps cst quelquefois bleualtre. Pendant le mois de juillet, tons ou presque tous les individus se tiennent sous lcs feuilles; mais, en aout et surtout en septembre, on ne les rencontre plus guère que sur les pousses.

Cet insecte, sa nymphe, et sourent mème les azufs, sont recou. verts d'une poussière pollineuse, blanchatre, laquelle est quelquefois si abondante que les fevilles et les fruits de l'arbre sur lequel ils se trouvent en sont tout couverts, an point, dit Kaltcmbach, que cettc poudre blanche salit les habits, quancl on vient à tourlier ces arbres.

Dans la Muselle, lc puceron du prunier csttrès-abondant, surtout sur les couetschiers et les mirabelliers. Je ne l'ai jamais rencontré sur le poiricr, ni même sur le poinmier. Sa forme ovoïde et allongée est très-caractéristiquc, clle suffit pour le distingucr 
des espéces voisines. Voici comment Degéer rend comple des observations qu'il a faites sur cette espèce de puceron : «Tous les pucerons aplères des feuilles du prunier sont des femelles qui pondlent des œufs en septembre; on les voit alors inquiètes et s'agiter sur les jeunes pousses des arbres, comme si elles voulaient cliercher une place convenable à la ponte des œufs. Elles préférent pour cela les petils enfoncements qui se trourent entre la tige et les yeux des boutons, e'est dans ces enfoncements quelles déposent leurs aufs l'un à eóté de l'autre et quelquefois aussi les uns sur les autres, de sorte que souvent il y en a de petits tas réunis eusemble. Aussitót qu'ils sout pondus, ils sont d'un vert foncé, mais plus tard ils devicnnent d'un noir bruuktre; la femelle mère, les recouvre ensuite d'une inatière cotonneuso blanchàtre qu'elle sécrète, comme les pucerons de l'aulne, par lo dessous du ventre et par les cótés de l'abdonien. Toutes les femelles que l'on ouvre a cette époque, ont le corps plein d'œufs... Pendant le temps que j'observais ceci sur le puceron du prunier, j'ai été assez heureux pour voir l'accouplement d'un mâle ailé avec une femelle non ailée... La forme du mále est celle des pucerons ailés en général; seulement l'abdomen est plus étroit, plus mince, sensiblement annelé et terminé par uue petite verrue; les cornicules sont très-courts, les yeux grands et les antennes passablenient grosses; enfin les ailes sunt une fois plus longnes que le corps, et de couleur noire mêlée de vert en dessus.

95. APHIS PYRX (Kooch).

Koch. Monogr. des Pucerons, page 60, n0 8, fig. 76, aile; 77, aptêre.

Vert jaunatre. La tète, une bande transversale sur le col, les bosses du corsclet, une tache transversale cntre les cotnicules, noires, ceux-ci courts, ainst que les antennes.

Le nom de A. pyri, de Boyer, étant passé dans la synonymie de l'Aphis pruni, Fabr., il n'y a plus d'inconvénient de conserver le nom assizné par M. Koch, à l'espèce que je vais faire connaitre en traduisunt ce que cet auteur en a rapportó : 
\& Le 25 mai, jobservai que quelques feuilles d'un poirier (Pyrus pyraster) étaient altaquécs á la partie inférieure par des pueerons, et que ces feuilles s'étaicnt roulées en dessous dans le sens de la cóte médiane, de manièrc que les deux cótés se touchaient. Dans ccs feuilles ainsi roulées se trouvaient des colonies de pucerons dont presque tous les individus étaient à l'état de nyınplıe; il y avait seulement deux ou trois vieillcs mères. Ces nymphes ćtaient sur lc point de se transformer, ct le mème jour, en cffet, il y en eut quelques-unes qui produisirent des puccrons ailés. Les vieilles mères ne paraissaient plus porter d'embryon, cependant elles étaient encore bombécs, quoique déja aplaties sur les bords. Leur forme est celle d'un ovoïde large el court, presque rond. Les premiers anneaux sont nettement accusés, ntais ceux de la partie postérieure sont réunis et connc soudés; la peau est tmate, les antennes courtcs, les quatre dernicrs articles courts et d'égale longucur, les corniculcs trc̀s-courts, presque cylindriques; la queue seulemeut ponetiforme, à peine visible; les pattes sont grandes et n'ont rien de particulier. Ces vicilles mèrcs sont de couleur brun-canelle, d'un brun plus clair sur les cótés, brun noir sur le dos avec une ligne dorsale moins colorće; tête, antenues, pattes ct cornicules noirs; les cuisses de la dernière paire de pattes sont un pell jauncs à la base.»

«Les larves sont d'un jaunc verdátre; les antennes, les pattes et les cornicules plus clairs que le corsclet; sur le dos de l'abdomen, deux lignes de taches transversales d'un vert-pré. Les individus ailés ont la forme et les caractères particulier's au genre. Les antennes sont sensiblement plus longues que chez les individus aptères, ct les deux articles qui suivent le premier, sont plus longs, annclés et dentés; les corniculcs, quoique courts, sont aussi plus longs que chez les aptères; la trompe cst trèslonguc chcz tous les individus; ellc dépasse le premier anneau de l'abdomen et ses articulations sont très-visibles. »

«La tête et les antennes des individus ailés sout noires; la trompe cst jaune obscur vers la pointe, mais avec l'extrénité des articles jauıàtrc; poitrine ct abdomon d'un vert jaunc avec unc bande 
transversale sur le col; la base du thorax et celle de la poitrine, une place eireulaire derrière la plaee de l'éeusson, trois laehes plus grandes et rondes sur les côtés, une grande tache arrondie entre les eoruieules, une bande transversale sur le bord postérieur des deux avant-derniers anneaux de l'abdomen, d'un vert luisant; les jambes sont noires; les euisses a leur naissance, et le milieu des jambes jaunatres; ailes irisées, le rouge et le violet les plus apparents; les nervures jaunes, la nervure costale brunâtre ainsi que le sigma; ees pueerons ailés sont agiles et prompts à s'envoler. 》

Pendant les mois d'aoùt et de septembre, j'ai eherelié cee puceronsur le poirier sauvage, sur les poiriers eultivés de nos environs et sur les autres arbres fruitiers, saus pouvoir en rencontrer un seul individu.

N. B. Avant de terminer ee qui est relatif aux puecrons qui font partie du genre Aphis, limité eomme Kalıembaeh l'a fait, je dois eneore mentionner une espèee qui ne me paraì décritc nulle part, et qui cependant se troure sur les poiriers et les pommiers. M. Goureau, qui l'a aussi tronvée sur les poiriers de Sautigny (département de l'Yonne), donne la description suivante des individus aptères: « Longueur, deux milliuètres. Noirs; antennes blanchatres à la bise (dans la plupart des individus il n'y a que le premier artiele de eette couleur), brunes a l'extrémité; tête et corps d'un noir un peu velouté; cornieules eourts, noirs; abdomen terminé par une petite queue; cuisses noires; tibias blanehitres, tarses bruns; bee noirutre alteignant les hanehes intermédiaires; en dessous, le ventre est d'un brun rougeảire.

"Ce pueeron vit en société nombreuse sous les feuilles du poirier qu'il courbe, raspe el déforne; écrasé il laisse mue taehe rouge conme du sang. Je l'ai observé le $1^{\text {er }}$ juin el j’ai élé témoin de l'aecouehement d'une mère aptère qui a mis au monde un petit, verdatre, sorti du ventre le derrière le premier, et qui remuait ses pattes de derrière ayant encore la tête dans le ventre de la mère. y 
Ce puceron se trouve dans lc département de la Moselle, sur les pomniers et sur les poiricrs, mais il nc parait pas $y$ vivre cn colonies aussi compactes et aussi homogènes que les autres espèccs de pucerons. Toujours il se rencontre au milieu dcs colonies de l'Aphis mali et toujours aussi en plus petit nombre que ces derniers. La description donnée par M. Goureau, s'appliqquc trèsbien à tous lcs individus que j'ai rencontrés; cependant je n'ai pu en Irouver qui, étant écrasés, aient produit des taches rouges commc du sang; l'intérieur de ces pucerons est d'un vert plus ou moins foncé, quelquefois presque noir, inais jamais ronge. Je dois cncore ajouter que je n'ai pas vu de ces pucerons noirs accoucher; par consćquent, je nc puis dire si, au moinent de leur naissance, les petits sont verts; mais ce que j'ai vu bien des fois, c'est que des petits pucerons, n'ayant pas encore changé de peau, étaient déjà d'un gris très-foncé ct presque noirs comne les mères dont ils étaient issus.

En consultant les planches de l'ouvrage de M. Koch, jc ne trouve que les figurcs 114,116 , qui puissent conscnir au puceron observé par M. Goureau et par moi, les dessins rcprésentent les femelles des Aphis laburni, Kalt., ct Aphis cerasi, Fabr., mais les descriptions ne corrcspondent pas à nos individus. Il faudrait connaître les insectes ailés pour cn détcrminer l'espècc qui devra, dans tous les cas, êtrc comprisc parmi les inscctes vivant sur le poirier.

\section{SCHIzONIURA (Hartig)*}

Kaltembach. Monographie des Pucerons, page 167.

Ce genre est un démembrcment du grand genre $A$ phis de Linné. Les insectes qui le composent, diffèrent des vrais pucerons par le cubitus des ailes, qui n'a qu'une branche simple, par des antennes de six articles et par l'absencc dc cornicules. Il est,

\footnotetext{
- Synonymie: ApHs (Linné); - Mrzoxylus (Blot.); - Rinden-IAUS (des Allemands).
} 
comme le suivant, placé dans la série de ccux qui ne renferment que des espèces vivipares. On n'en connait jusqu'à présent que six espèces.

Les Sclizoneura conuus vivent tous sur les arbres. Les uns (Sch. Reaumuri et tremula) sucent les pointes terminales des jeunes pousses, font erisper les fcuillcs, et celles-ci se réunissent ell une tête au milieu de laquelle les pucerons sont à l'abri; d'autres roulent ou plutỏt font rouler' les feuilles sur elles-mêmes. La Sch. Lanuginosa détermine, sur les fenilles, la formation de vessies velues dans lesquelles ils mettent leur géućration à l'abri, etc. Tous sout plus ou moins recouverts par une sécrćtion laineusc produile par l'insecte lui-mème. $\Lambda$ l'automne, on voit des mìres aptères qui sont fixées aux jeunes pousses oủ elles resteut ainsi sans mouvement jusqu'au printemps suivant; alors elles comuencent à pondre des jeunes qui ont des yeux extrèmement petits, nou saillants. Daus quelques espèces, on reınarque ‘u'il y a moins d'articles aux antennes des larves qu'à celles des insectes ailés qui viennent après. Les Schizoneura sont, d'après Kaltembacl, toujours vivipares, comme les Tctraneura et les Pemphigus; ecpendant, on n'a pas encore suivi leurs transformations d'une manière couplètc et l'on ne sait pas, par exemple, comment naissent les vieilles mères que l'on trouve en automne et qui pondent des petits au printemps.

Une espece de ce genre a été signalée counue vivant sur le poirier, c'est :

96. BCEIroNEURA IAKIGERA (Hausmanu).

Kaltembach. Monographie des Pucerons, no 2, page 169.

Synonymie: Aphis lanigera (Hausmann); - Aphis laniger (Illig.); - Mysoxylus (Anyou) ; - Puceron laniger; Wollig apfel blatt-luus (des Allemands); - Nyzoxylus mali (Blol.).

Longueur: un millimètre et demi à deux millimètres. Arténes: Corps de coulcur de micl, brun rougeảtee, rceot- 
vert en dessus d'une sécrétion blanche et laincuse; antennes très-courtes, d'un jaunc pàle, les trois derniers articles à peu près égaux el réunis ensemble, plus longs que le troisième; trompe blanclıàtre et noirc au bout, assez longue pour attcindre la troisième pairc de pattes; yeux très-petits, à peinc visibles ê bruns; pattes jaunâtres, tous les genoux bruns: les anneaux du corps sensiblement effacés. Pas de corniculcs, mais unc cicatricc circulairc à leur place; queue invisible.

Aıts: Antennes plus courtes que la tètect le thotax r'éunis variant du brun au noir; les deux premiers articles de la basc très-courts, le troisicme annclé de clair et plus long que les trois suivants; quatriẻme et einquiène égalensent annclés et de la mème longucur, le sixième plus petit que le précédent, lisse et elliptique; yeux très-grands ; trompe blanchåtre et altcignant la troisic̀me paire de pattes; tète et thorax noir brillanı, anneaux du col brunatres; abdomen couleur brun chocolat, sans cornicule ni queue; pattes grúles avec une teinte brunàtre plus foncéc sur les hanches, aux cuisses et à l'extrémité des tibias. Ailcs transparentes, avec toutes les nervures et le sigma d'un brun foncé, moins le cubitus qui est blanchàtre à l'origine de la fourche; le sigma est un peu plus clair vers le bord de l'aile.

Le Pueeron laniger vit le plus ordinairement sur les pommiers; cependant, M. Dubreuil dit positivement qu'il se propage également sur le poirier; Dalbrel dit aussi l'avoir vu sur le sorbier, sur l'alisier et même sur l'aubépine ; mais il ajoute ŗu'il n'a jamais vu d'exostoses sur ees arbres pas plus que sur le poirier. Dans le département de la Moselle, l'insecte lui-même n'a pas encore été observé, ni sur le pomnier, ni sur le poirier. Mais M. Thomas a vu depuis plusieurs années, du eóté de Lorry, des pommiers hauts-vents, ayant des clancres boursouflés et qui présentent lous les earactères qui ont été assignés aux protubéranees qui se voient sur le tronc des arbres envahis par ee pueeron. Ainsi que 
j'ai déjà eu oceasion de le dirc, eetle espèce parait être d'origine américaine. C'est vers 1812 qu'on a commencé à s'apereevoir de sa présenee daus les vergers de la Bretagne ; en 1820, daus eeux de la Nornandie. Il est exeessivement fécond et sa dissémination se fait, comme celle des autres pucerons, au moyen des individus qui sont ailés; mais, eomme cn outre des organes du vol, ces insectes ont le corps couvert d'un duvet très-fin el trèsléger, le vent les emporte souvent à des distances eonsidérables, c'est ec qui explique sun envahissement successif de l'Angleterre, de la Bretagne, de la Nurmandie, du nord de la Franec et enfin de la Belgique.

M. Audoin (Annales de la societé entomologique de France, tome IV, $\mathbb{1}^{\text {ro }}$ série, page 9 du bulletin) décrit ainsi les tubérosités produites par cet insecte : «La branclie envahie par le Puceron laniger ne présente d'abord aucune altération bien sensible; on voit á la surface quelques petites ondulations ou petites bossclures, et ordinairement un sillon plus ou ınoins ćlargi qui divise la branehe dans le sens longitudinal et dans une étendue de plusicur's pouccs quelquefois. C'est dans l'intérieur de ce sillon que sont logés et fixés au ponmier les nombreux pucerons qui attaquent les jeunes pousses; placés à la faee inférieurc de la branche, ils se trouvent ainsi à l'abri de la pluie. Cette première opération, produite sur les jets d'un pommier, n'est done pas d'abord bien frappante, et toutefois elle suffit pour modifier à janais la végétation de l'arbre. En effet, dès ec moment, la sève semble s'épaneher sur ec point et déjà la deuxième année on aperçoit une petite nodosité qui devient plus scnsible la troisième année, se fait remarquer davantage la quatrième et finit enfin au bout de 6,7 ou 8 ans par atteindre la grosseur du poing. Ces nodosités ont l'écorce à l'état normal et elles sont formées par des couehes ligneuses qui ne se sont développées que dn eòté où se trouvent les puecrons et y forment le tubercule dans lequel ehaque couche conscrve encore plus ou moins la trace du sillon formé, par les pucerons, quand elle était sous l'éeoree. \ Le puecron laniger se nourrit de la sìve de l'éeoree et de 
l'aubier des arbres sur lesquels il vit; comme ordinairement ils sont réunis en grand nombre dans la même place, l'écorce finit par se transformer cn une sorte de tissu cellulaire à cause de la multiplicitć des piquires. La sève affluant plus abondamment dans cette partie, une grande quantité s'cxtravase en purc perte et a u grand dommage de l'arbre; sur les branches ou sur lcs trones oủ l'ćcorce est plus épaisse et oủ la sève ne circule plus assez abondamment, les puccrons cherchent à s'introduire sous la eouche corticale en s'insinuant dans les crevasses, afiu d'y rellcontrer l'aubier ou le liber, plus tendre et plus riclie en sucs nutritifs. Il arrive mème quelquefois que eclte afflucnee de séve déterminc la formation dc pousses qui nuisent également à la fruetification de l'arbre. Pendant les hivers rigourcux, ecs puecrons eutrent daus le sol et vout se fixer sur les grosses racines de l'arbie.

C'est ordinaircment en dessous des jennes rameaux que sc fixent ees insectes, leur présence est facile à constater par la sćcrétion blanche et laineuse qui sc développe sur leur corps et qui, se détachant facilement, courre de ses débris toutes les parties de l'arbre voisines du lieu où ils vivent en famille. C'est plus partieulièrement contre le Puceron laniger que l'on doit cinployer les arrosages avec l'cau benzinće, la lessive, l'eau de chaux, l'cau du gaz, etc., ou le barbouillage au goudron, à l'huile, etc.

\section{PEDPEIGUS (Eartig)"}

Kaltcinbacl. Monographie des Pucerons, page 180.

Ce genre est, comme le précédent, un démembrement du genre Aphis de Linné; il en diffère seulenent par des antennes de six articles et par le cubitus des ailes, qui est sans ramifieation.

Les insectes qui appartienucnt à celte division, sont de

- Synonymie : ApuIs (Linné) ; - ERrosoma (IIeyden); -TuElaxed (D)alb.); WOLL-l,aus (des Allemands). 
très-petitc taille, plus ou moins rccouverts par une sécrćtion laincuse qui se renourelle a chaque changement de peau de l'inscete el qui est produite par des organes particuliers nun encore décrits. Les uns vivent dans l’intéricur de galles qui se forment à la suite des piquires faites par la trompe de l'animal; les autres vireut á découvert sur les plantes herbacées, sur le trone ou sur les racines des arbres sur lesquels ils détcrininent aussi quelquefois la formation d'exostose de nature analoguc a celles qui sont produites par lc Schizoneura lanigera. Ceux de ces insectes qui virent dans les galles sont plus longs à se transformer que les autres, ct tous paraissent aequérir des ailes (uoins cepcudant la mère fondatrice de la colonic qui est toujours aptère); dans cet état ailé, ils sortent de lcur retraite par une ouverture qui s'y forme naturellement et ront, sur d'autres arbres, fonder de nouvelles colonies en donnant naissance à des femelles aptères. Les espèces qui vivent à découvert sur les plantes, ne sc rencontrent que pendant fort peu de temps. Tous les individus de la première génération acquièrent des ailes, et l'on trourc, après leur dispersion, les dépouilles des nymplıes et le duvet qui recouvrait leur corps.

Kaltembach dit que les Pemphigus sont vivipares; cependant, il appelle sur cux l'attentiou des entomologistes; il ajoute qu'il $y$ a cncorc beaucoup a appreudre a leur sujet et il termine en disant qu'il eroit que dans certaines circonstances qu'il n'a pu déterminer, ees insectes sont ovipares.

La unère aptère d'une colonie de ces pucerons, ou au moins celle des espèces sur lesquelles on a pu faire des observatious, préseutc plusicurs singularités reuırquables; ainsi, son corps est plus gros quc. les autres individus adultes, et ses autennes ont moins d'articles que u'en ont les antennes des larres ou des insectes ailés qui proriennent de celles-ci.

Oı connait sept espèces europécnnes du genre Pemphigus: une vit sur les Grophalium erectum et Gn. germanicum; deux vivent dans des galles sur les Populus nigra et $P$. dilatala, etc. M. Aza-Fitclı en a fait connaitre une cspèce qui vit dans l'Amé- 
rique du nord, sur les arbres fruitiers, et plus partieulièrement sur les pominiers et sur les poiriers. Sa ressemblanee avee le pueeron laniger, son iulroduetion possible, probable méme, en Furope, sont des motifs qui mérilent de fixer notre altention, et éest ee qui m’a délerminé à donner l'histoire de eet inseete, d'apiès le travail de M. Aza-Fiıeh, auquel j'ai déjà fait plusieurs emprunts.

97. PAMPaIoUs PXRI (Aza-Fitch).

Axa-Filch, First report an the noxious, clc., page 9.

Synonymic: Eryosoma pyri (Fitch); - Pemphigus americamus (Walker); - The apple bool blight (des Américains).

Aptères. Longucur: un millimètre. Forme ovale, de couleur jaune pille; pattes petites et robustes, à peu près de mème longueur ; les antennes, qui ressemblent attx pattes, n'ont que cinq articles apparents et sont pointùes á leur extrérnité; l'abdomen est terminé par un filanent blane, cotomneux et diversement contourné, selon les individus; la blancheur de ees filaments les rend pereeptilles a l'wil nu et permet de suive leurs mouvements sur le trone de l'arbre où ils ne seraient pas visibles sans cela.

Autés. Longucur : y compris les ailes fermécs, cinq millimítres. Largeur des ailes, un millimètre. Le corps, les pattes et les antennes sont d'un noir de charbon; tout le dessus du corps est couvert d'une abondante séerétion colonncuse blanclıe ou d'un blanc blentitre; les ailes supérieures sont transparentes à l'extrémité, un peu enfumèes et paraissent recouvertes d'une poussière plus abondante a leur base, les nervures sont noires ou d'un brun foneć el le sigina de couleur enfuméc; les ailes inféricures plus transparcutes et lyyalines, avee les nervures d'un brun pàle.

Cette deseriplion est extraite de celle qui est insérée dans le 
mémoire de l'auteur amérieain, lequel donne en outre les dètails suivants sur cet insecte: "Vers le 29 oetobre 1849, j’étais oceupé à ranger un certain nombre de jeutes pommiers qui m’avaient été envoyés de la pépinière de Cilens-Falls, comté de Warren, quand, sur les racines de l'un d'eux j'observai quelques exeroissances vraimeut singulières. Pendant que je réfléehissais sur la eause qui les avait produites, $j$ 'aperçus presqu'entièrememt eaehé sous l'écorce des plus grands, un-pueeron, et en y regardant de plus près, j'en aperçus un second semblablement caclı́, l'un ćtait mort et l'autre eneore vivant. En cxaminant les erevasses de eette excroissance avec une loupe je vis qu'elles étaient oecupécs par des puccrons, si petits qu'ils n'étaient pas visibles à l'œil nu, et qui à n'en pas douter étaient les petits de eeux que j'avais aperçus d'abord. Vers la fin de l'automne, sur lesbranelies dans les taillis, $j$ 'avais déjà pris un grand nombre d'individus de la même espćee, et cependant il n’y avait pas de pommicrs à un mille à la ronde. Probablement ees insectes proventient des raeines du Thorn (Amelanehier du Canada, Petromeles Canadensis, J. Q.), et ee qui semble justifier eelte supposition, e'est que l'insecte dont il s'agit se trouve non-seulement sur les arbres de la famille des Pomacées, nais eneore sur d'autres arbres fruitiers et des arbres sauvages à feuilles eaduques.

\Cette maladie des jeuncs pommiers avait cléjá été signalée dans nos revues périodiques d'agricuiture, ct diflérentes reeherches avaient été faites sur l'insecte qui l'oeeasionne, mais sans donncr de résultats satisfaisants, parce que cet insecte appartient à une espèce nouvelle, différcnte de toutes celles qui ont ćté déeritcs jusqu'à présent et qui sont eonnues des horticulteurs et des pépiniéristes. Une communication de J. Fulton, du comté de Chester, dans l'IIorticulteur de Downing*, ferait penser que

- "Le principal but de mon ouvrage, dit M. Fulton, est d'attirer l'attention sur un sujet important et de demander tous les éclaireissements que l'un pourra me donner à ee sujet. En arrachant des arbres eet automne de 1848, je reunarqnai que quelques racines étaient pleines d'excroissances ou 
cette maladie s'élend sur une vaste étendue de notre pays et cause de grandes pertes à nos éleveurs.

2Une courte description de cet insecte fut publiée par moi daus le Catulogue des inseetes homoptères, déposé dans le cabinet d'histoirc naturelle de l'État, sous le nom d'Eriosoma prri. 'Tous ces puccrons, qui étaient naguèrc renfermés dans le gemre Eriosoma, de Lrach, et qui sont caractérisés par la simplicité du cubitus des ailes supériaures, forment maintenaul le genre Pemphigus, de llartig, auquel genre on doit nécessairement rapporter ret insecle. Plusicurs des autres cspèces de ce genre, aussi bien que cètte dernière, sont eonumes pour attaquer les racines des plantes. Je conserve à peine le plus léger doute qu'elle ne soit aussi la mème espèce que $M$. Walker a déerite, d'aprics les individus piovenant de la Nouvelle-Écosse, sous le nom de Pemphigus amerieanus, quoique la longueur qu'il lui assigne ( 4 lignes) soit bien supérieure à cclle que j'ai trouvée elicz les plus grands individus.

\C'est a nos pépiniéristes qu'il appartient de faile l'histoire

verrues, el couvertes d'un insecte laincux, tris-petit, dont un grand nombre était logé dains les blessures failes par la taille. Comme les arbresparaissaient vigoureux, je portai peu d'altention à ce fait, jusqu'à ce qu'un autre horticulteur vint appeler inon allention sur lui, en me disant que n'ayant pu suffire à une demande considélable de ponuniers, il avait èté dans plusieurs établissements pour en acheler, mais qu'il n'avait pa s'en procurer ume quantité suffisante parce qu'un très-grand nombre élait affecté de cettc inaladie, et qu'il avait été obligé de les refuser. Depuis lors, un jeune homme de mes amis revint de la Virginic oú il avait vendu et livré plusieurs milliers de ces arbres et me dit que là tous les arbres itaient dans le mène état ef qu'il n'avail cepeudant pas rcmarịiu que cette circonstance fut le unoins du monde prëjudiciable à leur santé en a leur vigueur, et que celui qui les avnit propagés ne sétait pas non flus aperẹu de ce vice de conformation. Cet insecte, continue M. Fulton, est-it le Puceron Laniger? s'il en cst ainsi, que peuvent faire les pépiniéristes pour se garantir d'une peste qui malheureuscment rest que trop counmune? J'ai trouvé cet insecte dans les arbres qui croissent dans un sol siliceux ou chisleux et rarement danz ceux qui croissent dans une terre grasse et marneuse. " 
de cet insectc, et des maladies qu'il occasionne, parce qu'ils peuvent l'observer mieux qu'on ne saurait le faire dans aucune autre profession. Les nœuds ou excroissances apparaisscnt aussi bien sur les grosses racines que sur le chevelu le plus délié; dans le seul cas qui soit arrivé à ma connaissance, la principale racinc d'un jeune arbre avait un lemi-pouce de diamètre à un pied de profondeur et à ce point, elle.était aux deux tiers cnveloppée par une excroissance de deux pouces le long et de trois pouces d'épaisseur en diamètre, elle élait attachée à la racine par un pied beaucoup plus petit que la base ${ }^{\star}$. La forme est irrégulière, bosscléc; la surface est de la inène couleur, brun jaunàtre, que la racine elle-même et se trouve partout parsemće de bosses de la grosscur d'un grain de moutarde à la grosseur d'un petit pois. En coupant l'un de ces nouds saillants, on trouve çu'il est d'une texture ligneuse très-dure et sans ancune cavité à l'intérienr; sur la principale racine, entre celte excroissance et la surface dn sol, se trouvait une srconde excroissance scmblable, nuais plus petite, et sur plusieurs des radicelles se trouvaient aussi de semblables nodosités variant de la grosseur d'un pois à celle d'une balle. $D$

๖ Ces excroissances sont, saus aucun doute, formées de la même manière que les gralles et toules les productions morbides des végétaux; l'insecte femelle se glisse sous terre en suivant les racines, probablement vers la fiu de l'automne, la elle dépose son paquet d'œufs et meurl, ces oufs s'ouvrent quand la terre devient chaude, au printemps suivant, et les jeunes pucerons enfonecnt leur trompe dans l'écorce de la racine pour en tirer leur nourriture. Ces piquires produisent une sorte d'irritation qui attire dans cette partic un flux surabondant de sìve et occasionne un développement anorinal du bois et dont il résulte les excroissances que nous avons décrites. Comme il arrive pour les autres familles, les pucerons continueut probablement is se

- Ici l'auteur donne la description de la figure au quart de grandeur naturelle, et qui représente l'excroissance dont il est question. 
multiplier sans le secours d'une nouvelle fécondation jusqu’à l'automne oủ des individus ailés apparaissent, quittent leur retraitc et après s'être accouplés à l'air libre, clıcrchent de nouvcaux arbres pour y propager leur espèce. Les autres, autant que je puis en juger par l'époque avancéc de la saison où je trouvai de jeunes pucerons sur les excroissances, restent sous leurs abris pendant tout l'hiver pour cuntinuer leurs opérations sur les mêmes racines pendant l'année suivante.

ע Quand un arbre cesse de croitre avec la vigueur ordinaire et que ses feuilles sont d'une teiute plus pále qu'à l'état nor'mal ou jaunátre, et qu'aucune blessure du trone ou autre cause de maladie ue peut être découverte, on doit soupģonner la présence de cet insecte sur ses racines; on doit alors fouiller la terre pour s'assurer s'il n'y a pas d'excroissances semblables à celles que nous avons décrites plus laut, et, si l'on en découvre, il scra bon de rejeter au loin la terre qui les recouvrait, autant qu'on le pourra sans inconvénient, et de l'arroser avec de l'cau de savon en assez grande quantité pour remplir les crevasses des excroissances, ear il n'y a pas à douter que tout insecte qui sera atteint par cette eau, périsse immédiatement. On mélera des cendres à la nouvelle terrc dont on recouvrira les racines; il est probable qu'en recourant à ces mesures un arbre malade peut ètre guéri dans le plus grand nombre des cas. C'est surtout dans les pépinières, sur les racines des jeunes arbres arrachés pour être trausplantés, que le Pemphigus pyri peut être découvert; il en est résulté que, dans notre comté, des milliers d'arbres ont dú çtre rejetćs; il est probable que celte prohibition deviendrait inutile si l'on arait la précaution de tremper leurs racines dans l'eau de saron, à moins ccpendant que ce puceron ne soit plus résistant que les espèces voisines qui vivent sur les branches ou les arbres. Ceci pcut être vérifié pas tous les horticulteurs. M. Downing recommande le mélange d'un boissean de cendres avce la terre dans laquelle on plantc ces arbres, cc qui, peut-ĉtrc, suffit pour produirc le même effet que l'imınersion des racines dans l'eau cle saron. $>$ 
Il est certain que cc qui précède ne saurait être applicable à ce qui est connu du Puceron laniger et l'on eomprendra difficilement les doutes exprimés á cc sujet par M. Fulıon. Cependant, pour plus de certitude à cet égard, il suffit de lirc la description très-longue et très-compliquée, que M. Aza-Fitcl fait des ailcs du Penphigus prri; eetle description quc j'ai eru devoir passer sous silence, s'applique si parfaitement aux ailes du genrc $P$ emphigus représentées par les figures 13 et 14, de l'ouvrage de Kalteınbach, que l'on serait tenté de croirc que l'auteur américain les a pris pour guide. Or, le Puceron laniger apparticnt au genre Schizoneura dont le cubilus a un raneau secondaire, tandis que dans les Pcmphigus, le eubitus est simple. Enfin, je ferai observer que M. Aza-Fitch supposc que la fenelle du puccron pond des aufs à l'automne, cc qui est cucore en contradietion avec Kaltembach qui range le geure Pempligus parmi les puccrons vivipares.

Bien que dans notre département on ait déji reçu de nómbreus arbres fruiliers de l'Amérique du Nord, il ne parait pas quion y ait encore obßervé ni les excroissances ni le puceron qui contribue à leur formation, est-ce uné raison suffisantc pour penser qu"il cn sera toujours ainsi? Je ne lc crois pas, en raison des cnvois eontinuels que l'on fait de l'Anérique du nord en Europe, d'arbres fruiticrs que l'on eommence à cultiver chez nous; c'est ce qui m’a déterminé à donner presqu'cu entier la description de M. Aza-Fitcl.

\section{trur. coccus (rinnd)*}

Amyot et Serville. Ilémiptères, suites à Buffon, page 628 .

Male: Antenues de dix articles au moins; pas de bee, deux ailes transpatentes assez longues; abdomen pourvu de deux filets assez longs.

Femelle: Corps épais, oblong ou globuleux, aptèes, com-

- Synonyinie. Cocienille; - Gallinsecte. 
posées de quatorze anncaux plus ou moins distinets; yeux très-petits; antennes conrtes, le neuf articles; bec cottrt, de trois articles naissant de la poitrine au milieu de l'insertion des quatre pattes antéricures; abdomen garni de deux i quatre filets courts ; pattes couttes, grèles, d'égale longueur ; larses d'un seul article visible.

Les eochenilles sont ees inseetes que l'on eonnait généralement sous les noms de Poux d'écorce, Punaise des bois, Tigre su' icorce, et qui, comme les pucerons, mériteut de fixer tout partieulièrement notre altention, tant à eause du domınage qu'clles nous eansent que par les particularités remarquables qui out été observées dans leur organisation, leur développement et leurs lıabitudes.

Il est peu de personnes qui n'aieut eu oecasion d'olserver, pendant le mois de juin ou de juillet, sur les branches de la vigne, du pêeher, de l'aubépine, du poirier ou du pommier, des sortes de galles hémisphériques d'une eouleur analogue à celle de l'écoree el souvent entourée à leur base d'un petil bourrelet blanchàtre plus ou moins abondant. En y regardant de près, et surtout en détaeliant ees sortes de eoques de la branehe sur laquelle elles semblent eollées, on ne tarde pas a y trouver toutes les traees qui earactérisent un insecte. C'est, qu'en effet, ees earapaces desséehées sont celles de femelles de eochenilles, lesquelles servent eneore aptès leur mort, à abriter les germes de la génération destinée à propager l'espèce l'aunée suivante.

Au milieu du tissu cotonneux dont nous avons parlé, et qui remplit tout l'espace recouvert par les débris de l'inseete, on irouve des centaines, quelquefois des milliers de petils grains de eoulcur ordinairement rougeàtre et qui sont des xufs, dont l'ćelosion aura lieu aux premiers beaux jours de l'aunéc suivante out, selon les espèees, pendant le courant de l'été. Les nouveaux nés sorteul par une petite ouverture l'estée béante, entre la carapace el l'écorce; Réaumur eroil que eetle ouverture existe déjá lors de l'aceouplement el que èest par là qu'il a lieu. 
Pendant les premiers jours, les jcunes larves, qui sont aptères, ayant encore besoin d'un abri, restent logées sous le :entre de la mère. Bientòt eependant, pcut-ĉtre après une première mue, elles se dispersellt et vont se fixer sur les jeunes pousses, sur lcs fenilles, etc, dans lesquellcs ellcs cnfoncent leur petite trompe pour pomper la sève ou les sucs propres de la plante sur laquelle elles doirent virre.

Pendant leur premier age, toutes les jeunes cochenilles se ressemblent, ct lcur facies leur donne l'apparence de petits eloportes. Ces larves, presquc inieroseopiqucs, ont six pattes grêles, deux antenncs très-courtes et une trompe qui parait implantée sur la poitrine. Pendant lc premicr àge, elles se meuvent avec facilité; ınais à l'automnc, pour celles qui n'ont qu'une génération, ou à une autre époque, pour celles qui en ont plusieurs, elles se déplacent unc dernière fois, et se fixent dans unc partie de la plante qu'elles ne doirent plus quilter.

Au moment de la fécondation, on voit apparaitre des individus ailés, excessivement pctits, bicn que produisant, dit-on, un léger bourdonnement. Ces insecles sont à ce qu'il parait les màles des cochenilles; ils n'ont que deux ailes, ils sont dépourvus de bec et ont l'abdomen terminé par deux appendices sétiformes.

Lcs obscrvations de Réaumur, de Degécr, de Geoffroy, de Macquart, de Sclımidbergcr, etc., scmblaient mettre liors de doute que les individus ailés dont il vient d'être question, ne fussent les máles des cochenillcs; eependant, je dois dire iei qu'un naturaliste de Naples a publić, il y a déjả une trentaine d'années, des obscrvations qui tendraient à établir que ees prétendus máles ne sont que des insectes parasitcs diptères appartenant au genre Cécidomyie, at quc les prétendus aecouplements observés n'étaient que l'opération de la ponte, faite par les femclles de ces diptères sur Ics cochenilles aux dépens desquelles doivent se nourrir les larves de ce parasite. Depuis les observations de M. Costa, rien n'cst venu confirmer d'une manière authentique les résultats annoucés par ce naturaliste; d'ailleurs, pour résoudre la question d'une manière complète, il faut, si les in- 
dividus ailés que nous avons décrits plus haut uc sont que des parasites, faire connaitre quel est le véritable mále de la cochenille. Quoiqu'il en soit, c'cst après cctte apparition des individus ailés que les femelles prennent, très-rapidement, un accroisscment considerable; les anneaux dc l'abdomen sc distendent, se gonflent, et bientót on voit apparaitrc autour du corps ce cordon blanc de consistance laineuse que j’ai déjà signalć.

Si, pendant quc ces femelles prenncnt lcur accroissement, on clıerche à les détacher de la partie sur laquelle élles sont fixées, on ne peut le faire sans leur arraelier le bee ou les pattes, tant ees organes sont enfoneés daus l'épiderıne de la plantc. Après deux ou trois jours, on reınarquc avec surprise qu'à mesure quc le duvet eotonneux augnente de volume, la mentbrane inférieure du ventre se rapproclie davantage de cellc du dos, parce que les oufs sont, à mcsure qu'ils sout pondus, poussés, non en dehors de l'animal, comme c'est le cas général, mais en dessous, entre la premièrc couche de sécrétion laineusc qui isolait primitivement l'abdomen de la plantc, ct les $\mathrm{mcm}-$ branes de l'abdomen, lesquclles se retirent de plus cn plus, se dessèchentet restent, après la mort de l'animal, collées au dos pour protéger les œufs jusqu'au moment de lcur éclosion, qui a lien d'ordinaire une douzaine de jours après la pontc; les jeuncs coehenilles, au sortir de l'œuf, sont extrêmement petites ct trèsagiles.

Certaines espèces de cochenilles produisent dcux ou trois mille aufs; an comprend qu'avcc une pareille fécondité, quelques femelles suffisent pour infestcr complćtement un arbre de jcunes larves qui, aussi bicn par la sève qu'elles absorbent, que par' les piquires nombreuses qui cn laissent perdre unc grande quantité, épuiscnt les arbres, les font languir et peuvent, dans certains cas, en détcrminer le dépérissemcit ct même la mort.

Les cochenilles ne se rencontrent que très-rarement sur les plantes annuelles; ce n'cst que sur les arbres qu'on les trouve le plus abondamment; les carapaces qui se trou- 
vent sur le jeune bois, sont celles de l'année, celles qui sont sur le vieux bois, sont celles des feınelles qui ont produit leur génération et qui ne sont plus préjudiciables à la plante. La température a unetrès-grande influence sur la propagation des cochenilles; c'est pour cela que dans les serres ehaudes, elles se multiplient en telle abondance, 'qu'elles finisseut par envahir des plantes de toutes sortes et mêrne des plantes aunuelles.

Les espèees qui attaquent les feuilles caduques des arbres, $y$ restent jusqu'ả l'automne*; si la feuille vient à tomber avant que le manque de sève, ou la température ait averti la jeune cochenille qu'il est tcmps d'énigrer, la larve alors, tombe avee la feuille; nais elle remonte d'elle-mêne sur lc tronc de l'arbre oì elle va chercher une place plus eonvenable. C'cst a cette époque de l'émigration des larves que l'on voit les jcuues pousses s'en couvrir d'un nombre considérable, au point que, nou-sculement ces insectes font du tort à l'arbre par la quantité de sève aspirée ou perdue, mais encore en ee qu'ils bouchent les por'cs de l'écorce et l'empêelient de respirer par les stomates.

Peudant l'hiver, les coehenilles paraissent engourdies et ne prennent pas de nourriture, aux mois de mars et d'avril, clles augmentent un peu de volume sans changer de plaec et subissent une dernière mue avant la fécondation; dans ce cas, la vicille peau se détache d'elle-même par lambeaux blanchàtres et plus ou inoins transparcnts, ou bicu elle restc attachée à la nouvelle et forme une deuxième tunique protectrice pour la ponte de la femelle.

La sève qui s'écoule des piqüres faites par les larves de cochenilles cst tellement abondante que, dans quelques circonstances, le sol de dessous eertains arbres semble êtrc mouillé par la roséc, ee qui attire une grande quantité de gueêpes et de

- Toutes les espèces de cochenilles ne sont pourtant pas nuisibics; quelques-unes sont employées dans les arts pour la matiere colorante qu'elles prodnisent (Cochenille du Nopal, Cochenille de Pologne), un autre la Coecus lacca, produit, dans l'Inde, la gomme laque, ctc. 
fourmis friandes de sues mielleux. C'est par la présenee de ecs Hyménoptères que I'on pourra plus faeilement déeouvrir eelle des eochenilles, dont la taille et la couleur ne permettent pas de les distinguer facilement de l'épiderme de Ia plante.

C'est le plus ordinairement sur les arbres élevés en espaliers que, dans les jardins, sc déreloppent plus aboudamment les eochenilles; e'est du eòté opposé à la lumière, à la base des jeunes pousses, près des boutons, qu'elles vont se fixer de préféreuce; ces agglomérations sout quelqucfois telles que l'écorce parait rugueuse et que plus tard, quand le duvet blane déborde la carapaee des femelles, on dirait un tissu laineux reeouvrant une partie de la branche.

Le noubre des espèees de eochenilles déerites n'est pas trèseonsidérable, un grand nombre d'arbres en nourrissent qui leur sont particulières ou qui peuvent à la fois vivro sur plusieurs essences. La déformation qui se produit dans les enveloppes de Ia femelle après l'aceouplensent, la petitesse exeessive des màles, ne permettent pas de distinguer faeilement les espèces voisines.

Plusieurs vivent sur les arbres fruitiers; les plantes de serres ehaudes sont envahies par le C. adonidum, celles des serres tempérées par les Coecus nerii et C. Lauri, ete. Les premières n'ont qu'une génération, tandis que celles-ci et quelques espices exotiques en ont plusieurs. L̀nfin, les arbres de nos forêts en nourrissent aussi quclques espèces, mais, dans ees eireonstanees, on n'a jamais eité u ue grande invasion de ces parasites.

La destruction des Cochenilles est encore plos diffieile que eelle des Puecrons on celle des Psylles. Les fumigations ne produisent rien sur elles, et les arrosages ou les lavages avee des solutions aeides, ou l'eau de savon, la lessive, etc., ne produisent d'effets sur elles que quand ces liquides sont eaustiques au point d'être nuisibles à la plante. La taille du priutemps, faite d'assez. bonnc heure pour enlever les jeunes bois avant la dispersion des jeunes est, de tous les moyens, le plus rationel, simon, le plus effieace. Pendaut l'été, quand on en déeouvrira qui auron 
échappé à la taille, il faudra les déıacher de l'écorce avee uue brosse ou un torchon rudc.

98. coccus MaII (Schranck).

Nordlinger : Die Kleinen feinde der Landwirthsehaft, page 316.

Synonymie : Cochenille du Pommier;-Apfel-schildlaus (des Allemands).

Màle : Ineonnu.

Femeile: Corps elliptique, de la forme d'un bouelier ayant le bord foliacé, plissé, ride, presque transparent; d'un brun blanchatre avee un bord blanc quand elle est arrivée à l'état adulte; d'un brun clair sans bordure quand elle est jeune.

Cette description cst la seule quc j'aie trouvée dans les auteurs, qui, en général, ont fait avec les cochcnilles comue avec les pueerons, c'cst-á-dirc qu'ils se sont coutentés de donner pour uous spécifiques aux espèces, le nouı de la plante sur laquelle ils rencontraient l'insecte, saus y ajouter unc descriplion suffisante ou mème une simple diagnose. D'ailleurs, comme nous sarons que les cochenillcs, une fois fixées et fécondées, elıangeut conti. nuellement de forme et de couleur, que celle-ei varic encore après la mort de l'inseete, il cn résulte qu'il est impossible de reconnaitrc de quelle espèce ont voulu parler Linné, Fabricius, Schranck, etc., surtout quand, comme cela arrivc pour les arbres fruiticrs, la mc cme plautc en uourrit plusieurs ou que la mêne eochcnillc vit à la fois sur plusicurs de ees arbres.

Fu $18: 38$ et cu 1859, j’ai trouvé sur les pommiers, sur les poiricrs, mais surtout sur l'aubépinc, une cochenille vivante, de coulcur verditrc, d'environ cinq uillimçtres de long sur quatre nillim. de large. La surface était irrégulièrement plissće ct rappelait parfaitcment, pour la forme et pour la couleur, la carapace de la tortue géométrique. Les $\propto u f s$ de cette cochcnille, quc je rapporte au $C$. mali des auteurs, som placés au milieu d'uu 
tissu cotonneux blanc; ils sont comme les oufs de la eoehenille de la vigne, de couleur rosée.

Depuis la mort de l'animal, eette coehenille a eomplétement ehangé de caraetères extérieurs et elle se rapproche singulièrement de eelle que M. Nordlinger décrit de la manière suivante :

* Sur le bois des pommiers de deux ou trois ans, j’ai trouvé une eochenille cle grandeur et de forme un peu différentes, mais morle; elle est plus ou moins convexe en avant, allant en se rétrécissant en arrière. Sur le premier tiers antérieur se troure, une paire de bosses plus ou moins visibles, une autre paire d'aussi grandes et brillantes vers le milien du eorps, enfin une troisième paire de bosses plus petites, en arrière; quelques plis transversaux plus ou moins apparents sur la circonférence du eorps. 》

Aux deux deseriptions qui préeèdent, M. Nordlinger ajoute encore: a Sur les branclies de poiriers de un à deux ans, vir, souvent en famille, une espèce de coelıenille aussi graude, presque ronde quoique bossue, d'un brun luisant. Une autre trèssemblable sur les cerisiers. Lc prunier en nourrit aussi une espèce. ,

D'après ee qui précède, on est porté à eroire que le Coceus mali est aussi une espèce eollective, ou bien que sa femelle vit indistinetement sur plusieurs sortes d'arbres fruitiers et qu'elle y revêt des formes très-varićes dont quelques-unes sont propres à tel ou tel arbre. Toutes ces ineertitudes ne pourront être levées que par la eonnaissanee des màles de cliaque espèce, ou, tout au moins, par l'étude des fcmelles à un àge où n'étant pas eneore fixées d'une manière définitive sur l'épiderme de la plante, clles peurent fourni: des caraetères moins variables que eeux qui, juscuu’à préscnt, ont été tirés de la fortme et de la couleur de leur earapace.

Dalbret, qui nomme Kermès, les inseetes que nous désignons sous le nom de Coehenille, donne les détails suivants sur leurs habitudes. a Le kcrmès cst eonnu des eultivateurs sous le nom de punaise (gallinscete, des a!tteurs). Ces inscctes se font 
remarquer aı printeuns par leur furme et leur fixité, Les plus grandes eoquilles ont truis lignes de long sur deux lignes de largeur, ce sont les vieux de !'année dernière et ils sont morts; mais les jeunes sont plus petits et de la eouleur de l'éeoree, ou à peu pres. On trouve ces cufs en nombre variable sous les femclles; ils éclosent en juin, plus ou moins avant dans le mois, selon la température, les petits sont d'un jaune pảle, avec six pattes très courtes; ils se dispersent et ront se fixer sous les feuilles....; les années chaudes $\mathrm{ct}$ sèches leur sont favorables ainsi que les expositions abritées contre les pluies battantes.

\section{IIV. IECANIUM (Illiger)".}

Amyot et Serville; llemipières, suiles ì Buffon, page 630 .

Ce genre est à peine distinct du précédeat, et l'on troure les contradictions les plus manifestes dans les diagnoses qu'en donnent plusieurs auterrs, tandis que d'autres entomologistes n'en produisent qu'aree doute les principaux caractères. Les mảles sont d'ailleurs aussi peu eonnus et aussi mal caractérisés dans ee genre que dans le précédent, ee n'est que pour ne pas embrouiller la synonymie qu'il aurait fallu établir, que j’ai adopté cette division faite dans le grand genre Coeeus, de Linné.

Les mours et les principales circonstances qui aecompagnent l'évolution de ces petits insectes, sont d'ailleur semblables á celles des Cochenilles, après l'aceouplement, les femelles se déforment eneore plus dans le genre Leeanium que dans le genre Coceus.

99. IECANIUM PYRI (Schranck).

Macquart, le seul des auteurs français qui se soit occupé d'ıne manière générale des insectes nuisibles, cite cet insecte sous le nom de Chermes pyri, Linné; mais il ne dit rien de ses habiludes. Dalbret cite nn Kermes pyri, mais sans non d'auteur et sans

- Synonymie: Coccus (Linné) ; - Cheames (Geollroy); - Keamés. 
aueune deseription. Aza-Fitel, dans l'ourrage que j’ai déjà eu occasion de citer, donne l'histoire du Lecanium pyri, de Schranck, qu'il désigne sous le noun anglais de Barck louse (Poux des écorces) et qui u’aurait été signalé comme existant dans l'Auérique du Nord, qu'en 1850, par le doeteur Harris, et en 1854 , par lui-même.

Par suite de l'éehange qui se fait, d'une grande quantité d'arbres fruitiers, eutre les deux eontiuents, il est possible que cette espèce leur soit devenue conumune à cause de la facilité avec laquelle les coclienilles, en général, se propagent. Grice à l'obligeanee de M. Le docteur Haro, je donne ici la traduetion du passage qui, dans l'ouvrage de M. Aza-Fiteh, coneerue le Lecanium pyri:

"Comme le poiricr est trés-voisin du pommier, il n'est pas étonuant que la plupart des insectes qui vivent sur l'un de ees arbres, se rencontrent aussi sur l'autre; uous avous déjả noté ce fait pour un grand nombre d'espèees, en étudiant les insectes du pommier. Mais, indépendanment des espćees communes aux dcux arbres, il y en a eependant d'autres qui sont propres à chacun et ne changent pas de domicile; excepté peut-être daus les eas rares d'une multiplication tellement considérable que "arbre qui leur est appropric par la nature, ne pent plus fournir à l'alimentation de tous les individus appelés à y vivre. De tous les inseetes qui sont partieuliers au poirier, le seul qui se soit jusqu'à présent offert à mon observation, est uue espèce de poux d'écoree, qui, ee qui est probable, est le mème que l'on trouve en Europe sur eet arbre et qui est appelé par Scliranch Coccus pyri et qui appartient au genre noderne des Lecanium. Cet inseete n'avait pas cneore été annoneé publiquenent de ce cóté de l'Atlantique, quand, ver's le commeneement de juillet $185 \%$ (si je ne ue trompe), j'en ai remarqqué en grande quantité sur les poiriers de la ville d'Albany et de Troie. Je fais cepcudant remarquer que le doetcur Harris, dans so n diseours à la Société de Pomologic américaine, dans le mo is de septembre deruier (page 8), mentionne accidentellement le fait que nos poiricrs souffrent quelquefois de la prósence des poux des écorces. , 
« La forme snus laquelle se présente cet inseete est eelle d'une écaille hémisphérique d'environ 0,20 (mesure américaine), en décembre. D'une couleur brun-marron, adlıérente à la portion inférieure de l'écorce et particulièrement sur les jeunes arbres dont la eroissance est chétive. Ces ćeailles sont les r'estes des fcmelles mortes en couvrant et protégeant leur fanille. Les unes sont d'une eouleur plus foncée que les autres, et l'on en voit de petites qui son! entièrement jaunes; mais elles ne sont pas uarquées de taches pảles conme ic sont beaucoup de nos poux d'écorce. Ces éeailles préseutent fréquemment de légères impressions, comme si on les avait pressées sur place avec la tête d'une épingle; leur bord extérienr est ridé comme on le voitdans la figure, souvent aussi il est marqué de bandes noiratres. Si on eulève ure de ees éeailles, il reste sur la branehe, unc tache blaıclie, de la dimension de l'inseete, on dirait qu'elle a été faite avec de la cliaux. A la partie inférieure d'une branclıe, daus la longueur de neuf pouces, il y avait trente de ces écailles el cinq taches blanches marquaut la place d'autant d'insectes qui avaient été enlevés. \

« A l'époque ou je remarquai ces écailles, les jeunes poux qu'clles contenaient étaient en action et les petits ressemblaient, à l'ocil nu, à des grains de poussière. J'en portai une de ces branches dans ma résidence et l'altachai à un jeune poiricr pour voir si ces insectcs pouraient vivre sur cet arbre, mais ils périrent tous, aucun d'eux n'ayant quitté la branehe sur laquelle je les arais trouvés. Autant que je pus m'en assurer au mois de mai suivant, les taehes resscmblaient a des taehes de chaux et Inalgr'é le temps qui s'était écoulé cepuis que ees taches étaient à découvert clles étaient encore visibles, les tempêtes et les gelécs de l'automnc et de l'hiver les avaient à peine dimiuuées. Audessous de ces écailles, les jeunes poux sont disséminés daus une matière eotonneuse, qui angmente peu à peu de volume et se fitit une issue par l'une des cxtrémités de l'écaille qu'elle détachc de la branche, comme on le voit dans la figure ci-jointc. Alors Ics jeunes poux sortent de cette natière 
et se répandent sur les éeorces lisses, apparaissant l'vil nu comme de petics taches ou de petits points blancs, Quand ils sont plus développés, ils sont un peu plus ovales, un peu aplatis, de la centième partie d'un pouee en longueur el ayant un tiers de moins de largcur; ils sont cntièrement blancs, munis de six pattes et dc dcux courtes antemes d'un blanc transparent; les antenncs sont commc des aiguilles, ou d'un égal dianètre dans toutc sa longueur et on à peu près le cinquième de la longueur du corps, elles sont composćes de plusieurs petits articles légèrcnient relus.

\& Je n'ai pas encole eu jusłu’à présent l'occasion de suivre plus loin l'histoire dc ees pelits inseetes, sans doutc que, comme pour les autres espéces du genre, les jeunes larves ne tardeut pas à se fixer sur l'éeorce et à augmenter un peu de volume; elles conscrucnt la méme forme pendant tout l'liver ct au premier printemps lcs màle's entrent dans l'état de clırysalids; bientôt aprics ils en sortent sous la forme de petites mouches munies seulement de deux ailes; pendant que les femelles, sans éprouver aucuu changement appréciable, grossissent peu à peu, jusqu’à ce qu'cllcs aient aequis lc vo'ume et la forme des ćeailles quc nous avons décritcs. 》

a Un insecte parasite qui appartient probablenent au geure Coccophagus de Westwood, de la famille des Chalcicide de l'ordie des Ilyménoptères, vil dans le corps des fcmellcs et se nourit de ses petits. Cettc larve est semblable à eclle que nous arous renırquée sous les écailles dı pou d'ćeorce du pommier, mais elle est plus grande. Ayant accompii toutes ses métamorphoses, elle sort par un trou qu'elle pratique dans l'éeaille. Plusieurs écailles ćlaient ainsi perforćes, le trou est rugucux, déchiré sur les bords et l'écaille elle-ıême était visiblenent décolorée tout autour, 》

* Cet insecte ne peut devenir essentiellement nuisible au poirier, que quand les femelles sont aussi nombrenses que dans le cas oì je les ai rencontrées. Aueun arbre ne pent croitre avec IIII parcil nombre de petits succurs insérés à la surfacc de toutes 
les jeunes branches. Un petit nombre de fenelles peut au contraire trouver sur chaque branehe une nourriture superflue. Heureusement qu'elles sont d'une taille assez grande jour être facilemcnt aperçues et détruites quand on inspecte aree attention les parties inférieures des jeunes braüclıes. C'est dans la dernière moitié de juin qu'il faut les ehercher, époquc où les femelles ont atteint tout leur développement et par conséquent oú il est plus facile de les découvrir; on les détache avee une brosse dure ou avee une éponge. Dans ce moment clles sont presque mortes et ue peuvent plus remonter; les petits, au contrairc, sout eneore trop jeunes pour se passer de l'abri de leur mère.

En comparant la description de l'iusecte dont parle M. AzaFitch, à eelle que donne, du Coccus mali, M. Nordlinger, on est conduit à penser que les deux auteurs ont déerit la même cspèee de cochenille sous deux nouns différents, l'un en decrivant les bosses de la carapace, l'autre en comparant les dépressions eomprises entre ces bosses, à des enfoncemeuts produits par la tête d'une épingle. Il est cependant bon de faire remarquer que M. Nordlinger dit que lc $C$. mali vit sur le pommier, tandis que M. Aza-Fitelı dit n'aroir observé le L. pjeri que sur le poirier.

Une autre observation très-importante, e'est que pour l'auteur américain, il seuble n'y avoir aueun doute dans son esprit sur l'jdentité du mâle du Lecanium prri, avec le petit inscete diptère qu'il a observé et qui, selon M. Costa, ne serait qu'uu parasite appartenant au genre Cécidomyie. Bien ccrainement les observations du naturaliste de Naples ne sont pas iueonnues au savant entomologiste d'Albany, et eependant celui-ci ne met pas en doute l'existenee du mảle de l’insectc qu'il a étudié. Ne serait. il pas extraordinaire de voir commettre la nuéme méprise, à la fois sur les deux eontinents surtout, quand, de part et d'autre, les entomologistes décrivent avee le plus grand soin les parasites des cochenilles. Pour M. Aza.Fitclı, comme pour Réaumur et Degécr, dont la finesse d'observation est incontcstée, les máles des cochenilles, des Lecanium, elc., sont biell ces petils diptères 
que, seul jusqu'ici, M. Costa a considérés comme les parasiles de ees insectes.

\section{ASPIDIOTUS (Bouche)*}

Bouche; Naturgesichte der Insecten, prenière partie, page 9.

Màle: Deux ailes transparentes et deux balanciers en massue; trompe courte; antennes Jongues proportionnelIenent, cornposées de neuf artieles, en forme de soies; organe génital mảle en alène, horizontal, composé de deux artieles dont le premier est eourt, gros et eylindrique; tarses de trois articles avee deux crochets.

Femelle: Corps clıarnu, arrondi ou allongé, peu convexe en lessus; la plupart du temps il ı'y a qu'une partic du corps qui devient eharnue; la téte demi-eireulaire, se grossit de telle sorte que les antennes finissent par disparaitre sous elle, la trompe est égalenient repoussée vers la poitrine, de sorte qu'elle parait sortir du milieu de l'inseete; les jambes s'atrophient égalemcut dans quelques espèees. La trompe, longue de la moitic du eorps, est organisece eomme eelle des Cochenilles.

Le genre Aspidiolus esı, comme le préeédent, un dénembrement du genre Coccus, de Linné. Aux earactẻres que lui assigne Bouché el qui ue le dislinguent pas assez des coehenilles, on ajoute encore que le corps de la femelle, nuême aprés la fécondation, reste plus ou moins plat el que les anneaux qui le composent sont toujours distincts.

Bouché donne, ainsi que nous l'avons vu, une deseription assez eumplète du mảle que III. Costa considère eomme un diplère parasile pour lequel il a mème eréé le geure Diaspis. L'anomalie que forment, dans l'ordre des Ilomopteres, les inseetes de la

- Synonymie : Coccus (Lin.); - Diaspis (Costa), 
famille des Coceiniens, dont les males nont que deux ailes et dont les femelles sont aptères, est remarquable en ee sens que les deux sexes de l'espèee s'éloignent également du type de la famille dans laquelle les entomologistes ont eru devoir placer ces insectes, tandis quc les anonialies que l'on observe daus lés autres ordres (Coléoptères, Iépidoptères, Hyménuptères... etc.) n'existent que dans l'un des sexes. Quelle que soit d'ailleurs la perfection que l'on cherehe a introduire dans nos mélhodes de elassification, on ne pourra jamais éviter ces sorles d'empiélements réciproques de certains groupes sur d'autres plus ou moins éloignés dans la sćric organique. Partout l'on reucontre de ecs types aubigus, qui paraissent appartenir a plusieurs fanilles naturelles et qui semblent eréćs pour rapproelıer les anneaux éloignés de la chaine et démontrer l'iṇutilité de nos efforts á constituer une série coutinue.

Après l'aceouplement, les femelles d'Aspidiotes deviennent plus allongées ou plus larges et elles pondent, toujours sous elles eomme le fout les eochenilles, des œufs elliptiques quine tardent pas à éclore si la température est favorable. Les larves qui proviennent de ces $\propto u f s$ vivent pendant quelques jours a l'abri de la rarapace de la mère el y subissent inème, selon Bonché, une première mue; plus tard elles se dispersent sur les feuilles, le jeune bois, cte, selon les espèces. Ces larves sont elliptiques, plates, le dessous de la poittine se termine par la trompe; les yeux sont saillants, petits et éeartés; les antennes, longues d'environ la moitié du eorps, ont six arlieles, dont les cinq preıniers sont arrondis, le sixiène allongé et terminé par deux fortes soies plus petites chez les máles que eliez les femelles; eorselet et abdomen ridés et glabres; les pattes sont plus courtes que eliez les individus adultes; enfin le dernier anneau de l'abdomen porte deux soics divergentes plus ou moins longues selon les espèces.

Les laryes d'Aspidiotes sont assez agiles tant qu'elles ne se sont pas fixées sur quelque partie de la plante; mais unc fois qu'elles le sont, elles ne changent plus de place, et se laissent plutdt 
arracher la trompe que de la retirer de l'épiderne dans lequel elle est enfoncéc. C'est ordinairement sur les arbustes appartenant aux fanilles des Laurinées, des Cactées ou des Magnolacées, que dans les serres chaudes ou tempérées, on rencontre les Aspidiotus; dans les jardins, on en trouve sur les rosiers et sur plusicurs espèces d'arbres fruitiers; enfin, dans les champs ou dans les forêts, c'est sur les arbres qui appartiennent à la famille des Ainentacécs (chẻne, saulc, peuplier, ctc.), que l'on en compte le plus grand nombre d'espèces. Une observation importante at faire iei, e'esı que, quel que soil le nombre des aspidiotes que l'on trouve st:r une plante, on ne yoit jamais la partie du végćlal où ils sont fixés, mouillée par la sère comme eela a licu pour les pucerons ou même les cochenilles; par conséquent, les fourmis n'étaut pas attirées par ce liquide sucré, on ne les rencoutre pas ródanı autour de ces insectes.

100. ABEXXOTUS coxanxTORMXs (Gmélin).

Synonymie : Coccus arborum linearis (Modéer); - Diaspis linearis (Costa); - Tigre sur bois (des Jardiniers); La Gallinsecle en forme de coquille (de Rénumur); La cochenillc en icaille de moulc (de Geoffroy); Cecolcpis (Amyot).

Femelle: Longue de deux millim. et deni à trois millim. et demi, large de un à deux millim. Corps ordinairement arqué, aminei en avant et ayant la forme d'une petite coquille du genre Mylilus ou d'une perite sangsue; de eouletrr brun grisàtre plus ou moins foncé selon la eouleur de l'épiderme sur lequel elle est fixée; partic antérieure, eorrespondant ì la tête, de eouleur plus elaire et ordinairement jaune ferrugineux.

Get insecte, dont le inåle m'est inconnu, se rencontre par milliers sur l'épiderme de nos poiriers; on le trouve aussi sur quelques autres arbres (Prunus spinosus, Mespylus oxvacantha, 
Mespylus germanica, ete.), mais jaurais en aussi grande abondance que sur lc poirier oú l'on voit souvent les individus se toucher et même se recouvrir les uns les autres. Je dois eependant faire observer ici que les individus que j’ai rencontrés sur le Prunellier sont d'une tailic beaucoup plus grande que ceux qui se trouvent sur les arbres fruitiers *.

Réaumur avait déjà remarqué eet iusecte et voici ee qu'il en dit (tome IV, mémoire 1, page 67):

« Il me reste encore à parler d'une espèce de gallinsccte qui me semble avoir un caractère propre à déterminer un nouveau genre de ces petits animaux, parce qu'on ne saurait guère les ramener à l'un des deux autres genres que nous avons fixés; le premier, celni que urous avons uommi la forme de bateau renversé, est cependant celui anquel clle a le plus de rapport; mais au lieu que les deux bouts des gallinsectes en bateau renversé sont à peu près également gros, celles que nous voulons faire connaitre ont un de leur bout minee et mème pointu par rapport à l'autre. Nous les nommerons des gallinsectes en forme de coqnille, parce que leur figure ressemble asscz à celle d'une de ces pièces dont deux ensemble forment la coquille entière d'une moule de mer; nos gallinsectes sont pourtant plus allongés par le plus mcnu de leurs bouts que ne le sont par le mène bout les moitiés des eoquilles auxqnelles nous les comparons, cle.

«Ces gallinscctcs eu eoquille sont extrêmement petits et m'en ont imposé pendant plısieurs années; je les ai pris d'abord pour une coquille qu'un très-petit inseete s'élait faite pour se métamorphoscr; les tronvant ensuite pleines d'œufs et ayant oublié que je les avais trouvécs remplies par un inscete, je crus qu'elles étaient un joli nid dans lequel un insecte atvait renfermé ses oufs, mais enfm j'ourris de ces nids dans un temps our les petits étaient éclos et

- Au Jardia-Botanique de Melz, M. Belhomme a observé cel Aspidiotus sur le Cormus paniculata el sur l'Evonymus verrueosa; à Paris, M. Belhoune l'a observé sur le Clatrastes tinctoria et sur l'Hamamelis virginiana. Tous ces arbres sont originaires de l'Amérique septentrionale. 
la figure de ees petits me les fit reennnaitre pour des gallinsectes naissants. \ La figure que donne ensuite Réaunur de eette sorte de coclienille, ne laisse aueun doute sur son identité avee eelui dont il est ici question.

Depuis Réaumur el Geoffroy, e’est a peine si l'on trnuve, dans la plupart des ourrages cientomologie publiés en France, quelque elose comeernant eet insecte. M. Amyol, dans ses Rhyncholes de France, ne fail que eiter lasynonymic des deux auteurs qui précèdent en indiquant à tnrt, eependant, les fig. 5,9 et 11 , de Réaumur, lesquelles représenteut le gallinsecte en bateau, au lieu des fig. 5, 6 et 7, qui se rapportent à l'. Ispidiotus conch)formis. Bouelıé, l'auteur du genre Aspidiolus, ne parait pas non plus avoir eonnu eet inseete, à moins cependant, ce qui est peu probable, qu'il ne l'ait eonfondu avee son Aspidiolus lauri, auquel il donne aussi la forme d'une petite conquille. M. Nordlinger, dans son ouvrage sur les inseetes nuisibles, ne fait qu'indiquer ect insecte; et, dans un Supplémcnt aux insectcs nuisibles de Ratzcburg, publié jar ent auteur, en 1856, il eite de nouveau un Aspidiotus de forme de petite eoquille (Mytilus) lequel vit sur le cornouiller, sur le frêne, sur le platane, sur le tilleul, sur le Juglans regia, etc., mais il ne l'indique pas sur les arbres fruiticrs.

En Ámérique, on rencontre aussi l'Aspidiolus conchyformis, snin histoire a été faite d'une manière remarquable par Aza-Fitelı. C'est d'apres ce dernier auteur que nous allons faire eonnaitre les détails intéressantẹ qui ont été publiés sur cet inseete nuisible, en faisant observer qu'il est assez extraordinaire d'aller cherelıer en Amérique l'listoire d'un insecte qui y a été iutroduit par les arbres quc nous y avons envoyés. Il est également nécessaire de faire renarquer que, dans le eomté d'Albany, e'est sur lc pommier que l'on trouve ordinairement l's spidiotus conchyformis, tandis que dans la Moselle on ne le reneontre que rarement sur cet arbre, probablement en raison du duvet assez scrré qui recouvre son jeune bois.

* Le pou d'écorec, dit M. Aza-Fitclı, est sans doute le plus 
peruicieux et le plus destructeur de tous les iusectes nuisibles connus jusqu'à présent dans nos contrécs comme vivant sur le pomınier. Répandu dans tous les états du nord, il cause partout la mort de beaucoup d'arbres ou nuit ì la santé et a la vigueur de beaucoup d'autres. Il sc présente sous la figure de pelites ćcailles, adhórentes a l'ćcorce, et ayant à peu près la forme de celles des moules, comme on le roil dans la figure ei-jointe. Il n'est pas rare de rencontrer des arbres dont l'écorce est litternlement couverte de ces écailles, depuis la racine jusqu'i l'extrémité des branches; quelques-uns inême, ne trouvant plus de place vacante pour se fixer, se portent sur les feuilles et même les fruits sur lesquels on en trouve souvent plusicurs. $Q$ uand un arbre continuc ainsi à être inf́rsté il dépérit d’annces en aunées et finit par mourip. C'est ce que j'ai observé surtout sur les jeunes arbres qui se trouvent isolés dans les champs; car alors les insectes ne trouvent pas d'autres sujets sur lesquels ils puissent émigrer et chercher une nourriture suffisante pour remplacer celle qui leur uranque sur l'arhre en souffiance. On a aussi remarqué que des arbres qui avaient été couverts de ees insectes peudant une ou deux années, et qui étaient arrivés a un point de dépérissement qui ne leur permettail plus de fournir une mourriture abondante? à ces insectes, avaient repris leur vigueur ordinaire apris petit nombre d'années, paree que les insectes parasites avaient abandonné l’arbre qu’ils avaient épuisé. Je ne saurais dire si, dans ce eas, l'insecte périt faute d'aliments ou s'il rmigre sur d'autres arbres, je crois cependaut que le premier fait, celui du dépérissement de l'inscete par faute de nourriture, est eelui qui arrive le plus souvent.

" Bien que eet msecte fasse un tort considérable aux arbres des vergers de l'état de New-York, ee n'est rien en comparaison des rarages qu'il exeree chez nos roisins de l'ouest. Dans les districts qui longent le lac de Miehigan, en particulier, il y produit mainteuant des dégàts dout l'intensité surpasse tout ce que l'on a dit jusqu'a plésent sur re pou d'écoree. C'est à peine si l'on trouve un seul arbre qui en soit exempt, et si l'on ne piend 
des niesures pour les tétruire, on est sủr de voir périr l'arbre un petit nomlsre d'années après leur invasion. 》

"Gcorges Kimball, Esq., de Kenosha, dans le Wisconsin, m’a donné les renseigneınents intéressants qui suivent sur l'introduction et la multiplication de ces insectes sur ces arbres: «Le pou 》 d'écorce parait avoir été amené ici en $\mathbf{1 8 4 0}$ par quatre jeunes 》poiriers que mon fils avait rajportés de Cleveland, Ohio. Ces ๖ arbres étaient chétifs et lcurs feuilles avaient une tcinte noi》) ràtre, l'écorce était littéralement recouverte de ces poux, s'en. » chevêtrant et se recouvrant cux-mêmes de manière.à former 》de larges écailles qui se détachaient par le choc de la pluic v et restaient souvent attachées en grappes après les feuilles de " l'arbre, jusqqu'à ce qu'enfin, en 1848 , ces arbres périrent n'ayaut $\searrow$ grandi que d'un pouce tous les ans pendant Jes trois dernicies "années. Mais avant celle mortalité, les insectes avaient plus ou » moins envalıi mes autres arbres. Bientót mềne, tons furent 》attcints, les plus jeunes plus que les plus ágés. Quelynes-uns " de res insectes allèrent se réfugier jusque sur mes poiriers, 》particulicrement sur un petit arbre qui se trourait ne porter 》 que de mauvais fruits. Ce dernier cn fut couvert autant que 》quelques-uns de mes pommiers. Nous n'a vons rien trouvé dans "I Ies livres, ni dans Ics rapports d'agriculture ou d'horticulture, ¿ qui puisse être rapporté à ces insectes, et de là sans doute notre " ignorance des moyens de les combattre. Dans notre localité on 》 fit des efforts pour organiser une société dont chaque membre 》) paicrait dix dollars, pour former une caisse d'encouragentent 》 pour les expérinentateur's et pour récompenser convenablement 》l'autcur dn meilleur remède. Un procédé secret dont l'expé* rience a d'ailleurs dénıontré l'inefficacité, fut aclieté partout dans 》 notre Comté moyenuant un dollar par individu. Espérant que " mes jeunes arlores plus vigourcux pourraient surmonter cette 》 infection dn pou d'écorce, je fis arracher et jeter au loin tous mes 》 vieux arbres, at: nombre de plus de trente, il m'en reste inainte" nant environ 150 dont aucun n'a plus de douze années et j'espère จ que ies remèdes que j’ai employés les préserveront de ces poux 
¿ d'écorce. Mais daus toute cette partie du Comté, les arbres sont 》 couverts et rongés par ces insectes; un arbre ne vit pas plus de ¿ trois ans après qu'il a été envalii, et partout l'on peut rencontrel 》 quelques arbres inorts et un grand nombre d'autres qui sout „assez malades pour que l'on désespère de les guérir. 》

"Cel insecte, continue M. Aza-Fitch, après avoir rapporté celte communication de son corresponciant, ne parait pas avoir pénétré dans l'ouest jusqu'à présent, au-delà des districts qui bordent le lac Michigan. J'ai renarqué que les vergers situés sur les bords dı Mississipi en sont exempls, ct dans me inspection sévére des arbres de la ferme de Ridge, appartenantà l'esquire Baldwiu, unoins de cent milles à l'ouest de Clicago, je les ai tous trouvés intacts. Mais, il ne saurait y avoir aucun dloute sur leur invasion future, graducllement accomplic, jusque dans ees contrées. Il est également à eraindre que pendant quelques années après l'apparition des poux d'ćcorces dans unc région, ils n'accomplissent les mêmes évolutions que celles qu'ils parcourent ınaintenant dans les environs du lac Michigan, car ces phéıoménes sont conımuns à tous les insectes nuisibles, c'est-à-dirc, qu'ils ne se multiplient et n'atteignent un développement considérable, que quand ils sont entièrement naturalisés. »

a Dans l'ouest ou admet généralement que cette coclienille est unc nourelle espèce particulière à ees contrées, parce qu'elle ne se trouve décrite daus aucun des livres acecssibles a la masse des lerteurs. Cicst par ces raisons que mon ami Robert W. Kennicotl, de Northfield (Illinois), a, dans une communicalion faice all mois de juin deruier à l'académic des sciences naturelles de Cleveland, et publiée daus ses mémoires, arec la figure des jeunes larves, décrit cette espèce sous le non de Coccus pyrus malus, sous lequel il est désigné maiutcuant dans quelques-unes de nos revues périodiques d'ugriculure de l'ouest. Mais cet insecte est bien celui que nous avous observé jusqu'à prósent daus l'est, il a tonjours élé regardé comme étant le mêur que celui qui est connu depuis longtemps et qui rit en Europe sur les pomniers et quelques autres arbres. Réaumur le décrivit en 1738 , et cet 
autcur l'indique comme vivant sur l'orme; il parait aussi avoir été décrit d'abord par Modéer et publié par lıi sous le nom de Coecus arborum linearis (ce qui littéralement signifie Cochenille liıéaire des écorees d'arbres). Il fut anssi désigné sous ce nom par Geoffroy et par les auteurs qui l'ont saivi. Gmélin parle du même insecte (au moins l'a-t-on supposé généralement) sous le nom de Coecus eonelyformis ou pou d'écorce, ayant la forme d'une eoquille de moulc. Le nom spécifique de Arborum linearis s'il est réellement employé ponr désigner l'insecte dont il est question et qui vit sur l'écorce du pommier, n'est certainement pas heureux, puisque cette espèce n'est pas du tout linéaire dans sa forme, qui est conique, comme presque toutes les espèces de cochenil!es qui attaquent les arbres comne eelle-ci. M. Costa a récemment réformé ce, nom, en retrancliant l'expression arborum qui n'était qu'un pléonasme, ınais le nom original doit être rejeté en raison de son peu de rapport avec les règles modernes de la nomenclature scientifique ${ }^{\star}$. Le nom de conchyformis douné par Gruélin, doit en eonséquence ètré préféré à cause de sa priorité et de sa justesse. Quelques autorités scientifiques pensent cependant que les $C$. conehyformis et $C$. linearis forment deux espices distinctes. Ces doutes touchant la question de savoir lequel de ces deux noms convient a notre pou de l'écorce du pomınier, seraient facilement levés s'il était prouvé que c'est la même espéce que celle qui vit en Europe, question que je suis à peine en état de résoudre avec le péu d'autorité sur ces insectes que j'ai entre les nains. Counne M. Curtis, le célebre entomo-

- Tout en admettant la justesse des observations faitcs pas ces auteurs relativement au nom spécifique, peu convenable, du reste, employé par Modéer, on ne saurait admeture la conclusion qui précède. Je ne sais trop ce que l'auteur entend par les rigles modernes de la nomenclatare scientifique, mais si elles sont conformes aux principes qu'il professe en ce monent, on peut en conclure qu'elles ne sont pas encore admiscs en Europe, car leur adoption conduirait, sans aucur doute, au bouleversement complet de oute nomenclature scientifique. 
logiste anglais, actuellement président de la Société entomologique de Londres, a donné unu séric d'articles sur plusieurs cspèces de ce geure, dans le troisiène volume des Cronicles gardener's, volume que je n'ai pas en ma possession, et comme j’ai déjà eu avec lui quelques rapports de correspondance, je lui enroyai récemment, pour avoir son opinion, quelques éclontillons de notre pou d'ćcoree dı pommicr et quelques autres, très-probablement de la même espèce de notre osier rouge (Cormussericea). Voiei un extrait de la réponse que j’en ai reçue: «J'ai examiné »avec soin ros échantillons, ils sont identiques et se rapportent 》au Coceus arbormm linearis, de Geoffroy, etje pense au Coceus „ conchyformis, de Ginélin, qui dans ee eas n'est qu'un synonyme; »vous avez raison de les placer dans le genre Aspidiolus, 》 J'espère que celte citation satisfera ceux de mes amis qui, jusqu'à présent, ont repoussé l'opinion émise par moi que eet inseete n'est pas nouveau, mais une espèce commune it notre contrée, à l'est, ct aussi à l'Europe. ఎ

«M. Rennie dit qu’il a trouvé ectte espèec en abondance sur les buissons de groseilliers. Je ne l'ai jamais trouvé sur les gruscilliers cultivés, mais quelquefois seulement sur notre gro seillier saurage (Ribes floridnm) et en assez grand nombre. Les écailles sont entièremeut scmblables à celles que l'on trouve sur lepommier, mais un peu plus pelites et d'une couleur d'un brun moins foncé, et quoiqu'elles ne soient pas souvent comınunes on le trouve aussi sur les branches des noyers, mais quelquefois si perites qu'elles sont inperceptibles à l'wil nu. Comme celles-ciforment évidemment une espèce nouvelle je prupuse de les désigner sous le Irom'de Poux d'écorce du noyer (Aspidiotıs juglandis). Mon ami, M. le docteur 'Todd, de Wlreeling, en Virginic, m'a envoyé des ćchantillons des espèces du même genre vivant sur les rosiers. Il dit a ce sujet : "Mes plus belles roses sont envahies par cette »vermine, elle tue infitliblement tuus les rosicrs sur lesquels elle » se développc. Cette espéce présente une écaille ronde, mollc, Dd'environ les cing centièmes d'un puuce de dianetrc, souvent »avec une légère tache jaune au ecntrc. C'cst probablement l' $A s-$ 
\piliotus rosø, de Bouché (Sehcedlich: gard.: ins., page 53) dé¿ crite dans l'édition anglaise du Traile de Kollar, page 179. - L'Aspidiolus conehyformis a environ la huitième partic d'nn pouee anglais de longueur, il a une forme ovale irrégulière, suuvent bounbée au milieu et plus ou moins courbée a la plus petite extrémité qui est pointuc, tandis que l'autre est arrondie; sa couleur est d'un brun passant sonvent an noir. La petite extréuité est pálc et jaunátre. L'insecte ressemble parfaitcment à une trèspetite éeaille de nıoule appliqquée sur l'écorce. Celte ressemblance est așsez frappante pour avoir été saisie par tout le inonde et lui avoir fait donnel le nom vulgaire de Pou d'écorce en coquille. Ces éeailles sont placées irrégulièrement, quoique le plus ordinairement elles se trouvent sur la longueur des branches, ayant leur petite extrénité dirigée vers le haut. Ces écailles sont les restes des corps des femelles recouvrant et protégeaut leurs œufs. Durant l'hiver et au printemps, ces œufs peuvent étte trouvés en soulevant l'écaille qui les recourre. Jeur nombre, pour chaque individu, est for 1 variable; eeux que j'ai couptés m'ont donné les nombre suivants : 15, 22, 56, 54, 58, 71, 86, 10\%. J'ai trouvé uniformément un plus grand nombre d'œuls sous les écailles des arbres vigoureux. Mais quand uı arbre en est recouvert de manière á dépérir et par conséquent à ne plus fournir une nourriture assez abondante, alors le nombre des nufs est moindre. 》

« Sous ces éeailles, j'ai souvent trouvé une larve ordinairement plus petite, longue de trois eentièmes de pouce, d'uue forme un peu ovale, arrondie á l'une de ses exlrémités et en pointe aiguë à l'autre, molle, de la couleur du miel, légèremeut transparente et luisante, avee une tacbe opaque brune vers le milieu, produite par les aliments contenus dans l'intestin et divisée en denx segments par des lignes trausversales légèrement urarquées. Ce ver est probablement la larve de quelque petit insecte Hyıćuoptère spécialement créé par la l'rovideuce pour détruire les aufs de ce pou d'écorce. Lil preure que ces œufs lui servellt de nourriture, e'est que quand ld larve est jeune, on trouve un 
eertain nombre d'wufs avec elle sous l'ćcaille, quand elle est plus grande au cuntraire les œufs sont plus rares. Les individus dont je viens de donner plus laut la mesure et la description n'avaient plus que deux œufs à cousommer. Que la larve soit grande ou petite elle parait remplir la cavité de l'écaille concurramuicnt avec les ocufs et je n'ai trouvé qu'unc seule fois ce parasite sur des arbres bien portants, sur lesquels cependant chaque écaille renfermait un grand nombẹc d'œufs. Sans doute celte larve reste sous les écailles du pou d'écoree pendant son premier ćtal et elle en sort en perçant un trou rond. La figure représente unc écaille amplifiće et perfurée par la surtie du parasite; la petite ligne qui est a druite représente la longueur naturelle de l’éeaille. »

* Les cufs d'Aspidiolus sont un peu moins grands que la centième partic d'un pouce, ils ont une forme ovale régulière, à peu près deux fois aussi longue que large, ils sont unous, upaques et peu luisants, la plupart sont blancs, les autres d'un jaune pảle. »

«Pas plus tard que le 12 mai, j'ai déjà trouvé des larves éeloses ct courant avec aetivité parmi les oufs, mais restant encore a l'abri sous l'écaille. En général, ee n'est guère que quiuze jours aprc̀s leur sọrtic de l'ouf que les jeuues larves se montrent au dehors et qu'elles quittent l'écaille puur se répandre sur l'éeorce. A l'ocil nu elles apparaissent coume de petits puints blanes uniformément répandus sur l'écorce lisse des branehes et ayant l'air d'y former une granulation appartenant à l'épiderme. Une personne a laquelle je faisais un jour remarquer ces puints blancs ne vuulait pas croire qu'ils ćtaicnt autre chose que des points naturels propres à l'écorre, jusquà ce qu'enfiu, par une attention suutenue elle fut parvenue à les roir se reumer sur l'éeorce. Au mourent de la sortie des larves de l'ouf, elles sont à peu près de la grandeur de la moitié dé cet ocuf, d'une forme ovale et d'une cou. leur jaune-foncé, ou aperçoil trois paires de paltes, deux placées antérieurcment, les autres postérieureuncnt et très-distinctes, clles marchent arec beaucoup d'animation et d'agilitć. Je n'ai 
pas suivi eet insecle a travers les phases subséquentes de sa vie avec assez de soin pour en donner l'histoire. »

* Le Fermier de la prairic et autres publications agricoles de l'ouest, ont fait connailre un grand nombre de remèdes pour la destruction du pou de l'écoree. Le remède secret qui a été préconisé dans toute cette contrée conme devant les détruire infailliblement était simplement une infusion de Quassia dont on arrosait ou aspergeait les arbres au moyen d'unc seringue. On ne tarda pas à s'apercevoir que ce procédé n'a vait aucu ne efficacité et qu'il n'avait d'utilité que quand on répandait ce liquide su. les jeunes nouvellement éclos, époque ì laquelle les infusions de tabac: ou l'eau de savon, bien plus écononiques, sont tout aussi efficaces. Les liquides dont nous venons de parler, ainsi qu'une lessive forte, l'eau de potasse, l'eau blanche, les cendres sèches, le soufre et je ne sais combien d'autres substances, out élé fréconisées par différents auteurs. Dans un dernier numéro du Fermier de Michigan (vol. 13, page 82), A-G. Hanford, rend un compte favorable des effets du goudron et de l'luile de lin battus ensemble et appliqués a chaud arec une brosse de feutre. Arant l'ćvolution des bourgeons, celte couclıe se desseche, se fendille, se détaclıe et emporte avec elle les ćeailles mortes. Des arbres traités par ce moyen ont grandi de deux pieds a deux pieds et demi l'été suivant, et cependant la eroissance de ces arbres n'avait pas dépassé quelques pouces pendant les auuées précédentes. Le remède précouisé par M. Kinball, de Kenoslı, est probablement l'un des plus efficaces et aussi conveuable que tout autre; cet horticulteur, fait bouillir des feuilles de tabac dans uuc forte lessive, jusqu'a ce que le tout soit réduit en une pulpe impalpable ec qui se fait asscz rapideurent, alors il y mêle du savou fondu (que l'on a fait refroidir; mais pas assez cependaut pour (qu'il se prenue en g̀clée) de manière à former du tout une unasse de la cousistance d'une bovillie épaissc; l'objet de cette manipulation est d'obtenir une preparation qui ne soit pas coleve de l'arbre par les premieres pluies, ce qui arrive ordinairenent aux couches faites avce la décoction de tabac que ces 
premières pluies enlèrent sourent el que les pluies suivantes enlèvent infailliblemeut. 》

"Les fibres du tabac unélangées à cette préparation, contribuent a la faire adhérer partout ou elle est appliquée, bien plus que ue le ferait toute autre substanee entièrenent soluble dans l'eau. On eommence a neltoyer les arbres afin que chaque branclie puisse être atteinte par le pinceau et on applique la préparation avant que les boutons ne se soient gonflés an printemps. Deux hommes strictement chargés de ee travail et arec lesquels on pourait compter que l'arbre serait enduit jusqu'á la deruière branclse, furent employés l'année dernière pendant quinze jours à enduire 130 arbres jeunes. Quand je vis ces arbres vers la fin de septeubıe, on voyait encore des traces de la composition qu'ou avait appliquée sur le tronc et sur les branclies, l'écorce de ces parties était en bonne santé. Les arbres araieut poussé avec vigueur, se portaient bien, et c'est à peine si sur les nouvelles brauches on royait ça et lả une écaille de pou d'ćcorce, tandis que sur les aucienues branches on n'en voyail aucune. Quoique les arbres détruits par les poux d'écorce fussent dans les earrés voisins, il parait que les insectes préfèrent mourig d'inanition plutót que de s'empoisonner en émiglant sur les autres arbres, de lả on peut conclure qu'il suffit d'une seule application bien faite avec cette composition pour détruire tous ces insectes et pour protéger les arbres saius contre l'invasion de ceux qui habitent les arbres du voisinage pendant une période de deux années; car, propres comme l'étaicut eu septembre les arbres qui avaient été envahis par le pou d'écorce, ils n'ont pas di avoir. besoin d'une nourelle eouclie au printeups suivant.

Cet extrait de l'ouvrige de M. Aza-litel est un peu longr sans doute, unais il est tellement intéressant et coutient tant de choses applieables à notıe pay's que je n'ai pas craint de le douner tout entiel.

Dans le département de la Moselle, je n'ai jamais rencontre d'écailles d'Aspidiotus ayant une couleur noile; ordinairenent un les trouve un plus grande adondance daus les environs des 
bourgeons ou a la naissance des jeunes branches, les oufs sont toujours blancs et leur nouble n'a jaıa is dépasse 88 , dans ccux que j'ai comptés provenant d'une seule fernclle ; à part ces différences de peu d'importance, toutes les autres particularités indiquées par l'autcur américain sont de la plus exacte resscmblance, et je u'heisite pas à r'cconnaitıe que c'est bien la mème espèce qui habite lcs deux conlinents; tout me fait supposer qu'elle a été trassportéc d'Furope aux Fitats-Unis avec les arbres fruiliers quon a introduits dans ces contrécs où ellc aura sins doute trouvé des condilions de dévcloppement plus favorables; c'est ce qui explique son extrêmc inultiplication. Quant au parasitc qui vit aux dépens des oufs de eet $A s p i-$ diotus, j’ai aussi trouvé a Metz des écailles d'Aspidiotus ayant un trou rond, ınais je n'ai rencontré dans l'iltéricur de ces dépouilles, ni cufs, ni larves.

Comme remćde cn dehors de ce qui est indiqué plus lıat, je dirai que le badigeon a la chaux, fait i l'automne et renouvelé au printemps, donne de très-bons résultats.

$-\operatorname{sing}$

Liste des Dipteres qui vivent sur te Poirier.

TIPULIENS.

Cecadomyia Mgra (Meigen). La larve vil dans les jeunes poircs et les - Pyucola (Nordling.). f fait tomber.

Assez nuisible.

- Pyr (Bouché). La larve vit dans les bourgeons terminaux des jeunes pousses.

Scisna pym (Sclunidberger). La larve vit dans les I jeunes poires et les - Schmidoenger (Kollar). $\int_{\text {Assez nuisible. }}^{\text {fait tomber. }}$ 


\section{IVI. CECIDOMYIA (Latreille)*}

Macquart; Diplères, suites a Buffon, Ed. Roret, t. 1, page 159.

Tète petite, lıémisphérique; pas de stenmates; palpes de deux, trois ou quatre arlicles; anternes recourbees vers l'extrémité, à articles nombreux, aussi longues que le corps dans les mảles, à articles sphériques et circulairemsent entourés de poils; cluez la fenclle les antennes sont plus eourtes, plus rapproclsées à leur insertion; 'le nombre des articles des antennes est assez variable* , ordinairement de douze ehez les femelles "l de vingt-qualre chez les máles ***; corselel ovoüde; abilomen composc de lıuit auneaux, eylindrique chez les màles, pointu elses les femelles et termiuc par une tariere plus ou moins longue; cette tarière est composéc de plusieurs artieles emboités les uns dans les autres comme le sont les tubes d'une lunette d'approelıc; ailes obtuses ou arrondies a l'extrémite, ayant le bor'cl postéricur garui de franges plus ou moins longues et Irois nervures sur le disque ***, dressees parallèlement pendant le repus; pattes longues proportionnellement, plus ou moins velues, premier article des tarses courts.

- Synonymie: Tipula (Linué); - Cécidomye.

- Beaneoup d'auteurs (Macquart, ß̣tanehard) disent que le nombre des arlieles varie de dix á trente-six, mais M. Bremi fail observer que ces organes étant très-délieats, il arrive souvent qu'ils sont ineomplets quand on les examine; e'est probablement ce qui est cause de la divergenee d'opiniou que l'on rencontre dans les anteurs rclativement in nombre des articles des antennes des Céeidonyies.

“. Dans le mile de la Cecidomyia ribesii M. Brémi n’a trouvé que douze articles.

.... M. Brémi dil que la Cocidomyia grandis (Meigen) a quatre nervures aux jiles, et une la Ceeidomyia formosa (Brénii) en a cinu. 
Les Cécidomyies sont des insectes de très-petite taille et dont la forme généralc rappelle eelle du cousin ordinaire que tout le monde connait. Ces insectes echappent, en raison de leur exiguité, aux yeux du vulgaire, mais il n'en est pas de méme de leurs travaux ou plutót des praduits dont la présence de lcurs larves détermine la formation. Trop sonvent aussi, leur noubre prodigicux suppléc à leur petite taille, el, dans certains cas, ces insectcs en apparence si chchifs, et incapables de rien, nous causent, a l'état de larves, des pertes considérables daus les récoltes du blé, du colza, ete.

Les mảles des Cécidomyies sont en général plus rares que les femelles; ils sont très-ardents, l'accouplement se fail ordinairement dans l'air, les deux sexes sout placés bout à bout et le inàle reticul la femelle au moyen d'appendices particuliers cn forme de pinees el relevés sur le dos en temps ordinaire; une fois le temps de l'accouplement passé, les máles ue tardent pas a mouril. Les femelles, excessivement nombreuses dans plusicur's espéces, ont une tarière ordinairement très-longue, mais qui le plus souvent n'apparait au dehors, qu'au moment de la ponte; une fois fécondées, ees femelles ne paraissent plus s'occuper que du soin de placer leurs $\alpha u f s$, et une fois ce soin accompli, elles meurent rapidement.

C'est, en général, sur les parties verles des végètanx que les Cédydomyies déposent leurs oufs eu lés enfonçant dans le prarenclyyme de la plante, au moyen de lemr longuc tarière rétractile. Ces œufs ne tardent pas à écloré les larves qui en résultent, ,ont apodes, anineies en avant, clles u'ont ni trompe ni mandihules, mais sculement des appendices en forme de levres qui, par leur réunion, constituent une surte de musean au moyen duquel la larve pompe la nourriture, nécessaircment liquide, qu'elle conjurunte au végétal sur Icquel l'ocuf a été pondu. Celle surion constamment excreće sur un mème point, y détermine une affluence du suc nourricier et donne lieu à des excroissanecs, a des boursoufflements et à des déformations dans lesquelles vivent les larres des Cécjdomyies a l'abri des aggents extérieurs. 
Les galles ou exeroissances qui sont produites par les insectes dont il cst ici question, ressembleıt assez à celles que déterıninent aussi les larves des Cynips, de l'ordre des Hyménoptères, sependant elles en différent par moins de régularité et moins de constanec dans leurs formes. Les galles prodnites par les larres de Céeidomyies sont simples et caduques comme le sont celles qui se développent sur les fcuilles du tillenl et qui sont produites par la Ciccidomyia tilliacea (Réaumur), ou persistantes comme celles du lıêtre, produites par la Cccidomyria fagi (IIartig), ou doubles, c'esta-dire visibles des deux còlés de la feuille, eomme eelles de la Cericlomyia polymorpha (Brénii) qui vit sur le tremble, etc., etc.

D'autres espèces de Céeidomyies pondent leurs oufs sur d'autres parties de la plante et y causent des altérations trèsvariées et très-imprortantes á étudier, selon la nature de l'organe qui est atteint et surtout snivant l'inportanec éeonomique qu'on y attaehc. Ainsi par exemple: la Cecidomyia salicina pond ses aufs sur les bourgeons terminaus du saule marceau et fait ainsi avorter les pousses de cet arbrisseau; la Cecidomyia verbasci pond ses $\propto$ fus sur les fleurs du bouillon blane et les empéehe de s'ouvrir; la Cecidomyia tritici pond ses cufs sur l'ovaire du blé et fait dépérir la graine; la Cecidomyia palustris produit le même cffet sur le vulpin des prés; la Cccillomyia nigra pond ses ucufs sur l'ovaire du poirier et les jeunes poires ne tardent pas à pourrir intérieurement puis à tomber sur le sol; la Cecidomyia brassicce pond ses œufs sur l'ovaire du colza el les siliques de la plante ayortent complétement, ou, si elles se développent, elles ue produisent que quelques graines; la Cecidomyia degeeri. pond ses oufs sur l'éeoree des jeunes rameaux du Salix purpurca el $y$ déternine la formation de boursouffures irrégulières ; eufin, par une cxecption singulière, la Cecidomy ia entomophila pond ses aufs sur les insecles que l'on conserve dans les colleetions.

Certaines larves de Céeidomyies vivent en soeiété plus ou unoins nombreuses combue celles du coquelieot, du lotier et du poirier; d'autres virent solitaires comme celles dubitre, du frène et du bouillon blane. Quaud, après un ou plusieurs elauge- 
ments de pcau, ees larves ont pareouru toutes les plases de leur développement, ce qui en général, se fait d'une maniére assez rapide, elles se elıangent en nymphes soit dans leur propre peau comme la Cécidomyie du blé, soit en se filant une coque soyeuse eomme la Céeidomyie du pin. Il y a eependant des larves qui, tout en ayant une filière, ne se filent pas de eoque de soie, soit par suite d'une sorte d'arrêt de développement, soit par toute autre influenee extérieure; M. Brémi a vu qu'une larve de Céeidomyie fileuse cesse la formation de son eoeon, si on l'inquiète pendant ee travail, et qu'elle se transforme néanmoins en nymplie et plus tard en insecte parfait.

La transformation de la larre en nymple a lieu soit dans la galle, l'exeroissanec, ou la cavité, dans laquelle a véeu la larve (Cecidomyia fagi, C. fraxini), soit en delırs de es organes (Cecidomyia nigra, C. Irilici) et dans des endroits propices pour passer l'hiver. C'est ordinairement dans la terre, à la lacine des plantes, ou quelquefois sous les feuilles tombées, sous les écorces, etc., que se fait cette métamorphose. Mais comme ces larves sont apodes et qu'elles ne peuvent avaneer que par un inouvement de reptation qui parait très-pénible à exécuter, pour leur permettıe de faire quelquefois de longs trajets, la nature eıploloie des procédés Irès-divers. A insi, quelques larves (Cecidomyia nigra) attendent que le fruit, dans lequel elles ont réeu, se détaelıe de lui-même de f'arbre, tombe sur le sol, s'ouvre ouse décompose et livre ainsi passageaux prisonnières; d'autres attendent la maturité du fruit (Cecidouy in brassice) et la déhiscence de ses valves pour se laisser tomber sur le sol; d'autres enfin (Cecidomyia triliei, Cecidonyia populi) quittent le grain de blé ou la feuille du peupliè. eu rappruchant leurs deux extréınités, puis en se détendant brus. quement se laneent en l'air, retombent sans se blesser sur le sol sur lequel elles reeommencent la même manœuvire jusqu'à ce qu'elles rencontrent ane place convenable pour y pénétrer et s'y ransformer en nymphes, immédiatement ou plus tard, et attendre, dans eet élat, la saison favorable a leur éclosion.

Les larres de Cécidomyies sont, en général, blanehes ou un 
peu verdàtres; eependant il y en a de grises, de jaunes et de rouges; leurs anneaux sont toujours bien inarqués et leurs formes eylindriques, coniques ou plus ou moins aplaties. Dans les nymplies qui ne se filent pas de corpues, on distingue facilement sous leur dernière peau, les ailes, les antenues et les pattes de l'insecte parfait. Dans quelques espéces, peut-être mème dans toutes, la tête et le thorax présentent des tubereules en forme de petites corues. D'après les réeentes observations de MM. Amblare et Laboulbène, ces cornieules servent probablement d'organes respiratoires aux nymplıes qui en sont pourvues.

Peu de Céeidomyies n'ont qn'une génération annuelle, beaucoup en ont deux, quelques-unes (Cecidomyia strobilana) en ont trois et même quatre. Il arrive aussi que l'on trouve a la fois, dans une galle liabitée par ces insectes, des larves prêtes à se transformer en nymphes, tandis que d'autres commeneent seulement leur évolution.

A l'état parfait, les Céeidomyies n'ont qu'une existenee de courte durée, quelques lieures seuleuent pour certaines espèces; pendant ee temps on les roit souvent réunies en groupes de plusieurs eentaines et voltiger comme fout les cousins pendaut les chaudes soirées d'été. C'est ordinairement à l'ombre, près des buissons ou des taillis, dans les prés et les lieux bat, humides et ehauds, qu'on les trouve le plus souvent à l'état parfait D'après ce qui préecde, on comprend pourquoi l'on ne reneontre ordinairement que des individus isolés qui, la plupart du temps, sont des femelles oeeupées de la poute de leurs aufs; e'est ce qui explique les contradictions qui existent dans les auteurs, relativement i la rareté proportionnelle des deux sexes et aussi en ce qui eomeerne les individus appartenaut à quelques espèces. Quniqu'en dise Meigen, éest plutót pendant l'été qu'au printemps qu’ou reneoutre le plus grand nombre des espèces à l’état parfait; leur itude est du reste assez difficile en raison de la petilesse des individus et strelout de leur fragilite, de la variation qui s'opère dans leurs couleurs après leur mort, ele.

Le nombre des espéces de Céeidomyies déjà déerites par les 
autcurs est très-considérable, mais beaucoup sont encore a décourrir, inême en France; et comme on en connaît à peine quelques espèces de l'Amérique du Nord et qu'on n'en connait aucunc des autres continenls, on cst frappé de la làche qu'aura à accomplir celui qui voudra entreprendre la monograplie de ces insectes intéressants.

102. Cвcidomyra migan (Meigen).

Macquart; Dipleres, suiles i Buffon, tome 1, page 161 .

Synonymic: Cécidomyie noire; -- Dic Schwartz Birngallemicke (en allemand).

Longueur: deux millimètres. Corps noir; thorax postérieurement d'un eendré elangeant, ì bandes noires; écusson gris; borts des segments ile l'abilomen, rougeàtre; balaueiers jaunảıres; ailes lorunàtres.

Cette description est copiéc daus M. Macquart, qui ne donne, daus l'ouvrage cité plus haut, aucun autıe reuseignement, tandis que, dans un aulre ouvrage du même autenr (Des arbres cl des arbrisseaux d'Europe, page 456), celte espèce de Cícillomyie cst indiquée conme virant (a l'état de larre sans doute) dans les chatons du bouleau, et plus loin (page 448) commc vivant sur le cliarme.

Cependant sous le nom de Cecidomyia migra (Meigeu), M. Nordlinger donne, d'après Schnidberger, la description d'un inscete dont il est impossible* de méconnaitre l'analogie, sinon l'identite, arec celui dont il est ici question.

Il est permis de supposer que Schmidberger a fail sa descrip-

- Voici les earactères que $\mathrm{M}$. Nordlinger assigne à cet insecte: Lnngueur : un à un millimètre et deıni. Antennes d'un brun nuir; corselet noir, cendré à la parlie pustérieure, une ligne noire sur le dos; censson grisâtre ; partie postérieure du corps, noiråtre, garnic d'incisions jannes; tariẻre de la femelle oussi Inngue que lout le corps, d'un jaune sale; jambes d'un gris pâle; ailes frales, ayont trnis nervures effacées. 
lion sur deux individus rivants qu'il avait à sit disposition, les ayant obtenus d'ćclosion, tandis que Maequart s'est serri d'inseetes morts, conservés dans sa collection. Quoiqu'il en soil, on a de la peine à eomprend’e la différenee, si tranelsée d’ailleurs, dans les habitudes que ces deux entomologistes attribuent à la Cecidomyra nigra (Meigen). Ces observations préliminaires terIninées; je donne, Ionjours d'après Selunidberger, l'histuire de l'insecte dont ce dernier auteur a suivi les métamorploses, en la complétant toutefois de unes propres observations.

Au moment où les pétales des fleurs du poirier eommencent a se montrer entre les sépales du ralice, c’est-à-dire vers le milien du mois (l'avril, cet inserte (la Cecidomyia nigra) se plaee perpendieulairement sur la fleur, perce les pétales, et, avee sa longue tarière, introduit un ou plusieurs neufs sur les étamines de la.fleur eneore ferméc. Selımidberger dit eependant ayoir ru ¡uelquefois cette Cécidomyie pondre ses eufs sur le caliee de la fleur. Ces oufs, réunis en las, all nombre de quinze á vingt, sont blanchatres, un peu transparents, allongés et pointus à un bout. L'éclosion se fait très-rapidement; Schmidberger a déjả trouvé, le quatriène jour après la ponte, de petites larves se frayant un passage pour gagner l'ovaire ou on les troure eomplétement installées avant l'épanouissement de la fleur; de eette façon elles évitent le contaet des rayons du soleil qui leur sont trés-nuisibles, anssi bien que la pluie qui leur est également muisible, bien qu'à un moindre degré. Arivées au centre de l'ovaire, elles continuent a en manger l'intérieur'; après quelques jours, elles ont atteint tout leur développement et n'altendent plus qu'une occasion favorable pour dèloger. Si la pluie survieut, les jeunes poires pourvissent, se fendillent e: laissent sorti. les jeunes larves qui n'ont qu'à se laisser tomlier sur le sol, 'ce qu'elles font sans se blesser, et elles s'enfoncent dans la terre oi elles se transforment en nymphes; s'il n'y a pas de pluie, efles attendent que la petite poire tombe naturellement; qu'elle se fendille et sé pourrisse par son eontaet avee le sol humide, dè mànière á.permettre aux jeunes larves de sortir 
facilemen', e'est lis le eas le plus ordinaire; quelquefois cnfin il arrive ou que les poires ne tombent pas, on qu'elles ne s'altìrent pas, et que cependant elles ne se dessèchent pas eomplétement, alors les jcunes larves y restent emprisonnées, s'y transformcut en nymphes mais ne donuent d'insectes parfaits que si plus tard il se produit des altérations qui leur onvreut une issue au dehors; car les Cécidomyies sont encore bien moins que leurs larves, en état de se frayer un passage à travers les parois, même trèsramollies, des jeunes puirettcs. Enfin, si le temps est sec, il arrive que les poires qui contiennent des larves de Cécidomyies, se dessèclıent complétement ct que cette séeheresse, jointe à la coutraction que subissent les poirettes, font périr toules les larves contenues dans ces jeunes fruits. Cette dcrnière circonstanee a surtont étć très-facile à observer, en 1858, dans certaius jardins le Plantières et de Vallières, où, dès la fin de mai, le Doyenné d'liver avait la moitié de ses fruits dessécliés et où l'on trouvait dans l'intérieur, durci et raccorni, les pctites larves mortes et desséchées de l'inseete que je rapporte, avee doute cepen. daut, à la Cecidomyia nigra, car je ne l’ai pu obtenir d’éclosion.

En 1831, dit Sehnidberger, la ponte ayant été faite du 15 au 18 avril, les larves avaient subi toutcs leurs tranformations du 14 au 20 mai; en 1832, l'évolution ne fut terminćc que du 20. au 26 mai, cn raison de la température froide du printemps de eette année. Il faut done quatre à cinq semaines pour que les larves de eette Cécidomyie aient atteint l'àge anquel elles cessent de prendre de la nourriture. En novembre, Srlimidberger trouva des larres déjà transformées en nymphes et serrées les uncs eontre les autres; elles étaient d'une couleur jaune-foncé et on distinguait parfaitement les pattes et les antennes de la future Cécidomyie.

C'est évidemment au printetnps que l'éelosion a lieu. L'autcur auquel j'emprunte ces détails les a vues éclore cliez lui en décembre, en janvier et en février; mais ees éclosions préeoces, déterminées, sans doute, par la température plus éievée du lieu ou se trouvaient les laryes, n'ont produit que des inseetes qui 
n'ont pas roulu sucer la nourriture qu'ou leur donnait et qui tous ont péri rapidement sans s'aceoupler.

- La Ciecidomyiu nigra décrite par Maequart existc dans notre département oú elle n'est mème pas très-rare an muis d'arril. Durant le mois de mai, on troure en abondanee dans certaines années (1857 et 1858) et dans quelques jardius de Plantières, de Vallières et de Saint-Julien, une grande quantité de poirettes provenant presque toutes d'arbres élevés ell quenouilles, et dans l'intérieur desquelles ae troment de petites larves, en nombre très-rariable; car, dans certaines poires, on n'en eompte que quninze a ringt, landis que dans d'autres, en 1858 surtout, j'en ai trouvé jusqu’à soixante-dix et même quatre-vingts. Ces larves sout jaunes, Iongues d'environ dcux millimetres el se changent en nymplies sans se filer de coque.

Ordinairement l'intérieur de la jeune poirette est mangé d'uue nanière assez régulière, c'est-à-dire que les larves, quelqu'cn soit le nombre, seticnnent à igale distance de l'épitlerme, mais quelquefois aussi elles altaquent celui-ci, alors le fruit se déforme, se courbe, "t si l'épiderme est tout à fait enlevé, les larves ainsi mises à jour, périssent arant leur complet développeınent Je n'ai pu, malgré plusieurs tentatives, obtenir l’éclusion de la Cícidomyie à laquelle ces larves appartiennent, et ne puis, par consíquent, assurer qu'elles soient bien celles dont Sehmidberger a suivi les métanorploses et qu'il rapporte à la Cecidomyia nigra de Meigen, et eela avec d'autant plus de doute que cet auteur ne donne pas la descriptioñ de la larve.

Le moyen, non d'éviter cet insecte, mais d'en diminuer le nombie, consiste tout simplement à rauasser au mois de mai, toutes les jeunes poires tombées, toutes eelles qui sont tachées et qui pendent eneore à l'arbre et à les écraser ou à les jeter au feu. 
102. araidom ria Frarcoza (Nordlinger).

Nordlinger : Die Kleine feinde, der Landwirtschaft, page 527.

Synonymie: Die Birne gallemuicke (en allemand).

Longueur, saus les autennes : deux millimètres. Antennes à artieles allongés, visiblement séparés et velus, d'un gris noiràtre; tète contractée, en forme de massue; dos et jambes d'un gris noiratre; ail's ayant les nervures ordinaires des autres Céeidomyies, d'un gris obseur, avec un duvet gris; balaneiers blanes; parlies postéricures du corps, parties postérieures de la poitrine de eouleur jaunc-brun sale dans les individus morts, l'un rouge vif dans les individus vivants.

Cette diagnose, dit M. Nordlinger, ne convient à aueune des espèces décrites par Meigen*, pour éviter toute confusion avec une autre espèce dont parle Ratzeburg et qui se trouve décrite par Bouché, sous le nom de Cecidomyia pyri, je lui ai donné le nom de Pyricola. Suivant cet auteur, cet insecte (la Cecidomyia prricola) a ćté obtenu d'éclosion avcc des larves contenues dans de jeunes poires avortées, et dans lesquelles se trouraient également des larves de la Sciara pyri; c'est cc qui me fail supposer qu'ellc a des habitudes analogues à eelles que nous avons fait connaitre pour la $C$. nigrn.

103. czcrDomTIA PTRI (Bouché).

Nordlinger; Die Kleine feinde der Laudwirschaft, page 527.

A propos de l'insecte précédent, M. Nordlinger cite la Cecidomyia pyri et il dit que eetle espèee a élé décritc par Bouché. Je ne possède que deux ouvrages de ce dernier autcur (Naturgeschichte der Schidllichen und Nützlichen Garden Insecten und, etc., Berlin 1833, et Natârgeschichte der Insecten beson-

- J'ajouterai que je ne lui trouve non plus aucun rapport avec les espèces déeriles par Macquars. 
ders in hinsich ihrer ersten Zuftünde, etc., Berlin 1834, Erste Liefernng), et dans aucun d'eux je ne trouve déerile ou mentionnée la Cecidomyia pyri.

Ratzeburg ne fait que eiter eet insecte, et Macquart, sans en dunner la deseription daus son Histoire des Diptères (suites à Bu(fon), eite celle Cécidomyie dans son Catalogue des arbres et arbrisseaux d'Europe, ou il ajoute : "Elle est quelquefois trèsnuisible au poirier en racoquillant l'extrétnité des jcunes tiges et en occasionnant la courbure du trone. \& D'après cela, il est probable que la Céeidonyie dont ce dernier auteur a voulu parler, pond ses œufs sur les bourgeons terminaux des jennes pousses du poirier, que les larves qui en éelosent déterminent l'avortement des feuilles gui le composent, que celles-ci restent à l'élat rudimentairc et qu'elles forment un abri daus lequel les jeunes larves achèvent leur évolution ainsi que cela se fait chez la Cecidonyia salicina. Malgré mes reeherches el celles de plusieurs jardiniers clévoués à mes travaux, je n’ai pu rencoulrer, sur le Poirier, de larves ayan dans leurs habitudes quelque rapport avee celles de la Cecidomyia pýri.

\section{IVII. SCIARA (Meigen)*.}

Macquart. Diptères, suites $a$ Buffon, lome 1, page 117.

Tète sphérique; trompe eourte; palpes de trois articles dislincts; yeux réniformes, rapprochés l'un de l'aulre, plaeés sur le vertex; antennes gréles, courtes, filiformes, les deux premier's articles presque cylindriques, séparés des autres quti sont peu distinets; ailes grandes, finement velues, arrondies, avee les eellules basilaires et marginales ćlroites; abdomen cylindrique dans les máles, pointu dans les femelles; jambes longues et minces, les euisses sillonnées an cóte interne, et les libias bi-épincux à l'extrémité.

- Synonymie: Molonnus (Latreille); - Tipula (Linnë). 
Le genre Sciara est coniposé d'un assez grand nombre d'espèces; toutes sont de petite taille et, en général, ınal décrites et ınal définies. Ces insectes voisins des Céeidomyics en diffèrent par leurs antennes qui ont seize articles et pas de poils disposés en verticilles. Les ailes sont le plus ordinairement de couleur sounbrę Les femelles ont aussi une tarière rétractile au noyen de laçucllc elles enfoneent leur's aufs daus différentes parties dcs végélanx ou dans le terreau qui résulte de la décomposilion des tissus lignenx.

Úne espèce, la Sciara thoma (Fabricius), vit en Suède, où ellc préseute le singulier phèuomène d'émigirations en quantité considérable et opérćes par des larres apodes réunies et comme agglutinées enscmble, formant un rubau de $10^{\circ}$ a 15 eentimètres de largeur, de deux à trois d'ćpaisseur et atteignant quelquefois la longueur de 10 a 12 mètres; d'aprés les petites dimensions des individns qui composent ces colonnes serpcutiformes, on peut évaluer lenr nombre à plusicurs millions.

Aucunc espèce de cciara n’a cncore éré obscrvée dans Ic déparıement de la Moselle, bicn certainement il y en existe, mais clles u'ont pas encore altiré l'attention, an moins à l'état parfait, des naturalistes qui ont exploré nos eontrées; on rencontre sonvent à l'état de larve celle d'une espćce de ce genre dans les poirctles calebassées, commc le sont eelles dans lesquelles vivent les larves de la Cecidonyia nigra.

104. SCIARA PYMI (Schnidberger).

Nordlinger; Die Kleine feinule der Landwirschafl, page 533.

Synonymie: Die Birn muckchen (en allemand).

Longueur: deux millimitres; largeur : un peu moitrs d'un millimètre. Téted'un brun noir ; anteunes et corselet noirs; articles des antenues au nombre de seize, eylindriques, les deux de la base plus gros que les autres; balanciers en forme de massuc et de couleur blanchıitre; partie postérieure de l'abdomen de couleur plombic, avee sept anneanx soyeux; 
jambes longues et minces. Le noale porte à l'extrémité de l'abdonien, des pinces formées de deux articles en massuc et tottles couvertes d'un duvet fin.

Cette deseription, dit M. Nordlinger, se rapporte assez bien alux individus que j'ai élevés; mais, c'el auteur ajoute que dans ceux qui sont desséchés, les antennes sont d'un gris noirätre et les jambes d'un jaune plus ou moins elair.

D'après Selımidberger, on trouve sur les fleurs de poirier, non encore épauouies, des femelles qui y enfoncent leurs nufs. Ceux-ei produisent des larves qui deseendent dans l'endoearpe, mangent tout ee qui s'y trouve et vecasionneut ainsi le dépérissement précoce du fruit. La jeune poire ainsi attaquèe, s'allonge, se contracte par le milieu et produit ces poirertes ealebassées dont nous avons déjà parlè à propos de la Cecidomyia nigra.

Les larves qui, selon Sehmidberger, appartiennent à la Sciara pyri, sont longues de trois millimètres et larges de uu nillim.; elles sont apodes, ont dix anneaux, la tête pointue et deux taehes noires sur le devant de celle ei. Elles arriveut à l'ètat adulte vers la fiu du noois de ıuai, alors elles abandonnent la jeuve poire, s'enfoncent dans la terre vil elles se transforment en nymphes dans une pelite cellule voutice. Quelquefois elles restent daus le fruit jusqu'en juill, sans être transformées en nymphes, et souvent, au commencement de l'hiver, on en reneontre encore quelques-unes qui sont malades et qui ne produisent pas de "ymphes. L'ćclosion de l'insecte parfait a ordinairement lieu, en eaptivité, du 15 aoủt au 15 septembre, mais Selunidberger ne dit pas à quelle époque elle a lieu à l'état libre.

En rapprochant ce qui précède de ce qqui a été dit à propos des larves de la Cccidomyia pyri on voit que, sauf la taille qui est diflérente, on peut eroire qu'il s'agit du même insecte. En lisant ce que Sehnuidberger a écrit à propos des Cecidomyia et des Sciura qui vivent sur les poiriers, on ne saurait cependant avoir de duutes sur ee yu'il en rapporte; car il a obtenu d'éclosion et avec les poireltes récoltées par lui d'abord, ell aoùt et 
en septembre, des Sciara prri; plus tard, et daus les mémes vases, des Cocidomyia nigru ou lout au moins l'espèce qu'il y rapporte. Cependaut, en raison des mours tout a fait differentes que Maequarl, donl persoune d'ailleurs ne saurait meltre en doute l'autorité èn ce quí concerue les Diptères, altribue à sá Cecillomyia nigra, "jui vit sur les chatous du elarine el du bouleau, des doutes que m'a comnnuiqués M. Bigol, autre autorité diptérologique et qui ue comuait pas de Sriara pyri, il est permis de peuser que des espéces roisines ont été confondues. De tout cela il résulte pour moi, qu'il y a ici, comme puur l'Allantus oethiops, de nombreux faits nuureaux a observer el que toutes ces incertitudes ne porront ètre levées que parr de nornbreuseśs expériences et l'élude des inseetes oblenus d'éelosions.

\section{SCIARA SCHMIDBgRGEI (Kollar).}

Nordlinger; Die Kleine feinde der Landurirschafı, yage 531.

\section{Synonymie: Sciare pyri mujor (Schmillherger).}

Longueur : Deux millimetres; largetur : un millimètre; ces dimensions sont eelle's de la femclle, le unále est plus minece el plus eour. Antennes noiritres et plus courtes que le corps; tète noire, corselet moir et brillant; palpes d'un gris-cendré ; abilomen noir dans le mále; brunâtre, avee des anneaux noirs, dans la fenelle; pattes eendrées, tarses noirs.

Selon Sehmidberger, celte espéce de Sciara a des mocurs semblables a celles que nous arous fail connaitre pour les Cecidomyia nigra et Sciura pyri. Cet auteur a ublenu, au mois de juillet, l'éelosion de eet insecte avee des larves qui vivaient aussi dans l'intérieur de poires nouvellement lormées.

I. Nordlinger dit '[u'il ue saurait admeltre que toutes le especes de Sciara et de Cecidonyia qui se rencontrent au prinlcmps sur les fleurs du poirier, y aillent toutes pour y déposer des auts. Il pense que beaueoup d'entre elles ue s'y tendent que pour y sucer le sue mielleux qui s'y truuve el qui constitue, comme on 
le sait, la nourriture de ces insectes à l'état parfait. Ces réflexions de M. Nordlinger viennent encorc eonfirancr les doutts que j'ai précédemment émis relativemcnt à la nomcnelature des espèces entomologiques qui eorrespondeut aux larves b!anclses, jaunes ou rougeátres et de taille variable qui se trouvent dans les jeuncs poircs*.

En terminant ce qui est rclatif aus insectes Diptères qui ont f́té jusqu'ici indiqués conımc étan! nuisibles au poirier, je dois faire observer qu'un bon nombre d'insectes Hyménoplères appartcuant aux genres Eulophus, IVisocampa, Plalygaster, etc., vivent en parasites sur les larves des insectes appartenaut particulièrcment a la tribu des Tipulaires. J'ajoutcrai encorc qu'outre les Diptires appartenant aux genres Cecidlomyin et Sciara, il y en a cncore dautres appartenant aux geurcs Bibio, Syrphus, ctc., qui vivent sur les arbres, mais sur lesqucls on ne possede aucun détail particulier. On sait sculenent que celles le ces especes qui appatlennent aut genre Bibio, pouraicnt bien ètre des cspèecs nuisibles aux plantes, l'une d'clles porte urêue le nou de Hirtea prri (Fabricius) ou Bibio Johannis (Meigen). Quant a celles qui appartiennent au geme Syrphus, on sait qu'elles ne vivent que de pucerons dont elles font une

C'est à l'une des espèces que nous venuns d'examiner quil faut rapporter l'article publić par M. Félix Arrame, dans la Presse du 28 novenbre 1857. Suivant eet autcur, on doit altribuer le manque de la recolte de poires de cette année i la prodigieuse inultiplication d'une espèce le larve yii les fait ealcbasser, pourrir cl tomber ivant leur maturité. seulement cet auteur coumet la faute énorme le dire que ees larves dounont naissonce à un papillon. Cel auteur ajoute encore une naïveté dont il fait bien certui. nement à tort remonter lit responsabilite i M. Blanelıard; selou lui, eu effet, cet entouologiste consulté sur les moyens i employer pour se préserver i l'avenir des ravages de cet insecte, aurait conseille de ramasser la terre de dessow. les arbres, a trois ou quatre centimedres d'ejuisseur el la transporler hors dh jurdin! Les lárves de Cícidoungies s'enfungaut souvent plus frofondément, il aurait follu dire huir ou dix centimètres de terre ì enlever. ce moven ust bon, sans doute, mais est-il praticable?? 
énorme cousommation, ainsi que nuus l'avons dit à l'histoire de ccs Hoinoptères.

L'histoire de tous les insectes dont je viens de parler, devrait comme eclle des Coccinelles, qui termine la prenicère partie des insectes du poirier, étre comprise daus ce travail, mais en présence du silence de presque tous les auteurs et du pétit nombre d'observations qu'il m'a été donué de faire sur eux, je n'ai pas osé l'entrcprendre en ce moment et j'ajourue la publication de ce qui les concerne jusqu'à la publication de la quattrième partie de l'histoire des insectes qui vircnt sur le poirier.

Fia de la ze Partie des Issectes nuisibles au Poirier. 


\section{TABLE ALPHABETIQUE}

\section{DES NOM DES INSICTES CITES}

Dans celle seconde parlie.

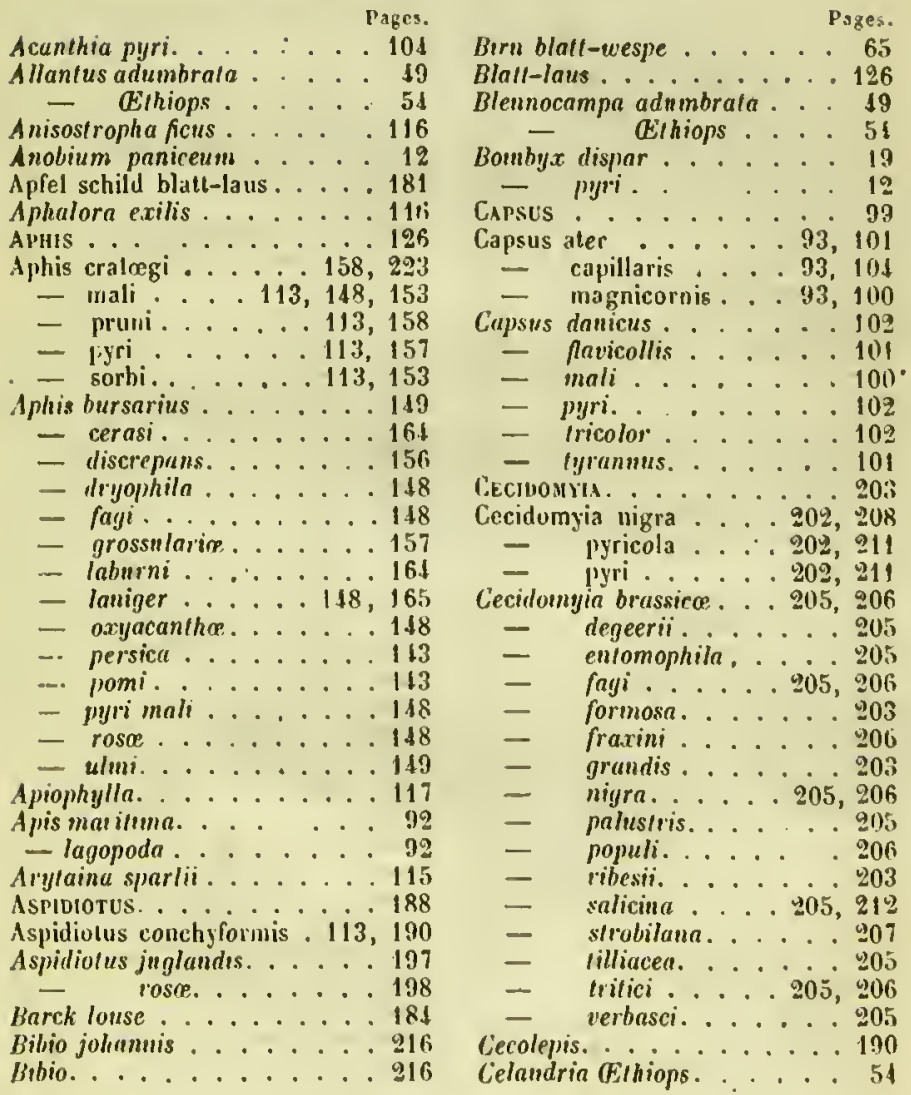


Gephaleiu nemorum . . . . 68 sylvalica. . . . 68

CePHUS

Cephus compressus . . \$1, 59

Cephus ubdoninalis . . . 60

- pygmous . . . . . 59

Chenses. . . ...... 19:3

Chermes ficus . . . . 116

- laricis ....... 148

- pyri $\ldots \ldots 117,183$

- pyri conimunis. . . 118

$=$ quercus. ...... 115

Clirysopa reticulata . . . . . . 36

Gigale. . . . . . . 22

Gimex aler. . . . . . 101

- baccuimm . . . . 98

- bicolor....... 95

- dissimilis. . . . . 96, 99

- Alavomuculalus. . .. 100

- juniperimun .... 97

- nubilosa ....... 94

- prasina ....... Ј6

- prosinus. . . . . 98

- pyri....... 102

- semifauus. . . 101

- tricolor . . . . 102

- verbasci... 98

Goccinelles. . . . 2 217

Coccophagus ...... 183

Coccus........ 183, 188

Coccus adonidum . . . . 180

- lauri....... 180

- mali....... 181

- ncrii....... 180

- pyrus malus. . . . 1!5

Coccus mali . . . . 113, 181

Cachenille........ 175

Cochenille du Nopul . . . . . 179

- de Pologne. . . . 179

- du pommier.... 181

- en ecuilles de moules 190

Conoceplulus miridissimus. . . " 1

Courtillière . . . 25, 6

Crabro. .......... 111

Criquet . . . . . y

Cyonels . . . . . . 93

Cydnus bicolor . . . . . . !3

DEcticus. . . . . .

Heclicus verrucivorus . 14,

Iliuspis lineuris ....... 188

Dyclionola pyri. . . . 161

Ephedrus ........ 101

Eird-Krebs . . . . . 96

Ert-Wolff . . . . . . 96

Eriocampa adumbra!a . . . 49
Eryosoma pyri...... 170

Eulophus......... 216

Euphylleura oloce . . 116, 124

Fausses chenilles . . . . 44

Faux puccron ....... 114

Flor-fiegen . . . . 33, 35

Furda formicaria. . . . . 149

Forficula ...... 11

Forficula auricularia. . . . 14

Porficule . . 15, 16

Fonmica. . . . . 69

Formica cunicularia.... $\$ 1,80$

- flava.... 41, i9

- fusca... . 41, 79

- rufa. . . . 11.77

Fourchelle. . . . . 16

Fourmi brume à corselel faure. 77

- des bois. . .... . 77

- fauve..... . 77

- jaunc...... . 79

- mincuse. . . . . 80

- noire cendrée .'. . . 79

Firelon........ 85

Gallinscc1c ........ 175

Galliusecle en coquille. . . 190

Grand suceur de poires . . . 120

Grunde sauterelle. . . . 21

GRTLLOTALPA . . . . . $2 \%$

Gryllolalpa nulgaris. . 14, 26

Griflus yryllo ialpa. . . . 26

- verrucivorus. . . . 24

- viridissimus. . . . 21

Guêpe. . . 81, 89

Guepre allcmande .... 87

- frelon..... 85

- gernanique ... 86

- sylvestre. . . . 88

- vulgaire. . . . . 86

Halmuespe .. . . . . . 59

Hèmérobe . . . . . . 33

Herwerobe perle . . . . . 35

HEMEROBHUS . . . . . 33

llemerobius Perla. . . . 26

Chrysops. . . . . 26

Hirlea pyri........ 216

Hornisse. . . . . . . 85

Ilyalopterus pruni . . . . 158

Kermes ......... 183

liermes pyri........ 183

Lecaniuat . . . . . 183

Lecaniun pyri .... 113, 183

lacyrunit perec-oreille . . . 16

Lion des pucerons. . . 33, 35

Livia juncorum ...... 115

Linilla ulicis. ....... 115 
Locusta. . . . . . 20, 23

Locustu veriucivora. . . . 24

Locusta viridissima . . 14, 21

LYDA . . . . . . . 63

Lyda circumcincta, . . . . 65

- clyprata. . . . . 65

- flaviventris. . . . . 65

- nemorum... . . 68

Lyda pyri . . . 41, 65, 68

- sylvatica. . . 41,68

Lygcus ......... 142

Lygueus flavicollis . . . . 101

tyrannus.... 101

Lymacodes . . . . . . 141

Megachile . . . 9 90

Megachile du poirier. . . . 92

Megachile pyrina . . 4 4, 92

Misocampa.......216

Mouches a scie . . . . . . 42

Myzoxilus mali ....... 165

Nemutus Ethiops . . . . 54

Noctua tridens . . . . 12

Ohrwurm ...... . 16

Ophion mercator . . 67

Panphilius sylvaticus. . . . 68

Paracletus cimiciformis. . . . 149

Penpilzus....... 168

Pemphigus americanus. . . . 180 bursarius. ... 149

Pempligus pyri ....113, 157

Pempliredon..... . . 141

Pentatoma . . 93, 95

Pentatoma Bihamata. . . 98

- confusa..... 98

- depressa. . . . 98

- dissemulable. . . 96

- eringii. . . . . 98

- juniperina . . . . 96

- viyricornis . . . . 98

- wilkinsonii . . . 98

Pentatonis baccarum . . 93, 98

- dissimilis. . . 93,96

- juniperuni. . . 93, 97

- prasina . . 9 93, 98

Perce-oreille. . . . . 15, 16

Petidia. . . . . 97

Pliysapus vulgatissima . . 39

Phytocoris capillaris. . . . 102

- clavicornis . . . 101

_ magnicornis. . . 100

- mali....... 100

Pliylloxeura coccinea. . . . 148

Piggudus. . . . . . 102

Pimpla slercorator. . . . . 63

Platiguster. . . . . . 216
Polistes. . . . . . . 89

Poliste. . . . . . 90

Polistus gallicus . . 41,80

Poud'ecorces. ........ 184

Prunifex.......... 176

PsillaA. . . . . . 114

Psylla alni . . 113, 113, 117

- apiophila . . 113, 119

- aurantiaca. . . 113,124

- pyri . . 113,113, 161

- pyricola. . . 113,119

-- pyrisuga . 113, 113, 120

- rubra.... 113,123

Psyllo luxi..........115

- erica...... 115

- exilis........ 110

-- ficus ........ 115

- olce......... 116

- pyri..... 120, 122

- similis ....... 116

- spartii....... 115

Psylle dc l'aulne....... 117

- du poirier. ...... 117

- ronge........ 123

Pucerou ......... 126

Puceron brun-cafe. . . . 153

- du pommier...... 148

- du prunier..... 158

- du sorbier..... 153

- laniger...... 165

Punaise à deux coulcurs . . 9 4

- i fraise untique. . 104

- bine di antennes et bords panachés . . 98

- des bois....... 176

- du poirier. . . . 104

- noirecítaches blanch. 94

- tiyre..... 101

- vertc. ..... 96

verte du cliou. . . 98

Reit Krote . . . . . . . 26

Rhinocoln erica . . . . . 115

Rhizobius pyri. . . . . 149

Nichmaûs . . . . . 26

Rinden-laus........ 164

Rothe ameise . . . . . 77

Sauterelle à coutelas. . . . . 21

Suterelle a subre. . . . . 24

verte .. . . 21

SchizoneuaA. .... 148, 164

Schizoweura lanigera.... 113

Schizoneura lanuginosa ... 165

- tremula ...... 165

- Rcuumuri.... 165

Schleeg wurm ...... 49 
SсIAR4........., 212

Seisra pyri..... 202, 213 - Schnidbersei. . 202, 215

Sciaru Thoma.. . . . . . 213

Schwariz birn galle mucke. . . 208

Selondria olro...... . 49

- Gilhiops. . . . 54

Sirex compressus . . . . . 60

Silophilus granarius . . . . 9, 12

Silinch fliege . . . . . . 35

Syrphus ......... 142

Taupe grillon. . . . . 26

TeNThIEDO. . . . . 41, 59,63

Tenihredo cerasi . . . . 52, 54 - fulvipes. .... 68

- hamorrhoüdalis. . 65

- prolongala. . . $\quad 60$

Tenthredo syluatica. . . . 68

Guthedo sumbrata . ¿ $41,50,54$

Telraneura ulmi. ..... 149

The apple boot hight. . . . 170

TurIPS. . . . . . . 37

Thrips des serres . . . . . . 40 - physapus. . . . . 39

Thrips vulgatissima . . . . 37, 39

- Homorroidalis . . 37. 40

Tigre......... 104

Tigre sur bois ....... 190
Tigre sur écorce....... 176

Tinea cereolella. . . . . . 9

- cognatella. . . . . . 66

Tivas. . . . . . . . 103

Tingis appendiceus. . . . . 104

Tingis pyri . . . 12, 104, 93

Tipuls ........ . 903

Trachelus compressus . . . . 60

Trana troglodyles ...... 149

Triozo urlica........ 115

Trilomegas. . . . . . . 94

Trilomegas bicolor. . . . . . 49

Vacuno coccinea. ..... 148

- digopliyla.... . 148

Ver limace. . . . . . . . . 54

Vespa. . . . . . . 81, 89

Vespa crabo. . . . 41,85

- Germanica. . . 41,86

- Sylvestris . . 41, 88

- vulgaris... . 41,87

Yespa gallica . . . . . . 90

- valgaris. . . . . . 86

Wold blall-wespe. . . . . . 68

Wort-bil. . . . . . . . 25

Werle. . . . . . . . 26

Wespe. . . . . . . . 81

Wollig opfel bloll-lous. . . . 165

IVoll-laus........ 168

Wierre........ 26 


\section{ERRATA.}

Page 20, ligne 19, au lieu de longues, lisez longs.

- 49, dernière ligne, au lieu de quarante-cing millim., lisez quatre à cing millimètres.

- 49, ligne 27, au lieu de 38 , lisez 60 .

- 53, - 23, au lien de Tenthédites, lisez Tenthrédites.

- 54, - 9, au lieu de 59 , lisez 61 .

- $59,-1$, au lieu de $X X X V I I$, , lisez $X X X I X$.

- 60, - 4, au lieu de 60, lisez 62.

- 65, - 28, an lien de $X X X I X$, lisez $X L$.

- 76, - 10, au lieu de de manière ì ce que, lisez de manière que.

- 86, - 15, au lien de Marquart, lisez Macquart.

- 113, entre les lignes 18 el 19, ajoute\% Cartasi (Kaltembach).

- 100, ligne 29, au lieu de Phytocooris, lisez Plylocoris.

- 102, - 14, au lieu de La Capsus, lisez le Capsns.

- 109, - 4, au lieu de suffit, lisez suffise.

- 110, - 8, au lieu de qu'ils le sont aux, lisez qu'elle l'est anx.

- 116, - 23, an lieu de Euphillura, lisez Eupluyllura.

- 118, - 13 et 28, au lieu de la Chermes, lisez. Ic Chermes.

- 121, - 4, an lien de ne soil pas, lisez n'est pas.

- 125, - 23, au lieu de devant, lisez avant.

- 128, - 21, au lieu de Jermaphroditisme, lisez Hermaphrodiane. 




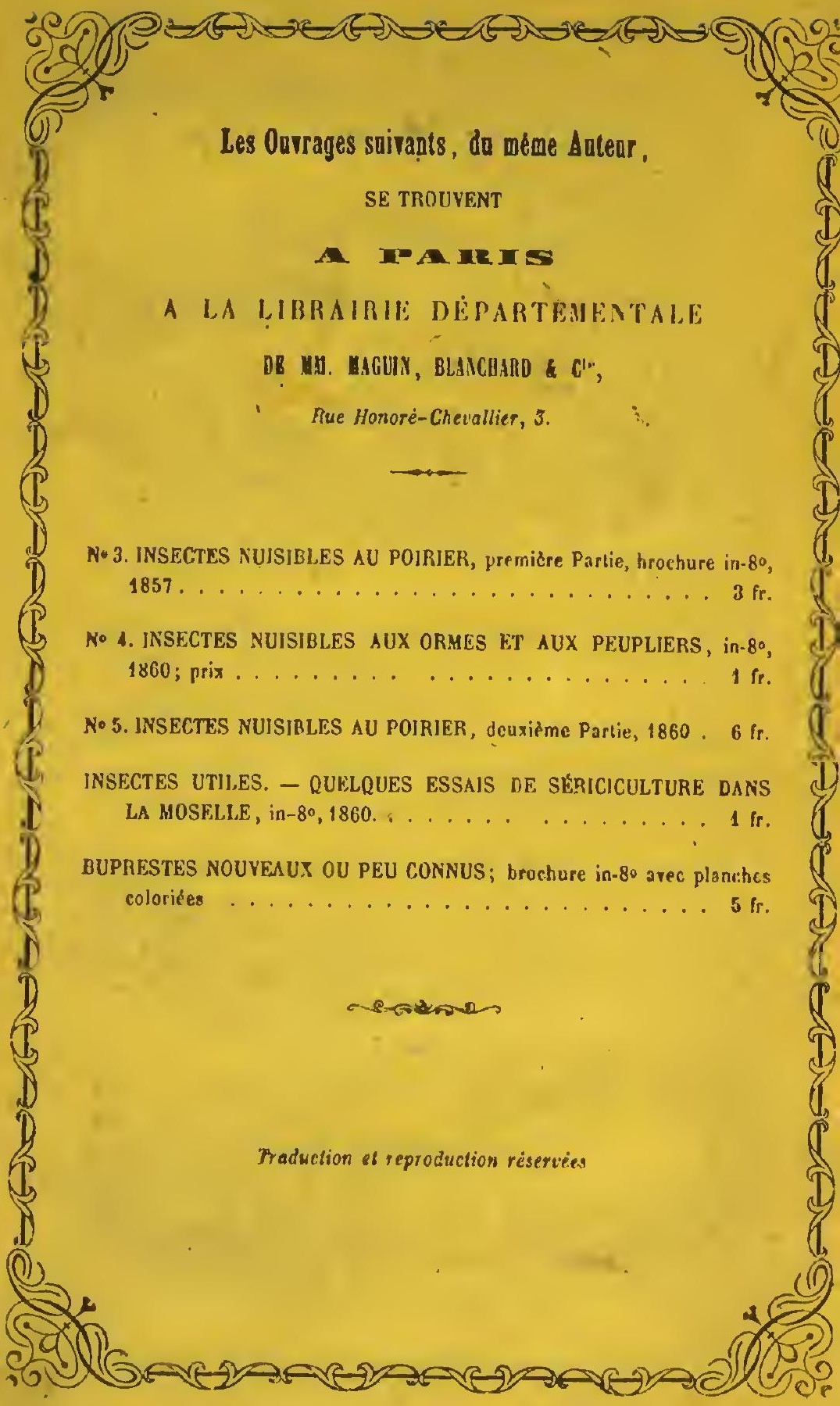



- $\quad$ : 